Prepared in cooperation with the Ohio Water Development Authority

\title{
Methods for Estimating Selected Low-Flow Statistics and Development of Annual Flow-Duration Statistics for Ohio
}

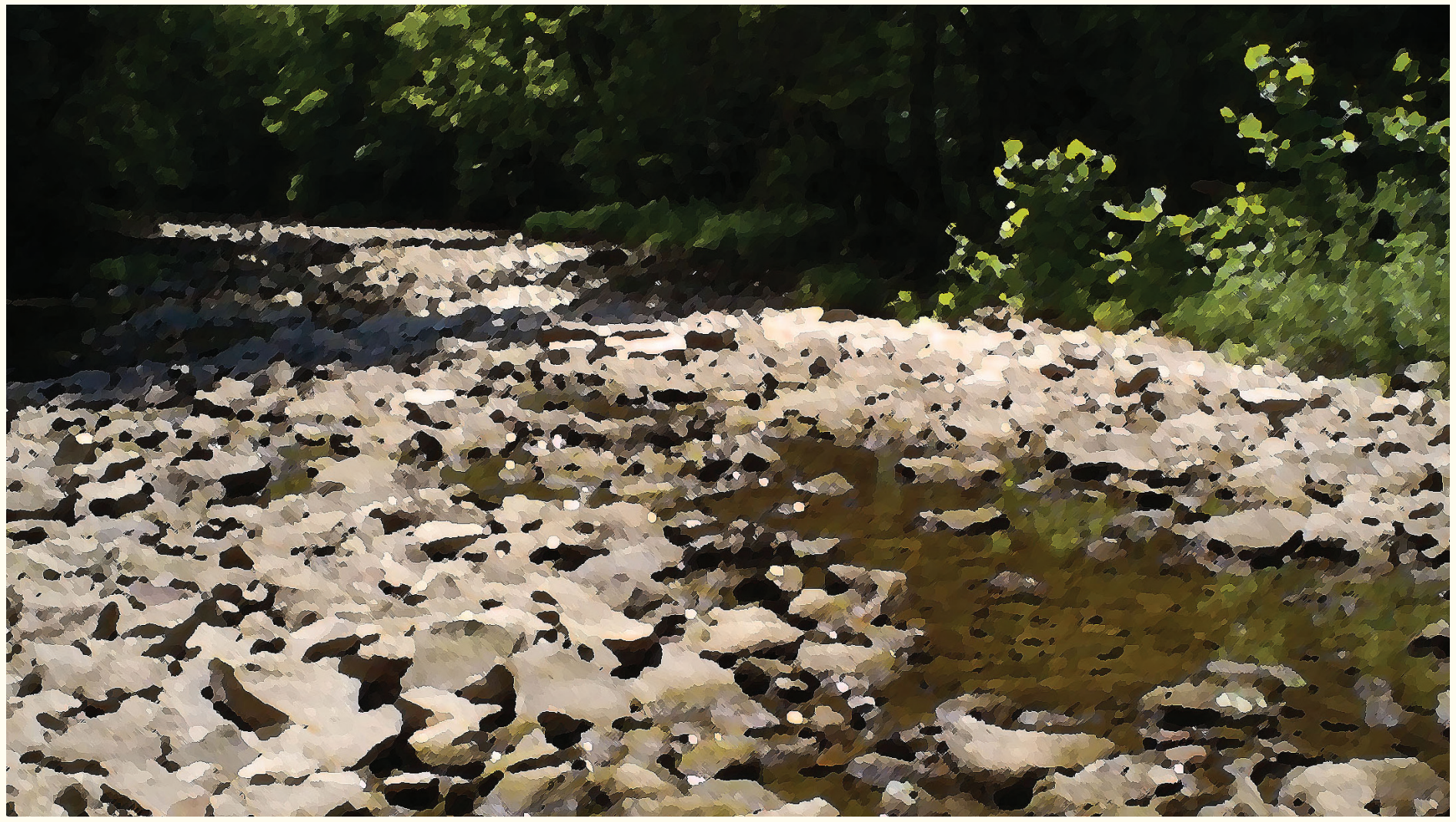

Scientific Investigations Report 2012-5138 
Cover. Stream channel during low-flow conditions on Big Walnut Creek at Sunbury, Ohio. (Rendering based on original photograph by Al Dillenburg, U.S. Geological Survey.) 


\section{Methods for Estimating Selected Low-Flow Statistics and Development of Annual Flow-Duration Statistics for Ohio}

By G.F. Koltun and Stephanie P. Kula

Prepared in cooperation with the Ohio Water Development Authority

Scientific Investigations Report 2012-5138

U.S. Department of the Interior

U.S. Geological Survey

U.S. Department of the Interior 


\section{KEN SALAZAR, Secretary}

\section{U.S. Geological Survey \\ Marcia K. McNutt, Director}

U.S. Geological Survey, Reston, Virginia: 2013

For more information on the USGS - the Federal source for science about the Earth,

its natural and living resources, natural hazards, and the environment—visit http://www.usgs.gov or call 1-888-ASK-USGS

For an overview of USGS information products, including maps, imagery, and publications, visit http://www.usgs.gov/pubprod

To order this and other USGS information products, visit http://store.usgs.gov

Any use of trade, product, or firm names is for descriptive purposes only and does not imply

endorsement by the U.S. Government.

Although this report is in the public domain, permission must be secured from the individual copyright owners to reproduce any copyrighted material contained within this report.

Suggested citation:

Koltun, G.F., and Kula, S.P., 2013, Methods for estimating selected low-flow statistics and development of annual flow-duration statistics for Ohio: U.S. Geological Survey Scientific Investigations Report 2012-5138, 195 p. 


\section{Contents}

Abstract

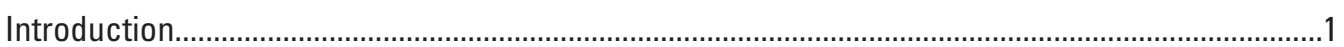

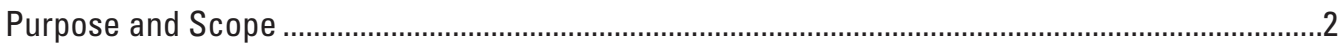

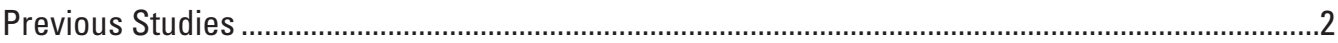

Sources of Low-Flow Statistics and Flow-Duration Data .............................................................

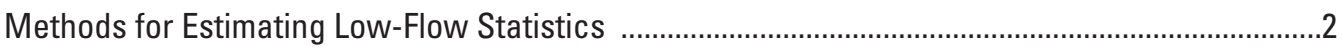

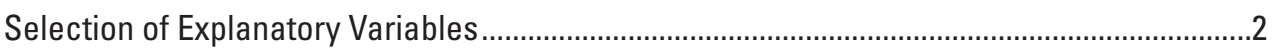

Data Used in Streamflow-Variability Index (SVI) Related Analyses ......................................7

Estimating SVI at Ungaged Sites.....................................................................................

Errors Associated with Estimating SVI at Ungaged Sites.....................................................8

Equations for Estimating Low-Flow Yield Statistics as a Function of SVI ..............................8

Model Error for Low-Flow-Yield Statistic Estimation Equations ...........................................11

Logistic-Regression Equations for Identification of Zero-Valued Low-Flow Statistics ..........12

Limitations of the Estimating Equations ……………………...................................................13

Methods for Annual Flow-Duration Analyses............................................................................

Results and Discussion of Annual Flow-Duration Analyses ........................................................16

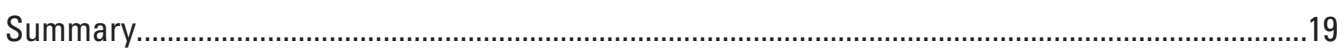

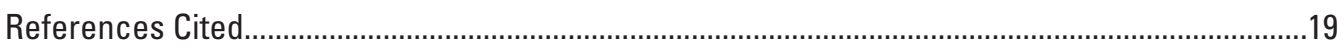

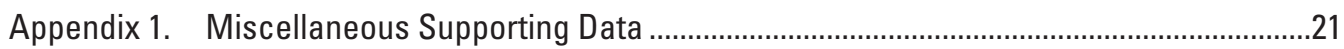

Appendix 2. Basin Characteristics Tested for Use in Low-Flow Regression Analyses

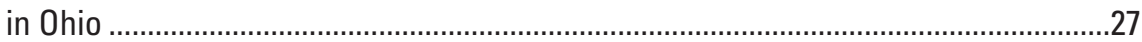

Appendix 3. Annual 1-Day and 7-Day Mean Flow-Duration Results for Sites with Streamflows that have No or Minimal Regulation...................................................31

Appendix 4. Annual Seasonal-Period Flow-Duration Results for Sites with Streamflows that have No or Minimal Regulation ...................................................................99

Appendix 5. Annual 1-Day and 7-Day Mean Flow-Duration Results for Sites with Streamflows that are Subject to Regulation............................................................135

Appendix 6. Annual Seasonal-Period Flow-Duration Results for Sites with Streamflows that are Subject to Regulation

\section{Figures}

1. Graph showing plot of streamflow-variability index as a function of 7-day, 10-year low-flow yield

2. Map showing spatial distribution of streamflow-variability index values determined from streamflow records for streamgages in Ohio and nearby portions of adjacent States

3. Generalized map of Ohio showing estimated spatial variation in streamflowvariability index

4. Boxplot showing streamflow-variability index residuals from the leave-one-out cross-validation analysis

5. Graph showing plot of the standard deviation of logarithms of annual 7-day, 10 -year low-flow values as a function of years of streamgage record. 
6. Graph showing plot of 7-day, 10-year low-flow yield as a function of streamflowvariability index, illustrating fit of the constrained polynomial equation.

7. Map showing low-flow regions used in regression equations for $090,10 \mathrm{~m}$, and D80m

8. Graphs illustrating the process used to compute selected quantiles of the a nnual $q_{p}$ series.

9. Map showing locations of streamflow-gaging stations with annual flow-duration analyses that have no or minimal streamflow regulation

10. Map showing locations of streamflow-gaging stations with annual flow-duration analyses that have streamflows that are subject to appreciable regulation . .18

1-1. Scatter plots of regression residuals as a function of predicted low-flow yields...........26

\section{Tables}

1. Data used to develop equations for estimating selected low-flow yield statistics for Ohio.-Continued... .3

2. Equations for estimating selected low-flow yield statistics for Ohio. . .9

3. Logistic-regression equations for estimating the annual probability of zero flow for selected

low-flow statistics for unregulated streams in Ohio

1-1. Streamflow-variability index (SVI) values for locations in Ohio and adjacent States used for SVI-related analyses.

2-1. Basin characteristics determined for selected streamgages in Ohio and adjacent States Available in Excel format. 


\section{Conversion Factors}

Inch/Pound to SI

\begin{tabular}{|c|c|c|}
\hline Multiply & By & To obtain \\
\hline \multicolumn{3}{|c|}{ Length } \\
\hline inch (in.) & 2.54 & centimeter $(\mathrm{cm})$ \\
\hline centimeter $(\mathrm{cm})$ & 0.3937 & inch (in.) \\
\hline inch (in.) & 25.4 & millimeter $(\mathrm{mm})$ \\
\hline foot $(\mathrm{ft})$ & 0.3048 & meter $(\mathrm{m})$ \\
\hline mile (mi) & 1.609 & kilometer $(\mathrm{km})$ \\
\hline \multicolumn{3}{|c|}{ Area } \\
\hline square foot $\left(\mathrm{ft}^{2}\right)$ & 929.0 & square centimeter $\left(\mathrm{cm}^{2}\right)$ \\
\hline square foot $\left(\mathrm{ft}^{2}\right)$ & 0.09290 & square meter $\left(\mathrm{m}^{2}\right)$ \\
\hline square mile $\left(\mathrm{mi}^{2}\right)$ & 259.0 & hectare (ha) \\
\hline square mile $\left(\mathrm{mi}^{2}\right)$ & 2.590 & square kilometer $\left(\mathrm{km}^{2}\right)$ \\
\hline \multicolumn{3}{|c|}{ Flow rate } \\
\hline cubic foot per second $\left(\mathrm{ft}^{3} / \mathrm{s}\right)$ & 0.02832 & cubic meter per second $\left(\mathrm{m}^{3} / \mathrm{s}\right)$ \\
\hline $\begin{array}{l}\text { cubic foot per second per square mile } \\
{\left[\left(\mathrm{ft}^{3} / \mathrm{s}\right) / \mathrm{mi}^{2}\right]}\end{array}$ & 0.01093 & $\begin{array}{l}\text { cubic meter per second per square } \\
\text { kilometer }\left[\left(\mathrm{m}^{3} / \mathrm{s}\right) / \mathrm{km}^{2}\right]\end{array}$ \\
\hline \multicolumn{3}{|c|}{ Hydraulic gradient } \\
\hline foot per mile (ft/mi) & 0.1894 & meter per kilometer $(\mathrm{m} / \mathrm{km})$ \\
\hline
\end{tabular}

Temperature in degrees Fahrenheit $\left({ }^{\circ} \mathrm{F}\right)$ may be converted to degrees Celsius $\left({ }^{\circ} \mathrm{C}\right)$ as follows:

${ }^{\circ} \mathrm{C}=\left({ }^{\circ} \mathrm{F}-32\right) / 1.8$

Horizontal coordinate information is referenced to the North American Datum of 1983 (NAD 83).

A climatic year is defined as the 12-month period from April 1 to March 31 and is designated by the calendar year in which it ends.

A water year is the 12-month period from 0ctober 1 through September 30 and is designated by the calendar year in which it ends.

\section{Abbreviations}

APE average absolute percent error

APEE annual percentage equaled or exceeded

ESRI Environmental Systems Research Institute

FDC flow-duration curves

HUC hydrologic unit code

IDW inverse-distance weighted

LCTR long-term continuous record

LFPR low-flow partial record

SAS Statistical Analysis System

SVI streamflow-variability index

USGS U.S. Geological Survey

WLS weighted least squares 



\title{
Methods for Estimating Selected Low-Flow Statistics and Development of Annual Flow-Duration Statistics for Ohio
}

\author{
By G.F. Koltun and Stephanie P. Kula
}

\section{Abstract}

This report presents the results of a study to develop methods for estimating selected low-flow statistics and for determining annual flow-duration statistics for Ohio streams. Regression techniques were used to develop equations for estimating 10-year recurrence-interval (10-percent annualnonexceedance probability) low-flow yields, in cubic feet per second per square mile, with averaging periods of 1,7 , 30 , and 90-day(s), and for estimating the yield corresponding to the long-term 80-percent duration flow. These equations, which estimate low-flow yields as a function of a streamflowvariability index, are based on previously published low-flow statistics for 79 long-term continuous-record streamgages with at least 10 years of data collected through water year 1997. When applied to the calibration dataset, average absolute percent errors for the regression equations ranged from 15.8 to 42.0 percent. The regression results have been incorporated into the U.S. Geological Survey (USGS) StreamStats application for Ohio (http://water.usgs.gov/osw/streamstats/ ohio.html) in the form of a yield grid to facilitate estimation of the corresponding streamflow statistics in cubic feet per second. Logistic-regression equations also were developed and incorporated into the USGS StreamStats application for Ohio for selected low-flow statistics to help identify occurrences of zero-valued statistics.

Quantiles of daily and 7-day mean streamflows were determined for annual and annual-seasonal (SeptemberNovember) periods for each complete climatic year of streamflow-gaging station record for 110 selected streamflowgaging stations with 20 or more years of record. The quantiles determined for each climatic year were the 99-, 98-, 95-, 90-, 80-, 75-, 70-, 60-, 50-, 40-, 30-, 25-, 20-, 10-, 5-, 2-, and 1-percent exceedance streamflows. Selected exceedance percentiles of the annual-exceedance percentiles were subsequently computed and tabulated to help facilitate consideration of the annual risk of exceedance or nonexceedance of annual and annual-seasonal-period flow-duration values. The quantiles are based on streamflow data collected through climatic year 2008.

\section{Introduction}

To address the needs for estimating low-flow statistics at ungaged locations in Ohio and for stochastically relevant annual flow-duration statistics, a study was conducted by the U.S. Geological Survey (USGS), in cooperation with the Ohio Water Development Authority, to develop methods for estimating selected low-flow statistics with regulatory significance ${ }^{1}$ and to compute and report stochastically relevant annual flow-duration statistics for Ohio streams.

Streamflow statistics are used for a variety of purposes. Among other uses, both low-flow and flow-duration statistics are particularly useful for making decisions about the suitability of a stream to act as an industrial or public water supply or as a suitable destination for waste effluents. These suitability questions frequently hinge on the impact of these uses on aquatic biota in the affected streams; thus, the statistics that have been adopted for regulatory purposes typically are biologically relevant. In spite of the widespread need for such statistics, the streamflow data required to compute these statistics have been collected only at a small number of locations in Ohio. Consequently, it is important that streamflow statistics be determined for gaged locations and that, whenever possible, suitable methods are developed to permit estimation of those statistics at ungaged locations.

Traditional flow-duration curves (FDCs) show the percentage of time that daily mean streamflow of a given magnitude was equaled or exceeded over a long period of time (many years). While traditional FDCs provide useful information about long-term streamflow exceedance characteristics, their utility is diminished when trying to answer questions about exceedance characteristics within a typical year. Another drawback of traditional FDCs is that they are not appropriate for extracting stochastic hydrologic information to address water-resources problems (Sugiyama and others, 2003).

${ }^{1}$ For the purposes of this report, regulatory-significant statistics are defined as those statistics that are used by regulators because of legal mandate or because of recommended best-management practices. 


\section{Purpose and Scope}

The purpose of this report is to (1) describe analytical methods and resulting equations for estimating 10-percent annual-nonexceedance probability of the 1-, 7-, 30-, and 90-day low-flow yields and 80-percent flow-duration yield and (2) describe methods used to compute annual flow-durationfrequency statistics for selected streamgages in Ohio and present the computed annual and annual-seasonal period flowduration frequency statistics. Information is provided to help the reader evaluate the accuracy and limitations of the regression equations, which have been incorporated into the USGS StreamStats application for Ohio (Koltun and others, 2006).

\section{Previous Studies}

Previous studies that produced regression equations for estimating one or more low-flow statistics for Ohio streams include those by Koltun and Whitehead (2002) and Koltun and Schwartz (1987). This report updates equations for estimating two of the low-flow statistics (the 7-day, 10-year and 30-day, 10-year low flows) presented by Koltun and Schwartz (1987) and includes equations for additional low-flow statistics (the 1-day, 10-year and 90-day, 10-year low flows, and the 80 -percent duration streamflow) that were not addressed in either study.

\section{Sources of Low-Flow Statistics and Flow-Duration Data}

Regression equations presented in this report are based on low-flow and duration statistics for selected Ohio streamgages with 10 or more years of record as reported by Straub (2001). The low-flow statistics and flow-duration characteristics reported by Straub (2001) were based on streamflow data collected through water year 1997. In contrast to the longterm flow-duration characteristics reported by Straub (2001), this report presents annual flow-duration characteristics in a stochastic framework based on a new analysis of daily streamflow data from streamgages with a minimum of 20 years of record.

\section{Methods for Estimating Low-Flow Statistics}

Equations for estimating low-flow statistics frequently are developed by means of regression techniques employing basin and (or) climatic characteristics as explanatory variables.
Forty-three potential explanatory variables ${ }^{2}$ were determined for this study and plotted against low-flow statistics and low-flow yields computed from long-term streamflow records to assess the nature and strength of covariate relations. Correlation and regression analyses were used to supplement the plots and explore secondary and interaction effects between explanatory variables.

\section{Selection of Explanatory Variables}

Ultimately, relations between a streamflow-variability index $(S V I)$ and low-flow yields (that is, the low flow with a $D$-day averaging period, and a $T$-year recurrence interval, in cubic feet per second, divided by the drainage area, in square miles) were found to be much stronger than any other covariate relations. SVI is a measure of the variability in streamflow resulting from variability in precipitation as mitigated by characteristics of the basin such as surface storage and groundwater discharge. All other things being equal, unregulated streams with relatively small SVIs tend to have proportionally more flow contributed from groundwater discharge (and (or) surface storage) than streams with larger SVIs (Searcy, 1959).

The SVI is defined as the standard deviation of the logarithms of the 19 streamflow values at 5-percent class intervals from 5 to 95 percent on the daily flow-duration curve for the analysis period (Searcy, 1959). The formula for the SVI discussed in this report is

$$
S V I=\sqrt{\frac{\sum_{i=5,5}^{95}\left(\log _{10}\left(D_{i}\right)-\overline{\log _{10}(D)}\right)^{2}}{18}}
$$

where

$$
\begin{aligned}
S V I & \text { is the streamflow-variability index, } \\
D_{i} & \text { is the } i \text { th percent duration streamflow }(i=5,10, \\
& 15, \ldots 95) \text {, and }
\end{aligned}
$$

In those rare cases where $D_{i}=0$, a value of 0.001 was substituted and the resulting $S V I$ was considered less well defined. Low-flow yield variables are identified in this document by the addition of an "m" to the low-flow statistic name (for example, the 7-day, 10-year low-flow $(Q 7,10)$ yield is referred to as Q7,10m).

\footnotetext{
${ }^{2}$ All the potential explanatory variables considered for this study-grouped into major categories of land cover, surficial soils and geology, climate, basin and channel morphology, hydrogeology, and streamflow variability - are discussed in appendix 2 .
} 


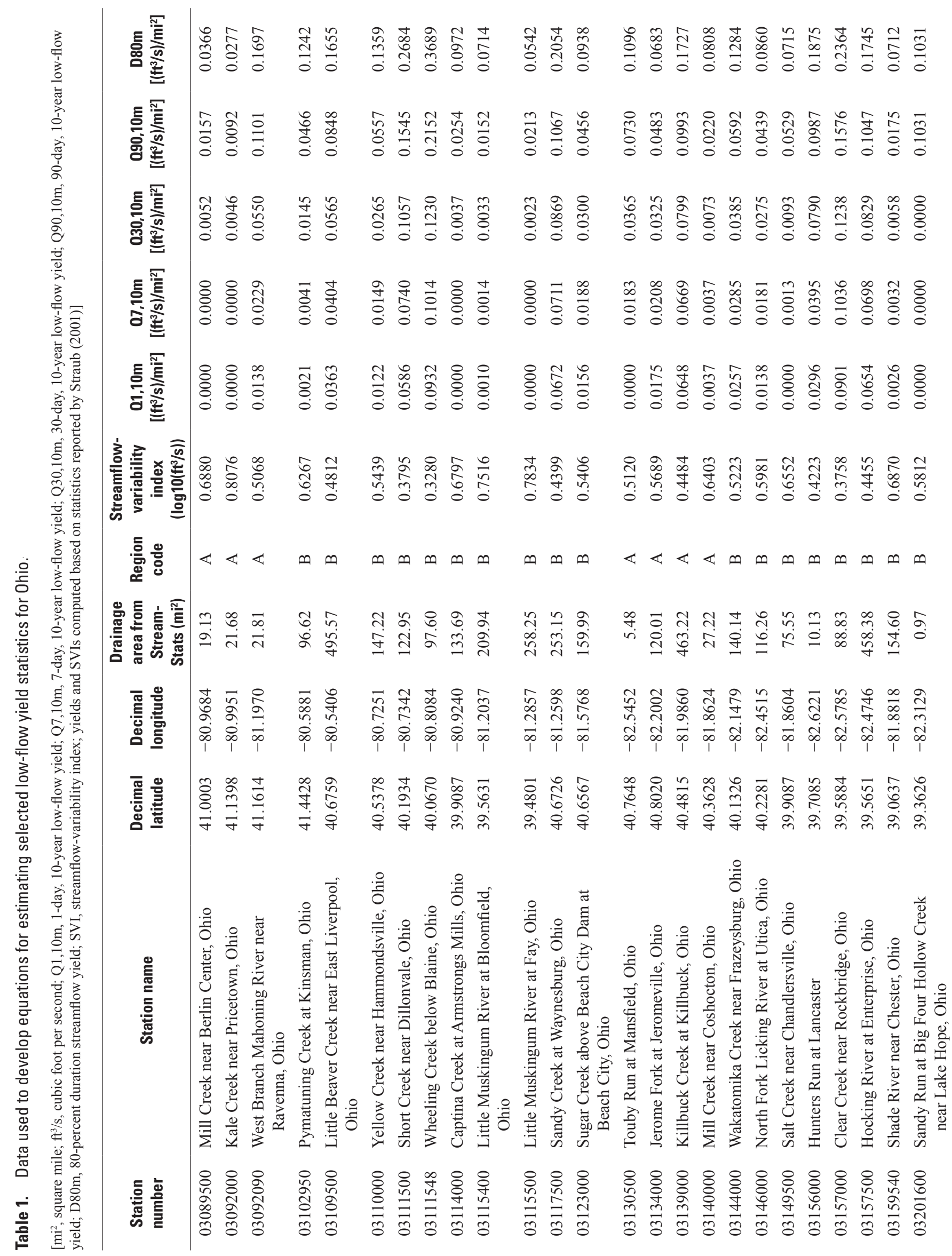




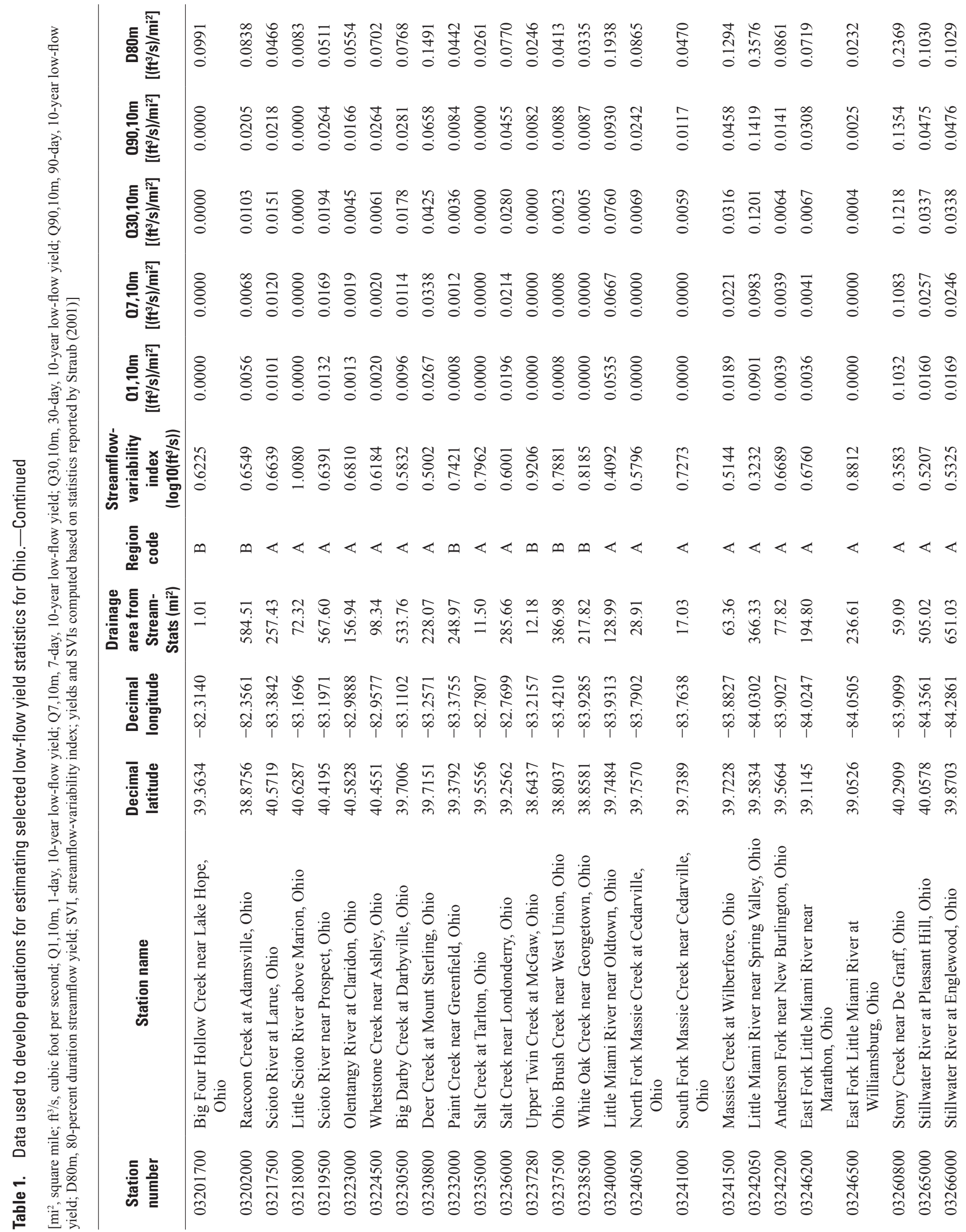




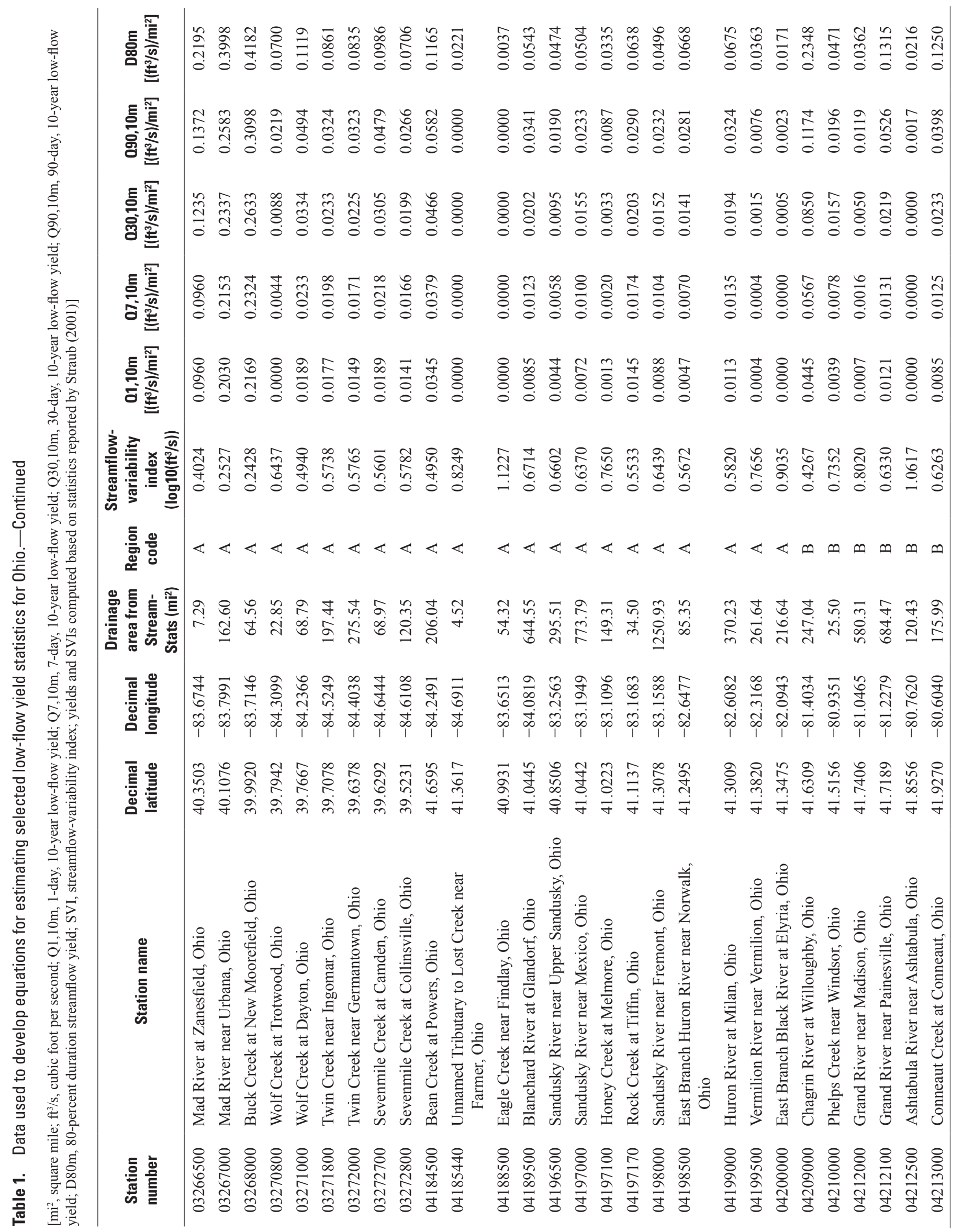


Figure 1. Graph showing plot of streamflow-variability index as a function of 7-day, 10-year low-flow yield.

Figure 2. Map showing spatial distribution of streamflow-variability index values determined from streamflow records for streamgages in Ohio and nearby portions of adjacent States (LTCR, long-term continuous record; LFPR, low-flow partial record).
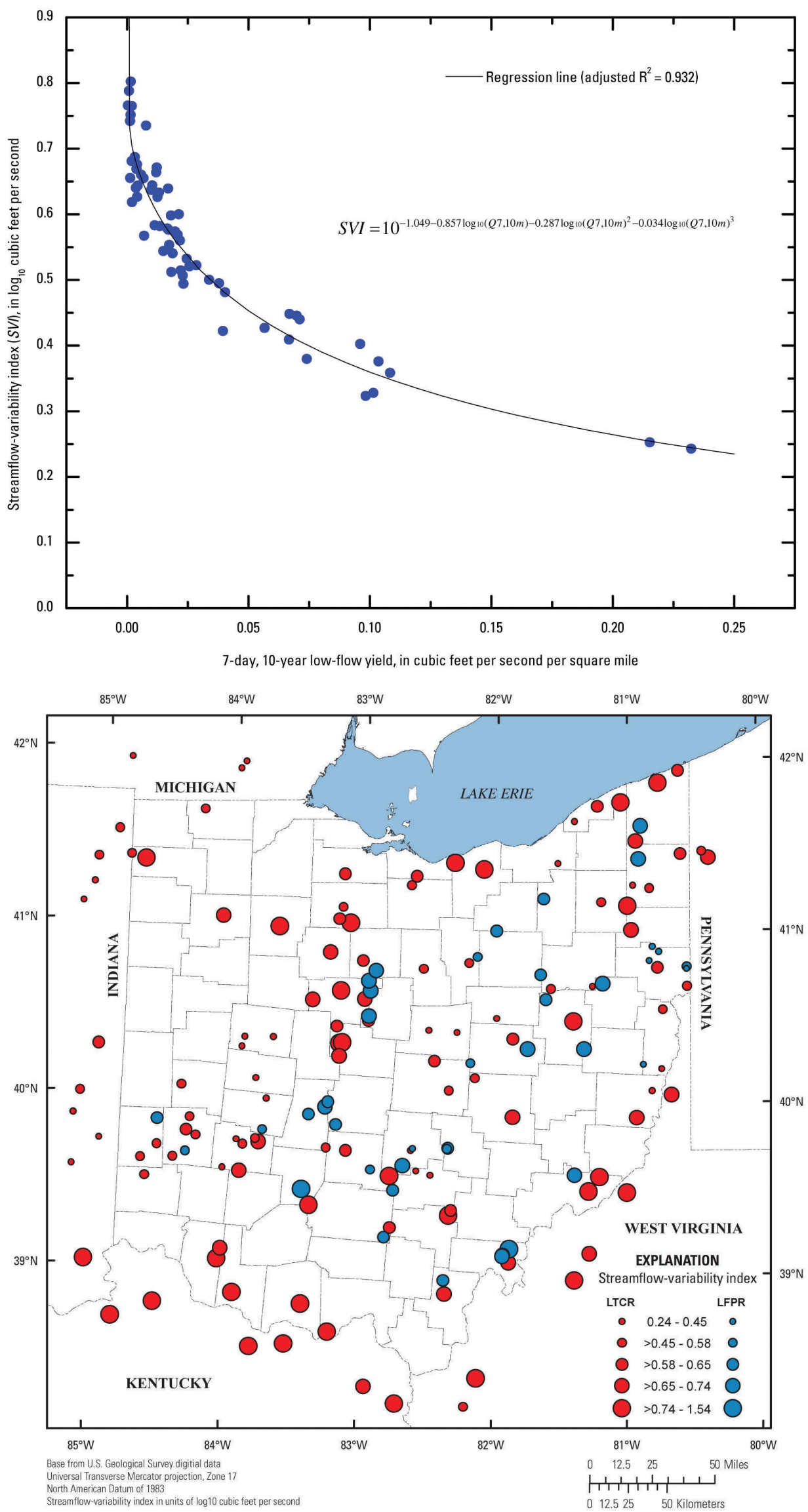


\section{Data Used in Streamflow-Variability Index (SVI) Related Analyses}

A variety of data sources were used to examine the relation between $S V I$ and low-flow statistics and to facilitate the estimation of SVI at ungaged sites. SVIs were obtained, computed, or estimated for a total of 162 sites (see appendix 1). Of those 162 sites, 121 were long-term continuous record (LTCR) sites with 10 or more years of record (93 in Ohio, 11 in Indiana, 6 in Kentucky, 6 in West Virginia, 3 in Michigan, and 2 in Pennsylvania) and 41 were low-flow partial record ${ }^{3}$ (LFPR) sites located in Ohio with published low-flow statistics. SVIs for LTCR and LFPR sites in Ohio are based on the lowflow statistics and periods of record published in Straub (2001). SVIs for LTCR sites in Indiana, Kentucky, West Virginia, Michigan, and Pennsylvania were either provided by the USGS Water Science Center in that State (in the cases of Indiana, Kentucky, and West Virginia) or were computed from approved streamflow records or statistics (in the cases of Michigan and Pennsylvania). As noted in subsequent discussions, subsets of the 162 site $S V I$ dataset were used for some analyses.

\section{Estimating $S V I$ at Ungaged Sites}

SVI must be regionalized in some fashion to permit its estimation at ungaged sites. Koltun and Whitehead (2002) developed a generalized grid-based spatial coverage of $S V I$ for Ohio that they used for that purpose. SVI was used by Koltun and Whitehead (2002) as an explanatory variable in equations they developed to estimate June-October mean monthly streamflows; the 25th-, 50th-, and 75th-percentile streamflows; and the harmonic mean streamflow. For this study, a new SVI grid coverage was created that is more spatially refined and reflects at-site $S V I$ information from more streamgages than the coverage developed by Koltun and Whitehead (2002).

The geostatistical techniques of kriging and cokriging were both examined as possible mechanisms for regionalizing SVI; however, a lack of wide-sense stationarity in SVI (possibly owing to localized hydrogeologic features that are poorly represented by available covariate datasets) made those methods unsuitable. This result is consistent with similar findings from a recent study by Martin and Arihood (2010) in which they observed LTCR streamgage data from Kentucky often indicated a change in $S V I$ values even though lithologic characteristics of surface geology did not change. They further concluded that specific lithologies did not necessarily result in consistent effects on groundwater discharge and low flow.

Examination of semivariogram plots (not shown) prepared when exploring options for kriging and cokriging indicated that sites that are closer together tend to have more

\footnotetext{
${ }^{3}$ Low-flow partial record (LFPR) sites are sites where periodic low-flow measurements are made for the purpose of developing a statistical relation with concurrent low flows at a long-term continuous record (LTCR) site. That statistical relation is used to estimate low-flow statistics for the LFPR site as a function of low-flow statistics computed for the LTCR site.
}

similar SVI values than sites that are further apart. Consequently, the decision was made to develop a spatial grid of $S V I$ by use of an inverse-distance-weighted (IDW) interpolation (Environmental Systems Research Institute, 2008) of the point SVI data. The IDW model, as applied, assumes that observations further away from a given location have their contributions to its spatial average reduced as a function of the square of the distance from that location. The IDW interpolation, which was based on the 12 nearest neighbors (in this case, sites with defined $S V I S)$ to each 1 square kilometer $\left(\mathrm{km}^{2}\right)$ grid cell, was done with the Environmental Systems Research Institute (ESRI) Spatial Analyst software.

To prepare the $S V I$ grid, LTCR sites (sites with 10 or more years of daily record) were selected from Ohio that were thought to be predominately free of low-flow regulation (that is, they had no significant regulation from upstream dams or other controlled lakes, and no significant low-flow regulation from water-supply withdrawals or wastewater discharges), along with comparable LTCR sites from adjacent States that were located near the State border of Ohio. SVIs determined from long-term streamflow records for the LTCR sites initially were plotted on a map (not shown) to assess spatial trends. Although there were visually identifiable spatial trends (for example a cluster of low SVIs at streamgages in a watershed known for relatively high groundwater discharge), it was apparent that, in some areas, SVIs can change appreciably over relatively short distances. Consequently, it was deemed important to obtain and use as much SVI data as possible to prepare the grid.

Since $S V I$ was strongly related to the target low-flow statistics, a relation between $S V I$ and $Q 7,10 m$ was determined and used to estimate $S V I$ at 41 LFPR sites (table 1-1) where Q7,10 previously had been estimated by Straub (2001) in order to expand the SVI dataset. Only data from LFPR sites with highly correlated LFPR-LTCR streamflow-measurement pairings (Pearson's r greater than 0.95) were used to help ensure high-quality $S V I$ estimates. The following polynomial equation relating $Q 7,10 m$ to $S V I$ was determined by use of ordinary least-square regression using data from 62 LTCR sites in Ohio (table 1) with non-zero $Q 7,10$ flows:

$$
S V I=10^{-1.049-0.857 X-0.287 X^{2}-0.034 X^{3}}
$$

where

$$
X=\log _{10}(Q 7,10 m) .
$$

Equation 2, which has an adjusted Pearson's correlation coefficient $\left(\mathrm{R}^{2}\right)$ of 0.93 , is plotted as the curve in figure 1, along with the observed values used to develop the equation.

The spatial distribution of SVIs for the 121 LTCR and 41 LFPR sites used to create the $S V I$ grid is shown in figure 2. Spatial gaps in the data are owing to either a lack of available or suitable streamgage data or areas where low flows are significantly regulated (and thus SVIs for streamgages in those areas are not representative of natural flows). The statewide spatial variation in estimated $S V I$ (as represented in the $S V I$ grid) is illustrated in figure 3 . 
Figure 3. Generalized map of Ohio showing estimated spatial variation in streamflowvariability index.

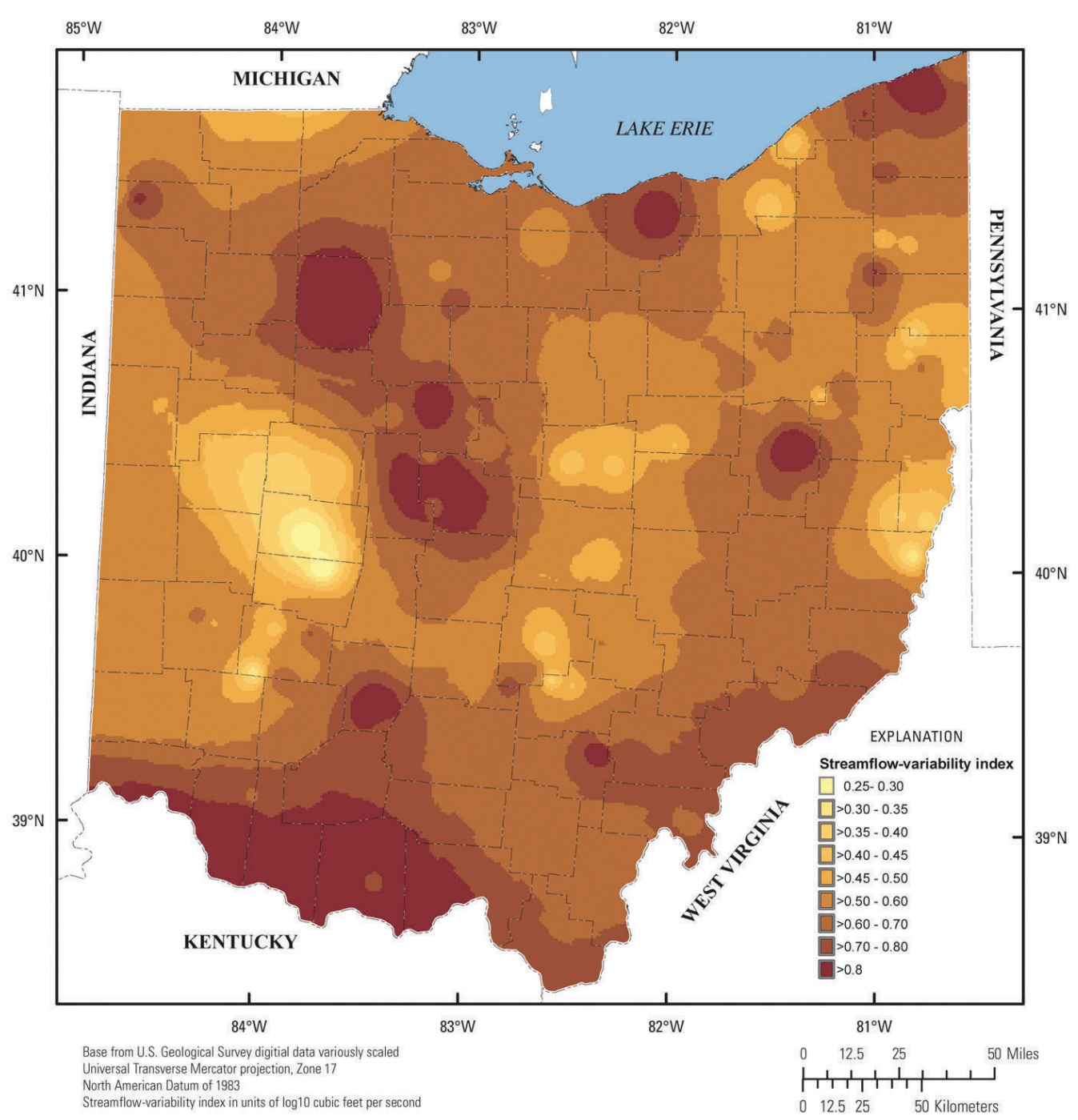

\section{Errors Associated with Estimating $S V I$ at Ungaged Sites}

A leave-one-out cross-validation technique was used to assess the nature of potential errors in SVI when using the grid to estimate $S V I$ at ungaged locations. Leave-one-out crossvalidation was done by (1) omitting the $S V I$ observation for a single streamgage, (2) using the IDW interpolation method to develop a new SVI grid based on the remaining streamgage data, (3) determining the new SVI grid value at the omitted streamgage location, and (4) computing the residual, which is the difference between the $S V I$ value determined from the original grid (typically about equal to the $S V I$ value observed for the streamgage) and the $S V I$ value determined in step 3. These 4 steps were repeated for each of the 162 streamgages whose data were used to create the original SVI grid, resulting in a set of 162 residuals. The residuals ranged from -0.51 to 0.63 with a mean and standard deviation of 0.01 and 0.16 , respectively. Fifty percent of the residuals were in the range of -0.08 to 0.06 equating to estimate errors ranging from about -15.4 to 8.5 percent of the observed at-site $S V I$ values (figure 4).

\section{Equations for Estimating Low-Flow Yield Statistics as a Function of $\mathbf{S V I}$}

The relations between $S V I$ and low-flow yields were determined by use of ordinary least-squares regression based on data collected through water year 1997 from 79 LTCR sites in Ohio (table 2). LFPR and LTCR sites in other States, sites with any known regulation (even if considered minor), and sites with less well defined SVIs were not used in developing the low-flow yield equations.

Weighted least-squares (WLS) regression is sometimes used to develop low-flow equations (Martin and Arihood, 2010; Wright and Ensminger, 2004); however, we chose not to use WLS regression because (a) there is considerable uncertainty about how best to weight low-flow estimates, 


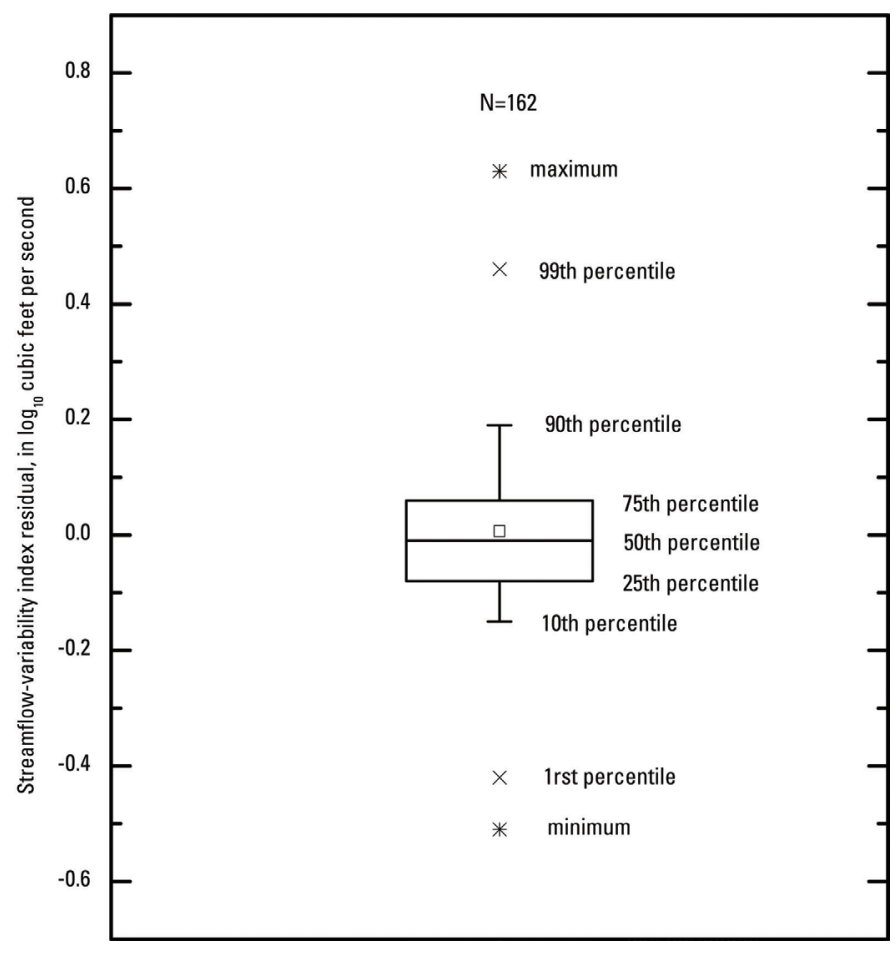

Figure 4. Boxplot showing streamflow-variability index residuals from the leave-one-out cross-validation analysis. (b) common weighting schemes that give more weight to observations determined from longer record lengths also will have the side effect of weighting the regressions toward characteristics associated with larger basins,${ }^{4}$ and (c) examination of the relation between the standard deviation of the logs of annual 7-day low flows versus the years of record for LTCR sites in Ohio indicated that although the standard deviation appeared to decrease somewhat with longer record length (as indicated by the downward-sloping regression line in figure 5, the decrease was not statistically significant (the slope of the regression line was not significantly different than 0 ) and record length explained only about 2 percent of the variation in standard deviation.

The relations between $S V I$ and the low-flow yields (in cubic feet per second per square mile $\left.\left(\mathrm{ft}^{3} / \mathrm{s}\right) / \mathrm{mi}^{2}\right)$ were not linear (for example, see fig. 6). Consequently, options for developing equations to estimate low-flow yields as a function of SVI involve either transformations of the model variables to fit a linear equation or fitting data to a non-linear or curvilinear equation. While logarithmic and square root transformations did a reasonable job of linearizing the relations for data pairs

${ }^{4}$ Because of their importance as water supplies and (or) flooding sources, streamgages in Ohio with large drainage areas also tend to have relatively long periods of record as compared to streamgages on smaller drainages.

Table 2. Equations for estimating selected low-flow yield statistics for Ohio.

$\left[\mathrm{R}^{2}\right.$, Pearson's correlation coefficient; all statistics are in units of cubic feet per second per square mile; Q1,10m, 1-day, 10-year low-flow yield; Q7,10m, 7-day, 10-year low-flow yield; Q30,10m, 30-day, 10-year low-flow yield; Q90,10m, 90-day, 10-year low-flow yield; D80m, 80-percent duration streamflow yield; R, low-flow region, use 0 for region A and 1 for region B; SVI, streamflow-variability index in units of base-10 logarithms of cubic feet per second; absolute percent errors do not reflect application of the logistic equations]

\begin{tabular}{|c|c|c|c|}
\hline $\begin{array}{l}\text { Equation } \\
\text { number }\end{array}$ & Equation & 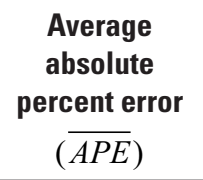 & $\begin{array}{c}\text { Adjusted } \\
\mathbf{R}^{2}\end{array}$ \\
\hline 1 & $\begin{array}{l}Q 1,10 m=0.795-3.740 S V I+6.633 S V I^{2}-5.234 S V I^{3}+1.543 S V I^{4} ; \text { if } \mathrm{SVI} \leq 0.80 \\
Q 1,10 m=0.0 ; \text { if } \mathrm{SVI}>0.80\end{array}$ & 38.7 & 0.93 \\
\hline 2 & $\begin{array}{l}Q 7,10 m=0.806-3.687 S V I+6.384 S V I^{2}-4.938 S V I^{3}+1.433 S V I^{4} ; \text { if } \mathrm{SVI} \leq 0.80 \\
Q 7,10 m=0.0 ; \text { if } \mathrm{SVI}>0.80\end{array}$ & 42.0 & .94 \\
\hline 3 & $\begin{array}{l}Q 30,10 m=0.783-3.245 S V I+5.073 S V I^{2}-3.535 S V I^{3}+0.924 S V I^{4} ; \text { if } \mathrm{SVI} \leq 0.91 \\
Q 30,10 m=0.0 ; \text { if } \mathrm{SVI}>0.91\end{array}$ & 34.2 & .95 \\
\hline 4 & $\begin{array}{l}Q 90,10 m=0.911-3.868 S V I+6.488 S V I^{2}-5.041 S V I^{3}+1.504 S V I^{4}+0.013 \mathrm{R} ; \text { if } \mathrm{SVI} \leq 1.0 \\
Q 90,10 m=0.0 ; \text { if SVI }>1.0\end{array}$ & 27.2 & .94 \\
\hline 5 & $D 80 m=e^{(0.221-4.715 S V I+0.350 R)}$ & 15.8 & .97 \\
\hline
\end{tabular}


Figure 5. Graph showing plot of the standard deviation of logarithms of annual 7-day, 10-year low-flow values as a function of years of streamgage record.

Figure 6. Graph showing plot of 7-day, 10-year low-flow yield as a function of streamflowvariability index, illustrating fit of the constrained polynomial equation.
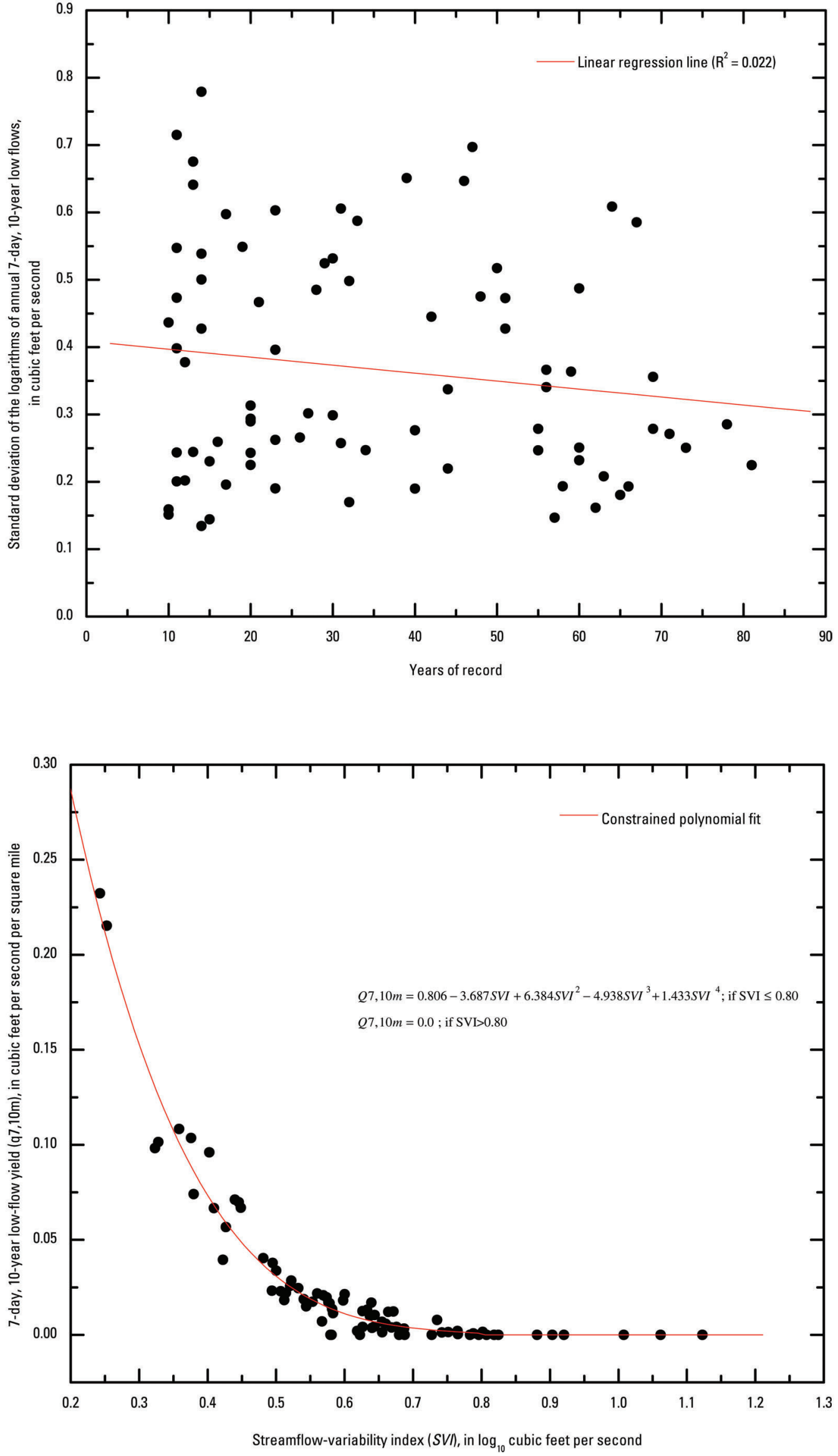
that did not contain zero-valued flow statistics, the logarithm of zero is undefined so data pairs with zero-valued statistics had to be omitted or a constant had to be added to the statistics for a logarithmic transformation to be used. Zeros are not a problem mathematically when using a square-root transformation; however, they can (and in this case did) produce undesirable effects on the regression owing to having a fairly wide range of $S V I$ values associated with zero-valued yields. Ultimately, it was decided that a polynomial model was the best model for most of the low-flow yield statistics because it permits all data pairs to be used without modification. For most low-flow yield statistics, a $4^{\text {th }}$ degree polynomial equation was fit; however, for $D 80 m$ (the 80-percent duration yield), a logarithmic equation was fit because all of the $D 80 \mathrm{~m}$ values in the calibration dataset were nonzero.

Residuals from all regression analyses were examined for normality and homoscedasticity (uniform variance around the regression line), and to assess spatial bias. The residuals for the statistics showed some indication of heteroscedasticity (fig. 1-1), in these cases indicating that estimate variance tended to increase somewhat with increasing predicted yields. Shapiro-Wilk tests (Shapiro and Wilk, 1965) indicated that, with the exception of $D 80 \mathrm{~m}$, the residuals for the low-flow statistics also deviated from normal at an alpha level of 0.05 , but normal quantile plots (not shown) indicated that they were only moderately aberrant in that respect. Several linearizing transformations (such as log and square root) were reexamined because of these issues; however, residuals from those models showed similar or worse indications of heteroscedasticity than the original model, so the original form of equation was selected. The regression analysis was repeated for the model forms shown in table 2 using an option to estimate the asymptotic covariance matrix of the estimates under the hypothesis of heteroscedasticity. Using that option results in the same parameter estimates as the ordinary least-squares regression but provides more robust estimates of their standard errors. Using robust standard errors did not change any of the conclusions from the original ordinary least-squares regression. It is worth noting that the regression models are valid for prediction in spite of the issues with heteroscedasticity and normality of the residuals; however, the variance of the prediction cannot be accurately determined.

The initial regression equations developed for $Q 90,10 \mathrm{~m}$ and $D 80 \mathrm{~m}$ had residuals that exhibited noticeable spatial bias (no appreciable spatial bias was evident for the other statistics); consequently, the State was ultimately broken up into two low-flow regions ( $A$ and $B$, fig. 7), and an indicator variable $(R)$, set to equal 0 for region $\mathrm{A}$ and set to equal 1 for region $\mathrm{B}$, was added to the regressions for these two statistics to account and correct for the observed bias. The regions were divided along 8-digit hydrologic unit code (HUC) boundaries. A variety of spatial datasets (for example, physiographic provinces, boundaries of glaciations, and maps of glacial-drift thickness) were examined in an attempt to help explain and locate appropriate boundaries for the observed regional trends in residuals. Ultimately, the transition in residual pattern from predominately positive residuals to predominately negative residuals appeared to most closely align with boundaries between areas of relatively high glacial-drift thickness and areas of relatively low or no glacial-drift thickness as defined by the Ohio Division of Geological Survey (2004).

The low-flow yield estimation equations were checked to ensure that estimated yields either decreased monotonically or were constant with increasing SVI over the range of anticipated $S V I$ values for Ohio. This was found to be true except at relatively high $S V I$ values for the polynomial equations. Examination of observed low-flow yields for $Q 1,10$ through $Q 30,10$ at LTCR sites showed that yields typically were zero when $S V I$ values exceeded thresholds ranging from about 0.8 to 1.0 , depending on the statistic being examined. Consequently, a constraint was applied to the yield equations that forced yields to be zero when those $S V I$ thresholds were exceeded. This resulted in low-flow yield estimates that were monotonically decreasing or constant (when yields reached zero) with increasing SVI. The yield curves also were examined to ensure that the progression of yield estimates for the 1-, 7-, 30-, and 90-day low flows at a given SVI was logical with respect to the statistics and durations being considered (for example, the yield for a given duration was always less than or equal to the yield for a longer duration for a particular SVI level).

The predictive equations are summarized in table 2 , and the underlying data used to develop those equations are listed in table 1. Values of $S V I$ and streamflow yields reported in table 2-1 are based on the low-flow statistics and periods of record listed for those streamgages in Straub (2001). The equations in table 2 are based on observations with $S V I$ magnitudes ranging from about 0.24 to $1.12 \log _{10}$ (cubic feet per second $\left.\left(\mathrm{ft}^{3} / \mathrm{s}\right)\right)$ for streamgages ranging in drainage area from approximately 0.97 to 1,250 square miles $\left(\mathrm{mi}^{2}\right)$. Figure 6 , which shows a scatter plot of observed SVI and $Q 7,10 \mathrm{~m}$ along with a curve representing the corresponding constrained polynomial equation (table 1 , equation 2 ), is typical of the fit associated with the constrained polynomial equations. The equations shown in table 2 were used to estimate low-flow yields for each cell in the $S V I$ grid discussed earlier, resulting in grids of low-flow yield estimates whose values can be multiplied by drainage area to estimate low-flow statistics (in $\mathrm{ft}^{3} / \mathrm{s}$ ) at ungaged sites.

\section{Model Error for Low-Flow-Yield Statistic Estimation Equations}

The low-flow yield grids were used to estimate low-flow yields at sites used for model calibration in order to assess the relation between observed and predicted values. Low-flow yields for the LTCR sites were estimated by determining the value corresponding to the low-flow yield grid cell at the location of the site. The low-flow yield estimates were used along 
Figure 7. Map showing lowflow regions used in regression equations for $090,10 \mathrm{~m}$, and D80m.

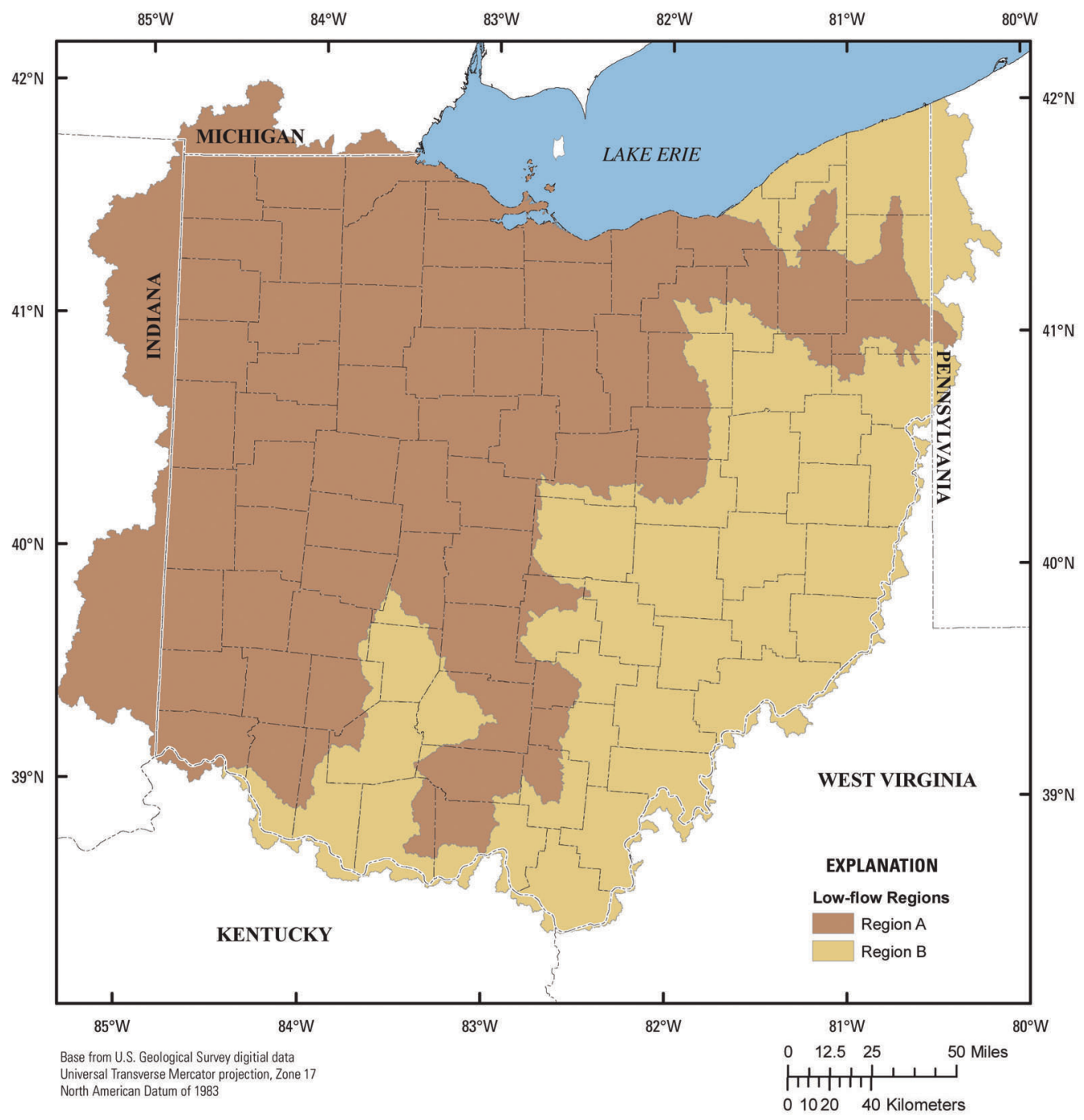

with the observed values to compute a measure of model error called the average absolute percent error $(\overline{A P E})$. The $\overline{A P E}$, which is reported in table 2, was determined as

$$
\overline{A P E}=\frac{1}{n} \sum_{i=1}^{n}\left|100 * \frac{\left(Y_{i}-\hat{Y}_{i}\right)}{Y_{i}}\right|
$$

where

$$
\begin{gathered}
Y_{i} \text { and } \hat{Y}_{i} \quad \begin{array}{l}
\text { are the } i \text { th observed and predicted low-flow } \\
\text { yields, and }
\end{array} \\
n \quad \text { is the number of observations. }
\end{gathered}
$$

When computing the $\overline{A P E}$ s, two allowances were made. First, if the observed and predicted flows were both zero, the percent error for that pair was said to be zero. Second, if the predicted flow was nonzero and the observed flow was zero, the percent error was undefined and therefore not used in the computation of the $\overline{A P E} . \overline{A P E}_{\mathrm{S}}$ ranged from a high of 42.0 percent for $Q 7,10 m$ to a low of 15.8 percent for $D 80 m$. It should be remembered that model error is not the only source of error for the low-flow yield estimate. There also will be error associated with the estimate of SVI.

\section{Logistic-Regression Equations for Identification of Zero-Valued Low-Flow Statistics}

Although the polynomial equations, coupled with the SVI threshold constraint, produce zero-valued statistic estimates for sites with high $S V I$ values, there remained some sites (11 for $Q 1,10 m, 8$ for $Q 7,10 m, 4$ for $Q 30,10 m$, and 3 for $Q 90,10 m$ ) in the calibration dataset with $S V I$ values lower than the thresholds that also had zero-valued statistics. In an attempt to improve identification of zero-valued statistics, logistic-regression equations (table 3 ) were developed to estimate the annual probability that the N-day, 10-year low flow is zero. Explanatory variables in the logistic-regression equations include the $S V I$ and the drainage area $(A)$. The results of the equations should be compared to the corresponding annualnonexceedance probability (in this case 0.10 ) because the logistic-regression equations were all developed for statistics 
with 10 -year recurrence intervals. All zero-valued statistics in the calibration dataset occurred in association with $S V I$ values greater than 0.50 ; therefore, at a minimum, the logisticregression equations should be used whenever a site has an estimated $S V I$ greater than 0.50 and a non-zero statistic is computed for the statistics listed in table 3 . It is recommended that the estimate for the statistic be revised to zero if the computed probability is greater than or equal to 0.10 .

Logistic-regression equations were developed for the $Q 1,10, Q 7,10$, and $Q 30,10$ statistics (table 3). Table 3 includes information about equation specificity (the percentage of non-zero statistics correctly identified), equation sensitivity (the percentage of zero-valued statistics correctly identified), and the overall percentage of correct classifications when classifying based on an annual-nonexceedance probability of 0.10 . No logistic-regression equations were developed for the $Q 90,10$ and $D 80$ statistics because there were either too few zero-valued statistics in the calibration dataset with which to develop reliable logistic equations (in the case of $Q 90,10$ ) or there were no zero-valued statistics (in the case of $D 80$ ) .

The low-flow yield equations (table 2) and logisticregression equations (table 3 ) have been incorporated into the StreamStats application for Ohio, which can accessed at http://water.usgs.gov/osw/streamstats/ohio.html. The StreamStats application for Ohio will automatically compute the drainage areas and low-flow statistics listed in table 2 using the same site-selection procedures used for other Ohio streamflow statistics, as described by Koltun and others (2006). StreamStats will convert the computed yields to streamflows, in units of cubic feet per second, by taking the product of the drainage area and yields.

\section{Limitations of the Estimating Equations}

The regression models are applicable to unregulated streams in Ohio that are not appreciably affected by local diversions or other forms of streamflow regulation. Large springs and sinks common to karst terrain in areas underlain by limestone can appreciably affect low flows. The regression models do not directly account for karst terrain, so model results can be erroneous when applied to basins with karst areas. The StreamStats application for Ohio makes no determination of streamflow regulation and provides no information on the presence of karst terrain; consequently, it is the user's responsibility to determine where and when the regression models can be suitably applied.

The estimating equations were developed by regressing streamflow statistics on explanatory variables. The $S V I$ was an explicit explanatory variable in the regression, and drainage area was an implicit explanatory variable in that it was used to compute the low-flow yields. SVI values for stations used in the regression analysis ranged from about 0.24 to $1.12 \log _{10}$ $\left(\mathrm{ft}^{3} / \mathrm{s}\right)$, and drainage areas ranged from about 1 to $1,250 \mathrm{mi}^{2}$ (table 1). The applicability of the regression equations is unknown when applied to basins with characteristics outside these ranges.

The SVI appeared to be much more strongly related to the low-flow statistics than the more traditional basin characteristics that were tested; however, there are some drawbacks to using $S V I$ as an explanatory variable. Unlike many traditional basin characteristics, $S V I$ is not a physical feature of a basin but instead reflects a variety of hydrologic processes integrated over an area draining to the point of interest. Consequently, $S V I$ could not be determined from readily available spatial datasets, so a new spatial dataset for $S V I$, consisting of a grid

Table 3. Logistic-regression equations for estimating the annual probability of zero flow for selected low-flow statistics for unregulated streams in Ohio.

$[A$, drainage area, in square miles; $p$, probability that the N-day, 10-year low flow does not exceed zero; $Q 1,10,1$-day, 10 -year low flow; $Q 7,10,7$-day, 10-year low flow; $Q 30,10,30$-day, 10-year low flow; $S V I$, streamflow-variability index; specificity, percentage of non-zero statistics correctly identified; sensitivity, percentage of zero-valued statistics correctly identified]

\begin{tabular}{ccccc}
\hline Statistic & Equation & $\begin{array}{c}\text { Percent } \\
\text { correct } \\
\text { at } \boldsymbol{p}=\mathbf{0 . 1 0}\end{array}$ & $\begin{array}{c}\text { Specificity } \\
\text { at } \boldsymbol{p}=\mathbf{0 . 1 0} \\
\text { (percent) }\end{array}$ & $\begin{array}{c}\text { Sensitivity } \\
\text { at } \boldsymbol{p}=\mathbf{0 . 1 0} \\
\text { (percent) }\end{array}$ \\
\hline Q1,10 & $p=\frac{e^{\left(-8.680+9.576 S V I-5.650 \times 10^{-3} A\right)}}{\left(1+e^{\left(-8.680+9.576 S V I-5.650 \times 10^{-3} A\right)}\right)}$ & 90.6 & 91.5 & 74.7 \\
$Q 7,10 \quad p=\frac{e^{\left(-9.973+10.35 S V I-5.020 \times 10^{-3} A\right)}}{\left(1+e^{\left(-9.973+10.35 S V I-5.020 \times 10^{-3} A\right)}\right)}$ & 93.7 & 94.6 & 73.6 \\
$Q 30,10$ & $p=\frac{e^{(-14.26+12.35 S V I)}}{\left(1+e^{(-14.26+12.35 S V I)}\right)}$ & 97.2 & 97.5 & 69.0 \\
\hline
\end{tabular}


composed of $1 \mathrm{~km}^{2}$ grid cells, was constructed to facilitate its estimation throughout the State. Although every effort was made to produce the best possible SVI spatial dataset, it must be remembered that $S V I$ is being estimated and so is subject to error. Those errors will be reflected in the low-flow yield estimates computed by StreamStats. Operation of a LTCR or LFPR streamgage at a site should be considered to provide a streamflow-based estimate of SVI for applications where uncertainties in the low-flow estimates will be poorly tolerated.

\section{Methods for Annual Flow-Duration Analyses}

Instead of using the traditional class-interval-based approach for defining FDCs described by Searcy (1959), a more direct and efficient quantile-estimation procedure, as recommend by Vogel and Fennessey (1994), was used to the compute annual flow-duration statistics discussed in this report. A program was written in the Statistical Analysis System (SAS) programming language (Version 9.1, SAS Institute Inc.) that computed and output selected quantiles of daily and 7-day-average streamflows for annual and seasonal periods for each climatic year in the station record that was complete (that is, it had no missing record). The season examined in this study is the period September-November, which, in Ohio, frequently contains the lowest streamflows during the year. The quantiles that were computed and output for each climatic year include the 99-, 98-, 95-, 90-, 80-, 75-, 70-, 60-, 50-, 40-, 30-, 25-, 20-, 10-, 5-, 2-, and 1-percent exceedance streamflows [defined as $100 P\left\{Q>q_{p}\right\}$, where $Q$ represents the daily mean (or 7-day average) streamflows during the climatic year or season, and $q_{p}$ is the value of the daily mean (or 7-day average) streamflow that equaled or exceeded $P$ percent of the time in the climatic year or season]. The $q_{p}$ values that were equaled or exceeded $n$ percent of the time over the analytical period are presented in data tables in appendixes 2-4 with $n$ shown in the first column labeled APEE (annual percentage equaled or exceeded).

The flow-duration quantiles were computed from the observed $Q$ time series by ranking the observed streamflows $[q(i), i=1, \ldots, n$ where $n=$ the number of days (or averaging periods) in the climatic year or season] and computing the quantiles (exceedance percentiles) using the type 4 quantileestimator option of the SAS UNIVARIATE procedure (SAS Institute Inc., 2011). The SAS type 4 quantile estimator is an $L$-moment-type estimator that provides weighted averages of adjacent order statistics (Vogel and Fennessey, 1994). The quantile estimator is defined as

$$
q_{p}=(1-\theta) q(i)+\theta q(i+1)
$$

where

$\theta \quad$ equals the fractional portion of $(n+1) p / 100$ and

$i \quad$ equals the integer portion of $(n+1) p / 100$ and where

$q(n+1) \quad$ is taken to be $q(n)$.

To permit assessment of whether a single theoretical distribution could be used to describe the distribution of annual $q_{p}$ series (that is, $q_{p}(i), i=1, \ldots, m$, where $m=$ the number of complete years of record), the SAS program was designed to plot ordered values of the observed distribution of annual $q_{p}$ values versus percentiles of selected theoretical distributions. The distributions plotted included the lognormal, Weibull, and gamma (also called Pearson Type III) distributions. These distributions were chosen, in part, based on recommendations made by Kroll and Vogel (2002). Ultimately, many of the annual $q_{p}$ series appeared to be reasonably well fit by one or more of the three distributions (with Weibull and gamma distributions providing good fit to many of the annual $q_{p}$ series); however, none of the distributions fit the annual series well for all annual-exceedance probabilities at all of the LTCR sites (including some unregulated sites with long periods $(>50$ years) of record. In particular, few of the high-percent exceedance-level series (such as the 95- or 98-percent exceeds series) were well fit.

A second program, written in the MATLAB programming language (The MathWorks Inc., 2010), was used to take the annual $q_{p}$ values determined with the SAS program and compute quantiles of those estimates. For example, assume a streamflow-gaging station had 30 complete climatic years of record. The SAS program would compute $q_{p}$ estimates for selected exceedance percentiles for each of the 30 years. The MATLAB program would then order the annual $q_{p}$ values and compute quantiles of the 30-year $q_{p}$ series. Quantiles of the annual $q_{p}$ series are represented by the symbol $q_{p, k}$; where $k$ is the percentage of time over the $n$ years of record that the annual $q_{p}$ value was exceeded (for example, $q_{50,90}$ is the annual $50^{\text {th }}$ percentile streamflow that was equaled or exceeded 90 percent of the time over the 30-year period). Initially, it was hoped that the $q_{p, k}$ estimates could be determined by fitting a theoretical distribution to the annual $q_{p}$ series; however, because the annual $q_{p}$ series for some annual-exceedance probabilities were poorly fit by the theoretical distributions for some LTCR streamflow-gaging stations, that approach was rejected in favor of estimating $q_{p, k}$ using the same quantile estimator (equation 4) used when analyzing the individual climatic-year series.

One of two predetermined sets of exceedance percentiles of the annual $q_{p}$ values were computed depending on the number of climatic years of record analyzed. If there were 50 or more years of record, the following exceedance percentiles were computed: $2,5,10,20,50,80,90,95$, and 98 percent. If there were less than 50 years of record, but greater than or equal to 20 years of record, exceedance percentiles of 5,10 , 
$20,30,50,70,80,90$, and 95 percent were computed (streamflow-gaging stations with less than 20 complete climatic years of record were not used). These two sets of exceedance percentiles were chosen so as to maximize the range of exceedance percentiles computed for a given record length yet still base all calculations on interpolations from computed quantiles.

The process used to compute the $q_{p, k}$ values is illustrated in figure 8 using data from the streamflow-gaging station on Wheeling Creek below Blaine, Ohio (station 03111548). The black lines in the lower plot of figure 8 represent the annualexceedance plots for each complete climatic year from 1983 through 2008. If one examines the magnitudes of streamflows corresponding to the annual 50-percent exceedance value (those lying along the red line in the lower plot), one can see that they range from 38 to $152 \mathrm{ft}^{3} / \mathrm{s}$. The computed exceedance percentiles for the annual values lying along that line (that is

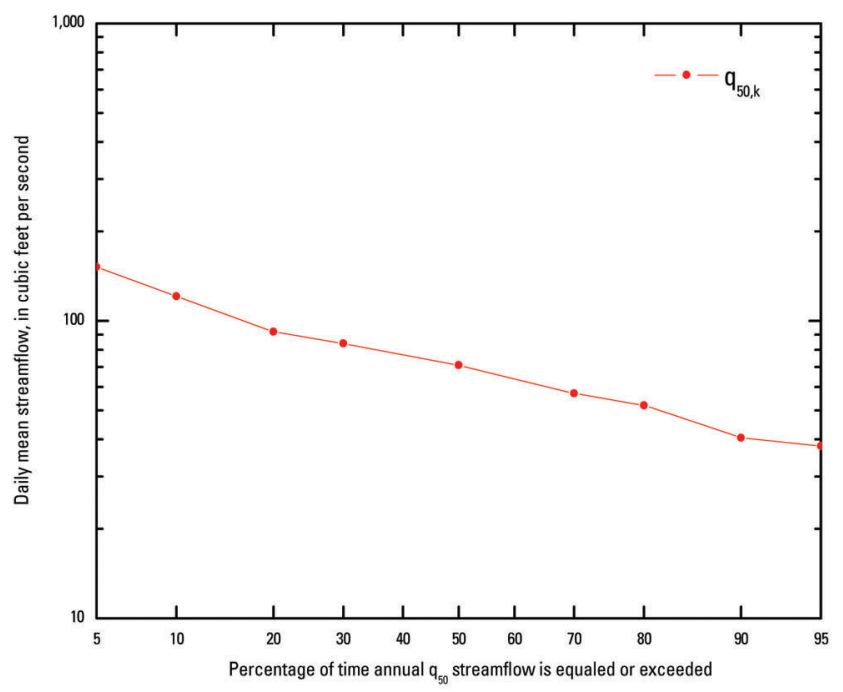

the $q_{50, k}$ values) are plotted as the red line in the upper left-hand plot and are tabulated in the row corresponding to an APEE value of 50 in appendix 3 (top table on page 381). If instead one examines the magnitudes of streamflows corresponding to the annual 90-percent exceedance value (those lying along the blue line in the lower plot), one can see that the computed exceedance percentiles range from 14 to about $70.2 \mathrm{ft}^{3} / \mathrm{s}$. The computed exceedance percentiles for the annual 90 -percent exceedance streamflows (that is the $q_{90, k}$ values) are plotted as the blue line in the upper right-hand plot and are tabulated in the row corresponding to an APEE value of 90 in appendix 3 (top table on page 38). If the top two plots were combined into one plot, one would observe that the blue line would lie below the red line, which is consistent with the expectation that the 90-percent exceedance streamflows should be less than or equal to the 50-percent exceedance streamflows.

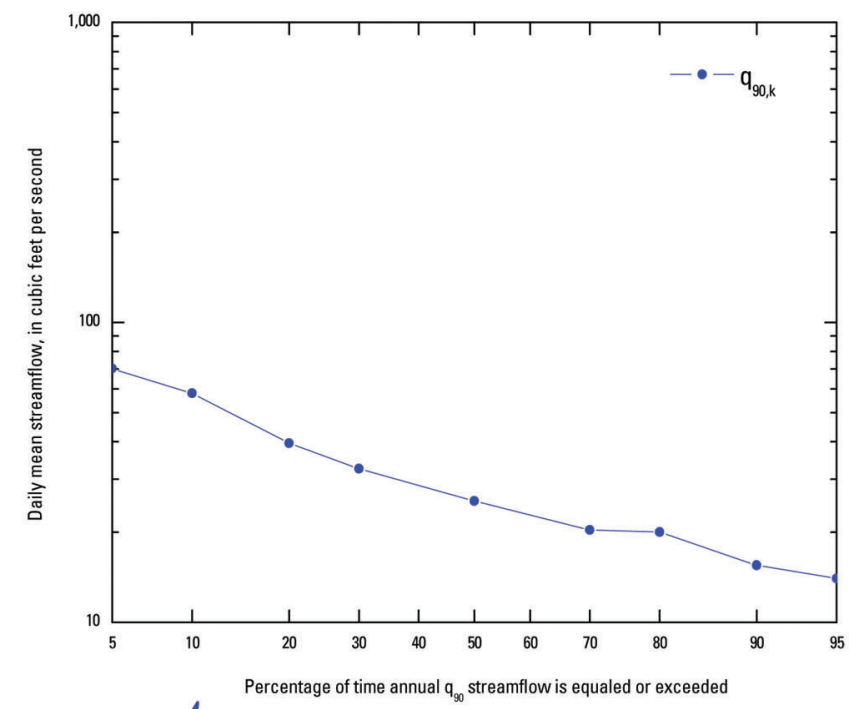

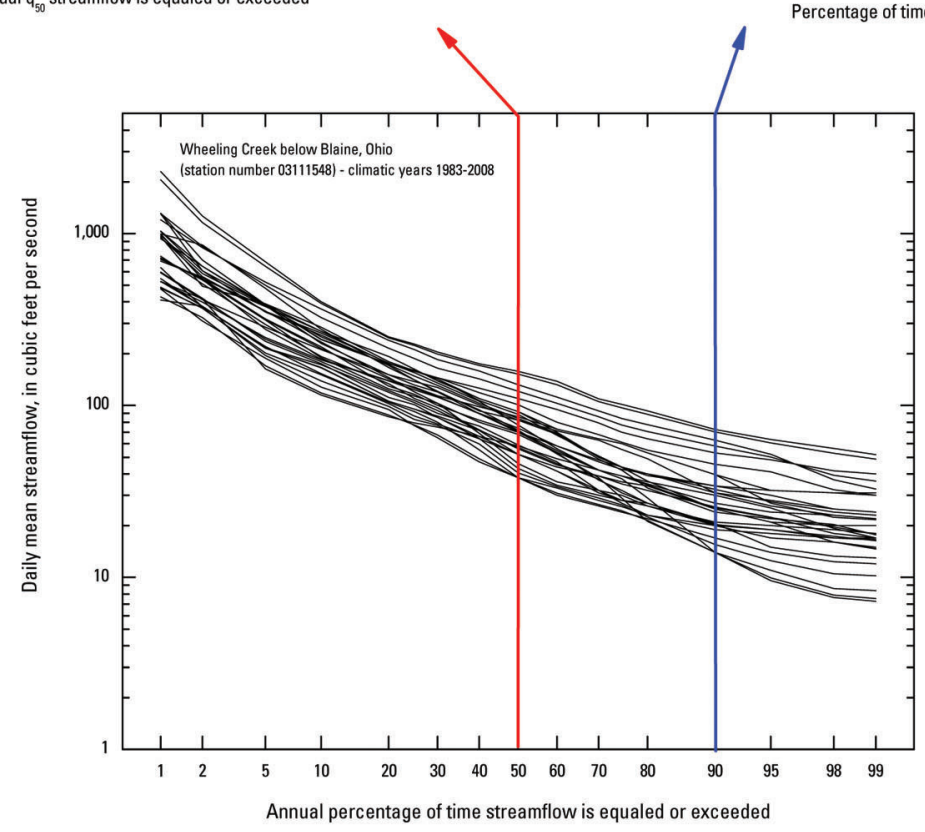

Figure 8. Graphs illustrating the process used to compute selected quantiles of the annual $q_{p}$ series. 


\section{Results and Discussion of Annual Flow-Duration Analyses}

Results of the annual and annual seasonal-period flow-duration analyses are presented in appendixes 3-6. Appendixes 3 and 4 contain results from the annual and annual seasonal-period analyses, respectively, for 67 to 70 LTCR sites $^{5}$ that have no or minimal regulation, whereas appendixes 5 and 6 contain results from the annual and annual seasonalperiod analyses, respectively, for 40 LTCR sites with streamflows that are subject to appreciable regulation. Maps showing the location of flow-duration sites that were thought to have no or minimal streamflow regulation and flow-duration sites that have streamflows that are subject to appreciable regulation are shown in figures 9 and 10, respectively.

The annual flow-duration characteristics presented in this report offer some advantages over traditional flow-duration characteristics. For example, one could construct a flowduration curve composed of medians of the annual-exceedance percentiles (that is $q_{50, k}$ values) to represent the distribution of daily streamflows in a median hypothetical year. Although the likelihood of actually observing such a year in nature is small (because all of the flow percentiles for the year would have to have a common annual-exceedance probability), the data support such analyses. Also, if a design calls for use of a specific annual-flow duration, one can consider the annual risk of exceedance or nonexceedance of that flow-duration value. Although risk-based assessments are possible, one should be particularly cautious of such interpretations for streams that are subject to regulation given that future changes in regulation could alter the flow-duration characteristics.

The following is an example of how the flow-duration data presented in this report might be used. For example, a

\footnotetext{
${ }^{5}$ Three sites had sufficient record to do annual seasonal-period analyses but did not have sufficient record to do annual analyses.
}

side-channel reservoir is being designed for a location adjacent to Wheeling Creek below Blaine, Ohio (station 03111548), and there is a flow-by requirement ${ }^{6}$ equal to the annual 80 -percent duration streamflow or the total streamflow, whichever is smaller. The amount of water that is required to flow by the reservoir can affect how rapidly the reservoir can be filled (since the flow-by requirement reduces the amount available for withdrawal); however, too small of a flow-by requirement may negatively impact downstream water quality and the health of aquatic biota.

If we examine the column corresponding to the annual 80-percent duration streamflow (that is, the daily mean streamflow that is equaled or exceeded 80 percent of the time in a given year) for Wheeling Creek below Blaine, Ohio (appendix 3, top table on page 38), we see that 50 percent of the time (row corresponding to APEE $=50$ ) the streamflow magnitude equaled or exceeded $52 \mathrm{ft}^{3} / \mathrm{s}$. That means that in about one-half of the years, the annual 80-percent duration streamflow was greater than $52 \mathrm{ft}^{3} / \mathrm{s}$. Should we wish to be more protective of the stream by assuring that the annual 80-percent duration streamflow that occurs in most years is allowed to pass by the reservoir, then we may wish to use an annual 80-percent duration streamflow that has a smaller annual-exceedance probability. For example, we could choose an annual 80-percent duration streamflow that was exceeded in only 10 percent of the years (row corresponding to APEE $=10$ ), which would increase the flow-by requirement to $166 \mathrm{ft}^{3} / \mathrm{s}$. Although increasing the flow-by requirement may provide greater downstream protection, it also may necessitate increasing the pump capacity and (or) volume of the reservoir so that there is sufficient water to meet demand at all times.

\footnotetext{
${ }^{6} \mathrm{~A}$ flow-by requirement is the minimum quantity of streamflow that must be allowed to flow by a point on a stream. Flow-by requirements typically are established to help ensure that there is sufficient streamflow downstream from an impoundment or withdrawal point on a stream to support ecological or water-use demands, or to supply other legally mandated minimum in-stream flows.
} 


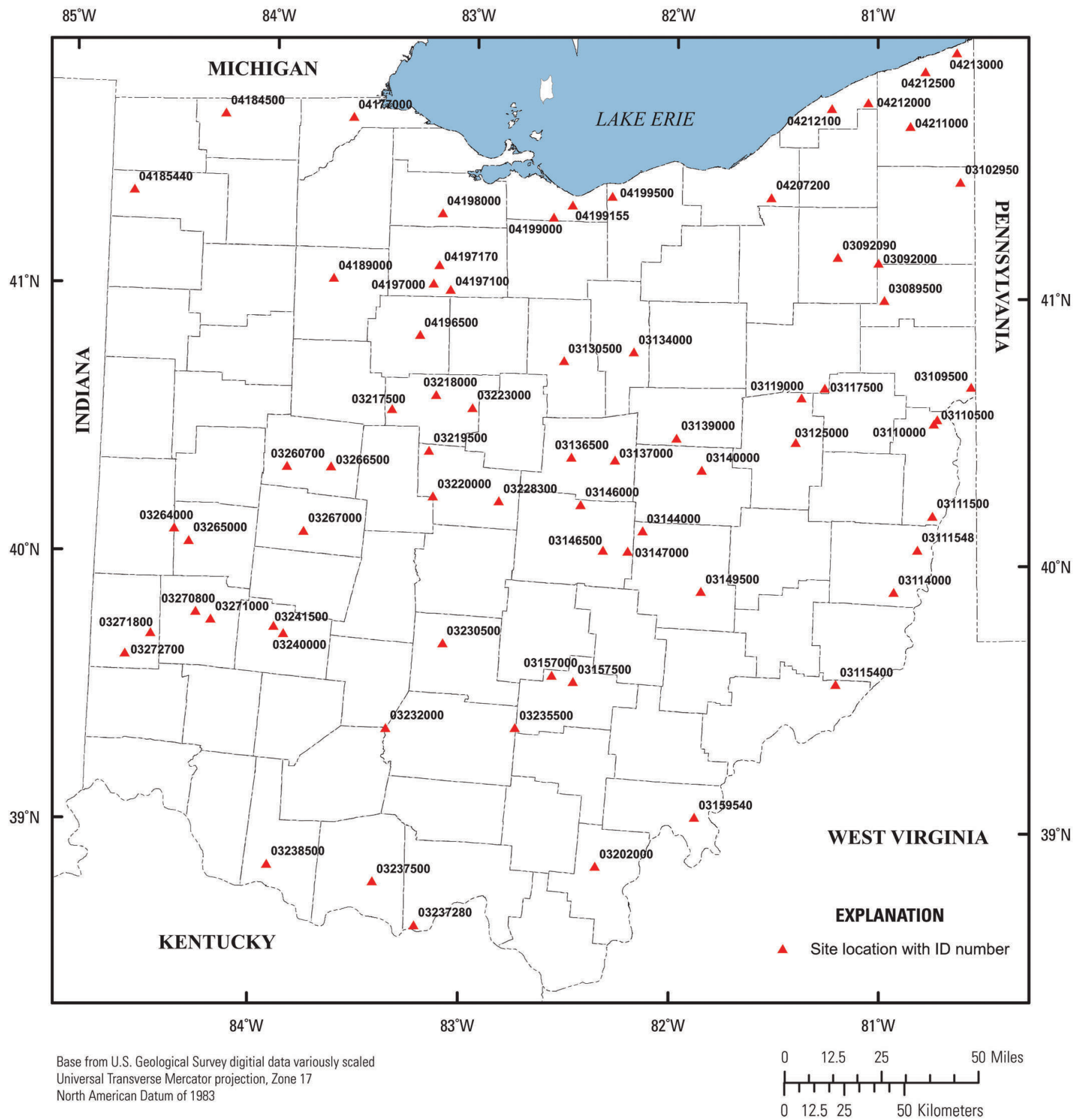

Figure 9. Map showing locations of streamflow-gaging stations with annual flow-duration analyses that have no or minimal streamflow regulation. 


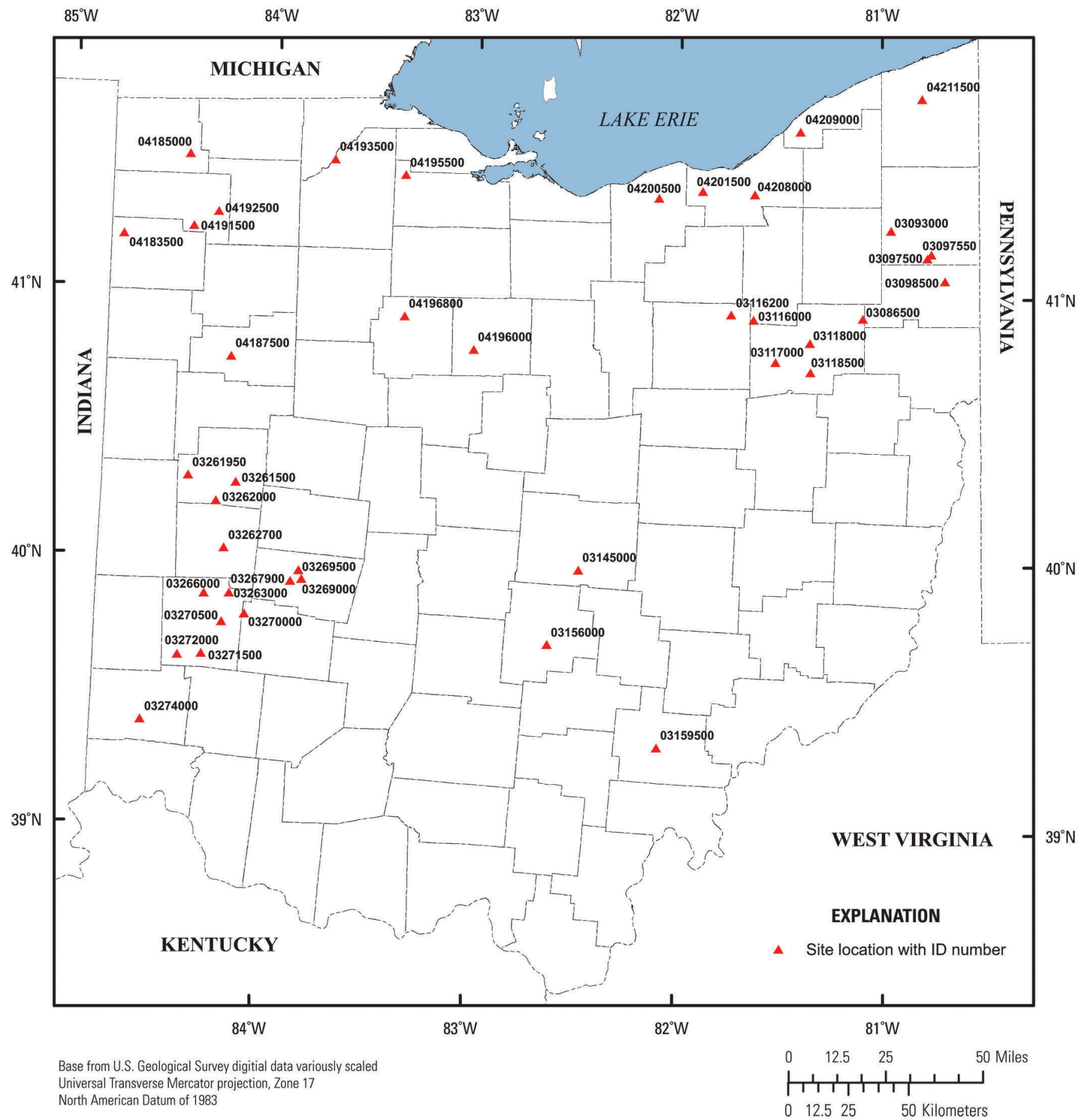

Figure 10. Map showing locations of streamflow-gaging stations with annual flow-duration analyses that have streamflows that are subject to appreciable regulation. 


\section{Summary}

Streamflow statistics are used for a variety of purposes. Among other uses, both low-flow and flow-duration statistics are particularly useful for making decisions about the suitability of a stream to act as an industrial or public water supply or as a suitable destination for waste effluents. In spite of the widespread need for such statistics, precomputed statistics are available for only a small number of locations in Ohio. Consequently, it is important that streamflow statistics be determined for suitable gaged locations, and it is highly desirable that methods be developed to permit estimation of those statistics at ungaged locations.

Ordinary least-squares regression techniques were used to develop equations for estimating 10-year recurrence-interval (10-percent annual-nonexceedance probability) low-flow yields, in cubic feet per second per square mile, with averaging periods of $1,7,30$, and 90 day(s), and for estimating the yield corresponding to the long-term 80 -percent duration flow. These equations, which estimate low-flow yields as a function of a streamflow-variability index (SVI), are based on lowflow statistics published by Straub (2001) for 79 long-term continuous record streamgages with data collected through water year 1997. Constraints were applied to the equations to ensure that zero-valued yields were computed when SVI magnitudes exceeded thresholds above which all low-flow yields for a given statistic were observed to be zero in the calibration dataset. The average absolute percent error for the constrained regression equations, when applied to the calibration dataset, ranged from 15.8 to 42.0 percent. The regression results have been incorporated into the U.S. Geological Survey StreamStats application for Ohio in the form of a yield grid to facilitate estimation of the corresponding streamflow statistics in cubic feet per second. Although the constrained polynomial regression equations presented in this report can produce zero-valued estimates, logistic-regression equations also were developed for selected low-flow statistics to help identify occurrences of zero-valued statistics.

Traditional flow-duration curves (FDCs) show the percentage of time that daily mean streamflow of a given magnitude was equaled or exceeded over a long period of time. While these traditional FDCs provide useful information about long-term streamflow-exceedance characteristics, their utility is diminished when trying to answer questions about exceedance characteristics within a typical year. To address the need for stochastically relevant annual flow-duration statistics, quantiles of daily and 7-day mean streamflows were determined for annual and annual-seasonal (September-November) periods for each complete climatic year of streamflow-gaging station record for 110 selected streamflow-gaging stations with 20 or more years of record. The quantiles determined for each climatic year were the 99-, 98-, 95-, 90-, 80-, 75-, 70-, 60-, 50-, 40-, 30-, 25-, 20-, 10-, 5-, 2-, and 1-percent exceedance streamflows. Selected exceedance percentiles of the annualexceedance percentiles were computed and are tabulated in this report to help facilitate consideration of the annual risk of exceedance or nonexceedance of annual and annual seasonalperiod flow-duration values.

\section{References Cited}

Environmental Systems Research Institute, 2008, ArcGIS Desktop 9.3 Help-How inverse distance weighted (IDW) interpolation works, accessed May 21, 2012, at http://webhelp.esri.com/arcgiSDEsktop/9.3/index. cfm? TopicName $=$ How_Inverse_Distance_Weighted (IDW)_interpolation_works.

Koltun, G.F., Kula, S.P., and Puskas, B.M., 2006, A streamflow statistics (StreamStats) Web application for Ohio: U.S. Geological Survey Scientific Investigations Report 2006-5312, 62 p.

Koltun, G.F., and Schwartz, R.R., 1987, Multiple-regression equations for estimating low flows at ungaged stream sites in Ohio: U.S. Geological Survey Water-Resources Investigations Report 86-4354, 39 p.

Koltun, G.F., and Whitehead, M.T., 2002, Techniques for estimating selected streamflow characteristics of rural unregulated streams in Ohio: U.S. Geological Survey WaterResources Investigations Report 2002-4068, 50 p.

Kroll, C.N., and Vogel, R.M., 2002, Probability distribution of low streamflow series in the United States: American Society of Civil Engineers, Journal of Hydrologic Engineering, v. 7, no. 2, p. 137-146.

Martin, G.R., and Arihood, L.D., 2010, Methods for estimating selected low-flow frequency statistics for unregulated streams in Kentucky: U.S. Geological Survey Scientific Investigations Report 2010-5217, 83 p., plus 2 pl.

Ohio Division of Geological Survey, 2004, Shaded drift-thickness map of Ohio: Ohio Department of Natural Resources, Division of Geological Survey Map SG-3, generalized page-size version with text, 3 p., scale 1:2,000,000.

SAS Institute, Inc., 2011, The UNIVARIATE Procedure-Calculating percentiles, accessed May 15, 2010, at http://support.sas.com/documentation/cdl/en/ procstat/63104/HTML/default/viewer.htm\#procstat univariate_sect028.htm.

Searcy, J.K., 1959, Manual of hydrology_Part 2, Low flow techniques_Flow-duration curves: U.S. Geological Survey Water-Supply Paper, 1542-A, 33 p.

Shapiro, S.S., and Wilk, M.B., 1965, An analysis of variance test for normality (complete samples): Biometrika, v. 52, no. 3/4, p. 591-611. 
Straub, D.E., 2001, Low-flow characteristics of streams in Ohio through water year 1997: U.S. Geological Survey Water-Resources Investigations Report 2001-4140, 415 p.

Sugiyama, Hironobu, Vudhivanich, Varawoot, Whitaker, A.C., and Lorsirirat, Kosit, 2003, Stochastic flow duration curves for evaluation of flow regimes in rivers: Journal of the American Water Resources Association, v. 39, no. 1, p. 47-58.

The MathWorks, Inc., 2010, MATLAB ver. 7.11 (R2010b)Data Analysis: Natick, Mass., The MathWorks, Inc. [variously paginated], accessed May 5, 2012, at http://www.mathworks.com/help/releases/R2010b/pdf_doc/ matlab/data_analysis.pdf.
Vogel, R.M., and Fennessey, N.M., 1994, Flow-duration curves-I, New interpretation and confidence intervals: American Society of Civil Engineers, Journal of Water Resources Planning and Management, v. 120, no. 4, p. 485-504.

Wright, L.S., and Ensminger, P.A., 2004, Regionalized regression equations for estimating low-flow characteristics for selected Louisiana streams: Louisiana Department of Transportation and Development Water Resources Technical Report No. 75, 33 p. 


\section{Appendix 1. Miscellaneous Supporting Data}

Table 1-1. Streamflow-variability index (SVI) values for locations in Ohio and adjacent States used for SVI-related analyses.

[Streamflow-variability index, in units of base-10 logarithms of cubic feet per second; LTCR, long-term continuous record; LFPR, low-flow partial record]

\begin{tabular}{|c|c|c|c|c|c|}
\hline Station number & Station name & $\begin{array}{l}\text { Decimal } \\
\text { latitude }\end{array}$ & $\begin{array}{l}\text { Decimal } \\
\text { longitude }\end{array}$ & $\begin{array}{l}\text { Streamflow- } \\
\text { variability } \\
\text { index }\end{array}$ & Site type \\
\hline 03089500 & Mill Creek near Berlin Center, Ohio & 41.000 & -80.968 & 0.688 & LTCR \\
\hline 03092000 & Kale Creek near Pricetown, Ohio & 41.140 & -80.995 & 0.808 & LTCR \\
\hline 03092090 & West Branch Mahoning River near Ravenna, Ohio & 41.161 & -81.197 & 0.507 & LTCR \\
\hline 03093000 & Eagle Creek at Phalanx Station, Ohio & 41.261 & -80.954 & 0.449 & LTCR \\
\hline 03094500 & Mahoning River at Warren, Ohio & 41.244 & -80.827 & 0.478 & LTCR \\
\hline 03102000 & Shenango River near Jamestown Pennsylvania & 41.458 & -80.425 & 0.492 & LTCR \\
\hline 03102500 & Little Shenango River at Greenville Pennsylvania & 41.422 & -80.376 & 0.673 & LTCR \\
\hline 03102950 & Pymatuning Creek at Kinsman, Ohio & 41.443 & -80.588 & 0.627 & LTCR \\
\hline 03108980 & Middle Fork Little Beaver Creek near Salem, Ohio & 40.906 & -80.805 & 0.379 & LFPR \\
\hline 03108985 & Cherry Valley Run at Leetonia, Ohio & 40.876 & -80.756 & 0.448 & LFPR \\
\hline 03108996 & Middle Fork Little Beaver Creek at Teegarden, Ohio & 40.822 & -80.827 & 0.439 & LFPR \\
\hline 03109000 & Lisbon Creek at Lisbon, Ohio & 40.782 & -80.765 & 0.621 & LTCR \\
\hline 03109395 & Bull Creek at Negley, Ohio & 40.788 & -80.545 & 0.484 & LFPR \\
\hline 03109400 & North Fork Little Beaver Creek near Negley, Ohio & 40.775 & -80.543 & 0.434 & LFPR \\
\hline 03109500 & Little Beaver Creek near East Liverpool, Ohio & 40.676 & -80.541 & 0.481 & LTCR \\
\hline 03110000 & Yellow Creek near Hammondsville, Ohio & 40.538 & -80.725 & 0.544 & LTCR \\
\hline 03111465 & Short Creek at Adena, Ohio & 40.219 & -80.873 & 0.395 & LFPR \\
\hline 03111500 & Short Creek near Dillonvale, Ohio & 40.193 & -80.734 & 0.380 & LTCR \\
\hline 03111548 & Wheeling Creek below Blaine, Ohio & 40.067 & -80.808 & 0.328 & LTCR \\
\hline 03112000 & Wheeling Creek at Elm Grove West Virginia & 40.045 & -80.661 & 0.708 & LTCR \\
\hline 03114000 & Captina Creek at Armstrongs Mills, Ohio & 39.909 & -80.924 & 0.680 & LTCR \\
\hline 03114500 & Middle Island Creek at Little, West Virginia & 39.475 & -80.997 & 0.751 & LTCR \\
\hline 03115400 & Little Muskingum River at Bloomfield, Ohio & 39.563 & -81.204 & 0.752 & LTCR \\
\hline 03115500 & Little Muskingum River at Fay, Ohio & 39.480 & -81.286 & 0.783 & LTCR \\
\hline 03115650 & East Fork Duck Creek at Lower Salem, Ohio & 39.574 & -81.390 & 0.669 & LFPR \\
\hline 03117310 & Pipe Run at Malvern, Ohio & 40.688 & -81.184 & 0.679 & LFPR \\
\hline 03117500 & Sandy Creek at Waynesburg, Ohio & 40.673 & -81.260 & 0.440 & LTCR \\
\hline 03122900 & Sugar Creek near West Lebanon, Ohio & 40.737 & -81.653 & 0.609 & LFPR \\
\hline 03123000 & $\begin{array}{l}\text { Sugar Creek above Beach City Dam at Beach City, } \\
\text { Ohio }\end{array}$ & 40.657 & -81.577 & 0.541 & LTCR \\
\hline 03123300 & South Fork Sugar Creek near Dundee, Ohio & 40.593 & -81.614 & 0.613 & LFPR \\
\hline 03125000 & Home Creek near New Philadelphia, Ohio & 40.468 & -81.403 & 1.037 & LTCR \\
\hline 03127100 & Crooked Creek near Stillwater, Ohio & 40.308 & -81.324 & 0.690 & LFPR \\
\hline 03129100 & White Eyes Creek near Fresno, Ohio & 40.305 & -81.750 & 0.673 & LFPR \\
\hline 03130500 & Touby Run at Mansfield, Ohio & 40.765 & -82.545 & 0.512 & LTCR \\
\hline 03134000 & Jerome Fork at Jeromeville, Ohio & 40.802 & -82.200 & 0.569 & LTCR \\
\hline 03134300 & Muddy Fork near Rowsburg, Ohio & 40.836 & -82.138 & 0.549 & LFPR \\
\hline 03136500 & Kokosing River at Mount Vernon, Ohio & 40.406 & -82.500 & 0.424 & LTCR \\
\hline 03137000 & Kokosing River at Millwood, Ohio & 40.398 & -82.286 & 0.434 & LTCR \\
\hline
\end{tabular}


Table 1-1. Streamflow-variability index (SVI) values for locations in Ohio and adjacent States used for SVI-related analyses.Continued

[Streamflow-variability index, in units of base-10 logarithms of cubic feet per second; LTCR, long-term continuous record; LFPR, low-flow partial record]

\begin{tabular}{|c|c|c|c|c|c|}
\hline Station number & Station name & $\begin{array}{l}\text { Decimal } \\
\text { latitude }\end{array}$ & $\begin{array}{l}\text { Decimal } \\
\text { longitude }\end{array}$ & $\begin{array}{l}\text { Streamflow- } \\
\text { variability } \\
\text { index }\end{array}$ & Site type \\
\hline 03138790 & Killbuck Creek at Burbank, Ohio & 40.990 & -81.995 & 0.650 & LFPR \\
\hline 03139000 & Killbuck Creek at Killbuck, Ohio & 40.481 & -81.986 & 0.448 & LTCR \\
\hline 03140000 & Mill Creek near Coshocton, Ohio & 40.363 & -81.862 & 0.640 & LTCR \\
\hline 03143760 & Wakatomika Creek near Perryton, Ohio & 40.220 & -82.181 & 0.511 & LFPR \\
\hline 03144000 & Wakatomika Creek near Frazeysburg, Ohio & 40.133 & -82.148 & 0.522 & LTCR \\
\hline 03146000 & North Fork Licking River at Utica, Ohio & 40.228 & -82.452 & 0.598 & LTCR \\
\hline 03146500 & Licking River near Newark, Ohio & 40.059 & -82.340 & 0.467 & LTCR \\
\hline 03149500 & Salt Creek near Chandlersville, Ohio & 39.909 & -81.860 & 0.655 & LTCR \\
\hline 03154500 & Reedy Creek near Reedy, West Virginia & 38.961 & -81.390 & 0.862 & LTCR \\
\hline 03155500 & Hughes River at Cisco, West Virginia & 39.119 & -81.277 & 0.739 & LTCR \\
\hline 03155895 & Hocking River at Union St at Lancaster, Ohio & 39.718 & -82.610 & 0.415 & LFPR \\
\hline 03156000 & Hunters Run at Lancaster, Ohio & 39.709 & -82.622 & 0.422 & LTCR \\
\hline 03156549 & Center Branch Rush Creek near Junction City, Ohio & 39.723 & -82.343 & 0.632 & LFPR \\
\hline 03156550 & Rush Creek near Junction City, Ohio & 39.720 & -82.350 & 0.552 & LFPR \\
\hline 03156900 & Clear Creek at Clearport, Ohio & 39.618 & -82.680 & 0.667 & LFPR \\
\hline 03157000 & Clear Creek near Rockbridge, Ohio & 39.588 & -82.578 & 0.376 & LTCR \\
\hline 03157500 & Hocking River at Enterprise, Ohio & 39.565 & -82.475 & 0.445 & LTCR \\
\hline 03159536 & West Branch Shade River at Chester, Ohio & 39.100 & -81.926 & 0.689 & LFPR \\
\hline 03159538 & Middle Branch Shade River at Chester, Ohio & 39.104 & -81.923 & 0.704 & LFPR \\
\hline 03159540 & Shade River near Chester, Ohio & 39.064 & -81.882 & 0.687 & LTCR \\
\hline 03159555 & East Branch Shade River near Tuppers Plains, Ohio & 39.141 & -81.877 & 0.753 & LFPR \\
\hline 03201600 & $\begin{array}{l}\text { Sandy Run ab Big Four Hollow Creek near Lake } \\
\text { Hope, Ohio }\end{array}$ & 39.363 & -82.313 & 0.581 & LTCR \\
\hline 03201700 & Big Four Hollow Creek near Lake Hope, Ohio & 39.363 & -82.314 & 0.623 & LTCR \\
\hline 03201800 & Sandy Run near Lake Hope, Ohio & 39.334 & -82.332 & 1.077 & LTCR \\
\hline 03201990 & Little Raccoon Creek near Vinton, Ohio & 38.953 & -82.366 & 0.616 & LFPR \\
\hline 03202000 & Raccoon Creek at Adamsville, Ohio & 38.876 & -82.356 & 0.655 & LTCR \\
\hline 03204000 & Guyandotte River at Branchland, West Virginia & 38.221 & -82.203 & 0.545 & LTCR \\
\hline 03204500 & Mud River near Milton, West Virginia & 38.388 & -82.113 & 0.820 & LTCR \\
\hline 03216500 & Little Sandy River at Grayson, Kentucky & 38.330 & -82.939 & 0.720 & LTCR \\
\hline 03216540 & East Fork Little Sandy River near Fallsburg, Kentucky & 38.234 & -82.709 & 0.800 & LTCR \\
\hline 03217500 & Scioto River at Larue, Ohio & 40.572 & -83.384 & 0.664 & LTCR \\
\hline 03218000 & Little Scioto River above Marion, Ohio & 40.629 & -83.170 & 1.008 & LTCR \\
\hline 03219500 & Scioto River near Prospect, Ohio & 40.420 & -83.197 & 0.639 & LTCR \\
\hline 03219590 & Bokes Creek near Warrensburg, Ohio & 40.322 & -83.175 & 1.539 & LTCR \\
\hline 03219600 & Eagon Run near Warrensburg, Ohio & 40.328 & -83.153 & 0.914 & LTCR \\
\hline 03220000 & Mill Creek near Bellepoint, Ohio & 40.248 & -83.174 & 0.689 & LTCR \\
\hline 03222500 & Olentangy River near New Winchester, Ohio & 40.747 & -82.905 & 0.670 & LFPR \\
\hline 03222700 & Mud Run near Caledonia, Ohio & 40.689 & -82.962 & 0.671 & LFPR \\
\hline 03222800 & Flat Run near Caledonia, Ohio & 40.631 & -82.948 & 0.715 & LFPR \\
\hline 03223000 & Olentangy River at Claridon, Ohio & 40.583 & -82.989 & 0.681 & LTCR \\
\hline
\end{tabular}


Table 1-1. Streamflow-variability index (SVI) values for locations in Ohio and adjacent States used for SVI-related analyses.Continued

[Streamflow-variability index, in units of base-10 logarithms of cubic feet per second; LTCR, long-term continuous record; LFPR, low-flow partial record]

\begin{tabular}{|c|c|c|c|c|c|}
\hline Station number & Station name & $\begin{array}{l}\text { Decimal } \\
\text { latitude }\end{array}$ & $\begin{array}{c}\text { Decimal } \\
\text { longitude }\end{array}$ & $\begin{array}{c}\text { Streamflow- } \\
\text { variability } \\
\text { index }\end{array}$ & Site type \\
\hline 03224000 & Shaw Creek at Shawtown, Ohio & 40.483 & -82.957 & 0.690 & LFPR \\
\hline 03224500 & Whetstone Creek near Ashley, Ohio & 40.455 & -82.958 & 0.618 & LTCR \\
\hline 03230230 & Big Darby Creek near West Jefferson, Ohio & 39.980 & -83.249 & 0.642 & LFPR \\
\hline 03230310 & Little Darby Creek at West Jefferson, Ohio & 39.951 & -83.269 & 0.651 & LFPR \\
\hline 03230400 & Big Darby Creek at Darbydale, Ohio & 39.850 & -83.189 & 0.630 & LFPR \\
\hline 03230500 & Big Darby Creek at Darbyville, Ohio & 39.701 & -83.110 & 0.583 & LTCR \\
\hline 03230600 & Hominy Creek at Circleville, Ohio & 39.591 & -82.924 & 0.542 & LFPR \\
\hline 03230745 & Deer Creek at US Route 142 near London, Ohio & 39.905 & -83.393 & 0.586 & LFPR \\
\hline 03230800 & Deer Creek at Mount Sterling, Ohio & 39.715 & -83.257 & 0.500 & LTCR \\
\hline 03231800 & Sugar Creek near Rock Mills, Ohio & 39.470 & -83.435 & 0.987 & LFPR \\
\hline 03232000 & Paint Creek near Greenfield, Ohio & 39.379 & -83.375 & 0.742 & LTCR \\
\hline 03235000 & Salt Creek at Tarlton, Ohio & 39.556 & -82.781 & 0.796 & LTCR \\
\hline 03235090 & Salt Creek at Adelphi, Ohio & 39.473 & -82.750 & 0.624 & LFPR \\
\hline 03236000 & Salt Creek near Londonderry, Ohio & 39.256 & -82.770 & 0.600 & LTCR \\
\hline 03236800 & Salt Creek at Richmond Dale, Ohio & 39.200 & -82.811 & 0.630 & LFPR \\
\hline 03237280 & Upper Twin Creek at McGaw, Ohio & 38.644 & -83.216 & 0.921 & LTCR \\
\hline 03237500 & Ohio Brush Creek near West Union, Ohio & 38.804 & -83.421 & 0.788 & LTCR \\
\hline 03237900 & Cabin Creek near Tollesboro, Kentucky & 38.568 & -83.537 & 1.150 & LTCR \\
\hline 03238500 & White Oak Creek near Georgetown, Ohio & 38.858 & -83.929 & 0.818 & LTCR \\
\hline 03239000 & Little Miami River near Selma, Ohio & 39.810 & -83.739 & 0.531 & LFPR \\
\hline 03240000 & Little Miami River near Oldtown, Ohio & 39.748 & -83.931 & 0.409 & LTCR \\
\hline 03240500 & North Fork Massie Creek at Cedarville, Ohio & 39.757 & -83.790 & 0.580 & LTCR \\
\hline 03241000 & South Fork Massie Creek near Cedarville, Ohio & 39.739 & -83.764 & 0.727 & LTCR \\
\hline 03241500 & Massies Creek at Wilberforce, Ohio & 39.723 & -83.883 & 0.514 & LTCR \\
\hline 03242050 & Little Miami River near Spring Valley, Ohio & 39.583 & -84.030 & 0.323 & LTCR \\
\hline 03242200 & Anderson Fork near New Burlington, Ohio & 39.566 & -83.903 & 0.669 & LTCR \\
\hline 03246200 & East Fork Little Miami River near Marathon, Ohio & 39.115 & -84.025 & 0.676 & LTCR \\
\hline 03246500 & East Fork Little Miami River at Williamsburg, Ohio & 39.053 & -84.050 & 0.881 & LTCR \\
\hline 03251000 & North Fork Licking River near Lewisburg, Kentucky & 38.549 & -83.794 & 1.250 & LTCR \\
\hline 03254400 & North Fork Grassy Creek near Piner, Kentucky & 38.792 & -84.514 & 1.350 & LTCR \\
\hline 03260700 & Bokengehalas Creek near De Graff, Ohio & 40.347 & -83.891 & 0.371 & LTCR \\
\hline 03260800 & Stony Creek near De Graff, Ohio & 40.291 & -83.910 & 0.358 & LTCR \\
\hline 03265000 & Stillwater River at Pleasant Hill, Ohio & 40.058 & -84.356 & 0.521 & LTCR \\
\hline 03266000 & Stillwater River at Englewood, Ohio & 39.870 & -84.286 & 0.533 & LTCR \\
\hline 03266500 & Mad River at Zanesfield, Ohio & 40.350 & -83.674 & 0.402 & LTCR \\
\hline 03267000 & Mad River near Urbana, Ohio & 40.108 & -83.799 & 0.253 & LTCR \\
\hline 03268000 & Buck Creek at New Moorefield, Ohio & 39.992 & -83.715 & 0.243 & LTCR \\
\hline 03270800 & Wolf Creek at Trotwood, Ohio & 39.794 & -84.310 & 0.644 & LTCR \\
\hline 03271000 & Wolf Creek at Dayton, Ohio & 39.767 & -84.237 & 0.494 & LTCR \\
\hline 03271400 & Bear Creek at Ellerton, Ohio & 39.673 & -84.310 & 0.545 & LFPR \\
\hline 03271736 & Twin Creek at Lewisburg, Ohio & 39.855 & -84.532 & 0.600 & LFPR \\
\hline
\end{tabular}


Table 1-1. Streamflow-variability index (SVI) values for locations in Ohio and adjacent States used for SVI-related analyses.Continued

[Streamflow-variability index, in units of base-10 logarithms of cubic feet per second; LTCR, long-term continuous record; LFPR, low-flow partial record]

\begin{tabular}{|c|c|c|c|c|c|}
\hline Station number & Station name & $\begin{array}{l}\text { Decimal } \\
\text { latitude }\end{array}$ & $\begin{array}{c}\text { Decimal } \\
\text { longitude }\end{array}$ & $\begin{array}{c}\text { Streamflow- } \\
\text { variability } \\
\text { index }\end{array}$ & Site type \\
\hline 03271800 & Twin Creek near Ingomar, Ohio & 39.708 & -84.525 & 0.574 & LTCR \\
\hline 03272000 & Twin Creek near Germantown, Ohio & 39.638 & -84.404 & 0.577 & LTCR \\
\hline 03272700 & Sevenmile Creek at Camden, Ohio & 39.629 & -84.644 & 0.560 & LTCR \\
\hline 03272800 & Sevenmile Creek at Collinsville, Ohio & 39.523 & -84.611 & 0.578 & LTCR \\
\hline 03274650 & Whitewater River near Economy, Indiana & 40.004 & -85.116 & 0.577 & LTCR \\
\hline 03274750 & Whitewater River near Hagerstown, Indiana & 39.874 & -85.163 & 0.332 & LTCR \\
\hline 03275000 & Whitewater River near Alpine, Indiana & 39.579 & -85.158 & 0.397 & LTCR \\
\hline 03275600 & East Fork Whitewater River at Abington, Indiana & 39.733 & -84.960 & 0.397 & LTCR \\
\hline 03276700 & South Hogan Creek near Dillsboro, Indiana & 39.030 & -85.038 & 0.818 & LTCR \\
\hline 03291500 & Eagle Creek at Glencoe, Kentucky & 38.705 & -84.824 & 1.350 & LTCR \\
\hline 03325500 & Mississinewa River near Ridgeville, Indiana & 40.280 & -84.992 & 0.644 & LTCR \\
\hline 04096515 & South Branch Hog Creek near Allen, Michigan & 41.949 & -84.828 & 0.395 & LTCR \\
\hline 04175700 & River Raisin near Tecumseh, Michigan & 41.944 & -83.945 & 0.316 & LTCR \\
\hline 04176000 & River Raisin near Adrian, Michigan & 41.905 & -83.981 & 0.343 & LTCR \\
\hline 04177720 & Fish Creek at Hamilton, Indiana & 41.532 & -84.904 & 0.500 & LTCR \\
\hline 04178000 & St. Joseph River near Newville, Indiana & 41.385 & -84.802 & 0.518 & LTCR \\
\hline 04179500 & Cedar Creek at Auburn, Indiana & 41.366 & -85.052 & 0.551 & LTCR \\
\hline 04180000 & Cedar Creek near Cedarville, Indiana & 41.219 & -85.076 & 0.447 & LTCR \\
\hline 04182810 & Spy Run Creek at Fort Wayne, Indiana & 41.105 & -85.153 & 0.415 & LTCR \\
\hline 04184500 & Bean Creek at Powers, Ohio & 41.659 & -84.249 & 0.495 & LTCR \\
\hline 04185440 & Unnamed Tributary to Lost Creek near Farmer, Ohio & 41.362 & -84.691 & 0.825 & LTCR \\
\hline 04188500 & Eagle Creek near Findlay, Ohio & 40.993 & -83.651 & 1.123 & LTCR \\
\hline 04189500 & Blanchard River at Glandorf, Ohio & 41.044 & -84.082 & 0.671 & LTCR \\
\hline 04196000 & Sandusky River near Bucyrus, Ohio & 40.804 & -83.006 & 0.632 & LTCR \\
\hline 04196500 & Sandusky River near Upper Sandusky, Ohio & 40.851 & -83.256 & 0.660 & LTCR \\
\hline 04197000 & Sandusky River near Mexico, Ohio & 41.044 & -83.195 & 0.637 & LTCR \\
\hline 04197100 & Honey Creek at Melmore, Ohio & 41.022 & -83.110 & 0.765 & LTCR \\
\hline 04197170 & Rock Creek at Tiffin, Ohio & 41.114 & -83.168 & 0.553 & LTCR \\
\hline 04198000 & Sandusky River near Fremont, Ohio & 41.308 & -83.159 & 0.644 & LTCR \\
\hline 04198500 & East Branch Huron River near Norwalk, Ohio & 41.249 & -82.648 & 0.567 & LTCR \\
\hline 04199000 & Huron River at Milan, Ohio & 41.301 & -82.608 & 0.582 & LTCR \\
\hline 04199500 & Vermilion River near Vermilion, Ohio & 41.382 & -82.317 & 0.766 & LTCR \\
\hline 04200000 & East Branch Black River at Elyria, Ohio & 41.348 & -82.094 & 0.903 & LTCR \\
\hline 04206211 & Park Creek at Bath Center, Ohio & 41.179 & -81.636 & 0.583 & LFPR \\
\hline 04207200 & Tinkers Creek at Bedford, Ohio & 41.384 & -81.527 & 0.420 & LTCR \\
\hline 04209000 & Chagrin River at Willoughby, Ohio & 41.631 & -81.403 & 0.427 & LTCR \\
\hline 04209500 & Grand River near North Bristol, Ohio & 41.413 & -80.912 & 0.661 & LFPR \\
\hline 04210000 & Phelps Creek near Windsor, Ohio & 41.516 & -80.935 & 0.735 & LTCR \\
\hline 04210500 & Grand River near Rome, Ohio & 41.606 & -80.894 & 0.653 & LFPR \\
\hline 04212000 & Grand River near Madison, Ohio & 41.741 & -81.046 & 0.802 & LTCR \\
\hline 04212100 & Grand River near Painesville, Ohio & 41.719 & -81.228 & 0.633 & LTCR \\
\hline
\end{tabular}


Table 1-1. Streamflow-variability index (SVI) values for locations in Ohio and adjacent States used for SVI-related analyses.Continued

[Streamflow-variability index, in units of base-10 logarithms of cubic feet per second; LTCR, long-term continuous record; LFPR, low-flow partial record]

\begin{tabular}{|c|c|c|c|c|c|}
\hline Station number & Station name & $\begin{array}{l}\text { Decimal } \\
\text { latitude }\end{array}$ & $\begin{array}{c}\text { Decimal } \\
\text { longitude }\end{array}$ & $\begin{array}{l}\text { Streamflow- } \\
\text { variability } \\
\text { index }\end{array}$ & Site type \\
\hline 04212500 & Ashtabula River near Ashtabula, Ohio & 41.856 & -80.762 & 1.062 & LTCR \\
\hline 04213000 & Conneaut Creek at Conneaut, Ohio & 41.927 & -80.604 & 0.626 & LTCR \\
\hline
\end{tabular}



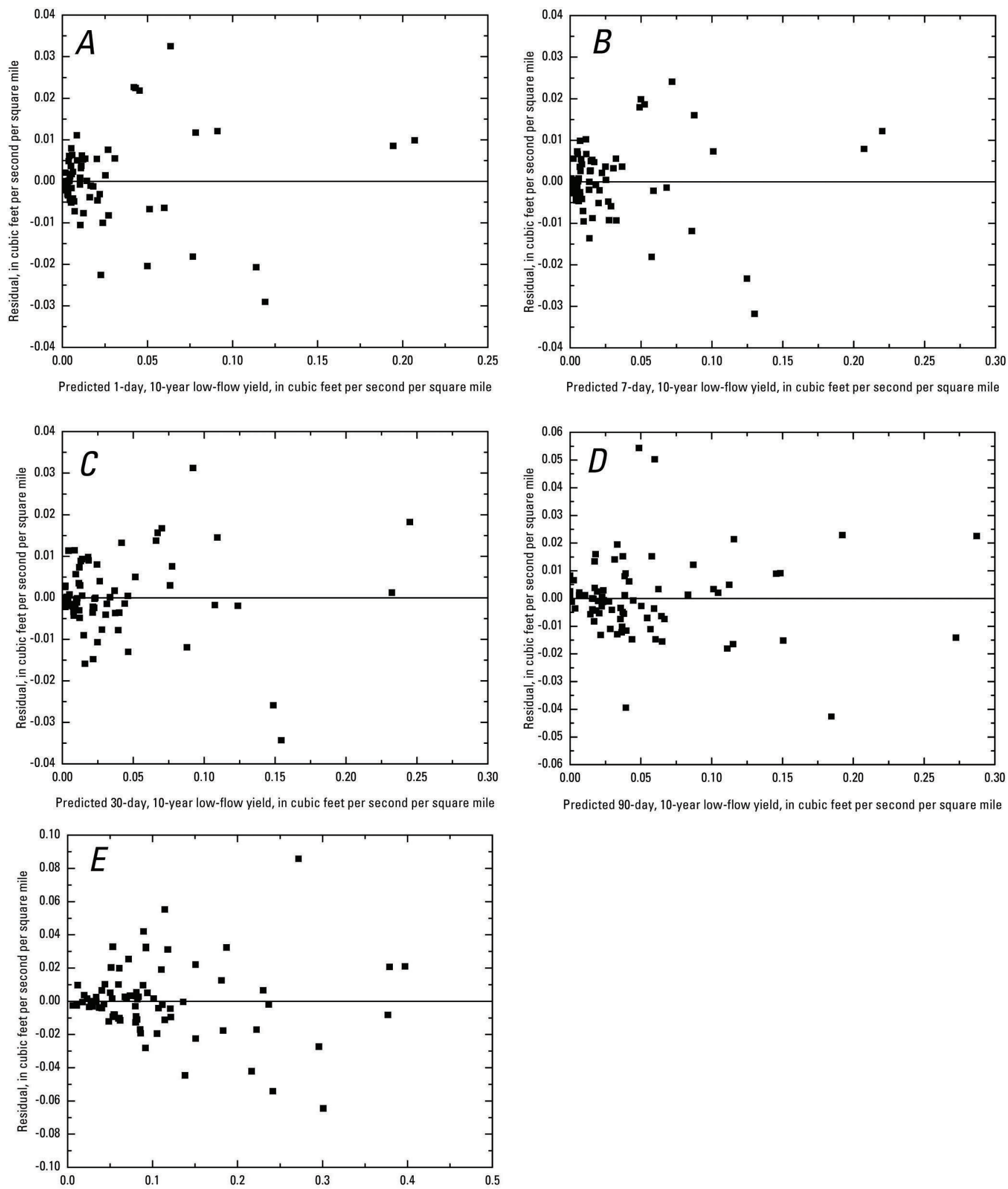

Predicted 80-percent duration flow yield, in cubic feet per second per square mile

Figure 1-1. Scatter plots of regression residuals as a function of predicted low-flow yields. A, 1-day, 10-year. B, 7-day, 10-year. $C, 30$-day, 10 -year. $D, 90$-day, 10-year. $E$, 80-percent duration low-flow yields. 


\section{Appendix 2. Basin Characteristics Tested for Use in Low-Flow Regression Analyses in Ohio}

\section{Location Variables}

Latitude of basin centroid ( Lat $_{c}$ ) - latitude of basin centroid, in decimal degrees.

Longitude of basin centroid ( Long $_{c}$ ) - longitude of basin centroid, in decimal degrees. Although all decimal longitudes in the study are west of the Prime Meridian and conventionally expressed as negative values, they were converted to positive values for the regression analyses.

\section{Hydrologic Variables}

Streamflow-variability index $(S V I)$ - streamflow-variability index at the outlet of the basin (index value) — determined by interpolating a grid (with 1,000 $\mathrm{ft}^{2}$ cells) from at-site values for the streamflow-variability index computed for 163 rural, unregulated streamflow-gaging stations in Ohio and nearby areas of adjacent States. Grid interpolation was done using an inverse distance-weighting (IDW) technique in the Spatial Analyst extension of ArcInfo. Interpolation was based on the 12 nearest neighboring streamflow-gaging stations. For further discussion on streamflow-variability index, see Koltun and Whitehead (2002).

Slope $_{10-85}\left(\mathrm{SL}_{10-85}\right)$ - channel slope, in feet per mile - computed by (1) determining the longest flow path from the point of interest to a topographic divide (which generally corresponded to the basin divide), (2) determining the elevation at 10 percent of the distance along the longest flow path upstream from the point at which the flow statistic is desired $\left(\mathrm{E}_{10}\right)$, (3) determining the elevation at 85 percent of the distance along the longest flow path upstream from the point at which the flow statistic is desired $\left(\mathrm{E}_{85}\right)$, (4) determining the length of the stream segment between points 10 and 85 percent of the distance along the longest flow path upstream from the point at which the flow statistic is desired $\left(\mathrm{L}_{10-85}\right)$, and then (5) dividing the change in elevation $\left(\mathrm{E}_{85}-\mathrm{E}_{10}\right)$ by $\mathrm{L}_{10-85}$.

Drainage area (DA_SS) — area, in square miles — determined by use of the StreamStats application for Ohio (Koltun and others, 2006). StreamStats uses a specially processed digital elevation model to determine drainage areas. StreamStats will compute drainage areas that are comparable to map-based determinations in most cases; however, limitations associated with digital elevation model resolution and (or) scale, as well as other possible factors, may result in erroneous drainage area determinations. It is the user's responsibility to verify that the drainage boundaries (and consequently the drainage area) derived by StreamStats are correct.
Longest flow path (LFPath) - flow path, the length, in miles - computed by determining the longest flow path from the point of interest to a topographic divide (which generally corresponded to the basin divide). StreamStats determines the longest flow path based on a specially processed digital elevation model that includes the streams of the 1:100,000 National Hydrography Dataset (U.S. Geological Survey, 2004).

\section{Land-Cover Variables}

Agriculture (Agric) - percentage of the basin classified as agriculture-determined by (1) summing the areas of the 2001 National Land Cover Dataset (NLCD) grid cells (U.S. Geological Survey, 2007) contained within the basin boundary that are classified as pasture/hay or cultivated crops (land-cover class codes 81 and 82, respectively), (2) dividing by the area of the basin, and then (3) multiplying by 100 .

Impervious (Imperv) - percentage of the basin classified as having impervious surfaces - determined by (1) summing the per-pixel estimates of the imperviousness of the land cover for all the areas of the 2001 NLCD (U.S. Geological Survey, 2007) contained within the basin boundary, (2) dividing by the number of cells in the basin, and then (3) multiplying by 100.

Open water (OpenWater) - percentage of the basin classified as open water - determined by (1) summing the areas of the 2001 NLCD grid cells (U.S. Geological Survey, 2007) contained within the basin boundary that are classified as open water (land-cover class code 1), (2) dividing by the area of the basin, and then (3) multiplying by 100.

Storage (W) - percentage of the basin classified as water and wetlands - determined by (1) summing the areas of the 2001 NLCD grid cells (U.S. Geological Survey, 2007) contained within the basin boundary that are classified as water, woody wetlands, and emergent herbaceous wetlands (land-cover class codes 11,90 , and 95, respectively), (2) dividing by the area of the basin, and then (3) multiplying by 100 .

Wetland (Wetland) — percentage of the basin classified as wetlands - determined by (1) summing the areas of the 2001 NLCD grid cells (U.S. Geological Survey, 2007) contained within the basin boundary that are classified as woody wetlands and emergent herbaceous wetlands (land-cover class codes 90 and 95, respectively), (2) dividing by the area of the basin, and then (3) multiplying by 100 . 
Vegetated (Veg) - percentage of the basin classified as vegetated - determined by (1) summing the areas of the 2001 NLCD grid cells (U.S. Geological Survey, 2007) contained within the basin boundary that are classified as deciduous forest, evergreen forest, mixed forest, developed, open space (lawn grasses), shrub/scrub, and grassland/herbaceous (landcover class codes $41,42,43,21,52$, and 71 respectively), (2) dividing by the area of the basin, and then (3) multiplying by 100 .

Forest (Forest) - percentage of the basin classified as forestdetermined by (1) summing the areas of the 2001 NLCD grid cells (U.S. Geological Survey, 2007) contained within the basin boundary that are classified as deciduous forest, evergreen forest, or mixed forest (land-cover class codes 41 , 42 , and 43 respectively), (2) dividing by the area of the basin, and then (3) multiplying by 100 .

Tree canopy (TreeCanopy) - percentage of the basin classified as having tree canopy-determined by (1) summing the per-pixel density of the tree cover of the 2001 NLCD (U.S. Geological Survey, 2007) contained within the basin boundary, (2) dividing by the number of cells in the basin, and then (3) multiplying by 100.

\section{Geologic Variables}

Primary rock carbonate or evaporite (CarbEvap1) percentage of the basin classified as carbonate or evaporite for the primary rock type - determined by (1) summing the areas of the Preliminary Integrated Geologic Map Databases for the United States (Nicholson and others, 2005a; Nicholson and others, 2005b; Dicken and others, 2005) contained within the basin boundary that are classified as carbonate or evaporite rock for the primary rock type, (2) dividing by the area of the basin, and then (3) multiplying by 100 .

Primary/secondary rock carbonate or evaporite rock (CarbEvap12) - percentage of the basin classified as carbonate or evaporite for the primary or secondary rock type - determined by (1) summing the areas of the Preliminary Integrated Geologic Map Databases for the United States (Nicholson and others, 2005a; Nicholson and others, 2005b; Dicken and others, 2005) contained within the basin boundary that are classified as carbonate or evaporite rock for the primary or secondary rock type, (2) dividing by the area of the basin, and then (3) multiplying by 100 .

Known karst (KarstKnown) - percentage of the basin classified as known karst areas - determined by (1) summing the areas of the State Geological Survey karst maps (Pavey and others, 1999; Shaffer and others, 2007) contained within the basin boundary that are classified as known karst, (2) dividing by the area of the basin, and then (3) multiplying by 100 .
Probable karst (KarstProb) - percentage of the basin classified as probable karst areas - determined by (1) summing the areas of the State Geological Survey karst maps (Pavey and others, 1999; Shaffer and others, 2007; Michigan Department of Environmental Quality and others, 2004) contained within the basin boundary that are classified as probable karst, (2) dividing by the area of the basin, and then (3) multiplying by 100 .

Drift thickness 0-50 feet (Drift_gt50 ) - percentage of the basin classified as having a $0-50$ feet glacial-drift thicknessdetermined by (1) standardizing and summing the areas of the State Geological Survey drift thickness maps (Ohio Department of Natural Resources, 2000; Dintaman and Gray, 2002; Michigan Department of Environmental Quality and others, 2004) contained within the basin boundary that are classified as having drift thickness of 0-50 feet, (2) dividing by the area of the basin, and then (3) multiplying by 100 .

Drift thickness greater than 50 feet (Drift_0_50)percentage of the basin classified as having greater than 50 feet glacial-drift thickness - determined by (1) standardizing and summing the areas of the State Geological Survey drift thickness maps (Ohio Department of Natural Resources, 2000; Dintaman and Gray, 2002; Michigan Department of Environmental Quality and others, 2004) contained within the basin boundary that are classified as having drift thickness of greater than 50 feet, (2) dividing by the area of the basin, and then (3) multiplying by 100 .

Glaciated (Glaciated) - percentage of the basin classified as having been glaciated - determined by (1) summing the glaciated areas (Soller and Packard, 1998) contained within the basin boundary that are classified as having been glaciated, (2) dividing by the area of the basin, and then (3) multiplying by 100 .

\section{Soil Variables}

Depth to water table (DpthWatTab) - area-weighted average STATSGO2 depth to water table, in centimetersdetermined by (1) multiplying each depth value, aggregated by dominant component, of the 2006 U.S. General Soil Map (STATSGO2) (U.S. Department of Agriculture-National Resources Conservation Service, 2006) as defined by the NRCS Soil Data Viewer 5.2 (U.S. Department of AgricultureNational Resources Conservation Service, 2008) by its area of the basin, (2) summing those values, and then (3) dividing by the total area of the basin.

\footnotetext{
Hydrologic soil group percentages (SoilGroupA, SoilGroupB, SoilGroupC, SoilGroupD, SoilGroupCD, or SoilGroupBD) - percentage of basin classified in each of the six STATSGO2 soil groups-determined by (1) summing the gridded areas of the 2006 U.S. General Soil Map
} 
(STATSGO2) (U.S. Department of Agriculture-National Resources Conservation Service, 2006) contained within the basin boundary that are classified as soil group A, B, C, D, $\mathrm{CD}$, or $\mathrm{BD}$, aggregated by dominant component, as defined by the Natural Resources Conversation Service (NRCS) Soil Data Viewer 5.2 (U.S. Department of Agriculture-National Resources Conservation Service, 2008), (2) dividing by the area of the basin, and then (3) multiplying by 100 .

Mean hydrologic soil group index (SoilGroupMean) - area weighted average STATSGO2 soil group index-determined by (1) assigning each soil group, aggregated by dominant component, of the 2006 U.S. General Soil Map (STATSGO2) (U.S. Department of Agriculture-National Resources Conservation Service, 2006) as defined by the NRCS Soil Data Viewer 5.2 (U.S. Department of Agriculture-National Resources Conservation Service, 2008) a numeric value: $\mathrm{A}[1], \mathrm{B}[3], \mathrm{C}$ or $\mathrm{BD}[5], \mathrm{CD}[6]$, and $\mathrm{D}[7]$, (2) multiplying each numeric class by its area of the basin, (3) summing those values, and then (4) dividing by the total area of the basin.

Soil drainage class percentages (DrainCl1, DrainCl2, DrainC13, DrainC14, DrainCl5) - percentage of basin classified in each of the five STATSGO2 soil drainage classes - determined by (1) summing the gridded areas of the 2006 U.S. General Soil Map (STATSGO2) (U.S. Department of Agriculture-National Resources Conservation Service, 2006), aggregated by dominant component as defined by the NRCS Soil Data Viewer 5.2 (U.S. Department of AgricultureNational Resources Conservation Service, 2008) contained within the basin boundary that are classified as drainage class Well drained, Moderately well drained, Somewhat poorly drained, Poorly drained, or Very poorly drained, (2) dividing by the area of the basin, and then (3) multiplying by 100 .

Mean soil drainage class index (DrainClMean) - area weighted average STATSGO2 soil group index-determined by (1) assigning each soil drainage class of the 2006 U.S. General Soil Map (STATSGO2) (U.S. Department of Agriculture-National Resources Conservation Service, 2006) as defined by the NRCS Soil Data Viewer 5.2 (U.S. Department of Agriculture-National Resources Conservation Service, 2008) a numeric value: well drained [1], Moderately well drained [2] Somewhat poorly drained [3], Poorly drained [4], or Very poorly drained [5], (2) multiplying each numeric class by its area of the basin, (3) summing those values, and then (4) dividing by the total area of the basin.

\section{Climatic Variables}

Mean annual precipitation 1 at the basin centroid (Precip1) - the value, in inches, of the grid cell from Whitehead's (2002) rasterized triangular irregular network of Harstine's (1991) isoline map of mean annual precipitation for the period 1931-80 that is collocated with the basin centroid.
Mean annual precipitation 2 at the basin centroid (Precip2) - the value, in inches, of the grid cell from the Parameter-elevation Regressions on Independent Slopes Model (PRISM) dataset of mean annual precipitation for the period 1971-2000 (PRISM Climate Group, 2006a) that is collocated with the basin centroid.

Basin-mean annual precipitation (MeanAnnP) - the value, in inches, determined by summing and averaging grid-cell values from the 1971-2000 mean annual precipitation PRISM dataset (PRISM Climate Group, 2006a) that are contained within the basin boundary.

Basin-mean July precipitation (MeanJuIP) - the value, in inches, determined by summing and averaging grid-cell values from the 1971-2000 mean July temperature PRISM dataset (PRISM Climate Group, 2006a) that are located within the basin boundary.

Basin-minimum Autumn precipitation (MinAutP) - the value, in inches, determined by (1) summing, for each grid cell, the values assigned to the average minimum September, October, and November grid cells from the 1971-2000 monthly average minimum precipitation PRISM datasets (PRISM Climate Group, 2006a) that are located within the basin boundary, and (2) selecting the minimum value of those grid-cell sums.

\section{Basin-mean minimum Autumn precipitation}

(MinAutP2) - the value, in inches, determined by (1) summing, for each grid cell, the values assigned to the average minimum September, October, and November grid cells from the 1971-2000 monthly average minimum precipitation PRISM datasets (PRISM Climate Group, 2006a) that are located within the basin boundary, and (2) dividing by the number of cells in the basin.

Basin-maximum Autumn temperature (MaxAutT) - the value, in degrees F, determined by (1) for each grid cell located within the basin, selecting the maximum temperature value from the average maximum September, October, and November temperature grids from the 1971-2000 PRISM dataset (PRISM Climate Group, 2006b), and (2) selecting the largest of the grid-cell maximum values.

\section{Basin-mean maximum Autumn temperature}

(MaxAutT2) - the value, in degrees F, determined by (1) for each grid cell located within the basin, selecting the maximum temperature value from the average maximum September, October, and November temperature grids from the 19712000 PRISM datasets (PRISM Climate Group, 2006b),

(2) summing the maximum values for each grid cell, and then, (3) dividing by the number of cells in the basin.

Table 2-1. Basin characteristics determined for selected streamgages in Ohio and adjacent States. (Available in Excel format. Click here to open table. 


\section{References Cited}

Dicken, C.L., Nicholson, S.W., Horton, J.D., Kinney, S.A., Gunther, Gregory, Foose, M.P., and Mueller, J.A.L., 2005, Preliminary integrated geologic map databases for the United States_-Delaware, Maryland, New York, Pennsylvania, and Virginia: U.S. Geological Survey Open-File Report 2005-1325, ver. 1.1, accessed November 18, 2009, at http://pubs.usgs.gov/of/2005/1325/.

Dintaman, C.R., and Gray, H.H., 2002, Thickness ranges of unconsolidated deposits in Indiana: Indiana Geological Survey digital data, 1:500,000, accessed July 27, 2009, at http://inmap.indiana.edu/download.html.

Harstine, L.J., 1991, Hydrologic atlas for Ohio-Average annual precipitation, temperature, streamflow, and water loss for a 50 year period, 1931-1980: Ohio Department of Natural Resources, Division of Water, Water Inventory Report 28, 13 p., 4pl.

Koltun, G.F., Kula, S.P., and Puskas, B.M., 2006, A streamflow statistics (StreamStats) Web application for Ohio: U.S. Geological Survey Scientific Investigations Report 2006-5312, 62 p.

Koltun, G.F., and Whitehead, M.T., 2002, Techniques for estimating selected streamflow characteristics of rural unregulated streams in Ohio: U.S. Geological Survey WaterResources Investigations Report 2002-4068, 50 p.

Michigan Department of Environmental Quality, U.S. Geological Survey, and Michigan State University, 2004, Aquifer characteristics of glacial drift-Data based on digitizing the 1981 Hydrological Atlas of Michigan-Plate 26, accessed August 4, 2009, at http://gwmap.rsgis.msu.edu.

Nicholson, S.W., Dicken, C.L., Foose, M.P., and Mueller, J.A.L., 2005a, Preliminary integrated geologic map databases for the United States-Minnesota, Wisconsin, Michigan, Illinois, and Indiana: U.S. Geological Survey Open-File Report 2004-1355, ver. 1.1, accessed November 18, 2009, at http://pubs.usgs.gov/of/2004/1355/.

Nicholson, S.W., Dicken, C.L., Horton, J.D., Labay, K.A., Foose, M.P., and Mueller, J.A.L., 2005b, Preliminary integrated geologic map databases for the United States-Kentucky, Ohio, Tennessee, and West Virginia: U. S. Geological Survey Open-File Report 2005-1324, ver. 1.1, accessed July 28, 2009, at http://pubs.usgs.gov/of/2005/1324/.

Ohio Department of Natural Resources, 2000, Unconsolidated aquifers of Ohio, accessed August 4, 2009, at http://www.dnr.state.oh.us/water/tabid/4230/Default.aspx.
Pavey, R.R., Hull, D.N., Brockman, C.S., Schumacher, G.A., Stith, D.A., Swinford, E.M., Sole, T.L., Vorbau, K.E., Kallini, K.D., Evans, E.E., Slucher, E.R., and Van Horn, R.G., 1999, Known and probable karst in Ohio (revised 10/2007, 5/2006, 2002): Ohio Division of Geological Survey Map EG-1, scale 1:500,000, 1 map and GIS files.

PRISM Climate Group at Oregon State University, 2006a, United States average monthly or annual precipitation, 1971-2000, accessed April 29, 2010, at http://www.prism.oregonstate.edu/products/matrix.phtml.

PRISM Climate Group at Oregon State University, 2006b, United States average monthly or annual maximum temperature, 1971-2000, accessed April 29, 2010, at http://www.prism.oregonstate.edu/products/matrix. phtml? vartype $=$ tmax .

Shaffer, N.R., Sowder, K.H., Weibel, C.P., Wolfe, M.E., and Paylor, R.L., 2007, Map showing industrial minerals operations and carbonate regions affected by solution features in Illinois, Indiana, Kentucky, and Ohio: Indiana Geological Survey Open-File Study 07-02, scale: 1:1,000,000.

Soller, D.R., and Packard, P.H., 1998, Digital representation of a map showing the thickness and character of quaternary sediments in the glaciated United States East of the Rocky Mountains: U.S. Geological Survey Digital Data Series DDS-38.

U.S. Department of Agriculture-Natural Resources Conservation Service, 2006, U.S. General Soil Map (STATSGO2), accessed July 20, 2009, at http://soildatamart.nrcs.usda.gov.

U.S. Department of Agriculture-Natural Resources Conservation Service, 2008, Soil Data Viewer 5.2 User Guide, accessed August 20, 2011, at ftp://ftp-fc.sc.egov.usda.gov/ NSSC/Soil_Data_Viewer/Soil_Data_Viewer_5_2_User Guide.pdf.

U.S. Geological Survey, 2004, The National Hydrography Dataset (NHD), 1:100,000, accessed June 3, 2006, at http://nhd.usgs.gov.

U.S. Geological Survey, 2007, National Land Cover Database 2001 (NLCD2001), ver. 1.0, accessed July 6, 2009, at http://www.mrlc.gov/nlcd2001_downloads.php.

Whitehead, M.T., 2002, Mean annual precipitation for Ohio, 1931-80: U.S. Geological Survey data, accessed June 3, 2006, at http://water.usgs.gov/lookup/getspatial?raintin. 


\section{Appendix 3. Annual 1-Day and 7-Day Mean Flow-Duration Results for Sites with Streamflows that have No or Minimal Regulation}

03089500 Mill Creek near Berlin Center, Ohio

Years analyzed $=29$

Climatic year range $=1942-70$

[APEE, annual percentage equaled or exceeded; $\mathrm{ft}^{3} / \mathrm{s}$, cubic foot per second]

\begin{tabular}{|c|c|c|c|c|c|c|c|c|c|}
\hline \multirow[b]{2}{*}{ APEE } & \multicolumn{9}{|c|}{ Daily mean streamflow $\left(\mathrm{ft}^{3} / \mathrm{s}\right)$ that was equaled or exceeded the indicated percentage of time at APEE level } \\
\hline & 95 & 90 & 80 & 70 & 50 & 30 & 20 & 10 & 5 \\
\hline 1 & 139. & 179. & 199. & 217. & 234. & 300. & 329. & 385 & 476. \\
\hline 2 & 76.2 & 94.4 & 101. & 123. & 162. & 194. & 225 & 273. & 292. \\
\hline 5 & 34.7 & 42.0 & 45.4 & 57.1 & 69.5 & 94.8 & 101. & 114 & 130. \\
\hline 10 & 15.0 & 19.4 & 24.8 & 27.0 & 32.0 & 44.3 & 48.8 & 63.0 & 72.8 \\
\hline 20 & 4.84 & 8.96 & 10.0 & 11.0 & 15.0 & 20.0 & 23.2 & 26.0 & 28.3 \\
\hline 25 & 3.23 & 6.00 & 7.03 & 8.40 & 10.0 & 14.5 & 18.0 & 19.5 & 21.0 \\
\hline 30 & 2.30 & 4.00 & 4.70 & 5.70 & 8.00 & 11.0 & 13.0 & 15.0 & 17.0 \\
\hline 40 & 1.68 & 2.00 & 2.60 & 3.00 & 4.26 & 6.80 & 8.06 & 8.80 & 11.8 \\
\hline 50 & 1.05 & 1.20 & 1.40 & 1.90 & 2.60 & 4.00 & 5.00 & 6.40 & 7.80 \\
\hline 60 & 0.75 & 0.84 & 1.00 & 1.20 & 1.80 & 2.48 & 2.94 & 4.90 & 5.25 \\
\hline 70 & 0.50 & 0.60 & 0.70 & 0.80 & 1.20 & 1.31 & 1.70 & 3.40 & 3.79 \\
\hline 75 & 0.40 & 0.40 & 0.56 & 0.60 & 0.90 & 1.20 & 1.40 & 2.28 & 3.13 \\
\hline 80 & 0.30 & 0.40 & 0.40 & 0.50 & 0.80 & 1.00 & 1.10 & 1.64 & 2.42 \\
\hline 90 & 0.10 & 0.19 & 0.20 & 0.30 & 0.40 & 0.60 & 0.80 & 1.00 & 1.75 \\
\hline 95 & 0.05 & 0.10 & 0.10 & 0.20 & 0.30 & 0.50 & 0.55 & 0.80 & 1.50 \\
\hline 98 & 0.00 & 0.00 & 0.10 & 0.10 & 0.21 & 0.40 & 0.50 & 0.70 & 1.32 \\
\hline 99 & 0.00 & 0.00 & 0.03 & 0.10 & 0.20 & 0.33 & 0.47 & 0.60 & 1.23 \\
\hline
\end{tabular}

\begin{tabular}{|c|c|c|c|c|c|c|c|c|c|}
\hline \multirow[b]{2}{*}{ APEE } & \multicolumn{9}{|c|}{ 7-day mean streamflow ( $\left.\mathrm{ft}^{3} / \mathrm{s}\right)$ that was equaled or exceeded the indicated percentage of time at APEE level } \\
\hline & 95 & 90 & 80 & 70 & 50 & 30 & 20 & 10 & 5 \\
\hline 1 & 80.1 & 91.7 & 103. & 105. & 135. & 166. & 231. & 261. & 300. \\
\hline 5 & 38.5 & 50.1 & 58.0 & 63.3 & 73.7 & 90.1 & 101. & 120 & 138. \\
\hline 10 & 19.4 & 25.6 & 31.3 & 38.3 & 50.4 & 59.4 & 68.1 & 78.3 & 80.0 \\
\hline 30 & 2.60 & 4.23 & 5.94 & 7.57 & 11.1 & 16.7 & 20.4 & 23.9 & 24.8 \\
\hline 40 & 1.71 & 1.93 & 2.89 & 3.24 & 5.11 & 9.86 & 11.6 & 16.3 & 18.9 \\
\hline 50 & 1.19 & 1.30 & 1.56 & 2.27 & 3.17 & 4.51 & 7.69 & 10.2 & 11.5 \\
\hline 60 & 0.81 & 0.96 & 1.13 & 1.56 & 2.17 & 3.09 & 4.00 & 6.81 & 7.76 \\
\hline 90 & 0.19 & 0.20 & 0.26 & 0.31 & 0.47 & 0.88 & 0.92 & 1.10 & 1.92 \\
\hline 95 & 0.07 & 0.11 & 0.16 & 0.24 & 0.36 & 0.59 & 0.71 & 0.93 & 1.79 \\
\hline 98 & 0.03 & 0.06 & 0.08 & 0.16 & 0.27 & 0.45 & 0.50 & 0.77 & 1.58 \\
\hline 99 & 0.01 & 0.03 & 0.04 & 0.11 & 0.23 & 0.39 & 0.49 & 0.67 & 1.46 \\
\hline
\end{tabular}




\section{Kale Creek near Pricetown, Ohio}

Years analyzed $=51$

Climatic year range $=1942-92$

[APEE, annual percentage equaled or exceeded; $\mathrm{ft}^{3} / \mathrm{s}$, cubic foot per second]

\begin{tabular}{|c|c|c|c|c|c|c|c|c|c|}
\hline \multirow[b]{2}{*}{ APEE } & \multicolumn{9}{|c|}{ Daily mean streamflow ( $\mathrm{ft}^{3} / \mathrm{s}$ ) that was equaled or exceeded the indicated percentage of time at APEE level } \\
\hline & 98 & 95 & 90 & 80 & 50 & 20 & 10 & 5 & 2 \\
\hline 1 & 75.4 & 182. & 211. & 261. & 367. & 494. & 577. & 727. & 883. \\
\hline 2 & 59.7 & 105. & 122 & 151. & 245. & 311. & 401. & 446. & 492. \\
\hline 5 & 38.7 & 39.9 & 54.4 & 68.4 & 104. & 144. & 165. & 189. & 221. \\
\hline 10 & 18.0 & 21.2 & 25.0 & 31.0 & 46.2 & 76.4 & 89.1 & 94.6 & 97.9 \\
\hline 20 & 5.06 & 6.65 & 9.02 & 12.9 & 21.6 & 29.8 & 35.8 & 40.9 & 43.9 \\
\hline 25 & 3.51 & 4.30 & 5.21 & 8.64 & 13.5 & 21.0 & 26.8 & 28.8 & 35.8 \\
\hline 30 & 2.60 & 2.65 & 3.53 & 6.13 & 10.2 & 16.6 & 19.2 & 20.9 & 28.7 \\
\hline 40 & 1.11 & 1.52 & 1.81 & 2.98 & 6.32 & 10.4 & 11.2 & 13.8 & 17.9 \\
\hline 50 & 0.70 & 0.80 & 1.01 & 1.42 & 3.50 & 6.90 & 7.50 & 9.08 & 12.9 \\
\hline 60 & 0.34 & 0.40 & 0.60 & 0.80 & 2.00 & 4.56 & 5.60 & 7.00 & 9.72 \\
\hline 70 & 0.10 & 0.21 & 0.30 & 0.40 & 1.20 & 2.59 & 4.12 & 5.15 & 6.58 \\
\hline 75 & 0.10 & 0.18 & 0.20 & 0.30 & 0.81 & 2.23 & 3.40 & 4.13 & 5.26 \\
\hline 80 & 0.04 & 0.10 & 0.20 & 0.23 & 0.59 & 1.76 & 2.68 & 3.16 & 3.70 \\
\hline 90 & 0.00 & 0.06 & 0.10 & 0.10 & 0.30 & 0.98 & 1.43 & 1.75 & 2.19 \\
\hline 95 & 0.00 & 0.02 & 0.07 & 0.10 & 0.20 & 0.73 & 0.93 & 1.16 & 1.82 \\
\hline 98 & 0.00 & 0.00 & 0.00 & 0.05 & 0.13 & 0.49 & 0.80 & 0.94 & 1.39 \\
\hline 99 & 0.00 & 0.00 & 0.00 & 0.03 & 0.11 & 0.36 & 0.70 & 0.91 & 1.26 \\
\hline
\end{tabular}

\begin{tabular}{|c|c|c|c|c|c|c|c|c|c|}
\hline \multirow[b]{2}{*}{ APEE } & \multicolumn{9}{|c|}{ 7-day mean streamflow ( $\mathrm{ft}^{3} / \mathrm{s}$ ) that was equaled or exceeded the indicated percentage of time at APEE level } \\
\hline & 98 & 95 & 90 & 80 & 50 & 20 & 10 & 5 & 2 \\
\hline 1 & 53.0 & 80.3 & 109. & 139. & 178. & 233. & 286. & 337. & 359. \\
\hline 2 & 41.1 & 69.5 & 90.9 & 119. & 142 & 195. & 216. & 268. & 311. \\
\hline 5 & 32.2 & 53.2 & 61.8 & 77.1 & 105 & 141. & 156. & 180 & 197. \\
\hline 10 & 21.9 & 25.2 & 30.3 & 45.4 & 68.1 & 96.0 & 103. & 114. & 130. \\
\hline 20 & 5.40 & 9.02 & 13.5 & 19.2 & 33.1 & 53.2 & 61.9 & 67.0 & 72.9 \\
\hline 25 & 4.12 & 4.90 & 7.27 & 13.5 & 21.9 & 40.2 & 48.8 & 52.0 & 59.1 \\
\hline 30 & 2.85 & 3.37 & 4.43 & 9.79 & 16.6 & 31.8 & 37.2 & 39.7 & 45.2 \\
\hline 40 & 1.52 & 1.68 & 2.19 & 3.77 & 8.80 & 17.1 & 21.2 & 29.0 & 30.6 \\
\hline 50 & 0.82 & 0.91 & 1.37 & 1.71 & 4.57 & 9.60 & 12.5 & 18.1 & 23.3 \\
\hline 60 & 0.43 & 0.57 & 0.64 & 0.90 & 2.54 & 6.35 & 7.48 & 11.9 & 15.7 \\
\hline 70 & 0.12 & 0.25 & 0.35 & 0.45 & 1.49 & 3.79 & 5.36 & 8.39 & 11.7 \\
\hline 75 & 0.06 & 0.20 & 0.27 & 0.35 & 0.88 & 3.26 & 4.27 & 6.49 & 9.05 \\
\hline 80 & 0.05 & 0.14 & 0.20 & 0.25 & 0.67 & 2.35 & 3.45 & 4.03 & 7.36 \\
\hline 90 & 0.00 & 0.06 & 0.10 & 0.14 & 0.34 & 1.19 & 1.65 & 2.34 & 3.73 \\
\hline 95 & 0.00 & 0.05 & 0.07 & 0.10 & 0.22 & 0.85 & 1.06 & 1.62 & 2.10 \\
\hline 98 & 0.00 & 0.02 & 0.05 & 0.06 & 0.16 & 0.66 & 0.90 & 1.10 & 1.79 \\
\hline 99 & 0.00 & 0.00 & 0.02 & 0.04 & 0.14 & 0.59 & 0.76 & 1.01 & 1.54 \\
\hline
\end{tabular}


03092090 West Branch Mahoning River near Ravenna, Ohio

Years analyzed $=27$

Climatic year range $=1966-92$

[APEE, annual percentage equaled or exceeded; $\mathrm{ft}^{3} / \mathrm{s}$, cubic foot per second]

\begin{tabular}{|c|c|c|c|c|c|c|c|c|c|}
\hline \multirow[b]{2}{*}{ APEE } & \multicolumn{9}{|c|}{ Daily mean streamflow (ft $3 / s)$ that was equaled or exceeded the indicated percentage of time at APEE level } \\
\hline & 95 & 90 & 80 & 70 & 50 & 30 & 20 & 10 & 5 \\
\hline 1 & 127. & 190. & 218. & 256. & 287. & 366. & 391. & 451. & 475. \\
\hline 2 & 87.8 & 119. & 160. & 175. & 213. & 244 & 250 & 304. & 353. \\
\hline 5 & 52.9 & 69.6 & 80.2 & 83.8 & 116. & 149. & 166. & 177. & 198. \\
\hline 10 & 30.3 & 39.5 & 44.4 & 47.7 & 65.0 & 78.4 & 89.8 & 107. & 112. \\
\hline 20 & 16.8 & 20.4 & 24.9 & 26.3 & 32.0 & 38.1 & 42.0 & 49.2 & 53.2 \\
\hline 25 & 13.2 & 16.2 & 19.8 & 21.4 & 23.5 & 29.6 & 34.0 & 39.0 & 42.3 \\
\hline 30 & 10.2 & 13.6 & 16.3 & 17.9 & 19.0 & 24.9 & 30.0 & 32.9 & 33.1 \\
\hline 40 & 7.21 & 9.80 & 11.6 & 12.0 & 14.6 & 18.6 & 21.0 & 23.5 & 25.8 \\
\hline 50 & 4.86 & 6.80 & 8.46 & 8.76 & 11.0 & 13.6 & 16.4 & 18.0 & 19.2 \\
\hline 60 & 3.34 & 4.68 & 6.00 & 6.60 & 8.20 & 9.94 & 12.0 & 13.4 & 16.2 \\
\hline 70 & 2.40 & 3.03 & 4.12 & 4.54 & 5.78 & 6.92 & 7.46 & 10.1 & 12.1 \\
\hline 75 & 2.04 & 2.60 & 3.32 & 3.84 & 4.85 & 5.76 & 6.20 & 9.11 & 9.82 \\
\hline 80 & 1.76 & 2.00 & 2.68 & 3.21 & 4.12 & 4.40 & 4.85 & 7.86 & 8.05 \\
\hline 90 & 0.82 & 1.19 & 1.40 & 1.78 & 2.50 & 2.93 & 3.26 & 4.13 & 4.63 \\
\hline 95 & 0.44 & 1.05 & 1.12 & 1.34 & 1.80 & 2.40 & 2.75 & 3.10 & 3.68 \\
\hline 98 & 0.31 & 0.71 & 0.94 & 0.99 & 1.40 & 1.90 & 2.14 & 2.76 & 3.12 \\
\hline 99 & 0.23 & 0.49 & 0.85 & 0.93 & 1.20 & 1.81 & 1.94 & 2.48 & 2.92 \\
\hline
\end{tabular}

\begin{tabular}{|c|c|c|c|c|c|c|c|c|c|}
\hline \multirow[b]{2}{*}{ APEE } & \multicolumn{9}{|c|}{ 7-day mean streamflow $\left(\mathrm{ft}^{3} / \mathrm{s}\right)$ that was equaled or exceeded the indicated percentage of time at APEE level } \\
\hline & 95 & 90 & 80 & 70 & 50 & 30 & 20 & 10 & 5 \\
\hline 1 & 78.2 & 91.1 & 126. & 160. & 181. & 206. & 219. & 234 & 268. \\
\hline 2 & 64.7 & 80.5 & 95.7 & 123. & 136. & 157. & 175 & 188. & 203. \\
\hline 5 & 51.4 & 64.5 & 83.2 & 85.0 & 96.0 & 114. & 128. & 141. & 151. \\
\hline 10 & 34.5 & 47.1 & 54.1 & 59.6 & 72.4 & 82.4 & 94.9 & 107. & 116. \\
\hline 20 & 16.5 & 25.4 & 29.6 & 30.7 & 41.0 & 54.5 & 60.2 & 70.0 & 79.8 \\
\hline 25 & 12.7 & 20.9 & 24.4 & 25.7 & 34.3 & 47.4 & 50.2 & 57.7 & 60.0 \\
\hline 30 & 10.4 & 16.6 & 19.7 & 21.7 & 25.7 & 37.2 & 41.0 & 47.3 & 49.1 \\
\hline 40 & 7.62 & 10.2 & 13.2 & 14.6 & 17.3 & 26.3 & 29.1 & 35.7 & 37.7 \\
\hline 50 & 5.67 & 7.63 & 9.50 & 10.5 & 12.8 & 17.3 & 20.6 & 25.4 & 27.0 \\
\hline 60 & 3.75 & 5.48 & 6.99 & 8.57 & 9.74 & 12.4 & 14.8 & 18.4 & 20.9 \\
\hline 70 & 2.50 & 3.29 & 4.77 & 5.30 & 7.08 & 7.95 & 9.54 & 12.4 & 17.1 \\
\hline 75 & 2.15 & 2.84 & 3.93 & 4.63 & 5.80 & 6.57 & 7.99 & 10.4 & 14.9 \\
\hline 80 & 1.91 & 2.48 & 3.24 & 3.84 & 4.61 & 5.54 & 6.06 & 8.51 & 12.0 \\
\hline 90 & 1.30 & 1.51 & 1.86 & 2.17 & 3.24 & 3.60 & 3.94 & 4.91 & 6.44 \\
\hline 95 & 0.71 & 1.07 & 1.25 & 1.46 & 2.33 & 2.88 & 3.23 & 3.71 & 4.37 \\
\hline 98 & 0.43 & 0.89 & 1.06 & 1.13 & 2.01 & 2.25 & 2.71 & 3.38 & 3.82 \\
\hline 99 & 0.33 & 0.62 & 0.97 & 1.05 & 1.64 & 2.07 & 2.35 & 3.09 & 3.51 \\
\hline
\end{tabular}


03102950 Pymatuning Creek at Kinsman, Ohio

Years analyzed $=28$

Climatic year range $=1966-93$

[APEE, annual percentage equaled or exceeded; $\mathrm{ft}^{3} / \mathrm{s}$, cubic foot per second]

\begin{tabular}{|c|c|c|c|c|c|c|c|c|c|}
\hline \multirow[b]{2}{*}{ APEE } & \multicolumn{9}{|c|}{ Daily mean streamflow $\left(\mathrm{ft}^{3} / \mathrm{s}\right)$ that was equaled or exceeded the indicated percentage of time at APEE level } \\
\hline & 95 & 90 & 80 & 70 & 50 & 30 & 20 & 10 & 5 \\
\hline 1 & 466. & 642. & 746. & 862. & 985. & 1030. & 1110 & 1240 & 1470 . \\
\hline 2 & 390. & 550. & 612. & 635. & 749. & 799. & 836. & 855. & 872. \\
\hline 5 & 290. & 363. & 408 & 451. & 514. & 568. & 623. & 680. & 707. \\
\hline 10 & 186. & 240 & 272. & 281. & 344. & 407. & 437. & 501. & 518. \\
\hline 20 & 87.6 & 117. & 138. & 178. & 194. & 228 & 255. & 274 & 308. \\
\hline 25 & 65.4 & 95.8 & 106. & 132. & 154. & 188. & 207. & 235. & 269. \\
\hline 30 & 48.3 & 76.8 & 83.8 & 97.7 & 121. & 155. & 168. & 201. & 225. \\
\hline 40 & 31.9 & 47.6 & 57.8 & 68.1 & 79.6 & 98.9 & 112. & 150. & 158. \\
\hline 50 & 21.1 & 32.3 & 40.0 & 44.1 & 51.0 & 71.5 & 79.2 & 110. & 114. \\
\hline 60 & 15.4 & 20.6 & 23.3 & 27.1 & 36.9 & 48.7 & 55.3 & 77.7 & 90.9 \\
\hline 70 & 8.42 & 9.57 & 12.6 & 16.0 & 22.0 & 33.0 & 37.0 & 51.2 & 61.6 \\
\hline 75 & 5.04 & 7.93 & 9.70 & 11.0 & 15.3 & 27.6 & 28.8 & 42.6 & 50.5 \\
\hline 80 & 3.43 & 6.22 & 7.06 & 8.20 & 11.1 & 18.6 & 24.0 & 31.2 & 34.4 \\
\hline 90 & 1.29 & 2.00 & 3.17 & 3.71 & 5.32 & 10.9 & 12.2 & 14.1 & 16.1 \\
\hline 95 & 0.43 & 1.07 & 1.47 & 2.26 & 3.40 & 6.58 & 7.04 & 7.44 & 8.80 \\
\hline 98 & 0.21 & 0.23 & 0.84 & 1.32 & 2.40 & 3.83 & 5.09 & 5.48 & 5.92 \\
\hline 99 & 0.13 & 0.17 & 0.71 & 1.07 & 2.10 & 3.23 & 3.99 & 4.38 & 4.79 \\
\hline
\end{tabular}

\begin{tabular}{|c|c|c|c|c|c|c|c|c|c|}
\hline \multirow[b]{2}{*}{ APEE } & \multicolumn{9}{|c|}{ 7-day mean streamflow ( $\mathrm{ft}^{3} / \mathrm{s}$ ) that was equaled or exceeded the indicated percentage of time at APEE level } \\
\hline & 95 & 90 & 80 & 70 & 50 & 30 & 20 & 10 & 5 \\
\hline 1 & 321. & 502. & 546. & 641. & 766. & 873. & 904. & 1070 . & 1160 \\
\hline 2 & 309. & 442. & 476. & 535. & 604. & 705. & 750 & 807. & 869. \\
\hline 5 & 251. & 326. & 380 & 411. & 446. & 503. & 530. & 595. & 615. \\
\hline 10 & 183. & 219. & 292. & 299. & 333. & 368. & 418. & 443. & 471. \\
\hline 20 & 94.4 & 126. & 154. & 170 . & 230 & 252. & 270 & 300 . & 305. \\
\hline 25 & 65.4 & 101. & 118. & 140 . & 180. & 200 & 209. & 258 & 260. \\
\hline 30 & 49.9 & 73.6 & 91.6 & 113. & 138. & 169. & 174. & 225 & 234. \\
\hline 40 & 33.0 & 50.5 & 63.0 & 75.0 & 88.0 & 118. & 126. & 174. & 184. \\
\hline 50 & 21.2 & 34.8 & 42.8 & 49.7 & 57.6 & 83.3 & 92.0 & 136. & 142. \\
\hline 60 & 15.3 & 20.1 & 25.7 & 31.5 & 39.7 & 55.5 & 63.3 & 104. & 111. \\
\hline 70 & 9.80 & 10.6 & 16.1 & 19.5 & 22.9 & 37.7 & 45.9 & 70.2 & 78.1 \\
\hline 75 & 5.37 & 8.41 & 11.0 & 14.1 & 17.7 & 31.6 & 36.3 & 55.6 & 59.7 \\
\hline 80 & 3.65 & 6.54 & 7.59 & 9.53 & 11.9 & 22.7 & 27.3 & 41.1 & 43.8 \\
\hline 90 & 1.38 & 2.15 & 3.84 & 4.27 & 6.23 & 14.0 & 15.2 & 16.9 & 20.1 \\
\hline 95 & 0.64 & 1.17 & 2.27 & 2.68 & 3.87 & 7.65 & 8.27 & 10.2 & 11.6 \\
\hline 98 & 0.26 & 0.35 & 0.93 & 1.80 & 3.06 & 4.51 & 5.78 & 6.91 & 8.48 \\
\hline 99 & 0.21 & 0.24 & 0.81 & 1.40 & 2.62 & 4.09 & 5.33 & 5.66 & 6.84 \\
\hline
\end{tabular}


03109500 Little Beaver Creek near East Liverpool, Ohio

Years analyzed $=93$

Climatic year range $=1916-2008$

[APEE, annual percentage equaled or exceeded; $\mathrm{ft}^{3} / \mathrm{s}$, cubic foot per second]

\begin{tabular}{|c|c|c|c|c|c|c|c|c|c|}
\hline \multirow[b]{2}{*}{ APEE } & \multicolumn{9}{|c|}{ Daily mean streamflow $\left(\mathrm{ft}^{3} / \mathrm{s}\right)$ that was equaled or exceeded the indicated percentage of time at APEE level } \\
\hline & 98 & 95 & 90 & 80 & 50 & 20 & 10 & 5 & 2 \\
\hline 1 & 1230. & 2090 & 2520 & 2860 & 4110. & 5250. & 6260. & 7050 . & 9230. \\
\hline 2 & 960. & 1290. & 1940. & 2120 & 2940. & 3910. & 4520 . & 5030 & 6730. \\
\hline 5 & 709. & 876. & 1110. & 1300 . & 1830 & 2440 & 2740 & 2920 . & 3330. \\
\hline 10 & 517. & 643. & 749. & 866. & 1230 & 1540 & 1740 . & 1900. & 2110 . \\
\hline 20 & 268. & 372. & 429. & 494. & 700 . & 936. & 1040. & 1150 & 1260. \\
\hline 25 & 206. & 301. & 350 . & 403. & 570. & 767. & 900. & 1000 & 1100. \\
\hline 30 & 161. & 222. & 289. & 328. & 479. & 670. & 765. & 864. & 962. \\
\hline 40 & 106. & 134. & 192. & 225. & 321. & 494. & 593. & 674. & 777. \\
\hline 50 & 67.6 & 98.7 & 127. & 160. & 245 & 392. & 453. & 525 & 665. \\
\hline 60 & 50.2 & 77.6 & 87.0 & 111. & 176. & 290. & 343. & 413. & 566. \\
\hline 70 & 38.7 & 54.5 & 64.7 & 83.6 & 130. & 206. & 264. & 314. & 477. \\
\hline 75 & 35.0 & 48.0 & 54.7 & 69.8 & 110. & 180. & 221. & 281. & 434. \\
\hline 80 & 30.4 & 41.7 & 46.4 & 58.4 & 92.0 & 131. & 182. & 249. & 391. \\
\hline 90 & 21.4 & 30.6 & 33.2 & 38.0 & 61.7 & 93.1 & 132. & 175 & 272. \\
\hline 95 & 17.5 & 24.5 & 26.4 & 31.8 & 47.4 & 78.6 & 106. & 136. & 207. \\
\hline 98 & 14.0 & 19.9 & 22.0 & 25.8 & 39.6 & 66.3 & 91.3 & 124. & 172. \\
\hline 99 & 12.9 & 17.1 & 20.7 & 24.0 & 35.7 & 60.2 & 81.5 & 109. & 155. \\
\hline
\end{tabular}

\begin{tabular}{|c|c|c|c|c|c|c|c|c|c|}
\hline \multirow[b]{2}{*}{ APEE } & \multicolumn{9}{|c|}{ 7-day mean streamflow ( $\mathrm{ft}^{3} / \mathrm{s}$ ) that was equaled or exceeded the indicated percentage of time at APEE level } \\
\hline & 98 & 95 & 90 & 80 & 50 & 20 & 10 & 5 & 2 \\
\hline 1 & 970. & 1340 & 1740 & 2040 & 2820 & 3970. & 4450. & 5060 & 6230. \\
\hline 2 & 844. & 1130 & 1420 . & 1670. & 2460 & 2970 . & 3420 . & 3810. & 4640. \\
\hline 5 & 700 & 834. & 1030. & 1280 & 1720 & 2150 & 2540 & 2760 & 3050 . \\
\hline 10 & 496. & 636. & 759. & 901. & 1220 & 1620. & 1790. & 2010 & 2230. \\
\hline 20 & 278 & 391. & 429. & 512. & 802. & 1040 & 1240 & 1390. & 1530. \\
\hline 25 & 195. & 320. & 375. & 431. & 632. & 890. & 1010 & 1190. & 1280 \\
\hline 30 & 161. & 241. & 309. & 341. & 526. & 777. & 835. & 1090 & 1150 \\
\hline 40 & 107. & 145. & 201. & 231. & 358. & 591. & 669. & 790 . & 911. \\
\hline 50 & 76.6 & 110. & 131. & 172. & 270. & 430. & 520. & 591. & 733. \\
\hline 60 & 52.1 & 80.9 & 93.8 & 115. & 189. & 310. & 399. & 467. & 612. \\
\hline 70 & 40.0 & 58.1 & 71.2 & 85.2 & 136. & 236. & 289. & 337. & 530. \\
\hline 75 & 37.3 & 52.2 & 58.7 & 72.5 & 120. & 196. & 232. & 312. & 487. \\
\hline 80 & 30.6 & 43.1 & 50.9 & 60.7 & 101. & 155. & 207. & 287. & 427. \\
\hline 90 & 24.3 & 31.2 & 35.3 & 40.7 & 64.6 & 103. & 146. & 210 & 349. \\
\hline 95 & 17.8 & 26.6 & 30.2 & 34.2 & 50.4 & 86.3 & 120. & 147. & 232. \\
\hline 98 & 14.6 & 21.5 & 24.7 & 28.9 & 43.1 & 75.7 & 105. & 137. & 196. \\
\hline 99 & 14.0 & 20.6 & 23.0 & 26.2 & 40.0 & 69.4 & 93.9 & 133. & 180. \\
\hline
\end{tabular}


03110000 Yellow Creek near Hammondsville, Ohio

Years analyzed $=68$

Climatic year range $=1941-2008$

[APEE, annual percentage equaled or exceeded; $\mathrm{ft}^{3} / \mathrm{s}$, cubic foot per second]

\begin{tabular}{|c|c|c|c|c|c|c|c|c|c|}
\hline \multirow[b]{2}{*}{ APEE } & \multicolumn{9}{|c|}{ Daily mean streamflow $\left(\mathrm{ft}^{3} / \mathrm{s}\right)$ that was equaled or exceeded the indicated percentage of time at APEE level } \\
\hline & 98 & 95 & 90 & 80 & 50 & 20 & 10 & 5 & 2 \\
\hline 1 & 594. & 719. & 832. & 926. & 1290. & 1710 . & 1890. & 2170 & 2980. \\
\hline 2 & 406. & 488. & 618. & 682. & 863. & 1170 . & 1350. & 1490. & 1740. \\
\hline 5 & 267. & 325. & 339. & 425. & 561. & 734. & 858. & 916. & 1170. \\
\hline 10 & 186. & 223. & 241. & 288 & 386. & 464. & 565. & 586. & 699. \\
\hline 20 & 104. & 120. & 131. & 160. & 235. & 286. & 317. & 361. & 403. \\
\hline 25 & 76.6 & 95.1 & 101. & 126. & 186. & 248 & 267. & 313. & 352. \\
\hline 30 & 49.4 & 68.1 & 81.1 & 102. & 153. & 206. & 229. & 265. & 306. \\
\hline 40 & 27.6 & 39.3 & 54.0 & 69.4 & 104. & 160. & 181. & 216. & 239. \\
\hline 50 & 17.1 & 24.1 & 35.7 & 42.0 & 73.0 & 121. & 146. & 164. & 192. \\
\hline 60 & 10.8 & 16.6 & 22.4 & 30.0 & 49.7 & 85.4 & 108. & 130. & 159. \\
\hline 70 & 7.44 & 10.5 & 15.0 & 20.0 & 35.9 & 59.6 & 73.8 & 100. & 125. \\
\hline 75 & 6.03 & 8.63 & 12.0 & 16.0 & 28.5 & 46.0 & 61.5 & 87.9 & 114. \\
\hline 80 & 5.04 & 7.05 & 8.99 & 13.0 & 22.3 & 38.0 & 46.0 & 79.5 & 98.6 \\
\hline 90 & 3.11 & 3.98 & 4.78 & 6.20 & 13.0 & 22.0 & 30.1 & 51.8 & 68.7 \\
\hline 95 & 1.71 & 2.52 & 3.43 & 4.08 & 9.30 & 16.3 & 25.1 & 37.3 & 56.2 \\
\hline 98 & 1.02 & 1.92 & 2.18 & 3.09 & 7.43 & 13.2 & 19.1 & 26.2 & 47.2 \\
\hline 99 & 0.88 & 1.66 & 1.90 & 2.86 & 6.25 & 12.1 & 16.8 & 24.2 & 42.9 \\
\hline
\end{tabular}

\begin{tabular}{|c|c|c|c|c|c|c|c|c|c|}
\hline \multirow[b]{2}{*}{ APEE } & \multicolumn{9}{|c|}{ 7-day mean streamflow (ft $\left.{ }^{3} / \mathrm{s}\right)$ that was equaled or exceeded the indicated percentage of time at APEE level } \\
\hline & 98 & 95 & 90 & 80 & 50 & 20 & 10 & 5 & 2 \\
\hline 1 & 419. & 495. & 521. & 645. & 871. & 1150 & 1440 & 1650 & 1800 \\
\hline 2 & 364. & 406. & 473. & 558. & 740 . & 865. & 962. & 1210. & 1520 \\
\hline 5 & 281. & 310. & 347. & 403. & 554. & 702. & 776. & 873. & 1160 \\
\hline 10 & 184. & 227. & 242. & 294. & 408. & 514. & 579. & 624. & 738. \\
\hline 20 & 98.8 & 115. & 143. & 172. & 269. & 333. & 372. & 429. & 464. \\
\hline 25 & 66.2 & 93.9 & 112. & 133. & 214. & 281. & 320. & 369. & 407. \\
\hline 30 & 49.5 & 69.1 & 83.6 & 106. & 171. & 232 & 262. & 325. & 354. \\
\hline 40 & 31.0 & 42.3 & 57.6 & 77.4 & 115. & 180. & 204. & 237. & 278. \\
\hline 50 & 15.4 & 26.2 & 36.6 & 43.8 & 77.8 & 129. & 161. & 180. & 217. \\
\hline 60 & 11.6 & 17.2 & 23.7 & 32.8 & 55.3 & 94.7 & 123. & 143. & 185. \\
\hline 70 & 7.69 & 12.0 & 17.6 & 21.3 & 39.9 & 64.5 & 81.6 & 113. & 145. \\
\hline 75 & 6.76 & 9.54 & 13.4 & 18.5 & 31.5 & 54.3 & 69.0 & 101. & 129. \\
\hline 80 & 5.84 & 7.47 & 10.2 & 13.3 & 25.9 & 41.9 & 54.5 & 87.0 & 117. \\
\hline 90 & 3.39 & 4.21 & 5.44 & 6.54 & 15.4 & 25.3 & 33.8 & 59.2 & 75.9 \\
\hline 95 & 2.20 & 2.67 & 3.66 & 4.56 & 10.6 & 18.8 & 28.0 & 46.3 & 63.1 \\
\hline 98 & 1.58 & 2.02 & 2.81 & 3.80 & 8.81 & 15.0 & 23.7 & 32.5 & 54.2 \\
\hline 99 & 1.02 & 1.89 & 2.36 & 3.40 & 7.46 & 13.6 & 19.9 & 28.7 & 52.0 \\
\hline
\end{tabular}


03111500 Short Creek near Dillonvale, Ohio

Years analyzed $=67$

Climatic year range $=1942-2008$

[APEE, annual percentage equaled or exceeded; $\mathrm{ft}^{3} / \mathrm{s}$, cubic foot per second]

\begin{tabular}{|c|c|c|c|c|c|c|c|c|c|}
\hline \multirow[b]{2}{*}{ APEE } & \multicolumn{9}{|c|}{ Daily mean streamflow $\left(\mathrm{ft}^{3} / \mathrm{s}\right)$ that was equaled or exceeded the indicated percentage of time at APEE level } \\
\hline & 98 & 95 & 90 & 80 & 50 & 20 & 10 & 5 & 2 \\
\hline 1 & 366. & 410. & 483. & 541. & 749. & 1210. & 1390. & 1530. & 2690 \\
\hline 2 & 278 & 294. & 337. & 401. & 542. & 821. & 925. & 1180 & 1420. \\
\hline 5 & 187. & 200 & 227. & 254. & 369. & 480 . & 579. & 715 . & 760. \\
\hline 10 & 136. & 147. & 167. & 194. & 265 & 335. & 408 & 429. & 479. \\
\hline 20 & 94.5 & 103. & 111. & 125. & 176. & 222. & 271. & 291. & 315. \\
\hline 25 & 80.5 & 81.9 & 93.4 & 106. & 148. & 197. & 235. & 256. & 287. \\
\hline 30 & 62.8 & 67.5 & 81.4 & 92.4 & 130. & 178. & 202. & 233. & 257. \\
\hline 40 & 37.7 & 43.8 & 55.5 & 68.6 & 99.2 & 143. & 162. & 195. & 220 \\
\hline 50 & 25.7 & 34.6 & 42.4 & 50.0 & 71.0 & 114. & 134. & 153. & 194. \\
\hline 60 & 19.1 & 28.6 & 30.0 & 37.4 & 58.4 & 91.7 & 113. & 127. & 171. \\
\hline 70 & 16.4 & 22.8 & 25.0 & 27.6 & 46.0 & 67.9 & 95.5 & 103. & 141. \\
\hline 75 & 14.7 & 20.4 & 22.4 & 24.6 & 39.0 & 60.4 & 85.6 & 93.6 & 129. \\
\hline 80 & 13.4 & 19.0 & 19.3 & 21.6 & 36.0 & 52.2 & 73.2 & 85.8 & 118. \\
\hline 90 & 12.0 & 13.0 & 14.9 & 16.6 & 26.0 & 39.4 & 55.6 & 68.6 & 89.3 \\
\hline 95 & 8.30 & 10.0 & 12.0 & 15.0 & 23.0 & 33.4 & 47.4 & 63.6 & 75.1 \\
\hline 98 & 4.84 & 7.28 & 9.96 & 13.0 & 20.0 & 29.6 & 42.8 & 56.5 & 70.8 \\
\hline 99 & 3.92 & 5.88 & 9.28 & 12.0 & 19.0 & 28.3 & 40.5 & 53.6 & 67.2 \\
\hline
\end{tabular}

\begin{tabular}{|c|c|c|c|c|c|c|c|c|c|}
\hline \multirow[b]{2}{*}{ APEE } & \multicolumn{9}{|c|}{ 7-day mean streamflow ( $\mathrm{ft}^{3} / \mathrm{s}$ ) that was equaled or exceeded the indicated percentage of time at APEE level } \\
\hline & 98 & 95 & 90 & 80 & 50 & 20 & 10 & 5 & 2 \\
\hline 1 & 249. & 288. & 313. & 384. & 573. & 833. & 972. & 1340 & 1470 . \\
\hline 2 & 220 & 262. & 274. & 327. & 467. & 685 & 782. & 872. & 1210 \\
\hline 5 & 189. & 194. & 207. & 244. & 363. & 481. & 577. & 655. & 844. \\
\hline 10 & 139. & 162. & 166. & 187. & 269. & 350 & 430 & 463. & 482. \\
\hline 20 & 95.1 & 101. & 114. & 126. & 185. & 249 & 296. & 334. & 348. \\
\hline 25 & 79.1 & 84.1 & 94.3 & 112. & 155. & 212 & 248 & 284. & 311. \\
\hline 30 & 64.5 & 69.5 & 79.2 & 93.0 & 138 & 189. & 223. & 250. & 286. \\
\hline 40 & 37.7 & 45.6 & 59.6 & 70.2 & 103. & 154. & 178. & 204. & 245. \\
\hline 50 & 26.0 & 35.4 & 45.3 & 51.0 & 79.1 & 122 & 146. & 165 & 212 \\
\hline 60 & 20.6 & 29.9 & 33.7 & 39.3 & 63.1 & 96.8 & 122. & 138. & 190. \\
\hline 70 & 17.1 & 24.2 & 27.1 & 29.9 & 48.3 & 75.8 & 102. & 118. & 164. \\
\hline 75 & 15.4 & 22.0 & 24.5 & 25.8 & 44.3 & 68.1 & 91.2 & 108. & 147. \\
\hline 80 & 14.3 & 20.0 & 21.0 & 23.1 & 38.3 & 56.3 & 79.5 & 96.9 & 132. \\
\hline 90 & 12.4 & 13.6 & 16.8 & 18.2 & 28.3 & 42.0 & 61.5 & 77.9 & 104. \\
\hline 95 & 8.86 & 11.0 & 13.0 & 15.6 & 24.1 & 35.9 & 49.8 & 67.6 & 87.1 \\
\hline 98 & 6.42 & 8.56 & 11.5 & 13.9 & 21.4 & 32.0 & 46.0 & 62.4 & 80.1 \\
\hline 99 & 5.46 & 7.46 & 10.7 & 13.5 & 20.4 & 30.2 & 44.4 & 60.8 & 77.0 \\
\hline
\end{tabular}


03111548 Wheeling Creek below Blaine, Ohio

Years analyzed $=24$

Climatic year range $=1983-2008$

[APEE, annual percentage equaled or exceeded; $\mathrm{ft}^{3} / \mathrm{s}$, cubic foot per second]

\begin{tabular}{|c|c|c|c|c|c|c|c|c|c|}
\hline \multirow[b]{2}{*}{ APEE } & \multicolumn{9}{|c|}{ Daily mean streamflow $\left(\mathrm{ft}^{3} / \mathrm{s}\right)$ that was equaled or exceeded the indicated percentage of time at APEE level } \\
\hline & 95 & 90 & 80 & 70 & 50 & 30 & 20 & 10 & 5 \\
\hline 1 & 428 & 481. & 526. & 593. & 720 . & 976. & 1030. & 1310. & 2050 \\
\hline 2 & 324. & 371. & 378. & 415. & 539. & 585. & 645. & 842. & 1160. \\
\hline 5 & 170 & 195. & 216. & 242. & 316. & 379. & 386. & 501. & 644. \\
\hline 10 & 118. & 139. & 166. & 180. & 215. & 250. & 270 & 325. & 392. \\
\hline 20 & 88.0 & 98.6 & 106. & 122. & 143. & 173. & 176. & 216. & 248 \\
\hline 25 & 76.9 & 83.0 & 95.0 & 107. & 126. & 148. & 159. & 187. & 224 \\
\hline 30 & 67.4 & 76.5 & 85.2 & 93.5 & 113. & 131. & 142. & 165. & 199. \\
\hline 40 & 49.0 & 59.6 & 66.0 & 71.9 & 89.8 & 103. & 110. & 143. & 171. \\
\hline 50 & 38.0 & 40.5 & 52.0 & 57.0 & 71.0 & 84.0 & 92.0 & 121. & 152. \\
\hline 60 & 30.8 & 33.7 & 41.4 & 46.0 & 54.7 & 69.0 & 73.0 & 103. & 132. \\
\hline 70 & 26.5 & 29.0 & 32.0 & 37.4 & 42.0 & 50.4 & 64.0 & 86.0 & 105. \\
\hline 75 & 24.4 & 26.4 & 28.0 & 31.0 & 39.0 & 44.0 & 60.0 & 77.0 & 97.1 \\
\hline 80 & 21.4 & 22.5 & 26.0 & 27.0 & 34.5 & 40.0 & 53.0 & 70.6 & 89.0 \\
\hline 90 & 14.0 & 15.5 & 20.0 & 20.3 & 25.4 & 32.5 & 39.6 & 58.0 & 70.2 \\
\hline 95 & 9.94 & 12.5 & 17.0 & 19.0 & 22.2 & 27.5 & 32.0 & 50.2 & 60.5 \\
\hline 98 & 7.88 & 10.5 & 16.0 & 17.0 & 19.7 & 24.0 & 31.0 & 39.3 & 52.7 \\
\hline 99 & 7.54 & 10.2 & 14.7 & 16.5 & 17.8 & 23.0 & 30.0 & 36.3 & 48.7 \\
\hline
\end{tabular}

\begin{tabular}{|c|c|c|c|c|c|c|c|c|c|}
\hline \multirow[b]{2}{*}{ APEE } & \multicolumn{9}{|c|}{ 7-day mean streamflow ( $\mathrm{ft}^{3} / \mathrm{s}$ ) that was equaled or exceeded the indicated percentage of time at APEE level } \\
\hline & 95 & 90 & 80 & 70 & 50 & 30 & 20 & 10 & 5 \\
\hline 1 & 290. & 303. & 344. & 375 & 481. & 609. & 729. & 1090. & 1220 \\
\hline 2 & 237. & 255. & 290. & 328. & 401. & 472. & 553. & 756. & 1030. \\
\hline 5 & 182. & 195. & 223. & 247. & 325. & 372. & 406. & 515. & 692. \\
\hline 10 & 127. & 144. & 172. & 186. & 222. & 262. & 286. & 343. & 388. \\
\hline 20 & 92.6 & 97.6 & 114. & 130. & 153. & 178. & 187. & 235. & 272 \\
\hline 25 & 77.6 & 82.9 & 100 & 119. & 133. & 164. & 169. & 207. & 253. \\
\hline 30 & 70.4 & 78.2 & 86.3 & 98.9 & 115. & 146. & 151. & 193. & 236. \\
\hline 40 & 52.0 & 61.8 & 68.3 & 74.9 & 90.7 & 111. & 121. & 163. & 200 \\
\hline 50 & 41.2 & 44.1 & 52.6 & 62.1 & 74.4 & 95.0 & 100. & 135. & 167. \\
\hline 60 & 33.1 & 34.4 & 45.3 & 49.0 & 59.4 & 76.2 & 81.9 & 119. & 145. \\
\hline 70 & 27.3 & 30.4 & 32.9 & 38.8 & 47.0 & 52.8 & 66.1 & 101. & 120. \\
\hline 75 & 25.0 & 28.1 & 29.3 & 33.4 & 43.2 & 47.6 & 61.9 & 89.1 & 110. \\
\hline 80 & 23.1 & 24.1 & 26.6 & 28.7 & 37.6 & 41.7 & 55.9 & 78.9 & 98.4 \\
\hline 90 & 15.4 & 18.5 & 20.9 & 22.2 & 28.3 & 34.6 & 40.4 & 64.7 & 80.6 \\
\hline 95 & 10.1 & 12.5 & 18.4 & 19.9 & 24.6 & 30.9 & 33.4 & 58.5 & 67.0 \\
\hline 98 & 8.20 & 11.1 & 17.1 & 18.9 & 21.8 & 25.9 & 32.0 & 48.7 & 61.8 \\
\hline 99 & 7.83 & 10.6 & 16.0 & 18.0 & 19.4 & 25.0 & 31.9 & 40.8 & 59.9 \\
\hline
\end{tabular}


03114000 Captina Creek at Armstrongs Mills, Ohio

Years analyzed $=52$

Climatic year range $=1927-2002$

[APEE, annual percentage equaled or exceeded; $\mathrm{ft}^{3} / \mathrm{s}$, cubic foot per second]

\begin{tabular}{|c|c|c|c|c|c|c|c|c|c|}
\hline \multirow[b]{2}{*}{ APEE } & \multicolumn{9}{|c|}{ Daily mean streamflow (ft $3 / s)$ that was equaled or exceeded the indicated percentage of time at APEE level } \\
\hline & 98 & 95 & 90 & 80 & 50 & 20 & 10 & 5 & 2 \\
\hline 1 & 344. & 1020. & 1120. & 1170. & 1530. & 1980. & 2380. & 2700 & 3360. \\
\hline 2 & 197. & 667. & 776. & 848. & 1070 & 1320 . & 1480 & 1730 . & 1800. \\
\hline 5 & 144. & 320 . & 412. & 481. & 615. & 754. & 854. & 966. & 1090. \\
\hline 10 & 101. & 185. & 239. & 289. & 373. & 469. & 550. & 571. & 592. \\
\hline 20 & 34.5 & 110. & 127. & 150. & 207. & 284. & 301. & 342. & 359. \\
\hline 25 & 30.3 & 84.9 & 100. & 115. & 165. & 232. & 254. & 272. & 326. \\
\hline 30 & 21.6 & 61.9 & 79.8 & 89.9 & 130. & 191. & 210 & 232. & 288 \\
\hline 40 & 11.0 & 36.4 & 47.9 & 57.8 & 91.8 & 140. & 158. & 182. & 230. \\
\hline 50 & 5.59 & 20.0 & 30.6 & 37.6 & 62.5 & 103. & 126. & 153. & 179. \\
\hline 60 & 2.20 & 9.58 & 16.0 & 21.5 & 38.6 & 71.4 & 93.0 & 119. & 141. \\
\hline 70 & 0.96 & 4.15 & 6.28 & 11.6 & 23.8 & 37.9 & 62.4 & 82.7 & 108. \\
\hline 75 & 0.35 & 3.00 & 4.27 & 9.29 & 17.0 & 29.5 & 52.1 & 67.4 & 95.9 \\
\hline 80 & 0.17 & 1.88 & 2.63 & 7.00 & 12.7 & 22.4 & 40.0 & 51.5 & 80.7 \\
\hline 90 & 0.01 & 0.29 & 1.08 & 2.08 & 5.97 & 12.8 & 20.1 & 31.7 & 58.0 \\
\hline 95 & 0.00 & 0.07 & 0.40 & 1.02 & 3.93 & 8.48 & 12.5 & 18.6 & 40.6 \\
\hline 98 & 0.00 & 0.00 & 0.03 & 0.36 & 2.13 & 5.48 & 7.40 & 10.6 & 29.0 \\
\hline 99 & 0.00 & 0.00 & 0.00 & 0.09 & 1.60 & 4.30 & 5.29 & 9.70 & 27.0 \\
\hline
\end{tabular}

\begin{tabular}{|c|c|c|c|c|c|c|c|c|c|}
\hline \multirow[b]{2}{*}{ APEE } & \multicolumn{9}{|c|}{ 7-day mean streamflow ( $\mathrm{ft}^{3} / \mathrm{s}$ ) that was equaled or exceeded the indicated percentage of time at APEE level } \\
\hline & 98 & 95 & 90 & 80 & 50 & 20 & 10 & 5 & 2 \\
\hline 1 & 202. & 600. & 655. & 701. & 928. & 1380. & 1720. & 1760 . & 1870 . \\
\hline 2 & 172. & 498. & 580. & 632. & 745. & 999. & 1090. & 1190. & 1290. \\
\hline 5 & 131. & 329. & 390. & 461. & 547. & 724. & 809. & 944. & 1060 . \\
\hline 10 & 100 & 199. & 273. & 327. & 420 & 511. & 545. & 609. & 705. \\
\hline 20 & 36.9 & 120 . & 151. & 182. & 247. & 307. & 364. & 413. & 430. \\
\hline 25 & 26.6 & 88.5 & 115. & 137. & 197. & 267. & 320 . & 344. & 372. \\
\hline 30 & 22.4 & 68.3 & 86.7 & 104. & 159. & 231. & 271. & 310. & 338. \\
\hline 40 & 13.3 & 36.4 & 50.7 & 67.2 & 109. & 174. & 199. & 231. & 276. \\
\hline 50 & 6.25 & 20.8 & 32.5 & 42.4 & 77.2 & 123. & 155. & 185. & 223. \\
\hline 60 & 3.10 & 10.9 & 17.5 & 26.1 & 48.0 & 81.1 & 117. & 144. & 170. \\
\hline 70 & 1.33 & 4.68 & 8.18 & 13.9 & 28.7 & 47.6 & 79.3 & 97.2 & 134. \\
\hline 75 & 0.69 & 3.17 & 5.29 & 10.2 & 21.2 & 35.5 & 61.2 & 82.7 & 116. \\
\hline 80 & 0.24 & 2.17 & 3.80 & 9.05 & 16.4 & 27.3 & 49.4 & 62.5 & 98.0 \\
\hline 90 & 0.01 & 0.50 & 1.41 & 2.66 & 7.98 & 15.5 & 25.9 & 36.2 & 73.8 \\
\hline 95 & 0.00 & 0.10 & 0.84 & 1.33 & 5.31 & 11.0 & 17.8 & 26.3 & 56.4 \\
\hline 98 & 0.00 & 0.00 & 0.09 & 0.69 & 3.56 & 7.64 & 10.2 & 17.2 & 42.0 \\
\hline 99 & 0.00 & 0.00 & 0.03 & 0.42 & 1.96 & 6.09 & 7.71 & 11.4 & 35.9 \\
\hline
\end{tabular}


03115400 Little Muskingum River at Bloomfield, Ohio

Years analyzed $=35$

Climatic year range $=1959-2008$

[APEE, annual percentage equaled or exceeded; $\mathrm{ft}^{3} / \mathrm{s}$, cubic foot per second]

\begin{tabular}{|c|c|c|c|c|c|c|c|c|c|}
\hline \multirow[b]{2}{*}{ APEE } & \multicolumn{9}{|c|}{ Daily mean streamflow $\left(\mathrm{ft}^{3} / \mathrm{s}\right)$ that was equaled or exceeded the indicated percentage of time at APEE level } \\
\hline & 95 & 90 & 80 & 70 & 50 & 30 & 20 & 10 & 5 \\
\hline 1 & 1920. & 2040 & 2200 & 2340 & 2740 & 3430. & 3680. & 4240 & 5470 \\
\hline 2 & 1270 & 1400 & 1430. & 1600 & 1970. & 2350 & 2660 & 2950 . & 4080 \\
\hline 5 & 680. & 748. & 830. & 934. & 1060. & 1250. & 1420 & 1610. & 1780 \\
\hline 10 & 354. & 402. & 460. & 518. & 579. & 722. & 816. & 941. & 1010 \\
\hline 20 & 184. & 199. & 220. & 271. & 311. & 400. & 444. & 483. & 579. \\
\hline 25 & 112. & 136. & 168. & 221. & 246. & 342. & 351. & 396. & 449. \\
\hline 30 & 81.2 & 99.4 & 134. & 165. & 200. & 280 & 297. & 342. & 379. \\
\hline 40 & 41.6 & 57.8 & 75.9 & 83.7 & 126. & 186. & 216. & 257. & 310. \\
\hline 50 & 22.4 & 28.5 & 40.2 & 44.0 & 81.0 & 117. & 168. & 199. & 239. \\
\hline 60 & 10.3 & 13.8 & 19.8 & 24.6 & 50.0 & 73.5 & 115. & 154. & 179. \\
\hline 70 & 3.35 & 6.46 & 11.2 & 14.8 & 26.8 & 44.4 & 66.2 & 111. & 124. \\
\hline 75 & 2.52 & 4.06 & 6.48 & 10.5 & 20.0 & 31.6 & 49.9 & 89.6 & 105. \\
\hline 80 & 1.84 & 2.75 & 4.20 & 5.16 & 13.0 & 22.6 & 38.8 & 69.7 & 83.9 \\
\hline 90 & 0.56 & 0.73 & 1.47 & 2.10 & 4.72 & 9.32 & 18.9 & 32.2 & 52.4 \\
\hline 95 & 0.23 & 0.42 & 0.80 & 1.16 & 3.73 & 6.14 & 9.88 & 19.7 & 40.0 \\
\hline 98 & 0.08 & 0.19 & 0.40 & 0.71 & 2.47 & 4.13 & 5.35 & 9.59 & 26.4 \\
\hline 99 & 0.05 & 0.08 & 0.32 & 0.56 & 2.10 & 3.08 & 4.00 & 8.22 & 19.8 \\
\hline
\end{tabular}

\begin{tabular}{|c|c|c|c|c|c|c|c|c|c|}
\hline \multirow[b]{2}{*}{ APEE } & \multicolumn{9}{|c|}{ 7-day mean streamflow (ft $\left.{ }^{3} / s\right)$ that was equaled or exceeded the indicated percentage of time at APEE level } \\
\hline & 95 & 90 & 80 & 70 & 50 & 30 & 20 & 10 & 5 \\
\hline 1 & 894. & 980. & 1210 & 1470 & 1730 & 1970. & 2380 & 3280 & 5150. \\
\hline 5 & 693. & 699. & 765. & 854. & 1050 & 1090. & 1150 . & 1490 & 1800 \\
\hline 10 & 411. & 497. & 590. & 598. & 685. & 805. & 860. & 959. & 1150. \\
\hline 30 & 87.7 & 126. & 150. & 199. & 252. & 368. & 403. & 448. & 505. \\
\hline 40 & 47.8 & 67.5 & 87.2 & 98.5 & 165. & 226. & 273. & 345. & 419. \\
\hline 50 & 27.5 & 33.2 & 44.7 & 49.5 & 93.0 & 156. & 212. & 259. & 328. \\
\hline 60 & 12.5 & 16.8 & 22.5 & 28.5 & 61.4 & 95.7 & 143. & 206. & 246. \\
\hline 90 & 0.61 & 0.88 & 1.57 & 2.42 & 6.74 & 11.8 & 24.8 & 45.4 & 68.0 \\
\hline 95 & 0.28 & 0.47 & 0.92 & 1.36 & 3.97 & 7.92 & 12.1 & 26.7 & 50.3 \\
\hline 98 & 0.16 & 0.27 & 0.46 & 0.84 & 3.40 & 6.03 & 8.76 & 13.4 & 35.7 \\
\hline 99 & 0.11 & 0.23 & 0.40 & 0.65 & 2.63 & 4.73 & 6.16 & 12.0 & 26.1 \\
\hline
\end{tabular}


03117500 Sandy Creek at Waynesburg, Ohio

Years analyzed $=70$

Climatic year range $=1939-2008$

[APEE, annual percentage equaled or exceeded; $\mathrm{ft}^{3} / \mathrm{s}$, cubic foot per second]

\begin{tabular}{|c|c|c|c|c|c|c|c|c|c|}
\hline \multirow[b]{2}{*}{ APEE } & \multicolumn{9}{|c|}{ Daily mean streamflow $\left(\mathrm{ft}^{3} / \mathrm{s}\right)$ that was equaled or exceeded the indicated percentage of time at APEE level } \\
\hline & 98 & 95 & 90 & 80 & 50 & 20 & 10 & 5 & 2 \\
\hline 1 & 756. & 929. & 1240 & 1410 . & 1990. & 2520 & 2800 & 3290. & 4100 \\
\hline 2 & 617. & 756. & 863. & 1170 & 1440. & 1850 & 2170 & 2330 & 2880 \\
\hline 5 & 409. & 477. & 573. & 651. & 920. & 1220 & 1380. & 1470 . & 1970. \\
\hline 10 & 292. & 350 . & 401. & 450 . & 633. & 820. & 922. & 1040 . & 1290. \\
\hline 20 & 159. & 188. & 225 & 257. & 368. & 509. & 592. & 664. & 764. \\
\hline 25 & 106. & 156. & 190. & 222. & 303. & 414. & 491. & 565. & 638. \\
\hline 30 & 81.5 & 120. & 154. & 179. & 263. & 360 . & 417. & 498. & 570. \\
\hline 40 & 56.3 & 72.0 & 92.8 & 122. & 197. & 275 & 338. & 386. & 458. \\
\hline 50 & 40.4 & 52.8 & 61.2 & 88.2 & 141. & 205. & 254. & 304. & 375. \\
\hline 60 & 31.5 & 40.2 & 46.0 & 62.1 & 108 & 164. & 199. & 246. & 319. \\
\hline 70 & 23.5 & 33.5 & 36.1 & 48.4 & 79.8 & 126. & 150. & 203. & 262. \\
\hline 75 & 22.9 & 32.0 & 33.0 & 42.1 & 71.5 & 107. & 132. & 185. & 232. \\
\hline 80 & 21.4 & 30.0 & 31.0 & 36.2 & 60.8 & 96.0 & 114. & 168. & 204. \\
\hline 90 & 19.0 & 23.6 & 25.0 & 29.0 & 44.8 & 68.8 & 89.2 & 132. & 161. \\
\hline 95 & 15.5 & 20.2 & 22.0 & 24.7 & 38.0 & 57.3 & 79.6 & 106. & 128. \\
\hline 98 & 14.1 & 17.7 & 20.0 & 23.0 & 33.2 & 52.9 & 74.8 & 85.0 & 110. \\
\hline 99 & 13.5 & 16.8 & 17.4 & 21.1 & 31.7 & 50.3 & 71.7 & 79.3 & 99.4 \\
\hline
\end{tabular}

\begin{tabular}{|c|c|c|c|c|c|c|c|c|c|}
\hline \multirow[b]{2}{*}{ APEE } & \multicolumn{9}{|c|}{ 7-day mean streamflow ( $\mathrm{ft}^{3} / \mathrm{s}$ ) that was equaled or exceeded the indicated percentage of time at APEE level } \\
\hline & 98 & 95 & 90 & 80 & 50 & 20 & 10 & 5 & 2 \\
\hline 1 & 572. & 738. & 897. & 1100 & 1470 . & 1940. & 2170 & 2500 & 2930. \\
\hline 2 & 541. & 666. & 706. & 892. & 1180. & 1520. & 1720. & 1780 . & 2190. \\
\hline 5 & 396. & 455 & 548. & 676. & 876. & 1140 & 1270 & 1370 . & 1750 . \\
\hline 10 & 281. & 340 & 415. & 450. & 630. & 829. & 968. & 1060. & 1230. \\
\hline 20 & 154. & 183. & 231. & 266. & 400. & 562. & 625. & 734. & 856. \\
\hline 25 & 105. & 148. & 195. & 229. & 335. & 469. & 532. & 611. & 742. \\
\hline 30 & 85.7 & 124. & 159. & 197. & 294. & 404 & 463. & 547. & 629. \\
\hline 40 & 57.6 & 73.8 & 107. & 126. & 213. & 303. & 364. & 436. & 522. \\
\hline 50 & 41.8 & 58.0 & 64.6 & 93.5 & 153. & 231. & 293. & 357. & 438. \\
\hline 60 & 30.2 & 40.3 & 47.4 & 61.1 & 114. & 181. & 208. & 296. & 352. \\
\hline 70 & 24.6 & 34.4 & 36.8 & 49.7 & 84.9 & 133. & 167. & 233. & 288. \\
\hline 75 & 23.0 & 31.7 & 33.8 & 42.2 & 73.6 & 119. & 146. & 214. & 261. \\
\hline 80 & 21.8 & 29.4 & 31.4 & 38.4 & 64.6 & 102. & 127. & 192. & 237. \\
\hline 90 & 18.5 & 23.8 & 25.6 & 29.7 & 47.7 & 74.4 & 94.3 & 145. & 184. \\
\hline 95 & 16.2 & 20.8 & 22.7 & 25.7 & 39.1 & 63.8 & 81.3 & 118. & 147. \\
\hline 98 & 14.9 & 19.0 & 21.2 & 24.8 & 35.1 & 56.1 & 77.0 & 95.6 & 121. \\
\hline 99 & 14.5 & 18.2 & 19.8 & 24.1 & 33.7 & 54.0 & 75.6 & 83.7 & 116. \\
\hline
\end{tabular}


03119000 Sandy Creek at Sandyville, Ohio

Years analyzed $=23$

Climatic year range $=1924-46$

[APEE, annual percentage equaled or exceeded; $\mathrm{ft}^{3} / \mathrm{s}$, cubic foot per second]

\begin{tabular}{|c|c|c|c|c|c|c|c|c|c|}
\hline \multirow[b]{2}{*}{ APEE } & \multicolumn{9}{|c|}{ Daily mean streamflow ( $\left.\mathrm{ft}^{3} / \mathrm{s}\right)$ that was equaled or exceeded the indicated percentage of time at APEE level } \\
\hline & 95 & 90 & 80 & 70 & 50 & 30 & 20 & 10 & 5 \\
\hline 1 & 1140. & 2270. & 2530. & 2920. & 3270. & 4050. & 4430. & 5490. & 7490. \\
\hline 2 & 897. & 1700. & 1870. & 2130 & 2400 & 3230. & 3730. & 4260. & 4710. \\
\hline 5 & 629. & 1020. & 1210. & 1260. & 1570 . & 1980. & 2030 & 2160 & 2390. \\
\hline 10 & 471. & 692. & 752. & 809. & 1080. & 1220. & 1310. & 1440 . & 1680. \\
\hline 20 & 268. & 399. & 447. & 479. & 656. & 770 . & 830. & 911. & 1120. \\
\hline 25 & 226. & 333. & 376. & 393. & 555. & 675. & 693. & 760. & 932. \\
\hline 30 & 176. & 268. & 300. & 314. & 481. & 554. & 598. & 663. & 801. \\
\hline 40 & 135. & 163. & 210 & 248. & 354. & 424. & 461. & 541. & 658. \\
\hline 50 & 112. & 129. & 167. & 187. & 268. & 326. & 352. & 423. & 530. \\
\hline 60 & 96.8 & 109. & 133. & 155. & 211. & 247. & 281. & 324. & 412. \\
\hline 70 & 89.8 & 99.3 & 117. & 129. & 174. & 198. & 206. & 248. & 298. \\
\hline 75 & 86.8 & 92.8 & 106. & 114. & 161. & 178. & 186. & 205. & 254. \\
\hline 80 & 83.3 & 88.2 & 93.0 & 105. & 139. & 156. & 166. & 171. & 214. \\
\hline 90 & 72.8 & 74.6 & 81.6 & 89.3 & 115. & 126. & 136. & 146. & 158. \\
\hline 95 & 65.1 & 67.6 & 76.2 & 83.7 & 103. & 113. & 123. & 132. & 138. \\
\hline 98 & 57.4 & 59.7 & 72.3 & 80.2 & 98.0 & 103. & 111. & 118. & 122. \\
\hline 99 & 53.4 & 56.9 & 71.6 & 74.3 & 90.0 & 98.3 & 102. & 113. & 118. \\
\hline
\end{tabular}

\begin{tabular}{|c|c|c|c|c|c|c|c|c|c|}
\hline \multirow[b]{2}{*}{ APEE } & \multicolumn{9}{|c|}{ 7-day mean streamflow (ft $\left.{ }^{3} / s\right)$ that was equaled or exceeded the indicated percentage of time at APEE level } \\
\hline & 95 & 90 & 80 & 70 & 50 & 30 & 20 & 10 & 5 \\
\hline 1 & 883. & 1390. & 1700 & 2330 & 2950. & 3150 & 3630. & 4080 & 5940. \\
\hline 5 & 576. & 1010 & 1130. & 1240 & 1380. & 1800. & 1890. & 2190 & 2380. \\
\hline 10 & 430. & 727. & 819. & 879. & 1070. & 1250 & 1330. & 1430. & 1650. \\
\hline 30 & 185. & 286. & 308. & 351. & 516. & 607. & 657. & 788. & 935. \\
\hline 40 & 136. & 167. & 234. & 273. & 348. & 459. & 528. & 639. & 738. \\
\hline 50 & 110. & 128. & 183. & 203. & 286. & 330. & 383. & 453. & 551. \\
\hline 60 & 95.2 & 108. & 140. & 161. & 228 & 278 & 309. & 354. & 455. \\
\hline 90 & 77.2 & 79.0 & 87.6 & 92.2 & 120. & 137. & 145. & 153. & 172. \\
\hline 95 & 68.6 & 73.0 & 80.5 & 88.2 & 110. & 118. & 132. & 142. & 151. \\
\hline 98 & 60.7 & 66.0 & 77.5 & 86.2 & 105. & 108. & 120 . & 131. & 133. \\
\hline 99 & 58.4 & 63.1 & 75.3 & 84.3 & 101. & 104. & 111. & 124. & 128. \\
\hline
\end{tabular}


03125000 Home Creek near New Philadelphia, Ohio

Years analyzed $=42$

Climatic year range $=1937-78$

[APEE, annual percentage equaled or exceeded; $\mathrm{ft}^{3} / \mathrm{s}$, cubic foot per second]

\begin{tabular}{|c|c|c|c|c|c|c|c|c|c|}
\hline \multirow[b]{2}{*}{ APEE } & \multicolumn{9}{|c|}{ Daily mean streamflow $\left(\mathrm{ft}^{3} / \mathrm{s}\right)$ that was equaled or exceeded the indicated percentage of time at APEE level } \\
\hline & 95 & 90 & 80 & 70 & 50 & 30 & 20 & 10 & 5 \\
\hline 1 & 8.67 & 8.86 & 9.34 & 11.3 & 15.3 & 18.8 & 21.5 & 25.1 & 30.4 \\
\hline 2 & 4.88 & 5.62 & 6.92 & 7.50 & 9.84 & 12.4 & 12.7 & 15.6 & 18.0 \\
\hline 5 & 2.49 & 3.14 & 3.27 & 3.73 & 5.14 & 6.10 & 6.85 & 7.53 & 8.17 \\
\hline 10 & 1.52 & 1.71 & 1.94 & 2.23 & 2.99 & 3.37 & 3.78 & 4.06 & 5.08 \\
\hline 20 & 0.71 & 0.82 & 1.08 & 1.12 & 1.55 & 1.90 & 1.99 & 2.27 & 2.82 \\
\hline 25 & 0.51 & 0.60 & 0.70 & 0.89 & 1.27 & 1.56 & 1.64 & 1.87 & 2.16 \\
\hline 30 & 0.32 & 0.40 & 0.53 & 0.64 & 0.93 & 1.23 & 1.45 & 1.58 & 1.86 \\
\hline 40 & 0.12 & 0.21 & 0.30 & 0.36 & 0.55 & 0.90 & 0.98 & 1.17 & 1.29 \\
\hline 50 & 0.05 & 0.10 & 0.14 & 0.20 & 0.30 & 0.60 & 0.71 & 0.83 & 0.87 \\
\hline 60 & 0.00 & 0.01 & 0.08 & 0.10 & 0.20 & 0.36 & 0.47 & 0.60 & 0.65 \\
\hline 70 & 0.00 & 0.00 & 0.02 & 0.06 & 0.10 & 0.20 & 0.27 & 0.39 & 0.45 \\
\hline 75 & 0.00 & 0.00 & 0.00 & 0.03 & 0.10 & 0.14 & 0.20 & 0.30 & 0.40 \\
\hline 80 & 0.00 & 0.00 & 0.00 & 0.02 & 0.07 & 0.10 & 0.12 & 0.25 & 0.32 \\
\hline 90 & 0.00 & 0.00 & 0.00 & 0.00 & 0.02 & 0.04 & 0.07 & 0.10 & 0.15 \\
\hline 95 & 0.00 & 0.00 & 0.00 & 0.00 & 0.00 & 0.02 & 0.05 & 0.09 & 0.10 \\
\hline 98 & 0.00 & 0.00 & 0.00 & 0.00 & 0.00 & 0.01 & 0.04 & 0.06 & 0.10 \\
\hline 99 & 0.00 & 0.00 & 0.00 & 0.00 & 0.00 & 0.01 & 0.02 & 0.04 & 0.06 \\
\hline
\end{tabular}

\begin{tabular}{|c|c|c|c|c|c|c|c|c|c|}
\hline \multirow[b]{2}{*}{ APEE } & \multicolumn{9}{|c|}{ 7-day mean streamflow ( $\left.\mathrm{ft}^{3} / \mathrm{s}\right)$ that was equaled or exceeded the indicated percentage of time at APEE level } \\
\hline & 95 & 90 & 80 & 70 & 50 & 30 & 20 & 10 & 5 \\
\hline 1 & 5.27 & 5.37 & 6.25 & 7.28 & 9.22 & 11.5 & 13.9 & 16.9 & 20.1 \\
\hline 5 & 2.45 & 3.22 & 3.82 & 3.96 & 5.22 & 6.71 & 7.05 & 8.00 & 8.67 \\
\hline 10 & 1.56 & 1.76 & 2.35 & 2.66 & 3.44 & 4.03 & 4.30 & 5.29 & 6.00 \\
\hline 30 & 0.35 & 0.42 & 0.64 & 0.71 & 1.18 & 1.51 & 1.67 & 1.90 & 2.57 \\
\hline 40 & 0.14 & 0.23 & 0.29 & 0.37 & 0.66 & 1.03 & 1.27 & 1.42 & 1.62 \\
\hline 50 & 0.07 & 0.10 & 0.18 & 0.24 & 0.41 & 0.67 & 0.92 & 1.10 & 1.15 \\
\hline 60 & 0.05 & 0.06 & 0.12 & 0.13 & 0.26 & 0.45 & 0.66 & 0.78 & 0.86 \\
\hline 90 & 0.00 & 0.00 & 0.00 & 0.01 & 0.02 & 0.08 & 0.10 & 0.16 & 0.29 \\
\hline 95 & 0.00 & 0.00 & 0.00 & 0.00 & 0.01 & 0.03 & 0.07 & 0.11 & 0.14 \\
\hline 98 & 0.00 & 0.00 & 0.00 & 0.00 & 0.00 & 0.02 & 0.04 & 0.09 & 0.10 \\
\hline 99 & 0.00 & 0.00 & 0.00 & 0.00 & 0.00 & 0.02 & 0.04 & 0.07 & 0.09 \\
\hline
\end{tabular}


03130500 Touby Run at Mansfield, Ohio

Years analyzed $=31$

Climatic year range $=1947-77$

[APEE, annual percentage equaled or exceeded; $\mathrm{ft}^{3} / \mathrm{s}$, cubic foot per second]

\begin{tabular}{|c|c|c|c|c|c|c|c|c|c|}
\hline \multirow[b]{2}{*}{ APEE } & \multicolumn{9}{|c|}{ Daily mean streamflow $\left(\mathrm{ft}^{3} / \mathrm{s}\right)$ that was equaled or exceeded the indicated percentage of time at APEE level } \\
\hline & 95 & 90 & 80 & 70 & 50 & 30 & 20 & 10 & 5 \\
\hline 1 & 28.9 & 30.7 & 41.5 & 52.8 & 64.3 & 80.6 & 85.1 & 106. & 139. \\
\hline 2 & 21.2 & 24.5 & 29.5 & 32.5 & 42.7 & 49.5 & 54.4 & 58.2 & 66.5 \\
\hline 5 & 10.2 & 12.7 & 15.1 & 18.3 & 21.0 & 24.5 & 25.5 & 27.6 & 30.9 \\
\hline 10 & 6.00 & 6.91 & 7.26 & 9.35 & 11.4 & 13.0 & 14.0 & 16.0 & 17.0 \\
\hline 20 & 2.78 & 2.97 & 3.70 & 4.32 & 5.92 & 6.38 & 7.08 & 8.33 & 9.74 \\
\hline 25 & 1.90 & 2.21 & 2.75 & 3.54 & 4.25 & 5.23 & 5.50 & 6.38 & 8.25 \\
\hline 30 & 1.60 & 1.76 & 1.94 & 2.50 & 3.40 & 4.05 & 4.40 & 4.93 & 7.10 \\
\hline 40 & 0.86 & 1.00 & 1.34 & 1.72 & 2.20 & 2.60 & 2.91 & 3.46 & 5.20 \\
\hline 50 & 0.52 & 0.62 & 0.94 & 1.20 & 1.60 & 1.90 & 2.18 & 2.58 & 3.64 \\
\hline 60 & 0.36 & 0.50 & 0.75 & 0.96 & 1.10 & 1.34 & 1.60 & 2.00 & 2.20 \\
\hline 70 & 0.26 & 0.30 & 0.52 & 0.70 & 0.93 & 1.00 & 1.29 & 1.50 & 1.64 \\
\hline 75 & 0.20 & 0.30 & 0.44 & 0.60 & 0.79 & 1.00 & 1.06 & 1.30 & 1.48 \\
\hline 80 & 0.20 & 0.22 & 0.33 & 0.50 & 0.71 & 0.80 & 0.92 & 1.02 & 1.34 \\
\hline 90 & 0.16 & 0.20 & 0.22 & 0.30 & 0.50 & 0.61 & 0.64 & 0.79 & 0.97 \\
\hline 95 & 0.10 & 0.14 & 0.20 & 0.22 & 0.40 & 0.50 & 0.58 & 0.62 & 0.74 \\
\hline 98 & 0.08 & 0.10 & 0.15 & 0.20 & 0.30 & 0.40 & 0.50 & 0.50 & 0.74 \\
\hline 99 & 0.06 & 0.10 & 0.10 & 0.12 & 0.30 & 0.36 & 0.48 & 0.50 & 0.61 \\
\hline
\end{tabular}

\begin{tabular}{|c|c|c|c|c|c|c|c|c|c|}
\hline \multirow[b]{2}{*}{ APEE } & \multicolumn{9}{|c|}{ 7-day mean streamflow ( $\mathrm{ft}^{3} / \mathrm{s}$ ) that was equaled or exceeded the indicated percentage of time at APEE level } \\
\hline & 95 & 90 & 80 & 70 & 50 & 30 & 20 & 10 & 5 \\
\hline 1 & 19.0 & 25.2 & 29.7 & 30.5 & 33.4 & 41.3 & 44.7 & 62.1 & 68.4 \\
\hline 2 & 13.1 & 16.1 & 21.5 & 23.0 & 25.8 & 32.1 & 35.9 & 40.7 & 47.9 \\
\hline 5 & 9.54 & 10.5 & 14.9 & 17.1 & 18.8 & 20.9 & 24.2 & 31.2 & 35.5 \\
\hline 10 & 6.53 & 6.67 & 9.41 & 11.7 & 13.8 & 14.6 & 17.0 & 17.8 & 19.2 \\
\hline 20 & 3.17 & 3.53 & 5.09 & 5.56 & 7.40 & 9.30 & 10.6 & 12.8 & 13.0 \\
\hline 25 & 2.47 & 3.00 & 3.48 & 4.31 & 6.07 & 6.72 & 7.60 & 9.71 & 11.5 \\
\hline 30 & 1.84 & 2.08 & 2.83 & 3.36 & 4.89 & 5.48 & 6.02 & 7.52 & 9.50 \\
\hline 40 & 1.01 & 1.20 & 1.75 & 2.33 & 2.94 & 3.93 & 4.23 & 4.67 & 6.89 \\
\hline 50 & 0.61 & 0.81 & 1.16 & 1.61 & 2.07 & 2.80 & 3.21 & 3.61 & 5.00 \\
\hline 60 & 0.44 & 0.55 & 0.94 & 1.25 & 1.47 & 2.16 & 2.43 & 2.83 & 3.63 \\
\hline 70 & 0.33 & 0.39 & 0.66 & 0.87 & 1.23 & 1.58 & 1.80 & 2.30 & 2.45 \\
\hline 75 & 0.27 & 0.33 & 0.50 & 0.77 & 1.04 & 1.31 & 1.51 & 1.88 & 2.19 \\
\hline 80 & 0.23 & 0.27 & 0.45 & 0.63 & 0.90 & 1.18 & 1.34 & 1.56 & 1.75 \\
\hline 90 & 0.20 & 0.21 & 0.30 & 0.50 & 0.74 & 0.82 & 0.88 & 1.02 & 1.16 \\
\hline 95 & 0.16 & 0.20 & 0.20 & 0.34 & 0.47 & 0.64 & 0.68 & 0.80 & 0.96 \\
\hline 98 & 0.13 & 0.15 & 0.18 & 0.26 & 0.40 & 0.53 & 0.58 & 0.68 & 0.87 \\
\hline 99 & 0.10 & 0.11 & 0.16 & 0.23 & 0.36 & 0.49 & 0.52 & 0.58 & 0.79 \\
\hline
\end{tabular}


03134000 Jerome Fork at Jeromeville, Ohio

Years analyzed $=23$

Climatic year range $=1926-48$

[APEE, annual percentage equaled or exceeded; $\mathrm{ft}^{3} / \mathrm{s}$, cubic foot per second]

\begin{tabular}{|c|c|c|c|c|c|c|c|c|c|}
\hline \multirow[b]{2}{*}{ APEE } & \multicolumn{9}{|c|}{ Daily mean streamflow (ft $3 / s)$ that was equaled or exceeded the indicated percentage of time at APEE level } \\
\hline & 95 & 90 & 80 & 70 & 50 & 30 & 20 & 10 & 5 \\
\hline 1 & 371. & 664. & 889. & 947. & 1290. & 1600. & 1940. & 2270 & 2440 \\
\hline 2 & 293. & 467. & 526. & 736. & 916. & 1130 & 1310 . & 1430 . & 1650 \\
\hline 5 & 127. & 190. & 310. & 369. & 420 . & 520. & 580. & 788. & 901. \\
\hline 10 & 71.8 & 98.4 & 148. & 169. & 203. & 260. & 288 & 398. & 479 . \\
\hline 20 & 39.4 & 43.9 & 60.2 & 67.5 & 95.2 & 130. & 149. & 197. & 237. \\
\hline 25 & 26.8 & 32.4 & 43.1 & 51.0 & 70.0 & 106. & 114. & 162. & 195. \\
\hline 30 & 16.8 & 24.2 & 30.8 & 37.6 & 55.0 & 86.8 & 91.8 & 127. & 167. \\
\hline 40 & 10.1 & 13.8 & 18.5 & 21.6 & 34.6 & 58.6 & 69.0 & 81.5 & 106. \\
\hline 50 & 7.84 & 10.2 & 12.0 & 15.2 & 25.0 & 40.8 & 46.0 & 60.2 & 79.4 \\
\hline 60 & 6.30 & 7.66 & 8.88 & 11.6 & 19.0 & 25.8 & 33.1 & 39.5 & 51.0 \\
\hline 70 & 4.98 & 5.90 & 6.98 & 9.44 & 15.0 & 18.0 & 23.8 & 28.1 & 30.4 \\
\hline 75 & 4.88 & 5.31 & 6.00 & 7.52 & 12.0 & 15.6 & 18.0 & 22.6 & 23.4 \\
\hline 80 & 4.46 & 4.80 & 5.59 & 6.36 & 11.0 & 12.8 & 15.4 & 19.6 & 20.0 \\
\hline 90 & 3.30 & 3.74 & 4.08 & 5.16 & 6.20 & 8.80 & 9.49 & 10.6 & 14.2 \\
\hline 95 & 2.91 & 3.16 & 3.50 & 3.84 & 5.00 & 6.04 & 7.16 & 9.60 & 11.6 \\
\hline 98 & 2.27 & 2.46 & 2.85 & 3.12 & 4.00 & 5.52 & 6.26 & 8.24 & 9.95 \\
\hline 99 & 2.04 & 2.28 & 2.65 & 2.91 & 4.00 & 4.96 & 6.16 & 7.92 & 9.57 \\
\hline
\end{tabular}

\begin{tabular}{|c|c|c|c|c|c|c|c|c|c|}
\hline \multirow[b]{2}{*}{ APEE } & \multicolumn{9}{|c|}{ 7-day mean streamflow $\left(\mathrm{ft}^{3} / \mathrm{s}\right)$ that was equaled or exceeded the indicated percentage of time at APEE level } \\
\hline & 95 & 90 & 80 & 70 & 50 & 30 & 20 & 10 & 5 \\
\hline 1 & 242. & 400. & 558. & 646. & 733. & 917. & 1000. & 1260 & 1370 \\
\hline 2 & 193. & 314. & 433. & 536. & 657. & 780 . & 886. & 1100 & 1250 \\
\hline 5 & 121. & 212 & 304. & 377. & 423. & 615 & 667. & 709. & 767. \\
\hline 10 & 82.9 & 126. & 188. & 226. & 259. & 312. & 339. & 421 & 486. \\
\hline 20 & 41.1 & 50.1 & 64.8 & 80.2 & 134. & 165. & 198. & 248 & 298. \\
\hline 25 & 30.6 & 32.7 & 49.3 & 61.5 & 96.3 & 137. & 161. & 218 & 243. \\
\hline 30 & 22.0 & 26.2 & 39.2 & 45.9 & 68.4 & 109. & 129. & 180. & 212 \\
\hline 40 & 10.6 & 15.0 & 20.0 & 27.3 & 43.3 & 76.7 & 86.7 & 106. & 141. \\
\hline 50 & 8.32 & 10.3 & 14.5 & 19.0 & 28.9 & 50.2 & 58.9 & 69.9 & 103. \\
\hline 60 & 6.97 & 8.40 & 9.27 & 12.1 & 23.4 & 32.3 & 43.8 & 49.2 & 71.3 \\
\hline 70 & 5.87 & 6.70 & 7.76 & 9.99 & 17.7 & 22.2 & 24.8 & 35.3 & 40.2 \\
\hline 75 & 5.29 & 5.73 & 6.80 & 8.77 & 14.1 & 17.0 & 21.0 & 29.1 & 29.7 \\
\hline 80 & 4.89 & 5.09 & 6.12 & 7.75 & 12.9 & 14.0 & 17.0 & 22.2 & 25.4 \\
\hline 90 & 3.91 & 4.26 & 4.45 & 5.46 & 7.30 & 9.85 & 11.4 & 12.6 & 15.3 \\
\hline 95 & 3.40 & 3.59 & 3.95 & 4.86 & 5.70 & 7.64 & 8.79 & 10.9 & 13.7 \\
\hline 98 & 2.80 & 3.08 & 3.21 & 3.38 & 4.49 & 5.79 & 7.41 & 10.1 & 11.8 \\
\hline 99 & 2.57 & 2.71 & 2.95 & 3.24 & 4.00 & 5.51 & 6.75 & 9.65 & 11.0 \\
\hline
\end{tabular}


03136500 Kokosing River at Mount Vernon, Ohio

Years analyzed $=57$

Climatic year range $=1953-2009$

[APEE, annual percentage equaled or exceeded; $\mathrm{ft}^{3} / \mathrm{s}$, cubic foot per second]

\begin{tabular}{|c|c|c|c|c|c|c|c|c|c|}
\hline \multirow[b]{2}{*}{ APEE } & \multicolumn{9}{|c|}{ Daily mean streamflow $\left(\mathrm{ft}^{3} / \mathrm{s}\right)$ that was equaled or exceeded the indicated percentage of time at APEE level } \\
\hline & 98 & 95 & 90 & 80 & 50 & 20 & 10 & 5 & 2 \\
\hline 1 & 821. & 946. & 1110. & 1380. & 1840. & 2590. & 2870 & 3220. & 3680. \\
\hline 2 & 562. & 745. & 839. & 945. & 1330. & 1710 . & 1910. & 1990. & 2350 \\
\hline 5 & 306. & 414. & 448. & 536. & 757. & 1070 . & 1120 & 1310 . & 1550. \\
\hline 10 & 180. & 223. & 301. & 328. & 463. & 652. & 745. & 837. & 1040. \\
\hline 20 & 80.0 & 118. & 168. & 198. & 275. & 371. & 418. & 446. & 499. \\
\hline 25 & 63.2 & 97.1 & 124. & 164. & 221. & 305. & 337. & 367. & 406. \\
\hline 30 & 49.8 & 75.2 & 104. & 126. & 188. & 260. & 300 . & 315. & 356. \\
\hline 40 & 37.8 & 54.2 & 61.6 & 89.6 & 143. & 185. & 235. & 255. & 283. \\
\hline 50 & 30.8 & 39.5 & 45.6 & 64.6 & 110. & 145. & 188. & 215. & 234. \\
\hline 60 & 27.0 & 31.5 & 38.2 & 48.8 & 78.6 & 109. & 149. & 169. & 180. \\
\hline 70 & 24.0 & 26.7 & 29.0 & 38.2 & 58.0 & 83.1 & 111. & 129. & 146. \\
\hline 75 & 21.3 & 23.0 & 27.0 & 32.8 & 50.0 & 76.2 & 99.8 & 111. & 134. \\
\hline 80 & 20.2 & 21.9 & 24.4 & 30.0 & 44.2 & 69.8 & 86.9 & 91.2 & 121. \\
\hline 90 & 15.3 & 18.8 & 20.8 & 25.6 & 35.0 & 56.0 & 62.4 & 71.3 & 85.6 \\
\hline 95 & 13.4 & 14.3 & 18.4 & 23.2 & 32.0 & 49.6 & 52.5 & 59.0 & 70.3 \\
\hline 98 & 11.4 & 12.3 & 16.4 & 20.0 & 28.3 & 42.7 & 47.3 & 49.3 & 58.9 \\
\hline 99 & 10.6 & 10.7 & 14.8 & 18.0 & 27.7 & 40.2 & 46.2 & 48.0 & 56.9 \\
\hline
\end{tabular}

\begin{tabular}{|c|c|c|c|c|c|c|c|c|c|}
\hline \multirow[b]{2}{*}{ APEE } & \multicolumn{9}{|c|}{ 7-day mean streamflow (ft $\left.{ }^{3} / s\right)$ that was equaled or exceeded the indicated percentage of time at APEE level } \\
\hline & 98 & 95 & 90 & 80 & 50 & 20 & 10 & 5 & 2 \\
\hline 1 & 414. & 616. & 678. & 871. & 1370 & 1670 & 1970. & 2560 & 3450 \\
\hline 5 & 287. & 397. & 449. & 553. & 735. & 953. & 1060. & 1180 & 1180. \\
\hline 10 & 199. & 221. & 326. & 374. & 494. & 667. & 723. & 799. & 898. \\
\hline 30 & 53.9 & 85.1 & 111. & 138. & 216. & 304. & 364. & 403. & 454. \\
\hline 40 & 38.3 & 56.1 & 62.5 & 94.5 & 159. & 219. & 274. & 292. & 353. \\
\hline 50 & 31.6 & 39.2 & 47.7 & 65.7 & 117. & 164. & 210 & 242. & 284 \\
\hline 60 & 27.3 & 32.4 & 36.5 & 49.1 & 83.1 & 121. & 161. & 206. & 216. \\
\hline 90 & 16.1 & 19.2 & 21.0 & 26.4 & 36.7 & 59.4 & 68.1 & 77.5 & 91.2 \\
\hline 95 & 14.0 & 14.7 & 18.4 & 24.7 & 33.0 & 51.9 & 56.6 & 64.2 & 78.3 \\
\hline 98 & 11.8 & 13.3 & 17.0 & 20.7 & 31.0 & 45.7 & 50.1 & 53.5 & 67.3 \\
\hline 99 & 10.9 & 12.7 & 15.7 & 19.1 & 29.6 & 42.9 & 48.6 & 51.0 & 63.5 \\
\hline
\end{tabular}


03137000 Kokosing River at Millwood, Ohio

Years analyzed $=52$

Climatic year range $=1922-73$

[APEE, annual percentage equaled or exceeded; $\mathrm{ft}^{3} / \mathrm{s}$, cubic foot per second]

\begin{tabular}{|c|c|c|c|c|c|c|c|c|c|}
\hline \multirow[b]{2}{*}{ APEE } & \multicolumn{9}{|c|}{ Daily mean streamflow ( $\mathrm{ft}^{3} / \mathrm{s}$ ) that was equaled or exceeded the indicated percentage of time at APEE level } \\
\hline & 98 & 95 & 90 & 80 & 50 & 20 & 10 & 5 & 2 \\
\hline 1 & 696. & 1410. & 2370. & 3020. & 4500. & 6160. & 6960. & 7710. & 9070. \\
\hline 2 & 572. & 825. & 1780 & 2280 & 2840 & 3920. & 4700. & 5180. & 5310. \\
\hline 5 & 383. & 523. & 943. & 1290. & 1740. & 2210 & 2750 . & 2850 . & 2940. \\
\hline 10 & 245 & 373. & 545. & 735. & 1080. & 1360. & 1570 . & 1730. & 1850. \\
\hline 20 & 141. & 203. & 287. & 419. & 619. & 750. & 952. & 1040. & 1160. \\
\hline 25 & 128. & 146. & 235 & 322. & 502. & 641. & 802. & 886. & 1000. \\
\hline 30 & 108. & 112. & 189. & 251. & 418. & 553. & 676. & 787. & 798. \\
\hline 40 & 72.2 & 88.4 & 117. & 159. & 278. & 404. & 467. & 609. & 638. \\
\hline 50 & 64.0 & 69.2 & 86.0 & 114. & 199. & 298. & 345. & 442. & 498. \\
\hline 60 & 55.1 & 59.0 & 70.0 & 92.4 & 152. & 213. & 278 & 332. & 400. \\
\hline 70 & 51.1 & 53.3 & 62.8 & 78.3 & 117. & 160. & 209. & 247. & 319. \\
\hline 75 & 49.1 & 51.0 & 60.5 & 72.6 & 97.8 & 136. & 181. & 210. & 292. \\
\hline 80 & 46.0 & 48.7 & 54.7 & 65.2 & 86.9 & 120. & 157. & 177. & 259. \\
\hline 90 & 42.0 & 43.5 & 45.3 & 58.6 & 75.0 & 96.9 & 122. & 137. & 173. \\
\hline 95 & 38.1 & 39.6 & 42.2 & 54.4 & 70.0 & 85.4 & 107. & 122. & 154. \\
\hline 98 & 36.0 & 36.8 & 39.0 & 46.2 & 64.8 & 80.8 & 94.6 & 113. & 130. \\
\hline 99 & 35.1 & 36.0 & 38.3 & 45.2 & 62.0 & 77.9 & 92.2 & 110. & 128. \\
\hline
\end{tabular}

\begin{tabular}{|c|c|c|c|c|c|c|c|c|c|}
\hline \multirow[b]{2}{*}{ APEE } & \multicolumn{9}{|c|}{ 7-day mean streamflow ( $\mathrm{ft}^{3} / \mathrm{s}$ ) that was equaled or exceeded the indicated percentage of time at APEE level } \\
\hline & 98 & 95 & 90 & 80 & 50 & 20 & 10 & 5 & 2 \\
\hline 1 & 504. & 868. & 1550. & 2230 & 2840 & 4220 & 5570. & 6350 & 8980. \\
\hline 2 & 453. & 716. & 1310 & 1970. & 2330 & 3050 . & 3860. & 4760 & 4900. \\
\hline 5 & 362. & 485 & 1120 & 1290. & 1710 & 2170 & 2570 & 2710 & 2920. \\
\hline 10 & 249. & 398. & 534. & 860. & 1160 & 1450 & 1730. & 1890. & 1940. \\
\hline 20 & 142. & 186. & 292. & 449. & 700 & 938. & 1090. & 1240 & 1270 . \\
\hline 25 & 121. & 149. & 237. & 364. & 578. & 739. & 869. & 1050 & 1140 \\
\hline 30 & 108. & 116. & 194. & 251. & 472 & 645. & 741. & 911. & 1050 \\
\hline 40 & 76.2 & 88.1 & 123. & 167. & 327. & 462. & 571. & 678. & 722. \\
\hline 50 & 63.9 & 70.4 & 93.7 & 119. & 222 & 347. & 389. & 493. & 581. \\
\hline 60 & 57.1 & 59.2 & 71.7 & 94.6 & 160. & 237. & 314. & 389. & 469. \\
\hline 70 & 51.3 & 54.2 & 63.7 & 81.6 & 126. & 176. & 238 & 272. & 338. \\
\hline 75 & 50.0 & 51.0 & 61.3 & 73.1 & 103. & 150. & 190. & 222. & 309. \\
\hline 80 & 48.0 & 49.1 & 56.5 & 65.5 & 93.4 & 128. & 165. & 191. & 283. \\
\hline 90 & 40.6 & 43.9 & 48.2 & 59.5 & 77.4 & 100. & 130. & 143. & 190. \\
\hline 95 & 37.9 & 40.6 & 44.6 & 55.3 & 73.4 & 89.1 & 111. & 127. & 169. \\
\hline 98 & 37.3 & 38.0 & 42.9 & 48.7 & 67.5 & 82.9 & 99.1 & 121. & 142. \\
\hline 99 & 37.0 & 37.2 & 41.6 & 46.4 & 65.3 & 80.4 & 96.5 & 117. & 133. \\
\hline
\end{tabular}


03139000 Killbuck Creek at Killbuck, Ohio

Years analyzed $=78$

Climatic year range $=1931-2008$

[APEE, annual percentage equaled or exceeded; $\mathrm{ft}^{3} / \mathrm{s}$, cubic foot per second]

\begin{tabular}{|c|c|c|c|c|c|c|c|c|c|}
\hline \multirow[b]{2}{*}{ APEE } & \multicolumn{9}{|c|}{ Daily mean streamflow $\left(\mathrm{ft}^{3} / \mathrm{s}\right)$ that was equaled or exceeded the indicated percentage of time at APEE level } \\
\hline & 98 & 95 & 90 & 80 & 50 & 20 & 10 & 5 & 2 \\
\hline 1 & 1260 & 1340 & 1630. & 2000 & 2530 & 3460. & 4200 & 4850 & 9010. \\
\hline 2 & 1010 & 1150 & 1440. & 1620. & 2100 & 2780 & 3140 . & 3340. & 3970. \\
\hline 5 & 660. & 783. & 913. & 1110 & 1470 & 1920. & 2220 & 2520 & 2630. \\
\hline 10 & 472. & 501. & 569. & 707. & 1020 & 1390. & 1510 . & 1690. & 1800. \\
\hline 20 & 218 & 287. & 320. & 402. & 588. & 879. & 1040 & 1170 & 1230. \\
\hline 25 & 157. & 216. & 238 & 320 . & 489. & 713. & 937. & 992. & 1050. \\
\hline 30 & 119. & 178. & 205. & 274 & 409. & 585. & 769. & 832. & 928. \\
\hline 40 & 81.9 & 96.5 & 133. & 177. & 305. & 443. & 511. & 632. & 676. \\
\hline 50 & 59.0 & 71.8 & 94.5 & 115. & 221. & 315. & 385. & 501. & 558. \\
\hline 60 & 47.3 & 61.4 & 76.8 & 89.7 & 158. & 231. & 315. & 405 & 458. \\
\hline 70 & 39.7 & 51.8 & 58.6 & 71.0 & 121. & 179. & 266. & 317. & 375. \\
\hline 75 & 35.9 & 46.8 & 51.0 & 65.3 & 103. & 154. & 229. & 277. & 352. \\
\hline 80 & 33.7 & 43.1 & 46.9 & 60.2 & 92.2 & 134. & 193. & 257. & 316. \\
\hline 90 & 29.2 & 31.0 & 38.9 & 47.8 & 74.0 & 104. & 135. & 197. & 256. \\
\hline 95 & 26.6 & 30.0 & 36.9 & 43.3 & 63.6 & 93.1 & 120 & 157. & 231. \\
\hline 98 & 24.3 & 28.0 & 33.3 & 38.0 & 57.0 & 81.8 & 99.4 & 144. & 176. \\
\hline 99 & 24.2 & 26.6 & 32.0 & 36.6 & 56.0 & 79.3 & 96.4 & 135. & 149. \\
\hline
\end{tabular}

\begin{tabular}{|c|c|c|c|c|c|c|c|c|c|}
\hline \multirow[b]{2}{*}{ APEE } & \multicolumn{9}{|c|}{ 7-day mean streamflow (ft $\left.{ }^{3} / s\right)$ that was equaled or exceeded the indicated percentage of time at APEE level } \\
\hline & 98 & 95 & 90 & 80 & 50 & 20 & 10 & 5 & 2 \\
\hline 1 & 983. & 1020. & 1350 & 1700 & 2160 & 2920 & 3640. & 4930. & 9130. \\
\hline 5 & 585. & 710. & 862. & 1030. & 1390. & 1820. & 2110. & 2280 & 2450 . \\
\hline 10 & 443. & 503. & 538. & 709. & 991. & 1330. & 1530. & 1620. & 1730. \\
\hline 30 & 134. & 189. & 213. & 282. & 442. & 645. & 796. & 857. & 915. \\
\hline 40 & 83.3 & 102. & 138. & 176. & 329. & 475 & 575. & 690. & 748. \\
\hline 50 & 60.4 & 81.6 & 103. & 126. & 238. & 338. & 412. & 559. & 618. \\
\hline 60 & 48.4 & 65.3 & 78.3 & 95.8 & 166. & 253. & 342. & 432. & 521. \\
\hline 90 & 29.5 & 33.5 & 41.3 & 51.2 & 75.8 & 114. & 143. & 214. & 293. \\
\hline 95 & 26.7 & 30.4 & 37.1 & 44.7 & 67.6 & 96.6 & 124. & 183. & 261. \\
\hline 98 & 25.2 & 29.1 & 35.2 & 40.5 & 57.8 & 89.9 & 111. & 163. & 211. \\
\hline 99 & 24.7 & 27.4 & 34.3 & 38.7 & 57.3 & 88.0 & 102. & 150. & 176. \\
\hline
\end{tabular}


03140000 Mill Creek near Coshocton, Ohio

Years analyzed $=72$

Climatic year range $=1937-2008$

[APEE, annual percentage equaled or exceeded; $\mathrm{ft}^{3} / \mathrm{s}$, cubic foot per second]

\begin{tabular}{|c|c|c|c|c|c|c|c|c|c|}
\hline \multirow[b]{2}{*}{ APEE } & \multicolumn{9}{|c|}{ Daily mean streamflow $\left(\mathrm{ft}^{3} / \mathrm{s}\right)$ that was equaled or exceeded the indicated percentage of time at APEE level } \\
\hline & 98 & 95 & 90 & 80 & 50 & 20 & 10 & 5 & 2 \\
\hline 1 & 130. & 144. & 165. & 193. & 291. & 408 & 462. & 478 & 571. \\
\hline 2 & 64.9 & 92.2 & 101. & 128. & 184. & 255. & 283. & 345. & 408. \\
\hline 5 & 38.9 & 49.3 & 55.8 & 71.2 & 97.3 & 136. & 152. & 160. & 212. \\
\hline 10 & 26.1 & 32.7 & 39.0 & 44.9 & 61.0 & 83.7 & 93.6 & 99.1 & 119. \\
\hline 20 & 14.5 & 16.5 & 19.6 & 25.5 & 37.1 & 48.0 & 53.2 & 56.4 & 64.1 \\
\hline 25 & 9.39 & 11.8 & 14.2 & 19.3 & 30.8 & 39.7 & 44.7 & 49.7 & 52.6 \\
\hline 30 & 6.79 & 7.78 & 11.0 & 14.6 & 25.1 & 32.5 & 38.1 & 40.3 & 44.5 \\
\hline 40 & 3.00 & 3.67 & 6.45 & 9.33 & 16.3 & 24.0 & 27.9 & 31.1 & 34.9 \\
\hline 50 & 1.84 & 2.15 & 3.32 & 5.00 & 10.8 & 18.4 & 20.7 & 24.0 & 28.2 \\
\hline 60 & 1.00 & 1.28 & 2.10 & 3.16 & 7.00 & 12.4 & 15.7 & 17.7 & 22.9 \\
\hline 70 & 0.55 & 0.83 & 1.22 & 1.78 & 4.28 & 7.78 & 10.0 & 13.3 & 18.2 \\
\hline 75 & 0.30 & 0.61 & 0.80 & 1.40 & 3.10 & 6.05 & 8.32 & 11.7 & 16.6 \\
\hline 80 & 0.25 & 0.44 & 0.60 & 1.20 & 2.20 & 4.89 & 7.11 & 10.2 & 13.6 \\
\hline 90 & 0.10 & 0.20 & 0.30 & 0.56 & 1.25 & 2.70 & 4.57 & 6.84 & 10.0 \\
\hline 95 & 0.10 & 0.10 & 0.15 & 0.30 & 1.00 & 2.03 & 3.11 & 5.48 & 8.80 \\
\hline 98 & 0.10 & 0.10 & 0.11 & 0.20 & 0.62 & 1.64 & 2.43 & 4.35 & 7.84 \\
\hline 99 & 0.08 & 0.10 & 0.10 & 0.20 & 0.51 & 1.51 & 2.05 & 4.10 & 7.16 \\
\hline
\end{tabular}

\begin{tabular}{|c|c|c|c|c|c|c|c|c|c|}
\hline \multirow[b]{2}{*}{ APEE } & \multicolumn{9}{|c|}{ 7-day mean streamflow ( $\mathrm{ft}^{3} / \mathrm{s}$ ) that was equaled or exceeded the indicated percentage of time at APEE level } \\
\hline & 98 & 95 & 90 & 80 & 50 & 20 & 10 & 5 & 2 \\
\hline 1 & 72.2 & 93.7 & 102. & 119. & 171. & 230 & 306. & 402. & 460. \\
\hline 2 & 63.1 & 78.6 & 90.1 & 101. & 143. & 190. & 222 & 256. & 289. \\
\hline 5 & 37.2 & 49.4 & 65.7 & 76.5 & 103. & 137. & 150 & 168 & 212 \\
\hline 10 & 25.7 & 36.3 & 41.5 & 49.9 & 69.0 & 93.8 & 101. & 114 & 134. \\
\hline 20 & 13.4 & 18.7 & 21.4 & 27.2 & 42.6 & 57.3 & 67.5 & 71.4 & 77.3 \\
\hline 25 & 9.49 & 12.3 & 16.3 & 21.7 & 34.7 & 48.1 & 55.4 & 60.8 & 67.3 \\
\hline 30 & 7.27 & 8.11 & 12.1 & 16.1 & 29.2 & 38.2 & 44.9 & 49.7 & 61.2 \\
\hline 40 & 3.36 & 3.92 & 7.12 & 10.6 & 18.9 & 28.3 & 33.6 & 38.6 & 44.9 \\
\hline 50 & 2.27 & 2.52 & 4.35 & 5.73 & 11.6 & 20.4 & 24.3 & 27.4 & 36.1 \\
\hline 60 & 1.30 & 1.44 & 2.55 & 3.90 & 7.42 & 14.8 & 18.1 & 20.4 & 28.6 \\
\hline 70 & 0.61 & 0.98 & 1.58 & 2.22 & 4.68 & 9.27 & 12.2 & 16.4 & 22.1 \\
\hline 75 & 0.33 & 0.71 & 1.04 & 1.65 & 3.69 & 6.97 & 10.0 & 13.8 & 19.8 \\
\hline 80 & 0.21 & 0.52 & 0.76 & 1.32 & 2.73 & 5.65 & 8.02 & 11.7 & 17.6 \\
\hline 90 & 0.11 & 0.27 & 0.36 & 0.70 & 1.59 & 3.31 & 5.73 & 8.34 & 12.4 \\
\hline 95 & 0.10 & 0.14 & 0.22 & 0.38 & 1.14 & 2.43 & 4.19 & 6.41 & 10.1 \\
\hline 98 & 0.10 & 0.10 & 0.14 & 0.26 & 0.81 & 1.91 & 3.02 & 5.22 & 9.35 \\
\hline 99 & 0.08 & 0.10 & 0.11 & 0.22 & 0.62 & 1.70 & 2.72 & 4.91 & 8.91 \\
\hline
\end{tabular}




\section{Wakatomika Creek near Frazeysburg, Ohio}

Years analyzed $=72$

Climatic year range $=1937-2008$

[APEE, annual percentage equaled or exceeded; $\mathrm{ft}^{3} / \mathrm{s}$, cubic foot per second]

\begin{tabular}{|c|c|c|c|c|c|c|c|c|c|}
\hline \multirow[b]{2}{*}{ APEE } & \multicolumn{9}{|c|}{ Daily mean streamflow $\left(\mathrm{ft}^{3} / \mathrm{s}\right)$ that was equaled or exceeded the indicated percentage of time at APEE level } \\
\hline & 98 & 95 & 90 & 80 & 50 & 20 & 10 & 5 & 2 \\
\hline 1 & 577. & 813. & 944. & 1070. & 1430. & 2020. & 2590. & 3190. & 3250 \\
\hline 2 & 374. & 511. & 575. & 764. & 974. & 1250. & 1480 & 1870 . & 2070 \\
\hline 5 & 218 & 269. & 355. & 400 & 540. & 680. & 761. & 849. & 1090. \\
\hline 10 & 137. & 174. & 203. & 256. & 332. & 435. & 478. & 531. & 649. \\
\hline 20 & 57.8 & 91.7 & 119. & 151. & 194. & 253. & 297. & 319. & 357. \\
\hline 25 & 36.3 & 72.4 & 95.6 & 119. & 161. & 213. & 244. & 261. & 284. \\
\hline 30 & 27.2 & 53.1 & 72.0 & 95.3 & 134. & 179. & 206. & 218. & 248 \\
\hline 40 & 18.7 & 27.9 & 45.4 & 63.5 & 96.1 & 131. & 151. & 163. & 188. \\
\hline 50 & 12.9 & 17.6 & 23.6 & 42.2 & 65.0 & 96.4 & 111. & 134. & 151. \\
\hline 60 & 9.52 & 13.7 & 15.0 & 25.0 & 46.0 & 65.0 & 78.0 & 106. & 122. \\
\hline 70 & 7.95 & 9.93 & 11.2 & 17.5 & 30.3 & 47.9 & 59.1 & 75.5 & 96.2 \\
\hline 75 & 7.09 & 8.71 & 10.3 & 14.0 & 25.0 & 38.9 & 47.4 & 63.0 & 87.0 \\
\hline 80 & 6.31 & 7.45 & 9.21 & 11.0 & 20.0 & 31.5 & 40.7 & 53.8 & 73.2 \\
\hline 90 & 4.49 & 5.30 & 6.24 & 8.06 & 12.8 & 21.8 & 28.0 & 34.2 & 51.9 \\
\hline 95 & 3.44 & 4.16 & 4.80 & 6.93 & 9.45 & 15.9 & 21.8 & 27.4 & 37.4 \\
\hline 98 & 2.84 & 3.37 & 4.16 & 5.61 & 8.32 & 13.8 & 18.7 & 23.4 & 31.0 \\
\hline 99 & 2.75 & 3.20 & 3.69 & 5.35 & 8.00 & 12.8 & 17.9 & 21.6 & 29.1 \\
\hline
\end{tabular}

\begin{tabular}{|c|c|c|c|c|c|c|c|c|c|}
\hline \multirow[b]{2}{*}{ APEE } & \multicolumn{9}{|c|}{ 7-day mean streamflow ( $\mathrm{ft}^{3} / \mathrm{s}$ ) that was equaled or exceeded the indicated percentage of time at APEE level } \\
\hline & 98 & 95 & 90 & 80 & 50 & 20 & 10 & 5 & 2 \\
\hline 1 & 381. & 537. & 600 & 721. & 1020. & 1480 & 1910. & 2040 & 2890 \\
\hline 2 & 324. & 462. & 509. & 573. & 772. & 1040. & 1180. & 1380. & 1610 \\
\hline 5 & 241. & 299. & 324. & 423. & 548. & 705. & 829. & 970. & 1070 \\
\hline 10 & 143. & 184. & 219. & 294. & 371. & 477. & 549. & 574. & 707. \\
\hline 20 & 50.2 & 95.2 & 119. & 160. & 225. & 291. & 343. & 348. & 409. \\
\hline 25 & 38.2 & 69.1 & 102. & 129. & 179. & 243. & 288 & 301. & 372. \\
\hline 30 & 25.2 & 57.9 & 73.3 & 109. & 153. & 199. & 240. & 259. & 322. \\
\hline 40 & 18.6 & 27.3 & 46.4 & 66.8 & 107. & 149. & 175. & 189. & 247. \\
\hline 50 & 13.3 & 17.9 & 25.8 & 44.1 & 74.1 & 107. & 133. & 145. & 194. \\
\hline 60 & 10.2 & 14.3 & 16.0 & 28.6 & 50.5 & 74.0 & 93.4 & 117. & 148. \\
\hline 70 & 8.24 & 10.4 & 12.1 & 18.6 & 33.6 & 51.6 & 68.2 & 81.9 & 113. \\
\hline 75 & 7.13 & 8.81 & 10.8 & 15.6 & 26.9 & 44.4 & 52.6 & 70.1 & 102. \\
\hline 80 & 6.53 & 7.93 & 9.54 & 12.5 & 22.1 & 35.6 & 45.1 & 58.0 & 88.2 \\
\hline 90 & 4.51 & 5.52 & 7.42 & 8.54 & 13.6 & 23.5 & 30.7 & 38.4 & 61.2 \\
\hline 95 & 3.50 & 4.33 & 5.33 & 7.38 & 10.6 & 18.2 & 24.1 & 32.2 & 44.2 \\
\hline 98 & 2.95 & 3.69 & 4.40 & 6.18 & 9.26 & 14.9 & 20.7 & 25.3 & 36.0 \\
\hline 99 & 2.83 & 3.47 & 3.97 & 5.69 & 8.71 & 13.8 & 19.4 & 24.3 & 32.2 \\
\hline
\end{tabular}


03146000 North Fork Licking River at Utica, Ohio

Years analyzed $=20$

Climatic year range $=1940-81$

[APEE, annual percentage equaled or exceeded; $\mathrm{ft}^{3} / \mathrm{s}$, cubic foot per second]

\begin{tabular}{|c|c|c|c|c|c|c|c|c|c|}
\hline \multirow[b]{2}{*}{ APEE } & \multicolumn{9}{|c|}{ Daily mean streamflow $\left(\mathrm{ft}^{3} / \mathrm{s}\right)$ that was equaled or exceeded the indicated percentage of time at APEE level } \\
\hline & 95 & 90 & 80 & 70 & 50 & 30 & 20 & 10 & 5 \\
\hline 1 & 814. & 904. & 1430. & 1540. & 1820. & 2260. & 2510. & 2950. & 2990. \\
\hline 2 & 573. & 753. & 863. & 993. & 1270 & 1440 . & 1710 . & 1770 . & 1780 \\
\hline 5 & 290. & 313. & 428. & 500. & 560. & 778. & 820. & 852. & 863. \\
\hline 10 & 142. & 172. & 198. & 238. & 292. & 402. & 455. & 482. & 506. \\
\hline 20 & 68.0 & 73.5 & 95.0 & 120. & 148. & 194. & 221. & 234. & 263. \\
\hline 25 & 45.7 & 58.6 & 70.5 & 96.8 & 111. & 150. & 169. & 179. & 201. \\
\hline 30 & 25.4 & 44.6 & 54.6 & 75.6 & 90.5 & 119. & 135. & 153. & 174. \\
\hline 40 & 8.91 & 30.1 & 34.5 & 50.9 & 61.6 & 82.0 & 89.7 & 111. & 127. \\
\hline 50 & 6.08 & 19.3 & 24.2 & 33.6 & 45.5 & 53.9 & 63.7 & 81.8 & 84.8 \\
\hline 60 & 4.25 & 7.38 & 16.4 & 21.2 & 29.5 & 37.0 & 44.7 & 62.0 & 62.0 \\
\hline 70 & 3.55 & 4.77 & 10.8 & 14.0 & 17.4 & 27.7 & 30.6 & 42.7 & 51.6 \\
\hline 75 & 3.43 & 4.23 & 8.28 & 10.6 & 13.5 & 22.5 & 24.0 & 31.3 & 45.3 \\
\hline 80 & 3.04 & 3.98 & 7.16 & 8.73 & 12.0 & 15.8 & 19.8 & 20.2 & 37.5 \\
\hline 90 & 1.86 & 3.04 & 4.65 & 6.09 & 7.14 & 9.77 & 11.8 & 14.9 & 25.2 \\
\hline 95 & 1.48 & 2.46 & 3.79 & 4.83 & 5.42 & 8.28 & 9.58 & 11.2 & 20.5 \\
\hline 98 & 1.06 & 2.24 & 3.46 & 3.86 & 4.34 & 5.92 & 7.44 & 8.92 & 15.0 \\
\hline 99 & 0.95 & 1.92 & 3.01 & 3.18 & 3.85 & 5.44 & 6.59 & 7.44 & 13.7 \\
\hline
\end{tabular}

\begin{tabular}{|c|c|c|c|c|c|c|c|c|c|}
\hline \multirow[b]{2}{*}{ APEE } & \multicolumn{9}{|c|}{ 7-day mean streamflow $\left(\mathrm{ft}^{3} / \mathrm{s}\right)$ that was equaled or exceeded the indicated percentage of time at APEE level } \\
\hline & 95 & 90 & 80 & 70 & 50 & 30 & 20 & 10 & 5 \\
\hline 1 & 556. & 596. & 696. & 773. & 967. & 1200. & 1350. & 1430. & 1490 \\
\hline 2 & 426. & 495. & 633. & 670. & 765. & 970. & 1040. & 1130. & 1280 \\
\hline 5 & 248 & 323. & 416. & 448. & 536. & 679. & 767. & 826. & 894. \\
\hline 10 & 163. & 244. & 294. & 304. & 362. & 496. & 528. & 554. & 559. \\
\hline 20 & 87.7 & 94.1 & 132. & 175. & 201. & 273. & 288. & 365. & 408. \\
\hline 25 & 58.7 & 74.5 & 87.6 & 138. & 170. & 204. & 236. & 276. & 330. \\
\hline 30 & 25.8 & 56.1 & 71.6 & 103. & 123. & 172. & 196. & 208. & 286. \\
\hline 40 & 9.70 & 33.9 & 42.4 & 61.8 & 82.4 & 107. & 129. & 139. & 166. \\
\hline 50 & 6.30 & 21.6 & 29.8 & 41.3 & 55.9 & 70.3 & 91.3 & 107. & 108. \\
\hline 60 & 4.21 & 8.00 & 18.5 & 26.0 & 37.5 & 43.6 & 63.6 & 75.1 & 80.4 \\
\hline 70 & 3.51 & 4.79 & 11.1 & 16.2 & 19.6 & 32.8 & 40.8 & 48.4 & 64.9 \\
\hline 75 & 3.31 & 4.07 & 8.98 & 12.8 & 15.0 & 26.4 & 30.0 & 37.1 & 58.1 \\
\hline 80 & 2.99 & 3.87 & 7.67 & 9.79 & 13.1 & 19.3 & 22.9 & 27.9 & 53.0 \\
\hline 90 & 2.12 & 3.17 & 5.20 & 6.29 & 7.61 & 11.1 & 13.0 & 15.7 & 30.9 \\
\hline 95 & 1.86 & 2.71 & 4.32 & 5.11 & 5.91 & 8.86 & 10.2 & 12.3 & 24.4 \\
\hline 98 & 1.47 & 2.50 & 3.59 & 4.48 & 5.31 & 7.88 & 8.93 & 9.58 & 18.3 \\
\hline 99 & 1.34 & 2.42 & 3.19 & 4.00 & 4.62 & 6.88 & 7.49 & 8.59 & 16.0 \\
\hline
\end{tabular}


03146500 Licking River near Newark, Ohio

Years analyzed $=67$

Climatic year range $=1940-2008$

[APEE, annual percentage equaled or exceeded; $\mathrm{ft}^{3} / \mathrm{s}$, cubic foot per second]

\begin{tabular}{|c|c|c|c|c|c|c|c|c|c|}
\hline \multirow[b]{2}{*}{ APEE } & \multicolumn{9}{|c|}{ Daily mean streamflow $\left(\mathrm{ft}^{3} / \mathrm{s}\right)$ that was equaled or exceeded the indicated percentage of time at APEE level } \\
\hline & 98 & 95 & 90 & 80 & 50 & 20 & 10 & 5 & 2 \\
\hline 1 & 2110 & 2990. & 3540. & 4240 & 5570 & 6960. & 8070. & 9230. & 10100. \\
\hline 2 & 1330. & 1930. & 2480 & 2880 & 3890. & 5180. & 5500. & 5920. & 7520. \\
\hline 5 & 725. & 935. & 1300. & 1620. & 2310 & 2980 . & 3360. & 3540. & 4700 . \\
\hline 10 & 435. & 547. & 775. & 950. & 1450 & 1850. & 2090. & 2240 & 2880 . \\
\hline 20 & 194. & 306. & 384. & 525. & 755. & 1060. & 1210 & 1310. & 1650. \\
\hline 25 & 161. & 234. & 301. & 418. & 631. & 889. & 990. & 1110 & 1420 . \\
\hline 30 & 131. & 191. & 245. & 337. & 530. & 768. & 843. & 943. & 1280. \\
\hline 40 & 82.7 & 123. & 155. & 234. & 375. & 532. & 636. & 707. & 953. \\
\hline 50 & 64.8 & 90.0 & 108. & 168. & 271. & 388. & 501. & 564. & 745. \\
\hline 60 & 54.0 & 62.9 & 85.0 & 128. & 191. & 282 & 359. & 454. & 578. \\
\hline 70 & 45.1 & 53.0 & 72.4 & 93.5 & 141. & 197. & 271. & 314. & 433. \\
\hline 75 & 42.4 & 50.4 & 67.2 & 78.6 & 120. & 172. & 224. & 262. & 373. \\
\hline 80 & 42.1 & 46.8 & 59.9 & 70.8 & 100. & 149. & 179. & 227. & 336. \\
\hline 90 & 40.0 & 44.2 & 53.3 & 60.8 & 78.0 & 114. & 135. & 168. & 248. \\
\hline 95 & 36.7 & 40.0 & 51.0 & 54.3 & 68.0 & 99.4 & 116. & 141. & 195. \\
\hline 98 & 33.3 & 38.6 & 48.3 & 52.0 & 63.0 & 91.0 & 102. & 125. & 176. \\
\hline 99 & 31.8 & 37.2 & 45.5 & 49.9 & 61.0 & 88.0 & 99.2 & 118. & 171. \\
\hline
\end{tabular}

\begin{tabular}{|c|c|c|c|c|c|c|c|c|c|}
\hline \multirow[b]{2}{*}{ APEE } & \multicolumn{9}{|c|}{ 7-day mean streamflow ( $\left.\mathrm{ft}^{3} / \mathrm{s}\right)$ that was equaled or exceeded the indicated percentage of time at APEE level } \\
\hline & 98 & 95 & 90 & 80 & 50 & 20 & 10 & 5 & 2 \\
\hline 1 & 1120 & 1770. & 2240 & 2660. & 3540. & 5130. & 6270. & 6720 & 7260. \\
\hline 5 & 690. & 1080 & 1330. & 1720 & 2190. & 2690. & 3150. & 3450 & 4020. \\
\hline 10 & 472. & 576. & 866. & 1130 & 1530. & 1920. & 2040. & 2310 & 2880. \\
\hline 30 & 131. & 217. & 254. & 389. & 623. & 927. & 1010. & 1150 & 1490. \\
\hline 40 & 83.2 & 133. & 154. & 251. & 422. & 644. & 777. & 838. & 1170. \\
\hline 50 & 64.4 & 97.2 & 111. & 177. & 303. & 483. & 559. & 657. & 939. \\
\hline 60 & 53.4 & 62.5 & 90.3 & 138. & 216. & 349. & 421. & 528. & 683. \\
\hline 90 & 39.6 & 45.5 & 53.6 & 61.8 & 81.9 & 121. & 144. & 193. & 291. \\
\hline 95 & 37.9 & 41.2 & 52.0 & 56.8 & 69.3 & 101. & 123. & 154. & 214. \\
\hline 98 & 34.9 & 39.7 & 49.3 & 54.5 & 65.7 & 93.6 & 111. & 131. & 181. \\
\hline 99 & 33.6 & 38.6 & 48.4 & 52.9 & 63.0 & 91.7 & 105. & 125. & 178. \\
\hline
\end{tabular}




\section{Licking River at Toboso, Ohio}

Years analyzed $=41$

Climatic year range $=1904-60$

[APEE, annual percentage equaled or exceeded; $\mathrm{ft}^{3} / \mathrm{s}$, cubic foot per second]

\begin{tabular}{|c|c|c|c|c|c|c|c|c|c|}
\hline \multirow[b]{2}{*}{ APEE } & \multicolumn{9}{|c|}{ Daily mean streamflow $\left(\mathrm{ft}^{3} / \mathrm{s}\right)$ that was equaled or exceeded the indicated percentage of time at APEE level } \\
\hline & 95 & 90 & 80 & 70 & 50 & 30 & 20 & 10 & 5 \\
\hline 1 & 1950. & 2890. & 4980. & 5420. & 6420. & 7470. & 8260. & 9850. & 11500 \\
\hline 2 & 1000 & 2120 & 3340 . & 3660 . & 4600. & 5510. & 6200. & 6900. & 7890. \\
\hline 5 & 605. & 1270 & 1930. & 2030 . & 2580 & 3170 . & 3460 . & 3850 . & 4020 . \\
\hline 10 & 371. & 819. & 1130. & 1270 . & 1510 & 1800 & 2050 & 2190 & 2390. \\
\hline 20 & 250. & 453. & 541. & 693. & 834. & 1000 & 1110 & 1270 & 1430. \\
\hline 25 & 191. & 356. & 437. & 516. & 665. & 827. & 932. & 1050 & 1170 . \\
\hline 30 & 150. & 279. & 350 . & 385. & 560. & 687. & 784. & 897. & 958. \\
\hline 40 & 107. & 158. & 234. & 270 & 402. & 511. & 576. & 613. & 675. \\
\hline 50 & 82.5 & 111. & 153. & 193. & 299. & 345. & 387. & 451. & 505. \\
\hline 60 & 69.3 & 80.2 & 116. & 134. & 224. & 261. & 300. & 322. & 343. \\
\hline 70 & 59.8 & 69.4 & 96.0 & 105. & 162. & 191. & 207. & 223. & 248. \\
\hline 75 & 56.8 & 66.4 & 89.4 & 95.9 & 138. & 159. & 178. & 199. & 208. \\
\hline 80 & 55.7 & 64.4 & 81.0 & 87.2 & 115. & 135. & 145. & 169. & 177. \\
\hline 90 & 52.4 & 59.0 & 68.0 & 75.3 & 92.0 & 102. & 108. & 128. & 146. \\
\hline 95 & 50.2 & 53.0 & 61.2 & 70.2 & 81.5 & 90.2 & 99.2 & 112. & 138. \\
\hline 98 & 47.7 & 48.7 & 54.8 & 66.4 & 73.0 & 82.8 & 95.6 & 104. & 119. \\
\hline 99 & 46.0 & 47.0 & 53.3 & 63.5 & 70.0 & 80.4 & 93.0 & 99.3 & 116. \\
\hline
\end{tabular}

\begin{tabular}{|c|c|c|c|c|c|c|c|c|c|}
\hline \multirow[b]{2}{*}{ APEE } & \multicolumn{9}{|c|}{ 7-day mean streamflow ( $\mathrm{ft}^{3} / \mathrm{s}$ ) that was equaled or exceeded the indicated percentage of time at APEE level } \\
\hline & 95 & 90 & 80 & 70 & 50 & 30 & 20 & 10 & 5 \\
\hline 1 & 1060. & 2320 & 2810 & 3150 . & 4360 & 5390. & 6010 & 7760 . & 9260. \\
\hline 2 & 848. & 1910. & 2330 & 2620 & 3220 . & 4200 & 4510 & 5260. & 5750. \\
\hline 5 & 707. & 1230 & 1920. & 2080 & 2320 & 3010 . & 3160. & 3470 . & 4080 . \\
\hline 10 & 392. & 827. & 1260. & 1450 . & 1750 & 2090 . & 2300 & 2370 & 2520 . \\
\hline 20 & 277. & 492. & 644. & 819. & 1020 & 1260 & 1390. & 1490 . & 1620. \\
\hline 25 & 233. & 403. & 508. & 634. & 809. & 971. & 1100 & 1280 & 1330. \\
\hline 30 & 181. & 301. & 399. & 446. & 666. & 770. & 913. & 1100 & 1170 \\
\hline 40 & 111. & 170 . & 226. & 280. & 459. & 561. & 636. & 725. & 829. \\
\hline 50 & 88.1 & 118. & 167. & 199. & 326. & 407. & 449. & 504. & 566. \\
\hline 60 & 68.7 & 82.3 & 120. & 150. & 247. & 293. & 340. & 365. & 412. \\
\hline 70 & 59.4 & 71.6 & 101. & 111. & 173. & 219. & 241. & 267. & 279 . \\
\hline 75 & 57.4 & 67.9 & 95.1 & 100. & 143. & 173. & 200. & 229. & 234. \\
\hline 80 & 55.8 & 66.0 & 86.4 & 92.0 & 119. & 148. & 159. & 194. & 208. \\
\hline 90 & 53.5 & 60.8 & 70.7 & 76.7 & 95.6 & 105. & 111. & 135. & 154. \\
\hline 95 & 50.5 & 57.7 & 63.5 & 71.4 & 83.6 & 94.8 & 100. & 119. & 138. \\
\hline 98 & 48.8 & 50.3 & 58.3 & 70.5 & 78.6 & 88.3 & 97.4 & 113. & 128. \\
\hline 99 & 48.1 & 48.5 & 57.0 & 68.9 & 73.0 & 85.6 & 96.2 & 112. & 122. \\
\hline
\end{tabular}




\section{Clear Creek near Rockbridge, Ohio}

Years analyzed $=69$

Climatic year range $=1940-2008$

[APEE, annual percentage equaled or exceeded; $\mathrm{ft}^{3} / \mathrm{s}$, cubic foot per second]

\begin{tabular}{|c|c|c|c|c|c|c|c|c|c|}
\hline \multirow[b]{2}{*}{ APEE } & \multicolumn{9}{|c|}{ Daily mean streamflow $\left(\mathrm{ft}^{3} / \mathrm{s}\right)$ that was equaled or exceeded the indicated percentage of time at APEE level } \\
\hline & 98 & 95 & 90 & 80 & 50 & 20 & 10 & 5 & 2 \\
\hline 1 & 215. & 345. & 518. & 580. & 768. & 1100 & 1400 & 1560. & 1720 \\
\hline 2 & 152. & 248 & 313. & 375. & 523. & 733. & 857. & 1030. & 1140. \\
\hline 5 & 90.6 & 135. & 167. & 210. & 300 . & 402. & 442. & 508. & 556. \\
\hline 10 & 67.2 & 93.7 & 105. & 125. & 170 . & 243. & 273. & 300 . & 377. \\
\hline 20 & 47.4 & 57.0 & 68.0 & 75.2 & 102. & 144. & 163. & 189. & 222. \\
\hline 25 & 41.0 & 47.5 & 56.3 & 63.5 & 88.5 & 125. & 140 . & 159. & 176. \\
\hline 30 & 32.8 & 38.0 & 47.9 & 55.2 & 76.2 & 105. & 122. & 136. & 154. \\
\hline 40 & 21.4 & 27.0 & 33.6 & 43.0 & 58.0 & 81.0 & 97.0 & 107. & 122. \\
\hline 50 & 18.8 & 21.3 & 25.0 & 34.0 & 44.0 & 66.0 & 76.0 & 87.0 & 97.2 \\
\hline 60 & 16.0 & 17.0 & 19.0 & 25.0 & 33.0 & 48.4 & 68.0 & 71.2 & 81.0 \\
\hline 70 & 13.4 & 15.0 & 16.0 & 19.0 & 27.0 & 36.0 & 46.0 & 59.0 & 68.6 \\
\hline 75 & 13.0 & 14.0 & 15.0 & 18.0 & 24.0 & 31.0 & 42.0 & 53.5 & 61.7 \\
\hline 80 & 12.0 & 13.0 & 14.0 & 17.0 & 22.0 & 28.0 & 38.0 & 48.3 & 55.6 \\
\hline 90 & 9.40 & 11.0 & 11.0 & 13.0 & 17.0 & 21.6 & 30.0 & 38.5 & 45.0 \\
\hline 95 & 8.21 & 9.70 & 11.0 & 12.0 & 16.0 & 20.0 & 27.0 & 32.6 & 38.6 \\
\hline 98 & 7.40 & 8.10 & 9.80 & 11.0 & 14.3 & 19.0 & 25.0 & 28.0 & 33.7 \\
\hline 99 & 5.98 & 7.70 & 9.20 & 10.0 & 14.0 & 18.0 & 22.0 & 25.8 & 32.1 \\
\hline
\end{tabular}

\begin{tabular}{|c|c|c|c|c|c|c|c|c|c|}
\hline \multirow[b]{2}{*}{ APEE } & \multicolumn{9}{|c|}{ 7-day mean streamflow ( $\mathrm{ft}^{3} / \mathrm{s}$ ) that was equaled or exceeded the indicated percentage of time at APEE level } \\
\hline & 98 & 95 & 90 & 80 & 50 & 20 & 10 & 5 & 2 \\
\hline 1 & 159. & 237. & 288. & 365. & 513. & 739. & 980. & 1050 & 1400 \\
\hline 2 & 136. & 192. & 260 & 287. & 412. & 548. & 631. & 838. & 1020. \\
\hline 5 & 101. & 137. & 169. & 221. & 292. & 395. & 461. & 491. & 608. \\
\hline 10 & 66.5 & 91.5 & 108. & 138. & 200. & 268. & 313. & 335. & 372. \\
\hline 20 & 49.4 & 59.9 & 69.7 & 80.9 & 119. & 170 . & 189. & 219. & 246. \\
\hline 25 & 44.4 & 49.3 & 59.9 & 69.3 & 97.3 & 149. & 160. & 189. & 225 \\
\hline 30 & 36.0 & 40.2 & 49.6 & 62.1 & 84.0 & 125 & 136. & 157. & 201. \\
\hline 40 & 21.2 & 28.3 & 36.3 & 46.7 & 63.7 & 90.3 & 110. & 129. & 152. \\
\hline 50 & 18.9 & 22.5 & 27.7 & 36.0 & 48.7 & 69.0 & 84.6 & 105. & 119. \\
\hline 60 & 16.5 & 17.0 & 20.1 & 26.3 & 35.9 & 54.5 & 71.3 & 81.7 & 95.1 \\
\hline 70 & 14.0 & 15.1 & 17.0 & 19.9 & 28.0 & 42.0 & 51.1 & 67.4 & 81.6 \\
\hline 75 & 13.5 & 14.1 & 15.9 & 18.7 & 25.1 & 35.4 & 45.7 & 59.4 & 71.9 \\
\hline 80 & 12.7 & 12.9 & 14.7 & 17.4 & 23.3 & 30.9 & 42.3 & 52.6 & 61.6 \\
\hline 90 & 9.57 & 11.3 & 12.0 & 14.1 & 17.9 & 24.1 & 35.0 & 42.4 & 50.9 \\
\hline 95 & 8.78 & 10.2 & 11.0 & 12.0 & 16.3 & 20.6 & 29.9 & 35.9 & 42.6 \\
\hline 98 & 8.38 & 9.33 & 10.4 & 11.1 & 15.3 & 19.1 & 27.0 & 31.4 & 37.8 \\
\hline 99 & 7.71 & 8.42 & 10.0 & 11.0 & 14.9 & 18.7 & 26.0 & 28.0 & 35.3 \\
\hline
\end{tabular}


03157500 Hocking River at Enterprise, Ohio

Years analyzed $=77$

Climatic year range $=1932-2008$

[APEE, annual percentage equaled or exceeded; $\mathrm{ft}^{3} / \mathrm{s}$, cubic foot per second]

\begin{tabular}{|c|c|c|c|c|c|c|c|c|c|}
\hline \multirow[b]{2}{*}{ APEE } & \multicolumn{9}{|c|}{ Daily mean streamflow $\left(\mathrm{ft}^{3} / \mathrm{s}\right)$ that was equaled or exceeded the indicated percentage of time at APEE level } \\
\hline & 98 & 95 & 90 & 80 & 50 & 20 & 10 & 5 & 2 \\
\hline 1 & 1200. & 1580. & 2210. & 2920. & 3940. & 5650. & 6940. & 7450. & 8940. \\
\hline 2 & 752. & 1210 & 1770 . & 2100 & 2800 & 3780 . & 4800. & 5570. & 5890. \\
\hline 5 & 479. & 763. & 962. & 1190. & 1680 & 2240 & 2380 & 3000 . & 3250 . \\
\hline 10 & 345. & 506. & 571. & 723. & 1030 & 1440 . & 1660. & 1760. & 1880 \\
\hline 20 & 221. & 280 & 326. & 430 . & 586. & 815. & 958. & 1030. & 1200. \\
\hline 25 & 174. & 231. & 268. & 342. & 468. & 661. & 811. & 856. & 1020. \\
\hline 30 & 132. & 179. & 216. & 278. & 387. & 563. & 681. & 743. & 862. \\
\hline 40 & 87.2 & 118. & 145 & 193. & 279. & 430 . & 524. & 589. & 682. \\
\hline 50 & 66.0 & 85.7 & 103. & 134. & 209. & 335. & 416. & 469. & 550. \\
\hline 60 & 54.6 & 64.4 & 70.8 & 93.6 & 151. & 255. & 333. & 381. & 436. \\
\hline 70 & 46.5 & 52.8 & 58.4 & 70.5 & 116. & 187. & 258. & 283. & 352. \\
\hline 75 & 42.8 & 47.8 & 53.2 & 63.8 & 101. & 161. & 222. & 249. & 311. \\
\hline 80 & 39.8 & 42.9 & 47.0 & 57.7 & 88.4 & 135. & 197. & 218 & 266. \\
\hline 90 & 32.0 & 35.5 & 39.8 & 48.5 & 68.0 & 97.8 & 129. & 162. & 203. \\
\hline 95 & 28.6 & 31.0 & 36.6 & 43.2 & 61.0 & 84.0 & 104. & 139. & 164. \\
\hline 98 & 28.6 & 29.3 & 32.0 & 38.3 & 55.3 & 77.4 & 97.2 & 120. & 144. \\
\hline 99 & 27.4 & 28.3 & 30.9 & 37.6 & 50.0 & 71.3 & 92.7 & 118. & 127. \\
\hline
\end{tabular}

\begin{tabular}{|c|c|c|c|c|c|c|c|c|c|}
\hline \multirow[b]{2}{*}{ APEE } & \multicolumn{9}{|c|}{ 7-day mean streamflow ( $\mathrm{ft}^{3} / \mathrm{s}$ ) that was equaled or exceeded the indicated percentage of time at APEE level } \\
\hline & 98 & 95 & 90 & 80 & 50 & 20 & 10 & 5 & 2 \\
\hline 1 & 872. & 1230 & 1600 & 2040 & 2830 & 3720. & 5750. & 6790 & 7820. \\
\hline 2 & 750 & 948. & 1270 & 1610 & 2250 & 3040 . & 3660. & 4180 & 5360. \\
\hline 5 & 439. & 771. & 927. & 1060 & 1560 & 2060 & 2430. & 2580 & 2750 . \\
\hline 10 & 333. & 491. & 628. & 769. & 1090. & 1450 & 1630. & 1880 & 2050 . \\
\hline 20 & 234 & 265. & 327. & 463. & 646. & 930. & 1080. & 1160 & 1280. \\
\hline 25 & 199. & 224. & 267. & 361. & 499. & 795. & 918. & 946. & 1130 \\
\hline 30 & 149. & 184. & 216. & 306. & 407. & 660. & 783. & 830. & 1030 \\
\hline 40 & 90.1 & 121. & 162. & 217. & 305. & 488. & 591. & 679. & 833. \\
\hline 50 & 68.2 & 88.8 & 120. & 146. & 227. & 382. & 470. & 537. & 646. \\
\hline 60 & 56.9 & 70.2 & 78.5 & 104. & 170 & 304. & 381. & 449. & 498. \\
\hline 70 & 46.5 & 55.0 & 63.3 & 77.1 & 126. & 210 & 290. & 342. & 431. \\
\hline 75 & 43.4 & 52.3 & 57.3 & 67.2 & 109. & 175. & 258. & 285. & 363. \\
\hline 80 & 40.5 & 47.5 & 51.6 & 60.5 & 94.7 & 155. & 222. & 248 & 298. \\
\hline 90 & 32.6 & 37.6 & 43.8 & 52.1 & 73.1 & 109. & 140. & 185. & 228. \\
\hline 95 & 29.4 & 34.5 & 37.6 & 45.2 & 64.4 & 95.3 & 118. & 151. & 187. \\
\hline 98 & 28.7 & 30.7 & 35.2 & 41.5 & 60.0 & 85.3 & 107. & 128. & 162. \\
\hline 99 & 28.2 & 29.8 & 31.7 & 38.4 & 56.7 & 80.0 & 102. & 121. & 144. \\
\hline
\end{tabular}


03159540 Shade River near Chester, Ohio

Years analyzed $=42$

Climatic year range $=1966-2008$

[APEE, annual percentage equaled or exceeded; $\mathrm{ft}^{3} / \mathrm{s}$, cubic foot per second]

\begin{tabular}{|c|c|c|c|c|c|c|c|c|c|}
\hline \multirow[b]{2}{*}{ APEE } & \multicolumn{9}{|c|}{ Daily mean streamflow $\left(\mathrm{ft}^{3} / \mathrm{s}\right)$ that was equaled or exceeded the indicated percentage of time at APEE level } \\
\hline & 95 & 90 & 80 & 70 & 50 & 30 & 20 & 10 & 5 \\
\hline 1 & 1100 & 1220 & 1530. & 1780. & 2060 & 2380 & 2560 & 3380. & 3530. \\
\hline 2 & 768. & 801. & 1130. & 1330. & 1530 . & 1680 & 1710 & 2080 & 2230 \\
\hline 5 & 386. & 443. & 515. & 597. & 723. & 893. & 1010 & 1100 & 1240 \\
\hline 10 & 176. & 228. & 255. & 306. & 358. & 434. & 524. & 596. & 659. \\
\hline 20 & 93.6 & 112. & 130. & 147. & 182. & 230. & 276. & 307. & 324. \\
\hline 25 & 77.9 & 81.5 & 98.8 & 116. & 146. & 187. & 222. & 252. & 262. \\
\hline 30 & 58.5 & 66.0 & 74.8 & 93.3 & 109. & 158. & 177. & 201. & 231. \\
\hline 40 & 28.2 & 45.9 & 51.6 & 60.2 & 75.1 & 108. & 127. & 155. & 161. \\
\hline 50 & 10.4 & 24.3 & 31.6 & 39.8 & 45.5 & 79.5 & 91.2 & 124. & 132. \\
\hline 60 & 4.07 & 11.0 & 19.6 & 20.4 & 28.0 & 51.1 & 59.4 & 89.2 & 102. \\
\hline 70 & 1.42 & 5.80 & 8.32 & 9.36 & 18.0 & 30.5 & 41.2 & 67.8 & 81.7 \\
\hline 75 & 1.23 & 4.09 & 5.64 & 7.56 & 13.9 & 26.0 & 30.2 & 49.8 & 67.6 \\
\hline 80 & 0.95 & 3.27 & 4.06 & 5.11 & 11.0 & 17.3 & 23.2 & 32.3 & 57.4 \\
\hline 90 & 0.53 & 1.30 & 1.84 & 2.44 & 5.46 & 9.33 & 13.0 & 20.1 & 36.6 \\
\hline 95 & 0.37 & 0.72 & 1.17 & 1.69 & 3.26 & 6.75 & 9.16 & 14.8 & 28.1 \\
\hline 98 & 0.26 & 0.41 & 0.85 & 1.21 & 1.93 & 4.94 & 6.56 & 10.0 & 22.2 \\
\hline 99 & 0.21 & 0.35 & 0.72 & 0.90 & 1.58 & 4.20 & 5.87 & 7.95 & 14.4 \\
\hline
\end{tabular}

\begin{tabular}{|c|c|c|c|c|c|c|c|c|c|}
\hline \multirow[b]{2}{*}{ APEE } & \multicolumn{9}{|c|}{ 7-day mean streamflow (ft $\left.{ }^{3} / \mathrm{s}\right)$ that was equaled or exceeded the indicated percentage of time at APEE level } \\
\hline & 95 & 90 & 80 & 70 & 50 & 30 & 20 & 10 & 5 \\
\hline 1 & 589. & 598. & 814. & 945. & 1060. & 1350 & 1560 & 1990. & 2600 \\
\hline 2 & 534. & 563. & 692. & 800 . & 890. & 1020 . & 1160 & 1590. & 1800. \\
\hline 5 & 403. & 436. & 542. & 599. & 690. & 761. & 833. & 948. & 993. \\
\hline 10 & 264. & 286. & 346. & 392. & 450. & 527. & 557. & 642. & 722. \\
\hline 20 & 114. & 145. & 172. & 188. & 264. & 326. & 352. & 398. & 463. \\
\hline 25 & 92.8 & 103. & 122. & 139. & 196. & 243. & 304. & 358. & 368. \\
\hline 30 & 60.5 & 81.1 & 94.2 & 109. & 138. & 199. & 264. & 279. & 331. \\
\hline 40 & 32.9 & 52.9 & 64.1 & 70.8 & 90.8 & 135. & 177. & 198. & 225 \\
\hline 50 & 13.0 & 26.8 & 37.3 & 44.2 & 58.2 & 98.7 & 116. & 156. & 173. \\
\hline 60 & 5.26 & 13.1 & 23.9 & 27.6 & 33.8 & 64.0 & 75.1 & 122. & 135. \\
\hline 70 & 1.54 & 7.86 & 11.4 & 12.3 & 22.9 & 42.6 & 54.6 & 92.0 & 111. \\
\hline 75 & 1.33 & 5.21 & 6.84 & 8.71 & 17.5 & 31.3 & 42.3 & 79.7 & 102. \\
\hline 80 & 1.15 & 3.76 & 4.64 & 6.95 & 13.6 & 23.4 & 31.3 & 52.4 & 86.0 \\
\hline 90 & 0.64 & 1.49 & 2.11 & 2.72 & 6.80 & 12.5 & 19.0 & 26.4 & 49.5 \\
\hline 95 & 0.38 & 0.79 & 1.27 & 1.77 & 4.85 & 9.05 & 12.1 & 19.8 & 37.2 \\
\hline 98 & 0.31 & 0.51 & 0.97 & 1.37 & 2.78 & 6.97 & 8.51 & 14.0 & 28.1 \\
\hline 99 & 0.27 & 0.44 & 0.89 & 1.16 & 2.10 & 5.57 & 7.70 & 9.91 & 24.2 \\
\hline
\end{tabular}


03202000 Raccoon Creek at Adamsville, Ohio

Years analyzed $=82$

Climatic year range $=1916-2008$

[APEE, annual percentage equaled or exceeded; $\mathrm{ft}^{3} / \mathrm{s}$, cubic foot per second]

\begin{tabular}{|c|c|c|c|c|c|c|c|c|c|}
\hline \multirow[b]{2}{*}{ APEE } & \multicolumn{9}{|c|}{ Daily mean streamflow $\left(\mathrm{ft}^{3} / \mathrm{s}\right)$ that was equaled or exceeded the indicated percentage of time at APEE level } \\
\hline & 98 & 95 & 90 & 80 & 50 & 20 & 10 & 5 & 2 \\
\hline 1 & 1550. & 2580. & 2820. & 3800. & 4900. & 6800. & 8230. & 8980. & 13200 \\
\hline 2 & 1310. & 2230 & 2430 & 3210 . & 4070 & 5140. & 5810. & 6630. & 7120 . \\
\hline 5 & 867. & 1230 & 1620 . & 2090 . & 2790 & 3480 . & 3640 . & 3940 . & 4170 . \\
\hline 10 & 457. & 784. & 951. & 1240 . & 1730 & 2230 & 2400 & 2510 & 2840 . \\
\hline 20 & 214 & 367. & 454. & 611. & 905. & 1170 & 1310 . & 1520 . & 1630. \\
\hline 25 & 161. & 270 . & 342. & 478. & 699. & 934. & 1030. & 1190. & 1350. \\
\hline 30 & 120. & 185. & 260. & 378. & 567. & 758. & 873. & 948. & 1140. \\
\hline 40 & 66.1 & 106. & 157. & 212. & 361. & 602. & 649. & 727. & 874. \\
\hline 50 & 32.5 & 59.8 & 78.7 & 96.4 & 224. & 422. & 494. & 538. & 625. \\
\hline 60 & 18.8 & 26.0 & 42.1 & 63.8 & 132. & 265. & 373. & 423. & 477. \\
\hline 70 & 10.4 & 13.3 & 28.3 & 42.0 & 77.3 & 165. & 230. & 266. & 395. \\
\hline 75 & 8.39 & 11.0 & 22.3 & 34.0 & 61.5 & 130. & 178. & 220 & 363. \\
\hline 80 & 6.71 & 8.10 & 16.3 & 26.3 & 50.0 & 107. & 132. & 175 & 330. \\
\hline 90 & 2.53 & 5.41 & 8.28 & 14.6 & 29.5 & 66.2 & 94.2 & 118. & 200. \\
\hline 95 & 1.90 & 3.36 & 6.20 & 9.51 & 23.5 & 46.0 & 64.9 & 89.4 & 164. \\
\hline 98 & 1.65 & 2.61 & 5.09 & 7.06 & 19.3 & 39.8 & 54.1 & 69.4 & 134. \\
\hline 99 & 1.47 & 2.42 & 4.03 & 6.15 & 17.8 & 35.4 & 51.7 & 60.7 & 114. \\
\hline
\end{tabular}

\begin{tabular}{|c|c|c|c|c|c|c|c|c|c|}
\hline \multirow[b]{2}{*}{ APEE } & \multicolumn{9}{|c|}{ 7-day mean streamflow ( $\mathrm{ft}^{3} / \mathrm{s}$ ) that was equaled or exceeded the indicated percentage of time at APEE level } \\
\hline & 98 & 95 & 90 & 80 & 50 & 20 & 10 & 5 & 2 \\
\hline 1 & 1260 & 1940. & 2170 & 3000 & 3840 & 5670. & 6850. & 7600 . & 10500 \\
\hline 2 & 1060 & 1640 & 1850. & 2600 & 3290. & 4450 & 4830. & 6040 & 7420 . \\
\hline 5 & 881. & 1210 & 1530. & 1780 . & 2430 & 2890 . & 3270 . & 3500 . & 3900. \\
\hline 10 & 462. & 865. & 960. & 1250. & 1660 & 2150 & 2310 & 2430 & 2610. \\
\hline 20 & 201. & 360. & 507. & 701. & 985. & 1330. & 1460 . & 1530. & 1740. \\
\hline 25 & 159. & 297. & 362. & 520. & 771. & 1030 & 1160 & 1330. & 1480. \\
\hline 30 & 113. & 176. & 276 & 392. & 638. & 880 . & 973. & 1130. & 1290. \\
\hline 40 & 63.0 & 112. & 166. & 231. & 386. & 684. & 724. & 778. & 979. \\
\hline 50 & 36.2 & 58.3 & 74.1 & 111. & 247. & 496. & 573. & 631. & 740. \\
\hline 60 & 16.8 & 27.4 & 45.4 & 70.9 & 142. & 287. & 409. & 464. & 520. \\
\hline 70 & 10.3 & 13.5 & 30.0 & 47.9 & 82.0 & 186. & 265. & 304. & 436. \\
\hline 75 & 8.07 & 11.7 & 22.2 & 33.6 & 64.9 & 153. & 196. & 255 & 394. \\
\hline 80 & 6.91 & 8.31 & 17.3 & 27.4 & 52.9 & 125. & 158. & 222. & 367. \\
\hline 90 & 2.65 & 5.66 & 9.19 & 14.7 & 31.9 & 76.2 & 102. & 138. & 244. \\
\hline 95 & 2.00 & 3.43 & 6.76 & 11.2 & 25.1 & 52.0 & 78.3 & 108. & 182. \\
\hline 98 & 1.74 & 2.73 & 5.61 & 7.78 & 21.8 & 43.7 & 64.2 & 96.4 & 151. \\
\hline 99 & 1.58 & 2.58 & 4.69 & 6.79 & 19.0 & 39.8 & 56.0 & 77.2 & 136. \\
\hline
\end{tabular}


03217500 Scioto River at La Rue, Ohio

Years analyzed $=20$

Climatic year range $=1927-50$

[APEE, annual percentage equaled or exceeded; $\mathrm{ft}^{3} / \mathrm{s}$, cubic foot per second]

\begin{tabular}{|c|c|c|c|c|c|c|c|c|c|}
\hline \multirow[b]{2}{*}{ APEE } & \multicolumn{9}{|c|}{ Daily mean streamflow $\left(\mathrm{ft}^{3} / \mathrm{s}\right)$ that was equaled or exceeded the indicated percentage of time at APEE level } \\
\hline & 95 & 90 & 80 & 70 & 50 & 30 & 20 & 10 & 5 \\
\hline 1 & 439. & 585. & 1710. & 1950. & 2570 & 3190. & 3780 . & 3960. & 4680 \\
\hline 2 & 283. & 378. & 1110. & 1340 . & 1700 & 2290 & 2500 & 2610 & 3170 . \\
\hline 5 & 168. & 214. & 633. & 715. & 955. & 1340 . & 1490. & 1690. & 1840. \\
\hline 10 & 105. & 145. & 281. & 359. & 481. & 679. & 857. & 955. & 974. \\
\hline 20 & 50.0 & 74.1 & 122. & 142. & 213. & 320. & 375. & 497. & 533. \\
\hline 25 & 35.5 & 56.1 & 88.4 & 98.4 & 154. & 244. & 284. & 378. & 416. \\
\hline 30 & 24.8 & 41.5 & 58.6 & 76.6 & 106. & 188. & 233. & 306. & 350. \\
\hline 40 & 11.3 & 17.0 & 34.2 & 42.1 & 69.0 & 116. & 155. & 223. & 232. \\
\hline 50 & 9.10 & 11.7 & 21.0 & 29.3 & 43.0 & 74.3 & 100. & 153. & 159. \\
\hline 60 & 6.98 & 8.76 & 14.6 & 19.1 & 26.2 & 47.8 & 70.2 & 105. & 110. \\
\hline 70 & 5.73 & 6.58 & 10.4 & 12.3 & 16.5 & 24.3 & 38.7 & 63.3 & 74.4 \\
\hline 75 & 3.98 & 5.80 & 8.56 & 10.3 & 14.0 & 17.4 & 30.4 & 48.7 & 63.3 \\
\hline 80 & 3.93 & 4.79 & 7.20 & 8.95 & 12.0 & 15.8 & 24.5 & 41.3 & 45.8 \\
\hline 90 & 3.81 & 3.93 & 5.00 & 5.78 & 8.25 & 10.7 & 16.8 & 23.5 & 29.4 \\
\hline 95 & 3.02 & 3.49 & 3.92 & 4.55 & 6.00 & 8.53 & 11.8 & 14.9 & 23.5 \\
\hline 98 & 3.01 & 3.21 & 3.71 & 3.93 & 4.60 & 7.64 & 8.90 & 14.8 & 15.9 \\
\hline 99 & 3.01 & 3.15 & 3.66 & 3.93 & 4.20 & 7.40 & 8.63 & 13.8 & 14.9 \\
\hline
\end{tabular}

\begin{tabular}{|c|c|c|c|c|c|c|c|c|c|}
\hline \multirow[b]{2}{*}{ APEE } & \multicolumn{9}{|c|}{ 7-day mean streamflow ( $\left.\mathrm{ft}^{3} / \mathrm{s}\right)$ that was equaled or exceeded the indicated percentage of time at APEE level } \\
\hline & 95 & 90 & 80 & 70 & 50 & 30 & 20 & 10 & 5 \\
\hline 1 & 227. & 377. & 1220. & 1410. & 1500 & 2020 & 2230 & 2460 & 3460 \\
\hline 2 & 193. & 349. & 878. & 969. & 1380 . & 1530 . & 1940. & 2070 & 2570 \\
\hline 5 & 164. & 206. & 584. & 716. & 901. & 1240 . & 1320 . & 1550. & 1590. \\
\hline 10 & 99.2 & 120. & 331. & 386. & 552. & 780 . & 897. & 962. & 1000 \\
\hline 20 & 54.3 & 75.0 & 148. & 168. & 261. & 417. & 518. & 589. & 614. \\
\hline 25 & 41.3 & 55.6 & 109. & 122. & 187. & 337. & 393. & 463. & 513. \\
\hline 30 & 32.9 & 45.0 & 68.7 & 93.5 & 142. & 236. & 339. & 400 & 448. \\
\hline 40 & 13.2 & 18.9 & 44.8 & 52.2 & 82.6 & 149. & 199. & 292. & 337. \\
\hline 50 & 7.96 & 12.7 & 25.3 & 31.3 & 55.6 & 89.8 & 125. & 208. & 237. \\
\hline 60 & 6.67 & 8.68 & 17.1 & 19.9 & 30.4 & 57.5 & 88.4 & 152. & 166. \\
\hline 70 & 5.71 & 6.61 & 11.3 & 13.1 & 18.1 & 35.6 & 45.7 & 86.1 & 108. \\
\hline 75 & 4.97 & 6.00 & 9.43 & 10.8 & 15.3 & 27.4 & 35.3 & 60.8 & 85.9 \\
\hline 80 & 4.44 & 5.19 & 8.17 & 9.83 & 12.7 & 20.4 & 28.6 & 50.1 & 65.7 \\
\hline 90 & 3.91 & 4.19 & 5.46 & 6.61 & 8.62 & 11.5 & 17.4 & 27.3 & 29.9 \\
\hline 95 & 3.31 & 3.75 & 4.21 & 5.54 & 6.88 & 9.27 & 12.3 & 16.3 & 24.7 \\
\hline 98 & 3.02 & 3.35 & 3.80 & 4.74 & 5.58 & 8.75 & 10.1 & 15.3 & 16.9 \\
\hline 99 & 3.01 & 3.24 & 3.73 & 4.32 & 5.15 & 8.49 & 9.33 & 15.0 & 16.6 \\
\hline
\end{tabular}




\section{Little Scioto River above Marion, Ohio}

Years analyzed $=32$

Climatic year range $=1939-70$

[APEE, annual percentage equaled or exceeded; $\mathrm{ft}^{3} / \mathrm{s}$, cubic foot per second]

\begin{tabular}{|c|c|c|c|c|c|c|c|c|c|}
\hline \multirow[b]{2}{*}{ APEE } & \multicolumn{9}{|c|}{ Daily mean streamflow $\left(\mathrm{ft}^{3} / \mathrm{s}\right)$ that was equaled or exceeded the indicated percentage of time at APEE level } \\
\hline & 95 & 90 & 80 & 70 & 50 & 30 & 20 & 10 & 5 \\
\hline 1 & 274. & 305. & 408. & 515. & 596. & 739. & 803. & 892. & 1210 \\
\hline 2 & 168. & 205. & 320. & 356. & 427. & 490 & 546. & 617. & 744. \\
\hline 5 & 86.4 & 116. & 144. & 175. & 229. & 299. & 325. & 353. & 383. \\
\hline 10 & 38.3 & 55.9 & 77.1 & 87.2 & 130. & 169. & 178. & 195. & 221. \\
\hline 20 & 16.0 & 19.6 & 30.7 & 38.6 & 57.5 & 79.3 & 89.6 & 94.5 & 111. \\
\hline 25 & 11.2 & 15.2 & 19.4 & 28.9 & 43.4 & 59.3 & 69.3 & 75.8 & 88.8 \\
\hline 30 & 5.76 & 8.15 & 12.0 & 21.7 & 30.0 & 47.2 & 55.7 & 63.0 & 71.8 \\
\hline 40 & 1.44 & 2.82 & 7.55 & 9.58 & 16.0 & 29.2 & 36.2 & 42.7 & 45.7 \\
\hline 50 & 0.46 & 0.80 & 3.00 & 4.94 & 9.80 & 18.2 & 24.0 & 30.0 & 31.3 \\
\hline 60 & 0.00 & 0.13 & 1.12 & 2.29 & 5.10 & 11.1 & 15.0 & 20.4 & 22.7 \\
\hline 70 & 0.00 & 0.00 & 0.66 & 0.80 & 2.05 & 5.42 & 7.52 & 13.1 & 14.9 \\
\hline 75 & 0.00 & 0.00 & 0.40 & 0.49 & 1.45 & 3.45 & 4.59 & 9.68 & 12.3 \\
\hline 80 & 0.00 & 0.00 & 0.30 & 0.30 & 0.75 & 1.98 & 3.28 & 7.80 & 10.0 \\
\hline 90 & 0.00 & 0.00 & 0.00 & 0.10 & 0.30 & 0.94 & 1.16 & 3.88 & 4.94 \\
\hline 95 & 0.00 & 0.00 & 0.00 & 0.00 & 0.15 & 0.41 & 0.71 & 2.43 & 3.87 \\
\hline 98 & 0.00 & 0.00 & 0.00 & 0.00 & 0.10 & 0.30 & 0.48 & 1.60 & 3.28 \\
\hline 99 & 0.00 & 0.00 & 0.00 & 0.00 & 0.03 & 0.20 & 0.37 & 1.08 & 2.50 \\
\hline
\end{tabular}

\begin{tabular}{|c|c|c|c|c|c|c|c|c|c|}
\hline \multirow[b]{2}{*}{ APEE } & \multicolumn{9}{|c|}{ 7-day mean streamflow ( $\mathrm{ft}^{3} / \mathrm{s}$ ) that was equaled or exceeded the indicated percentage of time at APEE level } \\
\hline & 95 & 90 & 80 & 70 & 50 & 30 & 20 & 10 & 5 \\
\hline 1 & 178. & 236. & 291. & 313. & 380. & 519. & 611. & 738. & 1030. \\
\hline 2 & 146. & 189. & 237. & 253. & 314. & 370 . & 452. & 533. & 573. \\
\hline 5 & 77.9 & 112. & 163. & 209. & 229. & 272 & 297. & 353. & 419. \\
\hline 10 & 39.2 & 44.6 & 87.7 & 125. & 148. & 187. & 197. & 224 & 238. \\
\hline 20 & 17.3 & 20.4 & 36.2 & 45.1 & 69.7 & 101. & 111. & 121. & 135. \\
\hline 25 & 11.3 & 15.0 & 19.4 & 33.9 & 51.2 & 71.3 & 80.4 & 94.2 & 107. \\
\hline 30 & 6.69 & 8.34 & 13.7 & 25.1 & 36.9 & 55.0 & 68.3 & 74.2 & 85.3 \\
\hline 40 & 1.65 & 2.78 & 7.18 & 10.3 & 17.5 & 35.2 & 44.5 & 52.7 & 57.8 \\
\hline 50 & 0.62 & 0.94 & 3.62 & 5.18 & 10.4 & 21.1 & 26.7 & 37.3 & 39.5 \\
\hline 60 & 0.03 & 0.22 & 1.17 & 2.31 & 5.28 & 12.8 & 19.0 & 27.4 & 29.2 \\
\hline 70 & 0.00 & 0.00 & 0.66 & 1.02 & 2.26 & 6.54 & 9.26 & 15.5 & 17.8 \\
\hline 75 & 0.00 & 0.00 & 0.41 & 0.61 & 1.53 & 3.74 & 5.63 & 12.5 & 13.2 \\
\hline 80 & 0.00 & 0.00 & 0.28 & 0.40 & 0.86 & 2.22 & 4.07 & 9.39 & 10.8 \\
\hline 90 & 0.00 & 0.00 & 0.07 & 0.17 & 0.37 & 0.97 & 1.57 & 5.08 & 6.33 \\
\hline 95 & 0.00 & 0.00 & 0.00 & 0.01 & 0.19 & 0.50 & 0.76 & 2.94 & 4.12 \\
\hline 98 & 0.00 & 0.00 & 0.00 & 0.00 & 0.14 & 0.39 & 0.61 & 1.63 & 3.69 \\
\hline 99 & 0.00 & 0.00 & 0.00 & 0.00 & 0.09 & 0.25 & 0.44 & 1.40 & 3.37 \\
\hline
\end{tabular}


03219500 Scioto River near Prospect, Ohio

Years analyzed $=75$

Climatic year range $=1926-2008$

[APEE, annual percentage equaled or exceeded; $\mathrm{ft}^{3} / \mathrm{s}$, cubic foot per second]

\begin{tabular}{|c|c|c|c|c|c|c|c|c|c|}
\hline \multirow[b]{2}{*}{ APEE } & \multicolumn{9}{|c|}{ Daily mean streamflow $\left(\mathrm{ft}^{3} / \mathrm{s}\right)$ that was equaled or exceeded the indicated percentage of time at APEE level } \\
\hline & 98 & 95 & 90 & 80 & 50 & 20 & 10 & 5 & 2 \\
\hline 1 & 1070. & 1670. & 2290 & 3180. & 4610 & 6330. & 7060 . & 7690. & 8440 \\
\hline 2 & 719. & 1190. & 1950. & 2340 & 3640. & 4710 . & 5540. & 6030. & 7010 \\
\hline 5 & 410. & 682. & 919. & 1470 . & 2140 & 3070 . & 3690. & 4030 . & 4850 \\
\hline 10 & 270 & 315. & 486. & 744. & 1300 & 1970. & 2400 & 2700 & 2850 \\
\hline 20 & 127. & 152. & 258. & 296. & 589. & 984. & 1230 & 1370 . & 1510 \\
\hline 25 & 94.2 & 115. & 173. & 215. & 434. & 739. & 921. & 1040 . & 1230. \\
\hline 30 & 72.4 & 90.8 & 126. & 171. & 346. & 555. & 706. & 817. & 977. \\
\hline 40 & 28.5 & 52.4 & 68.0 & 100. & 217. & 382. & 450. & 527. & 652. \\
\hline 50 & 22.2 & 27.8 & 41.2 & 55.8 & 140. & 264. & 304. & 371. & 459. \\
\hline 60 & 17.0 & 21.0 & 29.2 & 38.2 & 87.2 & 177. & 216. & 256. & 346. \\
\hline 70 & 15.0 & 17.0 & 20.2 & 28.2 & 49.8 & 118. & 147. & 189. & 276. \\
\hline 75 & 13.8 & 15.0 & 17.0 & 23.2 & 39.0 & 93.6 & 118. & 150. & 251. \\
\hline 80 & 13.2 & 14.0 & 16.0 & 21.0 & 31.0 & 75.8 & 97.6 & 122. & 218. \\
\hline 90 & 10.4 & 12.0 & 13.0 & 15.0 & 21.0 & 39.0 & 62.8 & 73.6 & 158. \\
\hline 95 & 8.31 & 10.2 & 11.0 & 13.0 & 19.0 & 27.2 & 45.5 & 55.7 & 118. \\
\hline 98 & 7.33 & 8.84 & 9.64 & 11.5 & 17.0 & 21.8 & 35.3 & 48.2 & 81.6 \\
\hline 99 & 6.51 & 7.99 & 8.69 & 11.0 & 16.0 & 20.5 & 29.9 & 46.3 & 66.8 \\
\hline
\end{tabular}

\begin{tabular}{|c|c|c|c|c|c|c|c|c|c|}
\hline \multirow[b]{2}{*}{ APEE } & \multicolumn{9}{|c|}{ 7-day mean streamflow ( $\left.\mathrm{ft}^{3} / \mathrm{s}\right)$ that was equaled or exceeded the indicated percentage of time at APEE level } \\
\hline & 98 & 95 & 90 & 80 & 50 & 20 & 10 & 5 & 2 \\
\hline 1 & 770. & 1140. & 1810. & 2420. & 3530. & 4860. & 5700. & 5900. & 7140 \\
\hline 5 & 421. & 604. & 850. & 1360. & 2060 . & 2770 & 3230. & 3810 & 4300 \\
\hline 10 & 230 & 275 & 539. & 864. & 1290. & 1930. & 2160. & 2650 & 2880 \\
\hline 30 & 72.8 & 98.8 & 124. & 201. & 417. & 704. & 883. & 973. & 1110. \\
\hline 40 & 26.7 & 57.3 & 75.4 & 109. & 262. & 445. & 567. & 708. & 805. \\
\hline 50 & 22.1 & 28.5 & 42.3 & 65.9 & 150. & 305. & 392. & 475. & 638. \\
\hline 60 & 17.9 & 22.3 & 30.3 & 41.3 & 94.1 & 209. & 264. & 312. & 470. \\
\hline 90 & 11.1 & 12.2 & 13.4 & 16.2 & 22.8 & 45.5 & 72.4 & 88.7 & 181. \\
\hline 95 & 8.98 & 10.7 & 11.1 & 13.6 & 20.1 & 30.1 & 54.6 & 69.1 & 148. \\
\hline 98 & 7.70 & 9.68 & 10.4 & 12.2 & 17.9 & 23.3 & 41.6 & 50.1 & 107. \\
\hline 99 & 7.56 & 9.10 & 9.91 & 11.8 & 17.0 & 21.7 & 36.2 & 47.1 & 89.5 \\
\hline
\end{tabular}


03220000 Mill Creek near Bellepoint, Ohio

Years analyzed $=65$

Climatic year range $=1944-2008$

[APEE, annual percentage equaled or exceeded; $\mathrm{ft}^{3} / \mathrm{s}$, cubic foot per second]

\begin{tabular}{|c|c|c|c|c|c|c|c|c|c|}
\hline \multirow[b]{2}{*}{ APEE } & \multicolumn{9}{|c|}{ Daily mean streamflow $\left(\mathrm{ft}^{3} / \mathrm{s}\right)$ that was equaled or exceeded the indicated percentage of time at APEE level } \\
\hline & 98 & 95 & 90 & 80 & 50 & 20 & 10 & 5 & 2 \\
\hline 1 & 746. & 899. & 1260. & 1600. & 2290. & 2930. & 3500. & 3870. & 3990. \\
\hline 2 & 349. & 592. & 901. & 976. & 1710 & 2170 & 2580 & 2780 & 2950 \\
\hline 5 & 131. & 226. & 356. & 511. & 797. & 1240 & 1310 & 1550. & 1630. \\
\hline 10 & 72.0 & 79.0 & 168. & 211. & 344. & 596. & 720 . & 788. & 1010. \\
\hline 20 & 29.3 & 36.4 & 53.9 & 81.7 & 145. & 232. & 271. & 342. & 362. \\
\hline 25 & 23.3 & 27.3 & 34.3 & 57.0 & 97.0 & 152. & 191. & 233. & 254 \\
\hline 30 & 15.7 & 20.9 & 24.6 & 40.4 & 74.4 & 123. & 142. & 185. & 208. \\
\hline 40 & 7.02 & 11.6 & 14.0 & 25.8 & 46.0 & 75.5 & 94.0 & 122. & 128. \\
\hline 50 & 4.50 & 6.50 & 9.96 & 15.4 & 32.0 & 53.7 & 62.0 & 77.6 & 84.4 \\
\hline 60 & 3.09 & 4.22 & 6.84 & 10.4 & 20.4 & 37.8 & 43.2 & 56.3 & 64.6 \\
\hline 70 & 2.06 & 3.05 & 5.23 & 6.73 & 12.0 & 24.8 & 30.0 & 38.7 & 47.4 \\
\hline 75 & 1.70 & 2.42 & 4.33 & 5.32 & 9.80 & 20.0 & 25.8 & 30.3 & 39.9 \\
\hline 80 & 1.36 & 2.00 & 3.43 & 4.48 & 7.70 & 16.0 & 19.8 & 25.7 & 34.8 \\
\hline 90 & 0.73 & 1.10 & 1.46 & 3.02 & 5.34 & 10.0 & 12.4 & 17.4 & 22.2 \\
\hline 95 & 0.53 & 0.80 & 0.96 & 1.28 & 4.34 & 7.42 & 9.26 & 13.6 & 17.6 \\
\hline 98 & 0.20 & 0.43 & 0.60 & 0.97 & 3.40 & 6.29 & 7.54 & 9.48 & 14.4 \\
\hline 99 & 0.10 & 0.22 & 0.49 & 0.87 & 3.23 & 5.63 & 6.41 & 7.22 & 12.5 \\
\hline
\end{tabular}

\begin{tabular}{|c|c|c|c|c|c|c|c|c|c|}
\hline \multirow[b]{2}{*}{ APEE } & \multicolumn{9}{|c|}{ 7-day mean streamflow ( $\mathrm{ft}^{3} / \mathrm{s}$ ) that was equaled or exceeded the indicated percentage of time at APEE level } \\
\hline & 98 & 95 & 90 & 80 & 50 & 20 & 10 & 5 & 2 \\
\hline 1 & 457. & 567. & 731. & 958. & 1410 & 1990. & 2100 & 2230 & 3490 \\
\hline 2 & 412 & 431 & 568. & 705 & 1050 & 1380 & 1630. & 1730. & 1800 \\
\hline 5 & 132. & 223. & 389. & 487. & 731. & 1020 & 1220 & 1340 & 1450. \\
\hline 10 & 66.3 & 82.6 & 214. & 286. & 466. & 710 . & 772. & 882. & 992. \\
\hline 20 & 30.1 & 37.8 & 65.3 & 117. & 213. & 393. & 469. & 511. & 553. \\
\hline 25 & 24.2 & 26.8 & 43.9 & 80.5 & 149. & 297. & 357. & 405. & 442. \\
\hline 30 & 17.7 & 23.0 & 28.8 & 48.5 & 109. & 214. & 269. & 326. & 365. \\
\hline 40 & 6.99 & 13.5 & 14.9 & 30.4 & 69.7 & 120. & 156. & 194. & 241. \\
\hline 50 & 4.70 & 7.41 & 10.7 & 18.7 & 41.2 & 77.0 & 103. & 118 & 157. \\
\hline 60 & 3.20 & 4.56 & 7.79 & 12.4 & 26.0 & 44.6 & 61.4 & 81.6 & 89.3 \\
\hline 70 & 2.12 & 3.32 & 5.08 & 7.30 & 14.2 & 31.5 & 38.8 & 50.8 & 64.2 \\
\hline 75 & 1.75 & 2.39 & 4.43 & 5.58 & 10.6 & 24.1 & 31.4 & 40.3 & 50.2 \\
\hline 80 & 1.45 & 2.07 & 3.47 & 5.07 & 8.41 & 20.3 & 23.5 & 30.0 & 43.8 \\
\hline 90 & 0.87 & 1.16 & 1.49 & 3.41 & 5.84 & 12.3 & 14.8 & 20.5 & 29.0 \\
\hline 95 & 0.56 & 0.79 & 1.05 & 1.60 & 4.43 & 8.49 & 11.0 & 15.4 & 23.1 \\
\hline 98 & 0.37 & 0.58 & 0.74 & 1.08 & 4.06 & 7.13 & 8.90 & 13.4 & 18.1 \\
\hline 99 & 0.27 & 0.38 & 0.70 & 1.03 & 3.79 & 6.75 & 7.75 & 10.8 & 15.8 \\
\hline
\end{tabular}


03223000 Olentangy River at Claridon, Ohio

Years analyzed $=51$

Climatic year range $=1947-97$

[APEE, annual percentage equaled or exceeded; $\mathrm{ft}^{3} / \mathrm{s}$, cubic foot per second]

\begin{tabular}{|c|c|c|c|c|c|c|c|c|c|}
\hline \multirow[b]{2}{*}{ APEE } & \multicolumn{9}{|c|}{ Daily mean streamflow $\left(\mathrm{ft}^{3} / \mathrm{s}\right)$ that was equaled or exceeded the indicated percentage of time at APEE level } \\
\hline & 98 & 95 & 90 & 80 & 50 & 20 & 10 & 5 & 2 \\
\hline 1 & 487. & 859. & 1020. & 1290. & 1740. & 2250. & 2520. & 3040. & 3560. \\
\hline 2 & 367. & 599. & 745. & 972. & 1310. & 1590. & 1820. & 2120 & 2220 \\
\hline 5 & 245 & 284. & 382. & 500. & 752. & 995. & 1100 & 1210 & 1260. \\
\hline 10 & 124. & 152. & 171. & 228 & 408. & 553. & 692. & 749. & 774. \\
\hline 20 & 43.2 & 65.8 & 75.2 & 106. & 169. & 253. & 317. & 348. & 383. \\
\hline 25 & 28.0 & 45.6 & 56.2 & 77.3 & 128. & 196. & 245. & 269. & 290. \\
\hline 30 & 19.6 & 33.6 & 40.4 & 57.9 & 98.4 & 155. & 191. & 213. & 247. \\
\hline 40 & 9.96 & 19.0 & 23.4 & 30.2 & 62.0 & 101. & 118. & 147. & 178. \\
\hline 50 & 6.13 & 9.56 & 13.6 & 18.4 & 46.0 & 70.4 & 81.2 & 106. & 137. \\
\hline 60 & 4.04 & 5.38 & 8.34 & 11.4 & 27.4 & 45.8 & 59.4 & 71.0 & 111. \\
\hline 70 & 2.62 & 3.12 & 5.78 & 7.30 & 17.0 & 32.2 & 41.4 & 54.1 & 93.7 \\
\hline 75 & 1.91 & 2.26 & 4.24 & 5.88 & 13.0 & 21.8 & 34.4 & 47.0 & 83.8 \\
\hline 80 & 1.50 & 1.60 & 2.90 & 4.54 & 9.60 & 17.6 & 28.6 & 39.2 & 69.9 \\
\hline 90 & 0.42 & 0.92 & 1.44 & 2.20 & 6.00 & 11.0 & 16.8 & 23.4 & 39.0 \\
\hline 95 & 0.01 & 0.32 & 0.72 & 1.24 & 4.53 & 9.15 & 13.0 & 16.6 & 26.3 \\
\hline 98 & 0.00 & 0.08 & 0.26 & 0.74 & 3.40 & 7.32 & 9.25 & 13.7 & 17.0 \\
\hline 99 & 0.00 & 0.05 & 0.22 & 0.63 & 2.97 & 6.37 & 7.89 & 9.26 & 15.8 \\
\hline
\end{tabular}

\begin{tabular}{|c|c|c|c|c|c|c|c|c|c|}
\hline \multirow[b]{2}{*}{ APEE } & \multicolumn{9}{|c|}{ 7-day mean streamflow ( $\mathrm{ft}^{3} / \mathrm{s}$ ) that was equaled or exceeded the indicated percentage of time at APEE level } \\
\hline & 98 & 95 & 90 & 80 & 50 & 20 & 10 & 5 & 2 \\
\hline 1 & 318. & 452. & 685. & 866. & 1160. & 1680. & 1970. & 2490 & 3420. \\
\hline 2 & 291. & 359. & 531. & 703. & 937. & 1230 & 1500 & 1570 & 1690. \\
\hline 5 & 231. & 272 & 347. & 448. & 680. & 839. & 956. & 1110 & 1290. \\
\hline 10 & 130 & 149. & 182. & 289. & 427. & 588. & 632. & 707. & 747. \\
\hline 20 & 43.3 & 68.1 & 98.2 & 134. & 216. & 348. & 415. & 469. & 482. \\
\hline 25 & 31.9 & 53.3 & 72.9 & 92.4 & 168. & 255. & 355. & 393. & 438. \\
\hline 30 & 21.0 & 38.0 & 44.0 & 68.7 & 124. & 207. & 283. & 312. & 362. \\
\hline 40 & 11.7 & 21.1 & 23.9 & 34.2 & 75.9 & 137. & 159. & 192. & 250 \\
\hline 50 & 6.77 & 11.0 & 13.2 & 19.9 & 54.3 & 91.6 & 103. & 139. & 165. \\
\hline 60 & 3.95 & 5.43 & 8.84 & 12.0 & 32.3 & 57.4 & 72.1 & 98.3 & 134. \\
\hline 70 & 2.58 & 3.45 & 5.87 & 8.13 & 18.4 & 39.3 & 53.7 & 68.5 & 117. \\
\hline 75 & 2.12 & 2.53 & 4.58 & 6.39 & 15.0 & 27.5 & 41.9 & 60.7 & 104. \\
\hline 80 & 1.58 & 1.95 & 3.41 & 5.30 & 10.8 & 19.2 & 32.1 & 49.4 & 90.5 \\
\hline 90 & 0.56 & 1.05 & 1.77 & 2.64 & 6.64 & 12.7 & 20.2 & 35.7 & 55.5 \\
\hline 95 & 0.01 & 0.47 & 0.81 & 1.69 & 5.23 & 9.75 & 14.2 & 18.6 & 29.4 \\
\hline 98 & 0.00 & 0.19 & 0.47 & 0.88 & 3.83 & 8.67 & 11.3 & 15.9 & 24.9 \\
\hline 99 & 0.00 & 0.10 & 0.29 & 0.71 & 3.24 & 7.51 & 9.00 & 14.3 & 19.9 \\
\hline
\end{tabular}


03228300 Big Walnut Creek at Sunbury, Ohio

Years analyzed $=20$

Climatic year range $=1989-2008$

[APEE, annual percentage equaled or exceeded; $\mathrm{ft}^{3} / \mathrm{s}$, cubic foot per second]

\begin{tabular}{|c|c|c|c|c|c|c|c|c|c|}
\hline \multirow[b]{2}{*}{ APEE } & \multicolumn{9}{|c|}{ Daily mean streamflow $\left(\mathrm{ft}^{3} / \mathrm{s}\right)$ that was equaled or exceeded the indicated percentage of time at APEE level } \\
\hline & 95 & 90 & 80 & 70 & 50 & 30 & 20 & 10 & 5 \\
\hline 1 & 572. & 707. & 1010. & 1110 & 1570 & 1800 & 1890. & 2060 & 2870 \\
\hline 2 & 342. & 623. & 652. & 727. & 1060 & 1220 & 1290. & 1510 . & 1610 \\
\hline 5 & 158. & 299. & 319. & 359. & 539. & 778. & 790. & 829. & 1040 \\
\hline 10 & 94.1 & 135. & 170. & 176. & 252. & 329. & 384. & 424. & 517. \\
\hline 20 & 33.0 & 53.6 & 78.1 & 85.1 & 111. & 155. & 170. & 197. & 212. \\
\hline 25 & 22.9 & 38.0 & 57.4 & 66.3 & 91.3 & 116. & 132. & 140 . & 148. \\
\hline 30 & 15.5 & 25.8 & 43.2 & 52.5 & 71.6 & 91.5 & 97.5 & 111. & 121. \\
\hline 40 & 2.96 & 11.6 & 28.6 & 36.3 & 46.5 & 59.6 & 65.9 & 74.9 & 81.7 \\
\hline 50 & 0.84 & 5.12 & 16.4 & 21.3 & 32.0 & 39.5 & 45.6 & 53.8 & 57.8 \\
\hline 60 & 0.34 & 1.53 & 8.10 & 10.2 & 16.5 & 25.3 & 29.2 & 38.1 & 43.7 \\
\hline 70 & 0.02 & 0.30 & 2.41 & 3.67 & 7.23 & 13.5 & 17.0 & 25.4 & 29.8 \\
\hline 75 & 0.01 & 0.11 & 0.96 & 1.92 & 5.00 & 10.8 & 13.0 & 20.3 & 22.4 \\
\hline 80 & 0.00 & 0.03 & 0.46 & 0.89 & 2.61 & 7.39 & 9.95 & 11.9 & 17.7 \\
\hline 90 & 0.00 & 0.00 & 0.06 & 0.13 & 0.49 & 3.56 & 3.86 & 5.34 & 8.02 \\
\hline 95 & 0.00 & 0.00 & 0.00 & 0.03 & 0.09 & 1.44 & 2.43 & 3.26 & 4.38 \\
\hline 98 & 0.00 & 0.00 & 0.00 & 0.00 & 0.03 & 0.34 & 1.16 & 2.29 & 2.97 \\
\hline 99 & 0.00 & 0.00 & 0.00 & 0.00 & 0.01 & 0.14 & 0.60 & 1.18 & 1.74 \\
\hline
\end{tabular}

\begin{tabular}{|c|c|c|c|c|c|c|c|c|c|}
\hline \multirow[b]{2}{*}{ APEE } & \multicolumn{9}{|c|}{ 7-day mean streamflow $\left(\mathrm{ft}^{3} / \mathrm{s}\right)$ that was equaled or exceeded the indicated percentage of time at APEE level } \\
\hline & 95 & 90 & 80 & 70 & 50 & 30 & 20 & 10 & 5 \\
\hline 1 & 426. & 508. & 562. & 608. & 765. & 868. & 926. & 1170 & 1640 \\
\hline 2 & 276. & 439. & 489. & 516. & 639. & 743. & 806. & 882. & 1160. \\
\hline 5 & 160. & 289. & 318. & 359. & 523. & 597. & 646. & 741. & 813. \\
\hline 10 & 109. & 181. & 203. & 210 & 354. & 399. & 443. & 475 & 484. \\
\hline 20 & 39.6 & 83.8 & 100. & 109. & 164. & 251. & 282. & 321. & 324. \\
\hline 25 & 23.0 & 48.2 & 78.9 & 86.3 & 134. & 198. & 222. & 249. & 278 \\
\hline 30 & 17.2 & 30.0 & 62.9 & 73.2 & 106. & 144. & 171. & 186. & 242. \\
\hline 40 & 2.79 & 13.6 & 34.3 & 46.9 & 68.1 & 80.6 & 96.9 & 128. & 170. \\
\hline 50 & 0.96 & 7.15 & 18.9 & 27.2 & 41.8 & 53.3 & 70.4 & 76.3 & 100 \\
\hline 60 & 0.33 & 1.68 & 10.8 & 12.5 & 20.7 & 32.4 & 41.1 & 52.4 & 64.0 \\
\hline 70 & 0.03 & 0.37 & 3.03 & 5.00 & 10.4 & 17.1 & 26.0 & 34.7 & 36.5 \\
\hline 75 & 0.02 & 0.20 & 1.09 & 2.59 & 6.84 & 13.0 & 19.0 & 26.0 & 29.6 \\
\hline 80 & 0.01 & 0.09 & 0.64 & 1.47 & 3.38 & 9.86 & 13.3 & 17.5 & 22.5 \\
\hline 90 & 0.00 & 0.01 & 0.08 & 0.18 & 0.92 & 3.92 & 5.20 & 9.11 & 11.3 \\
\hline 95 & 0.00 & 0.00 & 0.01 & 0.05 & 0.22 & 1.87 & 2.74 & 5.06 & 6.94 \\
\hline 98 & 0.00 & 0.00 & 0.00 & 0.01 & 0.08 & 1.01 & 1.64 & 4.16 & 4.91 \\
\hline 99 & 0.00 & 0.00 & 0.00 & 0.01 & 0.05 & 0.41 & 1.27 & 3.32 & 3.42 \\
\hline
\end{tabular}


03230500 Big Darby Creek at Darbyville, Ohio

Years analyzed $=83$

Climatic year range $=1922-2008$

[APEE, annual percentage equaled or exceeded; $\mathrm{ft}^{3} / \mathrm{s}$, cubic foot per second]

\begin{tabular}{|c|c|c|c|c|c|c|c|c|c|}
\hline \multirow[b]{2}{*}{ APEE } & \multicolumn{9}{|c|}{ Daily mean streamflow $\left(\mathrm{ft}^{3} / \mathrm{s}\right)$ that was equaled or exceeded the indicated percentage of time at APEE level } \\
\hline & 98 & 95 & 90 & 80 & 50 & 20 & 10 & 5 & 2 \\
\hline 1 & 459. & 1910. & 2470 & 3150. & 5130 & 6540. & 7280 & 8380. & 10200. \\
\hline 2 & 340 . & 1260. & 1640. & 2150 & 3440. & 4640 . & 5340. & 5940. & 7470 . \\
\hline 5 & 227. & 609. & 941. & 1180. & 1890. & 2720 . & 3090. & 3650 . & 3880 . \\
\hline 10 & 134. & 383. & 519. & 654. & 1060 & 1750 . & 1920. & 2110 & 2280 \\
\hline 20 & 62.4 & 179. & 258. & 360. & 546. & 918. & 1080 & 1130. & 1240 \\
\hline 25 & 50.6 & 141. & 195. & 265. & 425. & 730 . & 857. & 891. & 975. \\
\hline 30 & 42.0 & 95.0 & 155. & 215. & 350. & 597. & 708. & 733. & 820. \\
\hline 40 & 27.8 & 39.4 & 88.0 & 144. & 250 & 399. & 509. & 551. & 599. \\
\hline 50 & 21.4 & 30.0 & 54.2 & 93.6 & 180. & 281. & 354. & 398. & 453. \\
\hline 60 & 17.4 & 20.2 & 39.6 & 52.0 & 110. & 194. & 249. & 306. & 361. \\
\hline 70 & 14.6 & 18.0 & 29.4 & 37.8 & 76.0 & 133. & 173. & 246. & 276. \\
\hline 75 & 12.4 & 15.2 & 26.2 & 32.8 & 61.0 & 105. & 143. & 211. & 248 \\
\hline 80 & 9.64 & 13.3 & 20.3 & 27.0 & 48.0 & 83.4 & 126. & 181. & 211. \\
\hline 90 & 6.17 & 7.38 & 12.8 & 18.5 & 33.0 & 55.2 & 81.2 & 110. & 163. \\
\hline 95 & 4.08 & 5.55 & 8.22 & 14.5 & 27.0 & 44.0 & 59.7 & 84.9 & 132. \\
\hline 98 & 2.54 & 4.39 & 6.00 & 11.9 & 23.0 & 39.3 & 46.8 & 74.0 & 107. \\
\hline 99 & 2.19 & 3.12 & 5.39 & 10.5 & 21.7 & 38.0 & 43.0 & 68.5 & 89.8 \\
\hline
\end{tabular}

\begin{tabular}{|c|c|c|c|c|c|c|c|c|c|}
\hline \multirow[b]{2}{*}{ APEE } & \multicolumn{9}{|c|}{ 7-day mean streamflow (ft 3 /s) that was equaled or exceeded the indicated percentage of time at APEE level } \\
\hline & 98 & 95 & 90 & 80 & 50 & 20 & 10 & 5 & 2 \\
\hline 1 & 376. & 1350 & 1640 & 1980. & 3260 & 4790 & 5480. & 6450 & 9740. \\
\hline 5 & 228. & 663. & 897. & 1150 & 1950. & 2510 & 3020. & 3200 & 3590. \\
\hline 10 & 128. & 392. & 593. & 772. & 1210 & 1710. & 1930. & 2090 . & 2250 . \\
\hline 30 & 40.8 & 88.7 & 145. & 237. & 371. & 704. & 853. & 952. & 1100. \\
\hline 40 & 26.8 & 41.8 & 92.3 & 152. & 268. & 485. & 600. & 661. & 750. \\
\hline 50 & 21.5 & 30.9 & 54.9 & 99.6 & 193. & 321. & 441. & 512. & 552. \\
\hline 60 & 17.6 & 20.5 & 41.1 & 52.3 & 119. & 218 & 293. & 371. & 425. \\
\hline 90 & 6.09 & 7.71 & 13.5 & 18.4 & 35.6 & 60.3 & 87.7 & 118. & 180. \\
\hline 95 & 4.32 & 5.92 & 8.34 & 15.4 & 28.3 & 45.7 & 61.5 & 89.5 & 149. \\
\hline 98 & 3.39 & 4.67 & 6.78 & 12.8 & 24.6 & 41.4 & 56.4 & 77.1 & 131. \\
\hline 99 & 2.67 & 3.89 & 6.23 & 11.4 & 23.9 & 39.6 & 44.8 & 73.8 & 103. \\
\hline
\end{tabular}




\section{Paint Creek near Greenfield, Ohio}

Years analyzed $=51$

Climatic year range $=1927-2008$

[APEE, annual percentage equaled or exceeded; $\mathrm{ft}^{3} / \mathrm{s}$, cubic foot per second]

\begin{tabular}{|c|c|c|c|c|c|c|c|c|c|}
\hline \multirow[b]{2}{*}{ APEE } & \multicolumn{9}{|c|}{ Daily mean streamflow $\left(\mathrm{ft}^{3} / \mathrm{s}\right)$ that was equaled or exceeded the indicated percentage of time at APEE level } \\
\hline & 98 & 95 & 90 & 80 & 50 & 20 & 10 & 5 & 2 \\
\hline 1 & 273. & 538. & 772. & 1480. & 2560. & 3970. & 4430. & 5630. & 6190 \\
\hline 2 & 174. & 359. & 687. & 1050 & 1760 & 2660 & 3000 . & 3520 . & 4000 \\
\hline 5 & 97.0 & 175 . & 407. & 619. & 970. & 1430 . & 1670 . & 1950. & 2330 \\
\hline 10 & 52.6 & 118. & 208. & 432. & 589. & 877. & 983. & 1190. & 1330. \\
\hline 20 & 25.1 & 55.2 & 112. & 212. & 293. & 475. & 565. & 600 & 661. \\
\hline 25 & 16.9 & 42.4 & 88.9 & 151. & 220. & 384. & 443. & 462. & 521. \\
\hline 30 & 11.8 & 31.2 & 68.1 & 103. & 180. & 308. & 355. & 376. & 426. \\
\hline 40 & 5.46 & 14.2 & 29.8 & 62.4 & 128. & 207. & 256. & 261. & 324. \\
\hline 50 & 3.22 & 6.78 & 11.7 & 35.8 & 85.0 & 147. & 178. & 200 & 243. \\
\hline 60 & 2.47 & 3.16 & 5.70 & 22.6 & 51.0 & 106. & 130. & 156. & 196. \\
\hline 70 & 1.41 & 2.08 & 4.54 & 11.3 & 26.0 & 76.6 & 91.5 & 123. & 130. \\
\hline 75 & 0.72 & 1.46 & 3.78 & 7.58 & 20.0 & 58.2 & 73.4 & 107. & 111. \\
\hline 80 & 0.44 & 1.16 & 2.52 & 4.55 & 15.0 & 40.1 & 60.8 & 89.7 & 95.3 \\
\hline 90 & 0.20 & 0.29 & 1.04 & 2.21 & 8.04 & 19.0 & 35.2 & 56.1 & 69.9 \\
\hline 95 & 0.10 & 0.17 & 0.60 & 1.40 & 4.29 & 9.60 & 23.9 & 44.8 & 57.2 \\
\hline 98 & 0.03 & 0.12 & 0.25 & 0.90 & 2.80 & 7.50 & 13.1 & 29.8 & 40.9 \\
\hline 99 & 0.00 & 0.10 & 0.21 & 0.46 & 2.50 & 6.69 & 12.0 & 24.8 & 36.7 \\
\hline
\end{tabular}

\begin{tabular}{|c|c|c|c|c|c|c|c|c|c|}
\hline \multirow[b]{2}{*}{ APEE } & \multicolumn{9}{|c|}{ 7-day mean streamflow ( $\mathrm{ft}^{3} / \mathrm{s}$ ) that was equaled or exceeded the indicated percentage of time at APEE level } \\
\hline & 98 & 95 & 90 & 80 & 50 & 20 & 10 & 5 & 2 \\
\hline 1 & 206. & 452. & 630. & 1030. & 1880. & 3080. & 3390. & 4350. & 5290. \\
\hline 2 & 127. & 299. & 473. & 912. & 1360 . & 2100 & 2420 . & 2760 & 3540 \\
\hline 5 & 86.0 & 187. & 397. & 621. & 939. & 1370 . & 1740 . & 1820. & 1860 \\
\hline 10 & 47.8 & 120. & 240 & 441. & 650. & 910. & 1050 . & 1110 . & 1190. \\
\hline 20 & 20.9 & 58.9 & 114. & 219. & 323. & 558. & 607. & 715. & 789. \\
\hline 25 & 14.5 & 43.8 & 90.4 & 181. & 249. & 447. & 509. & 622. & 690. \\
\hline 30 & 12.2 & 32.9 & 62.5 & 127. & 205. & 370. & 433. & 504. & 534. \\
\hline 40 & 5.79 & 16.6 & 28.5 & 70.8 & 149. & 248 & 294. & 336. & 356. \\
\hline 50 & 3.08 & 7.17 & 11.7 & 41.8 & 90.7 & 166. & 223. & 243. & 273. \\
\hline 60 & 2.63 & 3.28 & 5.85 & 23.7 & 57.7 & 125. & 151. & 190. & 217. \\
\hline 70 & 1.31 & 2.03 & 4.79 & 10.7 & 32.0 & 84.7 & 104. & 145. & 173. \\
\hline 75 & 0.86 & 1.69 & 4.18 & 8.42 & 24.0 & 68.4 & 84.2 & 121. & 133. \\
\hline 80 & 0.48 & 1.11 & 3.27 & 5.61 & 17.1 & 47.5 & 69.0 & 97.8 & 118. \\
\hline 90 & 0.27 & 0.67 & 1.66 & 2.42 & 8.44 & 20.1 & 38.9 & 62.7 & 84.1 \\
\hline 95 & 0.16 & 0.50 & 0.78 & 1.75 & 5.19 & 10.6 & 27.3 & 48.9 & 68.1 \\
\hline 98 & 0.09 & 0.17 & 0.41 & 1.15 & 3.43 & 8.68 & 18.6 & 32.4 & 54.9 \\
\hline 99 & 0.05 & 0.14 & 0.24 & 0.77 & 2.73 & 7.90 & 12.8 & 27.4 & 49.5 \\
\hline
\end{tabular}


03235500 Tar Hollow Creek at Tar Hollow State Park, Ohio

Years analyzed $=31$

Climatic year range $=1947-77$

[APEE, annual percentage equaled or exceeded; $\mathrm{ft}^{3} / \mathrm{s}$, cubic foot per second]

\begin{tabular}{|c|c|c|c|c|c|c|c|c|c|}
\hline \multirow[b]{2}{*}{ APEE } & \multicolumn{9}{|c|}{ Daily mean streamflow (ft³/s) that was equaled or exceeded the indicated percentage of time at APEE level } \\
\hline & 95 & 90 & 80 & 70 & 50 & 30 & 20 & 10 & 5 \\
\hline 1 & 6.40 & 7.88 & 10.3 & 12.7 & 16.0 & 21.9 & 25.8 & 28.0 & 31.1 \\
\hline 2 & 4.19 & 5.61 & 6.59 & 7.97 & 9.84 & 13.4 & 15.4 & 16.4 & 21.1 \\
\hline 5 & 1.87 & 2.65 & 3.32 & 3.83 & 5.26 & 7.26 & 7.77 & 8.69 & 9.50 \\
\hline 10 & 1.12 & 1.62 & 2.06 & 2.30 & 2.93 & 3.84 & 4.24 & 4.80 & 5.20 \\
\hline 20 & 0.45 & 0.72 & 1.00 & 1.07 & 1.48 & 1.78 & 2.22 & 2.78 & 2.94 \\
\hline 25 & 0.20 & 0.42 & 0.57 & 0.75 & 1.00 & 1.44 & 1.82 & 2.24 & 2.38 \\
\hline 30 & 0.00 & 0.04 & 0.32 & 0.48 & 0.70 & 1.14 & 1.40 & 1.74 & 2.05 \\
\hline 40 & 0.00 & 0.00 & 0.01 & 0.20 & 0.40 & 0.69 & 0.80 & 1.24 & 1.52 \\
\hline 50 & 0.00 & 0.00 & 0.00 & 0.00 & 0.20 & 0.35 & 0.48 & 0.60 & 1.20 \\
\hline 60 & 0.00 & 0.00 & 0.00 & 0.00 & 0.06 & 0.16 & 0.20 & 0.29 & 0.64 \\
\hline 70 & 0.00 & 0.00 & 0.00 & 0.00 & 0.00 & 0.05 & 0.10 & 0.18 & 0.30 \\
\hline 75 & 0.00 & 0.00 & 0.00 & 0.00 & 0.00 & 0.02 & 0.06 & 0.10 & 0.17 \\
\hline 80 & 0.00 & 0.00 & 0.00 & 0.00 & 0.00 & 0.02 & 0.04 & 0.10 & 0.14 \\
\hline 90 & 0.00 & 0.00 & 0.00 & 0.00 & 0.00 & 0.00 & 0.00 & 0.02 & 0.04 \\
\hline 95 & 0.00 & 0.00 & 0.00 & 0.00 & 0.00 & 0.00 & 0.00 & 0.00 & 0.01 \\
\hline 98 & 0.00 & 0.00 & 0.00 & 0.00 & 0.00 & 0.00 & 0.00 & 0.00 & 0.00 \\
\hline 99 & 0.00 & 0.00 & 0.00 & 0.00 & 0.00 & 0.00 & 0.00 & 0.00 & 0.00 \\
\hline
\end{tabular}

\begin{tabular}{|c|c|c|c|c|c|c|c|c|c|}
\hline \multirow[b]{2}{*}{ APEE } & \multicolumn{9}{|c|}{ 7-day mean streamflow ( $\left.\mathrm{ft}^{3} / \mathrm{s}\right)$ that was equaled or exceeded the indicated percentage of time at APEE level } \\
\hline & 95 & 90 & 80 & 70 & 50 & 30 & 20 & 10 & 5 \\
\hline 1 & 4.17 & 4.81 & 5.70 & 6.86 & 9.16 & 11.8 & 14.1 & 18.7 & 25.1 \\
\hline 2 & 3.58 & 4.42 & 4.83 & 5.80 & 7.47 & 10.1 & 10.5 & 12.5 & 14.6 \\
\hline 5 & 2.87 & 3.44 & 3.63 & 3.96 & 5.47 & 7.05 & 7.78 & 8.33 & 9.37 \\
\hline 10 & 1.32 & 1.64 & 2.52 & 2.56 & 3.17 & 4.51 & 5.48 & 6.18 & 6.63 \\
\hline 20 & 0.35 & 0.81 & 1.23 & 1.37 & 1.74 & 2.29 & 2.99 & 3.45 & 3.95 \\
\hline 25 & 0.23 & 0.48 & 0.70 & 0.87 & 1.20 & 1.90 & 2.26 & 2.80 & 3.03 \\
\hline 30 & 0.05 & 0.11 & 0.48 & 0.61 & 0.84 & 1.66 & 2.01 & 2.20 & 2.55 \\
\hline 40 & 0.00 & 0.00 & 0.04 & 0.24 & 0.51 & 0.84 & 1.12 & 1.50 & 1.85 \\
\hline 50 & 0.00 & 0.00 & 0.00 & 0.08 & 0.24 & 0.46 & 0.54 & 0.83 & 1.32 \\
\hline 60 & 0.00 & 0.00 & 0.00 & 0.00 & 0.10 & 0.21 & 0.28 & 0.33 & 0.73 \\
\hline 70 & 0.00 & 0.00 & 0.00 & 0.00 & 0.01 & 0.09 & 0.14 & 0.19 & 0.37 \\
\hline 75 & 0.00 & 0.00 & 0.00 & 0.00 & 0.00 & 0.05 & 0.10 & 0.14 & 0.24 \\
\hline 80 & 0.00 & 0.00 & 0.00 & 0.00 & 0.00 & 0.03 & 0.05 & 0.10 & 0.19 \\
\hline 90 & 0.00 & 0.00 & 0.00 & 0.00 & 0.00 & 0.01 & 0.02 & 0.03 & 0.07 \\
\hline 95 & 0.00 & 0.00 & 0.00 & 0.00 & 0.00 & 0.00 & 0.00 & 0.01 & 0.03 \\
\hline 98 & 0.00 & 0.00 & 0.00 & 0.00 & 0.00 & 0.00 & 0.00 & 0.00 & 0.00 \\
\hline 99 & 0.00 & 0.00 & 0.00 & 0.00 & 0.00 & 0.00 & 0.00 & 0.00 & 0.00 \\
\hline
\end{tabular}


03237280 Upper Twin Creek at McGaw, Ohio

Years analyzed $=45$

Climatic year range $=1964-2008$

[APEE, annual percentage equaled or exceeded; $\mathrm{ft}^{3} / \mathrm{s}$, cubic foot per second]

\begin{tabular}{|c|c|c|c|c|c|c|c|c|c|}
\hline \multirow[b]{2}{*}{ APEE } & \multicolumn{9}{|c|}{ Daily mean streamflow (ft $3 / s)$ that was equaled or exceeded the indicated percentage of time at APEE level } \\
\hline & 95 & 90 & 80 & 70 & 50 & 30 & 20 & 10 & 5 \\
\hline 1 & 64.0 & 73.2 & 104. & 126. & 156. & 224. & 260. & 326. & 352. \\
\hline 2 & 43.6 & 53.6 & 66.8 & 74.0 & 104. & 138. & 162. & 190. & 212 \\
\hline 5 & 25.3 & 31.1 & 39.8 & 42.7 & 55.7 & 65.0 & 71.3 & 101. & 113. \\
\hline 10 & 15.3 & 16.8 & 23.6 & 26.1 & 30.0 & 40.0 & 42.0 & 53.0 & 56.7 \\
\hline 20 & 7.30 & 9.88 & 11.0 & 14.0 & 17.0 & 22.8 & 24.8 & 28.1 & 30.9 \\
\hline 25 & 5.88 & 7.44 & 8.27 & 9.74 & 14.0 & 17.4 & 19.9 & 23.4 & 24.7 \\
\hline 30 & 4.32 & 5.48 & 6.09 & 7.08 & 10.2 & 14.0 & 17.0 & 19.1 & 20.0 \\
\hline 40 & 1.00 & 1.90 & 2.93 & 3.70 & 5.96 & 9.08 & 11.5 & 13.4 & 15.4 \\
\hline 50 & 0.34 & 0.47 & 1.26 & 1.70 & 3.10 & 6.12 & 7.30 & 8.94 & 11.0 \\
\hline 60 & 0.10 & 0.19 & 0.48 & 0.80 & 1.54 & 2.62 & 3.79 & 5.22 & 7.97 \\
\hline 70 & 0.06 & 0.12 & 0.21 & 0.36 & 0.69 & 1.38 & 1.82 & 2.56 & 5.80 \\
\hline 75 & 0.01 & 0.05 & 0.13 & 0.21 & 0.42 & 0.89 & 1.18 & 1.68 & 4.55 \\
\hline 80 & 0.00 & 0.02 & 0.07 & 0.10 & 0.25 & 0.54 & 0.80 & 1.30 & 3.13 \\
\hline 90 & 0.00 & 0.00 & 0.00 & 0.04 & 0.09 & 0.29 & 0.38 & 0.61 & 1.70 \\
\hline 95 & 0.00 & 0.00 & 0.00 & 0.00 & 0.05 & 0.13 & 0.22 & 0.48 & 1.20 \\
\hline 98 & 0.00 & 0.00 & 0.00 & 0.00 & 0.02 & 0.11 & 0.15 & 0.35 & 0.88 \\
\hline 99 & 0.00 & 0.00 & 0.00 & 0.00 & 0.02 & 0.09 & 0.15 & 0.28 & 0.69 \\
\hline
\end{tabular}

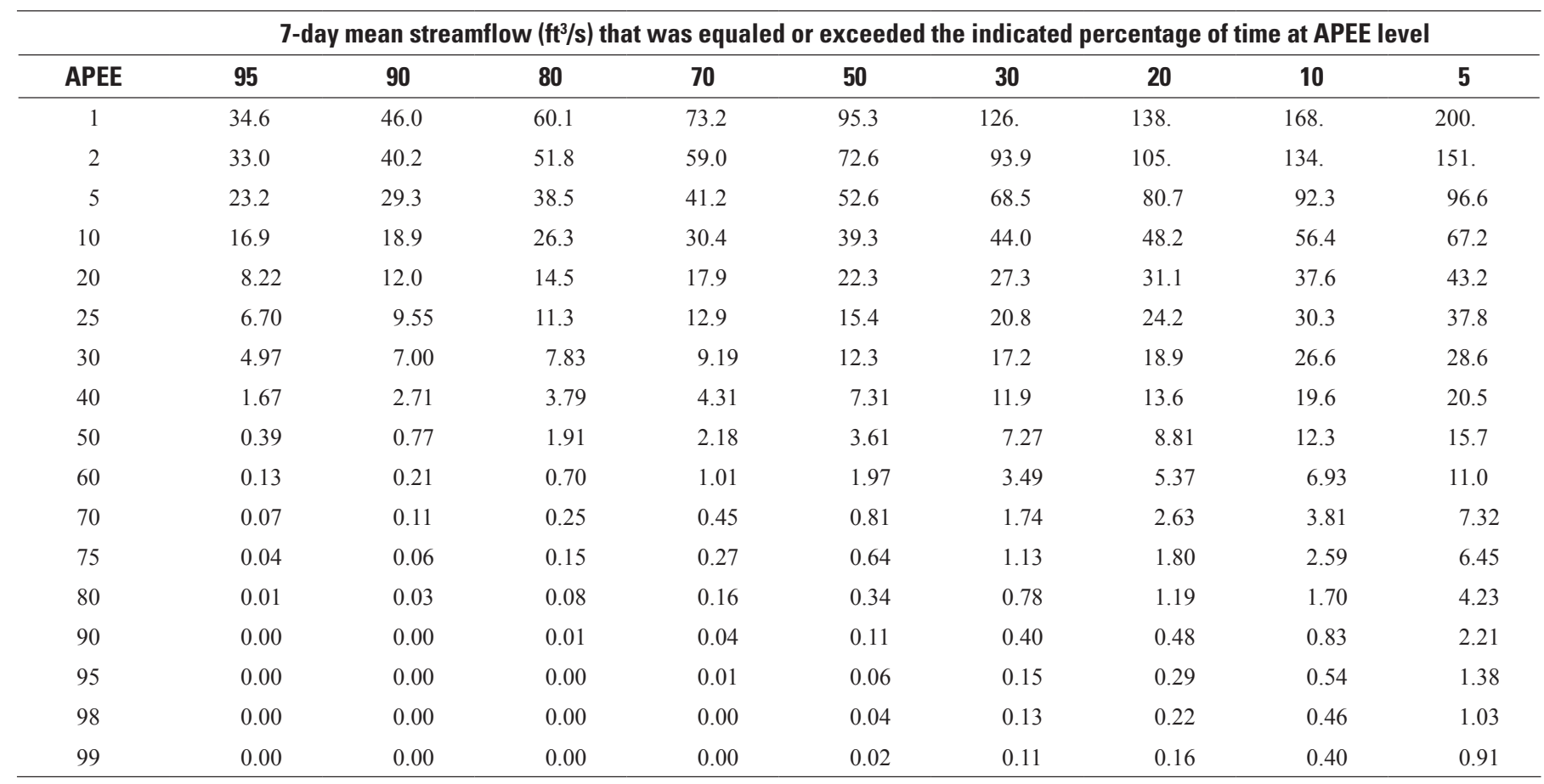




\section{Ohio Brush Creek near West Union, Ohio}

Years analyzed $=76$

Climatic year range $=1927-2008$

[APEE, annual percentage equaled or exceeded; $\mathrm{ft}^{3} / \mathrm{s}$, cubic foot per second]

\begin{tabular}{|c|c|c|c|c|c|c|c|c|c|}
\hline \multirow[b]{2}{*}{ APEE } & \multicolumn{9}{|c|}{ Daily mean streamflow $\left(\mathrm{ft}^{3} / \mathrm{s}\right)$ that was equaled or exceeded the indicated percentage of time at APEE level } \\
\hline & 98 & 95 & 90 & 80 & 50 & 20 & 10 & 5 & 2 \\
\hline 1 & 2000 & 3270 & 3760. & 4280 & 6130. & 8790. & 9930. & 11100 & 13200. \\
\hline 2 & 1040 & 1980. & 2390 & 2860 & 4180 . & 5540. & 6540 & 6860 & 7990. \\
\hline 5 & 487. & 857. & 1060. & 1310. & 2000 & 2500 & 2880 & 3380 . & 3820. \\
\hline 10 & 231. & 374. & 505. & 677. & 988. & 1310. & 1610 & 1940. & 2050 \\
\hline 20 & 123. & 177. & 232. & 316. & 451. & 617. & 796. & 895. & 1020 \\
\hline 25 & 89.1 & 135. & 183. & 228 & 352. & 470 . & 618. & 685. & 765. \\
\hline 30 & 58.8 & 104. & 142. & 174. & 264. & 374. & 486. & 549. & 611. \\
\hline 40 & 24.1 & 58.5 & 75.8 & 99.8 & 174. & 254. & 345. & 373. & 447. \\
\hline 50 & 11.7 & 16.0 & 24.9 & 54.8 & 108. & 168. & 236. & 265. & 301. \\
\hline 60 & 3.51 & 5.82 & 9.97 & 32.6 & 62.5 & 122. & 151. & 197. & 222 \\
\hline 70 & 1.38 & 2.90 & 5.07 & 13.8 & 35.9 & 73.2 & 99.4 & 129. & 168. \\
\hline 75 & 0.81 & 2.09 & 3.94 & 8.44 & 22.3 & 54.8 & 82.2 & 109. & 139. \\
\hline 80 & 0.63 & 1.48 & 2.58 & 6.12 & 16.0 & 38.8 & 68.9 & 76.1 & 110. \\
\hline 90 & 0.22 & 0.75 & 1.02 & 2.37 & 7.90 & 18.0 & 35.3 & 51.2 & 64.4 \\
\hline 95 & 0.13 & 0.26 & 0.55 & 0.88 & 4.78 & 11.2 & 22.0 & 31.5 & 49.2 \\
\hline 98 & 0.05 & 0.13 & 0.28 & 0.59 & 3.32 & 8.68 & 15.6 & 18.2 & 40.2 \\
\hline 99 & 0.05 & 0.13 & 0.20 & 0.46 & 2.55 & 8.00 & 12.9 & 14.5 & 36.2 \\
\hline
\end{tabular}

\begin{tabular}{|c|c|c|c|c|c|c|c|c|c|}
\hline \multirow[b]{2}{*}{ APEE } & \multicolumn{9}{|c|}{ 7-day mean streamflow ( $\mathrm{ft}^{3} / \mathrm{s}$ ) that was equaled or exceeded the indicated percentage of time at APEE level } \\
\hline & 98 & 95 & 90 & 80 & 50 & 20 & 10 & 5 & 2 \\
\hline 1 & 1080 & 1390. & 1980. & 2280 & 3090 . & 4630 & 6300 & 6700 & 11200 \\
\hline 2 & 922. & 1290. & 1590. & 1970. & 2510 & 3620. & 4480 . & 5480 & 6750 \\
\hline 5 & 508. & 990. & 1110 & 1340. & 1820 & 2450 & 2640 & 2830 & 3310. \\
\hline 10 & 270 & 553. & 693. & 853. & 1230. & 1640. & 1860. & 2040 & 2200 \\
\hline 20 & 141. & 234. & 329. & 438. & 661. & 957. & 1080 & 1310 & 1390. \\
\hline 25 & 107. & 177. & 242. & 336. & 516. & 739. & 917. & 1140 & 1200. \\
\hline 30 & 60.1 & 126. & 204. & 246. & 400. & 587. & 755. & 861. & 941. \\
\hline 40 & 32.4 & 64.2 & 92.9 & 129. & 246. & 382. & 512. & 595. & 661. \\
\hline 50 & 13.8 & 19.6 & 33.2 & 74.4 & 152. & 262. & 356. & 400 & 516. \\
\hline 60 & 4.11 & 6.07 & 11.7 & 44.6 & 86.0 & 169. & 219. & 299. & 334. \\
\hline 70 & 1.49 & 2.88 & 5.41 & 19.0 & 46.3 & 96.5 & 149. & 186. & 251. \\
\hline 75 & 0.92 & 2.06 & 4.03 & 10.9 & 31.9 & 72.0 & 112. & 135. & 213. \\
\hline 80 & 0.72 & 1.51 & 3.09 & 7.53 & 21.7 & 55.6 & 87.8 & 114. & 168. \\
\hline 90 & 0.22 & 0.79 & 1.17 & 2.97 & 10.1 & 30.1 & 48.5 & 66.4 & 82.9 \\
\hline 95 & 0.14 & 0.32 & 0.67 & 1.03 & 5.22 & 16.5 & 34.4 & 41.0 & 66.0 \\
\hline 98 & 0.09 & 0.19 & 0.35 & 0.79 & 3.95 & 11.2 & 20.6 & 29.2 & 55.4 \\
\hline 99 & 0.05 & 0.14 & 0.26 & 0.65 & 3.18 & 9.60 & 17.1 & 22.6 & 50.9 \\
\hline
\end{tabular}


03238500 White Oak Creek near Georgetown, Ohio

Years analyzed $=79$

Climatic year range $=1925-2008$

[APEE, annual percentage equaled or exceeded; $\mathrm{ft}^{3} / \mathrm{s}$, cubic foot per second]

\begin{tabular}{|c|c|c|c|c|c|c|c|c|c|}
\hline \multirow[b]{2}{*}{ APEE } & \multicolumn{9}{|c|}{ Daily mean streamflow $\left(\mathrm{ft}^{3} / \mathrm{s}\right)$ that was equaled or exceeded the indicated percentage of time at APEE level } \\
\hline & 98 & 95 & 90 & 80 & 50 & 20 & 10 & 5 & 2 \\
\hline 1 & 1280. & 2220. & 2520. & 3140. & 4500. & 5760. & 6400. & 7230 & 8180. \\
\hline 2 & 655. & 973. & 1650. & 2030 & 2930. & 3750 . & 4310. & 5250. & 5460. \\
\hline 5 & 194. & 375. & 518. & 784. & 1220 & 1760 . & 2140 & 2450 & 3010 . \\
\hline 10 & 83.2 & 136. & 245 & 362. & 517. & 793. & 1010. & 1140 & 1330. \\
\hline 20 & 36.4 & 62.0 & 101. & 130. & 193. & 281. & 387. & 430. & 535. \\
\hline 25 & 24.4 & 46.5 & 82.0 & 91.0 & 140. & 210 & 277. & 312. & 346. \\
\hline 30 & 16.1 & 34.0 & 58.7 & 68.0 & 109. & 155. & 216. & 225. & 260. \\
\hline 40 & 8.04 & 19.0 & 26.0 & 41.0 & 71.6 & 102. & 138. & 147. & 173. \\
\hline 50 & 3.24 & 7.40 & 11.0 & 26.0 & 43.0 & 70.0 & 97.0 & 103. & 124. \\
\hline 60 & 0.72 & 3.58 & 6.28 & 14.0 & 26.0 & 46.0 & 60.0 & 75.6 & 89.0 \\
\hline 70 & 0.12 & 1.00 & 3.40 & 7.93 & 16.0 & 27.8 & 43.0 & 55.0 & 60.4 \\
\hline 75 & 0.06 & 0.50 & 2.50 & 5.50 & 12.0 & 23.0 & 34.0 & 45.0 & 50.0 \\
\hline 80 & 0.00 & 0.10 & 0.90 & 3.64 & 7.86 & 18.0 & 26.6 & 35.0 & 39.4 \\
\hline 90 & 0.00 & 0.00 & 0.00 & 0.60 & 3.32 & 9.60 & 14.0 & 18.0 & 25.4 \\
\hline 95 & 0.00 & 0.00 & 0.00 & 0.22 & 1.60 & 5.13 & 11.0 & 13.0 & 18.2 \\
\hline 98 & 0.00 & 0.00 & 0.00 & 0.00 & 0.69 & 2.80 & 7.53 & 8.96 & 12.4 \\
\hline 99 & 0.00 & 0.00 & 0.00 & 0.00 & 0.46 & 2.57 & 4.99 & 7.02 & 9.74 \\
\hline
\end{tabular}

\begin{tabular}{|c|c|c|c|c|c|c|c|c|c|}
\hline \multirow[b]{2}{*}{ APEE } & \multicolumn{9}{|c|}{ 7-day mean streamflow ( $\mathrm{ft}^{3} / \mathrm{s}$ ) that was equaled or exceeded the indicated percentage of time at APEE level } \\
\hline & 98 & 95 & 90 & 80 & 50 & 20 & 10 & 5 & 2 \\
\hline 1 & 633. & 936. & 1180 & 1460 & 2180 & 2930. & 3580. & 3910. & 4490. \\
\hline 2 & 532. & 760 . & 1020. & 1240 & 1650 & 2110 & 2730 & 3330. & 3600. \\
\hline 5 & 267. & 623. & 760. & 887. & 1220 & 1540. & 1660. & 2080 & 2180 \\
\hline 10 & 118. & 199. & 406. & 561. & 756. & 1040 . & 1160. & 1330. & 1520. \\
\hline 20 & 46.2 & 93.1 & 156. & 235 & 353. & 577. & 675. & 798. & 865. \\
\hline 25 & 27.6 & 67.6 & 125. & 156. & 256. & 423. & 554. & 630. & 780 \\
\hline 30 & 17.5 & 47.3 & 78.7 & 111. & 188. & 326. & 475. & 491. & 644. \\
\hline 40 & 8.66 & 22.8 & 33.6 & 52.1 & 112. & 213. & 289. & 315. & 364. \\
\hline 50 & 3.76 & 9.33 & 16.4 & 34.0 & 64.0 & 120. & 169. & 211. & 270 \\
\hline 60 & 1.41 & 4.15 & 7.09 & 18.1 & 37.6 & 75.1 & 106. & 137. & 152. \\
\hline 70 & 0.32 & 1.46 & 4.40 & 9.16 & 21.1 & 38.6 & 67.6 & 85.1 & 111. \\
\hline 75 & 0.09 & 1.02 & 2.90 & 6.54 & 15.4 & 30.4 & 52.4 & 71.7 & 92.3 \\
\hline 80 & 0.05 & 0.32 & 1.39 & 4.56 & 11.8 & 22.9 & 37.7 & 52.6 & 73.4 \\
\hline 90 & 0.00 & 0.00 & 0.22 & 0.89 & 3.91 & 13.9 & 22.3 & 26.0 & 38.3 \\
\hline 95 & 0.00 & 0.00 & 0.00 & 0.40 & 2.27 & 7.39 & 13.8 & 17.7 & 29.3 \\
\hline 98 & 0.00 & 0.00 & 0.00 & 0.22 & 1.50 & 5.00 & 10.6 & 14.6 & 21.8 \\
\hline 99 & 0.00 & 0.00 & 0.00 & 0.14 & 0.85 & 3.97 & 7.53 & 11.4 & 17.7 \\
\hline
\end{tabular}


03240000 Little Miami River near Oldtown, Ohio

Years analyzed $=56$

Climatic year range $=1953-2008$

[APEE, annual percentage equaled or exceeded; $\mathrm{ft}^{3} / \mathrm{s}$, cubic foot per second]

\begin{tabular}{|c|c|c|c|c|c|c|c|c|c|}
\hline \multirow[b]{2}{*}{ APEE } & \multicolumn{9}{|c|}{ Daily mean streamflow $\left(\mathrm{ft}^{3} / \mathrm{s}\right)$ that was equaled or exceeded the indicated percentage of time at APEE level } \\
\hline & 98 & 95 & 90 & 80 & 50 & 20 & 10 & 5 & 2 \\
\hline 1 & 183. & 391. & 431. & 552. & 970. & 1540. & 2030. & 2300 & 2800 \\
\hline 2 & 135. & 279. & 313. & 393. & 654. & 933. & 1200 & 1300 & 1350. \\
\hline 5 & 92.9 & 166. & 210 & 236. & 387. & 549. & 597. & 690. & 774. \\
\hline 10 & 74.2 & 126. & 135. & 160. & 233. & 349. & 409. & 433. & 505. \\
\hline 20 & 50.3 & 68.5 & 85.3 & 106. & 149. & 225 & 264. & 279. & 282. \\
\hline 25 & 40.0 & 58.4 & 67.8 & 86.5 & 129. & 190. & 227. & 240. & 249. \\
\hline 30 & 34.5 & 48.8 & 55.1 & 73.6 & 112. & 165. & 201. & 208. & 215. \\
\hline 40 & 25.4 & 30.8 & 38.4 & 54.5 & 86.1 & 129. & 152. & 166. & 177. \\
\hline 50 & 18.4 & 21.9 & 28.8 & 39.2 & 69.0 & 104. & 126. & 134. & 149. \\
\hline 60 & 14.3 & 17.7 & 21.8 & 27.8 & 48.7 & 81.8 & 96.6 & 118. & 134. \\
\hline 70 & 11.4 & 14.9 & 16.1 & 21.4 & 36.0 & 59.9 & 77.9 & 99.9 & 123. \\
\hline 75 & 11.3 & 13.0 & 14.9 & 19.0 & 29.5 & 53.4 & 68.8 & 84.3 & 115. \\
\hline 80 & 10.1 & 11.8 & 13.3 & 17.0 & 27.5 & 46.9 & 57.6 & 76.5 & 108. \\
\hline 90 & 8.43 & 9.11 & 10.9 & 13.8 & 20.8 & 36.0 & 45.1 & 57.2 & 91.5 \\
\hline 95 & 6.87 & 8.21 & 9.26 & 12.0 & 19.0 & 31.2 & 41.2 & 49.2 & 83.5 \\
\hline 98 & 5.91 & 7.49 & 8.09 & 10.4 & 17.0 & 27.3 & 36.2 & 41.8 & 72.7 \\
\hline 99 & 5.68 & 7.17 & 7.89 & 9.98 & 16.0 & 25.7 & 35.6 & 38.0 & 68.1 \\
\hline
\end{tabular}

\begin{tabular}{|c|c|c|c|c|c|c|c|c|c|}
\hline \multirow[b]{2}{*}{ APEE } & \multicolumn{9}{|c|}{ 7-day mean streamflow ( $\mathrm{ft}^{3} / \mathrm{s}$ ) that was equaled or exceeded the indicated percentage of time at APEE level } \\
\hline & 98 & 95 & 90 & 80 & 50 & 20 & 10 & 5 & 2 \\
\hline 1 & 145. & 292. & 328. & 406. & 729. & 1140. & 1560. & 1710 & 1810 \\
\hline 2 & 129. & 228 & 261. & 341. & 511. & 805. & 1070 & 1160. & 1280 \\
\hline 5 & 92.2 & 171. & 191. & 245 & 393. & 538. & 622 & 769. & 789. \\
\hline 10 & 73.2 & 127. & 140 & 175. & 260 & 379. & 431. & 476. & 519. \\
\hline 20 & 50.7 & 67.0 & 87.3 & 109. & 163. & 260 & 294. & 309. & 318. \\
\hline 25 & 39.7 & 57.3 & 64.0 & 93.4 & 138. & 223. & 248. & 272. & 286. \\
\hline 30 & 34.0 & 46.9 & 56.3 & 76.6 & 120. & 193. & 218. & 234. & 256. \\
\hline 40 & 26.3 & 30.9 & 40.4 & 55.9 & 92.4 & 140. & 169. & 183. & 215. \\
\hline 50 & 20.1 & 22.0 & 30.2 & 39.4 & 70.7 & 109. & 132. & 154. & 164. \\
\hline 60 & 13.2 & 17.3 & 22.7 & 27.8 & 52.1 & 87.4 & 110. & 129. & 150. \\
\hline 70 & 11.7 & 14.4 & 16.2 & 21.5 & 37.9 & 66.4 & 84.5 & 104. & 137. \\
\hline 75 & 11.2 & 13.2 & 14.6 & 19.6 & 30.7 & 57.6 & 75.4 & 90.2 & 130. \\
\hline 80 & 10.6 & 11.8 & 13.2 & 17.2 & 27.9 & 49.9 & 61.3 & 81.8 & 122. \\
\hline 90 & 8.74 & 9.48 & 10.9 & 13.5 & 21.4 & 36.4 & 45.7 & 61.9 & 103. \\
\hline 95 & 7.33 & 8.36 & 9.48 & 12.0 & 18.9 & 33.0 & 41.7 & 51.5 & 94.1 \\
\hline 98 & 6.30 & 7.60 & 8.80 & 11.1 & 17.9 & 28.1 & 39.6 & 45.2 & 84.6 \\
\hline 99 & 5.85 & 7.47 & 8.20 & 10.6 & 17.4 & 27.2 & 38.3 & 43.3 & 73.1 \\
\hline
\end{tabular}




\section{Massies Creek at Wilberforce, Ohio}

Years analyzed $=56$

Climatic year range $=1953-2008$

[APEE, annual percentage equaled or exceeded; $\mathrm{ft}^{3} / \mathrm{s}$, cubic foot per second]

\begin{tabular}{|c|c|c|c|c|c|c|c|c|c|}
\hline \multirow[b]{2}{*}{ APEE } & \multicolumn{9}{|c|}{ Daily mean streamflow (ft $\left.{ }^{3} / \mathrm{s}\right)$ that was equaled or exceeded the indicated percentage of time at APEE level } \\
\hline & 98 & 95 & 90 & 80 & 50 & 20 & 10 & 5 & 2 \\
\hline 1 & 95.2 & 210. & 287. & 357. & 583. & 904. & 1040. & 1130 & 1720 \\
\hline 2 & 68.4 & 146. & 217. & 251. & 379. & 599. & 699. & 780 . & 884. \\
\hline 5 & 41.9 & 90.8 & 128. & 152. & 236. & 336. & 389. & 427. & 484. \\
\hline 10 & 30.1 & 56.8 & 79.3 & 93.9 & 144. & 209. & 241. & 261. & 301. \\
\hline 20 & 18.5 & 35.6 & 41.0 & 52.3 & 79.6 & 118. & 140. & 158. & 173. \\
\hline 25 & 14.5 & 24.9 & 30.5 & 43.1 & 66.3 & 96.9 & 112. & 130. & 138. \\
\hline 30 & 11.6 & 20.0 & 21.7 & 34.0 & 53.5 & 81.4 & 96.1 & 109. & 113. \\
\hline 40 & 6.70 & 11.2 & 16.4 & 24.4 & 40.6 & 62.0 & 72.5 & 77.3 & 91.3 \\
\hline 50 & 3.56 & 7.42 & 11.4 & 16.8 & 30.8 & 49.2 & 54.9 & 65.2 & 72.9 \\
\hline 60 & 2.25 & 5.16 & 6.81 & 11.4 & 21.0 & 35.2 & 42.3 & 54.3 & 57.7 \\
\hline 70 & 2.02 & 3.91 & 4.67 & 7.37 & 14.0 & 25.6 & 34.6 & 42.1 & 49.9 \\
\hline 75 & 1.98 & 3.10 & 4.17 & 6.25 & 11.5 & 21.2 & 30.6 & 38.1 & 46.7 \\
\hline 80 & 1.45 & 2.62 & 3.75 & 5.22 & 8.49 & 18.0 & 25.0 & 30.9 & 42.2 \\
\hline 90 & 0.81 & 1.78 & 2.64 & 3.62 & 5.96 & 10.6 & 16.3 & 19.3 & 33.8 \\
\hline 95 & 0.57 & 1.34 & 2.10 & 2.84 & 4.65 & 8.38 & 12.1 & 17.9 & 29.9 \\
\hline 98 & 0.48 & 1.11 & 1.69 & 2.40 & 4.35 & 7.29 & 10.0 & 15.3 & 22.5 \\
\hline 99 & 0.39 & 0.99 & 1.47 & 2.11 & 4.13 & 6.83 & 9.20 & 15.0 & 18.2 \\
\hline
\end{tabular}

\begin{tabular}{|c|c|c|c|c|c|c|c|c|c|}
\hline \multirow[b]{2}{*}{ APEE } & \multicolumn{9}{|c|}{ 7-day mean streamflow $\left(\mathrm{ft}^{3} / \mathrm{s}\right)$ that was equaled or exceeded the indicated percentage of time at APEE level } \\
\hline & 98 & 95 & 90 & 80 & 50 & 20 & 10 & 5 & 2 \\
\hline 1 & 75.3 & 161. & 201. & 262. & 426. & 605. & 834. & 963. & 1060 \\
\hline 2 & 62.3 & 121. & 171. & 216. & 305. & 490 & 522. & 641. & 697. \\
\hline 5 & 43.9 & 82.1 & 116. & 143. & 226. & 325. & 372. & 409. & 520. \\
\hline 10 & 31.1 & 64.9 & 76.4 & 96.5 & 157. & 226. & 271. & 282 & 300. \\
\hline 20 & 17.7 & 35.4 & 41.4 & 56.4 & 94.2 & 135. & 166. & 175. & 208. \\
\hline 25 & 14.2 & 25.4 & 30.3 & 44.4 & 74.9 & 115. & 138. & 154. & 177. \\
\hline 30 & 11.5 & 21.2 & 24.1 & 35.7 & 60.6 & 95.0 & 118. & 133. & 144. \\
\hline 40 & 7.50 & 12.2 & 16.8 & 26.1 & 45.8 & 70.6 & 87.0 & 90.6 & 111. \\
\hline 50 & 3.19 & 7.13 & 12.4 & 16.7 & 32.6 & 52.7 & 61.1 & 72.3 & 85.2 \\
\hline 60 & 2.51 & 5.45 & 7.11 & 11.8 & 21.7 & 39.1 & 47.0 & 58.9 & 67.2 \\
\hline 70 & 2.13 & 3.91 & 4.58 & 7.36 & 14.8 & 28.9 & 37.8 & 47.8 & 56.1 \\
\hline 75 & 2.01 & 3.21 & 4.18 & 6.49 & 11.7 & 23.1 & 33.5 & 42.1 & 52.6 \\
\hline 80 & 1.65 & 2.83 & 3.93 & 5.25 & 9.00 & 19.6 & 27.9 & 36.7 & 50.2 \\
\hline 90 & 0.97 & 1.78 & 2.82 & 3.91 & 6.61 & 11.9 & 17.0 & 21.6 & 39.9 \\
\hline 95 & 0.71 & 1.42 & 2.29 & 3.17 & 4.92 & 9.02 & 12.6 & 17.8 & 35.1 \\
\hline 98 & 0.50 & 1.19 & 1.89 & 2.59 & 4.56 & 7.97 & 11.3 & 17.0 & 28.1 \\
\hline 99 & 0.43 & 1.16 & 1.67 & 2.31 & 4.40 & 7.46 & 10.4 & 15.9 & 25.5 \\
\hline
\end{tabular}


03260700 Bokengehalas Creek near De Graff, Ohio

Years analyzed $=33$

Climatic year range $=1958-90$

[APEE, annual percentage equaled or exceeded; $\mathrm{ft}^{3} / \mathrm{s}$, cubic foot per second]

\begin{tabular}{|c|c|c|c|c|c|c|c|c|c|}
\hline \multirow[b]{2}{*}{ APEE } & \multicolumn{9}{|c|}{ Daily mean streamflow $\left(\mathrm{ft}^{3} / \mathrm{s}\right)$ that was equaled or exceeded the indicated percentage of time at APEE level } \\
\hline & 95 & 90 & 80 & 70 & 50 & 30 & 20 & 10 & 5 \\
\hline 1 & 109. & 151. & 176. & 217. & 277. & 324. & 356. & 390. & 507. \\
\hline 2 & 78.5 & 92.8 & 108. & 138. & 178. & 204. & 246. & 265. & 297. \\
\hline 5 & 42.8 & 59.2 & 68.5 & 78.8 & 105. & 125 & 137. & 170 . & 196. \\
\hline 10 & 30.4 & 34.5 & 44.0 & 53.8 & 66.8 & 82.6 & 89.9 & 117. & 130. \\
\hline 20 & 18.9 & 19.4 & 25.8 & 32.2 & 43.0 & 49.4 & 60.0 & 69.9 & 84.1 \\
\hline 25 & 14.1 & 16.4 & 21.4 & 27.4 & 37.5 & 41.6 & 51.8 & 61.5 & 71.9 \\
\hline 30 & 12.1 & 13.8 & 19.0 & 23.4 & 33.0 & 37.5 & 45.5 & 52.6 & 63.2 \\
\hline 40 & 8.68 & 10.0 & 14.0 & 16.2 & 23.6 & 29.0 & 36.4 & 43.0 & 50.3 \\
\hline 50 & 6.84 & 8.00 & 10.7 & 12.2 & 18.0 & 23.6 & 27.4 & 35.8 & 41.3 \\
\hline 60 & 5.61 & 6.60 & 7.97 & 9.82 & 15.0 & 18.8 & 22.0 & 28.8 & 31.5 \\
\hline 70 & 5.21 & 5.76 & 7.10 & 7.90 & 12.0 & 14.8 & 18.4 & 21.6 & 25.8 \\
\hline 75 & 4.70 & 5.60 & 6.66 & 7.56 & 11.0 & 13.0 & 16.6 & 20.0 & 22.6 \\
\hline 80 & 4.67 & 5.20 & 6.14 & 7.16 & 9.60 & 11.8 & 14.2 & 18.0 & 20.5 \\
\hline 90 & 4.11 & 4.57 & 5.60 & 6.00 & 7.96 & 9.41 & 11.2 & 13.8 & 14.6 \\
\hline 95 & 3.88 & 4.34 & 5.01 & 5.34 & 7.20 & 8.92 & 10.1 & 12.0 & 13.6 \\
\hline 98 & 3.41 & 4.00 & 4.29 & 4.72 & 6.40 & 7.98 & 9.56 & 10.2 & 13.3 \\
\hline 99 & 3.22 & 3.72 & 3.89 & 4.36 & 6.40 & 7.73 & 8.70 & 9.93 & 12.6 \\
\hline
\end{tabular}

\begin{tabular}{|c|c|c|c|c|c|c|c|c|c|}
\hline \multirow[b]{2}{*}{ APEE } & \multicolumn{9}{|c|}{ 7-day mean streamflow ( $\mathrm{ft}^{3} / \mathrm{s}$ ) that was equaled or exceeded the indicated percentage of time at APEE level } \\
\hline & 95 & 90 & 80 & 70 & 50 & 30 & 20 & 10 & 5 \\
\hline 1 & 68.0 & 84.6 & 109. & 157. & 180. & 241. & 259. & 295 & 364. \\
\hline 2 & 58.8 & 78.4 & 95.5 & 129. & 145. & 170 & 183. & 234. & 248 \\
\hline 5 & 43.4 & 53.1 & 67.7 & 82.1 & 105. & 124 & 133. & 168. & 186. \\
\hline 10 & 29.5 & 32.8 & 46.3 & 50.0 & 69.3 & 90.1 & 95.4 & 114. & 136. \\
\hline 20 & 16.9 & 20.3 & 27.3 & 33.6 & 47.9 & 55.7 & 63.2 & 85.4 & 93.6 \\
\hline 25 & 14.6 & 18.2 & 22.7 & 29.9 & 40.9 & 47.9 & 55.6 & 71.6 & 78.5 \\
\hline 30 & 12.5 & 14.5 & 20.1 & 25.4 & 35.7 & 40.0 & 50.0 & 59.9 & 69.8 \\
\hline 40 & 8.87 & 10.1 & 14.9 & 17.9 & 23.4 & 32.5 & 37.8 & 49.8 & 56.6 \\
\hline 50 & 6.67 & 8.12 & 10.8 & 13.5 & 18.7 & 25.9 & 31.3 & 39.4 & 48.0 \\
\hline 60 & 5.90 & 6.69 & 8.17 & 10.0 & 15.7 & 20.1 & 23.9 & 30.5 & 34.6 \\
\hline 70 & 5.35 & 6.02 & 7.40 & 8.39 & 12.6 & 15.9 & 20.5 & 23.1 & 29.0 \\
\hline 75 & 5.09 & 5.74 & 6.95 & 7.81 & 11.4 & 14.3 & 17.0 & 20.9 & 23.4 \\
\hline 80 & 4.80 & 5.53 & 6.49 & 7.28 & 9.86 & 12.5 & 15.2 & 19.3 & 21.4 \\
\hline 90 & 4.26 & 4.98 & 5.65 & 6.19 & 8.44 & 10.1 & 12.7 & 15.0 & 15.7 \\
\hline 95 & 3.96 & 4.43 & 5.30 & 5.55 & 7.29 & 9.55 & 11.1 & 12.8 & 14.4 \\
\hline 98 & 3.70 & 4.26 & 4.77 & 5.23 & 6.74 & 8.23 & 9.83 & 11.7 & 13.7 \\
\hline 99 & 3.47 & 3.93 & 4.40 & 5.01 & 6.57 & 8.13 & 9.60 & 10.5 & 13.4 \\
\hline
\end{tabular}




\section{Greenville Creek near Bradford, Ohio}

Years analyzed $=75$

Climatic year range $=1931-2008$

[APEE, annual percentage equaled or exceeded; $\mathrm{ft}^{3} / \mathrm{s}$, cubic foot per second]

\begin{tabular}{|c|c|c|c|c|c|c|c|c|c|}
\hline \multirow[b]{2}{*}{ APEE } & \multicolumn{9}{|c|}{ Daily mean streamflow $\left(\mathrm{ft}^{3} / \mathrm{s}\right)$ that was equaled or exceeded the indicated percentage of time at APEE level } \\
\hline & 98 & 95 & 90 & 80 & 50 & 20 & 10 & 5 & 2 \\
\hline 1 & 321. & 488. & 855. & 1190. & 1840. & 2490. & 3170. & 3390 . & 4000 \\
\hline 2 & 239. & 347. & 542. & 775. & 1310. & 1760 . & 2120 & 2590 . & 2720 \\
\hline 5 & 145. & 234. & 279. & 420 . & 732. & 1030. & 1160 & 1300 . & 1400 \\
\hline 10 & 98.9 & 149. & 176. & 216. & 426. & 569. & 677. & 719. & 748. \\
\hline 20 & 58.3 & 84.2 & 101. & 121. & 211. & 314. & 372. & 399. & 456. \\
\hline 25 & 45.6 & 68.6 & 82.6 & 101. & 175. & 253. & 295. & 329. & 374. \\
\hline 30 & 35.9 & 57.9 & 67.8 & 84.1 & 147. & 211. & 250 & 272. & 303. \\
\hline 40 & 21.6 & 37.2 & 50.2 & 57.1 & 105. & 154. & 182. & 202. & 221. \\
\hline 50 & 16.0 & 27.2 & 34.6 & 44.6 & 76.0 & 116. & 141. & 154. & 182. \\
\hline 60 & 14.7 & 21.7 & 28.2 & 35.5 & 58.0 & 91.9 & 105. & 118. & 152. \\
\hline 70 & 13.5 & 18.4 & 22.4 & 27.0 & 42.1 & 68.0 & 87.0 & 96.0 & 128. \\
\hline 75 & 13.5 & 15.0 & 20.8 & 24.0 & 38.0 & 58.6 & 70.0 & 87.2 & 118. \\
\hline 80 & 12.0 & 13.8 & 18.1 & 22.0 & 34.0 & 49.0 & 59.8 & 79.3 & 111. \\
\hline 90 & 9.85 & 12.6 & 14.0 & 17.0 & 27.0 & 37.6 & 46.6 & 64.5 & 89.0 \\
\hline 95 & 8.62 & 9.63 & 11.7 & 14.0 & 23.3 & 31.3 & 38.4 & 58.3 & 70.9 \\
\hline 98 & 7.18 & 8.25 & 10.7 & 12.1 & 21.0 & 28.9 & 33.6 & 51.1 & 58.1 \\
\hline 99 & 6.66 & 7.67 & 9.19 & 11.1 & 21.0 & 27.5 & 32.3 & 48.6 & 55.0 \\
\hline
\end{tabular}

\begin{tabular}{|c|c|c|c|c|c|c|c|c|c|}
\hline \multirow[b]{2}{*}{ APEE } & \multicolumn{9}{|c|}{ 7-day mean streamflow $\left(\mathrm{ft}^{3} / \mathrm{s}\right)$ that was equaled or exceeded the indicated percentage of time at APEE level } \\
\hline & 98 & 95 & 90 & 80 & 50 & 20 & 10 & 5 & 2 \\
\hline 1 & 213. & 396. & 508. & 833. & 1330. & 1720. & 2040. & 2670 & 2900 \\
\hline 2 & 197. & 324. & 403. & 693. & 966. & 1330. & 1570 & 1910. & 2380 \\
\hline 5 & 152. & 227. & 290. & 422. & 674. & 930. & 1050 & 1280. & 1390. \\
\hline 10 & 98.3 & 145. & 172. & 236. & 455. & 626. & 723. & 769. & 866. \\
\hline 20 & 53.5 & 86.7 & 101. & 137. & 262. & 363. & 450. & 493. & 524. \\
\hline 25 & 44.2 & 70.9 & 88.4 & 107. & 198. & 301. & 369. & 402. & 440. \\
\hline 30 & 35.2 & 62.1 & 76.3 & 85.2 & 161. & 239. & 309. & 328. & 369. \\
\hline 40 & 21.2 & 37.3 & 52.2 & 63.9 & 124. & 181. & 220. & 242 & 290. \\
\hline 50 & 16.5 & 26.9 & 34.9 & 49.0 & 88.4 & 136. & 165. & 187. & 217. \\
\hline 60 & 15.4 & 21.3 & 27.9 & 35.5 & 60.1 & 104. & 123. & 138. & 179. \\
\hline 70 & 14.4 & 18.9 & 22.9 & 28.4 & 45.5 & 77.9 & 93.3 & 109. & 149. \\
\hline 75 & 13.9 & 15.3 & 21.2 & 25.1 & 38.4 & 66.4 & 77.3 & 92.1 & 131. \\
\hline 80 & 13.0 & 13.9 & 19.4 & 22.8 & 34.9 & 55.0 & 65.3 & 84.6 & 122. \\
\hline 90 & 10.3 & 12.7 & 14.4 & 17.7 & 28.1 & 39.3 & 47.0 & 68.4 & 101. \\
\hline 95 & 9.00 & 10.3 & 11.9 & 14.4 & 24.2 & 34.4 & 41.1 & 61.6 & 84.9 \\
\hline 98 & 7.97 & 8.79 & 11.1 & 13.8 & 22.1 & 30.2 & 38.3 & 57.9 & 64.4 \\
\hline 99 & 7.14 & 8.20 & 10.5 & 13.0 & 21.7 & 29.3 & 35.5 & 54.1 & 60.7 \\
\hline
\end{tabular}




\section{Stillwater River at Pleasant Hill, Ohio}

Years analyzed $=85$

Climatic year range $=1917-2008$

[APEE, annual percentage equaled or exceeded; $\mathrm{ft}^{3} / \mathrm{s}$, cubic foot per second]

\begin{tabular}{|c|c|c|c|c|c|c|c|c|c|}
\hline \multirow[b]{2}{*}{ APEE } & \multicolumn{9}{|c|}{ Daily mean streamflow $\left(\mathrm{ft}^{3} / \mathrm{s}\right)$ that was equaled or exceeded the indicated percentage of time at APEE level } \\
\hline & 98 & 95 & 90 & 80 & 50 & 20 & 10 & 5 & 2 \\
\hline 1 & 1180. & 2460. & 3000. & 3820. & 5500. & 6590. & 8100. & 9890. & 12100. \\
\hline 2 & 712. & 1380 . & 1840. & 2400 & 3810. & 5120. & 6270 & 7040 & 8020. \\
\hline 5 & 457. & 648. & 847. & 1190. & 1940. & 2870 . & 3310 . & 3560 . & 3800. \\
\hline 10 & 288 & 330 . & 456. & 554. & 1040 . & 1450. & 1730 . & 2090 . & 2180 \\
\hline 20 & 151. & 167. & 200. & 267. & 544. & 713. & 899. & 1030. & 1100 \\
\hline 25 & 120. & 140. & 169. & 206. & 404. & 567. & 694. & 781. & 846. \\
\hline 30 & 89.6 & 110. & 145. & 167. & 329. & 451. & 538. & 609. & 680. \\
\hline 40 & 48.4 & 72.0 & 90.2 & 112. & 224. & 323. & 366. & 423. & 479. \\
\hline 50 & 36.9 & 47.8 & 63.0 & 86.2 & 152. & 228 & 267. & 328. & 353. \\
\hline 60 & 26.3 & 34.9 & 47.2 & 65.3 & 106. & 171. & 209. & 236. & 289. \\
\hline 70 & 21.4 & 29.3 & 37.9 & 48.2 & 76.6 & 126. & 164. & 182. & 232. \\
\hline 75 & 20.2 & 25.9 & 34.2 & 39.2 & 67.8 & 108. & 142. & 157. & 210 \\
\hline 80 & 18.6 & 24.0 & 29.1 & 34.6 & 57.2 & 91.3 & 113. & 141. & 190. \\
\hline 90 & 12.9 & 17.3 & 19.0 & 24.1 & 42.0 & 62.2 & 80.2 & 107. & 149. \\
\hline 95 & 10.8 & 12.5 & 14.2 & 18.0 & 34.0 & 56.0 & 66.8 & 85.8 & 125. \\
\hline 98 & 8.72 & 9.46 & 10.9 & 14.5 & 28.0 & 44.8 & 55.0 & 79.0 & 99.0 \\
\hline 99 & 7.63 & 8.76 & 9.98 & 12.2 & 27.3 & 42.8 & 53.5 & 73.6 & 86.2 \\
\hline
\end{tabular}

\begin{tabular}{|c|c|c|c|c|c|c|c|c|c|}
\hline \multirow[b]{2}{*}{ APEE } & \multicolumn{9}{|c|}{ 7-day mean streamflow ( $\mathrm{ft}^{3} / \mathrm{s}$ ) that was equaled or exceeded the indicated percentage of time at APEE level } \\
\hline & 98 & 95 & 90 & 80 & 50 & 20 & 10 & 5 & 2 \\
\hline 1 & 943. & 1260. & 1650. & 2350. & 3790. & 4870. & 5250. & 6040 & 8040 \\
\hline 2 & 773. & 1040 & 1240 & 2020 . & 2680 & 3670 . & 4150 & 4830 & 6590. \\
\hline 5 & 402. & 630. & 932. & 1260. & 1830 & 2620. & 2880 & 3510 & 3940. \\
\hline 10 & 305. & 361. & 475 & 666. & 1220. & 1700 . & 1950. & 2210 & 2450 \\
\hline 20 & 149. & 180. & 221. & 327. & 656. & 931. & 1160. & 1310 & 1410. \\
\hline 25 & 111. & 140. & 185. & 233. & 486. & 735. & 902. & 1070 & 1080. \\
\hline 30 & 93.8 & 118. & 147. & 188. & 402. & 585. & 720 . & 874. & 962. \\
\hline 40 & 48.0 & 73.1 & 96.5 & 128. & 260. & 408. & 519. & 596. & 709. \\
\hline 50 & 34.5 & 46.9 & 64.3 & 90.7 & 170 & 287. & 340. & 421. & 457. \\
\hline 60 & 25.2 & 35.9 & 47.9 & 66.4 & 116. & 203. & 245 & 290. & 349. \\
\hline 70 & 21.7 & 30.3 & 37.2 & 48.8 & 84.3 & 153. & 186. & 217. & 273. \\
\hline 75 & 20.7 & 26.1 & 33.6 & 41.0 & 70.0 & 124. & 159. & 177. & 240 \\
\hline 80 & 18.7 & 24.8 & 27.9 & 36.1 & 57.9 & 100 & 128. & 161. & 222. \\
\hline 90 & 15.1 & 18.7 & 20.6 & 24.9 & 44.3 & 69.6 & 91.4 & 111. & 175. \\
\hline 95 & 12.7 & 15.4 & 16.7 & 20.3 & 37.0 & 60.5 & 71.6 & 92.5 & 153. \\
\hline 98 & 9.73 & 11.1 & 14.1 & 16.7 & 32.1 & 49.9 & 59.5 & 85.0 & 122. \\
\hline 99 & 8.65 & 9.71 & 12.8 & 16.3 & 30.0 & 47.3 & 56.1 & 81.5 & 97.7 \\
\hline
\end{tabular}


03266500 Mad River at Zanesfield, Ohio

Years analyzed $=32$

Climatic year range $=1947-78$

[APEE, annual percentage equaled or exceeded; $\mathrm{ft}^{3} / \mathrm{s}$, cubic foot per second]

\begin{tabular}{|c|c|c|c|c|c|c|c|c|c|}
\hline \multirow[b]{2}{*}{ APEE } & \multicolumn{9}{|c|}{ Daily mean streamflow $\left(\mathrm{ft}^{3} / \mathrm{s}\right)$ that was equaled or exceeded the indicated percentage of time at APEE level } \\
\hline & 95 & 90 & 80 & 70 & 50 & 30 & 20 & 10 & 5 \\
\hline 1 & 28.1 & 43.0 & 45.3 & 60.0 & 70.2 & 88.5 & 95.1 & 106. & 127. \\
\hline 2 & 22.6 & 31.5 & 34.4 & 37.8 & 45.3 & 59.2 & 65.9 & 70.6 & 82.6 \\
\hline 5 & 11.1 & 12.6 & 15.9 & 17.9 & 24.0 & 30.2 & 35.0 & 45.0 & 52.9 \\
\hline 10 & 6.36 & 7.72 & 9.54 & 12.2 & 15.0 & 19.5 & 20.4 & 22.8 & 28.8 \\
\hline 20 & 4.13 & 4.51 & 5.64 & 7.00 & 9.54 & 10.3 & 14.0 & 14.7 & 16.7 \\
\hline 25 & 3.36 & 3.67 & 4.78 & 5.66 & 7.57 & 9.56 & 12.4 & 13.0 & 14.4 \\
\hline 30 & 2.52 & 2.93 & 3.84 & 4.79 & 6.30 & 8.22 & 11.0 & 12.0 & 13.0 \\
\hline 40 & 1.83 & 2.10 & 2.58 & 3.49 & 4.70 & 6.71 & 8.57 & 9.41 & 10.3 \\
\hline 50 & 1.43 & 1.53 & 1.86 & 2.58 & 3.60 & 5.10 & 5.74 & 6.72 & 7.89 \\
\hline 60 & 1.23 & 1.30 & 1.56 & 1.92 & 2.75 & 3.37 & 4.30 & 5.07 & 6.16 \\
\hline 70 & 0.96 & 1.10 & 1.26 & 1.77 & 2.09 & 2.62 & 3.18 & 4.10 & 4.96 \\
\hline 75 & 0.88 & 1.03 & 1.26 & 1.59 & 1.80 & 2.31 & 2.68 & 3.78 & 4.68 \\
\hline 80 & 0.84 & 1.00 & 1.11 & 1.49 & 1.60 & 2.20 & 2.42 & 3.39 & 4.23 \\
\hline 90 & 0.79 & 0.83 & 1.00 & 1.19 & 1.40 & 1.90 & 2.04 & 2.76 & 3.37 \\
\hline 95 & 0.71 & 0.80 & 0.90 & 1.10 & 1.30 & 1.80 & 1.96 & 2.35 & 2.87 \\
\hline 98 & 0.67 & 0.73 & 0.87 & 1.00 & 1.20 & 1.61 & 1.80 & 1.92 & 2.74 \\
\hline 99 & 0.64 & 0.73 & 0.82 & 0.90 & 1.15 & 1.51 & 1.76 & 1.88 & 2.58 \\
\hline
\end{tabular}

\begin{tabular}{|c|c|c|c|c|c|c|c|c|c|}
\hline \multirow[b]{2}{*}{ APEE } & \multicolumn{9}{|c|}{ 7-day mean streamflow (ft $\mathbf{3} / \mathrm{s})$ that was equaled or exceeded the indicated percentage of time at APEE level } \\
\hline & 95 & 90 & 80 & 70 & 50 & 30 & 20 & 10 & 5 \\
\hline 1 & 19.7 & 27.9 & 30.5 & 34.4 & 44.8 & 57.2 & 61.7 & 72.4 & 101. \\
\hline 2 & 14.2 & 20.4 & 26.6 & 28.5 & 32.2 & 42.3 & 44.5 & 55.4 & 63.3 \\
\hline 5 & 11.1 & 16.6 & 18.6 & 20.0 & 25.0 & 29.8 & 33.4 & 42.2 & 46.5 \\
\hline 10 & 6.34 & 6.80 & 11.0 & 13.6 & 15.5 & 19.7 & 24.7 & 27.8 & 31.5 \\
\hline 20 & 4.18 & 4.74 & 5.98 & 7.84 & 10.5 & 13.1 & 16.0 & 19.0 & 22.0 \\
\hline 25 & 3.54 & 4.05 & 5.18 & 6.22 & 8.90 & 10.9 & 14.2 & 15.9 & 18.7 \\
\hline 30 & 2.63 & 3.29 & 3.94 & 5.21 & 7.66 & 9.15 & 12.2 & 13.5 & 15.5 \\
\hline 40 & 1.82 & 2.16 & 2.49 & 3.92 & 5.10 & 7.23 & 9.16 & 10.1 & 12.2 \\
\hline 50 & 1.45 & 1.61 & 1.97 & 2.60 & 3.91 & 5.51 & 6.43 & 7.03 & 9.02 \\
\hline 60 & 1.19 & 1.32 & 1.66 & 2.06 & 2.76 & 3.56 & 4.89 & 5.63 & 7.10 \\
\hline 70 & 0.98 & 1.15 & 1.33 & 1.73 & 2.19 & 2.80 & 3.39 & 4.52 & 5.50 \\
\hline 75 & 0.94 & 1.09 & 1.25 & 1.64 & 1.96 & 2.48 & 2.98 & 3.99 & 5.02 \\
\hline 80 & 0.87 & 1.03 & 1.19 & 1.52 & 1.67 & 2.21 & 2.63 & 3.57 & 4.50 \\
\hline 90 & 0.79 & 0.89 & 1.07 & 1.22 & 1.46 & 1.93 & 2.13 & 2.98 & 3.63 \\
\hline 95 & 0.73 & 0.82 & 0.94 & 1.13 & 1.31 & 1.87 & 2.01 & 2.54 & 3.06 \\
\hline 98 & 0.69 & 0.79 & 0.90 & 1.04 & 1.24 & 1.78 & 1.86 & 2.06 & 2.84 \\
\hline 99 & 0.64 & 0.72 & 0.86 & 0.99 & 1.21 & 1.68 & 1.80 & 2.03 & 2.80 \\
\hline
\end{tabular}




\section{6

03267000 Mad River near Urbana, Ohio

Years analyzed $=69$

Climatic year range $=1926-2008$

[APEE, annual percentage equaled or exceeded; $\mathrm{ft}^{3} / \mathrm{s}$, cubic foot per second]

\begin{tabular}{|c|c|c|c|c|c|c|c|c|c|}
\hline \multirow[b]{2}{*}{ APEE } & \multicolumn{9}{|c|}{ Daily mean streamflow $\left(\mathrm{ft}^{3} / \mathrm{s}\right)$ that was equaled or exceeded the indicated percentage of time at APEE level } \\
\hline & 98 & 95 & 90 & 80 & 50 & 20 & 10 & 5 & 2 \\
\hline 1 & 233. & 315. & 410. & 488. & 808. & 1260 & 1440 & 1520 & 2100. \\
\hline 2 & 201. & 233. & 259. & 353. & 550. & 792. & 941. & 1090. & 1150. \\
\hline 5 & 155. & 160. & 178. & 233. & 345. & 497. & 579. & 629. & 666. \\
\hline 10 & 111. & 121. & 139. & 164. & 241. & 362. & 409. & 444. & 468. \\
\hline 20 & 79.4 & 88.0 & 107. & 127. & 183. & 275 & 298. & 309. & 326. \\
\hline 25 & 68.0 & 82.3 & 94.0 & 113. & 173. & 240 & 267. & 288 & 295. \\
\hline 30 & 58.0 & 74.5 & 85.0 & 98.8 & 158. & 219. & 246. & 265. & 271. \\
\hline 40 & 48.9 & 61.8 & 71.0 & 79.0 & 137. & 184. & 207. & 231. & 245. \\
\hline 50 & 42.6 & 53.0 & 58.0 & 70.5 & 120. & 160. & 182. & 197. & 221. \\
\hline 60 & 37.6 & 46.5 & 52.0 & 62.0 & 104. & 139. & 164. & 171. & 202. \\
\hline 70 & 35.3 & 44.0 & 46.8 & 57.0 & 90.0 & 121. & 144. & 153. & 189. \\
\hline 75 & 34.8 & 43.0 & 44.0 & 54.5 & 81.0 & 117. & 133. & 144. & 181. \\
\hline 80 & 33.8 & 41.2 & 43.0 & 52.0 & 78.0 & 106. & 123. & 134. & 173. \\
\hline 90 & 32.6 & 38.6 & 40.0 & 44.6 & 70.0 & 98.7 & 106. & 122. & 154. \\
\hline 95 & 30.4 & 35.3 & 37.3 & 41.0 & 67.0 & 90.0 & 98.3 & 116. & 149. \\
\hline 98 & 26.0 & 33.2 & 35.0 & 39.0 & 62.7 & 85.0 & 95.3 & 108. & 141. \\
\hline 99 & 25.4 & 31.5 & 34.7 & 39.0 & 60.7 & 83.0 & 94.0 & 103. & 138. \\
\hline
\end{tabular}

\begin{tabular}{|c|c|c|c|c|c|c|c|c|c|}
\hline \multirow[b]{2}{*}{ APEE } & \multicolumn{9}{|c|}{ 7-day mean streamflow $\left(\mathrm{ft}^{3} / \mathrm{s}\right)$ that was equaled or exceeded the indicated percentage of time at APEE level } \\
\hline & 98 & 95 & 90 & 80 & 50 & 20 & 10 & 5 & 2 \\
\hline 1 & 182. & 204. & 289. & 410 & 562. & 885. & 1020. & 1230 & 1540. \\
\hline 5 & 143. & 167. & 183. & 241. & 350. & 514. & 599. & 660. & 759. \\
\hline 10 & 107. & 120. & 140. & 177. & 274. & 397. & 438. & 449. & 500. \\
\hline 30 & 56.9 & 77.9 & 86.6 & 101. & 170 & 236. & 265. & 278. & 292. \\
\hline 40 & 50.0 & 63.8 & 72.1 & 80.7 & 140. & 195. & 221. & 238 & 260. \\
\hline 50 & 42.2 & 53.7 & 58.0 & 70.0 & 126. & 166. & 189. & 210 & 234. \\
\hline 60 & 37.4 & 46.9 & 52.9 & 62.9 & 106. & 148. & 167. & 180. & 211. \\
\hline 90 & 33.0 & 39.1 & 40.3 & 44.9 & 70.5 & 100 & 107. & 123. & 161. \\
\hline 95 & 31.1 & 36.6 & 38.3 & 42.1 & 67.4 & 93.7 & 101. & 118. & 154. \\
\hline 98 & 26.3 & 33.9 & 36.3 & 40.0 & 64.0 & 88.7 & 97.7 & 112. & 148. \\
\hline 99 & 25.9 & 32.4 & 36.0 & 38.9 & 62.3 & 87.0 & 97.3 & 105. & 144. \\
\hline
\end{tabular}


03270800 Wolf Creek at Trotwood, Ohio

Years analyzed $=23$

Climatic year range $=1963-85$

[APEE, annual percentage equaled or exceeded; $\mathrm{ft}^{3} / \mathrm{s}$, cubic foot per second]

\begin{tabular}{|c|c|c|c|c|c|c|c|c|c|}
\hline \multirow[b]{2}{*}{ APEE } & \multicolumn{9}{|c|}{ Daily mean streamflow (ft $3 / s)$ that was equaled or exceeded the indicated percentage of time at APEE level } \\
\hline & 95 & 90 & 80 & 70 & 50 & 30 & 20 & 10 & 5 \\
\hline 1 & 115. & 161. & 223. & 243. & 307. & 386. & 441. & 460. & 463. \\
\hline 2 & 51.4 & 82.1 & 128. & 157. & 205. & 222 & 261. & 349. & 378. \\
\hline 5 & 28.9 & 40.8 & 70.2 & 77.8 & 107. & 126. & 136. & 154. & 177. \\
\hline 10 & 15.6 & 20.3 & 31.2 & 44.2 & 50.2 & 64.8 & 75.9 & 85.3 & 87.0 \\
\hline 20 & 6.50 & 8.62 & 15.4 & 18.8 & 22.8 & 31.5 & 36.6 & 38.6 & 40.3 \\
\hline 25 & 4.52 & 6.86 & 11.6 & 12.6 & 18.5 & 23.8 & 29.1 & 30.8 & 31.0 \\
\hline 30 & 3.34 & 5.78 & 8.88 & 10.5 & 15.0 & 19.6 & 24.9 & 25.2 & 25.8 \\
\hline 40 & 2.00 & 3.26 & 5.54 & 7.04 & 11.0 & 12.8 & 17.1 & 18.6 & 19.8 \\
\hline 50 & 0.94 & 1.78 & 3.88 & 4.92 & 8.30 & 9.36 & 11.4 & 13.0 & 14.6 \\
\hline 60 & 0.57 & 1.07 & 1.96 & 3.71 & 5.60 & 6.68 & 8.39 & 9.71 & 10.8 \\
\hline 70 & 0.42 & 0.53 & 1.16 & 2.32 & 3.40 & 4.58 & 5.13 & 6.17 & 8.20 \\
\hline 75 & 0.40 & 0.45 & 0.96 & 1.80 & 2.90 & 3.79 & 3.90 & 5.00 & 7.40 \\
\hline 80 & 0.32 & 0.43 & 0.70 & 1.32 & 2.10 & 2.68 & 3.20 & 3.74 & 6.33 \\
\hline 90 & 0.22 & 0.32 & 0.37 & 0.63 & 1.10 & 1.27 & 1.52 & 2.20 & 4.60 \\
\hline 95 & 0.11 & 0.17 & 0.29 & 0.35 & 0.70 & 0.89 & 1.01 & 1.86 & 3.48 \\
\hline 98 & 0.01 & 0.07 & 0.20 & 0.25 & 0.46 & 0.70 & 0.84 & 1.62 & 2.33 \\
\hline 99 & 0.00 & 0.03 & 0.18 & 0.21 & 0.41 & 0.69 & 0.83 & 1.55 & 1.86 \\
\hline
\end{tabular}

\begin{tabular}{|c|c|c|c|c|c|c|c|c|c|}
\hline \multirow[b]{2}{*}{ APEE } & \multicolumn{9}{|c|}{ 7-day mean streamflow $\left(\mathrm{ft}^{3} / \mathrm{s}\right)$ that was equaled or exceeded the indicated percentage of time at APEE level } \\
\hline & 95 & 90 & 80 & 70 & 50 & 30 & 20 & 10 & 5 \\
\hline 1 & 64.5 & 85.2 & 139. & 153. & 193. & 228 & 258. & 275 & 304. \\
\hline 2 & 53.8 & 70.5 & 110. & 126. & 136. & 182. & 194. & 204. & 228 \\
\hline 5 & 33.3 & 51.4 & 66.9 & 85.3 & 97.7 & 114. & 121. & 142 & 154. \\
\hline 10 & 19.9 & 23.8 & 41.1 & 52.5 & 65.0 & 73.5 & 78.0 & 102. & 108. \\
\hline 20 & 5.90 & 9.39 & 19.9 & 26.5 & 31.6 & 40.2 & 55.8 & 58.3 & 58.7 \\
\hline 25 & 4.22 & 6.75 & 15.2 & 17.1 & 25.3 & 31.4 & 41.2 & 46.0 & 47.3 \\
\hline 30 & 3.23 & 5.86 & 10.7 & 12.7 & 19.0 & 27.7 & 31.8 & 38.6 & 39.1 \\
\hline 40 & 2.17 & 3.45 & 6.14 & 8.69 & 13.7 & 16.9 & 22.1 & 23.7 & 27.7 \\
\hline 50 & 1.24 & 2.16 & 4.22 & 5.37 & 9.24 & 11.2 & 14.5 & 16.1 & 17.9 \\
\hline 60 & 0.71 & 1.42 & 2.40 & 3.85 & 5.91 & 7.47 & 10.3 & 12.5 & 13.5 \\
\hline 70 & 0.50 & 0.62 & 1.50 & 2.55 & 3.95 & 5.34 & 6.08 & 7.87 & 10.3 \\
\hline 75 & 0.39 & 0.50 & 1.16 & 2.11 & 3.39 & 4.10 & 4.81 & 6.25 & 9.13 \\
\hline 80 & 0.29 & 0.47 & 0.95 & 1.51 & 2.66 & 3.10 & 4.05 & 4.68 & 7.85 \\
\hline 90 & 0.19 & 0.35 & 0.43 & 0.67 & 1.24 & 1.49 & 1.86 & 2.53 & 5.59 \\
\hline 95 & 0.12 & 0.24 & 0.31 & 0.46 & 0.87 & 1.07 & 1.26 & 2.07 & 4.43 \\
\hline 98 & 0.06 & 0.18 & 0.28 & 0.41 & 0.65 & 0.87 & 0.95 & 1.90 & 3.15 \\
\hline 99 & 0.02 & 0.10 & 0.21 & 0.30 & 0.59 & 0.80 & 0.87 & 1.78 & 2.80 \\
\hline
\end{tabular}


03271000 Wolf Creek at Dayton, Ohio

Years analyzed $=26$

Climatic year range $=1939-2008$

[APEE, annual percentage equaled or exceeded; $\mathrm{ft}^{3} / \mathrm{s}$, cubic foot per second]

\begin{tabular}{|c|c|c|c|c|c|c|c|c|c|}
\hline \multirow[b]{2}{*}{ APEE } & \multicolumn{9}{|c|}{ Daily mean streamflow $\left(\mathrm{ft}^{3} / \mathrm{s}\right)$ that was equaled or exceeded the indicated percentage of time at APEE level } \\
\hline & 95 & 90 & 80 & 70 & 50 & 30 & 20 & 10 & 5 \\
\hline 1 & 380. & 417. & 510. & 599. & 786. & 1180. & 1280 & 1510. & 1630. \\
\hline 2 & 223. & 238 & 305. & 385. & 489. & 675. & 787. & 947. & 1070 \\
\hline 5 & 98.1 & 114. & 131. & 197. & 245. & 324. & 394. & 436. & 506. \\
\hline 10 & 53.4 & 59.3 & 72.7 & 95.2 & 129. & 164. & 207. & 233. & 268. \\
\hline 20 & 31.6 & 34.2 & 37.9 & 45.5 & 72.0 & 89.2 & 107. & 116. & 124. \\
\hline 25 & 24.0 & 26.8 & 30.2 & 38.5 & 59.3 & 72.0 & 82.2 & 92.4 & 99.6 \\
\hline 30 & 18.4 & 23.0 & 25.3 & 32.6 & 49.1 & 58.0 & 65.3 & 77.9 & 82.4 \\
\hline 40 & 7.15 & 13.1 & 18.2 & 23.3 & 32.0 & 44.4 & 49.2 & 62.0 & 63.7 \\
\hline 50 & 5.01 & 8.57 & 13.8 & 17.0 & 21.5 & 33.0 & 38.2 & 49.3 & 51.9 \\
\hline 60 & 3.95 & 6.89 & 10.0 & 13.0 & 14.7 & 24.9 & 26.6 & 40.3 & 42.7 \\
\hline 70 & 3.51 & 5.55 & 6.80 & 9.82 & 12.0 & 16.9 & 20.6 & 30.3 & 34.6 \\
\hline 75 & 3.28 & 5.05 & 5.52 & 8.17 & 11.5 & 14.9 & 18.6 & 25.5 & 30.8 \\
\hline 80 & 2.91 & 4.40 & 4.54 & 7.26 & 10.0 & 13.0 & 16.0 & 22.8 & 27.6 \\
\hline 90 & 2.09 & 3.15 & 3.53 & 5.90 & 7.69 & 9.99 & 12.0 & 16.9 & 20.1 \\
\hline 95 & 1.59 & 2.28 & 2.82 & 5.15 & 6.35 & 8.83 & 10.5 & 14.0 & 16.2 \\
\hline 98 & 1.34 & 1.75 & 2.30 & 3.43 & 5.17 & 7.90 & 9.57 & 11.1 & 14.4 \\
\hline 99 & 1.24 & 1.35 & 2.16 & 3.40 & 5.00 & 7.66 & 8.67 & 10.7 & 12.8 \\
\hline
\end{tabular}

\begin{tabular}{|c|c|c|c|c|c|c|c|c|c|}
\hline \multirow[b]{2}{*}{ APEE } & \multicolumn{9}{|c|}{ 7-day mean streamflow (ft $\left.{ }^{3} / \mathrm{s}\right)$ that was equaled or exceeded the indicated percentage of time at APEE level } \\
\hline & 95 & 90 & 80 & 70 & 50 & 30 & 20 & 10 & 5 \\
\hline 1 & 215 & 247. & 366. & 389. & 502. & 604. & 731. & 883. & 1050 \\
\hline 2 & 165. & 221. & 260. & 314. & 345. & 517. & 529. & 614. & 752. \\
\hline 5 & 129. & 146. & 163. & 185. & 240 & 363. & 384. & 447. & 547. \\
\hline 10 & 71.0 & 79.5 & 96.4 & 129. & 161. & 228 & 245. & 284. & 328. \\
\hline 20 & 33.5 & 37.3 & 46.0 & 53.7 & 94.6 & 116. & 136. & 155. & 181. \\
\hline 25 & 25.2 & 31.5 & 37.3 & 41.9 & 77.4 & 89.3 & 99.6 & 122. & 152. \\
\hline 30 & 20.1 & 25.8 & 28.8 & 36.1 & 60.1 & 73.5 & 80.4 & 107. & 135. \\
\hline 40 & 7.22 & 14.5 & 20.8 & 26.2 & 38.8 & 53.9 & 58.8 & 76.3 & 82.8 \\
\hline 50 & 4.79 & 9.93 & 15.2 & 20.7 & 27.5 & 40.1 & 46.8 & 57.8 & 62.9 \\
\hline 60 & 4.02 & 7.25 & 10.7 & 14.4 & 17.0 & 31.5 & 34.1 & 48.8 & 55.3 \\
\hline 70 & 3.53 & 5.72 & 6.89 & 11.2 & 14.0 & 21.4 & 25.2 & 36.8 & 45.8 \\
\hline 75 & 3.25 & 5.21 & 5.92 & 9.17 & 12.6 & 17.9 & 22.9 & 33.4 & 40.4 \\
\hline 80 & 2.94 & 4.41 & 4.80 & 7.98 & 11.5 & 14.9 & 19.9 & 28.7 & 35.5 \\
\hline 90 & 2.16 & 3.20 & 3.76 & 6.02 & 8.18 & 11.1 & 13.8 & 20.4 & 23.9 \\
\hline 95 & 1.75 & 2.54 & 3.03 & 5.40 & 6.88 & 9.31 & 12.5 & 18.0 & 19.5 \\
\hline 98 & 1.54 & 1.81 & 2.50 & 3.69 & 5.94 & 8.60 & 10.4 & 13.5 & 16.5 \\
\hline 99 & 1.42 & 1.60 & 2.19 & 3.57 & 5.40 & 8.38 & 9.73 & 11.4 & 15.0 \\
\hline
\end{tabular}


03271800 Twin Creek near Ingomar, Ohio

Years analyzed $=36$

Climatic year range $=1963-98$

[APEE, annual percentage equaled or exceeded; $\mathrm{ft}^{3} / \mathrm{s}$, cubic foot per second]

\begin{tabular}{|c|c|c|c|c|c|c|c|c|c|}
\hline \multirow[b]{2}{*}{ APEE } & \multicolumn{9}{|c|}{ Daily mean streamflow (ft $3 / s)$ that was equaled or exceeded the indicated percentage of time at APEE level } \\
\hline & 95 & 90 & 80 & 70 & 50 & 30 & 20 & 10 & 5 \\
\hline 1 & 930. & 1110 & 1910. & 2110 & 2490 & 2880 & 3580. & 4070 & 4130. \\
\hline 2 & 434. & 719. & 943. & 1360 & 1710 & 2050 & 2330 & 2470 & 2700 \\
\hline 5 & 239. & 310. & 501. & 654. & 897. & 1060. & 1160. & 1350. & 1470 \\
\hline 10 & 143. & 190. & 270 & 325. & 421. & 534. & 592. & 766. & 862. \\
\hline 20 & 59.7 & 91.4 & 135. & 154. & 202. & 264. & 303. & 366. & 418. \\
\hline 25 & 48.4 & 74.4 & 102. & 118. & 162. & 204. & 231. & 296. & 341. \\
\hline 30 & 37.8 & 61.6 & 81.0 & 97.5 & 136. & 167. & 192. & 230 & 266. \\
\hline 40 & 20.4 & 38.6 & 52.0 & 67.4 & 98.5 & 110. & 135. & 163. & 184 \\
\hline 50 & 12.9 & 25.6 & 35.2 & 41.1 & 71.5 & 82.7 & 89.6 & 106. & 126. \\
\hline 60 & 9.32 & 13.4 & 20.6 & 24.2 & 48.2 & 60.6 & 65.0 & 75.5 & 106. \\
\hline 70 & 7.00 & 8.12 & 12.8 & 17.1 & 29.8 & 35.9 & 44.2 & 53.2 & 79.3 \\
\hline 75 & 6.08 & 6.94 & 11.4 & 15.1 & 23.8 & 29.5 & 33.2 & 45.7 & 68.1 \\
\hline 80 & 5.76 & 6.41 & 9.88 & 12.1 & 19.0 & 22.1 & 26.9 & 37.8 & 56.2 \\
\hline 90 & 4.09 & 4.63 & 6.14 & 8.03 & 11.5 & 16.0 & 18.6 & 24.0 & 33.4 \\
\hline 95 & 3.46 & 3.97 & 5.24 & 6.55 & 9.55 & 14.0 & 15.6 & 20.5 & 27.6 \\
\hline 98 & 2.99 & 3.54 & 4.72 & 5.93 & 8.45 & 12.3 & 13.2 & 18.1 & 21.0 \\
\hline 99 & 2.87 & 3.37 & 4.63 & 5.57 & 7.65 & 11.0 & 12.9 & 15.8 & 19.3 \\
\hline
\end{tabular}

\begin{tabular}{|c|c|c|c|c|c|c|c|c|c|}
\hline \multirow[b]{2}{*}{ APEE } & \multicolumn{9}{|c|}{ 7-day mean streamflow ( $\mathrm{ft}^{3} / \mathrm{s}$ ) that was equaled or exceeded the indicated percentage of time at APEE level } \\
\hline & 95 & 90 & 80 & 70 & 50 & 30 & 20 & 10 & 5 \\
\hline 1 & 567. & 672. & 1040 & 1310 . & 1540 & 1900. & 2180 & 2290 & 2690 \\
\hline 2 & 477. & 591. & 841. & 984. & 1160 & 1350 & 1490. & 1760. & 1970. \\
\hline 5 & 291. & 373. & 611. & 734. & 856. & 962. & 1040 & 1230 & 1330. \\
\hline 10 & 150. & 191. & 326. & 443. & 535. & 643. & 724. & 851. & 890. \\
\hline 20 & 65.4 & 98.3 & 172. & 202. & 278. & 383. & 410. & 492. & 557. \\
\hline 25 & 50.7 & 79.2 & 122. & 158. & 204. & 290. & 311. & 402. & 456. \\
\hline 30 & 40.5 & 65.7 & 90.0 & 117. & 167. & 220. & 250. & 329. & 386. \\
\hline 40 & 20.3 & 40.5 & 56.1 & 74.0 & 119. & 144. & 176. & 230 & 252. \\
\hline 50 & 12.9 & 26.9 & 36.5 & 45.2 & 82.1 & 99.4 & 105. & 148. & 157. \\
\hline 60 & 9.56 & 12.4 & 22.1 & 25.1 & 52.2 & 69.1 & 77.3 & 84.3 & 125. \\
\hline 70 & 6.94 & 8.10 & 13.3 & 18.4 & 34.3 & 42.0 & 51.1 & 62.3 & 96.0 \\
\hline 75 & 6.25 & 6.81 & 10.9 & 15.2 & 26.2 & 32.3 & 37.0 & 53.1 & 81.7 \\
\hline 80 & 5.71 & 6.35 & 10.1 & 13.1 & 19.3 & 24.8 & 28.8 & 41.2 & 70.7 \\
\hline 90 & 3.95 & 4.75 & 6.39 & 8.33 & 12.0 & 17.1 & 19.2 & 24.7 & 36.7 \\
\hline 95 & 3.44 & 4.01 & 5.53 & 6.96 & 10.1 & 14.7 & 16.5 & 21.4 & 31.4 \\
\hline 98 & 3.21 & 3.65 & 4.94 & 6.38 & 9.11 & 12.9 & 14.5 & 20.1 & 24.3 \\
\hline 99 & 3.09 & 3.42 & 4.91 & 5.76 & 8.63 & 12.1 & 13.8 & 18.2 & 21.2 \\
\hline
\end{tabular}


03272700 Sevenmile Creek at Camden, Ohio

Years analyzed $=35$

Climatic year range $=1971-2008$

[APEE, annual percentage equaled or exceeded; $\mathrm{ft}^{3} / \mathrm{s}$, cubic foot per second]

\begin{tabular}{|c|c|c|c|c|c|c|c|c|c|}
\hline \multirow[b]{2}{*}{ APEE } & \multicolumn{9}{|c|}{ Daily mean streamflow $\left(\mathrm{ft}^{3} / \mathrm{s}\right)$ that was equaled or exceeded the indicated percentage of time at APEE level } \\
\hline & 95 & 90 & 80 & 70 & 50 & 30 & 20 & 10 & 5 \\
\hline 1 & 389. & 433. & 570. & 652. & 844. & 1060. & 1290. & 1510. & 1730 \\
\hline 2 & 216. & 250 & 304. & 450 & 570. & 672. & 769. & 952. & 1060. \\
\hline 5 & 102. & 123. & 162. & 241. & 294. & 356. & 401. & 480. & 511. \\
\hline 10 & 65.5 & 73.4 & 101. & 121. & 170. & 198. & 213. & 284. & 293. \\
\hline 20 & 29.8 & 40.6 & 50.4 & 62.9 & 83.4 & 109. & 112. & 141. & 164. \\
\hline 25 & 23.4 & 32.9 & 42.0 & 50.2 & 68.0 & 83.3 & 95.3 & 113. & 132. \\
\hline 30 & 19.3 & 27.1 & 35.2 & 41.6 & 56.9 & 66.7 & 79.2 & 96.3 & 108. \\
\hline 40 & 12.4 & 16.4 & 22.9 & 31.8 & 41.6 & 49.1 & 57.8 & 69.4 & 74.2 \\
\hline 50 & 7.82 & 10.6 & 15.2 & 21.9 & 29.0 & 36.2 & 42.6 & 51.6 & 58.6 \\
\hline 60 & 5.02 & 5.79 & 7.39 & 10.7 & 20.0 & 24.5 & 29.9 & 40.0 & 47.2 \\
\hline 70 & 3.76 & 3.96 & 4.42 & 7.40 & 12.0 & 16.0 & 19.0 & 33.8 & 40.2 \\
\hline 75 & 3.10 & 3.31 & 3.88 & 5.91 & 9.00 & 11.0 & 15.0 & 30.8 & 35.2 \\
\hline 80 & 2.50 & 2.96 & 3.56 & 4.74 & 7.04 & 9.37 & 12.8 & 25.2 & 28.8 \\
\hline 90 & 1.86 & 1.99 & 2.61 & 3.04 & 4.92 & 6.30 & 7.15 & 17.4 & 18.7 \\
\hline 95 & 1.56 & 1.70 & 2.10 & 2.61 & 3.70 & 5.17 & 6.28 & 13.2 & 15.0 \\
\hline 98 & 1.27 & 1.57 & 1.77 & 2.08 & 3.10 & 4.78 & 5.17 & 11.1 & 12.5 \\
\hline 99 & 1.20 & 1.49 & 1.67 & 1.85 & 2.97 & 4.43 & 4.90 & 10.0 & 12.0 \\
\hline
\end{tabular}

\begin{tabular}{|c|c|c|c|c|c|c|c|c|c|}
\hline \multirow[b]{2}{*}{ APEE } & \multicolumn{9}{|c|}{ 7-day mean streamflow (ft $\left.{ }^{3} / \mathrm{s}\right)$ that was equaled or exceeded the indicated percentage of time at APEE level } \\
\hline & 95 & 90 & 80 & 70 & 50 & 30 & 20 & 10 & 5 \\
\hline 1 & 224. & 293. & 341. & 427. & 515. & 670. & 723. & 1080 & 1400 \\
\hline 2 & 209. & 239. & 266. & 313. & 388. & 515. & 611. & 664. & 856. \\
\hline 5 & 133. & 151. & 171. & 218. & 292. & 360 . & 387. & 456. & 525. \\
\hline 10 & 69.3 & 83.0 & 107. & 156. & 178. & 239. & 260 & 301. & 327. \\
\hline 20 & 31.9 & 46.4 & 66.7 & 72.8 & 107. & 131. & 143. & 182. & 252. \\
\hline 25 & 24.0 & 39.1 & 49.0 & 59.0 & 82.8 & 104. & 111. & 154. & 202. \\
\hline 30 & 20.1 & 30.7 & 38.5 & 46.2 & 64.4 & 88.5 & 91.5 & 135. & 151. \\
\hline 40 & 14.1 & 16.7 & 23.8 & 35.8 & 46.9 & 60.8 & 69.1 & 93.7 & 105. \\
\hline 50 & 9.74 & 11.2 & 16.4 & 22.8 & 32.9 & 41.5 & 47.3 & 62.8 & 79.9 \\
\hline 60 & 5.30 & 6.36 & 8.10 & 11.4 & 20.8 & 27.3 & 32.4 & 50.4 & 57.7 \\
\hline 70 & 4.08 & 4.34 & 4.78 & 8.48 & 12.5 & 16.9 & 20.7 & 41.1 & 50.5 \\
\hline 75 & 3.51 & 3.60 & 4.04 & 6.74 & 9.99 & 13.9 & 17.2 & 37.8 & 43.0 \\
\hline 80 & 3.01 & 3.16 & 3.81 & 4.93 & 8.36 & 11.1 & 13.9 & 31.9 & 38.8 \\
\hline 90 & 2.02 & 2.21 & 2.77 & 3.34 & 5.63 & 6.65 & 7.91 & 20.6 & 21.2 \\
\hline 95 & 1.61 & 1.84 & 2.33 & 2.74 & 4.03 & 5.41 & 6.95 & 16.1 & 17.4 \\
\hline 98 & 1.42 & 1.65 & 2.01 & 2.30 & 3.26 & 5.05 & 6.18 & 12.2 & 14.3 \\
\hline 99 & 1.33 & 1.59 & 1.81 & 2.14 & 3.14 & 4.73 & 5.73 & 10.9 & 13.3 \\
\hline
\end{tabular}


04177000 Ottawa River at University of Toledo, Toledo, Ohio

Years analyzed $=35$

Climatic year range $=1945-2008$

[APEE, annual percentage equaled or exceeded; $\mathrm{ft}^{3} / \mathrm{s}$, cubic foot per second]

\begin{tabular}{|c|c|c|c|c|c|c|c|c|c|}
\hline \multirow[b]{2}{*}{ APEE } & \multicolumn{9}{|c|}{ Daily mean streamflow $\left(\mathrm{ft}^{3} / \mathrm{s}\right)$ that was equaled or exceeded the indicated percentage of time at APEE level } \\
\hline & 95 & 90 & 80 & 70 & 50 & 30 & 20 & 10 & 5 \\
\hline 1 & 461. & 831. & 994. & 1110. & 1250. & 1610. & 1730. & 2090. & 2350 \\
\hline 2 & 408. & 555. & 741. & 789. & 908. & 1230 & 1470 . & 1600 & 1910. \\
\hline 5 & 243. & 302. & 422. & 460. & 550. & 772. & 832. & 980. & 1040. \\
\hline 10 & 122. & 150 . & 226. & 250 & 310. & 398. & 472. & 525. & 590. \\
\hline 20 & 47.1 & 72.1 & 106. & 111. & 158. & 179. & 200. & 231. & 285. \\
\hline 25 & 35.5 & 55.9 & 75.5 & 90.1 & 119. & 131. & 151. & 180. & 210 \\
\hline 30 & 28.8 & 46.3 & 53.6 & 69.6 & 92.6 & 100. & 115. & 139. & 164. \\
\hline 40 & 17.2 & 30.6 & 38.2 & 44.6 & 61.0 & 67.8 & 75.2 & 97.6 & 105. \\
\hline 50 & 10.5 & 19.4 & 25.6 & 30.0 & 45.0 & 49.0 & 55.0 & 69.4 & 73.2 \\
\hline 60 & 7.43 & 13.8 & 19.4 & 22.4 & 32.0 & 37.2 & 40.6 & 53.4 & 57.2 \\
\hline 70 & 5.48 & 9.83 & 14.2 & 15.0 & 18.0 & 27.2 & 30.8 & 38.2 & 48.6 \\
\hline 75 & 4.60 & 7.45 & 11.1 & 12.0 & 15.0 & 22.2 & 25.0 & 32.6 & 44.6 \\
\hline 80 & 3.90 & 5.72 & 8.73 & 9.68 & 12.0 & 17.7 & 21.8 & 28.3 & 39.8 \\
\hline 90 & 0.82 & 3.48 & 4.33 & 5.44 & 8.70 & 11.2 & 13.9 & 20.2 & 27.7 \\
\hline 95 & 0.00 & 2.22 & 2.94 & 4.30 & 7.03 & 8.98 & 10.8 & 16.5 & 20.0 \\
\hline 98 & 0.00 & 0.95 & 2.45 & 3.66 & 5.76 & 7.43 & 9.39 & 13.5 & 16.6 \\
\hline 99 & 0.00 & 0.69 & 2.10 & 2.94 & 5.16 & 7.12 & 9.23 & 12.4 & 15.6 \\
\hline
\end{tabular}

\begin{tabular}{|c|c|c|c|c|c|c|c|c|c|}
\hline \multirow[b]{2}{*}{ APEE } & \multicolumn{9}{|c|}{ 7-day mean streamflow ( $\mathrm{ft}^{3} / \mathrm{s}$ ) that was equaled or exceeded the indicated percentage of time at APEE level } \\
\hline & 95 & 90 & 80 & 70 & 50 & 30 & 20 & 10 & 5 \\
\hline 1 & 324. & 525. & 587. & 703. & 860. & 1050 & 1140 & 1480 & 1730 \\
\hline 2 & 274. & 446. & 551. & 609. & 683. & 861. & 978. & 1240 & 1360. \\
\hline 5 & 206. & 310. & 377. & 428. & 477. & 646. & 736. & 859. & 1020. \\
\hline 10 & 151. & 182. & 235 & 277. & 319. & 447. & 482. & 551. & 583. \\
\hline 20 & 61.8 & 72.2 & 120. & 149. & 182. & 228 & 258. & 325. & 349. \\
\hline 25 & 39.1 & 59.3 & 86.7 & 112. & 138. & 172. & 186. & 230. & 287. \\
\hline 30 & 25.9 & 48.6 & 60.7 & 84.3 & 109. & 133. & 152. & 190. & 230 \\
\hline 40 & 17.2 & 30.8 & 40.4 & 52.0 & 73.1 & 84.8 & 94.1 & 129. & 158. \\
\hline 50 & 11.9 & 21.5 & 29.9 & 32.9 & 49.4 & 61.0 & 70.1 & 89.7 & 101. \\
\hline 60 & 9.44 & 15.2 & 21.3 & 23.3 & 35.0 & 42.3 & 48.6 & 65.3 & 67.8 \\
\hline 70 & 7.51 & 11.8 & 16.3 & 17.7 & 22.8 & 31.4 & 34.7 & 47.1 & 55.2 \\
\hline 75 & 6.24 & 9.86 & 12.6 & 14.4 & 18.6 & 26.1 & 29.4 & 38.5 & 50.1 \\
\hline 80 & 4.31 & 6.92 & 9.54 & 12.1 & 15.0 & 20.6 & 25.6 & 32.8 & 45.1 \\
\hline 90 & 1.28 & 4.96 & 5.97 & 7.22 & 9.86 & 12.9 & 17.6 & 24.5 & 35.6 \\
\hline 95 & 0.05 & 2.89 & 4.04 & 5.24 & 7.81 & 10.6 & 14.2 & 20.9 & 29.4 \\
\hline 98 & 0.01 & 1.80 & 3.07 & 4.11 & 6.90 & 9.07 & 12.0 & 17.6 & 21.9 \\
\hline 99 & 0.00 & 1.30 & 2.96 & 3.91 & 6.24 & 8.68 & 9.61 & 16.1 & 19.1 \\
\hline
\end{tabular}




\section{Bean Creek at Powers, Ohio}

Years analyzed $=48$

Climatic year range $=1941-2008$

[APEE, annual percentage equaled or exceeded; $\mathrm{ft}^{3} / \mathrm{s}$, cubic foot per second]

\begin{tabular}{|c|c|c|c|c|c|c|c|c|c|}
\hline \multirow[b]{2}{*}{ APEE } & \multicolumn{9}{|c|}{ Daily mean streamflow $\left(\mathrm{ft}^{3} / \mathrm{s}\right)$ that was equaled or exceeded the indicated percentage of time at APEE level } \\
\hline & 95 & 90 & 80 & 70 & 50 & 30 & 20 & 10 & 5 \\
\hline 1 & 701. & 777. & 978. & 1110. & 1350. & 1750. & 2110. & 2380 & 2720 \\
\hline 2 & 478. & 536. & 731. & 784. & 1060. & 1310. & 1470 . & 1800. & 1990. \\
\hline 5 & 254 & 302. & 452. & 497. & 686. & 844. & 998. & 1160. & 1210. \\
\hline 10 & 159. & 193. & 263. & 338. & 430. & 523. & 608. & 700. & 756. \\
\hline 20 & 71.0 & 110. & 139. & 182. & 241. & 295. & 316. & 387. & 408. \\
\hline 25 & 53.0 & 89.6 & 113. & 139. & 195. & 240 & 259. & 302. & 332. \\
\hline 30 & 43.9 & 64.9 & 81.8 & 112. & 161. & 186. & 219. & 239. & 266. \\
\hline 40 & 25.8 & 36.5 & 47.4 & 67.0 & 111. & 132. & 146. & 178. & 185. \\
\hline 50 & 20.4 & 23.0 & 37.0 & 47.5 & 74.0 & 94.6 & 106. & 132. & 145. \\
\hline 60 & 15.4 & 19.7 & 26.8 & 36.7 & 53.2 & 70.6 & 78.6 & 98.0 & 112. \\
\hline 70 & 13.4 & 17.7 & 22.0 & 25.7 & 38.5 & 49.5 & 54.0 & 63.8 & 87.4 \\
\hline 75 & 12.9 & 14.9 & 19.0 & 22.7 & 32.5 & 41.0 & 46.0 & 57.5 & 75.8 \\
\hline 80 & 11.0 & 13.9 & 17.8 & 20.1 & 27.6 & 35.0 & 38.6 & 45.5 & 63.8 \\
\hline 90 & 8.66 & 10.0 & 14.0 & 15.7 & 20.0 & 24.3 & 28.4 & 35.1 & 39.5 \\
\hline 95 & 6.85 & 8.33 & 11.0 & 13.0 & 16.0 & 20.3 & 23.1 & 30.0 & 31.7 \\
\hline 98 & 6.38 & 7.19 & 9.82 & 11.7 & 14.0 & 18.5 & 20.1 & 26.1 & 28.5 \\
\hline 99 & 5.97 & 6.78 & 9.18 & 10.5 & 13.0 & 17.2 & 20.0 & 25.1 & 27.5 \\
\hline
\end{tabular}

\begin{tabular}{|c|c|c|c|c|c|c|c|c|c|}
\hline \multirow[b]{2}{*}{ APEE } & \multicolumn{9}{|c|}{ 7-day mean streamflow ( $\mathrm{ft}^{3} / \mathrm{s}$ ) that was equaled or exceeded the indicated percentage of time at APEE level } \\
\hline & 95 & 90 & 80 & 70 & 50 & 30 & 20 & 10 & 5 \\
\hline 1 & 519. & 642. & 741. & 890. & 1040. & 1330. & 1520 & 1750 & 1840 \\
\hline 2 & 356. & 518. & 609. & 683. & 852. & 1040 & 1260 & 1510 & 1590. \\
\hline 5 & 227. & 281. & 430 & 492. & 626. & 787. & 947. & 1060. & 1190. \\
\hline 10 & 155. & 168. & 302. & 344. & 425. & 553. & 611. & 756. & 789. \\
\hline 20 & 65.2 & 118. & 147. & 200 & 266. & 317. & 351. & 435. & 482. \\
\hline 25 & 52.7 & 98.2 & 112. & 161. & 211. & 263. & 292. & 343. & 395. \\
\hline 30 & 41.9 & 60.9 & 81.5 & 122. & 171. & 202. & 247. & 274. & 303. \\
\hline 40 & 24.0 & 35.8 & 48.9 & 68.4 & 119. & 147. & 163. & 203. & 216. \\
\hline 50 & 20.3 & 24.5 & 38.7 & 49.9 & 75.1 & 104. & 115. & 148. & 161. \\
\hline 60 & 16.0 & 19.2 & 27.3 & 36.0 & 51.9 & 74.8 & 84.2 & 104. & 131. \\
\hline 70 & 13.3 & 17.7 & 23.1 & 27.0 & 39.6 & 50.7 & 56.0 & 69.9 & 103. \\
\hline 75 & 12.5 & 14.7 & 19.7 & 23.0 & 33.9 & 42.8 & 47.7 & 59.6 & 86.8 \\
\hline 80 & 11.0 & 13.9 & 17.8 & 20.4 & 28.3 & 37.1 & 41.6 & 48.6 & 70.7 \\
\hline 90 & 8.78 & 10.5 & 14.5 & 16.5 & 20.6 & 25.5 & 29.7 & 36.7 & 41.5 \\
\hline 95 & 7.11 & 8.73 & 11.7 & 14.1 & 16.4 & 20.5 & 25.1 & 30.8 & 34.5 \\
\hline 98 & 6.72 & 7.33 & 10.8 & 12.2 & 14.5 & 18.9 & 21.6 & 27.2 & 30.2 \\
\hline 99 & 6.68 & 7.02 & 10.2 & 11.7 & 13.6 & 18.6 & 20.8 & 26.2 & 29.1 \\
\hline
\end{tabular}




\section{Unnamed Tributary to Lost Creek near Farmer, Ohio}

Years analyzed $=20$

Climatic year range $=1986-2008$

[APEE, annual percentage equaled or exceeded; $\mathrm{ft}^{3} / \mathrm{s}$, cubic foot per second]

\begin{tabular}{|c|c|c|c|c|c|c|c|c|c|}
\hline \multirow[b]{2}{*}{ APEE } & \multicolumn{9}{|c|}{ Daily mean streamflow ( $\left.\mathrm{ft}^{3} / \mathrm{s}\right)$ that was equaled or exceeded the indicated percentage of time at APEE level } \\
\hline & 95 & 90 & 80 & 70 & 50 & 30 & 20 & 10 & 5 \\
\hline 1 & 32.1 & 41.2 & 50.2 & 58.3 & 74.4 & 88.0 & 101. & 136. & 149. \\
\hline 2 & 17.4 & 25.9 & 28.3 & 36.8 & 46.1 & 56.5 & 63.5 & 66.9 & 83.8 \\
\hline 5 & 8.33 & 9.76 & 15.5 & 15.9 & 18.3 & 27.1 & 28.3 & 32.0 & 33.9 \\
\hline 10 & 3.43 & 4.04 & 5.69 & 6.87 & 8.97 & 11.3 & 12.0 & 13.7 & 14.0 \\
\hline 20 & 1.11 & 1.33 & 2.27 & 2.65 & 3.48 & 4.19 & 4.72 & 5.43 & 5.93 \\
\hline 25 & 0.80 & 0.89 & 1.66 & 1.76 & 2.28 & 3.14 & 3.25 & 4.10 & 4.25 \\
\hline 30 & 0.46 & 0.59 & 1.12 & 1.33 & 1.65 & 2.27 & 2.66 & 3.11 & 3.50 \\
\hline 40 & 0.26 & 0.36 & 0.65 & 0.71 & 0.99 & 1.49 & 1.76 & 1.98 & 2.09 \\
\hline 50 & 0.12 & 0.20 & 0.34 & 0.38 & 0.70 & 0.89 & 1.10 & 1.37 & 1.49 \\
\hline 60 & 0.09 & 0.10 & 0.15 & 0.21 & 0.46 & 0.57 & 0.70 & 0.95 & 1.09 \\
\hline 70 & 0.05 & 0.06 & 0.09 & 0.13 & 0.23 & 0.40 & 0.46 & 0.67 & 0.70 \\
\hline 75 & 0.02 & 0.05 & 0.07 & 0.09 & 0.18 & 0.35 & 0.40 & 0.55 & 0.60 \\
\hline 80 & 0.00 & 0.04 & 0.05 & 0.06 & 0.12 & 0.28 & 0.31 & 0.46 & 0.50 \\
\hline 90 & 0.00 & 0.00 & 0.02 & 0.03 & 0.07 & 0.12 & 0.16 & 0.28 & 0.30 \\
\hline 95 & 0.00 & 0.00 & 0.00 & 0.01 & 0.04 & 0.08 & 0.09 & 0.19 & 0.25 \\
\hline 98 & 0.00 & 0.00 & 0.00 & 0.00 & 0.03 & 0.06 & 0.08 & 0.13 & 0.21 \\
\hline 99 & 0.00 & 0.00 & 0.00 & 0.00 & 0.02 & 0.05 & 0.07 & 0.11 & 0.17 \\
\hline
\end{tabular}

\begin{tabular}{|c|c|c|c|c|c|c|c|c|c|}
\hline \multirow[b]{2}{*}{ APEE } & \multicolumn{9}{|c|}{ 7-day mean streamflow ( $\mathrm{ft}^{3} / \mathrm{s}$ ) that was equaled or exceeded the indicated percentage of time at APEE level } \\
\hline & 95 & 90 & 80 & 70 & 50 & 30 & 20 & 10 & 5 \\
\hline 1 & 19.3 & 21.2 & 26.2 & 31.0 & 35.5 & 42.5 & 48.0 & 59.7 & 67.1 \\
\hline 2 & 14.3 & 16.8 & 19.5 & 22.0 & 27.3 & 30.3 & 32.1 & 41.8 & 51.2 \\
\hline 5 & 9.16 & 10.6 & 14.0 & 16.3 & 20.5 & 25.2 & 26.3 & 34.4 & 35.0 \\
\hline 10 & 4.78 & 6.92 & 8.14 & 10.6 & 12.8 & 15.8 & 16.8 & 17.2 & 19.5 \\
\hline 20 & 1.65 & 2.09 & 4.19 & 4.84 & 6.30 & 9.04 & 10.2 & 10.9 & 11.1 \\
\hline 25 & 1.32 & 1.54 & 3.00 & 3.48 & 4.84 & 6.52 & 7.33 & 8.87 & 9.94 \\
\hline 30 & 0.90 & 1.10 & 1.97 & 2.59 & 3.47 & 4.48 & 5.38 & 6.41 & 8.13 \\
\hline 40 & 0.38 & 0.48 & 0.97 & 1.27 & 1.85 & 2.54 & 3.33 & 3.94 & 4.28 \\
\hline 50 & 0.15 & 0.28 & 0.49 & 0.62 & 1.04 & 1.29 & 1.98 & 2.64 & 3.36 \\
\hline 60 & 0.11 & 0.12 & 0.19 & 0.25 & 0.60 & 0.77 & 1.01 & 1.68 & 1.86 \\
\hline 70 & 0.06 & 0.08 & 0.09 & 0.15 & 0.30 & 0.54 & 0.64 & 0.94 & 1.02 \\
\hline 75 & 0.05 & 0.05 & 0.07 & 0.11 & 0.25 & 0.44 & 0.50 & 0.73 & 0.77 \\
\hline 80 & 0.03 & 0.04 & 0.05 & 0.08 & 0.18 & 0.35 & 0.42 & 0.58 & 0.62 \\
\hline 90 & 0.00 & 0.01 & 0.02 & 0.03 & 0.09 & 0.14 & 0.22 & 0.37 & 0.41 \\
\hline 95 & 0.00 & 0.00 & 0.01 & 0.02 & 0.06 & 0.09 & 0.14 & 0.24 & 0.31 \\
\hline 98 & 0.00 & 0.00 & 0.00 & 0.00 & 0.04 & 0.08 & 0.10 & 0.16 & 0.28 \\
\hline 99 & 0.00 & 0.00 & 0.00 & 0.00 & 0.03 & 0.07 & 0.08 & 0.13 & 0.27 \\
\hline
\end{tabular}




\section{Blanchard River near Findlay, Ohio}

Years analyzed $=79$

Climatic year range $=1924-2008$

[APEE, annual percentage equaled or exceeded; $\mathrm{ft}^{3} / \mathrm{s}$, cubic foot per second]

\begin{tabular}{|c|c|c|c|c|c|c|c|c|c|}
\hline \multirow[b]{2}{*}{ APEE } & \multicolumn{9}{|c|}{ Daily mean streamflow $\left(\mathrm{ft}^{3} / \mathrm{s}\right)$ that was equaled or exceeded the indicated percentage of time at APEE level } \\
\hline & 98 & 95 & 90 & 80 & 50 & 20 & 10 & 5 & 2 \\
\hline 1 & 626. & 941. & 1430. & 1900. & 3320. & 4330. & 5570. & 6180. & 8210 \\
\hline 2 & 484. & 791. & 1070 . & 1340. & 2350 & 3240 . & 3820 . & 4900. & 5620. \\
\hline 5 & 236. & 332. & 552. & 753. & 1170 . & 1960. & 2380 & 2860 & 3350. \\
\hline 10 & 128. & 163. & 216. & 346. & 574. & 1060. & 1330. & 1650. & 1740 \\
\hline 20 & 46.0 & 64.4 & 93.6 & 126. & 253. & 476. & 539. & 659. & 874. \\
\hline 25 & 33.8 & 54.0 & 67.0 & 91.5 & 185. & 346. & 405. & 471. & 615. \\
\hline 30 & 25.3 & 47.2 & 55.0 & 71.0 & 150. & 257. & 323. & 394. & 475 \\
\hline 40 & 11.8 & 17.2 & 36.6 & 44.0 & 96.0 & 175. & 221. & 241. & 309. \\
\hline 50 & 7.44 & 11.0 & 21.0 & 30.0 & 64.0 & 120. & 157. & 172. & 204. \\
\hline 60 & 5.36 & 9.50 & 13.0 & 22.0 & 45.0 & 75.0 & 95.0 & 125. & 157. \\
\hline 70 & 4.44 & 7.50 & 9.00 & 15.8 & 30.0 & 54.1 & 71.8 & 90.1 & 126. \\
\hline 75 & 3.70 & 6.50 & 8.20 & 12.0 & 26.0 & 44.8 & 66.0 & 77.0 & 112. \\
\hline 80 & 2.87 & 3.90 & 7.40 & 11.0 & 22.4 & 38.0 & 55.0 & 65.0 & 102. \\
\hline 90 & 1.80 & 3.20 & 5.40 & 7.40 & 15.4 & 24.6 & 40.0 & 46.6 & 74.8 \\
\hline 95 & 1.30 & 2.80 & 4.40 & 5.80 & 12.0 & 21.3 & 30.3 & 38.0 & 60.3 \\
\hline 98 & 1.14 & 2.20 & 3.30 & 4.30 & 10.0 & 18.0 & 24.3 & 33.3 & 48.2 \\
\hline 99 & 0.95 & 1.80 & 2.90 & 4.13 & 9.00 & 17.0 & 23.0 & 30.3 & 41.7 \\
\hline
\end{tabular}

\begin{tabular}{|c|c|c|c|c|c|c|c|c|c|}
\hline \multirow[b]{2}{*}{ APEE } & \multicolumn{9}{|c|}{ 7-day mean streamflow ( $\mathrm{ft}^{3} / \mathrm{s}$ ) that was equaled or exceeded the indicated percentage of time at APEE level } \\
\hline & 98 & 95 & 90 & 80 & 50 & 20 & 10 & 5 & 2 \\
\hline 1 & 372. & 711. & 922. & 1270 & 2170 & 2990. & 3560 & 4100 & 4730 \\
\hline 2 & 350 & 583. & 812. & 1050 & 1650 & 2260 & 3130. & 3730. & 3980. \\
\hline 5 & 223. & 347. & 550. & 720 & 1210 & 1620. & 2200 & 2510 & 2710 \\
\hline 10 & 106. & 165. & 245 & 407. & 752. & 1160. & 1440 & 1520. & 1740. \\
\hline 20 & 48.5 & 72.6 & 105. & 169. & 305. & 644. & 791. & 909. & 1030. \\
\hline 25 & 37.0 & 60.7 & 73.3 & 118. & 228. & 446. & 584. & 773. & 892. \\
\hline 30 & 26.8 & 52.9 & 57.6 & 79.4 & 182. & 364. & 460. & 580. & 704. \\
\hline 40 & 13.5 & 17.9 & 39.9 & 50.9 & 115. & 220. & 275. & 343. & 496. \\
\hline 50 & 7.05 & 11.4 & 21.8 & 32.6 & 76.0 & 137. & 183. & 232. & 297. \\
\hline 60 & 5.29 & 9.71 & 12.7 & 24.1 & 51.7 & 96.7 & 123. & 153. & 207. \\
\hline 70 & 4.60 & 7.37 & 9.77 & 16.6 & 32.9 & 63.9 & 79.4 & 119. & 159. \\
\hline 75 & 4.01 & 5.10 & 8.03 & 14.0 & 27.9 & 50.1 & 75.3 & 105. & 141. \\
\hline 80 & 3.23 & 4.50 & 7.50 & 11.9 & 24.3 & 44.4 & 65.3 & 84.7 & 128. \\
\hline 90 & 2.28 & 3.50 & 6.33 & 7.86 & 17.9 & 26.7 & 44.0 & 53.6 & 94.1 \\
\hline 95 & 1.74 & 3.01 & 4.73 & 6.26 & 13.3 & 22.0 & 34.4 & 43.0 & 73.8 \\
\hline 98 & 1.52 & 2.46 & 4.09 & 5.37 & 11.1 & 19.6 & 26.3 & 40.4 & 59.1 \\
\hline 99 & 1.35 & 2.21 & 3.43 & 4.86 & 10.7 & 18.7 & 25.6 & 37.0 & 57.8 \\
\hline
\end{tabular}


04196500 Sandusky River near Upper Sandusky, Ohio

Years analyzed $=64$

Climatic year range $=1922-2008$

[APEE, annual percentage equaled or exceeded; $\mathrm{ft}^{3} / \mathrm{s}$, cubic foot per second]

\begin{tabular}{|c|c|c|c|c|c|c|c|c|c|}
\hline \multirow[b]{2}{*}{ APEE } & \multicolumn{9}{|c|}{ Daily mean streamflow $\left(\mathrm{ft}^{3} / \mathrm{s}\right)$ that was equaled or exceeded the indicated percentage of time at APEE level } \\
\hline & 98 & 95 & 90 & 80 & 50 & 20 & 10 & 5 & 2 \\
\hline 1 & 577. & 1300. & 1670. & 1940. & 3040. & 3920. & 5000. & 5380. & 6240 \\
\hline 2 & 463. & 756. & 1230. & 1500 & 2190 & 2780 & 3180 . & 4230 & 5160. \\
\hline 5 & 247. & 341. & 537. & 780 . & 1140 & 1590. & 1910. & 2300 & 2880 \\
\hline 10 & 158. & 189. & 267. & 357. & 652. & 896. & 1090. & 1230 & 1330. \\
\hline 20 & 56.1 & 77.8 & 122. & 155. & 285. & 431. & 536. & 582. & 648. \\
\hline 25 & 34.1 & 57.3 & 84.8 & 112. & 219. & 337. & 413. & 468. & 484. \\
\hline 30 & 25.1 & 48.7 & 61.0 & 81.2 & 169. & 271. & 315. & 365. & 414. \\
\hline 40 & 13.5 & 21.7 & 31.5 & 49.6 & 114. & 179. & 209. & 243. & 268. \\
\hline 50 & 9.60 & 13.3 & 17.0 & 30.0 & 66.8 & 117. & 143. & 155. & 195. \\
\hline 60 & 7.00 & 8.30 & 11.9 & 21.0 & 41.6 & 82.4 & 107. & 121. & 139. \\
\hline 70 & 4.85 & 6.44 & 8.82 & 14.0 & 24.5 & 49.2 & 69.4 & 87.1 & 103. \\
\hline 75 & 3.93 & 5.33 & 7.65 & 11.0 & 20.5 & 40.0 & 56.5 & 68.9 & 90.0 \\
\hline 80 & 3.19 & 4.50 & 5.85 & 9.00 & 16.0 & 34.0 & 46.6 & 56.3 & 78.3 \\
\hline 90 & 1.88 & 2.63 & 3.40 & 5.60 & 11.0 & 21.0 & 26.4 & 32.3 & 51.0 \\
\hline 95 & 1.11 & 1.85 & 2.30 & 3.90 & 8.10 & 14.3 & 20.1 & 24.0 & 35.7 \\
\hline 98 & 0.89 & 1.22 & 1.90 & 2.80 & 6.45 & 12.0 & 16.2 & 20.3 & 29.7 \\
\hline 99 & 0.76 & 1.13 & 1.65 & 2.43 & 5.33 & 10.0 & 14.3 & 17.5 & 25.2 \\
\hline
\end{tabular}

\begin{tabular}{|c|c|c|c|c|c|c|c|c|c|}
\hline \multirow[b]{2}{*}{ APEE } & \multicolumn{9}{|c|}{ 7-day mean streamflow ( $\mathrm{ft}^{3} / \mathrm{s}$ ) that was equaled or exceeded the indicated percentage of time at APEE level } \\
\hline & 98 & 95 & 90 & 80 & 50 & 20 & 10 & 5 & 2 \\
\hline 1 & 383. & 779. & 1090. & 1340 & 2010 & 2630 & 2890 & 3570 & 3790. \\
\hline 2 & 322. & 700 & 850. & 1130. & 1520. & 1990. & 2290 & 3020. & 3230. \\
\hline 5 & 229. & 357. & 570. & 866. & 1140. & 1420 . & 1690. & 2250 & 2710 \\
\hline 10 & 165. & 175 & 270 & 464. & 722. & 934. & 1070 & 1310. & 1720 \\
\hline 20 & 63.3 & 79.7 & 139. & 175. & 353. & 564. & 664. & 744. & 848. \\
\hline 25 & 31.0 & 60.0 & 92.7 & 138. & 280 & 461. & 531. & 644. & 724. \\
\hline 30 & 24.0 & 48.0 & 63.2 & 88.4 & 207. & 388. & 451. & 515. & 609. \\
\hline 40 & 13.9 & 23.2 & 32.9 & 53.0 & 140. & 222. & 294. & 348. & 368. \\
\hline 50 & 9.95 & 14.4 & 18.1 & 33.4 & 84.7 & 147. & 185. & 220 & 261. \\
\hline 60 & 7.56 & 8.86 & 12.3 & 22.6 & 47.9 & 101. & 125. & 145. & 179. \\
\hline 70 & 5.12 & 7.01 & 9.86 & 13.4 & 27.1 & 61.4 & 87.6 & 103. & 137. \\
\hline 75 & 4.46 & 5.49 & 8.26 & 11.2 & 23.1 & 42.9 & 64.9 & 86.5 & 117. \\
\hline 80 & 3.62 & 4.78 & 6.79 & 10.1 & 18.5 & 35.1 & 57.5 & 75.0 & 102. \\
\hline 90 & 2.03 & 2.77 & 3.68 & 6.39 & 12.5 & 24.1 & 33.1 & 40.9 & 66.3 \\
\hline 95 & 1.42 & 2.14 & 2.36 & 4.60 & 8.95 & 17.7 & 22.4 & 27.9 & 41.8 \\
\hline 98 & 0.91 & 1.53 & 1.95 & 3.23 & 7.36 & 13.4 & 18.7 & 22.0 & 36.7 \\
\hline 99 & 0.86 & 1.34 & 1.77 & 2.76 & 6.60 & 12.3 & 16.4 & 20.9 & 35.5 \\
\hline
\end{tabular}




\section{Sandusky River near Mexico, Ohio}

Years analyzed $=55$

Climatic year range $=1923-81$

[APEE, annual percentage equaled or exceeded; $\mathrm{ft}^{3} / \mathrm{s}$, cubic foot per second]

\begin{tabular}{|c|c|c|c|c|c|c|c|c|c|}
\hline \multirow[b]{2}{*}{ APEE } & \multicolumn{9}{|c|}{ Daily mean streamflow $\left(\mathrm{ft}^{3} / \mathrm{s}\right)$ that was equaled or exceeded the indicated percentage of time at APEE level } \\
\hline & 98 & 95 & 90 & 80 & 50 & 20 & 10 & 5 & 2 \\
\hline 1 & 1320 & 2530 & 3720. & 4220. & 6010. & 8120. & 9060. & 10700 & 10900. \\
\hline 2 & 1000 & 1690. & 2590 & 3280 . & 4980. & 6430. & 7110 . & 8570. & 9180. \\
\hline 5 & 545. & 730. & 1160. & 1760. & 2930. & 4100. & 4400 & 4900. & 5930. \\
\hline 10 & 373. & 431. & 589. & 801. & 1380. & 2300 & 2740 & 3170 . & 4190. \\
\hline 20 & 166. & 185. & 249. & 331. & 623. & 1140 . & 1470 & 1660. & 1930. \\
\hline 25 & 82.9 & 141. & 186. & 244. & 499. & 800 . & 1070 . & 1220. & 1520. \\
\hline 30 & 59.7 & 110. & 137. & 175. & 359. & 598. & 779. & 916. & 1170. \\
\hline 40 & 37.4 & 53.6 & 75.4 & 105. & 239. & 387. & 466. & 596. & 726. \\
\hline 50 & 27.0 & 34.9 & 48.0 & 68.2 & 141. & 264. & 332. & 409. & 507. \\
\hline 60 & 21.0 & 26.6 & 34.0 & 49.2 & 94.6 & 173. & 223. & 281. & 317. \\
\hline 70 & 16.6 & 21.0 & 25.6 & 34.4 & 57.4 & 106. & 146. & 195. & 220 \\
\hline 75 & 14.4 & 18.6 & 22.6 & 30.8 & 45.8 & 91.3 & 124. & 154. & 184. \\
\hline 80 & 12.4 & 15.8 & 19.6 & 26.0 & 39.0 & 75.0 & 97.5 & 136. & 141. \\
\hline 90 & 9.00 & 11.4 & 13.2 & 18.0 & 26.6 & 51.9 & 60.5 & 79.9 & 106. \\
\hline 95 & 7.94 & 9.80 & 10.2 & 13.0 & 20.0 & 40.7 & 51.4 & 56.1 & 87.8 \\
\hline 98 & 4.82 & 7.44 & 8.50 & 9.93 & 17.0 & 37.4 & 43.0 & 47.0 & 73.6 \\
\hline 99 & 3.44 & 5.54 & 6.66 & 8.92 & 15.3 & 34.0 & 39.9 & 43.1 & 67.7 \\
\hline
\end{tabular}

\begin{tabular}{|c|c|c|c|c|c|c|c|c|c|}
\hline \multirow[b]{2}{*}{ APEE } & \multicolumn{9}{|c|}{ 7-day mean streamflow ( $\mathrm{ft}^{3} / \mathrm{s}$ ) that was equaled or exceeded the indicated percentage of time at APEE level } \\
\hline & 98 & 95 & 90 & 80 & 50 & 20 & 10 & 5 & 2 \\
\hline 1 & 839. & 1850 & 2440 & 3140. & 4700. & 6380 & 7230 & 8540. & 9800. \\
\hline 2 & 734. & 1400 & 2070 & 2430 & 3570 & 5030. & 5510. & 7140 & 7270 \\
\hline 5 & 468. & 654. & 1250 & 1810. & 2680 & 3650. & 4070 & 4670 & 5440. \\
\hline 10 & 347. & 411. & 601. & 943. & 1630. & 2320 . & 2690 & 3010 . & 3490 . \\
\hline 20 & 176. & 188. & 268. & 391. & 755. & 1350. & 1520 . & 1890. & 2200 \\
\hline 25 & 73.6 & 136. & 199. & 270. & 551. & 1060. & 1300 & 1520 . & 1820 . \\
\hline 30 & 56.7 & 105. & 149. & 195. & 441. & 818. & 1060 & 1260. & 1480. \\
\hline 40 & 37.1 & 52.3 & 75.8 & 116. & 245 & 454. & 650. & 844. & 919. \\
\hline 50 & 29.6 & 36.4 & 48.6 & 72.2 & 156. & 320. & 428 & 543. & 605. \\
\hline 60 & 22.8 & 27.3 & 35.1 & 47.6 & 106. & 204. & 270 & 360. & 397. \\
\hline 70 & 17.7 & 22.1 & 27.1 & 35.2 & 63.7 & 134. & 175. & 229. & 286. \\
\hline 75 & 15.5 & 17.6 & 24.5 & 31.5 & 46.4 & 99.8 & 146. & 200. & 223. \\
\hline 80 & 13.4 & 16.3 & 21.5 & 27.5 & 39.6 & 86.9 & 111. & 176. & 185. \\
\hline 90 & 9.50 & 11.5 & 14.4 & 18.0 & 29.4 & 59.2 & 65.9 & 85.9 & 126. \\
\hline 95 & 7.99 & 9.60 & 11.6 & 13.8 & 20.7 & 45.0 & 55.7 & 58.7 & 103. \\
\hline 98 & 5.76 & 7.88 & 9.30 & 12.3 & 17.6 & 39.7 & 49.2 & 50.8 & 98.3 \\
\hline 99 & 5.09 & 7.01 & 8.00 & 10.0 & 16.9 & 37.1 & 44.9 & 47.7 & 93.7 \\
\hline
\end{tabular}


04197100 Honey Creek at Melmore, Ohio

Years analyzed $=33$

Climatic year range $=1976-2008$

[APEE, annual percentage equaled or exceeded; $\mathrm{ft}^{3} / \mathrm{s}$, cubic foot per second]

\begin{tabular}{|c|c|c|c|c|c|c|c|c|c|}
\hline \multirow[b]{2}{*}{ APEE } & \multicolumn{9}{|c|}{ Daily mean streamflow $\left(\mathrm{ft}^{3} / \mathrm{s}\right)$ that was equaled or exceeded the indicated percentage of time at APEE level } \\
\hline & 95 & 90 & 80 & 70 & 50 & 30 & 20 & 10 & 5 \\
\hline 1 & 604. & 828. & 919. & 1230. & 1610 & 1880. & 2160 & 2700 & 2800 \\
\hline 2 & 381. & 719. & 795. & 930. & 1160 & 1290. & 1600 & 1990. & 2220 \\
\hline 5 & 194. & 326. & 477. & 501. & 678. & 847. & 912. & 1110 & 1250 \\
\hline 10 & 104. & 140 . & 216. & 285. & 340. & 469. & 566. & 718. & 792. \\
\hline 20 & 43.5 & 50.6 & 80.0 & 102. & 130. & 213. & 249. & 290. & 327. \\
\hline 25 & 31.9 & 38.2 & 57.4 & 72.6 & 101. & 155. & 173. & 213. & 228 \\
\hline 30 & 26.0 & 31.4 & 44.5 & 57.5 & 75.2 & 109. & 126. & 151. & 167. \\
\hline 40 & 12.7 & 17.2 & 26.6 & 34.3 & 49.2 & 64.6 & 76.8 & 96.9 & 116. \\
\hline 50 & 5.91 & 9.50 & 16.2 & 20.2 & 30.0 & 42.0 & 51.5 & 60.8 & 82.7 \\
\hline 60 & 3.68 & 5.04 & 9.42 & 13.0 & 20.4 & 31.4 & 37.4 & 41.2 & 65.0 \\
\hline 70 & 1.71 & 2.60 & 3.76 & 7.02 & 10.0 & 20.2 & 24.0 & 30.6 & 47.8 \\
\hline 75 & 1.13 & 1.66 & 2.92 & 4.73 & 8.98 & 13.6 & 20.0 & 26.6 & 39.2 \\
\hline 80 & 0.86 & 1.26 & 2.02 & 2.92 & 6.32 & 9.82 & 16.2 & 22.6 & 31.5 \\
\hline 90 & 0.44 & 0.78 & 1.34 & 1.50 & 3.20 & 5.38 & 7.73 & 14.6 & 17.0 \\
\hline 95 & 0.29 & 0.58 & 1.00 & 1.11 & 2.23 & 3.48 & 4.11 & 10.1 & 12.1 \\
\hline 98 & 0.17 & 0.45 & 0.67 & 0.95 & 1.63 & 2.76 & 3.25 & 6.54 & 9.34 \\
\hline 99 & 0.14 & 0.37 & 0.55 & 0.87 & 1.17 & 2.55 & 2.95 & 4.17 & 7.30 \\
\hline
\end{tabular}

\begin{tabular}{|c|c|c|c|c|c|c|c|c|c|}
\hline \multirow[b]{2}{*}{ APEE } & \multicolumn{9}{|c|}{ 7-day mean streamflow ( $\mathrm{ft}^{3} / \mathrm{s}$ ) that was equaled or exceeded the indicated percentage of time at APEE level } \\
\hline & 95 & 90 & 80 & 70 & 50 & 30 & 20 & 10 & 5 \\
\hline 1 & 422 & 524. & 678. & 786. & 1020. & 1290. & 1450 & 1680 & 1830. \\
\hline 2 & 312. & 416. & 525. & 634. & 817. & 943. & 1310. & 1500 & 1680 \\
\hline 5 & 178. & 344. & 389. & 467. & 633. & 707. & 918. & 1080 & 1160 \\
\hline 10 & 120. & 155 & 268. & 317. & 382. & 496. & 524. & 666. & 803. \\
\hline 20 & 48.1 & 64.3 & 99.6 & 123. & 188. & 276. & 312. & 390. & 419. \\
\hline 25 & 36.6 & 46.1 & 74.7 & 95.8 & 145. & 193. & 245. & 310 . & 335. \\
\hline 30 & 26.3 & 35.6 & 58.0 & 67.4 & 110. & 157. & 189. & 234. & 291. \\
\hline 40 & 14.7 & 17.7 & 34.8 & 41.1 & 65.6 & 98.2 & 118. & 150. & 171. \\
\hline 50 & 6.79 & 10.3 & 18.5 & 23.2 & 36.6 & 54.5 & 74.6 & 96.1 & 112. \\
\hline 60 & 4.16 & 6.05 & 10.0 & 14.7 & 22.7 & 38.4 & 50.7 & 56.2 & 85.5 \\
\hline 70 & 1.98 & 2.85 & 4.07 & 7.47 & 13.6 & 23.7 & 30.2 & 37.5 & 65.0 \\
\hline 75 & 1.21 & 1.91 & 3.35 & 5.31 & 11.0 & 17.3 & 23.9 & 31.9 & 55.9 \\
\hline 80 & 0.90 & 1.36 & 2.39 & 3.20 & 7.01 & 12.1 & 19.2 & 27.3 & 44.8 \\
\hline 90 & 0.47 & 0.81 & 1.52 & 1.82 & 3.53 & 6.14 & 10.8 & 17.8 & 25.5 \\
\hline 95 & 0.30 & 0.62 & 1.06 & 1.25 & 2.36 & 3.91 & 4.76 & 13.5 & 14.3 \\
\hline 98 & 0.19 & 0.51 & 0.79 & 0.98 & 1.90 & 2.98 & 3.83 & 9.12 & 11.6 \\
\hline 99 & 0.16 & 0.47 & 0.64 & 0.93 & 1.63 & 2.70 & 3.23 & 6.07 & 10.4 \\
\hline
\end{tabular}


04197170 Rock Creek at Tiffin, Ohio

Years analyzed $=25$

Climatic year range $=1984-2008$

[APEE, annual percentage equaled or exceeded; $\mathrm{ft}^{3} / \mathrm{s}$, cubic foot per second]

\begin{tabular}{|c|c|c|c|c|c|c|c|c|c|}
\hline \multirow[b]{2}{*}{ APEE } & \multicolumn{9}{|c|}{ Daily mean streamflow (ft³/s) that was equaled or exceeded the indicated percentage of time at APEE level } \\
\hline & 95 & 90 & 80 & 70 & 50 & 30 & 20 & 10 & 5 \\
\hline 1 & 147. & 221. & 347. & 449. & 491. & 590. & 731. & 942. & 1050. \\
\hline 2 & 107. & 133. & 204. & 268. & 345. & 383. & 504. & 608. & 704. \\
\hline 5 & 37.0 & 57.8 & 88.0 & 104. & 139. & 183. & 234. & 259. & 303. \\
\hline 10 & 16.6 & 19.8 & 34.5 & 36.2 & 60.0 & 84.5 & 86.0 & 119. & 149. \\
\hline 20 & 7.40 & 8.61 & 13.4 & 15.9 & 23.0 & 27.0 & 32.1 & 42.5 & 56.0 \\
\hline 25 & 6.27 & 6.88 & 10.2 & 12.8 & 15.5 & 19.6 & 20.8 & 29.6 & 38.6 \\
\hline 30 & 4.91 & 5.68 & 9.20 & 9.94 & 13.0 & 16.0 & 16.2 & 22.1 & 30.7 \\
\hline 40 & 3.13 & 3.54 & 6.18 & 7.00 & 8.32 & 11.0 & 11.0 & 14.0 & 17.8 \\
\hline 50 & 2.20 & 2.44 & 3.22 & 4.95 & 6.60 & 7.76 & 8.81 & 11.0 & 13.0 \\
\hline 60 & 1.73 & 1.86 & 2.32 & 3.33 & 4.38 & 5.41 & 6.12 & 8.72 & 9.85 \\
\hline 70 & 1.29 & 1.55 & 1.88 & 2.36 & 3.20 & 4.04 & 4.34 & 7.18 & 7.72 \\
\hline 75 & 1.19 & 1.40 & 1.70 & 1.99 & 2.80 & 3.28 & 3.76 & 6.57 & 6.81 \\
\hline 80 & 1.03 & 1.20 & 1.44 & 1.70 & 2.40 & 2.90 & 3.28 & 5.79 & 5.98 \\
\hline 90 & 0.77 & 0.88 & 1.10 & 1.28 & 1.80 & 2.12 & 2.54 & 4.02 & 4.44 \\
\hline 95 & 0.56 & 0.68 & 0.93 & 1.15 & 1.40 & 1.90 & 2.15 & 3.03 & 3.63 \\
\hline 98 & 0.39 & 0.47 & 0.78 & 1.00 & 1.13 & 1.53 & 1.93 & 2.44 & 3.27 \\
\hline 99 & 0.35 & 0.43 & 0.72 & 0.92 & 1.10 & 1.37 & 1.66 & 2.12 & 2.95 \\
\hline
\end{tabular}

\begin{tabular}{|c|c|c|c|c|c|c|c|c|c|}
\hline \multirow[b]{2}{*}{ APEE } & \multicolumn{9}{|c|}{ 7-day mean streamflow (ft $\left.{ }^{3} / \mathrm{s}\right)$ that was equaled or exceeded the indicated percentage of time at APEE level } \\
\hline & 95 & 90 & 80 & 70 & 50 & 30 & 20 & 10 & 5 \\
\hline 1 & 106. & 138. & 166. & 192. & 289. & 333. & 364. & 428 & 474. \\
\hline 2 & 65.3 & 90.1 & 125. & 167. & 207. & 256. & 323. & 378. & 419. \\
\hline 5 & 47.3 & 67.5 & 110 & 128. & 154. & 189. & 198. & 262. & 276. \\
\hline 10 & 26.6 & 34.9 & 48.1 & 75.9 & 90.3 & 109. & 126. & 186. & 206. \\
\hline 20 & 8.89 & 11.7 & 17.7 & 26.1 & 35.6 & 51.5 & 70.0 & 76.0 & 98.9 \\
\hline 25 & 7.13 & 8.30 & 15.1 & 21.0 & 28.4 & 38.3 & 46.5 & 60.2 & 79.4 \\
\hline 30 & 5.95 & 6.61 & 12.5 & 14.0 & 21.1 & 28.4 & 33.3 & 52.1 & 65.9 \\
\hline 40 & 3.20 & 3.56 & 7.47 & 8.89 & 12.8 & 16.8 & 17.7 & 29.1 & 34.7 \\
\hline 50 & 2.32 & 2.53 & 4.12 & 5.66 & 9.13 & 10.8 & 11.3 & 16.3 & 22.0 \\
\hline 60 & 1.83 & 1.93 & 2.56 & 3.72 & 4.91 & 7.06 & 8.47 & 12.8 & 17.6 \\
\hline 70 & 1.38 & 1.62 & 2.03 & 2.50 & 3.43 & 4.56 & 5.33 & 9.63 & 12.4 \\
\hline 75 & 1.24 & 1.52 & 1.74 & 2.16 & 2.93 & 3.87 & 4.44 & 7.84 & 9.87 \\
\hline 80 & 1.05 & 1.28 & 1.53 & 1.82 & 2.54 & 3.12 & 3.63 & 6.86 & 7.96 \\
\hline 90 & 0.84 & 0.92 & 1.17 & 1.37 & 1.83 & 2.25 & 2.70 & 4.76 & 5.17 \\
\hline 95 & 0.60 & 0.74 & 0.96 & 1.19 & 1.53 & 1.95 & 2.19 & 3.55 & 4.14 \\
\hline 98 & 0.47 & 0.64 & 0.84 & 1.12 & 1.21 & 1.79 & 2.10 & 2.63 & 3.34 \\
\hline 99 & 0.43 & 0.57 & 0.81 & 1.04 & 1.16 & 1.62 & 1.83 & 2.35 & 3.20 \\
\hline
\end{tabular}




\section{Sandusky River near Fremont, Ohio}

Years analyzed $=81$

Climatic year range $=1924-2008$

[APEE, annual percentage equaled or exceeded; $\mathrm{ft}^{3} / \mathrm{s}$, cubic foot per second]

\begin{tabular}{|c|c|c|c|c|c|c|c|c|c|}
\hline \multirow[b]{2}{*}{ APEE } & \multicolumn{9}{|c|}{ Daily mean streamflow $\left(\mathrm{ft}^{3} / \mathrm{s}\right)$ that was equaled or exceeded the indicated percentage of time at APEE level } \\
\hline & 98 & 95 & 90 & 80 & 50 & 20 & 10 & 5 & 2 \\
\hline 1 & 2670. & 4490. & 6220. & 7320. & 10900. & 14600. & 16800. & 20000 & 22300 \\
\hline 2 & 1780 . & 3270 & 4350 . & 5730 . & 8240. & 10800 . & 13600 . & 15300 . & 16400 \\
\hline 5 & 912. & 1670 . & 2280 & 3290 . & 5060 . & 6840. & 7920. & 9900. & 11000 \\
\hline 10 & 650. & 883. & 1170 . & 1510 . & 2500 & 4080 . & 5040 . & 6030 & 7090. \\
\hline 20 & 283. & 321. & 476. & 634. & 1150. & 1970. & 2600 & 2970 . & 3430 . \\
\hline 25 & 163. & 253. & 343. & 447. & 890. & 1460. & 1980. & 2250 & 2420 . \\
\hline 30 & 121. & 209. & 258. & 358. & 680. & 1100 & 1460. & 1630. & 1860. \\
\hline 40 & 69.3 & 101. & 150 . & 201. & 437. & 684. & 874. & 1010 & 1210. \\
\hline 50 & 48.6 & 65.7 & 81.6 & 120. & 286. & 477. & 564. & 706. & 825. \\
\hline 60 & 36.6 & 45.9 & 59.4 & 85.8 & 199. & 347. & 396. & 506. & 637. \\
\hline 70 & 28.1 & 37.0 & 43.4 & 59.5 & 121. & 229. & 298. & 368. & 486. \\
\hline 75 & 24.6 & 33.5 & 40.2 & 47.8 & 86.0 & 181. & 256. & 307. & 439. \\
\hline 80 & 21.6 & 28.6 & 33.0 & 40.0 & 71.2 & 140. & 212. & 265 & 404. \\
\hline 90 & 12.6 & 21.2 & 24.0 & 28.0 & 46.0 & 78.7 & 118. & 198. & 269. \\
\hline 95 & 9.94 & 13.1 & 17.1 & 22.9 & 39.3 & 66.2 & 88.8 & 132. & 193. \\
\hline 98 & 7.32 & 10.2 & 13.7 & 19.4 & 33.3 & 55.6 & 63.9 & 93.5 & 157. \\
\hline 99 & 6.20 & 10.1 & 12.4 & 17.1 & 30.0 & 49.5 & 59.1 & 89.2 & 140. \\
\hline
\end{tabular}

\begin{tabular}{|c|c|c|c|c|c|c|c|c|c|}
\hline \multirow[b]{2}{*}{ APEE } & \multicolumn{9}{|c|}{ 7-day mean streamflow ( $\mathrm{ft}^{3} / \mathrm{s}$ ) that was equaled or exceeded the indicated percentage of time at APEE level } \\
\hline & 98 & 95 & 90 & 80 & 50 & 20 & 10 & 5 & 2 \\
\hline 1 & 1710 . & 2970. & 4240 & 5230. & 7710 & 10900. & 12700 . & 14400 & 17800 \\
\hline 2 & 1580. & 2290 & 3280 . & 4230. & 6110 & 8280. & 10200 . & 12000 & 13400 \\
\hline 5 & 782. & 1790 & 2320 & 3050 . & 4630 & 6150. & 7210 . & 8760. & 9180. \\
\hline 10 & 560. & 841. & 1140 & 1730. & 2820 & 4150 . & 4800 . & 5570. & 6500. \\
\hline 20 & 280 & 331. & 561. & 755. & 1390. & 2350 & 3000 . & 3570 . & 3940. \\
\hline 25 & 146. & 252. & 376. & 548. & 1080 & 1830. & 2520 & 2890 . & 3200 . \\
\hline 30 & 118. & 221. & 296. & 413. & 816. & 1460. & 1910. & 2340 & 2610. \\
\hline 40 & 67.7 & 122. & 158. & 220. & 518. & 845. & 1240 & 1460. & 1530. \\
\hline 50 & 49.6 & 61.9 & 83.8 & 132. & 314. & 588. & 755 & 891. & 1080. \\
\hline 60 & 38.6 & 48.0 & 58.6 & 92.5 & 217. & 403. & 481. & 640. & 821. \\
\hline 70 & 31.1 & 39.5 & 43.6 & 60.7 & 132. & 261. & 349. & 462. & 624. \\
\hline 75 & 25.7 & 35.4 & 40.0 & 50.4 & 97.9 & 210 & 304. & 401. & 545. \\
\hline 80 & 22.5 & 31.0 & 34.2 & 42.6 & 77.3 & 170 & 245 & 321. & 476. \\
\hline 90 & 12.9 & 21.9 & 24.6 & 28.9 & 49.7 & 87.3 & 134. & 221. & 354. \\
\hline 95 & 11.0 & 15.8 & 18.1 & 24.4 & 40.6 & 69.4 & 97.1 & 138 & 239. \\
\hline 98 & 7.62 & 11.3 & 14.6 & 22.1 & 36.3 & 59.4 & 72.7 & 109. & 179. \\
\hline 99 & 7.00 & 10.9 & 13.3 & 20.8 & 33.9 & 53.7 & 69.4 & 97.9 & 162. \\
\hline
\end{tabular}


04199000 Huron River at Milan, Ohio

Years analyzed $=52$

Climatic year range $=1950-2008$

[APEE, annual percentage equaled or exceeded; $\mathrm{ft}^{3} / \mathrm{s}$, cubic foot per second]

\begin{tabular}{|c|c|c|c|c|c|c|c|c|c|}
\hline \multirow[b]{2}{*}{ APEE } & \multicolumn{9}{|c|}{ Daily mean streamflow $\left(\mathrm{ft}^{3} / \mathrm{s}\right)$ that was equaled or exceeded the indicated percentage of time at APEE level } \\
\hline & 98 & 95 & 90 & 80 & 50 & 20 & 10 & 5 & 2 \\
\hline 1 & 1340 & 1460. & 1990. & 2890 & 4100. & 5610. & 6620. & 7620 . & 8540 \\
\hline 2 & 831. & 1170 & 1400 & 1740 & 2780 & 3960. & 4430 & 5330. & 6110 \\
\hline 5 & 503. & 513. & 618. & 808. & 1390. & 1990. & 2280 & 2920. & 3490. \\
\hline 10 & 212. & 234. & 307. & 430 . & 677. & 1070. & 1310 . & 1580. & 1630. \\
\hline 20 & 69.5 & 128. & 151. & 201. & 334. & 527. & 624. & 797. & 845. \\
\hline 25 & 49.3 & 98.2 & 122. & 144. & 258. & 424. & 507. & 627. & 656. \\
\hline 30 & 34.6 & 82.5 & 92.6 & 116. & 201. & 341. & 403. & 486. & 503. \\
\hline 40 & 20.8 & 49.6 & 55.7 & 71.4 & 129. & 242. & 271. & 326. & 364. \\
\hline 50 & 14.8 & 29.0 & 35.5 & 45.6 & 90.0 & 163. & 190. & 237. & 257. \\
\hline 60 & 12.5 & 20.6 & 22.6 & 33.0 & 58.5 & 113. & 151. & 172. & 180. \\
\hline 70 & 10.2 & 15.8 & 19.0 & 24.9 & 39.5 & 72.7 & 86.0 & 125. & 141. \\
\hline 75 & 9.25 & 13.3 & 16.8 & 21.3 & 33.5 & 55.8 & 66.0 & 106. & 126. \\
\hline 80 & 7.99 & 11.7 & 15.0 & 17.1 & 28.0 & 47.5 & 58.2 & 93.0 & 110. \\
\hline 90 & 5.64 & 7.90 & 9.60 & 11.6 & 18.5 & 34.2 & 39.9 & 65.1 & 65.6 \\
\hline 95 & 5.02 & 5.56 & 6.55 & 9.02 & 15.0 & 25.2 & 33.8 & 43.7 & 50.1 \\
\hline 98 & 3.65 & 4.50 & 5.11 & 7.16 & 12.5 & 21.4 & 28.7 & 31.9 & 40.8 \\
\hline 99 & 3.18 & 4.04 & 4.49 & 6.30 & 11.0 & 20.0 & 24.7 & 29.2 & 37.2 \\
\hline
\end{tabular}

\begin{tabular}{|c|c|c|c|c|c|c|c|c|c|}
\hline \multirow[b]{2}{*}{ APEE } & \multicolumn{9}{|c|}{ 7-day mean streamflow (ft $\left.{ }^{3} / s\right)$ that was equaled or exceeded the indicated percentage of time at APEE level } \\
\hline & 98 & 95 & 90 & 80 & 50 & 20 & 10 & 5 & 2 \\
\hline 1 & 952. & 1190. & 1400 & 1710 . & 2340 & 3190 & 3980. & 4260 & 6450 \\
\hline 5 & 478. & 509. & 687. & 1010. & 1340. & 1880. & 2250 & 2460 & 2630 \\
\hline 10 & 301. & 331. & 354. & 495. & 888. & 1300. & 1510 . & 1750 & 2020 . \\
\hline 30 & 36.2 & 88.5 & 106. & 138. & 259. & 462. & 567. & 660. & 728. \\
\hline 40 & 21.1 & 59.0 & 66.8 & 83.6 & 167. & 332. & 402. & 464. & 544. \\
\hline 50 & 14.8 & 30.0 & 38.0 & 52.0 & 112. & 207. & 273. & 337. & 368. \\
\hline 60 & 13.1 & 21.6 & 26.4 & 38.6 & 68.3 & 150. & 185. & 233. & 258. \\
\hline 90 & 5.84 & 8.65 & 10.3 & 12.0 & 21.0 & 38.7 & 46.3 & 77.3 & 95.7 \\
\hline 95 & 5.39 & 5.79 & 7.28 & 9.60 & 16.2 & 30.3 & 38.0 & 52.4 & 61.1 \\
\hline 98 & 4.02 & 4.66 & 5.75 & 8.58 & 12.9 & 25.5 & 34.3 & 40.2 & 48.3 \\
\hline 99 & 3.88 & 4.49 & 5.11 & 7.57 & 12.4 & 23.3 & 30.0 & 34.9 & 47.2 \\
\hline
\end{tabular}


04199500 Vermilion River near Vermilion, Ohio

Years analyzed $=39$

Climatic year range $=1950-2008$

[APEE, annual percentage equaled or exceeded; $\mathrm{ft}^{3} / \mathrm{s}$, cubic foot per second]

\begin{tabular}{|c|c|c|c|c|c|c|c|c|c|}
\hline \multirow[b]{2}{*}{ APEE } & \multicolumn{9}{|c|}{ Daily mean streamflow $\left(\mathrm{ft}^{3} / \mathrm{s}\right)$ that was equaled or exceeded the indicated percentage of time at APEE level } \\
\hline & 95 & 90 & 80 & 70 & 50 & 30 & 20 & 10 & 5 \\
\hline 1 & 1500. & 2240. & 2410. & 2620. & 3280. & 3610. & 4470. & 5580. & 7650. \\
\hline 2 & 763. & 1250 & 1580 . & 1890. & 2230 & 2490 & 2830 & 4350 . & 5740. \\
\hline 5 & 320 . & 520. & 722 . & 893. & 1240 & 1310 . & 1710 . & 2250 & 2710 \\
\hline 10 & 168. & 254. & 312. & 495. & 582. & 860. & 1020. & 1150 & 1220 \\
\hline 20 & 83.6 & 117. & 145 & 208. & 269. & 360. & 411. & 532. & 625. \\
\hline 25 & 57.5 & 94.0 & 104. & 151. & 200 & 264. & 309. & 421. & 455. \\
\hline 30 & 45.2 & 65.2 & 74.0 & 112. & 153. & 213. & 234. & 341. & 361. \\
\hline 40 & 22.6 & 29.2 & 47.6 & 60.2 & 98.8 & 130. & 152. & 202. & 254. \\
\hline 50 & 12.0 & 17.0 & 29.0 & 39.0 & 62.5 & 95.0 & 111. & 137. & 170 . \\
\hline 60 & 8.00 & 8.04 & 14.4 & 23.0 & 39.0 & 63.0 & 76.4 & 101. & 120. \\
\hline 70 & 3.30 & 4.88 & 8.21 & 15.0 & 22.0 & 41.0 & 46.8 & 60.6 & 72.8 \\
\hline 75 & 2.55 & 3.85 & 5.55 & 10.0 & 15.5 & 28.5 & 33.0 & 42.5 & 62.8 \\
\hline 80 & 1.20 & 3.06 & 4.30 & 7.42 & 11.2 & 20.0 & 26.0 & 35.0 & 56.0 \\
\hline 90 & 0.07 & 1.00 & 2.26 & 3.00 & 5.10 & 11.0 & 13.6 & 20.7 & 28.0 \\
\hline 95 & 0.00 & 0.30 & 1.24 & 1.94 & 3.40 & 7.72 & 9.20 & 14.0 & 16.4 \\
\hline 98 & 0.00 & 0.10 & 0.70 & 1.20 & 2.30 & 5.50 & 6.93 & 9.63 & 11.0 \\
\hline 99 & 0.00 & 0.10 & 0.50 & 0.95 & 2.03 & 4.73 & 6.16 & 8.87 & 9.87 \\
\hline
\end{tabular}

\begin{tabular}{|c|c|c|c|c|c|c|c|c|c|}
\hline \multirow[b]{2}{*}{ APEE } & \multicolumn{9}{|c|}{ 7-day mean streamflow ( $\mathrm{ft}^{3} / \mathrm{s}$ ) that was equaled or exceeded the indicated percentage of time at APEE level } \\
\hline & 95 & 90 & 80 & 70 & 50 & 30 & 20 & 10 & 5 \\
\hline 1 & 1190. & 1220 & 1550. & 1640 & 1990. & 2380 & 3090. & 3490. & 7260 \\
\hline 2 & 830 . & 898. & 1140 & 1330. & 1520 . & 1950. & 2260 & 3030 . & 3410 \\
\hline 5 & 319. & 580. & 813. & 947. & 1150 & 1210 & 1300 & 2030 & 2510 \\
\hline 10 & 227. & 265. & 374. & 516. & 640. & 849. & 1040. & 1510 & 1570 \\
\hline 20 & 85.3 & 126. & 175. & 236. & 358. & 543. & 614. & 719. & 752. \\
\hline 25 & 68.3 & 96.6 & 132. & 180. & 254. & 411. & 481. & 567. & 613. \\
\hline 30 & 47.6 & 65.6 & 89.6 & 146. & 198. & 294. & 388. & 447. & 478 \\
\hline 40 & 25.6 & 29.9 & 50.4 & 72.6 & 125. & 187. & 216. & 290. & 334. \\
\hline 50 & 13.9 & 19.6 & 31.4 & 45.4 & 76.6 & 114. & 145 & 195. & 208. \\
\hline 60 & 8.43 & 9.97 & 16.4 & 28.9 & 48.0 & 86.6 & 98.7 & 132. & 139. \\
\hline 70 & 3.61 & 4.89 & 10.2 & 15.3 & 25.7 & 52.9 & 61.4 & 78.4 & 103. \\
\hline 75 & 2.43 & 3.97 & 6.89 & 10.6 & 18.4 & 36.1 & 42.9 & 55.3 & 78.7 \\
\hline 80 & 1.51 & 3.29 & 4.94 & 8.67 & 13.4 & 22.7 & 30.8 & 42.0 & 63.7 \\
\hline 90 & 0.19 & 1.44 & 2.63 & 3.64 & 6.65 & 14.1 & 16.4 & 25.5 & 45.5 \\
\hline 95 & 0.00 & 0.39 & 1.68 & 2.14 & 3.84 & 8.71 & 11.3 & 17.4 & 21.4 \\
\hline 98 & 0.00 & 0.19 & 0.99 & 1.47 & 2.60 & 6.67 & 8.19 & 12.7 & 15.3 \\
\hline 99 & 0.00 & 0.11 & 0.96 & 1.11 & 2.41 & 5.81 & 6.97 & 11.3 & 13.4 \\
\hline
\end{tabular}


04207200 Tinkers Creek at Bedford, Ohio

Years analyzed $=46$

Climatic year range $=1963-2008$

[APEE, annual percentage equaled or exceeded; $\mathrm{ft}^{3} / \mathrm{s}$, cubic foot per second]

\begin{tabular}{|c|c|c|c|c|c|c|c|c|c|}
\hline \multirow[b]{2}{*}{ APEE } & \multicolumn{9}{|c|}{ Daily mean streamflow $\left(\mathrm{ft}^{3} / \mathrm{s}\right)$ that was equaled or exceeded the indicated percentage of time at APEE level } \\
\hline & 95 & 90 & 80 & 70 & 50 & 30 & 20 & 10 & 5 \\
\hline 1 & 641. & 735. & 815. & 941. & 1140. & 1230. & 1280. & 1430. & 1540 \\
\hline 2 & 557. & 573. & 634. & 718. & 800 . & 918. & 957. & 1030 & 1130. \\
\hline 5 & 289. & 349. & 400 & 435. & 490. & 595. & 644. & 739. & 826. \\
\hline 10 & 189. & 208. & 253. & 279 & 307. & 381. & 436. & 475. & 519. \\
\hline 20 & 105. & 118. & 129. & 143. & 175. & 209. & 236. & 264. & 283. \\
\hline 25 & 88.0 & 93.3 & 99.7 & 120. & 135. & 166. & 180. & 206. & 215. \\
\hline 30 & 70.3 & 75.9 & 85.0 & 96.3 & 115. & 140. & 149. & 178. & 181. \\
\hline 40 & 42.4 & 54.6 & 61.4 & 68.9 & 86.8 & 100. & 112. & 134. & 140. \\
\hline 50 & 25.4 & 41.6 & 48.1 & 55.1 & 70.0 & 77.8 & 85.0 & 105. & 113. \\
\hline 60 & 17.7 & 28.8 & 38.9 & 43.0 & 53.0 & 61.8 & 68.4 & 81.0 & 90.1 \\
\hline 70 & 14.1 & 21.5 & 32.0 & 35.0 & 41.0 & 49.9 & 53.0 & 60.6 & 66.0 \\
\hline 75 & 11.7 & 18.5 & 28.8 & 30.1 & 35.4 & 43.0 & 46.3 & 52.2 & 58.2 \\
\hline 80 & 10.3 & 15.1 & 24.4 & 29.0 & 31.6 & 37.0 & 39.7 & 46.7 & 49.7 \\
\hline 90 & 8.86 & 11.0 & 19.0 & 21.6 & 25.0 & 27.9 & 30.6 & 35.0 & 36.5 \\
\hline 95 & 7.80 & 10.4 & 17.0 & 19.0 & 22.0 & 24.9 & 26.7 & 29.2 & 31.0 \\
\hline 98 & 7.20 & 9.12 & 14.4 & 16.1 & 20.0 & 22.0 & 23.0 & 26.3 & 27.6 \\
\hline 99 & 6.96 & 8.76 & 13.4 & 15.2 & 18.8 & 21.6 & 22.6 & 25.3 & 26.6 \\
\hline
\end{tabular}

\begin{tabular}{|c|c|c|c|c|c|c|c|c|c|}
\hline \multirow[b]{2}{*}{ APEE } & \multicolumn{9}{|c|}{ 7-day mean streamflow ( $\mathrm{ft}^{3} / \mathrm{s}$ ) that was equaled or exceeded the indicated percentage of time at APEE level } \\
\hline & 95 & 90 & 80 & 70 & 50 & 30 & 20 & 10 & 5 \\
\hline 1 & 411. & 473. & 550. & 606. & 703. & 814. & 852. & 981. & 1070 \\
\hline 2 & 372. & 429. & 464. & 497. & 583. & 637. & 704. & 778. & 835. \\
\hline 5 & 252 & 300. & 318 & 359. & 421 & 487. & 520. & 569. & 619. \\
\hline 10 & 174. & 210 & 241. & 264. & 306. & 355. & 403. & 424. & 464. \\
\hline 20 & 115. & 124. & 157. & 164. & 200. & 235. & 278. & 306. & 321. \\
\hline 25 & 97.9 & 108. & 119. & 135. & 158. & 200. & 222. & 264. & 293. \\
\hline 30 & 77.8 & 84.4 & 99.5 & 116. & 137. & 169. & 190. & 219. & 244. \\
\hline 40 & 46.4 & 57.6 & 71.5 & 86.5 & 101. & 127. & 144. & 171. & 194. \\
\hline 50 & 28.4 & 47.2 & 56.1 & 65.4 & 82.0 & 95.8 & 105. & 135 & 143. \\
\hline 60 & 18.7 & 35.7 & 46.4 & 51.9 & 63.0 & 73.5 & 80.6 & 106. & 114. \\
\hline 70 & 15.6 & 26.2 & 37.2 & 42.3 & 49.6 & 61.6 & 65.5 & 81.3 & 83.2 \\
\hline 75 & 13.9 & 22.0 & 33.3 & 37.7 & 43.5 & 54.2 & 57.5 & 67.6 & 71.0 \\
\hline 80 & 12.4 & 18.8 & 29.1 & 33.0 & 38.6 & 46.4 & 52.1 & 57.6 & 63.2 \\
\hline 90 & 10.5 & 14.0 & 22.9 & 25.7 & 29.6 & 35.7 & 39.3 & 44.6 & 45.9 \\
\hline 95 & 9.25 & 11.7 & 18.5 & 21.9 & 25.8 & 30.0 & 31.1 & 35.4 & 36.6 \\
\hline 98 & 7.65 & 10.7 & 16.0 & 18.4 & 23.5 & 26.1 & 28.5 & 31.3 & 32.4 \\
\hline 99 & 7.24 & 9.71 & 14.9 & 17.6 & 21.9 & 24.0 & 25.7 & 28.7 & 29.8 \\
\hline
\end{tabular}




\section{Rock Creek near Rock Creek, Ohio}

Years analyzed $=24$

Climatic year range $=1942-65$

[APEE, annual percentage equaled or exceeded; $\mathrm{ft}^{3} / \mathrm{s}$, cubic foot per second]

\begin{tabular}{|c|c|c|c|c|c|c|c|c|c|}
\hline \multirow[b]{2}{*}{ APEE } & \multicolumn{9}{|c|}{ Daily mean streamflow $\left(\mathrm{ft}^{3} / \mathrm{s}\right)$ that was equaled or exceeded the indicated percentage of time at APEE level } \\
\hline & 95 & 90 & 80 & 70 & 50 & 30 & 20 & 10 & 5 \\
\hline 1 & 500. & 542. & 698. & 784. & 931. & 1160. & 1260. & 1410 & 1490. \\
\hline 2 & 249 & 353. & 505. & 570. & 695. & 810 . & 921. & 973. & 981. \\
\hline 5 & 161. & 181. & 287. & 336. & 408 & 452. & 560. & 608. & 652. \\
\hline 10 & 74.5 & 103. & 147. & 157. & 205 & 243. & 300 . & 344. & 365. \\
\hline 20 & 26.4 & 34.1 & 53.6 & 61.3 & 86.9 & 105. & 119. & 133. & 158. \\
\hline 25 & 18.3 & 22.8 & 36.0 & 41.6 & 60.8 & 73.8 & 81.0 & 99.4 & 116. \\
\hline 30 & 14.0 & 14.6 & 24.2 & 29.4 & 42.6 & 56.0 & 63.4 & 76.5 & 89.0 \\
\hline 40 & 5.28 & 9.37 & 12.0 & 12.5 & 23.5 & 35.0 & 38.2 & 45.0 & 50.4 \\
\hline 50 & 2.63 & 3.20 & 5.35 & 6.50 & 10.3 & 18.5 & 21.0 & 28.0 & 31.0 \\
\hline 60 & 0.63 & 1.10 & 1.30 & 2.44 & 4.28 & 9.98 & 16.0 & 18.5 & 19.0 \\
\hline 70 & 0.00 & 0.05 & 0.30 & 0.95 & 1.94 & 2.77 & 5.80 & 10.1 & 11.6 \\
\hline 75 & 0.00 & 0.00 & 0.20 & 0.28 & 0.93 & 1.40 & 4.00 & 7.18 & 8.39 \\
\hline 80 & 0.00 & 0.00 & 0.00 & 0.00 & 0.60 & 0.85 & 2.60 & 4.80 & 5.57 \\
\hline 90 & 0.00 & 0.00 & 0.00 & 0.00 & 0.15 & 0.30 & 1.00 & 2.65 & 3.99 \\
\hline 95 & 0.00 & 0.00 & 0.00 & 0.00 & 0.00 & 0.15 & 0.30 & 1.71 & 2.98 \\
\hline 98 & 0.00 & 0.00 & 0.00 & 0.00 & 0.00 & 0.02 & 0.10 & 1.05 & 2.34 \\
\hline 99 & 0.00 & 0.00 & 0.00 & 0.00 & 0.00 & 0.00 & 0.07 & 0.68 & 2.22 \\
\hline
\end{tabular}

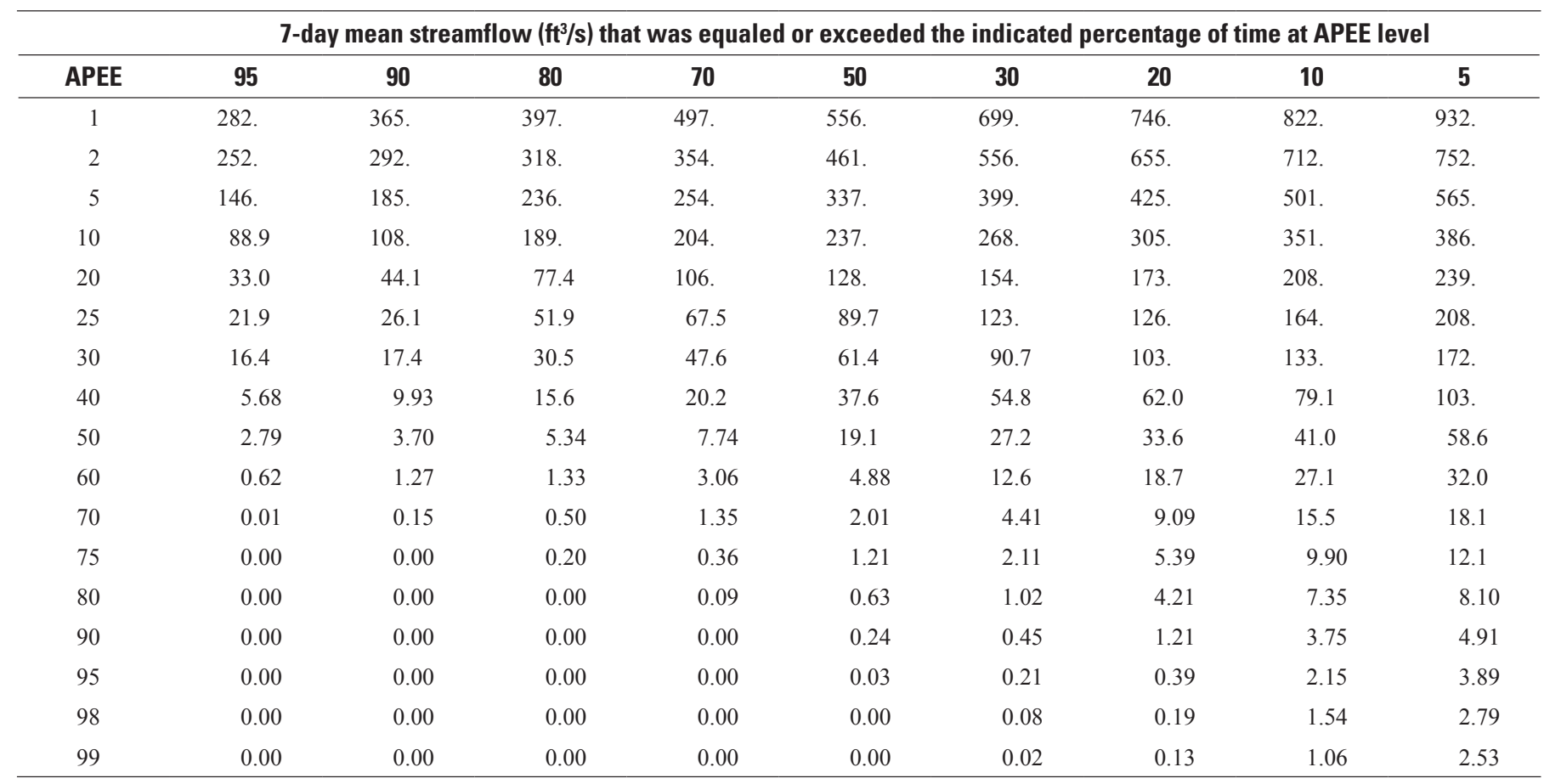




\section{Grand River near Madison, Ohio}

Years analyzed $=48$

Climatic year range $=1923-73$

[APEE, annual percentage equaled or exceeded; $\mathrm{ft}^{3} / \mathrm{s}$, cubic foot per second]

\begin{tabular}{|c|c|c|c|c|c|c|c|c|c|}
\hline \multirow[b]{2}{*}{ APEE } & \multicolumn{9}{|c|}{ Daily mean streamflow $\left(\mathrm{ft}^{3} / \mathrm{s}\right)$ that was equaled or exceeded the indicated percentage of time at APEE level } \\
\hline & 95 & 90 & 80 & 70 & 50 & 30 & 20 & 10 & 5 \\
\hline 1 & 3720. & 4300. & 4970. & 5370. & 6250 & 6690. & 7330 . & 7740 & 8590. \\
\hline 2 & 2680 & 3160 . & 3660. & 4180. & 4830 . & 5190. & 5550. & 6360. & 6780. \\
\hline 5 & 1450 & 1690. & 2370 & 2630 & 3090 . & 3510. & 3630. & 4560. & 4690. \\
\hline 10 & 908. & 1080 & 1320. & 1620. & 2010 & 2300 & 2590 & 2790 & 2890 \\
\hline 20 & 395. & 417. & 639. & 749. & 1010. & 1220 . & 1420 . & 1600 & 1710 . \\
\hline 25 & 242. & 317. & 398. & 516. & 673. & 932. & 1020. & 1290. & 1330. \\
\hline 30 & 158. & 208. & 271. & 368. & 513. & 689. & 796. & 955. & 1010. \\
\hline 40 & 82.1 & 109. & 144. & 200 & 295. & 388. & 459. & 580. & 625. \\
\hline 50 & 48.7 & 59.9 & 79.8 & 93.2 & 173. & 252 & 294. & 393. & 432. \\
\hline 60 & 26.4 & 27.9 & 40.0 & 48.0 & 73.2 & 159. & 184. & 255. & 277. \\
\hline 70 & 13.9 & 15.0 & 18.9 & 24.6 & 35.9 & 76.7 & 103. & 134. & 187. \\
\hline 75 & 9.89 & 12.0 & 15.8 & 17.9 & 25.4 & 49.3 & 70.4 & 108. & 146. \\
\hline 80 & 8.16 & 9.98 & 12.0 & 12.1 & 19.5 & 32.0 & 47.8 & 80.1 & 94.8 \\
\hline 90 & 3.26 & 4.64 & 6.36 & 7.52 & 10.5 & 16.5 & 22.8 & 31.0 & 43.0 \\
\hline 95 & 1.20 & 2.10 & 4.32 & 4.74 & 8.30 & 12.0 & 14.6 & 23.1 & 26.6 \\
\hline 98 & 0.42 & 1.20 & 2.14 & 3.14 & 6.90 & 9.40 & 11.1 & 17.2 & 24.2 \\
\hline 99 & 0.17 & 0.77 & 1.59 & 2.60 & 5.33 & 9.05 & 10.0 & 15.1 & 22.6 \\
\hline
\end{tabular}

\begin{tabular}{|c|c|c|c|c|c|c|c|c|c|}
\hline \multirow[b]{2}{*}{ APEE } & \multicolumn{9}{|c|}{ 7-day mean streamflow (ft $\left.{ }^{3} / \mathrm{s}\right)$ that was equaled or exceeded the indicated percentage of time at APEE level } \\
\hline & 95 & 90 & 80 & 70 & 50 & 30 & 20 & 10 & 5 \\
\hline 1 & 2390 & 2700 & 3180. & 3670. & 4270 & 4770 . & 5510. & 6160 & 6360 \\
\hline 2 & 1990. & 2280 & 2710 & 3040 . & 3580 . & 4110 & 4320 . & 5010. & 5160. \\
\hline 5 & 1410 & 1710 . & 2060 & 2420 & 2670 & 3060 . & 3210 & 3820 . & 3970. \\
\hline 10 & 948. & 1080 & 1390. & 1740 . & 2000 & 2210 & 2390 & 2600 & 2890 \\
\hline 20 & 460. & 546. & 720 . & 949. & 1170 & 1330. & 1550 & 1770 . & 1950. \\
\hline 25 & 282. & 357. & 522. & 661. & 897. & 1100. & 1200 & 1520. & 1620. \\
\hline 30 & 181. & 241. & 368. & 493. & 648. & 896. & 1010 & 1220 & 1340. \\
\hline 40 & 89.8 & 109. & 192. & 236. & 352. & 514. & 623. & 750. & 914. \\
\hline 50 & 50.7 & 61.5 & 86.0 & 99.8 & 193. & 310. & 347. & 521. & 601. \\
\hline 60 & 24.9 & 27.8 & 43.5 & 50.6 & 87.3 & 190. & 212. & 350. & 376. \\
\hline 70 & 13.5 & 15.3 & 21.6 & 25.9 & 38.0 & 100. & 130. & 161. & 239. \\
\hline 75 & 10.4 & 12.1 & 16.8 & 19.0 & 30.0 & 58.5 & 74.7 & 125. & 177. \\
\hline 80 & 8.51 & 9.62 & 12.6 & 13.6 & 22.0 & 38.0 & 59.1 & 95.5 & 131. \\
\hline 90 & 4.17 & 5.26 & 6.97 & 7.78 & 11.8 & 18.7 & 26.2 & 34.7 & 47.8 \\
\hline 95 & 1.88 & 2.98 & 4.89 & 5.67 & 8.88 & 12.8 & 16.1 & 25.2 & 29.1 \\
\hline 98 & 0.99 & 1.39 & 2.67 & 4.14 & 7.23 & 10.5 & 12.2 & 21.4 & 24.8 \\
\hline 99 & 0.36 & 1.04 & 2.01 & 3.44 & 6.31 & 9.99 & 11.3 & 17.9 & 23.9 \\
\hline
\end{tabular}




\section{Grand River near Painesville, Ohio}

Years analyzed $=34$

Climatic year range $=1975-2008$

[APEE, annual percentage equaled or exceeded; $\mathrm{ft}^{3} / \mathrm{s}$, cubic foot per second]

\begin{tabular}{|c|c|c|c|c|c|c|c|c|c|}
\hline \multirow[b]{2}{*}{ APEE } & \multicolumn{9}{|c|}{ Daily mean streamflow $\left(\mathrm{ft}^{3} / \mathrm{s}\right)$ that was equaled or exceeded the indicated percentage of time at APEE level } \\
\hline & 95 & 90 & 80 & 70 & 50 & 30 & 20 & 10 & 5 \\
\hline 1 & 5160. & 5600. & 6480. & 7030. & 7950. & 9580. & 10000. & 10300 & 10600 \\
\hline 2 & 3890. & 4350 & 5270. & 5470. & 6140 & 6900. & 7640 . & 8160. & 8880 \\
\hline 5 & 2350 & 2620 & 3080 . & 3580 . & 3960. & 4760 . & 5190. & 5730. & 6220 \\
\hline 10 & 1470 . & 1730 . & 1990. & 2240 & 2690 & 3340 . & 3530. & 3940 . & 4170 \\
\hline 20 & 822. & 914. & 1020. & 1220 & 1410. & 1900. & 2160 & 2350 & 2730 \\
\hline 25 & 603. & 699. & 786. & 970. & 1090. & 1550 & 1750 . & 1890. & 2210 \\
\hline 30 & 379. & 488. & 669. & 732. & 863. & 1100 & 1390. & 1610 . & 1870. \\
\hline 40 & 202. & 289. & 373. & 471. & 596. & 699. & 921. & 1170 . & 1320. \\
\hline 50 & 119. & 181. & 223. & 332. & 410. & 503. & 640. & 841. & 975. \\
\hline 60 & 60.4 & 104. & 151. & 204. & 264. & 323. & 412. & 583. & 629. \\
\hline 70 & 42.3 & 62.4 & 89.8 & 109. & 156. & 218 & 243. & 356. & 438. \\
\hline 75 & 30.1 & 38.3 & 70.0 & 78.0 & 109. & 174. & 202. & 284. & 327. \\
\hline 80 & 22.8 & 29.6 & 43.0 & 56.7 & 87.7 & 130. & 165. & 205 & 234 \\
\hline 90 & 11.7 & 14.5 & 25.0 & 29.0 & 50.5 & 73.3 & 82.4 & 95.8 & 115. \\
\hline 95 & 8.71 & 10.4 & 17.0 & 20.3 & 35.1 & 44.3 & 51.6 & 67.7 & 76.5 \\
\hline 98 & 6.33 & 7.70 & 13.0 & 15.3 & 26.2 & 35.7 & 38.3 & 51.6 & 58.2 \\
\hline 99 & 5.04 & 7.23 & 11.7 & 13.7 & 22.8 & 30.6 & 32.7 & 47.5 & 53.9 \\
\hline
\end{tabular}

\begin{tabular}{|c|c|c|c|c|c|c|c|c|c|}
\hline APEE & \multicolumn{9}{|c|}{ 7-day mean streamflow ( $\left.\mathrm{ft}^{3} / \mathrm{s}\right)$ that was equaled or exceeded the indicated percentage of time at APEE level } \\
\hline 1 & 3000. & 3550. & 4230. & 4860. & 6620. & 7310. & 7540. & 7920. & 8740. \\
\hline 5 & 2180. & 2410. & 2830. & 3130. & 3340. & 3770. & 4380 . & 4770 . & 5400 \\
\hline 10 & 1350 & 1840. & 2040 & 2250 & 2580 & 3070 . & 3340 . & 3570 . & 3950. \\
\hline 30 & 396. & 598. & 762. & 859. & 1070 . & 1290. & 1650. & 1810 . & 2240 \\
\hline 40 & 201. & 365. & 435. & 562. & 752. & 880 . & 1150 . & 1370 . & 1700 \\
\hline 50 & 116. & 195. & 266. & 400. & 482. & 586. & 842. & 1050. & 1190. \\
\hline 60 & 57.3 & 113. & 176. & 235. & 321. & 406. & 470. & 779. & 877. \\
\hline 90 & 13.7 & 17.1 & 26.6 & 32.3 & 57.2 & 84.0 & 114. & 136. & 154. \\
\hline 95 & 10.3 & 12.0 & 17.6 & 22.7 & 40.9 & 54.5 & 65.6 & 76.2 & 90.1 \\
\hline 98 & 7.76 & 9.46 & 14.6 & 18.7 & 31.1 & 45.1 & 54.6 & 58.1 & 71.9 \\
\hline 99 & 6.35 & 7.71 & 13.1 & 15.2 & 27.6 & 36.9 & 41.3 & 52.8 & 68.3 \\
\hline
\end{tabular}


04212500 Ashtabula River near Ashtabula, Ohio

Years analyzed $=47$

Climatic year range $=1925-78$

[APEE, annual percentage equaled or exceeded; $\mathrm{ft}^{3} / \mathrm{s}$, cubic foot per second]

\begin{tabular}{|c|c|c|c|c|c|c|c|c|c|}
\hline \multirow[b]{2}{*}{ APEE } & \multicolumn{9}{|c|}{ Daily mean streamflow $\left(\mathrm{ft}^{3} / \mathrm{s}\right)$ that was equaled or exceeded the indicated percentage of time at APEE level } \\
\hline & 95 & 90 & 80 & 70 & 50 & 30 & 20 & 10 & 5 \\
\hline 1 & 960. & 1110 & 1340. & 1470. & 1690. & 1930. & 2130 & 2600 & 2670 \\
\hline 2 & 710 . & 756. & 940. & 1110 & 1250. & 1440. & 1540 & 1690. & 1820 \\
\hline 5 & 416. & 447. & 532. & 617. & 743. & 857. & 936. & 1010. & 1080 \\
\hline 10 & 207. & 237. & 270 & 350 . & 395. & 481. & 501. & 551. & 643. \\
\hline 20 & 82.0 & 103. & 132. & 147. & 196. & 234. & 258. & 288. & 335. \\
\hline 25 & 60.8 & 64.5 & 90.5 & 106. & 135. & 171. & 192. & 217. & 237. \\
\hline 30 & 46.1 & 49.6 & 67.1 & 78.2 & 110. & 132. & 144. & 170. & 191. \\
\hline 40 & 22.0 & 30.8 & 36.8 & 45.2 & 70.0 & 84.2 & 97.0 & 119. & 125. \\
\hline 50 & 12.0 & 13.0 & 18.2 & 28.0 & 43.0 & 55.0 & 65.0 & 76.4 & 87.6 \\
\hline 60 & 4.28 & 4.78 & 6.75 & 8.24 & 21.0 & 32.4 & 42.4 & 48.2 & 56.0 \\
\hline 70 & 0.95 & 1.48 & 2.00 & 3.12 & 9.00 & 16.5 & 20.4 & 31.2 & 37.3 \\
\hline 75 & 0.18 & 0.62 & 1.16 & 1.50 & 4.00 & 10.0 & 13.0 & 24.0 & 26.8 \\
\hline 80 & 0.04 & 0.28 & 0.47 & 0.80 & 2.04 & 6.66 & 8.08 & 17.0 & 19.8 \\
\hline 90 & 0.00 & 0.00 & 0.00 & 0.12 & 0.50 & 1.62 & 2.72 & 6.52 & 8.14 \\
\hline 95 & 0.00 & 0.00 & 0.00 & 0.00 & 0.20 & 0.86 & 1.56 & 3.50 & 4.73 \\
\hline 98 & 0.00 & 0.00 & 0.00 & 0.00 & 0.00 & 0.49 & 1.04 & 2.55 & 3.44 \\
\hline 99 & 0.00 & 0.00 & 0.00 & 0.00 & 0.00 & 0.41 & 0.76 & 1.89 & 2.80 \\
\hline
\end{tabular}

\begin{tabular}{|c|c|c|c|c|c|c|c|c|c|}
\hline \multirow[b]{2}{*}{ APEE } & \multicolumn{9}{|c|}{ 7-day mean streamflow ( $\mathrm{ft}^{3} / \mathrm{s}$ ) that was equaled or exceeded the indicated percentage of time at APEE level } \\
\hline & 95 & 90 & 80 & 70 & 50 & 30 & 20 & 10 & 5 \\
\hline 1 & 536. & 668. & 736. & 834. & 1080. & 1230 & 1350. & 1500 & 1550. \\
\hline 2 & 469. & 566. & 657. & 685. & 813. & 930. & 994. & 1130 & 1210 \\
\hline 5 & 335. & 408 & 482 & 541. & 609. & 682. & 729. & 870. & 892. \\
\hline 10 & 243. & 285. & 322. & 398. & 432. & 488. & 528. & 618. & 632. \\
\hline 20 & 120. & 129. & 177. & 219. & 259. & 317. & 354. & 399. & 443. \\
\hline 25 & 82.9 & 98.7 & 140. & 164. & 212. & 257. & 281. & 314. & 351. \\
\hline 30 & 50.3 & 59.3 & 105 & 123. & 160. & 198. & 238. & 268. & 287. \\
\hline 40 & 25.7 & 34.6 & 51.3 & 63.0 & 92.9 & 111. & 147. & 195. & 205. \\
\hline 50 & 14.4 & 15.4 & 23.4 & 33.9 & 53.4 & 67.5 & 84.4 & 115. & 124. \\
\hline 60 & 4.48 & 5.12 & 7.42 & 9.19 & 26.8 & 41.9 & 51.9 & 76.6 & 80.9 \\
\hline 70 & 0.96 & 1.54 & 2.43 & 3.25 & 10.2 & 20.3 & 31.4 & 42.9 & 54.3 \\
\hline 75 & 0.31 & 0.77 & 1.37 & 2.02 & 4.73 & 14.1 & 21.0 & 32.9 & 43.3 \\
\hline 80 & 0.10 & 0.32 & 0.64 & 0.98 & 2.66 & 9.62 & 13.0 & 24.1 & 29.8 \\
\hline 90 & 0.00 & 0.00 & 0.06 & 0.20 & 0.61 & 1.88 & 3.31 & 8.71 & 10.0 \\
\hline 95 & 0.00 & 0.00 & 0.00 & 0.03 & 0.35 & 0.89 & 1.91 & 4.20 & 6.28 \\
\hline 98 & 0.00 & 0.00 & 0.00 & 0.00 & 0.06 & 0.67 & 1.27 & 3.58 & 4.12 \\
\hline 99 & 0.00 & 0.00 & 0.00 & 0.00 & 0.01 & 0.57 & 1.11 & 2.44 & 3.61 \\
\hline
\end{tabular}




\section{Conneaut Creek at Conneaut, Ohio}

Years analyzed $=71$

Climatic year range $=1923-2008$

[APEE, annual percentage equaled or exceeded; $\mathrm{ft}^{3} / \mathrm{s}$, cubic foot per second]

\begin{tabular}{|c|c|c|c|c|c|c|c|c|c|}
\hline \multirow[b]{2}{*}{ APEE } & \multicolumn{9}{|c|}{ Daily mean streamflow $\left(\mathrm{ft}^{3} / \mathrm{s}\right)$ that was equaled or exceeded the indicated percentage of time at APEE level } \\
\hline & 98 & 95 & 90 & 80 & 50 & 20 & 10 & 5 & 2 \\
\hline 1 & 1030. & 1530. & 1680. & 2050. & 2670. & 3350. & 3680. & 3940. & 5390 \\
\hline 2 & 859. & 1180. & 1310 . & 1440 & 1950. & 2530 & 2640. & 2790 . & 3510. \\
\hline 5 & 580. & 691. & 737. & 902. & 1200 & 1470 . & 1680. & 1820 . & 2030 . \\
\hline 10 & 358. & 381. & 427. & 526. & 713. & 892. & 984. & 1180 & 1400 \\
\hline 20 & 182. & 190. & 204. & 247. & 344. & 471. & 554. & 608. & 691. \\
\hline 25 & 133. & 154. & 163. & 198. & 273. & 370 . & 444. & 488. & 535. \\
\hline 30 & 97.5 & 120 . & 126. & 151. & 220. & 302. & 351. & 406. & 452. \\
\hline 40 & 56.9 & 68.3 & 78.3 & 100. & 150. & 205. & 229. & 286. & 314. \\
\hline 50 & 33.7 & 35.0 & 45.5 & 66.2 & 105. & 142. & 165. & 198. & 224. \\
\hline 60 & 15.0 & 17.6 & 22.2 & 38.0 & 69.0 & 107. & 119. & 140 . & 157. \\
\hline 70 & 7.27 & 9.01 & 14.2 & 18.0 & 36.8 & 72.2 & 89.2 & 102. & 115. \\
\hline 75 & 5.64 & 7.30 & 10.2 & 14.3 & 28.8 & 62.0 & 70.0 & 80.0 & 92.1 \\
\hline 80 & 4.50 & 5.64 & 7.64 & 11.0 & 21.0 & 49.8 & 55.8 & 59.4 & 67.9 \\
\hline 90 & 2.41 & 3.38 & 4.66 & 6.98 & 13.6 & 29.0 & 33.7 & 39.6 & 43.0 \\
\hline 95 & 1.43 & 2.42 & 3.45 & 5.08 & 11.0 & 19.6 & 24.9 & 30.4 & 39.3 \\
\hline 98 & 0.81 & 1.62 & 2.66 & 3.68 & 7.56 & 15.2 & 18.3 & 25.5 & 35.1 \\
\hline 99 & 0.53 & 1.46 & 2.28 & 3.25 & 7.00 & 13.0 & 17.8 & 23.2 & 34.0 \\
\hline
\end{tabular}

\begin{tabular}{|c|c|c|c|c|c|c|c|c|c|}
\hline \multirow[b]{2}{*}{ APEE } & \multicolumn{9}{|c|}{ 7-day mean streamflow ( $\mathrm{ft}^{3} / \mathrm{s}$ ) that was equaled or exceeded the indicated percentage of time at APEE level } \\
\hline & 98 & 95 & 90 & 80 & 50 & 20 & 10 & 5 & 2 \\
\hline 1 & 648. & 835. & 1150. & 1240. & 1750. & 2290. & 2640. & 2920. & 3380 \\
\hline 2 & 624. & 745. & 811. & 1040. & 1350. & 1570 . & 1780. & 1990. & 2520 \\
\hline 5 & 546. & 600 & 673. & 807. & 972. & 1200 & 1310. & 1390. & 1950. \\
\hline 10 & 389. & 416. & 452. & 560. & 724. & 884. & 1010 & 1080. & 1120 \\
\hline 20 & 204. & 258 & 279 & 318. & 463. & 588. & 664. & 742. & 808. \\
\hline 25 & 140. & 187. & 220 & 259 & 373. & 487. & 585. & 637. & 740. \\
\hline 30 & 105 & 149. & 158. & 202. & 301. & 421. & 478 & 544. & 657. \\
\hline 40 & 58.3 & 77.2 & 89.6 & 120. & 187. & 283. & 344. & 405. & 503. \\
\hline 50 & 30.9 & 37.3 & 48.8 & 74.8 & 129. & 194. & 243. & 294. & 362. \\
\hline 60 & 13.3 & 17.2 & 21.0 & 39.0 & 80.7 & 133. & 165. & 202. & 260. \\
\hline 70 & 7.90 & 9.80 & 14.0 & 18.9 & 42.4 & 92.9 & 106. & 136. & 161. \\
\hline 75 & 6.15 & 7.91 & 11.2 & 15.9 & 33.1 & 80.7 & 91.8 & 114. & 126. \\
\hline 80 & 4.93 & 5.98 & 8.50 & 11.0 & 24.1 & 62.2 & 76.0 & 80.9 & 100 \\
\hline 90 & 2.94 & 4.09 & 5.36 & 7.23 & 15.7 & 33.5 & 39.6 & 44.4 & 49.8 \\
\hline 95 & 2.10 & 2.80 & 3.90 & 5.66 & 12.3 & 21.8 & 28.9 & 37.9 & 42.5 \\
\hline 98 & 1.54 & 2.04 & 3.24 & 4.30 & 9.04 & 17.9 & 24.0 & 30.2 & 39.2 \\
\hline 99 & 0.97 & 1.89 & 2.65 & 3.91 & 8.24 & 15.3 & 21.2 & 26.3 & 37.6 \\
\hline
\end{tabular}



Appendix 4. Annual Seasonal-Period Flow-Duration Results for Sites with Streamflows that have No or Minimal Regulation 


\section{Mill Creek near Berlin Center, Ohio}

Years analyzed $=30$

Climatic year range $=1941-70$

[APEE, annual percentage equaled or exceeded; $\mathrm{ft}^{3} / \mathrm{s}$, cubic foot per second; Sep, September; Nov, November]

\begin{tabular}{|c|c|c|c|c|c|c|c|c|c|}
\hline \multirow[b]{2}{*}{ APEE } & \multicolumn{9}{|c|}{ Sep-Nov daily mean streamflow ( $\left.\mathrm{ft}^{3} / \mathrm{s}\right)$ that was equaled or exceeded the indicated percentage of time at APEE level } \\
\hline & 95 & 90 & 80 & 70 & 50 & 30 & 20 & 10 & 5 \\
\hline 1 & 2.24 & 2.80 & 8.78 & 10.0 & 22.5 & 46.8 & 83.8 & 141. & 373. \\
\hline 2 & 1.51 & 1.92 & 5.62 & 7.84 & 16.0 & 30.8 & 59.9 & 122 & 325 \\
\hline 5 & 1.08 & 1.30 & 2.58 & 4.22 & 6.97 & 14.2 & 23.7 & 46.0 & 76.8 \\
\hline 10 & 0.94 & 1.00 & 1.52 & 2.60 & 3.87 & 7.55 & 13.0 & 28.7 & 31.1 \\
\hline 20 & 0.66 & 0.81 & 1.02 & 1.76 & 2.48 & 3.38 & 5.62 & 15.2 & 18.9 \\
\hline 25 & 0.60 & 0.80 & 0.90 & 1.60 & 2.05 & 2.70 & 4.34 & 11.4 & 16.0 \\
\hline 30 & 0.56 & 0.68 & 0.72 & 1.39 & 1.80 & 2.27 & 3.77 & 9.09 & 12.4 \\
\hline 40 & 0.46 & 0.50 & 0.64 & 0.93 & 1.32 & 1.68 & 2.38 & 4.88 & 7.56 \\
\hline 50 & 0.35 & 0.40 & 0.60 & 0.73 & 1.15 & 1.37 & 1.84 & 3.42 & 5.19 \\
\hline 60 & 0.30 & 0.30 & 0.42 & 0.59 & 0.90 & 1.16 & 1.38 & 2.25 & 3.29 \\
\hline 70 & 0.20 & 0.30 & 0.31 & 0.43 & 0.60 & 0.81 & 1.05 & 1.38 & 2.32 \\
\hline 75 & 0.16 & 0.20 & 0.30 & 0.40 & 0.55 & 0.70 & 0.98 & 1.29 & 2.25 \\
\hline 80 & 0.16 & 0.20 & 0.21 & 0.40 & 0.50 & 0.60 & 0.89 & 1.28 & 1.85 \\
\hline 90 & 0.10 & 0.10 & 0.20 & 0.29 & 0.35 & 0.41 & 0.58 & 0.88 & 1.47 \\
\hline 95 & 0.06 & 0.10 & 0.11 & 0.20 & 0.30 & 0.40 & 0.50 & 0.77 & 1.31 \\
\hline 98 & 0.00 & 0.00 & 0.10 & 0.18 & 0.28 & 0.30 & 0.40 & 0.76 & 1.26 \\
\hline 99 & 0.00 & 0.00 & 0.10 & 0.10 & 0.20 & 0.30 & 0.40 & 0.68 & 1.21 \\
\hline
\end{tabular}

\section{Kale Creek near Pricetown, Ohio}

Years analyzed $=52$

Climatic year range $=1941-92$

[APEE, annual percentage equaled or exceeded; $\mathrm{ft}^{3} / \mathrm{s}$, cubic foot per second; Sep, September; Nov, November]

\begin{tabular}{|c|c|c|c|c|c|c|c|c|c|}
\hline \multirow[b]{2}{*}{ APEE } & \multicolumn{9}{|c|}{ Sep-Nov daily mean streamflow $\left(\mathrm{ft}^{3} / \mathrm{s}\right)$ that was equaled or exceeded the indicated percentage of time at APEE level } \\
\hline & 98 & 95 & 90 & 80 & 50 & 20 & 10 & 5 & 2 \\
\hline 1 & 0.52 & 0.93 & 1.61 & 11.0 & 114. & 326. & 446. & 689. & 789. \\
\hline 2 & 0.43 & 0.85 & 1.49 & 8.98 & 70.7 & 202. & 328. & 512. & 639. \\
\hline 5 & 0.35 & 0.58 & 0.98 & 4.52 & 29.5 & 103. & 120 . & 185. & 228 \\
\hline 10 & 0.31 & 0.40 & 0.78 & 2.46 & 11.3 & 39.7 & 70.0 & 96.3 & 140 . \\
\hline 20 & 0.30 & 0.36 & 0.47 & 1.22 & 5.16 & 16.4 & 27.6 & 42.6 & 58.7 \\
\hline 25 & 0.25 & 0.30 & 0.40 & 1.00 & 3.90 & 12.4 & 19.0 & 32.1 & 48.2 \\
\hline 30 & 0.20 & 0.27 & 0.36 & 0.76 & 2.91 & 8.88 & 16.3 & 26.2 & 34.8 \\
\hline 40 & 0.10 & 0.19 & 0.24 & 0.50 & 1.91 & 5.12 & 11.1 & 14.9 & 22.6 \\
\hline 50 & 0.05 & 0.13 & 0.20 & 0.37 & 1.30 & 3.88 & 8.50 & 11.0 & 16.8 \\
\hline 60 & 0.04 & 0.10 & 0.12 & 0.26 & 0.88 & 3.31 & 6.71 & 9.28 & 13.6 \\
\hline 70 & 0.00 & 0.09 & 0.10 & 0.20 & 0.54 & 2.04 & 4.10 & 7.50 & 10.8 \\
\hline 75 & 0.00 & 0.07 & 0.10 & 0.20 & 0.40 & 1.74 & 3.34 & 6.98 & 9.76 \\
\hline 80 & 0.00 & 0.06 & 0.10 & 0.18 & 0.37 & 1.25 & 2.55 & 6.18 & 7.88 \\
\hline 90 & 0.00 & 0.01 & 0.06 & 0.10 & 0.22 & 0.76 & 1.82 & 3.45 & 5.87 \\
\hline 95 & 0.00 & 0.00 & 0.01 & 0.06 & 0.20 & 0.61 & 1.47 & 2.14 & 5.81 \\
\hline 98 & 0.00 & 0.00 & 0.00 & 0.04 & 0.17 & 0.59 & 1.30 & 1.78 & 5.47 \\
\hline 99 & 0.00 & 0.00 & 0.00 & 0.04 & 0.14 & 0.57 & 1.27 & 1.67 & 4.43 \\
\hline
\end{tabular}


03092090 West Branch Mahoning River near Ravenna, Ohio

Years analyzed $=28$

Climatic year range $=1965-92$

[APEE, annual percentage equaled or exceeded; $\mathrm{ft}^{3} / \mathrm{s}$, cubic foot per second; Sep, September; Nov, November]

\begin{tabular}{|c|c|c|c|c|c|c|c|c|c|}
\hline \multirow[b]{2}{*}{ APEE } & \multicolumn{9}{|c|}{ Sep-Nov daily mean streamflow $\left(\mathrm{ft}^{3} / \mathrm{s}\right)$ that was equaled or exceeded the indicated percentage of time at APEE level } \\
\hline & 95 & 90 & 80 & 70 & 50 & 30 & 20 & 10 & 5 \\
\hline 1 & 17.6 & 24.7 & 58.0 & 89.1 & 158. & 255. & 330. & 540. & 1180 \\
\hline 2 & 17.2 & 22.3 & 42.0 & 69.7 & 88.3 & 208. & 225. & 355. & 465. \\
\hline 5 & 12.6 & 16.6 & 23.9 & 30.4 & 51.7 & 98.6 & 143. & 176. & 212. \\
\hline 10 & 9.15 & 11.4 & 15.0 & 18.6 & 33.0 & 52.9 & 68.2 & 98.6 & 135. \\
\hline 20 & 5.09 & 6.97 & 8.97 & 12.0 & 17.0 & 26.3 & 33.1 & 42.4 & 52.7 \\
\hline 25 & 4.24 & 6.18 & 7.42 & 11.7 & 14.0 & 20.6 & 24.2 & 27.6 & 38.5 \\
\hline 30 & 3.90 & 5.49 & 6.08 & 8.82 & 11.0 & 16.4 & 18.1 & 22.7 & 29.0 \\
\hline 40 & 2.64 & 3.64 & 4.36 & 5.45 & 8.79 & 10.4 & 15.0 & 16.9 & 24.2 \\
\hline 50 & 1.36 & 2.79 & 3.08 & 4.36 & 6.80 & 7.56 & 9.60 & 14.4 & 19.1 \\
\hline 60 & 1.05 & 2.05 & 2.60 & 3.57 & 4.63 & 5.83 & 7.35 & 12.2 & 15.6 \\
\hline 70 & 0.78 & 1.49 & 2.21 & 2.73 & 3.64 & 4.40 & 5.74 & 10.6 & 13.2 \\
\hline 75 & 0.71 & 1.39 & 1.94 & 2.37 & 3.20 & 3.81 & 5.10 & 9.73 & 12.2 \\
\hline 80 & 0.64 & 1.33 & 1.63 & 2.15 & 2.77 & 3.35 & 4.76 & 8.94 & 11.4 \\
\hline 90 & 0.49 & 0.98 & 1.22 & 1.40 & 2.05 & 2.70 & 4.16 & 6.02 & 9.25 \\
\hline 95 & 0.45 & 0.95 & 1.00 & 1.18 & 1.60 & 2.10 & 2.96 & 4.10 & 4.82 \\
\hline 98 & 0.41 & 0.82 & 0.88 & 0.99 & 1.39 & 1.93 & 2.54 & 3.32 & 4.62 \\
\hline 99 & 0.39 & 0.74 & 0.83 & 0.93 & 1.30 & 1.93 & 2.50 & 3.08 & 4.40 \\
\hline
\end{tabular}

\section{Pymatuning Creek at Kinsman, Ohio}

Years analyzed $=29$

Climatic year range $=1965-93$

[APEE, annual percentage equaled or exceeded; $\mathrm{ft}^{3} / \mathrm{s}$, cubic foot per second; Sep, September; Nov, November]

\begin{tabular}{|c|c|c|c|c|c|c|c|c|c|}
\hline \multirow[b]{2}{*}{ APEE } & \multicolumn{9}{|c|}{ Sep-Nov daily mean streamflow $\left(\mathrm{ft}^{3} / \mathrm{s}\right)$ that was equaled or exceeded the indicated percentage of time at APEE level } \\
\hline & 95 & 90 & 80 & 70 & 50 & 30 & 20 & 10 & 5 \\
\hline 1 & 35.0 & 107. & 146. & 260. & 443. & 603. & 723. & 1070. & 2280 \\
\hline 2 & 34.6 & 75.1 & 144. & 240 & 424. & 559. & 664. & 923. & 1730. \\
\hline 5 & 29.5 & 52.4 & 138. & 197. & 347. & 464. & 506. & 682. & 782. \\
\hline 10 & 25.3 & 44.8 & 93.2 & 135. & 256. & 290 & 338. & 445. & 555. \\
\hline 20 & 19.0 & 33.2 & 48.2 & 72.2 & 101. & 184. & 208. & 272. & 393. \\
\hline 25 & 17.5 & 29.0 & 37.0 & 44.0 & 88.0 & 144. & 169. & 207. & 282 \\
\hline 30 & 16.0 & 23.4 & 27.0 & 33.8 & 69.8 & 111. & 150. & 196. & 265. \\
\hline 40 & 9.25 & 12.2 & 16.4 & 23.0 & 44.4 & 78.2 & 107. & 139. & 203. \\
\hline 50 & 5.95 & 7.80 & 11.0 & 14.0 & 24.0 & 41.0 & 83.0 & 93.0 & 157. \\
\hline 60 & 5.03 & 6.48 & 8.80 & 11.0 & 16.8 & 33.0 & 56.0 & 64.8 & 129. \\
\hline 70 & 4.28 & 5.20 & 6.22 & 7.90 & 11.0 & 28.0 & 39.0 & 56.0 & 103. \\
\hline 75 & 3.60 & 4.50 & 5.10 & 5.70 & 9.60 & 20.0 & 37.0 & 50.0 & 87.0 \\
\hline 80 & 3.17 & 3.74 & 4.80 & 5.20 & 8.48 & 16.0 & 30.6 & 45.4 & 73.9 \\
\hline 90 & 1.11 & 2.00 & 2.56 & 3.26 & 5.72 & 11.0 & 24.0 & 36.0 & 50.9 \\
\hline 95 & 0.66 & 1.36 & 1.68 & 2.66 & 4.40 & 8.06 & 17.2 & 24.0 & 25.4 \\
\hline 98 & 0.46 & 0.99 & 1.38 & 1.85 & 3.20 & 6.78 & 11.6 & 19.5 & 22.2 \\
\hline 99 & 0.39 & 0.94 & 1.10 & 1.60 & 3.20 & 5.70 & 9.80 & 17.0 & 20.5 \\
\hline
\end{tabular}




\section{Little Beaver Creek near East Liverpool, Ohio}

Years analyzed $=94$

Climatic year range $=1915-2008$

[APEE, annual percentage equaled or exceeded; $\mathrm{ft}^{3} / \mathrm{s}$, cubic foot per second; Sep, September; Nov, November]

\begin{tabular}{|c|c|c|c|c|c|c|c|c|c|}
\hline \multirow[b]{2}{*}{ APEE } & \multicolumn{9}{|c|}{ Sep-Nov daily mean streamflow $\left(\mathrm{ft}^{3} / \mathrm{s}\right)$ that was equaled or exceeded the indicated percentage of time at APEE level } \\
\hline & 98 & 95 & 90 & 80 & 50 & 20 & 10 & 5 & 2 \\
\hline 1 & 121. & 157. & 291. & 394. & 882. & 2480. & 3950. & 6400. & 14800. \\
\hline 2 & 97.7 & 138. & 200 & 334. & 728. & 2200 & 3490. & 4950. & 8440. \\
\hline 5 & 68.7 & 87.8 & 140 . & 206. & 449. & 1320. & 2140 & 2880. & 4040 . \\
\hline 10 & 53.1 & 69.8 & 112. & 159. & 275 & 803. & 1340. & 1460. & 2670. \\
\hline 20 & 44.9 & 54.2 & 82.8 & 108. & 186. & 408. & 787. & 855. & 1300. \\
\hline 25 & 42.5 & 50.3 & 71.5 & 94.0 & 160. & 354. & 638. & 778. & 1070. \\
\hline 30 & 39.7 & 47.3 & 66.1 & 82.4 & 142. & 278 & 551. & 666. & 958. \\
\hline 40 & 37.5 & 39.2 & 52.8 & 65.2 & 113. & 220 & 395. & 512. & 734. \\
\hline 50 & 33.7 & 35.8 & 45.5 & 59.0 & 96.5 & 199. & 290. & 416. & 587. \\
\hline 60 & 28.8 & 33.0 & 39.9 & 49.0 & 80.4 & 159. & 226. & 379. & 510. \\
\hline 70 & 26.7 & 31.6 & 34.3 & 43.6 & 68.5 & 129. & 191. & 321. & 440. \\
\hline 75 & 25.1 & 29.8 & 32.5 & 42.0 & 58.5 & 112. & 168. & 310. & 422. \\
\hline 80 & 24.0 & 29.1 & 31.1 & 38.4 & 53.2 & 102. & 148. & 299. & 384. \\
\hline 90 & 21.1 & 24.8 & 26.6 & 32.0 & 46.5 & 82.8 & 132. & 267. & 337. \\
\hline 95 & 19.2 & 22.5 & 24.0 & 29.2 & 40.8 & 72.0 & 128. & 199. & 293. \\
\hline 98 & 18.2 & 21.4 & 22.0 & 27.0 & 38.2 & 64.5 & 122. & 175. & 254. \\
\hline 99 & 17.4 & 19.8 & 22.0 & 27.0 & 36.0 & 61.0 & 105. & 144. & 249. \\
\hline
\end{tabular}

\section{Yellow Creek near Hammondsville, Ohio}

Years analyzed $=68$

Climatic year range $=1941-2008$

[APEE, annual percentage equaled or exceeded; $\mathrm{ft}^{3} / \mathrm{s}$, cubic foot per second; Sep, September; Nov, November]

\begin{tabular}{|c|c|c|c|c|c|c|c|c|c|}
\hline \multirow[b]{2}{*}{ APEE } & \multicolumn{9}{|c|}{ Sep-Nov daily mean streamflow $\left(\mathrm{ft}^{3} / \mathrm{s}\right)$ that was equaled or exceeded the indicated percentage of time at APEE level } \\
\hline & 98 & 95 & 90 & 80 & 50 & 20 & 10 & 5 & 2 \\
\hline 1 & 20.0 & 39.5 & 71.1 & 135. & 313. & 789. & 1050 & 1960. & 5840. \\
\hline 2 & 16.8 & 35.8 & 62.6 & 97.8 & 243. & 580. & 798. & 1290. & 4290 . \\
\hline 5 & 14.1 & 24.8 & 42.5 & 66.1 & 143. & 368. & 530. & 706. & 1160. \\
\hline 10 & 9.73 & 17.0 & 32.2 & 44.4 & 92.2 & 251. & 292. & 475. & 839. \\
\hline 20 & 7.13 & 11.8 & 22.9 & 27.2 & 58.1 & 163. & 197. & 274. & 320. \\
\hline 25 & 6.61 & 9.98 & 19.9 & 24.0 & 49.5 & 128. & 171. & 225. & 286. \\
\hline 30 & 5.71 & 8.87 & 15.4 & 21.4 & 42.5 & 107. & 140. & 195. & 241. \\
\hline 40 & 4.91 & 6.57 & 10.2 & 15.8 & 31.7 & 69.4 & 107. & 164. & 196. \\
\hline 50 & 4.20 & 5.45 & 8.37 & 12.8 & 26.0 & 56.4 & 93.8 & 131. & 145. \\
\hline 60 & 3.50 & 4.64 & 6.14 & 9.30 & 19.9 & 38.4 & 74.2 & 105. & 124. \\
\hline 70 & 2.38 & 4.03 & 4.76 & 7.07 & 15.8 & 31.4 & 59.9 & 88.9 & 106. \\
\hline 75 & 2.04 & 3.75 & 4.17 & 6.62 & 13.0 & 27.2 & 55.2 & 81.3 & 101. \\
\hline 80 & 1.71 & 3.14 & 3.70 & 5.18 & 12.0 & 25.2 & 48.0 & 74.2 & 89.5 \\
\hline 90 & 1.13 & 2.09 & 2.48 & 4.26 & 9.03 & 20.3 & 31.0 & 59.6 & 69.3 \\
\hline 95 & 0.88 & 1.81 & 1.99 & 3.48 & 7.67 & 16.2 & 28.0 & 49.8 & 62.7 \\
\hline 98 & 0.88 & 1.48 & 1.88 & 2.92 & 6.65 & 13.9 & 26.1 & 47.9 & 59.4 \\
\hline 99 & 0.88 & 1.44 & 1.80 & 2.68 & 6.35 & 13.2 & 26.1 & 46.9 & 57.5 \\
\hline
\end{tabular}




\section{Yellow Creek at Hammondsville, Ohio}

Years analyzed $=20$

Climatic year range $=1915-34$

[APEE, annual percentage equaled or exceeded; $\mathrm{ft}^{3} / \mathrm{s}$, cubic foot per second; Sep, September; Nov, November]

\begin{tabular}{|c|c|c|c|c|c|c|c|c|c|}
\hline \multirow[b]{2}{*}{ APEE } & \multicolumn{9}{|c|}{ Sep-Nov daily mean streamflow $\left(\mathrm{ft}^{3} / \mathrm{s}\right)$ that was equaled or exceeded the indicated percentage of time at APEE level } \\
\hline & 95 & 90 & 80 & 70 & 50 & 30 & 20 & 10 & 5 \\
\hline 1 & 17.3 & 65.7 & 146. & 181. & 314. & 1090. & 1860. & 4690. & 7130 \\
\hline 2 & 13.3 & 64.4 & 98.3 & 153. & 246. & 789. & 1660. & 2600 & 4220 \\
\hline 5 & 10.2 & 32.9 & 51.2 & 65.8 & 129. & 404. & 624. & 1360. & 2070 \\
\hline 10 & 6.75 & 20.4 & 41.0 & 49.2 & 86.1 & 261. & 327. & 689. & 1010 \\
\hline 20 & 4.35 & 11.9 & 26.8 & 30.3 & 54.1 & 91.8 & 197. & 427. & 592. \\
\hline 25 & 3.63 & 9.20 & 21.6 & 24.6 & 38.5 & 76.3 & 94.0 & 332. & 528. \\
\hline 30 & 3.63 & 8.98 & 18.9 & 21.2 & 31.7 & 64.7 & 89.0 & 288. & 393. \\
\hline 40 & 3.01 & 7.13 & 12.4 & 15.9 & 24.7 & 52.5 & 73.6 & 173. & 321. \\
\hline 50 & 1.83 & 2.57 & 9.14 & 12.5 & 22.0 & 35.6 & 67.8 & 124. & 269. \\
\hline 60 & 1.57 & 2.12 & 7.19 & 9.26 & 17.9 & 26.4 & 57.2 & 82.0 & 224. \\
\hline 70 & 1.17 & 1.59 & 4.45 & 7.47 & 14.0 & 20.0 & 48.7 & 60.4 & 196. \\
\hline 75 & 1.01 & 1.39 & 4.30 & 6.52 & 13.0 & 18.0 & 40.4 & 57.2 & 167. \\
\hline 80 & 0.81 & 1.20 & 4.24 & 6.00 & 12.2 & 16.3 & 35.4 & 55.0 & 167. \\
\hline 90 & 0.54 & 1.01 & 3.25 & 5.24 & 10.1 & 13.7 & 23.6 & 52.1 & 138. \\
\hline 95 & 0.42 & 0.83 & 3.06 & 3.98 & 9.15 & 12.0 & 20.5 & 48.5 & 116. \\
\hline 98 & 0.22 & 0.63 & 2.00 & 2.49 & 7.13 & 10.5 & 17.4 & 44.7 & 84.6 \\
\hline 99 & 0.22 & 0.55 & 1.28 & 2.03 & 7.00 & 10.0 & 16.6 & 42.0 & 45.8 \\
\hline
\end{tabular}

\section{Short Creek near Dillonvale, Ohio}

Years analyzed $=68$

Climatic year range $=1941-2008$

[APEE, annual percentage equaled or exceeded; $\mathrm{ft}^{3} / \mathrm{s}$, cubic foot per second; Sep, September; Nov, November]

\begin{tabular}{|c|c|c|c|c|c|c|c|c|c|}
\hline \multirow[b]{2}{*}{ APEE } & \multicolumn{9}{|c|}{ Sep-Nov daily mean streamflow $\left(\mathrm{ft}^{3} / \mathrm{s}\right)$ that was equaled or exceeded the indicated percentage of time at APEE level } \\
\hline & 98 & 95 & 90 & 80 & 50 & 20 & 10 & 5 & 2 \\
\hline 1 & 48.9 & 67.5 & 94.3 & 118. & 285. & 468. & 972. & 1870 . & 3230 \\
\hline 2 & 37.9 & 54.7 & 73.3 & 97.2 & 177. & 339. & 635. & 1580. & 2790 \\
\hline 5 & 25.6 & 38.2 & 56.9 & 64.0 & 114. & 225 & 359. & 555. & 994. \\
\hline 10 & 19.6 & 31.3 & 39.2 & 46.1 & 82.3 & 159. & 235. & 284 & 499. \\
\hline 20 & 16.9 & 24.8 & 27.0 & 32.8 & 55.9 & 104. & 170 & 200. & 289. \\
\hline 25 & 16.5 & 23.0 & 25.0 & 30.0 & 51.0 & 93.4 & 150. & 187. & 233. \\
\hline 30 & 15.1 & 20.4 & 23.0 & 27.3 & 47.8 & 82.8 & 134. & 149. & 214. \\
\hline 40 & 14.1 & 18.6 & 20.9 & 24.0 & 40.6 & 71.2 & 111. & 130. & 184. \\
\hline 50 & 13.4 & 17.5 & 19.0 & 22.0 & 35.5 & 63.0 & 100 & 106. & 148. \\
\hline 60 & 12.8 & 14.9 & 18.0 & 20.0 & 31.8 & 53.2 & 80.2 & 96.9 & 132. \\
\hline 70 & 12.0 & 13.9 & 16.9 & 18.0 & 29.5 & 47.1 & 69.7 & 88.6 & 120. \\
\hline 75 & 11.0 & 12.4 & 16.0 & 18.0 & 28.0 & 43.4 & 66.0 & 84.0 & 116. \\
\hline 80 & 9.35 & 11.9 & 14.9 & 16.8 & 25.7 & 40.5 & 59.5 & 81.0 & 113. \\
\hline 90 & 6.25 & 9.96 & 12.9 & 14.0 & 22.5 & 35.8 & 52.8 & 73.5 & 106. \\
\hline 95 & 4.57 & 8.10 & 11.5 & 13.0 & 21.0 & 32.8 & 49.7 & 71.1 & 97.2 \\
\hline 98 & 3.64 & 7.47 & 9.75 & 12.7 & 19.3 & 31.0 & 47.5 & 64.2 & 82.9 \\
\hline 99 & 3.26 & 6.35 & 9.75 & 12.0 & 18.5 & 30.8 & 45.1 & 62.2 & 79.5 \\
\hline
\end{tabular}


03111548 Wheeling Creek below Blaine, Ohio

Years analyzed $=25$

Climatic year range $=1983-2008$

[APEE, annual percentage equaled or exceeded; $\mathrm{ft}^{3} / \mathrm{s}$, cubic foot per second; Sep, September; Nov, November]

\begin{tabular}{|c|c|c|c|c|c|c|c|c|c|}
\hline \multirow[b]{2}{*}{ APEE } & \multicolumn{9}{|c|}{ Sep-Nov daily mean streamflow $\left(\mathrm{ft}^{3} / \mathrm{s}\right)$ that was equaled or exceeded the indicated percentage of time at APEE level } \\
\hline & 95 & 90 & 80 & 70 & 50 & 30 & 20 & 10 & 5 \\
\hline 1 & 54.1 & 90.6 & 142. & 194. & 243. & 475. & 554. & 1550 & 3590. \\
\hline 2 & 48.4 & 56.3 & 107. & 136. & 202. & 313. & 447. & 901. & 2420 . \\
\hline 5 & 39.4 & 43.6 & 76.3 & 83.8 & 131. & 205. & 234. & 535. & 935. \\
\hline 10 & 33.4 & 34.9 & 41.8 & 60.7 & 98.6 & 130. & 168. & 281. & 429. \\
\hline 20 & 24.9 & 28.3 & 31.7 & 44.4 & 68.0 & 85.9 & 106. & 142. & 224. \\
\hline 25 & 23.6 & 26.2 & 28.6 & 40.4 & 64.0 & 77.6 & 92.2 & 105. & 180. \\
\hline 30 & 22.9 & 24.6 & 27.5 & 38.1 & 58.8 & 68.4 & 73.8 & 96.4 & 164. \\
\hline 40 & 21.3 & 22.6 & 23.6 & 26.8 & 44.2 & 49.5 & 64.2 & 84.1 & 142. \\
\hline 50 & 18.6 & 20.0 & 22.0 & 24.8 & 36.0 & 43.4 & 59.4 & 73.6 & 123. \\
\hline 60 & 15.5 & 19.0 & 21.0 & 23.6 & 29.6 & 38.0 & 53.0 & 68.6 & 106. \\
\hline 70 & 13.9 & 16.6 & 18.4 & 20.8 & 26.0 & 35.8 & 44.9 & 63.0 & 94.8 \\
\hline 75 & 11.9 & 14.6 & 18.4 & 20.0 & 24.0 & 34.6 & 40.4 & 61.2 & 92.1 \\
\hline 80 & 10.9 & 14.0 & 15.6 & 18.8 & 24.0 & 31.4 & 37.3 & 57.6 & 89.9 \\
\hline 90 & 8.01 & 10.7 & 14.4 & 17.8 & 20.0 & 26.6 & 34.4 & 50.3 & 78.6 \\
\hline 95 & 7.74 & 10.6 & 13.4 & 16.6 & 20.0 & 24.4 & 31.4 & 36.6 & 62.2 \\
\hline 98 & 7.33 & 9.98 & 12.9 & 13.8 & 18.8 & 23.4 & 30.7 & 35.2 & 56.6 \\
\hline 99 & 7.33 & 9.84 & 12.2 & 13.8 & 18.0 & 23.4 & 30.0 & 35.2 & 55.4 \\
\hline
\end{tabular}

03114000 Captina Creek at Armstrongs Mills, Ohio

Years analyzed $=54$

Climatic year range $=1926-2002$

[APEE, annual percentage equaled or exceeded; $\mathrm{ft}^{3} / \mathrm{s}$, cubic foot per second; Sep, September; Nov, November]

\begin{tabular}{|c|c|c|c|c|c|c|c|c|c|}
\hline \multirow[b]{2}{*}{ APEE } & \multicolumn{9}{|c|}{ Sep-Nov daily mean streamflow $\left(\mathrm{ft}^{3} / \mathrm{s}\right)$ that was equaled or exceeded the indicated percentage of time at APEE level } \\
\hline & 98 & 95 & 90 & 80 & 50 & 20 & 10 & 5 & 2 \\
\hline 1 & 15.0 & 66.3 & 88.0 & 197. & 423. & 1340. & 2050 & 3830. & 4950 \\
\hline 2 & 6.58 & 38.9 & 64.7 & 117. & 333. & 689. & 1180. & 2610 & 4610 \\
\hline 5 & 3.46 & 18.8 & 40.9 & 60.8 & 167. & 417. & 615. & 1260. & 1980. \\
\hline 10 & 2.34 & 12.6 & 22.8 & 38.0 & 88.5 & 253. & 361. & 543. & 720. \\
\hline 20 & 1.46 & 5.22 & 11.8 & 21.6 & 51.8 & 124. & 206. & 287. & 395. \\
\hline 25 & 1.18 & 4.90 & 9.55 & 18.0 & 42.5 & 102. & 153. & 239. & 349. \\
\hline 30 & 1.15 & 4.15 & 8.55 & 15.0 & 35.8 & 80.0 & 128. & 204. & 278. \\
\hline 40 & 0.71 & 2.66 & 4.65 & 9.84 & 21.3 & 47.0 & 85.2 & 141. & 199. \\
\hline 50 & 0.42 & 1.20 & 2.80 & 6.90 & 16.5 & 32.0 & 65.5 & 124. & 156. \\
\hline 60 & 0.11 & 0.65 & 1.94 & 5.24 & 11.4 & 24.0 & 56.4 & 105. & 125. \\
\hline 70 & 0.10 & 0.33 & 1.44 & 3.40 & 7.82 & 19.0 & 46.1 & 90.5 & 109. \\
\hline 75 & 0.10 & 0.25 & 1.21 & 2.30 & 6.85 & 17.0 & 42.5 & 83.5 & 101. \\
\hline 80 & 0.01 & 0.10 & 0.64 & 1.64 & 6.04 & 16.4 & 39.0 & 76.3 & 89.8 \\
\hline 90 & 0.00 & 0.07 & 0.21 & 0.92 & 4.59 & 11.0 & 29.3 & 63.1 & 70.5 \\
\hline 95 & 0.00 & 0.00 & 0.12 & 0.37 & 3.64 & 8.60 & 24.0 & 46.0 & 59.0 \\
\hline 98 & 0.00 & 0.00 & 0.00 & 0.28 & 2.72 & 7.44 & 18.7 & 38.0 & 54.4 \\
\hline 99 & 0.00 & 0.00 & 0.00 & 0.28 & 2.15 & 7.10 & 18.0 & 37.8 & 52.9 \\
\hline
\end{tabular}


03115400 Little Muskingum River at Bloomfield, Ohio

Years analyzed $=37$

Climatic year range $=1958-2008$

[APEE, annual percentage equaled or exceeded; $\mathrm{ft}^{3} / \mathrm{s}$, cubic foot per second; Sep, September; Nov, November]

\begin{tabular}{|c|c|c|c|c|c|c|c|c|c|}
\hline \multirow[b]{2}{*}{ APEE } & \multicolumn{9}{|c|}{ Sep-Nov daily mean streamflow $\left(\mathrm{ft}^{3} / \mathrm{s}\right)$ that was equaled or exceeded the indicated percentage of time at APEE level } \\
\hline & 95 & 90 & 80 & 70 & 50 & 30 & 20 & 10 & 5 \\
\hline 1 & 45.1 & 84.0 & 339. & 403. & 900. & 1280 & 2190. & 5110. & 8720. \\
\hline 2 & 42.2 & 71.1 & 210 & 330 . & 655. & 940. & 1690. & 4550 . & 5760. \\
\hline 5 & 20.6 & 44.5 & 103. & 146. & 262. & 493. & 932. & 1290. & 2260 \\
\hline 10 & 15.1 & 25.3 & 32.5 & 79.8 & 140. & 266. & 462. & 604. & 945. \\
\hline 20 & 4.67 & 6.07 & 13.2 & 36.4 & 68.2 & 130. & 227. & 345. & 563. \\
\hline 25 & 3.26 & 4.68 & 9.84 & 28.8 & 60.0 & 103. & 191. & 281. & 420. \\
\hline 30 & 2.65 & 4.16 & 8.20 & 20.0 & 47.4 & 79.8 & 156. & 226. & 339. \\
\hline 40 & 1.25 & 2.14 & 5.73 & 12.0 & 28.6 & 49.7 & 106. & 160. & 246. \\
\hline 50 & 0.95 & 1.74 & 4.76 & 6.88 & 20.0 & 33.6 & 63.6 & 118 & 196. \\
\hline 60 & 0.67 & 1.00 & 3.13 & 4.94 & 14.8 & 21.5 & 30.2 & 90.7 & 143. \\
\hline 70 & 0.32 & 0.72 & 1.99 & 3.08 & 9.64 & 14.4 & 20.0 & 65.3 & 114. \\
\hline 75 & 0.30 & 0.60 & 1.32 & 2.64 & 8.20 & 11.8 & 19.2 & 53.4 & 102. \\
\hline 80 & 0.29 & 0.48 & 0.96 & 1.95 & 5.22 & 9.89 & 18.4 & 48.3 & 93.1 \\
\hline 90 & 0.18 & 0.31 & 0.60 & 1.15 & 3.50 & 7.02 & 13.0 & 37.9 & 64.0 \\
\hline 95 & 0.08 & 0.11 & 0.39 & 0.81 & 2.60 & 4.77 & 7.16 & 31.8 & 46.0 \\
\hline 98 & 0.03 & 0.09 & 0.32 & 0.65 & 1.75 & 3.78 & 5.98 & 27.0 & 37.3 \\
\hline 99 & 0.02 & 0.09 & 0.31 & 0.54 & 1.50 & 3.06 & 5.50 & 25.8 & 36.2 \\
\hline
\end{tabular}

03117500 Sandy Creek at Waynesburg, Ohio

Years analyzed $=70$

Climatic year range $=1939-2008$

[APEE, annual percentage equaled or exceeded; $\mathrm{ft}^{3} / \mathrm{s}$, cubic foot per second; Sep, September; Nov, November]

\begin{tabular}{|c|c|c|c|c|c|c|c|c|c|}
\hline \multirow[b]{2}{*}{ APEE } & \multicolumn{9}{|c|}{ Sep-Nov daily mean streamflow $\left(\mathrm{ft}^{3} / \mathrm{s}\right)$ that was equaled or exceeded the indicated percentage of time at APEE leve } \\
\hline & 98 & 95 & 90 & 80 & 50 & 20 & 10 & 5 & 2 \\
\hline 1 & 36.9 & 45.8 & 95.7 & 234. & 459. & 980. & 1720. & 2590 & 4460. \\
\hline 2 & 34.8 & 40.5 & 86.7 & 168. & 435. & 819. & 1470 . & 2130 & 3900 . \\
\hline 5 & 27.8 & 38.1 & 61.6 & 110. & 238. & 652. & 952. & 1400 & 2210 . \\
\hline 10 & 24.6 & 36.5 & 44.0 & 79.6 & 166. & 409. & 617. & 888. & 1240. \\
\hline 20 & 22.6 & 33.8 & 38.0 & 53.0 & 112. & 257. & 408. & 553. & 679. \\
\hline 25 & 22.2 & 31.7 & 36.0 & 50.2 & 102. & 228 & 355. & 433. & 513. \\
\hline 30 & 21.8 & 31.1 & 35.0 & 44.2 & 93.7 & 206. & 309. & 391. & 466. \\
\hline 40 & 21.2 & 27.1 & 31.0 & 37.6 & 79.5 & 160. & 229. & 332. & 353. \\
\hline 50 & 20.4 & 25.6 & 28.0 & 34.6 & 68.0 & 130. & 186. & 272. & 303. \\
\hline 60 & 19.0 & 24.3 & 26.1 & 33.0 & 54.0 & 108. & 167. & 234. & 264. \\
\hline 70 & 17.1 & 22.8 & 24.6 & 30.0 & 46.3 & 82.4 & 131. & 183. & 235. \\
\hline 75 & 15.9 & 21.0 & 23.1 & 28.0 & 44.0 & 78.6 & 119. & 164. & 226. \\
\hline 80 & 15.5 & 20.7 & 23.1 & 26.7 & 42.0 & 72.6 & 116. & 154. & 213. \\
\hline 90 & 14.6 & 19.2 & 21.0 & 24.0 & 36.2 & 61.4 & 77.8 & 120. & 188. \\
\hline 95 & 13.7 & 17.0 & 19.0 & 21.9 & 34.5 & 54.9 & 71.9 & 116. & 152. \\
\hline 98 & 12.4 & 16.0 & 19.0 & 21.1 & 33.3 & 51.8 & 70.6 & 109. & 138. \\
\hline 99 & 12.0 & 15.6 & 18.0 & 20.4 & 32.5 & 50.8 & 68.5 & 108. & 134. \\
\hline
\end{tabular}


03119000 Sandy Creek at Sandyville, Ohio

Years analyzed $=24$

Climatic year range $=1923-46$

[APEE, annual percentage equaled or exceeded; $\mathrm{ft}^{3} / \mathrm{s}$, cubic foot per second; Sep, September; Nov, November]

\begin{tabular}{|c|c|c|c|c|c|c|c|c|c|}
\hline \multirow[b]{2}{*}{ APEE } & \multicolumn{9}{|c|}{ Sep-Nov daily mean streamflow ( $\left.\mathrm{ft}^{3} / \mathrm{s}\right)$ that was equaled or exceeded the indicated percentage of time at APEE level } \\
\hline & 95 & 90 & 80 & 70 & 50 & 30 & 20 & 10 & 5 \\
\hline 1 & 132. & 238. & 457. & 597. & 914. & 1720. & 2030 & 3170 & 4500 . \\
\hline 2 & 130. & 225. & 420 & 445. & 767. & 1580. & 1940. & 3040 & 4040. \\
\hline 5 & 115. & 141. & 230 . & 332. & 401. & 743. & 1180. & 1480 & 2460 . \\
\hline 10 & 103. & 122. & 165. & 236. & 295. & 490. & 682. & 984. & 1720 . \\
\hline 20 & 96.2 & 105. & 142. & 150. & 206. & 301. & 380. & 629. & 1040. \\
\hline 25 & 93.8 & 102. & 128. & 141. & 193. & 244. & 282. & 540. & 893. \\
\hline 30 & 92.2 & 100. & 123. & 134. & 180. & 215. & 252. & 488. & 840. \\
\hline 40 & 88.8 & 93.2 & 113. & 122. & 151. & 187. & 213. & 405. & 660. \\
\hline 50 & 86.5 & 90.5 & 103. & 116. & 131. & 161. & 200 & 330. & 572. \\
\hline 60 & 78.0 & 84.0 & 95.0 & 106. & 123. & 153. & 189. & 252. & 482. \\
\hline 70 & 72.5 & 80.6 & 88.0 & 99.4 & 114. & 143. & 162. & 196. & 415. \\
\hline 75 & 69.8 & 79.0 & 86.0 & 95.0 & 109. & 138. & 159. & 170. & 367. \\
\hline 80 & 67.5 & 75.1 & 85.0 & 89.3 & 108. & 128. & 138. & 151. & 323. \\
\hline 90 & 58.8 & 69.7 & 80.2 & 82.1 & 99.9 & 109. & 124. & 133. & 269. \\
\hline 95 & 54.8 & 66.0 & 75.6 & 77.5 & 93.7 & 103. & 117. & 128. & 239. \\
\hline 98 & 49.1 & 59.8 & 68.4 & 73.7 & 84.9 & 97.2 & 110. & 121. & 207. \\
\hline 99 & 46.0 & 58.5 & 65.0 & 72.0 & 79.0 & 95.0 & 99.0 & 110. & 120. \\
\hline
\end{tabular}

03125000 Home Creek near New Philadelphia, Ohio

Years analyzed $=43$

Climatic year range $=1937-79$

[APEE, annual percentage equaled or exceeded; $\mathrm{ft}^{3} / \mathrm{s}$, cubic foot per second; Sep, September; Nov, November]

\begin{tabular}{|c|c|c|c|c|c|c|c|c|c|}
\hline \multirow[b]{2}{*}{ APEE } & \multicolumn{9}{|c|}{ Sep-Nov daily mean streamflow $\left(\mathrm{ft}^{3} / \mathrm{s}\right)$ that was equaled or exceeded the indicated percentage of time at APEE level } \\
\hline & 95 & 90 & 80 & 70 & 50 & 30 & 20 & 10 & 5 \\
\hline 1 & 0.32 & 0.54 & 0.93 & 1.24 & 3.20 & 7.80 & 12.6 & 18.2 & 23.0 \\
\hline 2 & 0.15 & 0.42 & 0.52 & 0.87 & 2.04 & 5.37 & 9.01 & 10.6 & 13.9 \\
\hline 5 & 0.04 & 0.11 & 0.29 & 0.49 & 0.94 & 2.99 & 3.53 & 4.73 & 5.03 \\
\hline 10 & 0.01 & 0.07 & 0.12 & 0.30 & 0.50 & 1.58 & 2.08 & 2.68 & 3.12 \\
\hline 20 & 0.00 & 0.02 & 0.08 & 0.17 & 0.27 & 0.71 & 1.17 & 1.44 & 1.49 \\
\hline 25 & 0.00 & 0.00 & 0.05 & 0.10 & 0.22 & 0.56 & 0.97 & 1.20 & 1.28 \\
\hline 30 & 0.00 & 0.00 & 0.04 & 0.10 & 0.20 & 0.46 & 0.73 & 1.03 & 1.17 \\
\hline 40 & 0.00 & 0.00 & 0.02 & 0.04 & 0.12 & 0.29 & 0.50 & 0.70 & 0.87 \\
\hline 50 & 0.00 & 0.00 & 0.00 & 0.03 & 0.10 & 0.15 & 0.40 & 0.56 & 0.69 \\
\hline 60 & 0.00 & 0.00 & 0.00 & 0.02 & 0.08 & 0.12 & 0.25 & 0.41 & 0.56 \\
\hline 70 & 0.00 & 0.00 & 0.00 & 0.00 & 0.04 & 0.10 & 0.14 & 0.31 & 0.45 \\
\hline 75 & 0.00 & 0.00 & 0.00 & 0.00 & 0.03 & 0.10 & 0.10 & 0.26 & 0.43 \\
\hline 80 & 0.00 & 0.00 & 0.00 & 0.00 & 0.02 & 0.08 & 0.10 & 0.23 & 0.41 \\
\hline 90 & 0.00 & 0.00 & 0.00 & 0.00 & 0.01 & 0.06 & 0.10 & 0.12 & 0.20 \\
\hline 95 & 0.00 & 0.00 & 0.00 & 0.00 & 0.00 & 0.04 & 0.07 & 0.10 & 0.13 \\
\hline 98 & 0.00 & 0.00 & 0.00 & 0.00 & 0.00 & 0.03 & 0.05 & 0.10 & 0.12 \\
\hline 99 & 0.00 & 0.00 & 0.00 & 0.00 & 0.00 & 0.02 & 0.04 & 0.10 & 0.12 \\
\hline
\end{tabular}


03130500 Touby Run at Mansfield, Ohio

Years analyzed $=32$

Climatic year range $=1946-77$

[APEE, annual percentage equaled or exceeded; $\mathrm{ft}^{3} / \mathrm{s}$, cubic foot per second; Sep, September; Nov, November]

\begin{tabular}{|c|c|c|c|c|c|c|c|c|c|}
\hline \multirow[b]{2}{*}{ APEE } & \multicolumn{9}{|c|}{ Sep-Nov daily mean streamflow $\left(\mathrm{ft}^{3} / \mathrm{s}\right)$ that was equaled or exceeded the indicated percentage of time at APEE level } \\
\hline & 95 & 90 & 80 & 70 & 50 & 30 & 20 & 10 & 5 \\
\hline 1 & 1.75 & 3.64 & 6.06 & 7.95 & 14.0 & 44.0 & 57.0 & 90.9 & 113. \\
\hline 2 & 0.84 & 2.39 & 4.20 & 5.02 & 13.2 & 27.5 & 32.2 & 44.1 & 65.7 \\
\hline 5 & 0.63 & 1.19 & 2.17 & 3.12 & 6.65 & 9.20 & 15.2 & 19.7 & 23.7 \\
\hline 10 & 0.47 & 0.89 & 1.36 & 1.89 & 3.74 & 5.03 & 7.58 & 12.0 & 14.4 \\
\hline 20 & 0.30 & 0.53 & 0.86 & 1.28 & 1.90 & 2.50 & 3.70 & 4.73 & 7.71 \\
\hline 25 & 0.27 & 0.50 & 0.76 & 1.27 & 1.60 & 1.90 & 2.32 & 3.28 & 6.35 \\
\hline 30 & 0.27 & 0.34 & 0.71 & 0.98 & 1.37 & 1.60 & 1.84 & 2.75 & 5.04 \\
\hline 40 & 0.20 & 0.30 & 0.70 & 0.79 & 1.05 & 1.31 & 1.48 & 2.28 & 3.09 \\
\hline 50 & 0.20 & 0.20 & 0.50 & 0.54 & 0.93 & 1.11 & 1.24 & 1.67 & 2.11 \\
\hline 60 & 0.20 & 0.20 & 0.42 & 0.50 & 0.80 & 0.97 & 1.22 & 1.37 & 1.62 \\
\hline 70 & 0.17 & 0.20 & 0.30 & 0.40 & 0.65 & 0.77 & 0.98 & 1.27 & 1.43 \\
\hline 75 & 0.17 & 0.20 & 0.30 & 0.40 & 0.57 & 0.76 & 0.86 & 1.14 & 1.33 \\
\hline 80 & 0.10 & 0.14 & 0.20 & 0.29 & 0.50 & 0.62 & 0.83 & 1.03 & 1.23 \\
\hline 90 & 0.09 & 0.10 & 0.20 & 0.24 & 0.40 & 0.50 & 0.62 & 0.78 & 1.00 \\
\hline 95 & 0.08 & 0.10 & 0.20 & 0.20 & 0.38 & 0.50 & 0.51 & 0.66 & 0.81 \\
\hline 98 & 0.05 & 0.10 & 0.11 & 0.20 & 0.30 & 0.41 & 0.50 & 0.53 & 0.79 \\
\hline 99 & 0.04 & 0.09 & 0.10 & 0.19 & 0.30 & 0.40 & 0.50 & 0.50 & 0.73 \\
\hline
\end{tabular}

\section{Jerome Fork at Jeromeville, Ohio}

Years analyzed $=24$

Climatic year range $=1925-48$

[APEE, annual percentage equaled or exceeded; $\mathrm{ft}^{3} / \mathrm{s}$, cubic foot per second; Sep, September; Nov, November]

\begin{tabular}{|c|c|c|c|c|c|c|c|c|c|}
\hline \multirow[b]{2}{*}{ APEE } & \multicolumn{9}{|c|}{ Sep-Nov daily mean streamflow ( $\left.\mathrm{ft}^{3} / \mathrm{s}\right)$ that was equaled or exceeded the indicated percentage of time at APEE level } \\
\hline & 95 & 90 & 80 & 70 & 50 & 30 & 20 & 10 & 5 \\
\hline 1 & 20.0 & 29.0 & 71.0 & 119. & 224. & 505. & 658. & 952. & 1090. \\
\hline 2 & 18.1 & 27.7 & 35.7 & 72.8 & 155. & 361. & 462. & 694. & 1050 \\
\hline 5 & 10.9 & 15.3 & 21.4 & 35.1 & 60.4 & 209. & 262. & 437. & 633. \\
\hline 10 & 7.68 & 10.5 & 13.8 & 17.0 & 32.2 & 86.3 & 167. & 221. & 298. \\
\hline 20 & 6.28 & 8.00 & 9.10 & 11.8 & 17.8 & 42.0 & 56.0 & 111. & 160. \\
\hline 25 & 6.13 & 7.65 & 8.00 & 10.2 & 15.5 & 29.5 & 42.0 & 90.0 & 140 . \\
\hline 30 & 5.68 & 7.20 & 7.80 & 9.95 & 14.5 & 24.5 & 37.0 & 79.9 & 114. \\
\hline 40 & 5.05 & 6.25 & 6.80 & 8.70 & 12.1 & 17.2 & 28.0 & 67.7 & 88.0 \\
\hline 50 & 4.88 & 5.50 & 6.00 & 7.40 & 10.0 & 14.0 & 22.0 & 50.0 & 63.8 \\
\hline 60 & 4.05 & 4.50 & 5.40 & 6.45 & 8.52 & 11.9 & 19.0 & 38.9 & 47.8 \\
\hline 70 & 3.63 & 3.90 & 4.80 & 5.75 & 7.60 & 10.9 & 15.0 & 26.9 & 36.2 \\
\hline 75 & 3.63 & 3.90 & 4.80 & 5.30 & 6.80 & 10.7 & 13.0 & 23.3 & 31.4 \\
\hline 80 & 3.43 & 3.60 & 4.70 & 5.20 & 6.20 & 10.0 & 12.0 & 20.9 & 27.4 \\
\hline 90 & 3.05 & 3.45 & 4.10 & 4.45 & 5.65 & 7.65 & 11.0 & 14.0 & 20.6 \\
\hline 95 & 2.35 & 2.90 & 3.68 & 3.91 & 4.45 & 6.88 & 9.20 & 11.3 & 15.3 \\
\hline 98 & 2.23 & 2.70 & 3.20 & 3.45 & 4.43 & 6.20 & 7.70 & 9.82 & 11.8 \\
\hline 99 & 2.10 & 2.70 & 3.20 & 3.45 & 4.35 & 6.20 & 7.70 & 9.40 & 9.85 \\
\hline
\end{tabular}


03136500 Kokosing River at Mount Vernon, Ohio

Years analyzed $=57$

Climatic year range $=1953-2009$

[APEE, annual percentage equaled or exceeded; $\mathrm{ft}^{3} / \mathrm{s}$, cubic foot per second; Sep, September; Nov, November]

\begin{tabular}{|c|c|c|c|c|c|c|c|c|c|}
\hline \multirow[b]{2}{*}{ APEE } & \multicolumn{9}{|c|}{ Sep-Nov daily mean streamflow $\left(\mathrm{ft}^{3} / \mathrm{s}\right)$ that was equaled or exceeded the indicated percentage of time at APEE level } \\
\hline & 98 & 95 & 90 & 80 & 50 & 20 & 10 & 5 & 2 \\
\hline 1 & 24.5 & 40.5 & 56.0 & 90.6 & 325. & 1090. & 1500 & 2220 & 5130 \\
\hline 2 & 24.5 & 31.4 & 53.0 & 80.3 & 292. & 769. & 1290. & 1900. & 3720. \\
\hline 5 & 22.7 & 26.9 & 42.3 & 57.0 & 187. & 412 & 912. & 1310 . & 1470 \\
\hline 10 & 21.3 & 24.8 & 37.6 & 48.3 & 126. & 234. & 418. & 866. & 962. \\
\hline 20 & 21.2 & 22.9 & 31.6 & 40.7 & 83.8 & 143. & 216. & 330. & 428. \\
\hline 25 & 20.2 & 22.8 & 30.0 & 38.6 & 74.0 & 123. & 170 . & 252. & 357. \\
\hline 30 & 20.2 & 22.8 & 29.0 & 37.2 & 66.8 & 104. & 141. & 188. & 310. \\
\hline 40 & 19.2 & 22.7 & 28.0 & 32.6 & 48.0 & 85.2 & 121. & 141. & 247. \\
\hline 50 & 18.3 & 21.8 & 24.8 & 30.0 & 43.0 & 71.0 & 93.2 & 123. & 205 \\
\hline 60 & 17.3 & 20.8 & 23.4 & 28.0 & 39.0 & 62.9 & 87.2 & 106. & 178. \\
\hline 70 & 15.8 & 18.8 & 21.0 & 25.8 & 35.6 & 59.6 & 76.9 & 97.0 & 153. \\
\hline 75 & 15.2 & 17.8 & 19.8 & 25.0 & 34.0 & 56.4 & 71.6 & 93.1 & 139. \\
\hline 80 & 13.7 & 15.9 & 19.8 & 24.0 & 33.0 & 54.4 & 64.6 & 87.3 & 128. \\
\hline 90 & 12.3 & 14.0 & 18.0 & 21.1 & 31.0 & 49.5 & 57.6 & 72.1 & 106. \\
\hline 95 & 11.5 & 14.0 & 16.7 & 19.6 & 29.0 & 45.8 & 52.2 & 59.2 & 77.0 \\
\hline 98 & 9.90 & 13.0 & 15.6 & 17.9 & 27.0 & 43.5 & 49.4 & 53.2 & 70.3 \\
\hline 99 & 9.47 & 13.0 & 14.8 & 17.6 & 27.0 & 43.2 & 49.2 & 52.3 & 69.3 \\
\hline
\end{tabular}

03137000 Kokosing River at Millwood, Ohio

Years analyzed $=53$

Climatic year range $=1921-73$

[APEE, annual percentage equaled or exceeded; $\mathrm{ft}^{3} / \mathrm{s}$, cubic foot per second; Sep, September; Nov, November]

\begin{tabular}{|c|c|c|c|c|c|c|c|c|c|}
\hline \multirow[b]{2}{*}{ APEE } & \multicolumn{9}{|c|}{ Sep-Nov daily mean streamflow $\left(\mathrm{ft}^{3} / \mathrm{s}\right)$ that was equaled or exceeded the indicated percentage of time at APEE level } \\
\hline & 98 & 95 & 90 & 80 & 50 & 20 & 10 & 5 & 2 \\
\hline 1 & 55.8 & 86.0 & 127. & 185. & 769. & 1820. & 3620 & 5430. & 7800. \\
\hline 2 & 55.6 & 85.0 & 105. & 153. & 618. & 1470 . & 2680 & 4610 & 6760. \\
\hline 5 & 53.0 & 65.4 & 84.2 & 110. & 342. & 780 . & 1520 . & 2470 & 3250 . \\
\hline 10 & 52.2 & 59.9 & 71.9 & 90.5 & 218. & 438. & 640. & 1560. & 1950. \\
\hline 20 & 50.0 & 53.6 & 63.9 & 79.6 & 144. & 276. & 368. & 800. & 1080. \\
\hline 25 & 49.1 & 52.1 & 61.8 & 76.4 & 124. & 223. & 281. & 636. & 782. \\
\hline 30 & 48.2 & 50.7 & 61.4 & 74.0 & 113. & 191. & 247. & 533. & 732. \\
\hline 40 & 45.2 & 48.0 & 59.8 & 70.9 & 96.2 & 156. & 214 & 334. & 614. \\
\hline 50 & 43.2 & 46.0 & 56.8 & 66.2 & 84.0 & 123. & 154. & 267. & 577. \\
\hline 60 & 42.2 & 44.0 & 52.4 & 63.2 & 81.0 & 111. & 133. & 229. & 492. \\
\hline 70 & 39.2 & 43.2 & 49.4 & 58.6 & 78.0 & 100. & 120. & 211. & 307. \\
\hline 75 & 38.2 & 42.8 & 47.2 & 57.8 & 77.0 & 95.6 & 117. & 193. & 274. \\
\hline 80 & 38.1 & 39.7 & 45.6 & 55.6 & 74.0 & 92.0 & 113. & 172. & 248. \\
\hline 90 & 37.0 & 37.7 & 41.8 & 51.6 & 69.0 & 85.3 & 101. & 142. & 180. \\
\hline 95 & 35.6 & 36.0 & 41.4 & 49.6 & 66.0 & 80.2 & 98.2 & 123. & 130. \\
\hline 98 & 34.2 & 36.0 & 39.8 & 46.8 & 64.0 & 78.0 & 96.1 & 107. & 126. \\
\hline 99 & 34.2 & 36.0 & 38.0 & 46.0 & 64.0 & 77.2 & 95.2 & 106. & 121. \\
\hline
\end{tabular}


03139000 Killbuck Creek at Killbuck, Ohio

Years analyzed $=79$

Climatic year range $=1930-2008$

[APEE, annual percentage equaled or exceeded; $\mathrm{ft}^{3} / \mathrm{s}$, cubic foot per second; Sep, September; Nov, November]

\begin{tabular}{|c|c|c|c|c|c|c|c|c|c|}
\hline \multirow[b]{2}{*}{ APEE } & \multicolumn{9}{|c|}{ Sep-Nov daily mean streamflow $\left(\mathrm{ft}^{3} / \mathrm{s}\right)$ that was equaled or exceeded the indicated percentage of time at APEE level } \\
\hline & 98 & 95 & 90 & 80 & 50 & 20 & 10 & 5 & 2 \\
\hline 1 & 47.2 & 104. & 160. & 273. & 681. & 1120. & 1570 & 2210. & 4880. \\
\hline 2 & 45.1 & 93.9 & 124. & 229. & 577. & 955. & 1450 & 2120. & 3620 . \\
\hline 5 & 43.2 & 61.8 & 89.2 & 144. & 367. & 663. & 1070 & 1850. & 2820 . \\
\hline 10 & 39.2 & 51.6 & 70.8 & 123. & 224. & 481. & 752. & 1300. & 1720 . \\
\hline 20 & 37.0 & 48.0 & 59.6 & 93.8 & 140. & 332. & 564. & 804. & 1050. \\
\hline 25 & 35.6 & 47.0 & 57.0 & 77.0 & 128. & 284. & 490. & 640. & 873. \\
\hline 30 & 35.0 & 47.0 & 53.0 & 71.4 & 122. & 223. & 338. & 543. & 693. \\
\hline 40 & 32.3 & 44.0 & 51.0 & 64.0 & 100. & 170. & 299. & 434. & 542. \\
\hline 50 & 30.4 & 43.0 & 47.0 & 62.0 & 89.0 & 151. & 224. & 382. & 436. \\
\hline 60 & 29.2 & 38.0 & 44.0 & 53.0 & 82.0 & 134. & 198. & 300. & 378. \\
\hline 70 & 28.0 & 33.0 & 42.0 & 50.6 & 76.0 & 118. & 168. & 261. & 348. \\
\hline 75 & 26.6 & 31.0 & 40.0 & 47.0 & 72.0 & 107. & 153. & 245. & 334. \\
\hline 80 & 26.6 & 30.4 & 38.0 & 45.0 & 71.0 & 99.8 & 144. & 235. & 321. \\
\hline 90 & 25.2 & 30.0 & 35.2 & 42.0 & 61.8 & 92.0 & 125. & 178. & 275 . \\
\hline 95 & 24.2 & 28.6 & 34.0 & 38.6 & 54.0 & 87.4 & 114. & 157. & 250. \\
\hline 98 & 24.1 & 27.5 & 33.0 & 37.0 & 52.8 & 83.5 & 107. & 153. & 232. \\
\hline 99 & 23.6 & 27.0 & 32.0 & 37.0 & 52.0 & 82.0 & 107. & 149. & 222. \\
\hline
\end{tabular}

\section{Mill Creek near Coshocton, Ohio}

Years analyzed $=72$

Climatic year range $=1937-2008$

[APEE, annual percentage equaled or exceeded; $\mathrm{ft}^{3} / \mathrm{s}$, cubic foot per second; Sep, September; Nov, November]

\begin{tabular}{|c|c|c|c|c|c|c|c|c|c|}
\hline \multirow[b]{2}{*}{ APEE } & \multicolumn{9}{|c|}{ Sep-Nov daily mean streamflow $\left(\mathrm{ft}^{3} / \mathrm{s}\right)$ that was equaled or exceeded the indicated percentage of time at APEE level } \\
\hline & 98 & 95 & 90 & 80 & 50 & 20 & 10 & 5 & 2 \\
\hline 1 & 1.92 & 5.17 & 7.81 & 23.2 & 65.0 & 167. & 408. & 556. & 1400 \\
\hline 2 & 1.34 & 4.38 & 7.32 & 15.3 & 49.8 & 107. & 266. & 350 & 430 . \\
\hline 5 & 0.71 & 2.28 & 2.90 & 7.84 & 22.2 & 51.5 & 103. & 134. & 192. \\
\hline 10 & 0.44 & 1.50 & 1.93 & 4.46 & 11.9 & 29.0 & 55.0 & 84.1 & 124. \\
\hline 20 & 0.30 & 0.74 & 1.34 & 2.34 & 6.80 & 18.4 & 36.1 & 44.8 & 51.3 \\
\hline 25 & 0.30 & 0.60 & 1.20 & 2.00 & 5.80 & 16.4 & 28.4 & 36.4 & 43.8 \\
\hline 30 & 0.25 & 0.53 & 1.13 & 1.85 & 5.04 & 12.3 & 23.8 & 30.4 & 37.3 \\
\hline 40 & 0.20 & 0.42 & 0.82 & 1.41 & 3.22 & 9.24 & 16.5 & 24.4 & 30.6 \\
\hline 50 & 0.10 & 0.36 & 0.50 & 1.20 & 2.50 & 7.20 & 13.1 & 18.3 & 23.6 \\
\hline 60 & 0.10 & 0.27 & 0.46 & 0.95 & 2.09 & 5.46 & 12.1 & 15.2 & 19.1 \\
\hline 70 & 0.10 & 0.20 & 0.30 & 0.69 & 1.60 & 4.13 & 8.69 & 12.0 & 16.2 \\
\hline 75 & 0.10 & 0.17 & 0.26 & 0.63 & 1.30 & 3.74 & 6.97 & 11.3 & 15.2 \\
\hline 80 & 0.10 & 0.10 & 0.24 & 0.54 & 1.12 & 2.87 & 5.66 & 9.93 & 14.1 \\
\hline 90 & 0.10 & 0.10 & 0.17 & 0.33 & 0.93 & 2.04 & 3.87 & 8.47 & 11.6 \\
\hline 95 & 0.10 & 0.10 & 0.10 & 0.25 & 0.72 & 1.90 & 3.48 & 8.11 & 10.8 \\
\hline 98 & 0.05 & 0.10 & 0.10 & 0.20 & 0.58 & 1.72 & 3.11 & 7.87 & 9.35 \\
\hline 99 & 0.05 & 0.10 & 0.10 & 0.20 & 0.52 & 1.62 & 2.94 & 7.51 & 9.02 \\
\hline
\end{tabular}




\section{Wakatomika Creek near Frazeysburg, Ohio}

Years analyzed $=73$

Climatic year range $=1936-2008$

[APEE, annual percentage equaled or exceeded; $\mathrm{ft}^{3} / \mathrm{s}$, cubic foot per second; Sep, September; Nov, November]

\begin{tabular}{|c|c|c|c|c|c|c|c|c|c|}
\hline \multirow[b]{2}{*}{ APEE } & \multicolumn{9}{|c|}{ Sep-Nov daily mean streamflow ( $\left.\mathrm{ft}^{3} / \mathrm{s}\right)$ that was equaled or exceeded the indicated percentage of time at APEE level } \\
\hline & 98 & 95 & 90 & 80 & 50 & 20 & 10 & 5 & 2 \\
\hline 1 & 12.9 & 18.1 & 36.0 & 97.6 & 387. & 803. & 1320. & 1750. & 5480 \\
\hline 2 & 10.9 & 14.6 & 32.1 & 89.1 & 248. & 609. & 949. & 1280 & 3460 \\
\hline 5 & 9.06 & 12.0 & 26.9 & 45.1 & 126. & 310. & 561. & 656. & 923. \\
\hline 10 & 8.06 & 11.0 & 20.2 & 26.4 & 69.2 & 196. & 278 & 355. & 420. \\
\hline 20 & 7.10 & 9.20 & 13.9 & 19.4 & 45.2 & 111. & 150. & 192. & 218. \\
\hline 25 & 6.64 & 9.02 & 11.8 & 17.0 & 40.0 & 92.0 & 136. & 159. & 182. \\
\hline 30 & 6.52 & 8.56 & 10.8 & 16.4 & 34.0 & 83.5 & 118. & 141. & 159. \\
\hline 40 & 6.05 & 7.55 & 9.88 & 13.2 & 26.0 & 56.7 & 85.3 & 103. & 121. \\
\hline 50 & 5.24 & 6.28 & 8.56 & 11.0 & 21.0 & 48.2 & 69.0 & 88.9 & 101. \\
\hline 60 & 4.82 & 5.73 & 7.96 & 10.0 & 17.8 & 37.7 & 53.6 & 70.8 & 88.7 \\
\hline 70 & 4.03 & 5.19 & 6.72 & 8.70 & 13.6 & 31.0 & 45.0 & 52.2 & 80.0 \\
\hline 75 & 3.59 & 4.79 & 5.88 & 8.18 & 12.0 & 27.0 & 37.8 & 48.5 & 74.8 \\
\hline 80 & 3.44 & 4.32 & 5.33 & 7.53 & 11.0 & 24.0 & 32.6 & 45.7 & 68.4 \\
\hline 90 & 2.86 & 3.64 & 4.72 & 6.77 & 9.48 & 19.6 & 25.3 & 32.2 & 59.9 \\
\hline 95 & 2.75 & 3.20 & 4.18 & 5.99 & 8.62 & 17.7 & 21.2 & 29.4 & 51.9 \\
\hline 98 & 2.65 & 3.20 & 3.74 & 5.48 & 8.08 & 15.9 & 19.1 & 27.5 & 47.4 \\
\hline 99 & 2.65 & 3.20 & 3.48 & 5.20 & 8.00 & 15.2 & 18.6 & 25.9 & 46.6 \\
\hline
\end{tabular}

03146000 North Fork Licking River at Utica, Ohio

Years analyzed $=22$

Climatic year range $=1939-81$

[APEE, annual percentage equaled or exceeded; $\mathrm{ft}^{3} / \mathrm{s}$, cubic foot per second; Sep, September; Nov, November]

\begin{tabular}{|c|c|c|c|c|c|c|c|c|c|}
\hline \multirow[b]{2}{*}{ APEE } & \multicolumn{9}{|c|}{ Sep-Nov daily mean streamflow $\left(\mathrm{ft}^{3} / \mathrm{s}\right)$ that was equaled or exceeded the indicated percentage of time at APEE level } \\
\hline & 95 & 90 & 80 & 70 & 50 & 30 & 20 & 10 & 5 \\
\hline 1 & 5.43 & 19.4 & 75.6 & 270. & 413. & 829. & 1520 & 3000 . & 6200 \\
\hline 2 & 5.35 & 17.2 & 62.5 & 187. & 303. & 621. & 1160 & 2190 & 3220 . \\
\hline 5 & 4.53 & 10.1 & 41.5 & 68.1 & 138. & 236. & 332. & 1070 . & 1530. \\
\hline 10 & 4.31 & 6.32 & 24.0 & 43.2 & 83.7 & 144. & 150. & 406. & 531. \\
\hline 20 & 4.00 & 5.38 & 15.2 & 22.7 & 44.7 & 72.4 & 80.7 & 166. & 218. \\
\hline 25 & 3.66 & 5.02 & 11.0 & 19.5 & 36.5 & 56.3 & 66.4 & 135. & 181. \\
\hline 30 & 3.63 & 4.82 & 9.03 & 17.6 & 30.1 & 47.0 & 51.1 & 120. & 159. \\
\hline 40 & 3.52 & 4.41 & 7.24 & 13.8 & 22.2 & 32.3 & 35.8 & 89.3 & 113. \\
\hline 50 & 3.23 & 4.06 & 6.56 & 9.09 & 13.0 & 20.0 & 25.6 & 66.7 & 93.9 \\
\hline 60 & 2.83 & 3.66 & 5.95 & 8.08 & 10.4 & 16.1 & 18.0 & 56.6 & 81.0 \\
\hline 70 & 2.36 & 3.13 & 5.45 & 6.42 & 8.45 & 12.3 & 15.4 & 46.4 & 67.1 \\
\hline 75 & 2.09 & 2.83 & 5.00 & 5.94 & 7.90 & 10.3 & 14.4 & 30.7 & 61.2 \\
\hline 80 & 1.89 & 2.61 & 4.58 & 5.49 & 7.05 & 9.87 & 12.6 & 19.7 & 53.3 \\
\hline 90 & 1.44 & 2.32 & 3.55 & 4.20 & 5.95 & 8.31 & 9.58 & 17.2 & 47.4 \\
\hline 95 & 1.26 & 2.19 & 3.12 & 3.45 & 5.18 & 6.68 & 8.59 & 15.9 & 35.3 \\
\hline 98 & 1.11 & 2.08 & 2.60 & 2.98 & 4.73 & 6.46 & 7.34 & 11.4 & 26.2 \\
\hline 99 & 0.96 & 2.08 & 2.60 & 2.98 & 4.65 & 6.43 & 7.04 & 11.4 & 24.7 \\
\hline
\end{tabular}


03146500 Licking River near Newark, Ohio

Years analyzed $=69$

Climatic year range $=1939-2008$

[APEE, annual percentage equaled or exceeded; $\mathrm{ft}^{3} / \mathrm{s}$, cubic foot per second; Sep, September; Nov, November]

\begin{tabular}{|c|c|c|c|c|c|c|c|c|c|}
\hline \multirow[b]{2}{*}{ APEE } & \multicolumn{9}{|c|}{ Sep-Nov daily mean streamflow $\left(\mathrm{ft}^{3} / \mathrm{s}\right)$ that was equaled or exceeded the indicated percentage of time at APEE level } \\
\hline & 98 & 95 & 90 & 80 & 50 & 20 & 10 & 5 & 2 \\
\hline 1 & 67.8 & 126. & 201. & 299. & 1700. & 3440. & 5180. & 7240. & 13100. \\
\hline 2 & 60.7 & 116. & 200. & 265. & 1270 . & 2600 & 4830. & 6020. & 12200 . \\
\hline 5 & 55.7 & 86.3 & 137. & 224. & 625. & 1410 & 2940 . & 3780. & 4880 . \\
\hline 10 & 51.0 & 63.7 & 77.2 & 188. & 378. & 869. & 1150 . & 2140. & 3160 . \\
\hline 20 & 44.4 & 54.6 & 64.6 & 110. & 249. & 544. & 733. & 1130. & 1270 . \\
\hline 25 & 44.4 & 51.5 & 62.0 & 99.0 & 205. & 416. & 622. & 793. & 1110 . \\
\hline 30 & 42.4 & 50.7 & 62.0 & 83.0 & 153. & 315. & 518. & 634. & 972. \\
\hline 40 & 42.4 & 48.0 & 57.0 & 68.4 & 108. & 209. & 367. & 523. & 714. \\
\hline 50 & 40.8 & 47.0 & 55.0 & 66.0 & 93.0 & 167. & 268. & 395. & 538. \\
\hline 60 & 40.4 & 45.5 & 54.0 & 62.0 & 81.8 & 151. & 226. & 317. & 453. \\
\hline 70 & 40.0 & 42.5 & 53.0 & 58.0 & 77.0 & 141. & 182. & 260. & 394. \\
\hline 75 & 37.4 & 42.0 & 52.0 & 56.0 & 75.0 & 127. & 162. & 212. & 352. \\
\hline 80 & 36.9 & 42.0 & 52.0 & 54.4 & 70.8 & 108 & 146. & 186. & 316. \\
\hline 90 & 34.0 & 39.5 & 49.0 & 53.0 & 66.2 & 93.4 & 125. & 158. & 271. \\
\hline 95 & 32.0 & 38.6 & 48.4 & 51.2 & 65.0 & 92.0 & 107. & 150. & 232. \\
\hline 98 & 31.7 & 36.0 & 43.0 & 50.0 & 62.8 & 87.0 & 99.7 & 142. & 214. \\
\hline 99 & 30.0 & 36.0 & 43.0 & 49.0 & 62.0 & 87.0 & 98.0 & 137. & 212. \\
\hline
\end{tabular}

\section{Licking River at Toboso, Ohio}

Years analyzed $=42$

Climatic year range $=1904-60$

[APEE, annual percentage equaled or exceeded; $\mathrm{ft}^{3} / \mathrm{s}$, cubic foot per second; Sep, September; Nov, November]

\begin{tabular}{|c|c|c|c|c|c|c|c|c|c|}
\hline \multirow[b]{2}{*}{ APEE } & \multicolumn{9}{|c|}{ Sep-Nov daily mean streamflow $\left(\mathrm{ft}^{3} / \mathrm{s}\right)$ that was equaled or exceeded the indicated percentage of time at APEE level } \\
\hline & 95 & 90 & 80 & 70 & 50 & 30 & 20 & 10 & 5 \\
\hline 1 & 90.8 & 183. & 286. & 424. & 1050. & 2090. & 2830 & 4340. & 5710. \\
\hline 2 & 81.5 & 175. & 267. & 350. & 849. & 1450 . & 2190. & 3840 . & 4190. \\
\hline 5 & 74.5 & 124. & 206. & 256. & 475. & 836. & 1050. & 2200 . & 3220 . \\
\hline 10 & 68.9 & 91.4 & 138. & 171. & 303. & 490. & 622. & 1130. & 1810. \\
\hline 20 & 65.8 & 73.8 & 116. & 130. & 201. & 315. & 383. & 554. & 699. \\
\hline 25 & 65.3 & 70.6 & 101. & 123. & 175. & 285 & 303. & 471. & 528. \\
\hline 30 & 63.2 & 68.9 & 96.5 & 116. & 148. & 237. & 268. & 414. & 442. \\
\hline 40 & 61.2 & 66.0 & 83.4 & 98.9 & 118. & 167. & 215 & 284. & 353. \\
\hline 50 & 57.3 & 64.2 & 78.0 & 85.0 & 109. & 134. & 153. & 214. & 294. \\
\hline 60 & 54.8 & 60.2 & 74.7 & 84.6 & 97.3 & 111. & 133. & 190. & 235. \\
\hline 70 & 51.8 & 59.0 & 71.5 & 80.4 & 92.5 & 100. & 112. & 141. & 197. \\
\hline 75 & 50.6 & 55.6 & 69.2 & 73.9 & 88.0 & 98.2 & 104. & 140. & 188. \\
\hline 80 & 50.3 & 53.5 & 64.6 & 72.9 & 85.2 & 94.4 & 102. & 136. & 173. \\
\hline 90 & 48.1 & 50.5 & 61.0 & 69.4 & 77.3 & 90.9 & 98.1 & 116. & 156. \\
\hline 95 & 46.1 & 49.0 & 60.2 & 68.0 & 74.0 & 86.4 & 94.8 & 112. & 149. \\
\hline 98 & 44.3 & 48.3 & 58.9 & 64.9 & 72.8 & 84.4 & 92.7 & 111. & 129. \\
\hline 99 & 44.3 & 46.6 & 57.2 & 64.0 & 72.0 & 84.1 & 92.4 & 108. & 128. \\
\hline
\end{tabular}


03149500 Salt Creek near Chandlersville, Ohio

Years analyzed $=20$

Climatic year range $=1935-2008$

[APEE, annual percentage equaled or exceeded; $\mathrm{ft}^{3} / \mathrm{s}$, cubic foot per second; Sep, September; Nov, November]

\begin{tabular}{|c|c|c|c|c|c|c|c|c|c|}
\hline \multirow[b]{2}{*}{ APEE } & \multicolumn{9}{|c|}{ Sep-Nov daily mean streamflow $\left(\mathrm{ft}^{3} / \mathrm{s}\right)$ that was equaled or exceeded the indicated percentage of time at APEE level } \\
\hline & 95 & 90 & 80 & 70 & 50 & 30 & 20 & 10 & 5 \\
\hline 1 & 25.3 & 31.7 & 82.0 & 109. & 285. & 601. & 864. & 2000 & 4000 \\
\hline 2 & 19.2 & 21.8 & 47.0 & 88.2 & 192. & 306. & 794. & 1110 & 3230 . \\
\hline 5 & 7.18 & 9.40 & 31.0 & 50.5 & 86.0 & 152. & 374. & 511. & 762. \\
\hline 10 & 6.15 & 7.13 & 11.8 & 26.1 & 40.9 & 86.1 & 169. & 242. & 290. \\
\hline 20 & 5.11 & 5.29 & 8.38 & 12.5 & 21.7 & 43.1 & 87.7 & 130. & 152. \\
\hline 25 & 4.41 & 4.67 & 7.38 & 11.0 & 19.0 & 33.7 & 69.4 & 115. & 134. \\
\hline 30 & 4.14 & 4.31 & 6.36 & 8.91 & 16.5 & 28.1 & 58.0 & 91.2 & 113. \\
\hline 40 & 3.13 & 3.49 & 5.49 & 6.74 & 12.5 & 23.2 & 46.2 & 62.6 & 68.9 \\
\hline 50 & 2.71 & 2.82 & 3.44 & 5.83 & 9.05 & 17.4 & 31.6 & 48.4 & 50.0 \\
\hline 60 & 1.51 & 1.79 & 3.04 & 4.39 & 5.74 & 13.8 & 24.0 & 37.7 & 42.4 \\
\hline 70 & 0.72 & 1.00 & 2.26 & 3.00 & 3.54 & 7.74 & 16.5 & 29.0 & 34.0 \\
\hline 75 & 0.49 & 0.81 & 2.10 & 2.52 & 3.10 & 7.33 & 14.6 & 22.9 & 30.6 \\
\hline 80 & 0.35 & 0.80 & 1.02 & 2.16 & 2.60 & 6.71 & 10.5 & 18.4 & 27.0 \\
\hline 90 & 0.04 & 0.13 & 0.42 & 1.14 & 1.86 & 4.22 & 5.72 & 16.0 & 22.9 \\
\hline 95 & 0.03 & 0.11 & 0.29 & 0.81 & 1.49 & 3.74 & 4.42 & 12.5 & 19.7 \\
\hline 98 & 0.03 & 0.10 & 0.24 & 0.74 & 1.30 & 3.34 & 3.99 & 10.3 & 16.4 \\
\hline 99 & 0.01 & 0.10 & 0.24 & 0.73 & 1.30 & 3.21 & 3.96 & 9.04 & 14.7 \\
\hline
\end{tabular}

\section{Clear Creek near Rockbridge, Ohio}

Years analyzed $=70$

Climatic year range $=1939-2008$

[APEE, annual percentage equaled or exceeded; $\mathrm{ft}^{3} / \mathrm{s}$, cubic foot per second; Sep, September; Nov, November]

\begin{tabular}{|c|c|c|c|c|c|c|c|c|c|}
\hline \multirow[b]{2}{*}{ APEE } & \multicolumn{9}{|c|}{ Sep-Nov daily mean streamflow $\left(\mathrm{ft}^{3} / \mathrm{s}\right)$ that was equaled or exceeded the indicated percentage of time at APEE level } \\
\hline & 98 & 95 & 90 & 80 & 50 & 20 & 10 & 5 & 2 \\
\hline 1 & 20.4 & 22.1 & 32.2 & 70.6 & 165. & 365. & 771. & 1150 & 2020 \\
\hline 2 & 17.9 & 21.3 & 28.1 & 50.1 & 128. & 282. & 569. & 960. & 1140 \\
\hline 5 & 15.4 & 18.1 & 22.8 & 35.1 & 70.4 & 156. & 376. & 446. & 625. \\
\hline 10 & 14.0 & 16.1 & 19.8 & 25.5 & 48.3 & 102. & 160. & 282. & 332. \\
\hline 20 & 13.4 & 15.0 & 18.0 & 20.7 & 35.9 & 62.9 & 93.8 & 139. & 191. \\
\hline 25 & 13.0 & 15.0 & 17.1 & 19.2 & 32.0 & 55.0 & 79.2 & 113. & 157. \\
\hline 30 & 13.0 & 14.6 & 16.1 & 18.1 & 28.5 & 47.7 & 67.6 & 98.6 & 134. \\
\hline 40 & 12.4 & 13.6 & 15.1 & 18.0 & 23.1 & 34.8 & 53.3 & 69.8 & 108. \\
\hline 50 & 12.0 & 13.0 & 14.1 & 17.0 & 21.0 & 31.0 & 48.0 & 60.8 & 93.1 \\
\hline 60 & 12.0 & 12.0 & 14.0 & 16.0 & 19.0 & 27.0 & 42.9 & 54.5 & 83.4 \\
\hline 70 & 10.1 & 11.6 & 12.6 & 14.1 & 18.0 & 24.9 & 39.0 & 46.3 & 74.5 \\
\hline 75 & 9.84 & 11.0 & 12.0 & 14.0 & 17.5 & 23.8 & 35.4 & 42.8 & 70.7 \\
\hline 80 & 9.00 & 11.0 & 11.0 & 13.0 & 16.5 & 22.0 & 31.2 & 39.6 & 67.8 \\
\hline 90 & 8.16 & 9.53 & 10.3 & 11.4 & 15.0 & 19.2 & 28.0 & 35.1 & 55.7 \\
\hline 95 & 7.86 & 8.13 & 9.93 & 11.0 & 14.6 & 19.0 & 25.5 & 33.0 & 45.5 \\
\hline 98 & 6.27 & 7.98 & 9.44 & 10.2 & 13.9 & 18.0 & 23.8 & 30.7 & 41.3 \\
\hline 99 & 6.27 & 7.98 & 9.43 & 10.0 & 13.5 & 17.8 & 23.0 & 28.0 & 40.7 \\
\hline
\end{tabular}


03157500 Hocking River at Enterprise, Ohio

Years analyzed $=78$

Climatic year range $=1931-2008$

[APEE, annual percentage equaled or exceeded; $\mathrm{ft}^{3} / \mathrm{s}$, cubic foot per second; Sep, September; Nov, November]

\begin{tabular}{|c|c|c|c|c|c|c|c|c|c|}
\hline \multirow[b]{2}{*}{ APEE } & \multicolumn{9}{|c|}{ Sep-Nov daily mean streamflow $\left(\mathrm{ft}^{3} / \mathrm{s}\right)$ that was equaled or exceeded the indicated percentage of time at APEE level } \\
\hline & 98 & 95 & 90 & 80 & 50 & 20 & 10 & 5 & 2 \\
\hline 1 & 70.3 & 93.3 & 145. & 345. & 929. & 2200 & 3780 & 4620. & 7650. \\
\hline 2 & 63.1 & 91.6 & 110. & 240 & 689. & 1930. & 3230 & 3550. & 5460. \\
\hline 5 & 55.0 & 74.1 & 83.6 & 154. & 365. & 933. & 1650 & 2200 . & 3500 . \\
\hline 10 & 47.8 & 62.6 & 76.3 & 98.2 & 252. & 538. & 815. & 1350. & 1580. \\
\hline 20 & 44.2 & 53.4 & 62.0 & 72.9 & 148. & 313. & 449. & 800. & 891. \\
\hline 25 & 43.1 & 50.8 & 59.7 & 68.0 & 139. & 247. & 370. & 706. & 753. \\
\hline 30 & 40.3 & 49.8 & 57.8 & 66.2 & 127. & 214. & 338. & 612. & 654. \\
\hline 40 & 38.6 & 48.7 & 51.9 & 62.2 & 105 & 161. & 284. & 465. & 528. \\
\hline 50 & 36.5 & 41.9 & 46.9 & 56.0 & 88.0 & 134. & 228. & 300. & 446. \\
\hline 60 & 35.5 & 39.0 & 44.8 & 54.0 & 77.8 & 129. & 184. & 262. & 374. \\
\hline 70 & 32.3 & 36.5 & 39.9 & 51.8 & 70.5 & 118. & 166. & 225. & 338. \\
\hline 75 & 30.2 & 33.9 & 38.7 & 45.8 & 68.0 & 107. & 154. & 206. & 319. \\
\hline 80 & 29.0 & 32.4 & 37.7 & 44.9 & 66.0 & 99.3 & 151. & 188. & 292. \\
\hline 90 & 28.6 & 30.0 & 36.5 & 41.8 & 60.1 & 82.2 & 120. & 158. & 226. \\
\hline 95 & 28.0 & 29.0 & 33.7 & 39.6 & 55.5 & 76.8 & 114. & 132. & 202. \\
\hline 98 & 26.5 & 27.0 & 30.9 & 36.8 & 53.3 & 75.2 & 106. & 117. & 179. \\
\hline 99 & 26.2 & 27.0 & 30.9 & 36.8 & 52.0 & 75.0 & 101. & 116. & 175. \\
\hline
\end{tabular}

\section{Shade River near Chester, Ohio}

Years analyzed $=44$

Climatic year range $=1965-2008$

[APEE, annual percentage equaled or exceeded; $\mathrm{ft}^{3} / \mathrm{s}$, cubic foot per second; Sep, September; Nov, November]

\begin{tabular}{|c|c|c|c|c|c|c|c|c|c|}
\hline \multirow[b]{2}{*}{ APEE } & \multicolumn{9}{|c|}{ Sep-Nov daily mean streamflow $\left(\mathrm{ft}^{3} / \mathrm{s}\right)$ that was equaled or exceeded the indicated percentage of time at APEE level } \\
\hline & 95 & 90 & 80 & 70 & 50 & 30 & 20 & 10 & 5 \\
\hline 1 & 31.3 & 64.0 & 205. & 347. & 479. & 898. & 1780 & 2490 & 2860 \\
\hline 2 & 26.2 & 61.1 & 138. & 211. & 411. & 796. & 1280. & 2130 & 2340 \\
\hline 5 & 16.3 & 35.5 & 59.4 & 79.4 & 204. & 425. & 556. & 1060. & 1670. \\
\hline 10 & 12.1 & 18.9 & 26.8 & 41.6 & 99.3 & 224 & 295. & 448. & 771. \\
\hline 20 & 5.27 & 9.05 & 14.6 & 21.4 & 49.5 & 83.8 & 108. & 228 & 250 \\
\hline 25 & 4.45 & 7.80 & 12.0 & 15.0 & 37.5 & 67.0 & 84.0 & 189. & 200. \\
\hline 30 & 3.26 & 6.62 & 9.04 & 12.7 & 28.2 & 51.9 & 66.8 & 163. & 172. \\
\hline 40 & 1.43 & 4.45 & 6.06 & 8.24 & 18.1 & 29.8 & 44.4 & 108. & 133. \\
\hline 50 & 0.98 & 2.50 & 4.10 & 5.15 & 10.2 & 22.5 & 37.0 & 82.5 & 108. \\
\hline 60 & 0.66 & 1.80 & 3.08 & 3.68 & 7.80 & 16.5 & 32.0 & 65.4 & 96.1 \\
\hline 70 & 0.45 & 1.13 & 1.96 & 3.06 & 5.95 & 13.0 & 24.6 & 39.0 & 75.5 \\
\hline 75 & 0.45 & 1.00 & 1.80 & 2.45 & 5.55 & 12.0 & 18.0 & 32.0 & 68.8 \\
\hline 80 & 0.42 & 0.82 & 1.50 & 2.09 & 5.30 & 11.0 & 15.4 & 27.4 & 59.5 \\
\hline 90 & 0.32 & 0.56 & 1.10 & 1.45 & 3.66 & 7.58 & 12.0 & 18.2 & 36.5 \\
\hline 95 & 0.25 & 0.47 & 0.89 & 1.13 & 3.18 & 6.10 & 7.84 & 13.0 & 27.4 \\
\hline 98 & 0.22 & 0.39 & 0.70 & 0.85 & 2.32 & 5.29 & 5.87 & 11.1 & 23.8 \\
\hline 99 & 0.21 & 0.38 & 0.68 & 0.77 & 2.20 & 4.70 & 5.80 & 11.1 & 21.5 \\
\hline
\end{tabular}


03202000 Raccoon Creek at Adamsville, Ohio

Years analyzed $=86$

Climatic year range $=1915-2008$

[APEE, annual percentage equaled or exceeded; $\mathrm{ft}^{3} / \mathrm{s}$, cubic foot per second; Sep, September; Nov, November]

\begin{tabular}{|c|c|c|c|c|c|c|c|c|c|}
\hline \multirow[b]{2}{*}{ APEE } & \multicolumn{9}{|c|}{ Sep-Nov daily mean streamflow $\left(\mathrm{ft}^{3} / \mathrm{s}\right)$ that was equaled or exceeded the indicated percentage of time at APEE level } \\
\hline & 98 & 95 & 90 & 80 & 50 & 20 & 10 & 5 & 2 \\
\hline 1 & 14.7 & 30.9 & 72.4 & 132. & 721. & 2060. & 3850. & 4880 & 6750. \\
\hline 2 & 14.5 & 27.9 & 65.2 & 102. & 647. & 1970. & 3760 . & 4750 & 6660. \\
\hline 5 & 13.5 & 22.8 & 49.0 & 86.4 & 383. & 1280. & 2850 & 3350 . & 5260. \\
\hline 10 & 11.7 & 15.1 & 34.4 & 63.5 & 216. & 793. & 1620. & 2240 & 2740. \\
\hline 20 & 7.55 & 11.2 & 25.8 & 40.4 & 113. & 465. & 703. & 1020 . & 1330. \\
\hline 25 & 6.74 & 9.48 & 23.4 & 35.4 & 96.0 & 368. & 577. & 793. & 928. \\
\hline 30 & 5.08 & 8.79 & 21.5 & 32.8 & 76.5 & 287. & 497. & 652. & 817. \\
\hline 40 & 4.05 & 7.92 & 16.0 & 25.6 & 60.6 & 186. & 365. & 458. & 591. \\
\hline 50 & 3.32 & 7.15 & 13.7 & 22.0 & 44.5 & 139. & 238 & 324. & 523. \\
\hline 60 & 2.55 & 5.78 & 8.67 & 18.4 & 35.3 & 109. & 176. & 247. & 439. \\
\hline 70 & 2.11 & 4.49 & 6.88 & 16.2 & 29.8 & 77.2 & 137. & 189. & 377. \\
\hline 75 & 1.97 & 4.00 & 6.51 & 12.4 & 28.0 & 73.0 & 125. & 168. & 349. \\
\hline 80 & 1.90 & 3.45 & 6.43 & 9.80 & 25.8 & 64.8 & 110. & 152. & 256. \\
\hline 90 & 1.72 & 2.67 & 5.64 & 7.59 & 21.2 & 51.4 & 79.1 & 99.5 & 214. \\
\hline 95 & 1.61 & 2.51 & 4.17 & 6.80 & 19.3 & 41.6 & 65.2 & 86.5 & 173. \\
\hline 98 & 1.38 & 2.19 & 3.77 & 6.44 & 17.3 & 38.9 & 56.9 & 82.2 & 156. \\
\hline 99 & 1.32 & 2.10 & 3.77 & 6.44 & 16.0 & 37.6 & 54.8 & 80.9 & 154. \\
\hline
\end{tabular}

03217500 Scioto River at La Rue, Ohio

Years analyzed $=22$

Climatic year range $=1926-50$

[APEE, annual percentage equaled or exceeded; $\mathrm{ft}^{3} / \mathrm{s}$, cubic foot per second; Sep, September; Nov, November]

\begin{tabular}{|c|c|c|c|c|c|c|c|c|c|}
\hline \multirow[b]{2}{*}{ APEE } & \multicolumn{9}{|c|}{ Sep-Nov daily mean streamflow $\left(\mathrm{ft}^{3} / \mathrm{s}\right)$ that was equaled or exceeded the indicated percentage of time at APEE level } \\
\hline & 95 & 90 & 80 & 70 & 50 & 30 & 20 & 10 & 5 \\
\hline 1 & 13.0 & 22.3 & 48.4 & 78.9 & 282. & 1130. & 1290 & 2610 & 3550 \\
\hline 2 & 11.4 & 17.2 & 43.2 & 68.2 & 220 & 830 . & 1060 & 2080 & 3160 . \\
\hline 5 & 9.37 & 14.2 & 28.1 & 30.8 & 157. & 341. & 803. & 1060 . & 1680. \\
\hline 10 & 7.64 & 10.7 & 19.0 & 21.8 & 60.4 & 249. & 348. & 558. & 1200. \\
\hline 20 & 6.91 & 8.85 & 14.0 & 16.9 & 22.0 & 103. & 183. & 335. & 587. \\
\hline 25 & 5.82 & 8.15 & 13.0 & 15.9 & 19.5 & 67.8 & 150. & 222. & 444. \\
\hline 30 & 5.78 & 7.34 & 12.0 & 12.9 & 18.5 & 56.8 & 118. & 186. & 341. \\
\hline 40 & 4.37 & 6.05 & 9.60 & 11.9 & 14.2 & 25.8 & 74.6 & 143. & 251. \\
\hline 50 & 4.01 & 5.77 & 9.00 & 10.0 & 12.0 & 18.3 & 36.2 & 111. & 186. \\
\hline 60 & 3.95 & 5.04 & 7.63 & 8.47 & 10.5 & 17.8 & 26.0 & 76.8 & 152. \\
\hline 70 & 3.82 & 3.93 & 5.95 & 7.90 & 8.90 & 16.0 & 22.2 & 44.8 & 121. \\
\hline 75 & 3.65 & 3.93 & 5.44 & 6.90 & 8.75 & 14.0 & 15.8 & 31.1 & 114. \\
\hline 80 & 3.09 & 3.69 & 5.21 & 6.00 & 8.50 & 12.6 & 14.8 & 28.9 & 102. \\
\hline 90 & 3.05 & 3.48 & 4.12 & 4.95 & 6.50 & 9.10 & 13.9 & 25.1 & 78.6 \\
\hline 95 & 3.02 & 3.29 & 3.90 & 4.18 & 5.80 & 8.55 & 13.0 & 23.9 & 62.0 \\
\hline 98 & 2.83 & 3.18 & 3.90 & 3.98 & 4.60 & 8.05 & 11.3 & 22.8 & 50.4 \\
\hline 99 & 2.80 & 2.80 & 3.36 & 3.89 & 4.20 & 8.00 & 11.1 & 22.8 & 46.9 \\
\hline
\end{tabular}




\section{Little Scioto River above Marion, Ohio}

Years analyzed $=33$

Climatic year range $=1938-70$

[APEE, annual percentage equaled or exceeded; $\mathrm{ft}^{3} / \mathrm{s}$, cubic foot per second; Sep, September; Nov, November]

\begin{tabular}{|c|c|c|c|c|c|c|c|c|c|}
\hline \multirow[b]{2}{*}{ APEE } & \multicolumn{9}{|c|}{ Sep-Nov daily mean streamflow $\left(\mathrm{ft}^{3} / \mathrm{s}\right)$ that was equaled or exceeded the indicated percentage of time at APEE level } \\
\hline & 95 & 90 & 80 & 70 & 50 & 30 & 20 & 10 & 5 \\
\hline 1 & 0.00 & 0.22 & 2.18 & 11.6 & 31.0 & 99.0 & 164. & 347. & 400 \\
\hline 2 & 0.00 & 0.15 & 1.99 & 11.6 & 27.0 & 72.5 & 144. & 269. & 333. \\
\hline 5 & 0.00 & 0.00 & 1.40 & 4.27 & 13.8 & 38.4 & 75.0 & 136. & 160. \\
\hline 10 & 0.00 & 0.00 & 0.96 & 2.81 & 8.82 & 27.8 & 40.8 & 75.0 & 91.6 \\
\hline 20 & 0.00 & 0.00 & 0.76 & 1.26 & 3.14 & 10.0 & 20.2 & 32.8 & 46.6 \\
\hline 25 & 0.00 & 0.00 & 0.58 & 0.84 & 2.00 & 7.10 & 15.0 & 24.6 & 36.2 \\
\hline 30 & 0.00 & 0.00 & 0.40 & 0.63 & 1.70 & 5.35 & 10.9 & 18.0 & 24.9 \\
\hline 40 & 0.00 & 0.00 & 0.26 & 0.40 & 1.10 & 3.03 & 6.86 & 12.4 & 16.5 \\
\hline 50 & 0.00 & 0.00 & 0.18 & 0.30 & 0.90 & 1.76 & 5.10 & 8.88 & 11.3 \\
\hline 60 & 0.00 & 0.00 & 0.08 & 0.20 & 0.60 & 1.32 & 3.07 & 5.44 & 7.26 \\
\hline 70 & 0.00 & 0.00 & 0.00 & 0.02 & 0.40 & 0.85 & 1.27 & 4.27 & 5.36 \\
\hline 75 & 0.00 & 0.00 & 0.00 & 0.00 & 0.40 & 0.66 & 0.91 & 3.88 & 4.64 \\
\hline 80 & 0.00 & 0.00 & 0.00 & 0.00 & 0.30 & 0.58 & 0.73 & 3.57 & 4.32 \\
\hline 90 & 0.00 & 0.00 & 0.00 & 0.00 & 0.20 & 0.41 & 0.62 & 1.90 & 3.66 \\
\hline 95 & 0.00 & 0.00 & 0.00 & 0.00 & 0.10 & 0.35 & 0.53 & 1.39 & 3.00 \\
\hline 98 & 0.00 & 0.00 & 0.00 & 0.00 & 0.00 & 0.30 & 0.46 & 1.24 & 2.77 \\
\hline 99 & 0.00 & 0.00 & 0.00 & 0.00 & 0.00 & 0.28 & 0.45 & 1.12 & 1.93 \\
\hline
\end{tabular}

\section{Scioto River near Prospect, Ohio}

Years analyzed $=77$

Climatic year range $=1925-2008$

[APEE, annual percentage equaled or exceeded; $\mathrm{ft}^{3} / \mathrm{s}$, cubic foot per second; Sep, September; Nov, November]

\begin{tabular}{|c|c|c|c|c|c|c|c|c|c|}
\hline \multirow[b]{2}{*}{ APEE } & \multicolumn{9}{|c|}{ Sep-Nov daily mean streamflow $\left(\mathrm{ft}^{3} / \mathrm{s}\right)$ that was equaled or exceeded the indicated percentage of time at APEE level } \\
\hline & 98 & 95 & 90 & 80 & 50 & 20 & 10 & 5 & 2 \\
\hline 1 & 26.8 & 39.0 & 45.8 & 65.2 & 483. & 2300. & 3950. & 4970. & 5870 \\
\hline 2 & 24.8 & 33.5 & 43.1 & 59.0 & 395. & 2110 & 3600 . & 4550 . & 5410. \\
\hline 5 & 20.9 & 27.4 & 34.2 & 43.5 & 229. & 1380 . & 2370 . & 3600 & 4260. \\
\hline 10 & 19.1 & 22.2 & 25.8 & 36.0 & 152. & 815. & 1650. & 2300 & 3080 . \\
\hline 20 & 16.6 & 17.9 & 19.9 & 28.0 & 79.8 & 344. & 657. & 1210 . & 1970. \\
\hline 25 & 15.6 & 16.9 & 19.6 & 25.6 & 57.0 & 257. & 484. & 877. & 1530. \\
\hline 30 & 15.0 & 16.8 & 17.3 & 24.2 & 47.4 & 219. & 346. & 630. & 1090. \\
\hline 40 & 13.6 & 14.9 & 16.2 & 21.0 & 36.2 & 129. & 245 & 407. & 724. \\
\hline 50 & 12.1 & 13.9 & 15.0 & 18.6 & 29.0 & 85.6 & 210 & 290. & 449. \\
\hline 60 & 11.0 & 12.7 & 13.8 & 16.5 & 25.0 & 70.1 & 137. & 228 & 350. \\
\hline 70 & 9.78 & 11.0 & 12.0 & 14.0 & 23.0 & 52.0 & 110 & 177. & 270. \\
\hline 75 & 9.62 & 11.0 & 11.8 & 13.0 & 21.0 & 45.8 & 91.4 & 166. & 234. \\
\hline 80 & 8.37 & 10.9 & 11.0 & 13.0 & 20.4 & 41.4 & 77.6 & 114. & 207. \\
\hline 90 & 7.53 & 9.21 & 10.0 & 12.0 & 18.0 & 32.3 & 46.6 & 81.2 & 163. \\
\hline 95 & 7.03 & 8.33 & 8.96 & 11.0 & 17.0 & 26.2 & 43.7 & 58.2 & 117. \\
\hline 98 & 5.80 & 7.03 & 8.24 & 10.9 & 15.8 & 21.3 & 40.4 & 46.7 & 81.5 \\
\hline 99 & 5.06 & 6.74 & 7.58 & 9.38 & 15.0 & 21.0 & 32.6 & 46.5 & 73.6 \\
\hline
\end{tabular}




\section{Mill Creek near Bellepoint, Ohio}

Years analyzed $=66$

Climatic year range $=1943-2008$

[APEE, annual percentage equaled or exceeded; $\mathrm{ft}^{3} / \mathrm{s}$, cubic foot per second; Sep, September; Nov, November]

\begin{tabular}{|c|c|c|c|c|c|c|c|c|c|}
\hline \multirow[b]{2}{*}{ APEE } & \multicolumn{9}{|c|}{ Sep-Nov daily mean streamflow ( $\left.\mathrm{ft}^{3} / \mathrm{s}\right)$ that was equaled or exceeded the indicated percentage of time at APEE level } \\
\hline & 98 & 95 & 90 & 80 & 50 & 20 & 10 & 5 & 2 \\
\hline 1 & 6.27 & 10.1 & 14.4 & 33.4 & 303. & 1510. & 2390 & 3340. & 3900. \\
\hline 2 & 5.49 & 8.06 & 12.0 & 26.0 & 203. & 1370 . & 2100 & 2800 & 3250 . \\
\hline 5 & 3.79 & 5.19 & 8.73 & 15.5 & 113. & 645. & 1240 & 1630. & 1970. \\
\hline 10 & 2.65 & 4.27 & 6.55 & 10.9 & 51.5 & 203. & 405. & 720 . & 1140. \\
\hline 20 & 2.04 & 3.36 & 5.64 & 8.11 & 22.9 & 78.6 & 138. & 232. & 260. \\
\hline 25 & 1.97 & 3.04 & 5.55 & 7.70 & 20.0 & 62.6 & 110. & 168. & 230 \\
\hline 30 & 1.93 & 2.44 & 5.09 & 6.39 & 15.5 & 43.0 & 87.3 & 121. & 169. \\
\hline 40 & 1.70 & 1.96 & 3.67 & 5.38 & 11.0 & 25.8 & 45.6 & 75.2 & 101. \\
\hline 50 & 1.37 & 1.64 & 2.31 & 4.78 & 8.45 & 18.2 & 32.9 & 55.2 & 69.2 \\
\hline 60 & 1.02 & 1.30 & 1.58 & 4.18 & 7.40 & 15.6 & 26.5 & 47.7 & 51.3 \\
\hline 70 & 0.85 & 1.02 & 1.30 & 3.42 & 6.41 & 12.6 & 21.6 & 37.8 & 41.9 \\
\hline 75 & 0.80 & 0.90 & 1.20 & 2.92 & 5.95 & 10.5 & 18.3 & 30.6 & 34.3 \\
\hline 80 & 0.67 & 0.81 & 1.06 & 1.96 & 5.74 & 9.68 & 14.9 & 25.2 & 30.1 \\
\hline 90 & 0.31 & 0.60 & 0.70 & 1.47 & 4.38 & 7.52 & 11.1 & 15.9 & 22.8 \\
\hline 95 & 0.12 & 0.50 & 0.70 & 1.06 & 3.60 & 6.83 & 7.93 & 13.5 & 17.7 \\
\hline 98 & 0.03 & 0.37 & 0.64 & 0.80 & 2.98 & 5.87 & 6.80 & 10.3 & 15.4 \\
\hline 99 & 0.03 & 0.24 & 0.40 & 0.80 & 2.80 & 5.46 & 6.46 & 9.65 & 14.7 \\
\hline
\end{tabular}

03223000 Olentangy River at Claridon, Ohio

Years analyzed $=52$

Climatic year range $=1946-97$

[APEE, annual percentage equaled or exceeded; $\mathrm{ft}^{3} / \mathrm{s}$, cubic foot per second; Sep, September; Nov, November]

\begin{tabular}{|c|c|c|c|c|c|c|c|c|c|}
\hline \multirow[b]{2}{*}{ APEE } & \multicolumn{9}{|c|}{ Sep-Nov daily mean streamflow $\left(\mathrm{ft}^{3} / \mathrm{s}\right)$ that was equaled or exceeded the indicated percentage of time at APEE level } \\
\hline & 98 & 95 & 90 & 80 & 50 & 20 & 10 & 5 & 2 \\
\hline 1 & 8.14 & 11.5 & 24.2 & 41.2 & 229. & 1160. & 1450 & 1850 & 2580 \\
\hline 2 & 5.12 & 9.11 & 22.4 & 30.9 & 205 & 968. & 1380. & 1630. & 2350 \\
\hline 5 & 3.54 & 6.54 & 13.6 & 17.7 & 111. & 421. & 906. & 1190. & 1320. \\
\hline 10 & 2.70 & 4.66 & 8.65 & 11.9 & 58.1 & 238 & 361. & 764. & 928. \\
\hline 20 & 2.20 & 3.23 & 6.01 & 8.09 & 27.8 & 127. & 189. & 298. & 405. \\
\hline 25 & 1.71 & 2.68 & 5.51 & 7.46 & 22.5 & 90.8 & 156. & 212. & 306. \\
\hline 30 & 1.41 & 2.23 & 4.94 & 6.46 & 19.2 & 70.2 & 117. & 169. & 260. \\
\hline 40 & 0.86 & 1.69 & 3.32 & 5.15 & 13.6 & 31.5 & 69.4 & 109. & 197. \\
\hline 50 & 0.73 & 1.40 & 2.86 & 4.36 & 9.70 & 20.0 & 48.6 & 93.0 & 125. \\
\hline 60 & 0.44 & 1.06 & 2.00 & 3.40 & 6.80 & 16.0 & 32.9 & 74.0 & 104. \\
\hline 70 & 0.14 & 0.86 & 1.33 & 2.51 & 6.16 & 12.0 & 27.4 & 58.3 & 95.7 \\
\hline 75 & 0.03 & 0.57 & 0.96 & 1.83 & 5.60 & 11.0 & 24.6 & 51.1 & 73.8 \\
\hline 80 & 0.01 & 0.46 & 0.86 & 1.34 & 5.09 & 9.94 & 17.7 & 39.8 & 42.7 \\
\hline 90 & 0.00 & 0.15 & 0.43 & 0.88 & 3.66 & 8.41 & 11.8 & 24.9 & 32.1 \\
\hline 95 & 0.00 & 0.06 & 0.30 & 0.72 & 3.11 & 6.94 & 10.4 & 19.1 & 26.6 \\
\hline 98 & 0.00 & 0.03 & 0.23 & 0.54 & 2.70 & 6.39 & 9.27 & 17.4 & 21.6 \\
\hline 99 & 0.00 & 0.02 & 0.23 & 0.53 & 2.70 & 6.32 & 9.07 & 17.4 & 20.0 \\
\hline
\end{tabular}


03228300 Big Walnut Creek at Sunbury, Ohio

Years analyzed $=21$

Climatic year range $=1988-2008$

[APEE, annual percentage equaled or exceeded; $\mathrm{ft}^{3} / \mathrm{s}$, cubic foot per second; Sep, September; Nov, November]

\begin{tabular}{|c|c|c|c|c|c|c|c|c|c|}
\hline \multirow[b]{2}{*}{ APEE } & \multicolumn{9}{|c|}{ Sep-Nov daily mean streamflow $\left(\mathrm{ft}^{3} / \mathrm{s}\right)$ that was equaled or exceeded the indicated percentage of time at APEE level } \\
\hline & 95 & 90 & 80 & 70 & 50 & 30 & 20 & 10 & 5 \\
\hline 1 & 1.25 & 12.0 & 28.2 & 175. & 407. & 691. & 896. & 1540 & 3050 \\
\hline 2 & 0.93 & 8.24 & 23.7 & 106. & 258. & 510. & 742. & 1240 & 1920. \\
\hline 5 & 0.53 & 3.62 & 11.3 & 26.9 & 124. & 239. & 353. & 712 . & 943. \\
\hline 10 & 0.21 & 1.90 & 6.22 & 13.8 & 68.2 & 110. & 162. & 272. & 318. \\
\hline 20 & 0.08 & 0.84 & 3.79 & 5.98 & 16.0 & 56.5 & 71.7 & 106. & 121. \\
\hline 25 & 0.04 & 0.52 & 2.96 & 4.28 & 9.40 & 46.0 & 50.6 & 76.6 & 88.7 \\
\hline 30 & 0.04 & 0.44 & 1.20 & 3.02 & 7.20 & 35.0 & 43.6 & 51.7 & 70.0 \\
\hline 40 & 0.01 & 0.15 & 0.66 & 2.27 & 3.80 & 24.4 & 32.2 & 34.8 & 48.9 \\
\hline 50 & 0.00 & 0.06 & 0.34 & 0.72 & 2.60 & 12.4 & 19.6 & 29.4 & 39.0 \\
\hline 60 & 0.00 & 0.02 & 0.14 & 0.26 & 1.08 & 6.63 & 12.1 & 21.6 & 32.0 \\
\hline 70 & 0.00 & 0.01 & 0.05 & 0.09 & 0.67 & 5.14 & 8.15 & 11.2 & 25.5 \\
\hline 75 & 0.00 & 0.00 & 0.02 & 0.07 & 0.40 & 3.04 & 6.36 & 8.98 & 22.5 \\
\hline 80 & 0.00 & 0.00 & 0.01 & 0.06 & 0.30 & 2.38 & 4.69 & 7.14 & 20.0 \\
\hline 90 & 0.00 & 0.00 & 0.00 & 0.01 & 0.16 & 0.86 & 2.19 & 4.10 & 16.6 \\
\hline 95 & 0.00 & 0.00 & 0.00 & 0.01 & 0.05 & 0.38 & 1.80 & 2.52 & 7.55 \\
\hline 98 & 0.00 & 0.00 & 0.00 & 0.00 & 0.04 & 0.24 & 0.92 & 1.46 & 6.68 \\
\hline 99 & 0.00 & 0.00 & 0.00 & 0.00 & 0.04 & 0.22 & 0.67 & 1.14 & 5.97 \\
\hline
\end{tabular}

03230500 Big Darby Creek at Darbyville, Ohio

Years analyzed $=85$

Climatic year range $=1921-2008$

[APEE, annual percentage equaled or exceeded; $\mathrm{ft}^{3} / \mathrm{s}$, cubic foot per second; Sep, September; Nov, November]

\begin{tabular}{|c|c|c|c|c|c|c|c|c|c|}
\hline \multirow[b]{2}{*}{ APEE } & \multicolumn{9}{|c|}{ Sep-Nov daily mean streamflow $\left(\mathrm{ft}^{3} / \mathrm{s}\right)$ that was equaled or exceeded the indicated percentage of time at APEE level } \\
\hline & 98 & 95 & 90 & 80 & 50 & 20 & 10 & 5 & 2 \\
\hline 1 & 22.6 & 26.2 & 46.2 & 96.0 & 527. & 2400 & 5030 & 5540. & 7530. \\
\hline 2 & 21.8 & 25.4 & 41.4 & 81.8 & 466. & 1980. & 4460. & 4750 . & 6860. \\
\hline 5 & 19.9 & 24.5 & 36.2 & 64.5 & 278. & 1210 & 2990. & 3650 . & 3900. \\
\hline 10 & 16.2 & 22.0 & 34.5 & 51.6 & 160. & 547. & 1560. & 1950. & 2690. \\
\hline 20 & 14.8 & 18.0 & 29.4 & 40.4 & 85.6 & 264. & 669. & 941. & 1100. \\
\hline 25 & 12.7 & 17.3 & 28.6 & 37.2 & 68.0 & 222 & 429. & 705. & 861. \\
\hline 30 & 10.7 & 15.3 & 25.9 & 33.7 & 63.0 & 170. & 361. & 607. & 711. \\
\hline 40 & 8.83 & 12.4 & 21.7 & 28.2 & 47.4 & 133. & 266. & 393. & 490. \\
\hline 50 & 7.88 & 11.0 & 18.0 & 24.0 & 41.0 & 98.8 & 159. & 294. & 398. \\
\hline 60 & 6.19 & 8.98 & 15.8 & 19.4 & 35.8 & 85.7 & 125. & 257. & 341. \\
\hline 70 & 5.24 & 8.27 & 12.0 & 18.4 & 32.6 & 69.4 & 102. & 214. & 279. \\
\hline 75 & 4.52 & 7.56 & 11.2 & 16.2 & 31.0 & 63.6 & 91.4 & 182. & 248. \\
\hline 80 & 4.19 & 6.39 & 9.78 & 15.5 & 29.0 & 50.1 & 81.5 & 168. & 228. \\
\hline 90 & 3.49 & 4.61 & 7.52 & 13.0 & 25.0 & 44.1 & 69.8 & 132. & 194. \\
\hline 95 & 2.58 & 3.72 & 6.48 & 12.0 & 23.0 & 40.9 & 60.8 & 95.6 & 155. \\
\hline 98 & 1.85 & 3.48 & 6.00 & 11.6 & 21.0 & 40.0 & 56.3 & 73.9 & 129. \\
\hline 99 & 1.76 & 3.42 & 5.80 & 10.2 & 21.0 & 39.0 & 56.0 & 69.4 & 123. \\
\hline
\end{tabular}




\section{Paint Creek near Greenfield, Ohio}

Years analyzed $=55$

Climatic year range $=1926-2008$

[APEE, annual percentage equaled or exceeded; $\mathrm{ft}^{3} / \mathrm{s}$, cubic foot per second; Sep, September; Nov, November]

\begin{tabular}{|c|c|c|c|c|c|c|c|c|c|}
\hline \multirow[b]{2}{*}{ APEE } & \multicolumn{9}{|c|}{ Sep-Nov daily mean streamflow $\left(\mathrm{ft}^{3} / \mathrm{s}\right)$ that was equaled or exceeded the indicated percentage of time at APEE level } \\
\hline & 98 & 95 & 90 & 80 & 50 & 20 & 10 & 5 & 2 \\
\hline 1 & 5.11 & 6.88 & 15.4 & 60.6 & 360. & 1160. & 1980. & 4610 & 5180. \\
\hline 2 & 3.66 & 4.88 & 14.3 & 41.8 & 248 & 863. & 1880. & 3730. & 4640 . \\
\hline 5 & 2.43 & 4.52 & 8.30 & 18.7 & 132. & 571. & 1300 & 1800. & 2570 \\
\hline 10 & 1.46 & 3.39 & 5.88 & 13.0 & 77.8 & 335. & 665. & 1230. & 1900. \\
\hline 20 & 1.10 & 2.60 & 4.28 & 8.40 & 35.6 & 144. & 299. & 567. & 821. \\
\hline 25 & 0.97 & 2.32 & 3.54 & 6.46 & 29.0 & 129. & 259 & 448. & 558. \\
\hline 30 & 0.95 & 2.09 & 3.08 & 5.41 & 24.2 & 119. & 233. & 393. & 476. \\
\hline 40 & 0.69 & 1.46 & 2.61 & 4.50 & 18.0 & 85.4 & 172. & 302. & 320. \\
\hline 50 & 0.51 & 1.00 & 1.82 & 3.84 & 14.0 & 56.0 & 95.0 & 235 & 240 \\
\hline 60 & 0.32 & 0.50 & 1.37 & 2.92 & 11.8 & 43.4 & 73.9 & 183. & 201. \\
\hline 70 & 0.21 & 0.37 & 1.08 & 2.40 & 8.30 & 34.6 & 58.0 & 143. & 165. \\
\hline 75 & 0.20 & 0.29 & 0.96 & 1.96 & 7.00 & 28.4 & 53.8 & 132. & 149. \\
\hline 80 & 0.15 & 0.28 & 0.78 & 1.40 & 5.62 & 18.8 & 47.6 & 122. & 148. \\
\hline 90 & 0.11 & 0.19 & 0.46 & 1.02 & 3.46 & 14.8 & 32.7 & 70.0 & 114. \\
\hline 95 & 0.02 & 0.15 & 0.36 & 0.62 & 2.60 & 9.10 & 28.5 & 55.8 & 99.3 \\
\hline 98 & 0.01 & 0.10 & 0.25 & 0.45 & 2.20 & 7.25 & 25.8 & 45.2 & 96.0 \\
\hline 99 & 0.01 & 0.10 & 0.21 & 0.40 & 2.20 & 7.12 & 24.8 & 44.4 & 95.9 \\
\hline
\end{tabular}

\section{Tar Hollow Creek at Tar Hollow State Park, Ohio}

Years analyzed $=32$

Climatic year range $=1946-77$

[APEE, annual percentage equaled or exceeded; $\mathrm{ft}^{3} / \mathrm{s}$, cubic foot per second; Sep, September; Nov, November]

\begin{tabular}{|c|c|c|c|c|c|c|c|c|c|}
\hline \multirow[b]{2}{*}{ APEE } & \multicolumn{9}{|c|}{ Sep-Nov daily mean streamflow $\left(\mathrm{ft}^{3} / \mathrm{s}\right)$ that was equaled or exceeded the indicated percentage of time at APEE level } \\
\hline & 95 & 90 & 80 & 70 & 50 & 30 & 20 & 10 & 5 \\
\hline 1 & 0.00 & 0.00 & 0.00 & 0.27 & 1.65 & 4.13 & 6.96 & 11.7 & 14.0 \\
\hline 2 & 0.00 & 0.00 & 0.00 & 0.27 & 1.24 & 3.92 & 4.79 & 5.90 & 8.43 \\
\hline 5 & 0.00 & 0.00 & 0.00 & 0.00 & 0.42 & 1.07 & 1.41 & 2.62 & 5.01 \\
\hline 10 & 0.00 & 0.00 & 0.00 & 0.00 & 0.24 & 0.44 & 0.59 & 0.98 & 3.16 \\
\hline 20 & 0.00 & 0.00 & 0.00 & 0.00 & 0.05 & 0.21 & 0.34 & 0.49 & 1.91 \\
\hline 25 & 0.00 & 0.00 & 0.00 & 0.00 & 0.01 & 0.20 & 0.33 & 0.40 & 1.64 \\
\hline 30 & 0.00 & 0.00 & 0.00 & 0.00 & 0.00 & 0.12 & 0.24 & 0.40 & 1.27 \\
\hline 40 & 0.00 & 0.00 & 0.00 & 0.00 & 0.00 & 0.06 & 0.14 & 0.27 & 0.64 \\
\hline 50 & 0.00 & 0.00 & 0.00 & 0.00 & 0.00 & 0.03 & 0.11 & 0.20 & 0.35 \\
\hline 60 & 0.00 & 0.00 & 0.00 & 0.00 & 0.00 & 0.01 & 0.08 & 0.10 & 0.21 \\
\hline 70 & 0.00 & 0.00 & 0.00 & 0.00 & 0.00 & 0.00 & 0.04 & 0.10 & 0.13 \\
\hline 75 & 0.00 & 0.00 & 0.00 & 0.00 & 0.00 & 0.00 & 0.02 & 0.09 & 0.11 \\
\hline 80 & 0.00 & 0.00 & 0.00 & 0.00 & 0.00 & 0.00 & 0.02 & 0.04 & 0.11 \\
\hline 90 & 0.00 & 0.00 & 0.00 & 0.00 & 0.00 & 0.00 & 0.00 & 0.03 & 0.07 \\
\hline 95 & 0.00 & 0.00 & 0.00 & 0.00 & 0.00 & 0.00 & 0.00 & 0.03 & 0.05 \\
\hline 98 & 0.00 & 0.00 & 0.00 & 0.00 & 0.00 & 0.00 & 0.00 & 0.02 & 0.04 \\
\hline 99 & 0.00 & 0.00 & 0.00 & 0.00 & 0.00 & 0.00 & 0.00 & 0.01 & 0.04 \\
\hline
\end{tabular}




\section{Upper Twin Creek at McGaw, Ohio}

Years analyzed $=46$

Climatic year range $=1963-2008$

[APEE, annual percentage equaled or exceeded; $\mathrm{ft}^{3} / \mathrm{s}$, cubic foot per second; Sep, September; Nov, November]

\begin{tabular}{|c|c|c|c|c|c|c|c|c|c|}
\hline \multirow[b]{2}{*}{ APEE } & \multicolumn{9}{|c|}{ Sep-Nov daily mean streamflow ( $\mathrm{ft} 3 / \mathrm{s}$ ) that was equaled or exceeded the indicated percentage of time at APEE level } \\
\hline & 95 & 90 & 80 & 70 & 50 & 30 & 20 & 10 & 5 \\
\hline 1 & 0.61 & 0.90 & 3.78 & 9.91 & 36.0 & 103. & 184. & 297. & 374. \\
\hline 2 & 0.53 & 0.84 & 3.53 & 5.00 & 18.9 & 83.6 & 98.8 & 189. & 246. \\
\hline 5 & 0.23 & 0.56 & 1.51 & 3.11 & 8.47 & 27.3 & 43.3 & 73.4 & 80.7 \\
\hline 10 & 0.12 & 0.21 & 0.73 & 1.37 & 5.41 & 13.1 & 19.4 & 31.2 & 37.3 \\
\hline 20 & 0.04 & 0.11 & 0.27 & 0.64 & 2.89 & 4.70 & 7.40 & 17.2 & 24.2 \\
\hline 25 & 0.01 & 0.09 & 0.21 & 0.48 & 1.80 & 3.76 & 6.00 & 13.6 & 20.6 \\
\hline 30 & 0.00 & 0.08 & 0.16 & 0.41 & 1.16 & 3.07 & 4.06 & 12.5 & 15.4 \\
\hline 40 & 0.00 & 0.00 & 0.08 & 0.21 & 0.77 & 1.85 & 2.28 & 5.97 & 9.34 \\
\hline 50 & 0.00 & 0.00 & 0.04 & 0.13 & 0.43 & 0.78 & 1.62 & 2.65 & 7.21 \\
\hline 60 & 0.00 & 0.00 & 0.02 & 0.09 & 0.24 & 0.58 & 1.15 & 2.04 & 5.77 \\
\hline 70 & 0.00 & 0.00 & 0.01 & 0.05 & 0.13 & 0.34 & 0.79 & 1.78 & 4.18 \\
\hline 75 & 0.00 & 0.00 & 0.00 & 0.03 & 0.11 & 0.29 & 0.63 & 1.60 & 3.26 \\
\hline 80 & 0.00 & 0.00 & 0.00 & 0.02 & 0.07 & 0.27 & 0.51 & 1.41 & 2.62 \\
\hline 90 & 0.00 & 0.00 & 0.00 & 0.00 & 0.03 & 0.18 & 0.30 & 0.69 & 1.10 \\
\hline 95 & 0.00 & 0.00 & 0.00 & 0.00 & 0.02 & 0.14 & 0.22 & 0.51 & 0.85 \\
\hline 98 & 0.00 & 0.00 & 0.00 & 0.00 & 0.02 & 0.10 & 0.19 & 0.43 & 0.65 \\
\hline 99 & 0.00 & 0.00 & 0.00 & 0.00 & 0.02 & 0.10 & 0.18 & 0.42 & 0.64 \\
\hline
\end{tabular}

\section{Ohio Brush Creek near West Union, Ohio}

Years analyzed $=78$

Climatic year range $=1926-2008$

[APEE, annual percentage equaled or exceeded; $\mathrm{ft}^{3} / \mathrm{s}$, cubic foot per second; Sep, September; Nov, November]

\begin{tabular}{|c|c|c|c|c|c|c|c|c|c|}
\hline \multirow[b]{2}{*}{ APEE } & \multicolumn{9}{|c|}{ Sep-Nov daily mean streamflow $\left(\mathrm{ft}^{3} / \mathrm{s}\right)$ that was equaled or exceeded the indicated percentage of time at APEE level } \\
\hline & 98 & 95 & 90 & 80 & 50 & 20 & 10 & 5 & 2 \\
\hline 1 & 3.49 & 11.8 & 63.8 & 330. & 1920. & 4900. & 8080. & 8890. & 13400 \\
\hline 2 & 3.22 & 8.72 & 49.7 & 191. & 1330. & 3360 . & 4990. & 8150. & 10200. \\
\hline 5 & 2.27 & 4.90 & 22.7 & 82.9 & 483. & 1480 . & 2080 . & 2950. & 5500. \\
\hline 10 & 2.05 & 4.35 & 14.1 & 33.2 & 210 & 668. & 947. & 1330. & 2210 \\
\hline 20 & 1.27 & 2.98 & 7.35 & 16.9 & 85.1 & 244. & 429. & 555. & 906. \\
\hline 25 & 1.00 & 2.65 & 6.64 & 15.2 & 61.5 & 178. & 307. & 435. & 700 \\
\hline 30 & 0.93 & 2.35 & 4.16 & 10.3 & 48.5 & 151. & 256. & 343. & 579. \\
\hline 40 & 0.82 & 1.97 & 2.89 & 6.88 & 30.7 & 104. & 172. & 223. & 353. \\
\hline 50 & 0.32 & 1.10 & 1.68 & 4.60 & 15.5 & 58.4 & 123. & 154. & 263. \\
\hline 60 & 0.22 & 0.90 & 1.20 & 3.47 & 11.2 & 44.4 & 82.7 & 124. & 203. \\
\hline 70 & 0.19 & 0.67 & 0.95 & 1.97 & 7.44 & 29.4 & 63.5 & 95.3 & 161. \\
\hline 75 & 0.14 & 0.54 & 0.80 & 1.58 & 6.05 & 25.6 & 57.9 & 81.5 & 141. \\
\hline 80 & 0.13 & 0.48 & 0.68 & 1.34 & 5.28 & 22.8 & 49.5 & 76.1 & 123. \\
\hline 90 & 0.06 & 0.20 & 0.32 & 0.87 & 3.94 & 18.2 & 34.1 & 64.1 & 84.0 \\
\hline 95 & 0.06 & 0.13 & 0.20 & 0.83 & 3.27 & 13.5 & 27.1 & 51.1 & 66.8 \\
\hline 98 & 0.06 & 0.11 & 0.20 & 0.74 & 2.59 & 10.8 & 21.6 & 49.2 & 57.5 \\
\hline 99 & 0.06 & 0.11 & 0.20 & 0.57 & 2.55 & 10.0 & 18.4 & 49.1 & 56.4 \\
\hline
\end{tabular}




\section{White Oak Creek near Georgetown, Ohio}

Years analyzed $=81$

Climatic year range $=1924-2008$

[APEE, annual percentage equaled or exceeded; $\mathrm{ft}^{3} / \mathrm{s}$, cubic foot per second; Sep, September; Nov, November]

\begin{tabular}{|c|c|c|c|c|c|c|c|c|c|}
\hline \multirow[b]{2}{*}{ APEE } & \multicolumn{9}{|c|}{ Sep-Nov daily mean streamflow $\left(\mathrm{ft}^{3} / \mathrm{s}\right)$ that was equaled or exceeded the indicated percentage of time at APEE level } \\
\hline & 98 & 95 & 90 & 80 & 50 & 20 & 10 & 5 & 2 \\
\hline 1 & 5.76 & 9.03 & 22.4 & 116. & 970. & 4510. & 5920. & 7190. & 9130. \\
\hline 2 & 5.60 & 7.31 & 18.7 & 104. & 705 . & 2910 . & 4210 & 5580. & 6610 \\
\hline 5 & 2.54 & 4.14 & 11.2 & 30.7 & 218. & 1150 & 1890. & 3000 . & 3800. \\
\hline 10 & 0.72 & 2.54 & 6.26 & 14.4 & 91.2 & 451. & 637. & 938. & 1400 \\
\hline 20 & 0.10 & 0.79 & 3.28 & 8.88 & 34.2 & 148. & 196. & 303. & 383. \\
\hline 25 & 0.06 & 0.51 & 2.80 & 8.12 & 27.0 & 99.2 & 144. & 203. & 282 \\
\hline 30 & 0.06 & 0.40 & 2.24 & 6.66 & 22.0 & 77.2 & 111. & 164. & 200 \\
\hline 40 & 0.00 & 0.21 & 1.51 & 5.18 & 13.2 & 46.8 & 72.4 & 106. & 124. \\
\hline 50 & 0.00 & 0.11 & 0.42 & 3.58 & 9.00 & 32.6 & 53.2 & 75.4 & 92.8 \\
\hline 60 & 0.00 & 0.00 & 0.20 & 1.96 & 4.80 & 22.7 & 34.1 & 46.3 & 74.5 \\
\hline 70 & 0.00 & 0.00 & 0.10 & 0.78 & 3.72 & 16.0 & 25.2 & 35.5 & 46.9 \\
\hline 75 & 0.00 & 0.00 & 0.10 & 0.72 & 3.00 & 12.6 & 22.4 & 29.6 & 42.1 \\
\hline 80 & 0.00 & 0.00 & 0.00 & 0.40 & 1.90 & 11.0 & 19.3 & 23.0 & 36.8 \\
\hline 90 & 0.00 & 0.00 & 0.00 & 0.10 & 0.92 & 7.22 & 11.8 & 18.3 & 27.9 \\
\hline 95 & 0.00 & 0.00 & 0.00 & 0.00 & 0.62 & 4.97 & 9.10 & 15.0 & 20.6 \\
\hline 98 & 0.00 & 0.00 & 0.00 & 0.00 & 0.40 & 3.76 & 7.62 & 13.9 & 17.7 \\
\hline 99 & 0.00 & 0.00 & 0.00 & 0.00 & 0.40 & 3.12 & 7.38 & 13.7 & 16.7 \\
\hline
\end{tabular}

03240000 Little Miami River near Oldtown, Ohio

Years analyzed $=57$

Climatic year range $=1952-2008$

[APEE, annual percentage equaled or exceeded; $\mathrm{ft}^{3} / \mathrm{s}$, cubic foot per second; Sep, September; Nov, November]

\begin{tabular}{|c|c|c|c|c|c|c|c|c|c|}
\hline \multirow[b]{2}{*}{ APEE } & \multicolumn{9}{|c|}{ Sep-Nov daily mean streamflow ( $\left.\mathrm{ft}^{3} / \mathrm{s}\right)$ that was equaled or exceeded the indicated percentage of time at APEE level } \\
\hline & 98 & 95 & 90 & 80 & 50 & 20 & 10 & 5 & 2 \\
\hline 1 & 13.5 & 19.6 & 23.0 & 34.0 & 209. & 570. & 939. & 1660 & 2910 \\
\hline 2 & 13.3 & 16.5 & 22.0 & 32.0 & 164. & 458. & 756. & 1490. & 2360 \\
\hline 5 & 12.7 & 14.9 & 20.8 & 25.0 & 99.8 & 220. & 519. & 587. & 715. \\
\hline 10 & 12.2 & 13.9 & 19.0 & 23.9 & 60.8 & 133. & 237. & 371. & 385. \\
\hline 20 & 11.3 & 13.0 & 17.6 & 20.6 & 42.0 & 75.5 & 131. & 200. & 232. \\
\hline 25 & 11.2 & 12.0 & 16.6 & 20.0 & 34.0 & 68.8 & 112. & 168. & 209. \\
\hline 30 & 11.2 & 12.0 & 15.6 & 18.6 & 31.0 & 64.6 & 99.5 & 151. & 187. \\
\hline 40 & 10.2 & 11.9 & 14.6 & 17.1 & 29.0 & 53.5 & 74.4 & 127. & 165. \\
\hline 50 & 9.28 & 10.9 & 13.0 & 16.6 & 26.0 & 48.8 & 64.6 & 118. & 146. \\
\hline 60 & 8.61 & 10.8 & 12.6 & 14.6 & 23.0 & 41.9 & 58.0 & 97.1 & 129. \\
\hline 70 & 8.18 & 9.81 & 11.0 & 13.0 & 21.0 & 37.8 & 53.6 & 74.1 & 119. \\
\hline 75 & 7.88 & 9.61 & 10.8 & 13.0 & 20.0 & 36.4 & 51.4 & 69.3 & 115. \\
\hline 80 & 7.01 & 8.80 & 9.56 & 12.0 & 19.0 & 36.0 & 49.4 & 64.6 & 109. \\
\hline 90 & 6.06 & 7.85 & 8.58 & 11.7 & 18.0 & 32.0 & 42.0 & 48.5 & 101. \\
\hline 95 & 5.77 & 7.29 & 7.98 & 10.8 & 16.6 & 27.8 & 38.2 & 47.8 & 96.3 \\
\hline 98 & 5.34 & 6.76 & 7.43 & 9.80 & 15.8 & 26.5 & 36.2 & 46.2 & 93.4 \\
\hline 99 & 3.76 & 5.28 & 7.18 & 9.42 & 15.0 & 26.0 & 36.0 & 45.1 & 91.5 \\
\hline
\end{tabular}




\section{Massies Creek at Wilberforce, Ohio}

Years analyzed $=57$

Climatic year range $=1952-2008$

[APEE, annual percentage equaled or exceeded; $\mathrm{ft}^{3} / \mathrm{s}$, cubic foot per second; Sep, September; Nov, November]

\begin{tabular}{|c|c|c|c|c|c|c|c|c|c|}
\hline \multirow[b]{2}{*}{ APEE } & \multicolumn{9}{|c|}{ Sep-Nov daily mean streamflow $\left(\mathrm{ft}^{3} / \mathrm{s}\right)$ that was equaled or exceeded the indicated percentage of time at APEE level } \\
\hline & 98 & 95 & 90 & 80 & 50 & 20 & 10 & 5 & 2 \\
\hline 1 & 3.32 & 5.90 & 10.8 & 17.0 & 123. & 378. & 608. & 998. & 1350 \\
\hline 2 & 3.11 & 5.67 & 8.43 & 14.3 & 99.6 & 325. & 531. & 781. & 1230 \\
\hline 5 & 2.74 & 5.02 & 6.78 & 10.3 & 59.8 & 142. & 336. & 445 & 570. \\
\hline 10 & 2.27 & 4.37 & 5.75 & 8.26 & 34.6 & 91.6 & 144. & 225 & 312. \\
\hline 20 & 2.11 & 4.06 & 5.06 & 7.02 & 21.0 & 46.5 & 82.3 & 118. & 151. \\
\hline 25 & 2.11 & 3.74 & 4.88 & 6.46 & 16.0 & 35.8 & 58.8 & 102. & 124. \\
\hline 30 & 1.99 & 3.31 & 4.67 & 6.22 & 13.4 & 30.2 & 51.6 & 93.4 & 94.1 \\
\hline 40 & 1.99 & 3.09 & 4.10 & 5.52 & 11.0 & 21.0 & 41.3 & 66.2 & 84.6 \\
\hline 50 & 1.62 & 2.89 & 3.76 & 5.12 & 9.70 & 19.0 & 34.4 & 54.7 & 65.2 \\
\hline 60 & 1.49 & 2.59 & 3.20 & 4.22 & 8.78 & 16.4 & 27.0 & 46.5 & 57.2 \\
\hline 70 & 1.12 & 1.71 & 2.46 & 3.84 & 7.00 & 14.8 & 21.4 & 38.8 & 49.0 \\
\hline 75 & 0.68 & 1.55 & 2.26 & 3.60 & 6.00 & 13.0 & 20.2 & 36.2 & 45.6 \\
\hline 80 & 0.60 & 1.37 & 1.90 & 3.18 & 6.00 & 10.4 & 16.5 & 32.9 & 43.2 \\
\hline 90 & 0.50 & 1.18 & 1.76 & 2.66 & 5.30 & 7.97 & 14.2 & 22.3 & 36.8 \\
\hline 95 & 0.41 & 1.05 & 1.50 & 2.36 & 4.02 & 7.22 & 12.6 & 19.2 & 34.1 \\
\hline 98 & 0.40 & 0.98 & 1.48 & 2.25 & 3.78 & 6.82 & 9.60 & 15.3 & 31.1 \\
\hline 99 & 0.39 & 0.90 & 1.48 & 2.12 & 3.70 & 6.50 & 8.82 & 15.2 & 29.6 \\
\hline
\end{tabular}

\section{Bokengehalas Creek near De Graff, Ohio}

Years analyzed $=34$

Climatic year range $=1957-90$

[APEE, annual percentage equaled or exceeded; $\mathrm{ft}^{3} / \mathrm{s}$, cubic foot per second; Sep, September; Nov, November]

\begin{tabular}{|c|c|c|c|c|c|c|c|c|c|}
\hline \multirow[b]{2}{*}{ APEE } & \multicolumn{9}{|c|}{ Sep-Nov daily mean streamflow $\left(\mathrm{ft}^{3} / \mathrm{s}\right)$ that was equaled or exceeded the indicated percentage of time at APEE level } \\
\hline & 95 & 90 & 80 & 70 & 50 & 30 & 20 & 10 & 5 \\
\hline 1 & 11.8 & 13.0 & 19.0 & 29.5 & 52.5 & 103. & 149. & 309. & 375. \\
\hline 2 & 8.96 & 10.8 & 15.6 & 24.4 & 42.5 & 76.8 & 85.9 & 226. & 333. \\
\hline 5 & 6.81 & 8.42 & 12.0 & 16.5 & 26.4 & 45.3 & 56.8 & 116. & 186. \\
\hline 10 & 5.80 & 7.20 & 8.88 & 13.0 & 19.9 & 29.8 & 38.2 & 51.1 & 116. \\
\hline 20 & 5.68 & 6.47 & 7.80 & 9.84 & 14.3 & 19.0 & 22.0 & 34.5 & 68.8 \\
\hline 25 & 5.27 & 6.35 & 7.50 & 9.20 & 13.0 & 16.5 & 21.0 & 30.0 & 59.5 \\
\hline 30 & 5.27 & 6.13 & 7.30 & 8.84 & 11.6 & 15.5 & 20.0 & 24.6 & 53.5 \\
\hline 40 & 4.88 & 5.24 & 6.50 & 7.51 & 9.82 & 14.0 & 17.0 & 20.5 & 41.1 \\
\hline 50 & 4.50 & 5.10 & 6.20 & 6.95 & 8.85 & 12.5 & 15.0 & 19.0 & 31.3 \\
\hline 60 & 4.43 & 4.90 & 5.80 & 6.48 & 8.39 & 10.5 & 14.0 & 17.0 & 26.8 \\
\hline 70 & 4.10 & 4.60 & 5.60 & 6.10 & 7.88 & 10.5 & 12.6 & 16.0 & 24.6 \\
\hline 75 & 4.10 & 4.50 & 5.50 & 5.80 & 7.80 & 9.95 & 12.0 & 15.0 & 23.0 \\
\hline 80 & 3.95 & 4.45 & 5.30 & 5.65 & 7.47 & 9.86 & 12.0 & 13.0 & 15.8 \\
\hline 90 & 3.49 & 4.20 & 4.92 & 5.30 & 6.86 & 9.10 & 10.0 & 12.0 & 13.8 \\
\hline 95 & 3.47 & 3.94 & 4.76 & 5.07 & 6.48 & 8.65 & 9.80 & 11.5 & 13.0 \\
\hline 98 & 3.25 & 3.85 & 4.27 & 4.93 & 6.37 & 8.14 & 9.07 & 10.9 & 12.8 \\
\hline 99 & 3.25 & 3.85 & 4.10 & 4.85 & 6.15 & 8.10 & 9.00 & 10.4 & 12.0 \\
\hline
\end{tabular}


03264000 Greenville Creek near Bradford, Ohio

Years analyzed $=77$

Climatic year range $=1930-2008$

[APEE, annual percentage equaled or exceeded; $\mathrm{ft}^{3} / \mathrm{s}$, cubic foot per second; Sep, September; Nov, November]

\begin{tabular}{|c|c|c|c|c|c|c|c|c|c|}
\hline \multirow[b]{2}{*}{ APEE } & \multicolumn{9}{|c|}{ Sep-Nov daily mean streamflow ( $\left.\mathrm{ft}^{3} / \mathrm{s}\right)$ that was equaled or exceeded the indicated percentage of time at APEE level } \\
\hline & 98 & 95 & 90 & 80 & 50 & 20 & 10 & 5 & 2 \\
\hline 1 & 23.4 & 26.9 & 38.8 & 65.6 & 230. & 1140. & 1890. & 2830 & 4130 \\
\hline 2 & 22.5 & 25.9 & 35.3 & 59.8 & 198. & 969. & 1780 & 2430 & 3760. \\
\hline 5 & 19.5 & 22.9 & 30.8 & 44.8 & 123. & 476. & 861. & 1220 & 2100 \\
\hline 10 & 17.1 & 21.5 & 26.0 & 36.5 & 72.4 & 261. & 434. & 527. & 820. \\
\hline 20 & 15.6 & 19.7 & 25.2 & 30.6 & 52.6 & 134. & 206. & 237. & 385. \\
\hline 25 & 15.0 & 18.7 & 23.8 & 29.0 & 45.0 & 94.8 & 169. & 194. & 326. \\
\hline 30 & 14.6 & 18.6 & 23.6 & 27.8 & 42.0 & 79.0 & 138. & 160. & 267. \\
\hline 40 & 14.0 & 16.9 & 21.8 & 25.0 & 37.0 & 60.6 & 98.8 & 124. & 194. \\
\hline 50 & 12.1 & 14.9 & 18.8 & 23.2 & 34.0 & 52.4 & 84.4 & 108. & 150. \\
\hline 60 & 12.1 & 13.9 & 15.6 & 21.0 & 29.0 & 47.7 & 63.7 & 89.0 & 134. \\
\hline 70 & 10.9 & 12.0 & 13.5 & 19.0 & 27.0 & 41.0 & 54.0 & 79.0 & 121. \\
\hline 75 & 10.5 & 11.0 & 12.8 & 18.0 & 26.0 & 38.2 & 51.4 & 76.5 & 116. \\
\hline 80 & 9.46 & 10.9 & 12.0 & 15.8 & 24.0 & 35.4 & 47.8 & 73.7 & 111. \\
\hline 90 & 8.37 & 9.00 & 11.0 & 14.6 & 22.0 & 31.5 & 41.0 & 65.3 & 102. \\
\hline 95 & 7.73 & 8.29 & 9.97 & 13.0 & 21.2 & 29.6 & 36.8 & 62.5 & 94.6 \\
\hline 98 & 6.49 & 7.40 & 9.07 & 12.1 & 20.0 & 28.0 & 34.7 & 61.5 & 86.7 \\
\hline 99 & 6.20 & 7.38 & 8.20 & 10.6 & 19.0 & 27.4 & 33.0 & 60.5 & 82.5 \\
\hline
\end{tabular}

03265000 Stillwater River at Pleasant Hill, Ohio

Years analyzed $=87$

Climatic year range $=1916-2008$

[APEE, annual percentage equaled or exceeded; $\mathrm{ft}^{3} / \mathrm{s}$, cubic foot per second; Sep, September; Nov, November]

\begin{tabular}{|c|c|c|c|c|c|c|c|c|c|}
\hline \multirow[b]{2}{*}{ APEE } & \multicolumn{9}{|c|}{ Sep-Nov daily mean streamflow $\left(\mathrm{ft}^{3} / \mathrm{s}\right)$ that was equaled or exceeded the indicated percentage of time at APEE level } \\
\hline & 98 & 95 & 90 & 80 & 50 & 20 & 10 & 5 & 2 \\
\hline 1 & 27.6 & 41.4 & 68.8 & 116. & 608. & 4040. & 6740. & 8320. & 11200 . \\
\hline 2 & 26.9 & 37.4 & 67.2 & 107. & 375. & 3100 . & 5860. & 6440. & 8740 . \\
\hline 5 & 23.6 & 32.4 & 56.8 & 78.6 & 215. & 1350. & 3170 . & 4150 . & 5200. \\
\hline 10 & 22.5 & 30.2 & 45.0 & 64.5 & 133. & 719. & 1250 & 1870 . & 2660 . \\
\hline 20 & 22.0 & 27.6 & 37.6 & 50.6 & 106. & 338. & 526. & 932. & 1350. \\
\hline 25 & 22.0 & 26.2 & 35.8 & 47.6 & 84.0 & 265. & 448. & 755. & 1230. \\
\hline 30 & 21.8 & 25.4 & 34.3 & 44.8 & 78.2 & 206. & 311. & 566. & 1010. \\
\hline 40 & 20.8 & 24.2 & 30.5 & 40.0 & 62.0 & 139. & 232 & 414. & 659. \\
\hline 50 & 15.0 & 21.0 & 26.8 & 36.0 & 55.0 & 105. & 203. & 291. & 418. \\
\hline 60 & 13.6 & 19.0 & 23.4 & 31.5 & 49.0 & 91.9 & 159. & 203. & 317. \\
\hline 70 & 12.5 & 17.0 & 19.9 & 26.0 & 43.0 & 73.6 & 117. & 186. & 264. \\
\hline 75 & 11.8 & 16.0 & 18.6 & 24.0 & 40.0 & 68.0 & 110. & 175. & 236. \\
\hline 80 & 11.0 & 14.0 & 16.3 & 22.6 & 38.0 & 64.8 & 97.2 & 166. & 214. \\
\hline 90 & 8.91 & 10.1 & 13.8 & 16.2 & 33.0 & 52.7 & 64.6 & 145. & 167. \\
\hline 95 & 7.93 & 9.87 & 11.5 & 14.0 & 28.6 & 48.0 & 60.9 & 128. & 153. \\
\hline 98 & 7.13 & 8.50 & 9.54 & 12.8 & 25.7 & 41.9 & 55.2 & 116. & 141. \\
\hline 99 & 5.52 & 7.88 & 9.00 & 12.0 & 24.0 & 41.0 & 55.0 & 112. & 137. \\
\hline
\end{tabular}


03266500 Mad River at Zanesfield, Ohio

Years analyzed $=34$

Climatic year range $=1946-79$

[APEE, annual percentage equaled or exceeded; $\mathrm{ft}^{3} / \mathrm{s}$, cubic foot per second; Sep, September; Nov, November]

\begin{tabular}{|c|c|c|c|c|c|c|c|c|c|}
\hline \multirow[b]{2}{*}{ APEE } & \multicolumn{9}{|c|}{ Sep-Nov daily mean streamflow $\left(\mathrm{ft}^{3} / \mathrm{s}\right)$ that was equaled or exceeded the indicated percentage of time at APEE level } \\
\hline & 95 & 90 & 80 & 70 & 50 & 30 & 20 & 10 & 5 \\
\hline 1 & 1.68 & 2.25 & 3.20 & 4.80 & 10.4 & 22.5 & 32.0 & 79.5 & 114. \\
\hline 2 & 1.36 & 1.79 & 2.80 & 4.19 & 7.59 & 13.9 & 22.8 & 41.3 & 67.0 \\
\hline 5 & 1.28 & 1.52 & 1.94 & 2.80 & 5.19 & 8.35 & 11.2 & 16.2 & 30.0 \\
\hline 10 & 1.17 & 1.40 & 1.80 & 2.15 & 3.67 & 4.62 & 7.08 & 9.36 & 17.6 \\
\hline 20 & 1.08 & 1.23 & 1.56 & 1.85 & 2.71 & 3.25 & 4.10 & 5.54 & 9.52 \\
\hline 25 & 0.97 & 1.20 & 1.50 & 1.80 & 2.40 & 3.10 & 3.70 & 5.00 & 8.13 \\
\hline 30 & 0.97 & 1.20 & 1.50 & 1.62 & 2.20 & 2.85 & 3.14 & 4.55 & 7.07 \\
\hline 40 & 0.95 & 1.16 & 1.50 & 1.50 & 2.00 & 2.35 & 2.60 & 3.92 & 6.01 \\
\hline 50 & 0.95 & 1.10 & 1.30 & 1.40 & 1.75 & 2.20 & 2.30 & 3.60 & 4.85 \\
\hline 60 & 0.93 & 1.00 & 1.10 & 1.40 & 1.55 & 2.05 & 2.20 & 3.40 & 4.49 \\
\hline 70 & 0.90 & 1.00 & 1.10 & 1.30 & 1.45 & 1.95 & 2.16 & 2.95 & 4.14 \\
\hline 75 & 0.80 & 0.95 & 1.00 & 1.20 & 1.40 & 1.90 & 2.10 & 2.80 & 4.00 \\
\hline 80 & 0.80 & 0.84 & 1.00 & 1.20 & 1.35 & 1.90 & 2.10 & 2.74 & 3.32 \\
\hline 90 & 0.77 & 0.80 & 0.99 & 1.05 & 1.30 & 1.80 & 1.90 & 2.10 & 3.03 \\
\hline 95 & 0.70 & 0.78 & 0.90 & 0.98 & 1.20 & 1.65 & 1.80 & 2.05 & 2.95 \\
\hline 98 & 0.68 & 0.77 & 0.90 & 0.93 & 1.18 & 1.55 & 1.80 & 1.94 & 2.85 \\
\hline 99 & 0.68 & 0.77 & 0.90 & 0.93 & 1.10 & 1.55 & 1.80 & 1.90 & 2.85 \\
\hline
\end{tabular}

\section{Mad River near Urbana, Ohio}

Years analyzed $=72$

Climatic year range $=1925-2008$

[APEE, annual percentage equaled or exceeded; $\mathrm{ft}^{3} / \mathrm{s}$, cubic foot per second; Sep, September; Nov, November]

\begin{tabular}{|c|c|c|c|c|c|c|c|c|c|}
\hline \multirow[b]{2}{*}{ APEE } & \multicolumn{9}{|c|}{ Sep-Nov daily mean streamflow $\left(\mathrm{ft}^{3} / \mathrm{s}\right)$ that was equaled or exceeded the indicated percentage of time at APEE level } \\
\hline & 98 & 95 & 90 & 80 & 50 & 20 & 10 & 5 & 2 \\
\hline 1 & 39.4 & 52.0 & 71.6 & 92.4 & 177. & 639. & 950. & 1070. & 1490. \\
\hline 2 & 39.0 & 50.9 & 66.1 & 79.1 & 153. & 409. & 670. & 844. & 1160. \\
\hline 5 & 38.1 & 49.4 & 60.6 & 70.6 & 121. & 231. & 401 & 562. & 780. \\
\hline 10 & 37.9 & 48.2 & 55.7 & 66.1 & 103. & 166. & 255. & 349. & 514. \\
\hline 20 & 37.0 & 46.0 & 50.3 & 58.6 & 90.0 & 144. & 163. & 258. & 342. \\
\hline 25 & 36.0 & 45.0 & 48.0 & 57.0 & 85.0 & 133. & 159. & 229. & 285. \\
\hline 30 & 36.0 & 43.6 & 47.3 & 55.6 & 80.5 & 122. & 152. & 221. & 256. \\
\hline 40 & 35.1 & 42.7 & 46.0 & 53.2 & 78.0 & 117. & 138. & 186. & 229. \\
\hline 50 & 35.1 & 41.0 & 45.0 & 51.6 & 75.5 & 111. & 131. & 166. & 215. \\
\hline 60 & 33.6 & 40.0 & 43.0 & 50.6 & 73.0 & 107. & 122. & 157. & 203. \\
\hline 70 & 32.2 & 38.7 & 42.1 & 48.6 & 71.5 & 101. & 118. & 147. & 179. \\
\hline 75 & 32.2 & 38.3 & 39.3 & 47.6 & 70.0 & 100. & 115. & 134. & 173. \\
\hline 80 & 31.9 & 36.4 & 38.3 & 47.6 & 68.4 & 97.6 & 114. & 129. & 163. \\
\hline 90 & 31.8 & 35.0 & 38.3 & 45.2 & 65.1 & 96.0 & 101. & 117. & 148. \\
\hline 95 & 30.3 & 34.7 & 37.0 & 43.6 & 63.8 & 93.2 & 99.1 & 114. & 146. \\
\hline 98 & 29.8 & 34.0 & 35.6 & 42.8 & 62.9 & 92.3 & 98.6 & 111. & 143. \\
\hline 99 & 29.8 & 34.0 & 35.3 & 41.6 & 62.5 & 91.8 & 98.1 & 111. & 142. \\
\hline
\end{tabular}


03270800 Wolf Creek at Trotwood, Ohio

Years analyzed $=24$

Climatic year range $=1962-85$

[APEE, annual percentage equaled or exceeded; $\mathrm{ft}^{3} / \mathrm{s}$, cubic foot per second; Sep, September; Nov, November]

\begin{tabular}{|c|c|c|c|c|c|c|c|c|c|}
\hline \multirow[b]{2}{*}{ APEE } & \multicolumn{9}{|c|}{ Sep-Nov daily mean streamflow $\left(\mathrm{ft}^{3} / \mathrm{s}\right)$ that was equaled or exceeded the indicated percentage of time at APEE level } \\
\hline & 95 & 90 & 80 & 70 & 50 & 30 & 20 & 10 & 5 \\
\hline 1 & 0.85 & 3.40 & 14.0 & 24.5 & 63.0 & 126. & 259. & 517. & 597. \\
\hline 2 & 0.75 & 3.06 & 11.5 & 15.8 & 44.1 & 96.9 & 204. & 368. & 421. \\
\hline 5 & 0.67 & 2.32 & 6.00 & 7.54 & 15.2 & 42.5 & 95.8 & 151. & 268. \\
\hline 10 & 0.53 & 1.39 & 4.00 & 4.62 & 8.66 & 20.6 & 31.0 & 66.6 & 94.2 \\
\hline 20 & 0.42 & 1.13 & 2.20 & 3.18 & 5.54 & 12.3 & 14.0 & 25.4 & 30.5 \\
\hline 25 & 0.40 & 0.85 & 1.70 & 2.50 & 3.95 & 8.35 & 11.0 & 18.0 & 26.3 \\
\hline 30 & 0.40 & 0.80 & 1.60 & 2.20 & 3.42 & 6.08 & 9.30 & 13.5 & 20.1 \\
\hline 40 & 0.40 & 0.67 & 1.40 & 1.72 & 2.66 & 4.22 & 6.16 & 9.05 & 13.2 \\
\hline 50 & 0.25 & 0.58 & 1.20 & 1.40 & 2.00 & 3.10 & 3.90 & 5.45 & 9.65 \\
\hline 60 & 0.25 & 0.48 & 0.86 & 1.05 & 1.53 & 2.43 & 3.10 & 4.23 & 9.39 \\
\hline 70 & 0.16 & 0.36 & 0.43 & 0.69 & 1.08 & 1.45 & 2.16 & 3.65 & 8.43 \\
\hline 75 & 0.13 & 0.25 & 0.36 & 0.54 & 1.00 & 1.30 & 1.90 & 3.30 & 7.80 \\
\hline 80 & 0.13 & 0.23 & 0.34 & 0.54 & 0.86 & 1.20 & 1.90 & 2.61 & 7.10 \\
\hline 90 & 0.01 & 0.09 & 0.28 & 0.34 & 0.64 & 0.89 & 1.14 & 1.80 & 5.54 \\
\hline 95 & 0.01 & 0.06 & 0.24 & 0.30 & 0.53 & 0.81 & 0.90 & 1.74 & 4.58 \\
\hline 98 & 0.00 & 0.02 & 0.18 & 0.29 & 0.45 & 0.64 & 0.78 & 1.55 & 3.89 \\
\hline 99 & 0.00 & 0.02 & 0.17 & 0.29 & 0.40 & 0.57 & 0.78 & 1.55 & 3.58 \\
\hline
\end{tabular}

\section{Wolf Creek at Dayton, Ohio}

Years analyzed $=29$

Climatic year range $=1938-2008$

[APEE, annual percentage equaled or exceeded; $\mathrm{ft}^{3} / \mathrm{s}$, cubic foot per second; Sep, September; Nov, November]

\begin{tabular}{|c|c|c|c|c|c|c|c|c|c|}
\hline \multirow[b]{2}{*}{ APEE } & \multicolumn{9}{|c|}{ Sep-Nov daily mean streamflow ( $\left.\mathrm{ft}^{3} / \mathrm{s}\right)$ that was equaled or exceeded the indicated percentage of time at APEE level } \\
\hline & 95 & 90 & 80 & 70 & 50 & 30 & 20 & 10 & 5 \\
\hline 1 & 6.35 & 10.0 & 22.0 & 59.0 & 144. & 410. & 509. & 1010 & 1250 \\
\hline 2 & 5.89 & 8.99 & 17.8 & 50.6 & 84.4 & 327. & 485. & 660. & 1030 \\
\hline 5 & 4.83 & 6.96 & 14.4 & 30.0 & 71.1 & 117. & 192. & 337. & 580. \\
\hline 10 & 4.50 & 6.10 & 13.0 & 20.0 & 32.6 & 62.2 & 83.2 & 153. & 226. \\
\hline 20 & 4.20 & 5.40 & 8.84 & 12.0 & 19.6 & 36.0 & 40.2 & 68.0 & 73.4 \\
\hline 25 & 4.00 & 5.40 & 8.10 & 10.0 & 17.0 & 28.0 & 34.0 & 47.0 & 62.5 \\
\hline 30 & 3.80 & 5.08 & 7.60 & 10.0 & 14.0 & 24.4 & 26.4 & 45.0 & 55.9 \\
\hline 40 & 3.40 & 4.80 & 5.82 & 7.90 & 12.0 & 17.0 & 21.0 & 36.4 & 47.1 \\
\hline 50 & 2.85 & 4.20 & 5.10 & 7.00 & 11.0 & 15.0 & 18.0 & 34.0 & 39.5 \\
\hline 60 & 2.60 & 3.40 & 4.80 & 6.40 & 9.78 & 13.0 & 16.8 & 26.0 & 33.9 \\
\hline 70 & 2.25 & 2.90 & 4.08 & 6.06 & 8.56 & 12.6 & 14.0 & 21.0 & 29.3 \\
\hline 75 & 2.15 & 2.70 & 3.90 & 5.90 & 8.00 & 12.0 & 12.0 & 20.0 & 27.0 \\
\hline 80 & 1.99 & 2.50 & 3.60 & 5.54 & 7.70 & 11.0 & 12.0 & 18.0 & 24.4 \\
\hline 90 & 1.46 & 1.92 & 3.02 & 5.02 & 6.70 & 9.60 & 11.0 & 17.0 & 19.5 \\
\hline 95 & 1.31 & 1.58 & 2.40 & 4.58 & 6.22 & 9.06 & 11.0 & 14.6 & 16.6 \\
\hline 98 & 1.15 & 1.30 & 2.37 & 4.10 & 6.00 & 8.10 & 9.95 & 13.0 & 15.5 \\
\hline 99 & 1.15 & 1.20 & 2.20 & 4.10 & 6.00 & 8.10 & 9.70 & 13.0 & 15.5 \\
\hline
\end{tabular}


03271800 Twin Creek near Ingomar, Ohio

Years analyzed $=37$

Climatic year range $=1962-98$

[APEE, annual percentage equaled or exceeded; $\mathrm{ft}^{3} / \mathrm{s}$, cubic foot per second; Sep, September; Nov, November]

\begin{tabular}{|c|c|c|c|c|c|c|c|c|c|}
\hline \multirow[b]{2}{*}{ APEE } & \multicolumn{9}{|c|}{ Sep-Nov daily mean streamflow $\left(\mathrm{ft}^{3} / \mathrm{s}\right)$ that was equaled or exceeded the indicated percentage of time at APEE level } \\
\hline & 95 & 90 & 80 & 70 & 50 & 30 & 20 & 10 & 5 \\
\hline 1 & 11.6 & 16.8 & 36.8 & 89.2 & 248. & 1210. & 1560 & 2880 & 4370 \\
\hline 2 & 10.8 & 15.9 & 25.5 & 74.1 & 177. & 817. & 1430 . & 2570 & 3250. \\
\hline 5 & 10.6 & 12.3 & 19.3 & 48.6 & 104. & 440 . & 650. & 1130. & 1700 \\
\hline 10 & 9.31 & 11.6 & 15.0 & 25.7 & 64.8 & 192. & 287. & 402 & 638. \\
\hline 20 & 6.20 & 9.69 & 11.0 & 20.2 & 35.2 & 92.2 & 112. & 157. & 219. \\
\hline 25 & 6.20 & 8.72 & 10.6 & 19.4 & 27.0 & 42.4 & 85.4 & 110 & 195. \\
\hline 30 & 6.20 & 8.05 & 10.6 & 17.7 & 24.4 & 32.4 & 64.7 & 92.5 & 162. \\
\hline 40 & 5.80 & 7.42 & 9.61 & 15.0 & 19.0 & 24.7 & 36.1 & 64.8 & 91.2 \\
\hline 50 & 4.96 & 6.28 & 8.48 & 13.0 & 17.0 & 20.6 & 27.2 & 46.6 & 79.3 \\
\hline 60 & 4.25 & 5.08 & 6.73 & 8.95 & 14.8 & 18.0 & 21.4 & 38.0 & 69.0 \\
\hline 70 & 3.87 & 4.49 & 6.05 & 8.27 & 13.0 & 16.6 & 19.4 & 28.1 & 44.7 \\
\hline 75 & 3.60 & 4.14 & 5.88 & 7.60 & 12.0 & 15.6 & 18.4 & 25.4 & 38.5 \\
\hline 80 & 3.49 & 3.98 & 5.38 & 7.22 & 11.0 & 15.0 & 16.2 & 25.0 & 30.8 \\
\hline 90 & 3.28 & 3.66 & 5.09 & 6.55 & 9.12 & 13.0 & 15.0 & 19.4 & 27.4 \\
\hline 95 & 3.12 & 3.39 & 4.80 & 6.12 & 8.20 & 11.8 & 14.2 & 16.4 & 26.2 \\
\hline 98 & 2.77 & 3.14 & 4.60 & 5.87 & 7.52 & 10.6 & 12.8 & 15.4 & 24.3 \\
\hline 99 & 2.77 & 3.14 & 4.48 & 5.52 & 7.10 & 10.0 & 12.8 & 15.4 & 23.5 \\
\hline
\end{tabular}

\section{Sevenmile Creek at Camden, Ohio}

Years analyzed $=36$

Climatic year range $=1971-2008$

[APEE, annual percentage equaled or exceeded; $\mathrm{ft}^{3} / \mathrm{s}$, cubic foot per second; Sep, September; Nov, November]

\begin{tabular}{|c|c|c|c|c|c|c|c|c|c|}
\hline \multirow[b]{2}{*}{ APEE } & \multicolumn{9}{|c|}{ Sep-Nov daily mean streamflow $\left(\mathrm{ft}^{3} / \mathrm{s}\right)$ that was equaled or exceeded the indicated percentage of time at APEE level } \\
\hline & 95 & 90 & 80 & 70 & 50 & 30 & 20 & 10 & 5 \\
\hline 1 & 7.07 & 10.7 & 25.6 & 55.9 & 169. & 511. & 801. & 1060 & 1420 \\
\hline 2 & 6.25 & 9.24 & 15.4 & 36.7 & 118. & 437. & 573. & 826. & 1140 \\
\hline 5 & 5.65 & 6.97 & 9.82 & 21.9 & 67.6 & 178. & 277. & 384. & 493. \\
\hline 10 & 4.86 & 5.30 & 7.50 & 13.3 & 33.1 & 86.3 & 118. & 179 . & 242. \\
\hline 20 & 3.67 & 4.27 & 5.25 & 9.43 & 21.3 & 36.6 & 56.8 & 83.9 & 94.8 \\
\hline 25 & 3.56 & 3.94 & 4.82 & 7.84 & 17.5 & 24.9 & 41.3 & 69.6 & 79.5 \\
\hline 30 & 3.53 & 3.67 & 4.54 & 7.31 & 12.4 & 21.3 & 36.8 & 59.9 & 70.3 \\
\hline 40 & 3.24 & 3.51 & 4.05 & 5.92 & 9.02 & 14.9 & 17.6 & 43.4 & 51.7 \\
\hline 50 & 2.59 & 3.07 & 3.60 & 5.50 & 7.25 & 9.47 & 13.6 & 35.9 & 43.2 \\
\hline 60 & 2.32 & 2.56 & 3.16 & 3.86 & 6.26 & 7.82 & 11.0 & 28.5 & 36.9 \\
\hline 70 & 1.69 & 2.17 & 2.66 & 3.42 & 4.99 & 6.68 & 9.48 & 24.1 & 29.8 \\
\hline 75 & 1.67 & 2.01 & 2.54 & 3.20 & 4.50 & 6.49 & 8.02 & 21.6 & 25.9 \\
\hline 80 & 1.57 & 1.94 & 2.40 & 3.00 & 3.95 & 6.33 & 7.20 & 17.9 & 22.7 \\
\hline 90 & 1.46 & 1.68 & 2.05 & 2.51 & 3.27 & 5.25 & 6.26 & 14.5 & 17.2 \\
\hline 95 & 1.25 & 1.59 & 1.88 & 2.16 & 3.10 & 4.65 & 5.55 & 11.6 & 15.2 \\
\hline 98 & 1.14 & 1.50 & 1.74 & 2.07 & 2.89 & 4.49 & 5.27 & 10.6 & 14.0 \\
\hline 99 & 1.14 & 1.50 & 1.70 & 2.01 & 2.85 & 4.49 & 5.22 & 10.4 & 14.0 \\
\hline
\end{tabular}


04177000 Ottawa River at University of Toledo, Toledo, Ohio

Years analyzed $=36$

Climatic year range $=1945-2008$

[APEE, annual percentage equaled or exceeded; $\mathrm{ft}^{3} / \mathrm{s}$, cubic foot per second; Sep, September; Nov, November]

\begin{tabular}{|c|c|c|c|c|c|c|c|c|c|}
\hline \multirow[b]{2}{*}{ APEE } & \multicolumn{9}{|c|}{ Sep-Nov daily mean streamflow $\left(\mathrm{ft}^{3} / \mathrm{s}\right)$ that was equaled or exceeded the indicated percentage of time at APEE level } \\
\hline & 95 & 90 & 80 & 70 & 50 & 30 & 20 & 10 & 5 \\
\hline 1 & 52.7 & 64.1 & 97.0 & 158. & 416. & 650. & 888. & 1320 & 2150 \\
\hline 2 & 35.2 & 56.4 & 83.2 & 148. & 312. & 566. & 708. & 1290. & 2100 \\
\hline 5 & 26.5 & 33.8 & 56.4 & 79.6 & 169. & 255. & 495. & 875. & 1270 \\
\hline 10 & 18.2 & 28.2 & 35.1 & 60.8 & 96.5 & 152. & 284. & 490. & 769. \\
\hline 20 & 8.51 & 17.6 & 23.6 & 30.2 & 40.1 & 76.8 & 119. & 177. & 266. \\
\hline 25 & 7.18 & 15.7 & 21.0 & 25.0 & 34.5 & 49.3 & 83.6 & 134. & 215. \\
\hline 30 & 6.43 & 13.4 & 17.0 & 21.1 & 30.0 & 42.0 & 66.7 & 113. & 172. \\
\hline 40 & 5.04 & 10.0 & 13.4 & 16.0 & 21.7 & 28.0 & 47.9 & 76.8 & 116. \\
\hline 50 & 3.76 & 7.19 & 11.0 & 13.0 & 16.5 & 22.9 & 34.0 & 62.9 & 81.2 \\
\hline 60 & 2.89 & 5.25 & 9.51 & 10.0 & 11.9 & 16.8 & 26.2 & 48.0 & 56.5 \\
\hline 70 & 2.48 & 4.16 & 7.17 & 8.32 & 9.06 & 12.9 & 21.4 & 34.7 & 48.6 \\
\hline 75 & 0.86 & 3.44 & 5.20 & 7.03 & 8.45 & 11.9 & 19.8 & 32.0 & 44.8 \\
\hline 80 & 0.00 & 2.48 & 4.00 & 5.71 & 8.15 & 10.8 & 16.9 & 27.9 & 44.3 \\
\hline 90 & 0.00 & 1.55 & 3.20 & 4.93 & 6.56 & 8.79 & 13.4 & 19.6 & 36.1 \\
\hline 95 & 0.00 & 0.76 & 2.26 & 4.04 & 6.10 & 7.47 & 10.6 & 16.4 & 22.6 \\
\hline 98 & 0.00 & 0.64 & 1.62 & 2.92 & 4.36 & 7.03 & 9.45 & 14.9 & 19.6 \\
\hline 99 & 0.00 & 0.56 & 1.54 & 2.43 & 4.10 & 6.65 & 9.04 & 14.6 & 17.9 \\
\hline
\end{tabular}

\section{Bean Creek at Powers, Ohio}

Years analyzed $=49$

Climatic year range $=1940-2008$

[APEE, annual percentage equaled or exceeded; $\mathrm{ft}^{3} / \mathrm{s}$, cubic foot per second; Sep, September; Nov, November]

\begin{tabular}{|c|c|c|c|c|c|c|c|c|c|}
\hline \multirow[b]{2}{*}{ APEE } & \multicolumn{9}{|c|}{ Sep-Nov daily mean streamflow $\left(\mathrm{ft}^{3} / \mathrm{s}\right)$ that was equaled or exceeded the indicated percentage of time at APEE level } \\
\hline & 95 & 90 & 80 & 70 & 50 & 30 & 20 & 10 & 5 \\
\hline 1 & 20.0 & 26.0 & 45.0 & 88.0 & 328. & 539. & 655. & 930. & 1080 \\
\hline 2 & 18.2 & 25.2 & 44.2 & 77.1 & 245 & 469. & 535. & 766. & 890. \\
\hline 5 & 16.7 & 24.0 & 39.4 & 56.8 & 139. & 229. & 351. & 474. & 520. \\
\hline 10 & 16.5 & 22.0 & 32.0 & 46.6 & 77.2 & 136. & 238. & 300. & 338. \\
\hline 20 & 14.3 & 21.0 & 27.0 & 34.6 & 49.6 & 87.0 & 144. & 182. & 206. \\
\hline 25 & 13.5 & 20.0 & 26.0 & 32.0 & 42.0 & 71.0 & 125. & 158. & 172. \\
\hline 30 & 13.5 & 19.4 & 24.8 & 29.0 & 36.2 & 65.0 & 84.8 & 138. & 154. \\
\hline 40 & 12.0 & 16.0 & 21.0 & 26.0 & 32.0 & 55.4 & 66.0 & 110 & 121. \\
\hline 50 & 11.5 & 15.0 & 18.0 & 24.0 & 30.0 & 47.0 & 57.0 & 90.0 & 96.0 \\
\hline 60 & 10.5 & 13.0 & 17.0 & 21.0 & 26.8 & 37.8 & 45.0 & 55.0 & 80.4 \\
\hline 70 & 9.48 & 11.0 & 16.0 & 18.0 & 22.0 & 32.0 & 40.0 & 43.0 & 59.8 \\
\hline 75 & 8.60 & 11.0 & 14.0 & 17.0 & 20.0 & 28.0 & 37.0 & 43.0 & 54.0 \\
\hline 80 & 7.51 & 9.50 & 13.0 & 15.4 & 19.0 & 28.0 & 32.0 & 41.0 & 48.2 \\
\hline 90 & 6.95 & 8.40 & 11.0 & 13.0 & 17.0 & 23.0 & 26.2 & 34.0 & 44.6 \\
\hline 95 & 6.60 & 7.44 & 10.0 & 13.0 & 15.0 & 20.2 & 24.6 & 31.2 & 37.0 \\
\hline 98 & 6.27 & 6.67 & 10.0 & 11.8 & 14.8 & 19.0 & 24.0 & 27.8 & 34.5 \\
\hline 99 & 5.95 & 6.40 & 10.0 & 11.0 & 14.0 & 19.0 & 24.0 & 27.0 & 34.5 \\
\hline
\end{tabular}




\section{Unnamed Tributary to Lost Creek near Farmer, Ohio}

Years analyzed $=22$

Climatic year range $=1985-2008$

[APEE, annual percentage equaled or exceeded; $\mathrm{ft}^{3} / \mathrm{s}$, cubic foot per second; Sep, September; Nov, November]

\begin{tabular}{|c|c|c|c|c|c|c|c|c|c|}
\hline \multirow[b]{2}{*}{ APEE } & \multicolumn{9}{|c|}{ Sep-Nov daily mean streamflow $\left(\mathrm{ft}^{3} / \mathrm{s}\right)$ that was equaled or exceeded the indicated percentage of time at APEE level } \\
\hline & 95 & 90 & 80 & 70 & 50 & 30 & 20 & 10 & 5 \\
\hline 1 & 0.58 & 3.63 & 8.10 & 21.5 & 37.0 & 82.4 & 106. & 138. & 201. \\
\hline 2 & 0.22 & 1.44 & 5.38 & 13.4 & 28.5 & 60.3 & 68.8 & 78.0 & 95.1 \\
\hline 5 & 0.08 & 0.32 & 1.73 & 2.53 & 11.1 & 21.4 & 24.5 & 44.5 & 46.2 \\
\hline 10 & 0.05 & 0.15 & 0.46 & 0.74 & 3.50 & 7.82 & 12.4 & 21.4 & 22.6 \\
\hline 20 & 0.05 & 0.09 & 0.19 & 0.25 & 0.92 & 2.66 & 4.35 & 6.86 & 11.8 \\
\hline 25 & 0.04 & 0.07 & 0.17 & 0.22 & 0.61 & 1.33 & 2.70 & 4.84 & 9.15 \\
\hline 30 & 0.04 & 0.06 & 0.11 & 0.16 & 0.36 & 1.00 & 2.08 & 2.98 & 6.58 \\
\hline 40 & 0.04 & 0.04 & 0.06 & 0.12 & 0.20 & 0.52 & 0.72 & 1.53 & 1.87 \\
\hline 50 & 0.03 & 0.03 & 0.05 & 0.08 & 0.14 & 0.31 & 0.50 & 1.00 & 1.45 \\
\hline 60 & 0.02 & 0.03 & 0.03 & 0.04 & 0.12 & 0.22 & 0.38 & 0.71 & 0.94 \\
\hline 70 & 0.00 & 0.01 & 0.03 & 0.04 & 0.09 & 0.16 & 0.33 & 0.38 & 0.74 \\
\hline 75 & 0.00 & 0.00 & 0.02 & 0.03 & 0.08 & 0.15 & 0.30 & 0.32 & 0.66 \\
\hline 80 & 0.00 & 0.00 & 0.02 & 0.03 & 0.07 & 0.13 & 0.26 & 0.30 & 0.63 \\
\hline 90 & 0.00 & 0.00 & 0.01 & 0.01 & 0.05 & 0.10 & 0.17 & 0.27 & 0.38 \\
\hline 95 & 0.00 & 0.00 & 0.00 & 0.01 & 0.05 & 0.08 & 0.14 & 0.19 & 0.24 \\
\hline 98 & 0.00 & 0.00 & 0.00 & 0.01 & 0.04 & 0.08 & 0.10 & 0.16 & 0.24 \\
\hline 99 & 0.00 & 0.00 & 0.00 & 0.01 & 0.03 & 0.07 & 0.10 & 0.16 & 0.23 \\
\hline
\end{tabular}

\section{Blanchard River near Findlay, Ohio}

Years analyzed $=82$

Climatic year range $=1923-2008$

[APEE, annual percentage equaled or exceeded; $\mathrm{ft}^{3} / \mathrm{s}$, cubic foot per second; Sep, September; Nov, November]

\begin{tabular}{|c|c|c|c|c|c|c|c|c|c|}
\hline \multirow[b]{2}{*}{ APEE } & \multicolumn{9}{|c|}{ Sep-Nov daily mean streamflow $\left(\mathrm{ft}^{3} / \mathrm{s}\right)$ that was equaled or exceeded the indicated percentage of time at APEE level } \\
\hline & 98 & 95 & 90 & 80 & 50 & 20 & 10 & 5 & 2 \\
\hline 1 & 11.2 & 18.0 & 20.6 & 50.6 & 506. & 2110. & 2840 & 4470. & 5130. \\
\hline 2 & 8.73 & 13.8 & 19.6 & 45.9 & 306. & 1830. & 2530 & 3690 . & 4690. \\
\hline 5 & 7.37 & 11.5 & 14.7 & 30.6 & 149. & 960. & 1500 . & 2430 & 3280. \\
\hline 10 & 6.55 & 10.0 & 12.1 & 22.8 & 80.1 & 378. & 831. & 1560 . & 2380 \\
\hline 20 & 4.83 & 7.15 & 10.0 & 16.4 & 42.5 & 183. & 282. & 626. & 1420 \\
\hline 25 & 4.75 & 6.30 & 9.56 & 14.6 & 34.5 & 131. & 212. & 390. & 820. \\
\hline 30 & 4.19 & 5.74 & 8.86 & 12.0 & 31.7 & 107. & 169. & 257. & 635. \\
\hline 40 & 3.32 & 5.00 & 7.09 & 10.0 & 25.2 & 60.3 & 122. & 174. & 423. \\
\hline 50 & 2.83 & 4.40 & 6.39 & 9.18 & 22.0 & 47.0 & 93.3 & 112. & 290. \\
\hline 60 & 2.51 & 3.86 & 5.88 & 7.89 & 19.4 & 40.3 & 63.7 & 97.6 & 194. \\
\hline 70 & 2.06 & 3.52 & 4.93 & 7.00 & 17.0 & 32.5 & 52.0 & 86.7 & 149. \\
\hline 75 & 1.92 & 3.12 & 4.65 & 6.84 & 14.5 & 26.4 & 47.7 & 80.1 & 133. \\
\hline 80 & 1.49 & 2.95 & 4.56 & 6.26 & 13.4 & 24.8 & 45.1 & 75.0 & 118. \\
\hline 90 & 1.26 & 2.62 & 3.38 & 4.86 & 11.5 & 20.1 & 35.4 & 50.1 & 74.0 \\
\hline 95 & 1.07 & 2.03 & 2.94 & 4.17 & 10.5 & 18.4 & 29.1 & 46.9 & 58.4 \\
\hline 98 & 0.97 & 1.83 & 2.42 & 3.76 & 9.44 & 17.4 & 23.0 & 39.0 & 55.0 \\
\hline 99 & 0.86 & 1.66 & 2.26 & 3.66 & 9.40 & 16.4 & 23.0 & 38.7 & 54.4 \\
\hline
\end{tabular}


04196500 Sandusky River near Upper Sandusky, Ohio

Years analyzed $=65$

Climatic year range $=1921-2008$

[APEE, annual percentage equaled or exceeded; $\mathrm{ft}^{3} / \mathrm{s}$, cubic foot per second; Sep, September; Nov, November]

\begin{tabular}{|c|c|c|c|c|c|c|c|c|c|}
\hline \multirow[b]{2}{*}{ APEE } & \multicolumn{9}{|c|}{ Sep-Nov daily mean streamflow $\left(\mathrm{ft}^{3} / \mathrm{s}\right)$ that was equaled or exceeded the indicated percentage of time at APEE level } \\
\hline & 98 & 95 & 90 & 80 & 50 & 20 & 10 & 5 & 2 \\
\hline 1 & 20.3 & 26.0 & 35.6 & 78.4 & 391. & 1580. & 2420 & 3020. & 4180. \\
\hline 2 & 11.9 & 22.0 & 29.3 & 65.4 & 304. & 1340. & 2220 & 2710 & 3260. \\
\hline 5 & 7.28 & 16.7 & 23.8 & 32.6 & 141. & 683. & 1180. & 1610. & 2100 \\
\hline 10 & 5.68 & 13.1 & 16.1 & 21.8 & 71.0 & 450 . & 775. & 835. & 1410 . \\
\hline 20 & 4.39 & 8.16 & 10.6 & 15.7 & 42.6 & 205. & 333. & 417. & 698. \\
\hline 25 & 3.95 & 6.52 & 9.34 & 14.2 & 37.0 & 146. & 264. & 343. & 554. \\
\hline 30 & 3.71 & 5.59 & 8.62 & 12.2 & 31.4 & 112. & 209. & 292. & 452. \\
\hline 40 & 3.20 & 4.80 & 7.60 & 9.64 & 23.2 & 71.8 & 124. & 187. & 322. \\
\hline 50 & 2.42 & 4.64 & 6.46 & 8.28 & 19.0 & 39.6 & 96.6 & 151. & 254. \\
\hline 60 & 2.05 & 3.17 & 4.38 & 7.27 & 14.0 & 29.0 & 70.2 & 105. & 196. \\
\hline 70 & 1.63 & 2.39 & 3.35 & 6.16 & 12.6 & 26.4 & 59.2 & 80.1 & 144. \\
\hline 75 & 1.23 & 2.33 & 2.96 & 5.62 & 10.0 & 24.0 & 50.0 & 66.7 & 129. \\
\hline 80 & 1.12 & 2.12 & 2.46 & 5.02 & 9.94 & 22.2 & 41.8 & 55.8 & 117. \\
\hline 90 & 0.97 & 1.29 & 1.96 & 3.74 & 8.50 & 15.2 & 26.0 & 34.6 & 102. \\
\hline 95 & 0.81 & 1.15 & 1.78 & 2.84 & 6.50 & 13.6 & 19.6 & 27.1 & 81.1 \\
\hline 98 & 0.68 & 1.11 & 1.56 & 2.24 & 5.43 & 11.8 & 17.3 & 25.3 & 67.0 \\
\hline 99 & 0.60 & 1.00 & 1.46 & 1.92 & 4.60 & 11.0 & 16.4 & 24.2 & 31.4 \\
\hline
\end{tabular}

04197000 Sandusky River near Mexico, Ohio

Years analyzed $=57$

Climatic year range $=1923-81$

[APEE, annual percentage equaled or exceeded; $\mathrm{ft}^{3} / \mathrm{s}$, cubic foot per second; Sep, September; Nov, November]

\begin{tabular}{|c|c|c|c|c|c|c|c|c|c|}
\hline \multirow[b]{2}{*}{ APEE } & \multicolumn{9}{|c|}{ Sep-Nov daily mean streamflow $\left(\mathrm{ft}^{3} / \mathrm{s}\right)$ that was equaled or exceeded the indicated percentage of time at APEE level } \\
\hline & 98 & 95 & 90 & 80 & 50 & 20 & 10 & 5 & 2 \\
\hline 1 & 27.5 & 41.7 & 59.6 & 96.6 & 680. & 2420. & 4330 & 6090 & 7230 \\
\hline 2 & 26.5 & 41.5 & 55.8 & 87.9 & 598. & 2090 . & 4160. & 6010 & 6980. \\
\hline 5 & 23.1 & 36.1 & 47.1 & 57.4 & 232. & 1460. & 2780 & 4860 . & 5390. \\
\hline 10 & 17.3 & 28.0 & 35.3 & 45.4 & 152. & 770 . & 1320 . & 2210 & 3830 \\
\hline 20 & 15.3 & 23.3 & 27.3 & 37.1 & 84.2 & 316. & 554. & 1050 & 2020 \\
\hline 25 & 15.2 & 19.6 & 26.0 & 36.0 & 74.0 & 246. & 404. & 728. & 1710 . \\
\hline 30 & 15.2 & 16.7 & 25.2 & 31.6 & 66.8 & 190. & 321. & 617. & 1430 \\
\hline 40 & 12.4 & 14.1 & 22.2 & 29.0 & 50.2 & 125. & 235. & 419. & 1000 \\
\hline 50 & 10.3 & 12.0 & 18.6 & 22.6 & 39.0 & 87.6 & 147. & 273. & 574. \\
\hline 60 & 9.16 & 11.8 & 15.8 & 19.0 & 33.8 & 69.8 & 107. & 188. & 426. \\
\hline 70 & 8.61 & 10.9 & 13.4 & 17.0 & 28.0 & 58.6 & 86.8 & 154. & 319. \\
\hline 75 & 8.50 & 10.8 & 12.6 & 16.0 & 24.0 & 57.0 & 73.6 & 142. & 280. \\
\hline 80 & 7.99 & 9.90 & 10.9 & 14.2 & 24.0 & 52.4 & 71.4 & 131. & 260. \\
\hline 90 & 5.98 & 7.98 & 9.46 & 12.0 & 19.0 & 45.5 & 58.0 & 102. & 200. \\
\hline 95 & 4.30 & 5.90 & 7.66 & 9.37 & 16.6 & 37.9 & 49.4 & 87.9 & 174. \\
\hline 98 & 2.37 & 5.61 & 6.78 & 8.89 & 14.8 & 34.3 & 47.8 & 62.4 & 89.6 \\
\hline 99 & 2.37 & 5.38 & 5.96 & 8.52 & 14.0 & 34.0 & 44.4 & 61.6 & 89.4 \\
\hline
\end{tabular}


04197100 Honey Creek at Melmore, Ohio

Years analyzed $=33$

Climatic year range $=1976-2008$

[APEE, annual percentage equaled or exceeded; $\mathrm{ft}^{3} / \mathrm{s}$, cubic foot per second; Sep, September; Nov, November]

\begin{tabular}{|c|c|c|c|c|c|c|c|c|c|}
\hline \multirow[b]{2}{*}{ APEE } & \multicolumn{9}{|c|}{ Sep-Nov daily mean streamflow $\left(\mathrm{ft}^{3} / \mathrm{s}\right)$ that was equaled or exceeded the indicated percentage of time at APEE level } \\
\hline & 95 & 90 & 80 & 70 & 50 & 30 & 20 & 10 & 5 \\
\hline 1 & 12.9 & 30.4 & 50.0 & 125. & 528. & 764. & 1020 & 1290. & 2060 \\
\hline 2 & 11.3 & 28.6 & 39.1 & 100. & 387. & 685. & 936. & 1170 & 1530. \\
\hline 5 & 6.71 & 10.6 & 20.9 & 58.9 & 142. & 385. & 586. & 753. & 959. \\
\hline 10 & 4.65 & 6.30 & 13.7 & 24.8 & 68.0 & 216. & 265. & 505. & 641. \\
\hline 20 & 2.32 & 3.96 & 6.87 & 8.31 & 22.6 & 65.8 & 100. & 263. & 337. \\
\hline 25 & 2.09 & 3.40 & 4.02 & 6.78 & 16.0 & 47.2 & 73.0 & 132. & 268. \\
\hline 30 & 1.74 & 2.31 & 3.33 & 6.10 & 13.4 & 38.6 & 55.3 & 84.9 & 222. \\
\hline 40 & 1.17 & 1.50 & 2.28 & 4.25 & 9.24 & 25.0 & 33.6 & 58.0 & 102. \\
\hline 50 & 0.95 & 1.18 & 1.96 & 2.94 & 6.60 & 20.0 & 24.4 & 38.8 & 69.9 \\
\hline 60 & 0.65 & 0.96 & 1.60 & 1.96 & 5.30 & 9.86 & 20.2 & 28.0 & 49.4 \\
\hline 70 & 0.44 & 0.86 & 1.26 & 1.50 & 3.96 & 8.06 & 16.1 & 22.0 & 36.1 \\
\hline 75 & 0.38 & 0.73 & 1.10 & 1.34 & 3.60 & 6.36 & 15.2 & 19.6 & 31.8 \\
\hline 80 & 0.31 & 0.66 & 1.00 & 1.26 & 3.20 & 5.54 & 10.3 & 17.6 & 28.9 \\
\hline 90 & 0.19 & 0.53 & 0.75 & 1.01 & 2.40 & 3.20 & 5.11 & 13.6 & 15.6 \\
\hline 95 & 0.15 & 0.38 & 0.58 & 0.88 & 1.46 & 2.86 & 4.66 & 11.8 & 13.6 \\
\hline 98 & 0.13 & 0.30 & 0.51 & 0.71 & 1.27 & 2.63 & 4.14 & 10.5 & 12.6 \\
\hline 99 & 0.13 & 0.26 & 0.46 & 0.65 & 1.10 & 2.54 & 4.06 & 9.84 & 12.3 \\
\hline
\end{tabular}

\section{Rock Creek at Tiffin, Ohio}

Years analyzed $=26$

Climatic year range $=1983-2008$

[APEE, annual percentage equaled or exceeded; $\mathrm{ft}^{3} / \mathrm{s}$, cubic foot per second; Sep, September; Nov, November]

\begin{tabular}{|c|c|c|c|c|c|c|c|c|c|}
\hline \multirow[b]{2}{*}{ APEE } & \multicolumn{9}{|c|}{ Sep-Nov daily mean streamflow $\left(\mathrm{ft}^{3} / \mathrm{s}\right)$ that was equaled or exceeded the indicated percentage of time at APEE level } \\
\hline & 95 & 90 & 80 & 70 & 50 & 30 & 20 & 10 & 5 \\
\hline 1 & 3.69 & 4.67 & 7.58 & 34.7 & 131. & 295. & 373. & 609. & 962. \\
\hline 2 & 3.52 & 4.39 & 6.77 & 33.4 & 91.1 & 207. & 302. & 386. & 807. \\
\hline 5 & 2.48 & 3.33 & 4.97 & 10.6 & 26.6 & 80.4 & 118. & 246 & 489. \\
\hline 10 & 2.07 & 2.52 & 3.89 & 5.42 & 11.1 & 24.8 & 54.7 & 133. & 200 . \\
\hline 20 & 1.81 & 2.00 & 2.78 & 3.53 & 4.91 & 7.99 & 22.9 & 40.7 & 77.3 \\
\hline 25 & 1.68 & 2.00 & 2.46 & 3.30 & 4.15 & 6.49 & 14.7 & 24.3 & 52.9 \\
\hline 30 & 1.55 & 1.88 & 2.25 & 2.55 & 3.24 & 5.84 & 12.1 & 17.6 & 35.1 \\
\hline 40 & 1.51 & 1.71 & 1.90 & 2.00 & 2.87 & 4.82 & 6.76 & 11.7 & 17.7 \\
\hline 50 & 1.17 & 1.30 & 1.58 & 1.90 & 2.40 & 3.96 & 4.94 & 9.37 & 12.6 \\
\hline 60 & 1.07 & 1.20 & 1.37 & 1.58 & 1.94 & 3.19 & 3.97 & 8.02 & 9.20 \\
\hline 70 & 0.89 & 1.07 & 1.20 & 1.40 & 1.58 & 2.49 & 3.40 & 7.30 & 7.61 \\
\hline 75 & 0.84 & 0.98 & 1.14 & 1.21 & 1.45 & 2.39 & 3.06 & 6.86 & 7.06 \\
\hline 80 & 0.78 & 0.93 & 1.04 & 1.20 & 1.37 & 2.20 & 2.82 & 6.09 & 6.39 \\
\hline 90 & 0.52 & 0.63 & 0.87 & 1.10 & 1.21 & 2.08 & 2.38 & 3.94 & 4.85 \\
\hline 95 & 0.42 & 0.58 & 0.76 & 0.94 & 1.10 & 1.85 & 2.23 & 3.42 & 4.32 \\
\hline 98 & 0.36 & 0.53 & 0.72 & 0.82 & 1.00 & 1.70 & 2.09 & 3.23 & 4.18 \\
\hline 99 & 0.35 & 0.50 & 0.70 & 0.79 & 1.00 & 1.70 & 2.02 & 3.10 & 3.81 \\
\hline
\end{tabular}




\section{Sandusky River near Fremont, Ohio}

Years analyzed $=83$

Climatic year range $=1923-2008$

[APEE, annual percentage equaled or exceeded; $\mathrm{ft}^{3} / \mathrm{s}$, cubic foot per second; Sep, September; Nov, November]

\begin{tabular}{|c|c|c|c|c|c|c|c|c|c|}
\hline \multirow[b]{2}{*}{ APEE } & \multicolumn{9}{|c|}{ Sep-Nov daily mean streamflow $\left(\mathrm{ft}^{3} / \mathrm{s}\right)$ that was equaled or exceeded the indicated percentage of time at APEE level } \\
\hline & 98 & 95 & 90 & 80 & 50 & 20 & 10 & 5 & 2 \\
\hline 1 & 39.4 & 68.8 & 86.4 & 288. & 1550. & 5670. & 8620. & 10900. & 15300. \\
\hline 2 & 38.8 & 61.9 & 76.3 & 263. & 1190. & 4770 . & 7930. & 9620. & 14200 . \\
\hline 5 & 33.9 & 55.2 & 69.8 & 149. & 694. & 2770 . & 5330. & 7160 & 10000 . \\
\hline 10 & 26.5 & 49.6 & 59.6 & 96.3 & 347. & 1560. & 3470 . & 4660 & 6500. \\
\hline 20 & 24.4 & 38.2 & 47.3 & 65.3 & 161. & 591. & 1350. & 2820 & 3710 . \\
\hline 25 & 23.0 & 32.6 & 44.2 & 59.8 & 131. & 481. & 877. & 2330. & 2750 . \\
\hline 30 & 21.7 & 30.8 & 40.8 & 54.0 & 106. & 365. & 700 . & 1770 . & 2230 . \\
\hline 40 & 19.1 & 28.2 & 34.8 & 46.2 & 83.0 & 244. & 465. & 741. & 1530. \\
\hline 50 & 14.7 & 24.2 & 32.0 & 41.0 & 67.0 & 178. & 367. & 494. & 1060. \\
\hline 60 & 12.7 & 21.4 & 27.8 & 36.3 & 57.0 & 134. & 275. & 402. & 615. \\
\hline 70 & 12.6 & 19.1 & 22.8 & 29.9 & 51.0 & 107. & 216. & 341. & 455. \\
\hline 75 & 11.7 & 17.4 & 22.0 & 28.0 & 47.0 & 103. & 191. & 309. & 407. \\
\hline 80 & 11.4 & 15.2 & 18.3 & 26.9 & 43.2 & 91.6 & 151. & 238. & 358. \\
\hline 90 & 9.46 & 13.0 & 15.0 & 22.6 & 35.0 & 73.8 & 107. & 180. & 282. \\
\hline 95 & 7.41 & 11.1 & 13.0 & 20.4 & 33.0 & 60.8 & 91.6 & 142. & 260. \\
\hline 98 & 5.41 & 9.92 & 11.3 & 16.5 & 29.2 & 52.3 & 89.1 & 123. & 172. \\
\hline 99 & 5.41 & 9.52 & 11.0 & 16.0 & 28.0 & 50.2 & 88.8 & 117. & 168. \\
\hline
\end{tabular}

\section{Huron River at Milan, Ohio}

Years analyzed $=53$

Climatic year range $=1950-2008$

[APEE, annual percentage equaled or exceeded; $\mathrm{ft}^{3} / \mathrm{s}$, cubic foot per second; Sep, September; Nov, November]

\begin{tabular}{|c|c|c|c|c|c|c|c|c|c|}
\hline \multirow[b]{2}{*}{ APEE } & \multicolumn{9}{|c|}{ Sep-Nov daily mean streamflow $\left(\mathrm{ft}^{3} / \mathrm{s}\right)$ that was equaled or exceeded the indicated percentage of time at APEE level } \\
\hline & 98 & 95 & 90 & 80 & 50 & 20 & 10 & 5 & 2 \\
\hline 1 & 25.1 & 28.1 & 43.2 & 103. & 976. & 2150. & 3960. & 5160. & 7720 . \\
\hline 2 & 20.4 & 25.5 & 40.2 & 99.5 & 685. & 1440 . & 3090. & 4330. & 5370 \\
\hline 5 & 16.5 & 22.3 & 30.8 & 51.7 & 248. & 645. & 1190. & 2010 . & 3280. \\
\hline 10 & 14.6 & 21.4 & 24.8 & 41.0 & 159. & 343. & 567. & 975. & 2160 \\
\hline 20 & 13.2 & 15.7 & 19.4 & 33.8 & 73.6 & 159. & 286. & 463. & 811. \\
\hline 25 & 13.1 & 14.0 & 18.4 & 28.8 & 60.0 & 119. & 213. & 383. & 679. \\
\hline 30 & 12.1 & 13.7 & 18.4 & 27.9 & 51.4 & 96.8 & 182. & 296. & 598. \\
\hline 40 & 10.3 & 12.1 & 16.4 & 22.8 & 36.0 & 72.6 & 152. & 224. & 398. \\
\hline 50 & 8.77 & 10.6 & 15.4 & 19.8 & 30.0 & 62.2 & 91.8 & 177. & 271. \\
\hline 60 & 6.79 & 9.63 & 12.9 & 16.0 & 24.8 & 51.0 & 67.1 & 147. & 175. \\
\hline 70 & 5.87 & 8.17 & 11.0 & 12.0 & 22.0 & 45.0 & 56.7 & 123. & 136. \\
\hline 75 & 5.59 & 6.60 & 8.86 & 11.0 & 20.0 & 41.2 & 52.4 & 107. & 126. \\
\hline 80 & 5.21 & 5.58 & 8.50 & 11.0 & 17.4 & 38.0 & 47.0 & 93.0 & 110. \\
\hline 90 & 3.70 & 4.87 & 6.38 & 8.98 & 14.0 & 30.6 & 39.9 & 49.6 & 69.0 \\
\hline 95 & 3.28 & 4.27 & 5.32 & 7.24 & 12.2 & 24.8 & 35.4 & 44.5 & 63.6 \\
\hline 98 & 3.05 & 3.85 & 4.29 & 6.09 & 12.0 & 21.8 & 32.2 & 38.9 & 61.1 \\
\hline 99 & 3.05 & 3.67 & 4.08 & 6.00 & 12.0 & 21.0 & 32.2 & 38.3 & 58.0 \\
\hline
\end{tabular}




\section{Old Woman Creek at Berlin Rd near Huron, Ohio}

Years analyzed $=21$

Climatic year range $=1987-2008$

[APEE, annual percentage equaled or exceeded; $\mathrm{ft}^{3} / \mathrm{s}$, cubic foot per second; Sep, September; Nov, November]

\begin{tabular}{|c|c|c|c|c|c|c|c|c|c|}
\hline \multirow[b]{2}{*}{ APEE } & \multicolumn{9}{|c|}{ Sep-Nov daily mean streamflow $\left(\mathrm{ft}^{3} / \mathrm{s}\right)$ that was equaled or exceeded the indicated percentage of time at APEE level } \\
\hline & 95 & 90 & 80 & 70 & 50 & 30 & 20 & 10 & 5 \\
\hline 1 & 2.76 & 5.68 & 17.6 & 91.8 & 184. & 248. & 335. & 438 & 449. \\
\hline 2 & 2.54 & 4.97 & 13.3 & 51.6 & 102. & 161. & 212 & 306. & 426. \\
\hline 5 & 1.56 & 3.17 & 5.87 & 15.6 & 46.4 & 71.2 & 85.6 & 113. & 152. \\
\hline 10 & 0.46 & 1.52 & 2.42 & 5.48 & 20.0 & 31.8 & 42.8 & 59.2 & 60.7 \\
\hline 20 & 0.26 & 1.08 & 1.28 & 1.94 & 5.18 & 11.8 & 13.6 & 27.5 & 34.6 \\
\hline 25 & 0.15 & 0.89 & 1.03 & 1.30 & 4.10 & 8.32 & 9.60 & 20.2 & 23.8 \\
\hline 30 & 0.08 & 0.58 & 0.78 & 1.10 & 3.08 & 5.99 & 7.62 & 15.4 & 17.0 \\
\hline 40 & 0.02 & 0.23 & 0.57 & 0.73 & 2.12 & 3.62 & 5.47 & 9.35 & 12.2 \\
\hline 50 & 0.00 & 0.06 & 0.22 & 0.53 & 1.30 & 2.64 & 4.04 & 7.02 & 8.23 \\
\hline 60 & 0.00 & 0.00 & 0.07 & 0.38 & 0.84 & 1.88 & 2.54 & 4.54 & 6.41 \\
\hline 70 & 0.00 & 0.00 & 0.00 & 0.14 & 0.59 & 1.37 & 2.18 & 3.20 & 4.22 \\
\hline 75 & 0.00 & 0.00 & 0.00 & 0.12 & 0.42 & 1.19 & 1.92 & 2.68 & 3.34 \\
\hline 80 & 0.00 & 0.00 & 0.00 & 0.02 & 0.26 & 1.05 & 1.68 & 2.37 & 2.81 \\
\hline 90 & 0.00 & 0.00 & 0.00 & 0.00 & 0.16 & 0.47 & 1.19 & 1.88 & 2.11 \\
\hline 95 & 0.00 & 0.00 & 0.00 & 0.00 & 0.00 & 0.23 & 0.44 & 1.00 & 1.86 \\
\hline 98 & 0.00 & 0.00 & 0.00 & 0.00 & 0.00 & 0.11 & 0.41 & 0.81 & 1.68 \\
\hline 99 & 0.00 & 0.00 & 0.00 & 0.00 & 0.00 & 0.08 & 0.41 & 0.73 & 1.52 \\
\hline
\end{tabular}

\section{Vermilion River near Vermilion, Ohio}

Years analyzed $=39$

Climatic year range $=1950-2008$

[APEE, annual percentage equaled or exceeded; $\mathrm{ft}^{3} / \mathrm{s}$, cubic foot per second; Sep, September; Nov, November]

\begin{tabular}{|c|c|c|c|c|c|c|c|c|c|}
\hline \multirow[b]{2}{*}{ APEE } & \multicolumn{9}{|c|}{ Sep-Nov daily mean streamflow $\left(\mathrm{ft}^{3} / \mathrm{s}\right)$ that was equaled or exceeded the indicated percentage of time at APEE level } \\
\hline & 95 & 90 & 80 & 70 & 50 & 30 & 20 & 10 & 5 \\
\hline 1 & 6.60 & 19.0 & 84.0 & 286. & 637. & 1430. & 1520. & 2300 & 3340 \\
\hline 2 & 5.76 & 14.8 & 56.2 & 186. & 500. & 1070 & 1300 . & 1730 & 3060 . \\
\hline 5 & 4.76 & 8.72 & 24.6 & 120. & 250 & 461. & 588. & 959. & 1310. \\
\hline 10 & 3.82 & 7.36 & 20.6 & 63.0 & 132. & 225 & 294. & 503. & 563. \\
\hline 20 & 2.30 & 4.70 & 14.6 & 32.8 & 49.0 & 93.2 & 126. & 191. & 196. \\
\hline 25 & 2.00 & 4.20 & 13.0 & 18.0 & 37.0 & 70.0 & 115. & 138. & 157. \\
\hline 30 & 1.60 & 3.80 & 9.20 & 13.0 & 30.4 & 60.0 & 90.4 & 115. & 137. \\
\hline 40 & 1.10 & 2.90 & 6.60 & 8.26 & 20.2 & 42.0 & 60.4 & 81.2 & 98.2 \\
\hline 50 & 1.00 & 2.50 & 4.70 & 6.70 & 15.0 & 33.0 & 38.0 & 56.0 & 84.0 \\
\hline 60 & 0.08 & 2.28 & 3.54 & 5.32 & 10.0 & 20.8 & 30.8 & 43.8 & 71.6 \\
\hline 70 & 0.00 & 0.80 & 2.70 & 4.20 & 6.92 & 16.0 & 25.8 & 34.8 & 56.6 \\
\hline 75 & 0.00 & 0.50 & 2.30 & 3.00 & 4.30 & 14.0 & 22.0 & 30.0 & 48.0 \\
\hline 80 & 0.00 & 0.30 & 1.90 & 2.24 & 3.90 & 10.0 & 19.0 & 28.0 & 32.8 \\
\hline 90 & 0.00 & 0.20 & 1.30 & 1.50 & 2.92 & 6.90 & 9.42 & 15.4 & 16.0 \\
\hline 95 & 0.00 & 0.10 & 0.77 & 1.02 & 2.46 & 5.80 & 8.30 & 11.0 & 14.0 \\
\hline 98 & 0.00 & 0.10 & 0.48 & 0.90 & 2.10 & 5.35 & 7.38 & 9.22 & 12.8 \\
\hline 99 & 0.00 & 0.10 & 0.46 & 0.73 & 2.10 & 5.10 & 7.10 & 9.10 & 12.0 \\
\hline
\end{tabular}




\section{Tinkers Creek at Bedford, Ohio}

Years analyzed $=46$

Climatic year range $=1963-2008$

[APEE, annual percentage equaled or exceeded; $\mathrm{ft}^{3} / \mathrm{s}$, cubic foot per second; Sep, September; Nov, November]

\begin{tabular}{|c|c|c|c|c|c|c|c|c|c|}
\hline \multirow[b]{2}{*}{ APEE } & \multicolumn{9}{|c|}{ Sep-Nov daily mean streamflow $\left(\mathrm{ft}^{3} / \mathrm{s}\right)$ that was equaled or exceeded the indicated percentage of time at APEE level } \\
\hline & 95 & 90 & 80 & 70 & 50 & 30 & 20 & 10 & 5 \\
\hline 1 & 112. & 196. & 340. & 485. & 686. & 862. & 958. & 1230 & 2030 \\
\hline 2 & 89.8 & 171. & 273. & 361. & 474. & 726. & 798. & 1040 . & 1370 . \\
\hline 5 & 48.8 & 127. & 146. & 226. & 340 . & 543. & 580. & 631. & 674. \\
\hline 10 & 38.0 & 83.6 & 103. & 126. & 238 & 303. & 349. & 385. & 494. \\
\hline 20 & 25.2 & 50.9 & 61.8 & 94.0 & 127. & 163. & 179. & 237. & 283. \\
\hline 25 & 21.7 & 43.5 & 52.8 & 72.0 & 103. & 122. & 143. & 191. & 215. \\
\hline 30 & 20.0 & 38.8 & 44.4 & 55.6 & 85.2 & 96.8 & 117. & 150. & 185. \\
\hline 40 & 15.2 & 25.8 & 37.5 & 45.3 & 62.3 & 73.7 & 81.0 & 110. & 138. \\
\hline 50 & 11.1 & 22.1 & 32.2 & 39.1 & 48.0 & 60.0 & 65.2 & 81.3 & 117. \\
\hline 60 & 9.99 & 19.5 & 28.0 & 30.5 & 37.5 & 48.7 & 54.0 & 67.8 & 97.2 \\
\hline 70 & 9.38 & 17.9 & 24.2 & 28.0 & 33.8 & 41.6 & 45.6 & 55.3 & 76.8 \\
\hline 75 & 8.78 & 16.2 & 21.4 & 26.1 & 32.0 & 37.9 & 40.0 & 46.2 & 65.4 \\
\hline 80 & 8.52 & 14.5 & 21.0 & 25.1 & 28.4 & 33.0 & 37.4 & 42.3 & 55.8 \\
\hline 90 & 7.59 & 11.2 & 18.0 & 19.3 & 24.1 & 27.9 & 30.8 & 36.3 & 40.7 \\
\hline 95 & 7.20 & 10.3 & 16.2 & 18.0 & 21.1 & 24.6 & 27.2 & 31.2 & 33.8 \\
\hline 98 & 6.91 & 10.0 & 14.3 & 16.7 & 19.4 & 23.8 & 26.0 & 28.8 & 31.1 \\
\hline 99 & 6.65 & 9.46 & 13.4 & 16.0 & 19.0 & 23.0 & 25.6 & 28.0 & 30.3 \\
\hline
\end{tabular}

\section{Rock Creek near Rock Creek, Ohio}

Years analyzed $=24$

Climatic year range $=1942-65$

[APEE, annual percentage equaled or exceeded; $\mathrm{ft}^{3} / \mathrm{s}$, cubic foot per second; Sep, September; Nov, November]

\begin{tabular}{|c|c|c|c|c|c|c|c|c|c|}
\hline \multirow[b]{2}{*}{ APEE } & \multicolumn{9}{|c|}{ Sep-Nov daily mean streamflow ( $\left.\mathrm{ft}^{3} / \mathrm{s}\right)$ that was equaled or exceeded the indicated percentage of time at APEE level } \\
\hline & 95 & 90 & 80 & 70 & 50 & 30 & 20 & 10 & 5 \\
\hline 1 & 1.40 & 2.70 & 12.0 & 61.0 & 208. & 578. & 904. & 1090 & 1680 \\
\hline 2 & 1.21 & 2.45 & 9.28 & 36.6 & 133. & 366. & 482. & 719. & 918. \\
\hline 5 & 0.72 & 1.70 & 5.14 & 13.6 & 45.9 & 134. & 181. & 419. & 540. \\
\hline 10 & 0.14 & 1.10 & 2.14 & 3.97 & 21.3 & 68.4 & 101. & 184. & 318. \\
\hline 20 & 0.00 & 0.15 & 1.18 & 1.75 & 7.23 & 29.9 & 40.2 & 106. & 173. \\
\hline 25 & 0.00 & 0.00 & 0.80 & 1.45 & 5.10 & 17.5 & 26.0 & 87.0 & 125. \\
\hline 30 & 0.00 & 0.00 & 0.60 & 1.07 & 2.76 & 13.7 & 18.0 & 64.4 & 94.6 \\
\hline 40 & 0.00 & 0.00 & 0.30 & 0.51 & 1.49 & 7.45 & 10.2 & 38.7 & 52.7 \\
\hline 50 & 0.00 & 0.00 & 0.00 & 0.15 & 0.80 & 4.60 & 7.90 & 21.0 & 35.3 \\
\hline 60 & 0.00 & 0.00 & 0.00 & 0.10 & 0.49 & 2.58 & 4.90 & 7.84 & 19.3 \\
\hline 70 & 0.00 & 0.00 & 0.00 & 0.00 & 0.18 & 0.53 & 2.44 & 4.90 & 14.1 \\
\hline 75 & 0.00 & 0.00 & 0.00 & 0.00 & 0.10 & 0.25 & 2.00 & 4.60 & 12.5 \\
\hline 80 & 0.00 & 0.00 & 0.00 & 0.00 & 0.10 & 0.25 & 1.32 & 4.32 & 10.8 \\
\hline 90 & 0.00 & 0.00 & 0.00 & 0.00 & 0.00 & 0.05 & 0.40 & 2.25 & 3.65 \\
\hline 95 & 0.00 & 0.00 & 0.00 & 0.00 & 0.00 & 0.00 & 0.30 & 1.85 & 3.34 \\
\hline 98 & 0.00 & 0.00 & 0.00 & 0.00 & 0.00 & 0.00 & 0.20 & 1.75 & 3.08 \\
\hline 99 & 0.00 & 0.00 & 0.00 & 0.00 & 0.00 & 0.00 & 0.20 & 1.75 & 2.95 \\
\hline
\end{tabular}




\section{Grand River near Madison, Ohio}

Years analyzed $=49$

Climatic year range $=1922-73$

[APEE, annual percentage equaled or exceeded; $\mathrm{ft}^{3} / \mathrm{s}$, cubic foot per second; Sep, September; Nov, November]

\begin{tabular}{|c|c|c|c|c|c|c|c|c|c|}
\hline \multirow[b]{2}{*}{ APEE } & \multicolumn{9}{|c|}{ Sep-Nov daily mean streamflow $\left(\mathrm{ft}^{3} / \mathrm{s}\right)$ that was equaled or exceeded the indicated percentage of time at APEE level } \\
\hline & 95 & 90 & 80 & 70 & 50 & 30 & 20 & 10 & 5 \\
\hline 1 & 37.0 & 66.0 & 284. & 575. & 1630. & 4080. & 4880. & 5900. & 8270 \\
\hline 2 & 36.5 & 62.6 & 238. & 416. & 1460 . & 3080 & 4200 . & 5210. & 6860 \\
\hline 5 & 31.9 & 49.0 & 125. & 190. & 720 . & 1930. & 2300 & 3080 . & 3990. \\
\hline 10 & 23.8 & 28.4 & 88.2 & 109. & 361. & 951. & 1670 . & 1910. & 2820 \\
\hline 20 & 17.1 & 20.6 & 33.0 & 50.6 & 125. & 327. & 790 . & 1130 & 1870. \\
\hline 25 & 15.0 & 18.0 & 21.0 & 35.0 & 96.0 & 252. & 645. & 1010 & 1700 \\
\hline 30 & 13.7 & 15.0 & 19.0 & 31.4 & 74.8 & 208. & 451. & 793. & 1460 \\
\hline 40 & 9.16 & 11.2 & 15.0 & 20.6 & 39.0 & 127. & 184. & 590. & 910. \\
\hline 50 & 6.55 & 9.10 & 11.0 & 14.0 & 24.0 & 53.0 & 107. & 352. & 582. \\
\hline 60 & 4.87 & 6.42 & 9.00 & 11.0 & 18.0 & 40.0 & 59.0 & 150. & 400 \\
\hline 70 & 3.80 & 5.20 & 7.28 & 8.20 & 13.6 & 28.0 & 33.0 & 112. & 239. \\
\hline 75 & 3.35 & 4.90 & 6.70 & 7.20 & 12.0 & 22.0 & 30.0 & 87.0 & 159. \\
\hline 80 & 2.34 & 4.50 & 5.80 & 6.30 & 11.0 & 15.0 & 25.4 & 70.8 & 133. \\
\hline 90 & 1.33 & 2.34 & 3.90 & 5.42 & 8.08 & 11.4 & 12.2 & 22.0 & 34.5 \\
\hline 95 & 0.78 & 1.16 & 3.36 & 4.02 & 7.20 & 10.0 & 11.0 & 19.0 & 33.3 \\
\hline 98 & 0.20 & 0.77 & 2.02 & 3.75 & 5.95 & 9.00 & 10.0 & 16.8 & 21.5 \\
\hline 99 & 0.20 & 0.60 & 1.60 & 3.20 & 5.40 & 8.00 & 9.80 & 16.0 & 21.5 \\
\hline
\end{tabular}

\section{Grand River near Painesville, Ohio}

Years analyzed $=35$

Climatic year range $=1974-2008$

[APEE, annual percentage equaled or exceeded; $\mathrm{ft}^{3} / \mathrm{s}$, cubic foot per second; Sep, September; Nov, November]

\begin{tabular}{|c|c|c|c|c|c|c|c|c|c|}
\hline \multirow[b]{2}{*}{ APEE } & \multicolumn{9}{|c|}{ Sep-Nov daily mean streamflow $\left(\mathrm{ft}^{3} / \mathrm{s}\right)$ that was equaled or exceeded the indicated percentage of time at APEE level } \\
\hline & 95 & 90 & 80 & 70 & 50 & 30 & 20 & 10 & 5 \\
\hline 1 & 218. & 838. & 1510. & 3380. & 4490. & 6320. & 7340 & 8000. & 11400 \\
\hline 2 & 196. & 782. & 1310. & 2850 & 4290 & 5530. & 6070. & 7370 . & 8920. \\
\hline 5 & 158. & 386. & 921. & 2050 . & 2630 & 3880 . & 4510 . & 5590. & 6620. \\
\hline 10 & 119. & 283. & 675. & 1150 & 1670 & 2360 & 2730 & 4130. & 4990. \\
\hline 20 & 82.1 & 152. & 213. & 615. & 954. & 1310 . & 1870 . & 2390 & 2800 \\
\hline 25 & 75.4 & 124. & 189. & 385. & 698. & 1030 & 1380. & 1840 & 2310 \\
\hline 30 & 70.8 & 92.6 & 159. & 258 & 488. & 820 . & 1100 & 1670. & 1980. \\
\hline 40 & 59.5 & 72.5 & 106. & 134. & 314. & 542. & 755. & 1220 . & 1470 \\
\hline 50 & 42.6 & 50.2 & 88.8 & 104. & 203. & 374. & 454. & 871. & 1230. \\
\hline 60 & 27.0 & 34.3 & 54.9 & 79.6 & 118. & 254. & 325. & 627. & 907. \\
\hline 70 & 15.4 & 20.8 & 36.6 & 53.8 & 80.6 & 190. & 231. & 442. & 663. \\
\hline 75 & 13.6 & 16.6 & 30.6 & 41.0 & 68.0 & 147. & 179. & 318. & 524. \\
\hline 80 & 11.8 & 14.4 & 26.5 & 31.2 & 58.0 & 115. & 139. & 255. & 414 \\
\hline 90 & 9.21 & 10.7 & 17.4 & 24.0 & 46.2 & 60.8 & 103. & 140. & 282 \\
\hline 95 & 7.16 & 9.15 & 14.7 & 19.2 & 28.6 & 48.6 & 56.4 & 97.7 & 128 \\
\hline 98 & 6.62 & 8.61 & 12.9 & 16.0 & 25.0 & 38.7 & 50.8 & 79.8 & 106. \\
\hline 99 & 6.62 & 8.44 & 12.0 & 15.0 & 25.0 & 38.4 & 49.8 & 74.2 & 97.2 \\
\hline
\end{tabular}


04212500 Ashtabula River near Ashtabula, Ohio

Years analyzed $=51$

Climatic year range $=1924-79$

[APEE, annual percentage equaled or exceeded; $\mathrm{ft}^{3} / \mathrm{s}$, cubic foot per second; Sep, September; Nov, November]

\begin{tabular}{|c|c|c|c|c|c|c|c|c|c|}
\hline \multirow[b]{2}{*}{ APEE } & \multicolumn{9}{|c|}{ Sep-Nov daily mean streamflow $\left(\mathrm{ft}^{3} / \mathrm{s}\right)$ that was equaled or exceeded the indicated percentage of time at APEE level } \\
\hline & 98 & 95 & 90 & 80 & 50 & 20 & 10 & 5 & 2 \\
\hline 1 & 13.0 & 19.0 & 55.0 & 109. & 900. & 1680. & 2290 & 3790. & 6050 \\
\hline 2 & 12.2 & 15.5 & 38.5 & 82.8 & 444. & 1370 . & 1710 & 2490 . & 3490. \\
\hline 5 & 6.10 & 7.53 & 16.0 & 45.2 & 225 & 812. & 1160 & 1420 . & 2170 \\
\hline 10 & 3.45 & 5.52 & 8.18 & 23.3 & 128. & 426. & 722. & 910. & 1130. \\
\hline 20 & 1.65 & 3.50 & 4.37 & 7.06 & 66.8 & 204. & 370 . & 493. & 794. \\
\hline 25 & 1.41 & 2.54 & 3.28 & 4.38 & 38.0 & 145. & 311. & 404. & 639. \\
\hline 30 & 1.14 & 1.30 & 2.80 & 3.68 & 22.8 & 113. & 252. & 257. & 462. \\
\hline 40 & 0.14 & 0.51 & 1.41 & 2.40 & 12.0 & 84.6 & 145. & 186. & 318. \\
\hline 50 & 0.01 & 0.32 & 0.50 & 1.10 & 5.40 & 51.0 & 83.0 & 128. & 224. \\
\hline 60 & 0.00 & 0.06 & 0.20 & 0.50 & 3.20 & 28.0 & 56.4 & 91.9 & 189. \\
\hline 70 & 0.00 & 0.00 & 0.10 & 0.18 & 1.80 & 14.4 & 36.0 & 54.9 & 156. \\
\hline 75 & 0.00 & 0.00 & 0.00 & 0.10 & 1.40 & 12.4 & 25.0 & 44.0 & 124. \\
\hline 80 & 0.00 & 0.00 & 0.00 & 0.00 & 1.00 & 8.66 & 14.9 & 36.5 & 106. \\
\hline 90 & 0.00 & 0.00 & 0.00 & 0.00 & 0.32 & 3.10 & 7.47 & 19.7 & 39.3 \\
\hline 95 & 0.00 & 0.00 & 0.00 & 0.00 & 0.20 & 2.54 & 6.66 & 9.92 & 13.0 \\
\hline 98 & 0.00 & 0.00 & 0.00 & 0.00 & 0.20 & 1.62 & 4.58 & 6.71 & 10.9 \\
\hline 99 & 0.00 & 0.00 & 0.00 & 0.00 & 0.20 & 1.52 & 4.38 & 6.12 & 10.9 \\
\hline
\end{tabular}

\section{Conneaut Creek at Conneaut, Ohio}

Years analyzed $=73$

Climatic year range $=1922-2008$

[APEE, annual percentage equaled or exceeded; $\mathrm{ft}^{3} / \mathrm{s}$, cubic foot per second; Sep, September; Nov, November]

\begin{tabular}{|c|c|c|c|c|c|c|c|c|c|}
\hline \multirow[b]{2}{*}{ APEE } & \multicolumn{9}{|c|}{ Sep-Nov daily mean streamflow $\left(\mathrm{ft}^{3} / \mathrm{s}\right)$ that was equaled or exceeded the indicated percentage of time at APEE level } \\
\hline & 98 & 95 & 90 & 80 & 50 & 20 & 10 & 5 & 2 \\
\hline 1 & 43.4 & 48.5 & 140. & 441. & 1670. & 2950. & 3590. & 7640 & 9550. \\
\hline 2 & 36.4 & 40.7 & 122 & 302. & 1290. & 2460 & 3080 . & 4130. & 6300 \\
\hline 5 & 24.5 & 31.2 & 55.0 & 191. & 720 . & 1420. & 1780 . & 2030 . & 2500 \\
\hline 10 & 20.8 & 26.6 & 41.8 & 116. & 409. & 855. & 1220 & 1420 . & 1540 \\
\hline 20 & 17.0 & 18.0 & 25.0 & 39.8 & 208. & 433. & 605. & 786. & 921. \\
\hline 25 & 15.0 & 16.0 & 20.0 & 35.2 & 156. & 330. & 518. & 603. & 766. \\
\hline 30 & 12.0 & 14.8 & 17.6 & 22.1 & 115. & 268. & 412. & 506. & 660. \\
\hline 40 & 8.64 & 10.8 & 12.5 & 17.2 & 61.2 & 187. & 310. & 384. & 491. \\
\hline 50 & 6.18 & 7.64 & 9.76 & 13.8 & 32.0 & 131. & 208. & 261. & 366. \\
\hline 60 & 4.52 & 6.44 & 7.70 & 11.0 & 23.0 & 86.6 & 147. & 186. & 302. \\
\hline 70 & 3.63 & 5.07 & 6.44 & 7.64 & 19.0 & 58.9 & 107. & 142. & 239 \\
\hline 75 & 3.28 & 4.38 & 5.88 & 6.88 & 16.0 & 44.6 & 85.8 & 121. & 223. \\
\hline 80 & 3.08 & 3.95 & 5.05 & 6.28 & 13.4 & 36.5 & 68.8 & 98.4 & 193. \\
\hline 90 & 2.15 & 2.97 & 3.38 & 4.47 & 10.0 & 25.4 & 51.9 & 68.1 & 114. \\
\hline 95 & 1.65 & 2.08 & 2.78 & 3.76 & 8.50 & 20.4 & 40.3 & 44.3 & 71.8 \\
\hline 98 & 0.91 & 1.71 & 2.30 & 3.09 & 7.00 & 17.2 & 31.7 & 37.3 & 43.8 \\
\hline 99 & 0.83 & 1.71 & 2.20 & 3.06 & 7.00 & 17.0 & 29.8 & 37.3 & 42.6 \\
\hline
\end{tabular}




\section{Appendix 5. Annual 1-Day and 7-Day Mean Flow-Duration Results for Sites with Streamflows that are Subject to Regulation}

03086500 Mahoning River at Alliance, Ohio

Years analyzed $=51$

Climatic year range $=1942-92$

[APEE, annual percentage equaled or exceeded; $\mathrm{ft}^{3} / \mathrm{s}$, cubic foot per second]

\begin{tabular}{|c|c|c|c|c|c|c|c|c|c|}
\hline \multirow[b]{2}{*}{ APEE } & \multicolumn{9}{|c|}{ Daily mean streamflow (ft $\left.{ }^{3} / \mathrm{s}\right)$ that was equaled or exceeded the indicated percentage of time at APEE level } \\
\hline & 98 & 95 & 90 & 80 & 50 & 20 & 10 & 5 & 2 \\
\hline 1 & 190. & 434. & 511. & 676. & 937. & 1210. & 1360. & 1820. & 2880 \\
\hline 2 & 165. & 299. & 365. & 475. & 666. & 867. & 925. & 1010 & 1130. \\
\hline 5 & 96.2 & 148. & 186. & 231. & 365. & 493. & 597. & 620. & 725. \\
\hline 10 & 67.0 & 86.6 & 110. & 132. & 204. & 307. & 365. & 395. & 423. \\
\hline 20 & 29.1 & 35.0 & 52.4 & 69.4 & 101. & 154. & 170. & 203. & 220. \\
\hline 25 & 24.0 & 24.0 & 39.6 & 54.0 & 80.0 & 126. & 144. & 163. & 170. \\
\hline 30 & 18.0 & 20.6 & 30.4 & 40.2 & 64.9 & 104. & 121. & 135. & 151. \\
\hline 40 & 9.77 & 11.7 & 14.4 & 26.0 & 46.2 & 71.2 & 88.6 & 98.0 & 113. \\
\hline 50 & 3.74 & 4.80 & 9.36 & 15.4 & 33.0 & 54.2 & 65.0 & 75.4 & 84.6 \\
\hline 60 & 0.63 & 2.24 & 5.70 & 10.0 & 23.0 & 40.4 & 47.6 & 57.2 & 61.9 \\
\hline 70 & 0.00 & 0.50 & 2.94 & 6.56 & 17.0 & 28.1 & 35.6 & 44.6 & 48.0 \\
\hline 75 & 0.00 & 0.06 & 2.08 & 4.46 & 13.0 & 24.5 & 30.8 & 39.6 & 45.8 \\
\hline 80 & 0.00 & 0.00 & 0.26 & 2.85 & 10.0 & 20.1 & 25.8 & 34.2 & 39.8 \\
\hline 90 & 0.00 & 0.00 & 0.00 & 0.00 & 6.87 & 13.6 & 17.2 & 22.4 & 25.0 \\
\hline 95 & 0.00 & 0.00 & 0.00 & 0.00 & 4.10 & 10.7 & 14.6 & 17.0 & 22.8 \\
\hline 98 & 0.00 & 0.00 & 0.00 & 0.00 & 3.70 & 8.96 & 12.3 & 14.5 & 18.8 \\
\hline 99 & 0.00 & 0.00 & 0.00 & 0.00 & 3.30 & 8.16 & 11.7 & 12.4 & 16.5 \\
\hline
\end{tabular}

\begin{tabular}{|c|c|c|c|c|c|c|c|c|c|}
\hline \multirow[b]{2}{*}{ APEE } & \multicolumn{9}{|c|}{ 7-day mean streamflow ( $\left.\mathrm{ft}^{3} / \mathrm{s}\right)$ that was equaled or exceeded the indicated percentage of time at APEE level } \\
\hline & 98 & 95 & 90 & 80 & 50 & 20 & 10 & 5 & 2 \\
\hline 1 & 145. & 275. & 379. & 431. & 618. & 751. & 849. & 1130. & 1290. \\
\hline 2 & 126. & 240 & 261. & 363. & 486. & 594. & 669. & 773. & 1130. \\
\hline 5 & 88.5 & 141. & 189. & 231. & 332. & 454. & 477. & 562. & 604. \\
\hline 10 & 57.8 & 96.9 & 119. & 142. & 247. & 312. & 384. & 400 & 447. \\
\hline 20 & 29.4 & 40.5 & 57.2 & 78.3 & 129. & 197. & 244. & 272. & 279 \\
\hline 25 & 23.0 & 29.1 & 42.7 & 63.6 & 94.5 & 166. & 182. & 213. & 220 \\
\hline 30 & 19.2 & 20.2 & 36.8 & 50.5 & 74.9 & 133. & 160. & 170 . & 186. \\
\hline 40 & 9.33 & 11.4 & 16.6 & 25.6 & 50.8 & 91.8 & 114. & 135. & 141. \\
\hline 50 & 4.90 & 5.43 & 11.6 & 17.4 & 38.0 & 68.5 & 85.9 & 98.7 & 111. \\
\hline 60 & 1.22 & 3.70 & 6.42 & 11.9 & 27.0 & 50.3 & 57.4 & 70.7 & 81.2 \\
\hline 70 & 0.61 & 1.66 & 4.32 & 7.59 & 17.1 & 34.2 & 43.3 & 55.3 & 63.5 \\
\hline 75 & 0.02 & 0.70 & 3.24 & 5.87 & 14.3 & 27.9 & 38.3 & 49.3 & 55.3 \\
\hline 80 & 0.00 & 0.24 & 1.82 & 4.06 & 11.9 & 23.4 & 32.7 & 43.8 & 48.9 \\
\hline 90 & 0.00 & 0.00 & 0.00 & 0.57 & 7.14 & 16.6 & 22.1 & 28.7 & 34.1 \\
\hline 95 & 0.00 & 0.00 & 0.00 & 0.22 & 5.51 & 11.9 & 16.2 & 20.2 & 25.2 \\
\hline 98 & 0.00 & 0.00 & 0.00 & 0.00 & 4.27 & 9.99 & 13.8 & 17.8 & 20.9 \\
\hline 99 & 0.00 & 0.00 & 0.00 & 0.00 & 4.07 & 9.13 & 12.9 & 16.2 & 20.6 \\
\hline
\end{tabular}




\section{Eagle Creek at Phalanx Station, Ohio}

Years analyzed $=78$

Climatic year range $=1927-2008$

[APEE, annual percentage equaled or exceeded; $\mathrm{ft}^{3} / \mathrm{s}$, cubic foot per second]

\begin{tabular}{|c|c|c|c|c|c|c|c|c|c|}
\hline \multirow[b]{2}{*}{ APEE } & \multicolumn{9}{|c|}{ Daily mean streamflow $\left(\mathrm{ft}^{3} / \mathrm{s}\right)$ that was equaled or exceeded the indicated percentage of time at APEE level } \\
\hline & 98 & 95 & 90 & 80 & 50 & 20 & 10 & 5 & 2 \\
\hline 1 & 440. & 559. & 735 . & 856. & 1150. & 1480. & 1590. & 1770 & 2100 \\
\hline 2 & 315. & 412. & 530. & 625. & 760. & 1060. & 1180. & 1260 & 1410 . \\
\hline 5 & 166. & 219. & 257. & 343. & 442. & 590. & 687. & 731. & 868. \\
\hline 10 & 89.2 & 113. & 138. & 175. & 255. & 344. & 401. & 481. & 547. \\
\hline 20 & 47.4 & 55.0 & 65.4 & 93.2 & 132. & 186. & 214. & 243. & 277. \\
\hline 25 & 35.0 & 43.4 & 52.9 & 75.5 & 103. & 147. & 178. & 195. & 230 \\
\hline 30 & 28.9 & 36.8 & 42.9 & 57.9 & 84.4 & 120. & 151. & 160. & 185. \\
\hline 40 & 21.7 & 26.0 & 30.5 & 39.0 & 62.6 & 88.3 & 110. & 118. & 145. \\
\hline 50 & 16.3 & 19.9 & 22.9 & 27.8 & 48.0 & 66.0 & 84.0 & 91.6 & 118. \\
\hline 60 & 13.3 & 16.9 & 18.9 & 22.8 & 35.2 & 52.5 & 62.1 & 72.5 & 94.4 \\
\hline 70 & 10.7 & 13.0 & 15.6 & 19.4 & 27.0 & 37.2 & 46.2 & 55.3 & 71.6 \\
\hline 75 & 9.72 & 12.0 & 13.9 & 16.9 & 23.0 & 33.2 & 38.3 & 49.0 & 65.1 \\
\hline 80 & 8.30 & 9.99 & 12.8 & 15.0 & 20.0 & 27.0 & 35.1 & 43.0 & 56.1 \\
\hline 90 & 6.63 & 7.85 & 9.29 & 11.0 & 15.0 & 20.7 & 24.1 & 28.9 & 34.7 \\
\hline 95 & 5.19 & 6.69 & 7.12 & 9.36 & 12.0 & 18.1 & 21.1 & 25.1 & 28.1 \\
\hline 98 & 3.58 & 5.19 & 6.32 & 8.16 & 11.0 & 16.1 & 19.4 & 23.0 & 25.4 \\
\hline 99 & 2.64 & 3.90 & 5.76 & 7.05 & 9.90 & 15.2 & 18.0 & 20.8 & 24.0 \\
\hline
\end{tabular}

\begin{tabular}{|c|c|c|c|c|c|c|c|c|c|}
\hline \multirow[b]{2}{*}{ APEE } & \multicolumn{9}{|c|}{ 7-day mean streamflow ( $\mathrm{ft}^{3} / \mathrm{s}$ ) that was equaled or exceeded the indicated percentage of time at APEE level } \\
\hline & 98 & 95 & 90 & 80 & 50 & 20 & 10 & 5 & 2 \\
\hline 1 & 260. & 364. & 444. & 516. & 725. & 927. & 1050. & 1220 & 1480 \\
\hline 2 & 200 & 301. & 371. & 435. & 606. & 692. & 765. & 920. & 1080 \\
\hline 5 & 154. & 206. & 256. & 317. & 413. & 517. & 564. & 614. & 756. \\
\hline 10 & 93.6 & 127. & 138. & 223. & 284. & 387. & 431. & 472. & 482. \\
\hline 20 & 55.4 & 61.5 & 85.9 & 118. & 168. & 248. & 276. & 315. & 355. \\
\hline 25 & 37.9 & 51.0 & 64.9 & 91.0 & 133. & 197. & 215. & 254. & 291. \\
\hline 30 & 31.6 & 40.0 & 53.3 & 66.8 & 105. & 162. & 185. & 239. & 253. \\
\hline 40 & 23.1 & 27.8 & 31.4 & 42.6 & 72.8 & 99.3 & 134. & 158. & 192. \\
\hline 50 & 16.9 & 19.9 & 24.4 & 31.8 & 54.5 & 75.8 & 102. & 132. & 146. \\
\hline 60 & 13.7 & 16.8 & 19.7 & 23.7 & 39.6 & 56.5 & 78.3 & 89.4 & 119. \\
\hline 70 & 10.6 & 13.2 & 16.1 & 19.4 & 29.0 & 43.1 & 53.0 & 66.4 & 96.1 \\
\hline 75 & 9.55 & 12.0 & 14.4 & 17.3 & 25.1 & 37.5 & 43.5 & 59.1 & 83.5 \\
\hline 80 & 8.69 & 10.8 & 12.7 & 15.6 & 21.1 & 31.5 & 37.9 & 48.6 & 72.6 \\
\hline 90 & 7.29 & 8.29 & 9.95 & 12.1 & 15.5 & 23.2 & 28.7 & 35.5 & 48.8 \\
\hline 95 & 6.55 & 7.16 & 8.05 & 10.4 & 13.5 & 19.1 & 23.1 & 28.7 & 32.9 \\
\hline 98 & 5.39 & 6.47 & 7.24 & 9.41 & 12.2 & 17.3 & 21.2 & 25.2 & 27.3 \\
\hline 99 & 5.02 & 6.14 & 6.89 & 8.76 & 11.5 & 16.9 & 20.2 & 23.3 & 26.3 \\
\hline
\end{tabular}


03097500 Meander Creek at Mineral Ridge, Ohio

Years analyzed $=21$

Climatic year range $=1930-50$

[APEE, annual percentage equaled or exceeded; $\mathrm{ft}^{3} / \mathrm{s}$, cubic foot per second]

\begin{tabular}{|c|c|c|c|c|c|c|c|c|c|}
\hline \multirow[b]{2}{*}{ APEE } & \multicolumn{9}{|c|}{ Daily mean streamflow $\left(\mathrm{ft}^{3} / \mathrm{s}\right)$ that was equaled or exceeded the indicated percentage of time at APEE level } \\
\hline & 95 & 90 & 80 & 70 & 50 & 30 & 20 & 10 & 5 \\
\hline 1 & 121. & 289. & 379. & 476. & 641. & 840. & 912. & 1050. & 1130. \\
\hline 2 & 87.5 & 178. & 278. & 329. & 445. & 565. & 747. & 787. & 791. \\
\hline 5 & 50.1 & 82.4 & 106. & 121. & 211. & 266. & 284 & 417. & 464. \\
\hline 10 & 7.36 & 17.3 & 41.1 & 56.2 & 88.0 & 147. & 162. & 210 & 235. \\
\hline 20 & 2.05 & 3.44 & 4.29 & 6.64 & 26.0 & 53.6 & 66.2 & 78.4 & 87.2 \\
\hline 25 & 1.48 & 1.52 & 2.19 & 3.31 & 8.75 & 27.6 & 39.2 & 55.3 & 59.6 \\
\hline 30 & 1.16 & 1.20 & 1.58 & 2.11 & 5.40 & 13.1 & 21.8 & 38.6 & 44.8 \\
\hline 40 & 0.81 & 0.92 & 1.14 & 1.56 & 2.50 & 5.24 & 6.54 & 15.0 & 22.3 \\
\hline 50 & 0.62 & 0.80 & 0.94 & 1.28 & 1.60 & 2.04 & 3.10 & 5.20 & 8.73 \\
\hline 60 & 0.52 & 0.70 & 0.80 & 0.90 & 1.20 & 1.64 & 1.80 & 3.66 & 4.91 \\
\hline 70 & 0.51 & 0.62 & 0.70 & 0.70 & 0.80 & 1.44 & 1.66 & 3.32 & 4.15 \\
\hline 75 & 0.41 & 0.52 & 0.70 & 0.70 & 0.80 & 1.40 & 1.60 & 3.04 & 3.67 \\
\hline 80 & 0.41 & 0.50 & 0.60 & 0.61 & 0.70 & 1.30 & 1.46 & 2.72 & 3.09 \\
\hline 90 & 0.40 & 0.40 & 0.50 & 0.50 & 0.60 & 1.08 & 1.26 & 1.84 & 2.08 \\
\hline 95 & 0.30 & 0.32 & 0.40 & 0.40 & 0.60 & 0.98 & 1.12 & 1.60 & 1.78 \\
\hline 98 & 0.21 & 0.30 & 0.31 & 0.33 & 0.50 & 0.82 & 1.06 & 1.45 & 1.50 \\
\hline 99 & 0.03 & 0.12 & 0.30 & 0.30 & 0.47 & 0.71 & 1.00 & 1.39 & 1.50 \\
\hline
\end{tabular}

\begin{tabular}{|c|c|c|c|c|c|c|c|c|c|}
\hline \multirow[b]{2}{*}{ APEE } & \multicolumn{9}{|c|}{ 7-day mean streamflow ( $\mathrm{ft}^{3} / \mathrm{s}$ ) that was equaled or exceeded the indicated percentage of time at APEE level } \\
\hline & 95 & 90 & 80 & 70 & 50 & 30 & 20 & 10 & 5 \\
\hline 1 & 110. & 257. & 321. & 374. & 537. & 629. & 723. & 1100 & 1160. \\
\hline 2 & 85.4 & 189. & 232 & 310. & 368. & 436. & 501. & 584. & 819. \\
\hline 5 & 53.5 & 102. & 141. & 161. & 244. & 311. & 366. & 415. & 439. \\
\hline 10 & 15.3 & 23.4 & 61.5 & 73.9 & 122. & 185. & 196. & 266. & 288. \\
\hline 20 & 1.86 & 3.07 & 5.99 & 8.20 & 32.1 & 70.1 & 94.3 & 121. & 123. \\
\hline 25 & 1.28 & 1.58 & 2.49 & 3.97 & 14.3 & 47.4 & 62.8 & 76.9 & 92.5 \\
\hline 30 & 1.04 & 1.09 & 1.61 & 2.07 & 7.63 & 26.8 & 33.7 & 55.3 & 65.0 \\
\hline 40 & 0.79 & 0.91 & 1.13 & 1.84 & 3.03 & 7.03 & 10.7 & 28.9 & 33.0 \\
\hline 50 & 0.69 & 0.83 & 0.96 & 1.39 & 1.80 & 2.70 & 4.44 & 5.55 & 15.6 \\
\hline 60 & 0.58 & 0.75 & 0.86 & 0.95 & 1.20 & 1.76 & 2.01 & 4.16 & 5.05 \\
\hline 70 & 0.53 & 0.67 & 0.73 & 0.79 & 0.89 & 1.50 & 1.69 & 3.50 & 4.27 \\
\hline 75 & 0.50 & 0.61 & 0.69 & 0.75 & 0.80 & 1.42 & 1.62 & 3.19 & 3.90 \\
\hline 80 & 0.48 & 0.55 & 0.65 & 0.71 & 0.76 & 1.39 & 1.55 & 2.85 & 3.44 \\
\hline 90 & 0.43 & 0.46 & 0.54 & 0.60 & 0.67 & 1.17 & 1.36 & 1.83 & 2.34 \\
\hline 95 & 0.37 & 0.40 & 0.48 & 0.51 & 0.64 & 1.05 & 1.26 & 1.65 & 1.93 \\
\hline 98 & 0.19 & 0.35 & 0.42 & 0.44 & 0.60 & 0.96 & 1.19 & 1.52 & 1.56 \\
\hline 99 & 0.15 & 0.21 & 0.39 & 0.41 & 0.59 & 0.93 & 1.18 & 1.46 & 1.55 \\
\hline
\end{tabular}




\section{Mahoning River at Ohio Edison P Plt at Niles, Ohio}

Years analyzed $=21$

Climatic year range $=1988-2008$

[APEE, annual percentage equaled or exceeded; $\mathrm{ft}^{3} / \mathrm{s}$, cubic foot per second]

\begin{tabular}{|c|c|c|c|c|c|c|c|c|c|}
\hline \multirow[b]{2}{*}{ APEE } & \multicolumn{9}{|c|}{ Daily mean streamflow ( $\left.\mathrm{ft}^{3} / \mathrm{s}\right)$ that was equaled or exceeded the indicated percentage of time at APEE level } \\
\hline & 95 & 90 & 80 & 70 & 50 & 30 & 20 & 10 & 5 \\
\hline 1 & 1550. & 2200. & 2780. & 3970. & 5320. & 5650. & 5780. & 7250 & 7730. \\
\hline 2 & 1240 & 1780 . & 2400 . & 3170. & 3630. & 4580 . & 4820 & 5870 & 6740. \\
\hline 5 & 805. & 1150 & 1750. & 2420 & 2700 & 3560 . & 3770 . & 4500 & 4960. \\
\hline 10 & 631. & 795. & 1150. & 1760. & 2060 & 2970. & 3070 . & 3370 & 3700. \\
\hline 20 & 481. & 523. & 691. & 1040. & 1220 . & 2190. & 2270 & 2410 & 2680. \\
\hline 25 & 456. & 468. & 557. & 767. & 982. & 1730. & 2060 & 2180 & 2380 . \\
\hline 30 & 440. & 442. & 484. & 604. & 785. & 1280. & 1850 & 1970. & 2270 . \\
\hline 40 & 397. & 401. & 431. & 507. & 568. & 903. & 1460. & 1650 & 1790. \\
\hline 50 & 356. & 369. & 393. & 419. & 486. & 724. & 1120 & 1220 & 1410. \\
\hline 60 & 317. & 342. & 362. & 379. & 447. & 554. & 831. & 976. & 1160. \\
\hline 70 & 262. & 311. & 336. & 355. & 390. & 445. & 624. & 751. & 876. \\
\hline 75 & 249. & 298. & 325. & 333. & 379. & 428. & 558. & 632. & 710. \\
\hline 80 & 238 & 275. & 302. & 310. & 351. & 418. & 510. & 558. & 598. \\
\hline 90 & 215 & 246. & 257. & 273. & 314. & 351. & 392. & 420 & 455. \\
\hline 95 & 204. & 225. & 231. & 236. & 276. & 309. & 333. & 368. & 404. \\
\hline 98 & 194. & 201. & 209. & 226. & 257. & 282. & 299. & 341. & 353. \\
\hline 99 & 189. & 191. & 204. & 217. & 249. & 271. & 289. & 317. & 332. \\
\hline
\end{tabular}

\begin{tabular}{|c|c|c|c|c|c|c|c|c|c|}
\hline APEE & \multicolumn{9}{|c|}{ 7-day mean streamflow ( $\left.\mathrm{ft}^{3} / \mathrm{s}\right)$ that was equaled or exceeded the indicated percentage of time at APEE level } \\
\hline 1 & 1120. & 1350. & 2030. & 2940. & 3480. & 4190. & 4990. & 5570. & 5710. \\
\hline 5 & 744. & 1060. & 1540. & 2200. & 2550. & 3310. & 3330. & 4300 . & 4730 . \\
\hline 10 & 590. & 826. & 1140. & 1680 & 2070 & 2870 . & 2900 & 3270 . & 3820 . \\
\hline 30 & 441. & 465. & 522. & 660. & 847. & 1350. & 1900. & 2040 & 2190. \\
\hline 40 & 406. & 429. & 461. & 531. & 626. & 951. & 1550. & 1770 . & 1880. \\
\hline 50 & 377. & 396. & 410. & 461. & 511. & 739. & 1250 & 1420 . & 1490. \\
\hline 60 & 331. & 354. & 383. & 404. & 477. & 602. & 974. & 1100 & 1250. \\
\hline 90 & 222. & 252. & 271. & 284 & 323. & 384. & 415. & 459. & 516. \\
\hline 95 & 211. & 236. & 239. & 252. & 291. & 338. & 376. & 410. & 443. \\
\hline 98 & 204. & 216. & 222. & 235 & 273. & 302. & 339. & 378. & 392. \\
\hline 99 & 200. & 205. & 216. & 224 & 259. & 292. & 313. & 342. & 358. \\
\hline
\end{tabular}


03116000 Tuscarawas River at Clinton, Ohio

Years analyzed $=38$

Climatic year range $=1940-77$

[APEE, annual percentage equaled or exceeded; $\mathrm{ft}^{3} / \mathrm{s}$, cubic foot per second]

\begin{tabular}{|c|c|c|c|c|c|c|c|c|c|}
\hline \multirow[b]{2}{*}{ APEE } & \multicolumn{9}{|c|}{ Daily mean streamflow $\left(\mathrm{ft}^{3} / \mathrm{s}\right)$ that was equaled or exceeded the indicated percentage of time at APEE level } \\
\hline & 95 & 90 & 80 & 70 & 50 & 30 & 20 & 10 & 5 \\
\hline 1 & 633. & 694. & 764. & 850. & 983. & 1210 & 1360. & 1510. & 1610 \\
\hline 2 & 367. & 558. & 623. & 691. & 817. & 943. & 1070. & 1270 & 1380. \\
\hline 5 & 209. & 257. & 403. & 452. & 546. & 614. & 657. & 838. & 892. \\
\hline 10 & 145. & 161. & 219. & 254. & 316. & 398. & 450 . & 511. & 559. \\
\hline 20 & 93.4 & 108. & 121. & 143. & 169. & 218. & 270 & 289. & 377. \\
\hline 25 & 87.6 & 91.9 & 105. & 115. & 141. & 187. & 221. & 238. & 318. \\
\hline 30 & 70.7 & 82.2 & 95.0 & 99.9 & 119. & 155. & 179. & 198. & 261. \\
\hline 40 & 59.5 & 68.7 & 74.3 & 82.6 & 96.0 & 120. & 140. & 155. & 188. \\
\hline 50 & 53.4 & 58.9 & 66.8 & 71.0 & 84.5 & 98.6 & 113. & 136. & 154. \\
\hline 60 & 46.6 & 53.8 & 58.9 & 63.7 & 74.0 & 85.6 & 95.2 & 120. & 132. \\
\hline 70 & 42.7 & 48.9 & 52.9 & 59.1 & 66.0 & 77.9 & 80.6 & 96.6 & 111. \\
\hline 75 & 41.6 & 46.5 & 49.8 & 55.5 & 62.4 & 74.7 & 77.6 & 89.6 & 103. \\
\hline 80 & 40.1 & 45.0 & 48.8 & 52.7 & 59.0 & 70.6 & 74.4 & 84.0 & 92.2 \\
\hline 90 & 36.7 & 40.7 & 42.0 & 46.7 & 54.6 & 65.2 & 68.6 & 74.7 & 77.4 \\
\hline 95 & 33.7 & 36.9 & 40.0 & 42.4 & 51.0 & 62.0 & 64.7 & 70.1 & 74.1 \\
\hline 98 & 30.8 & 34.0 & 38.0 & 39.0 & 48.3 & 58.7 & 63.9 & 68.0 & 71.0 \\
\hline 99 & 28.8 & 33.0 & 36.5 & 37.8 & 47.8 & 57.2 & 61.1 & 66.8 & 70.0 \\
\hline
\end{tabular}

\begin{tabular}{|c|c|c|c|c|c|c|c|c|c|}
\hline \multirow[b]{2}{*}{ APEE } & \multicolumn{9}{|c|}{ 7-day mean streamflow ( $\mathrm{ft}^{3} / \mathrm{s}$ ) that was equaled or exceeded the indicated percentage of time at APEE level } \\
\hline & 95 & 90 & 80 & 70 & 50 & 30 & 20 & 10 & 5 \\
\hline 1 & 436. & 542. & 588. & 630. & 807. & 962. & 1010 & 1120 & 1160 \\
\hline 2 & 373. & 432 & 487. & 527. & 636. & 741. & 829. & 989. & 1060 \\
\hline 5 & 195. & 234. & 361. & 396. & 484. & 573. & 626. & 739. & 821. \\
\hline 10 & 133. & 149. & 233. & 274. & 337. & 378. & 420 & 497. & 564. \\
\hline 20 & 93.2 & 113. & 134. & 159. & 204. & 236 & 275 & 330 . & 369. \\
\hline 25 & 88.4 & 101. & 106. & 122 & 164. & 205 & 242. & 270 & 331. \\
\hline 30 & 73.8 & 85.9 & 98.1 & 108. & 138. & 179. & 199. & 221. & 286. \\
\hline 40 & 59.3 & 68.4 & 76.4 & 85.8 & 103. & 133. & 153. & 173. & 210 \\
\hline 50 & 54.3 & 61.6 & 68.6 & 72.9 & 89.6 & 104 & 120 & 145 & 181. \\
\hline 60 & 46.2 & 54.8 & 59.5 & 66.3 & 77.1 & 89.7 & 102. & 133. & 145. \\
\hline 70 & 43.0 & 49.3 & 55.3 & 59.8 & 67.7 & 82.1 & 89.1 & 107. & 123. \\
\hline 75 & 42.1 & 47.8 & 52.6 & 55.8 & 64.8 & 77.6 & 83.6 & 97.5 & 106. \\
\hline 80 & 41.0 & 45.7 & 50.0 & 53.8 & 60.6 & 73.3 & 80.4 & 88.4 & 94.6 \\
\hline 90 & 37.3 & 41.6 & 44.3 & 48.5 & 56.2 & 67.2 & 70.9 & 77.2 & 80.4 \\
\hline 95 & 35.7 & 39.3 & 41.5 & 45.7 & 52.5 & 63.4 & 67.3 & 72.5 & 75.4 \\
\hline 98 & 32.8 & 36.1 & 39.5 & 42.4 & 51.3 & 61.0 & 64.9 & 71.0 & 72.3 \\
\hline 99 & 31.8 & 35.3 & 38.9 & 41.2 & 50.4 & 60.2 & 64.4 & 69.4 & 71.0 \\
\hline
\end{tabular}




\section{Chippewa Creek at Easton, Ohio}

Years analyzed $=20$

Climatic year range $=1961-80$

[APEE, annual percentage equaled or exceeded; $\mathrm{ft}^{3} / \mathrm{s}$, cubic foot per second]

\begin{tabular}{|c|c|c|c|c|c|c|c|c|c|}
\hline \multirow[b]{2}{*}{ APEE } & \multicolumn{9}{|c|}{ Daily mean streamflow $\left(\mathrm{ft}^{3} / \mathrm{s}\right)$ that was equaled or exceeded the indicated percentage of time at APEE level } \\
\hline & 95 & 90 & 80 & 70 & 50 & 30 & 20 & 10 & 5 \\
\hline 1 & 805. & 899. & 1060. & 1110. & 1270. & 1490. & 1690. & 2320 & 2530 \\
\hline 2 & 547. & 633. & 780 . & 886. & 984. & 1070 . & 1160 & 1210 & 1390. \\
\hline 5 & 212 & 249. & 386. & 510. & 580. & 671. & 728. & 783. & 871. \\
\hline 10 & 119. & 127. & 166. & 252. & 336. & 415. & 486. & 542. & 634. \\
\hline 20 & 43.2 & 49.3 & 92.4 & 121. & 151. & 194. & 247. & 342. & 364. \\
\hline 25 & 27.7 & 38.6 & 71.8 & 86.3 & 120 . & 149. & 200. & 276. & 286. \\
\hline 30 & 20.3 & 31.0 & 56.9 & 67.3 & 95.6 & 125. & 159. & 228. & 242 \\
\hline 40 & 13.5 & 24.3 & 39.9 & 42.9 & 69.3 & 96.3 & 108. & 162. & 167. \\
\hline 50 & 11.4 & 20.7 & 27.0 & 27.6 & 52.5 & 77.5 & 85.0 & 110. & 122. \\
\hline 60 & 10.0 & 14.4 & 19.4 & 22.0 & 39.5 & 54.4 & 61.9 & 64.9 & 98.6 \\
\hline 70 & 9.05 & 10.3 & 13.2 & 17.0 & 27.9 & 34.1 & 43.8 & 58.5 & 79.8 \\
\hline 75 & 8.64 & 9.66 & 12.2 & 14.0 & 24.0 & 26.9 & 38.4 & 49.0 & 68.5 \\
\hline 80 & 8.22 & 8.65 & 11.2 & 12.3 & 19.5 & 24.2 & 33.2 & 42.4 & 51.5 \\
\hline 90 & 6.53 & 7.16 & 8.86 & 9.32 & 14.5 & 18.9 & 22.9 & 30.0 & 32.9 \\
\hline 95 & 6.20 & 6.22 & 8.04 & 8.67 & 12.5 & 15.7 & 19.6 & 26.4 & 28.9 \\
\hline 98 & 4.80 & 4.99 & 6.86 & 7.99 & 10.9 & 13.7 & 16.7 & 25.5 & 27.0 \\
\hline 99 & 3.93 & 4.60 & 6.29 & 7.69 & 10.3 & 13.2 & 15.6 & 25.2 & 26.0 \\
\hline
\end{tabular}

\begin{tabular}{|c|c|c|c|c|c|c|c|c|c|}
\hline \multirow[b]{2}{*}{ APEE } & \multicolumn{9}{|c|}{ 7-day mean streamflow ( $\mathrm{ft}^{3} / \mathrm{s}$ ) that was equaled or exceeded the indicated percentage of time at APEE level } \\
\hline & 95 & 90 & 80 & 70 & 50 & 30 & 20 & 10 & 5 \\
\hline 1 & 562. & 696. & 771. & 819. & 991. & 1240. & 1360. & 1470 & 2400 \\
\hline 2 & 486. & 524. & 623. & 675. & 838. & 907. & 941. & 1200 . & 1440. \\
\hline 5 & 208. & 224. & 377. & 447. & 551. & 623. & 654. & 740. & 747. \\
\hline 10 & 110. & 131. & 185 & 285 & 347. & 399. & 424. & 502. & 580. \\
\hline 20 & 41.1 & 46.9 & 96.2 & 135. & 185. & 234. & 279. & 364. & 430. \\
\hline 25 & 26.3 & 39.9 & 73.1 & 101. & 140 . & 174. & 226. & 317. & 351. \\
\hline 30 & 20.3 & 33.5 & 61.3 & 72.8 & 112. & 151. & 168. & 272. & 287. \\
\hline 40 & 14.9 & 25.5 & 41.6 & 46.8 & 77.9 & 112. & 121. & 178. & 197. \\
\hline 50 & 11.5 & 21.1 & 28.2 & 29.4 & 57.0 & 82.6 & 96.2 & 129. & 153. \\
\hline 60 & 9.99 & 15.8 & 21.6 & 22.8 & 43.5 & 61.2 & 75.8 & 84.2 & 114. \\
\hline 70 & 9.33 & 11.5 & 14.4 & 17.6 & 32.4 & 40.2 & 49.7 & 61.2 & 89.6 \\
\hline 75 & 8.94 & 10.2 & 13.1 & 15.3 & 27.1 & 30.0 & 45.5 & 58.7 & 76.6 \\
\hline 80 & 8.23 & 9.34 & 12.5 & 13.7 & 22.9 & 27.2 & 37.7 & 51.5 & 63.0 \\
\hline 90 & 7.47 & 8.06 & 10.1 & 10.7 & 15.5 & 20.5 & 25.0 & 32.3 & 38.5 \\
\hline 95 & 6.16 & 6.58 & 8.32 & 9.12 & 13.9 & 17.0 & 21.6 & 29.0 & 33.4 \\
\hline 98 & 5.76 & 6.04 & 7.25 & 8.70 & 11.9 & 15.0 & 18.3 & 27.9 & 31.4 \\
\hline 99 & 4.92 & 5.89 & 7.08 & 8.23 & 10.8 & 13.9 & 17.5 & 27.2 & 29.8 \\
\hline
\end{tabular}


03117000 Tuscarawas River at Massillon, Ohio

Years analyzed $=71$

Climatic year range $=1938-2008$

[APEE, annual percentage equaled or exceeded; $\mathrm{ft}^{3} / \mathrm{s}$, cubic foot per second]

\begin{tabular}{|c|c|c|c|c|c|c|c|c|c|}
\hline \multirow[b]{2}{*}{ APEE } & \multicolumn{9}{|c|}{ Daily mean streamflow $\left(\mathrm{ft}^{3} / \mathrm{s}\right)$ that was equaled or exceeded the indicated percentage of time at APEE level } \\
\hline & 98 & 95 & 90 & 80 & 50 & 20 & 10 & 5 & 2 \\
\hline 1 & 1390. & 1840. & 1930. & 2470. & 3380. & 3980. & 4910. & 5610. & 6540. \\
\hline 2 & 1010 & 1260. & 1640. & 1990. & 2600 & 3250 & 3590. & 4270. & 4770 . \\
\hline 5 & 640. & 700 . & 889. & 1250. & 1710 & 2230 & 2570. & 2930. & 3270 . \\
\hline 10 & 412. & 471. & 541. & 704. & 1020 & 1460. & 1810. & 1910. & 2190. \\
\hline 20 & 207. & 305. & 344. & 406. & 584. & 840. & 1080. & 1160. & 1250. \\
\hline 25 & 172. & 255. & 281. & 331. & 469. & 650. & 856. & 934. & 977. \\
\hline 30 & 151. & 208. & 232. & 279. & 398. & 541. & 712. & 749. & 835. \\
\hline 40 & 109. & 150. & 177. & 203. & 309. & 403. & 529. & 564. & 690. \\
\hline 50 & 97.4 & 125. & 140 . & 160. & 246. & 329. & 404. & 460. & 580. \\
\hline 60 & 90.9 & 113. & 121. & 138. & 194. & 268. & 313. & 367. & 466. \\
\hline 70 & 85.0 & 99.5 & 104. & 122. & 163. & 219. & 267. & 305. & 382. \\
\hline 75 & 82.0 & 94.0 & 96.8 & 111. & 149. & 200. & 240. & 278. & 333. \\
\hline 80 & 79.2 & 83.6 & 92.6 & 104. & 137. & 183. & 215. & 246. & 301. \\
\hline 90 & 71.1 & 73.9 & 79.7 & 91.4 & 114. & 146. & 170. & 200. & 230 . \\
\hline 95 & 63.5 & 66.6 & 73.1 & 82.8 & 102. & 132. & 147. & 173. & 195. \\
\hline 98 & 55.6 & 62.9 & 67.2 & 75.4 & 95.0 & 120. & 134. & 151. & 170. \\
\hline 99 & 52.9 & 59.1 & 64.2 & 72.5 & 88.7 & 114. & 125. & 140. & 164. \\
\hline
\end{tabular}

\begin{tabular}{|c|c|c|c|c|c|c|c|c|c|}
\hline \multirow[b]{2}{*}{ APEE } & \multicolumn{9}{|c|}{ 7-day mean streamflow ( $\mathrm{ft}^{3} / \mathrm{s}$ ) that was equaled or exceeded the indicated percentage of time at APEE level } \\
\hline & 98 & 95 & 90 & 80 & 50 & 20 & 10 & 5 & 2 \\
\hline 1 & 943. & 1250 & 1480 & 1740 & 2490 & 3220 & 3690 & 4230. & 5050. \\
\hline 2 & 890. & 1060 & 1150 & 1550. & 2010 & 2570 & 3050 & 3500 . & 3940. \\
\hline 5 & 584. & 697. & 786. & 1180. & 1470 . & 1870 . & 2180 & 2490. & 2850 . \\
\hline 10 & 402. & 460. & 541. & 757. & 988. & 1410 & 1610 & 1740. & 2020. \\
\hline 20 & 208. & 316. & 348. & 454. & 643. & 907. & 1130. & 1200. & 1290. \\
\hline 25 & 171. & 268. & 297. & 348. & 540. & 788. & 986. & 1080. & 1100. \\
\hline 30 & 157. & 221. & 246. & 298. & 450. & 632. & 814. & 933. & 982. \\
\hline 40 & 113. & 161. & 185. & 214. & 355. & 452. & 593. & 697. & 829. \\
\hline 50 & 98.4 & 128. & 150. & 167. & 271. & 357. & 481. & 559. & 700. \\
\hline 60 & 91.4 & 117. & 127. & 141. & 208. & 294. & 365. & 444. & 559. \\
\hline 70 & 84.8 & 102. & 109. & 130. & 177. & 236. & 296. & 336. & 459. \\
\hline 75 & 83.1 & 96.7 & 101. & 118. & 163. & 215. & 275. & 295. & 415. \\
\hline 80 & 81.5 & 87.7 & 95.3 & 112. & 148 & 196. & 236. & 268. & 360. \\
\hline 90 & 71.9 & 77.0 & 88.6 & 97.1 & 121. & 160. & 180. & 224. & 276. \\
\hline 95 & 64.5 & 71.6 & 76.5 & 88.2 & 110. & 139. & 157. & 191. & 227. \\
\hline 98 & 57.5 & 66.1 & 73.0 & 80.9 & 102. & 132. & 143. & 172. & 197. \\
\hline 99 & 56.2 & 64.0 & 69.2 & 76.9 & 98.7 & 125. & 138. & 145. & 187. \\
\hline
\end{tabular}


03118000 Middle Branch Nimishillen Creek at Canton, Ohio

Years analyzed $=67$

Climatic year range $=1942-2008$

[APEE, annual percentage equaled or exceeded; $\mathrm{ft}^{3} / \mathrm{s}$, cubic foot per second]

\begin{tabular}{|c|c|c|c|c|c|c|c|c|c|}
\hline \multirow[b]{2}{*}{ APEE } & \multicolumn{9}{|c|}{ Daily mean streamflow $\left(\mathrm{ft}^{3} / \mathrm{s}\right)$ that was equaled or exceeded the indicated percentage of time at APEE level } \\
\hline & 98 & 95 & 90 & 80 & 50 & 20 & 10 & 5 & 2 \\
\hline 1 & 90.5 & 146. & 154. & 237. & 319. & 420. & 511. & 589. & 810. \\
\hline 2 & 67.6 & 98.3 & 109. & 157. & 236. & 300 . & 351. & 422. & 482. \\
\hline 5 & 47.1 & 52.5 & 67.6 & 82.3 & 139. & 181. & 210. & 231. & 306. \\
\hline 10 & 30.5 & 36.6 & 42.2 & 51.2 & 80.4 & 122. & 144. & 165. & 193. \\
\hline 20 & 13.4 & 22.8 & 25.8 & 30.0 & 49.6 & 71.3 & 86.3 & 98.6 & 107. \\
\hline 25 & 9.53 & 15.6 & 21.0 & 25.0 & 42.0 & 60.4 & 73.7 & 82.1 & 89.5 \\
\hline 30 & 7.96 & 11.0 & 18.0 & 20.6 & 36.9 & 52.1 & 66.0 & 72.6 & 76.8 \\
\hline 40 & 5.11 & 6.24 & 11.2 & 13.8 & 28.0 & 40.0 & 52.2 & 57.7 & 62.3 \\
\hline 50 & 3.94 & 4.90 & 7.04 & 9.80 & 20.0 & 31.6 & 39.0 & 48.6 & 54.2 \\
\hline 60 & 3.00 & 3.76 & 5.07 & 7.50 & 15.0 & 24.4 & 32.5 & 39.2 & 45.3 \\
\hline 70 & 2.34 & 2.80 & 3.66 & 5.86 & 12.0 & 20.8 & 25.4 & 32.6 & 38.0 \\
\hline 75 & 1.89 & 2.40 & 3.27 & 4.96 & 11.0 & 18.0 & 22.2 & 28.6 & 33.6 \\
\hline 80 & 1.27 & 2.24 & 2.88 & 4.26 & 9.10 & 16.4 & 20.4 & 25.6 & 29.3 \\
\hline 90 & 0.58 & 1.56 & 2.16 & 2.98 & 6.70 & 13.0 & 15.4 & 19.3 & 22.0 \\
\hline 95 & 0.48 & 1.31 & 1.68 & 2.10 & 5.60 & 10.6 & 13.1 & 15.9 & 17.9 \\
\hline 98 & 0.40 & 1.16 & 1.30 & 1.82 & 5.00 & 9.24 & 12.0 & 12.0 & 15.4 \\
\hline 99 & 0.39 & 1.04 & 1.19 & 1.65 & 4.20 & 8.64 & 11.1 & 11.9 & 13.3 \\
\hline
\end{tabular}

\begin{tabular}{|c|c|c|c|c|c|c|c|c|c|}
\hline \multirow[b]{2}{*}{ APEE } & \multicolumn{9}{|c|}{ 7-day mean streamflow ( $\mathrm{ft}^{3} / \mathrm{s}$ ) that was equaled or exceeded the indicated percentage of time at APEE level } \\
\hline & 98 & 95 & 90 & 80 & 50 & 20 & 10 & 5 & 2 \\
\hline 1 & 66.0 & 85.1 & 113. & 134. & 198. & 271. & 306. & 349. & 598. \\
\hline 2 & 61.9 & 76.5 & 85.5 & 118. & 171. & 218 & 251. & 326. & 391. \\
\hline 5 & 44.7 & 51.9 & 59.0 & 91.2 & 126. & 162 & 195. & 230. & 275 \\
\hline 10 & 30.9 & 36.7 & 44.4 & 57.4 & 90.0 & 123. & 145. & 163. & 181. \\
\hline 20 & 12.8 & 24.0 & 27.4 & 34.4 & 56.3 & 84.7 & 102. & 109. & 120. \\
\hline 25 & 9.61 & 17.4 & 23.1 & 27.2 & 47.0 & 69.7 & 86.0 & 96.5 & 104. \\
\hline 30 & 8.43 & 13.8 & 19.9 & 23.8 & 40.9 & 61.7 & 72.0 & 87.2 & 90.4 \\
\hline 40 & 5.31 & 6.51 & 12.6 & 15.5 & 31.3 & 46.0 & 57.7 & 70.1 & 75.0 \\
\hline 50 & 3.83 & 5.02 & 7.10 & 10.8 & 23.6 & 35.8 & 47.9 & 56.6 & 61.9 \\
\hline 60 & 3.14 & 4.03 & 4.99 & 8.17 & 17.4 & 27.5 & 37.4 & 44.8 & 53.7 \\
\hline 70 & 2.41 & 2.83 & 3.71 & 6.23 & 13.6 & 23.2 & 29.1 & 37.0 & 45.4 \\
\hline 75 & 2.03 & 2.54 & 3.38 & 5.44 & 11.2 & 21.0 & 25.9 & 33.2 & 40.3 \\
\hline 80 & 1.56 & 2.26 & 3.09 & 4.49 & 9.65 & 18.4 & 22.7 & 29.6 & 37.4 \\
\hline 90 & 0.79 & 1.68 & 2.21 & 3.22 & 6.87 & 14.0 & 17.9 & 22.3 & 24.6 \\
\hline 95 & 0.53 & 1.43 & 1.87 & 2.59 & 6.14 & 11.3 & 14.2 & 18.5 & 20.7 \\
\hline 98 & 0.42 & 1.31 & 1.50 & 1.95 & 5.64 & 9.94 & 13.0 & 14.1 & 17.1 \\
\hline 99 & 0.41 & 1.19 & 1.37 & 1.85 & 4.86 & 9.13 & 11.8 & 12.8 & 15.5 \\
\hline
\end{tabular}


03118500 Nimishillen Creek at North Industry, Ohio

Years analyzed $=87$

Climatic year range $=1922-2008$

[APEE, annual percentage equaled or exceeded; $\mathrm{ft}^{3} / \mathrm{s}$, cubic foot per second]

\begin{tabular}{|c|c|c|c|c|c|c|c|c|c|}
\hline \multirow[b]{2}{*}{ APEE } & \multicolumn{9}{|c|}{ Daily mean streamflow ( $\left.\mathrm{ft}^{3} / \mathrm{s}\right)$ that was equaled or exceeded the indicated percentage of time at APEE level } \\
\hline & 98 & 95 & 90 & 80 & 50 & 20 & 10 & 5 & 2 \\
\hline 1 & 373. & 698. & 762. & 920. & 1390. & 1780. & 2180. & 2400 . & 3210. \\
\hline 2 & 287. & 490. & 563. & 666. & 983. & 1270 & 1510. & 1810. & 2210. \\
\hline 5 & 226. & 287. & 316. & 374. & 541. & 757. & 870. & 1050. & 1180. \\
\hline 10 & 164. & 202. & 215 & 273. & 351. & 503. & 551. & 661. & 787. \\
\hline 20 & 102. & 119. & 138. & 173. & 228. & 322. & 369. & 420 . & 448. \\
\hline 25 & 89.1 & 97.7 & 115. & 151. & 200. & 284. & 322. & 360. & 383. \\
\hline 30 & 75.8 & 87.4 & 99.7 & 131. & 178. & 252. & 288. & 323. & 346. \\
\hline 40 & 54.8 & 67.4 & 82.0 & 102. & 145. & 210 & 238 & 269. & 294. \\
\hline 50 & 46.6 & 54.0 & 70.7 & 88.4 & 125 & 183. & 198. & 229. & 255. \\
\hline 60 & 40.3 & 47.0 & 62.5 & 71.2 & 107. & 150. & 171. & 200 & 220. \\
\hline 70 & 35.0 & 39.8 & 55.4 & 63.0 & 91.0 & 131. & 149. & 174. & 194. \\
\hline 75 & 32.5 & 36.8 & 50.0 & 59.6 & 86.0 & 121. & 140 . & 160. & 186. \\
\hline 80 & 29.8 & 33.5 & 47.0 & 56.0 & 77.2 & 114. & 130. & 150 & 170. \\
\hline 90 & 24.9 & 30.0 & 40.3 & 49.1 & 70.0 & 103. & 110 . & 127. & 144. \\
\hline 95 & 22.8 & 27.4 & 37.0 & 45.2 & 65.0 & 91.5 & 106. & 116. & 133. \\
\hline 98 & 20.3 & 24.1 & 33.6 & 42.4 & 59.3 & 87.8 & 98.1 & 110. & 120. \\
\hline 99 & 19.7 & 23.3 & 29.8 & 40.0 & 57.3 & 85.0 & 93.7 & 106. & 113. \\
\hline
\end{tabular}

\begin{tabular}{|c|c|c|c|c|c|c|c|c|c|}
\hline \multirow[b]{2}{*}{ APEE } & \multicolumn{9}{|c|}{ 7-day mean streamflow ( $\mathrm{ft}^{3} / \mathrm{s}$ ) that was equaled or exceeded the indicated percentage of time at APEE level } \\
\hline & 98 & 95 & 90 & 80 & 50 & 20 & 10 & 5 & 2 \\
\hline 1 & 292. & 394. & 489. & 618. & 882. & 1140 & 1280 & 1550 & 1700. \\
\hline 2 & 267. & 369. & 424. & 527. & 674. & 910. & 1020. & 1230 & 1530 \\
\hline 5 & 221. & 289. & 303. & 388 & 531. & 681. & 784. & 906. & 1240 \\
\hline 10 & 176. & 201. & 226. & 274. & 384. & 523. & 601. & 690. & 762. \\
\hline 20 & 105. & 120. & 147. & 189. & 260 & 372. & 407. & 477. & 507. \\
\hline 25 & 91.5 & 104. & 123. & 167. & 229 & 316. & 349. & 428. & 470 . \\
\hline 30 & 80.5 & 91.1 & 109. & 147. & 202. & 289. & 319. & 395. & 429. \\
\hline 40 & 56.7 & 71.3 & 89.6 & 113. & 159. & 233. & 265. & 318. & 355. \\
\hline 50 & 48.0 & 57.7 & 74.9 & 90.0 & 138. & 197. & 226 & 266. & 302. \\
\hline 60 & 42.4 & 50.7 & 66.1 & 73.8 & 114. & 166. & 189. & 224. & 262. \\
\hline 70 & 37.1 & 45.0 & 58.1 & 67.0 & 96.1 & 149. & 164. & 193. & 227. \\
\hline 75 & 36.1 & 40.9 & 54.2 & 63.9 & 91.3 & 138. & 155. & 182. & 209. \\
\hline 80 & 31.7 & 37.2 & 49.7 & 59.8 & 85.7 & 128. & 145 & 170 & 193. \\
\hline 90 & 27.4 & 31.3 & 43.8 & 52.8 & 73.4 & 111. & 124. & 143. & 168. \\
\hline 95 & 25.7 & 30.1 & 41.1 & 48.9 & 68.3 & 101. & 116. & 128. & 141. \\
\hline 98 & 22.2 & 26.6 & 36.5 & 45.5 & 64.4 & 91.7 & 106. & 118. & 131. \\
\hline 99 & 21.9 & 25.6 & 35.3 & 44.5 & 62.9 & 90.0 & 99.4 & 110. & 123. \\
\hline
\end{tabular}




\section{South Fork Licking River near Hebron, Ohio}

Years analyzed $=48$

Climatic year range $=1940-2008$

[APEE, annual percentage equaled or exceeded; $\mathrm{ft}^{3} / \mathrm{s}$, cubic foot per second]

\begin{tabular}{|c|c|c|c|c|c|c|c|c|c|}
\hline \multirow[b]{2}{*}{ APEE } & \multicolumn{9}{|c|}{ Daily mean streamflow $\left(\mathrm{ft}^{3} / \mathrm{s}\right)$ that was equaled or exceeded the indicated percentage of time at APEE level } \\
\hline & 95 & 90 & 80 & 70 & 50 & 30 & 20 & 10 & 5 \\
\hline 1 & 848. & 927. & 1040. & 1180. & 1370. & 1680. & 1900. & 2190 & 2410 \\
\hline 2 & 619. & 670. & 799. & 847. & 1070. & 1240 & 1420 & 1690. & 1840. \\
\hline 5 & 321. & 375. & 435. & 530. & 687. & 827. & 889. & 995. & 1130. \\
\hline 10 & 179. & 211. & 271. & 326. & 407. & 536. & 564. & 603. & 675. \\
\hline 20 & 81.6 & 104. & 131. & 159. & 235. & 293. & 348. & 379. & 412. \\
\hline 25 & 61.4 & 76.0 & 92.3 & 126. & 186. & 215. & 282. & 328. & 340. \\
\hline 30 & 45.7 & 59.4 & 69.6 & 99.2 & 145. & 181. & 217. & 266. & 287. \\
\hline 40 & 25.5 & 36.2 & 52.6 & 57.0 & 87.5 & 110. & 134. & 165. & 186. \\
\hline 50 & 14.3 & 23.4 & 34.8 & 38.7 & 54.0 & 67.6 & 87.5 & 115. & 134. \\
\hline 60 & 8.55 & 16.4 & 21.9 & 24.8 & 36.4 & 42.3 & 62.0 & 74.4 & 91.0 \\
\hline 70 & 6.99 & 9.16 & 15.2 & 18.0 & 21.0 & 29.1 & 39.2 & 53.0 & 61.2 \\
\hline 75 & 6.15 & 7.69 & 10.7 & 15.0 & 17.0 & 24.0 & 31.0 & 43.3 & 46.4 \\
\hline 80 & 4.85 & 5.77 & 8.82 & 11.1 & 14.5 & 20.2 & 25.4 & 35.0 & 37.0 \\
\hline 90 & 2.70 & 4.20 & 6.24 & 7.58 & 9.56 & 14.0 & 17.2 & 21.7 & 24.4 \\
\hline 95 & 1.88 & 3.43 & 4.75 & 6.10 & 8.20 & 10.6 & 15.0 & 17.1 & 18.2 \\
\hline 98 & 1.49 & 2.18 & 3.82 & 5.38 & 6.80 & 8.35 & 11.5 & 15.0 & 15.2 \\
\hline 99 & 0.98 & 1.83 & 3.46 & 4.92 & 6.35 & 7.76 & 9.97 & 14.0 & 14.7 \\
\hline
\end{tabular}

\begin{tabular}{|c|c|c|c|c|c|c|c|c|c|}
\hline \multirow[b]{2}{*}{ APEE } & \multicolumn{9}{|c|}{ 7-day mean streamflow ( $\mathrm{ft}^{3} / \mathrm{s}$ ) that was equaled or exceeded the indicated percentage of time at APEE level } \\
\hline & 95 & 90 & 80 & 70 & 50 & 30 & 20 & 10 & 5 \\
\hline 1 & 453. & 547. & 643. & 789. & 936. & 1170 & 1380. & 1490. & 1680 \\
\hline 2 & 402. & 499. & 540 & 614. & 802. & 906. & 1050 & 1230 & 1280 \\
\hline 5 & 329. & 374. & 419. & 475 & 572. & 691. & 778. & 907. & 1000 \\
\hline 10 & 195. & 238 & 300 . & 332. & 430. & 508. & 528. & 548. & 642. \\
\hline 20 & 103. & 129. & 160. & 180. & 261. & 323. & 376. & 397. & 433. \\
\hline 25 & 66.3 & 90.6 & 123. & 147. & 212. & 279 & 310. & 356. & 369. \\
\hline 30 & 51.2 & 66.9 & 92.9 & 118. & 173. & 244. & 268. & 308. & 323. \\
\hline 40 & 28.4 & 44.7 & 60.3 & 79.6 & 119. & 161. & 186. & 203. & 233. \\
\hline 50 & 16.6 & 27.4 & 40.9 & 47.1 & 70.3 & 97.8 & 127. & 148. & 171. \\
\hline 60 & 10.3 & 19.6 & 24.5 & 31.6 & 44.4 & 58.0 & 78.7 & 109. & 122. \\
\hline 70 & 7.75 & 10.8 & 17.8 & 20.8 & 26.6 & 37.5 & 55.5 & 71.7 & 83.3 \\
\hline 75 & 5.91 & 8.18 & 14.4 & 17.2 & 20.6 & 29.9 & 42.6 & 62.3 & 65.9 \\
\hline 80 & 5.31 & 6.86 & 10.2 & 13.0 & 16.1 & 22.4 & 29.3 & 45.5 & 53.5 \\
\hline 90 & 3.46 & 4.53 & 6.45 & 8.33 & 10.6 & 15.9 & 19.0 & 26.1 & 32.2 \\
\hline 95 & 2.51 & 3.98 & 4.95 & 6.77 & 8.49 & 12.3 & 16.6 & 19.7 & 21.9 \\
\hline 98 & 1.84 & 3.01 & 4.24 & 5.97 & 7.66 & 9.95 & 13.9 & 15.9 & 16.5 \\
\hline 99 & 1.64 & 2.75 & 4.06 & 5.68 & 7.24 & 8.88 & 12.5 & 15.0 & 15.3 \\
\hline
\end{tabular}


03156000 Hunters Run at Lancaster, Ohio

Years analyzed $=23$

Climatic year range $=1956-78$

[APEE, annual percentage equaled or exceeded; $\mathrm{ft}^{3} / \mathrm{s}$, cubic foot per second]

\begin{tabular}{|c|c|c|c|c|c|c|c|c|c|}
\hline \multirow[b]{2}{*}{ APEE } & \multicolumn{9}{|c|}{ Daily mean streamflow $\left(\mathrm{ft}^{3} / \mathrm{s}\right)$ that was equaled or exceeded the indicated percentage of time at APEE level } \\
\hline & 95 & 90 & 80 & 70 & 50 & 30 & 20 & 10 & 5 \\
\hline 1 & 30.8 & 46.7 & 62.1 & 76.8 & 104. & 123. & 128. & 172. & 196. \\
\hline 2 & 21.2 & 27.6 & 32.9 & 45.5 & 66.7 & 77.8 & 81.5 & 90.2 & 128. \\
\hline 5 & 11.1 & 14.6 & 19.9 & 26.1 & 34.7 & 37.8 & 44.4 & 47.9 & 52.1 \\
\hline 10 & 7.79 & 9.72 & 13.8 & 16.0 & 21.0 & 23.1 & 24.7 & 28.6 & 29.0 \\
\hline 20 & 4.32 & 6.68 & 8.45 & 8.97 & 12.8 & 14.8 & 15.2 & 17.6 & 19.6 \\
\hline 25 & 3.76 & 5.76 & 6.84 & 7.41 & 8.70 & 12.8 & 13.2 & 14.6 & 17.4 \\
\hline 30 & 3.34 & 5.10 & 5.70 & 6.32 & 7.79 & 10.8 & 12.0 & 13.0 & 15.4 \\
\hline 40 & 2.42 & 3.62 & 4.26 & 4.52 & 6.30 & 8.52 & 10.0 & 11.2 & 12.8 \\
\hline 50 & 1.86 & 2.58 & 3.20 & 3.80 & 4.55 & 6.76 & 7.88 & 9.32 & 10.8 \\
\hline 60 & 1.22 & 1.56 & 2.36 & 2.82 & 3.20 & 4.72 & 6.11 & 8.14 & 9.10 \\
\hline 70 & 1.00 & 1.24 & 1.78 & 2.20 & 2.38 & 3.06 & 4.65 & 6.22 & 6.90 \\
\hline 75 & 0.90 & 1.14 & 1.50 & 1.82 & 2.10 & 2.58 & 4.20 & 5.46 & 6.22 \\
\hline 80 & 0.80 & 0.96 & 1.38 & 1.50 & 1.82 & 2.18 & 3.68 & 4.68 & 5.44 \\
\hline 90 & 0.70 & 0.74 & 1.08 & 1.10 & 1.40 & 1.82 & 2.46 & 3.64 & 4.00 \\
\hline 95 & 0.45 & 0.54 & 0.79 & 1.00 & 1.20 & 1.38 & 2.04 & 2.74 & 3.41 \\
\hline 98 & 0.23 & 0.51 & 0.68 & 0.72 & 1.00 & 1.28 & 1.74 & 2.00 & 3.20 \\
\hline 99 & 0.17 & 0.41 & 0.50 & 0.67 & 1.00 & 1.20 & 1.69 & 1.89 & 3.13 \\
\hline
\end{tabular}

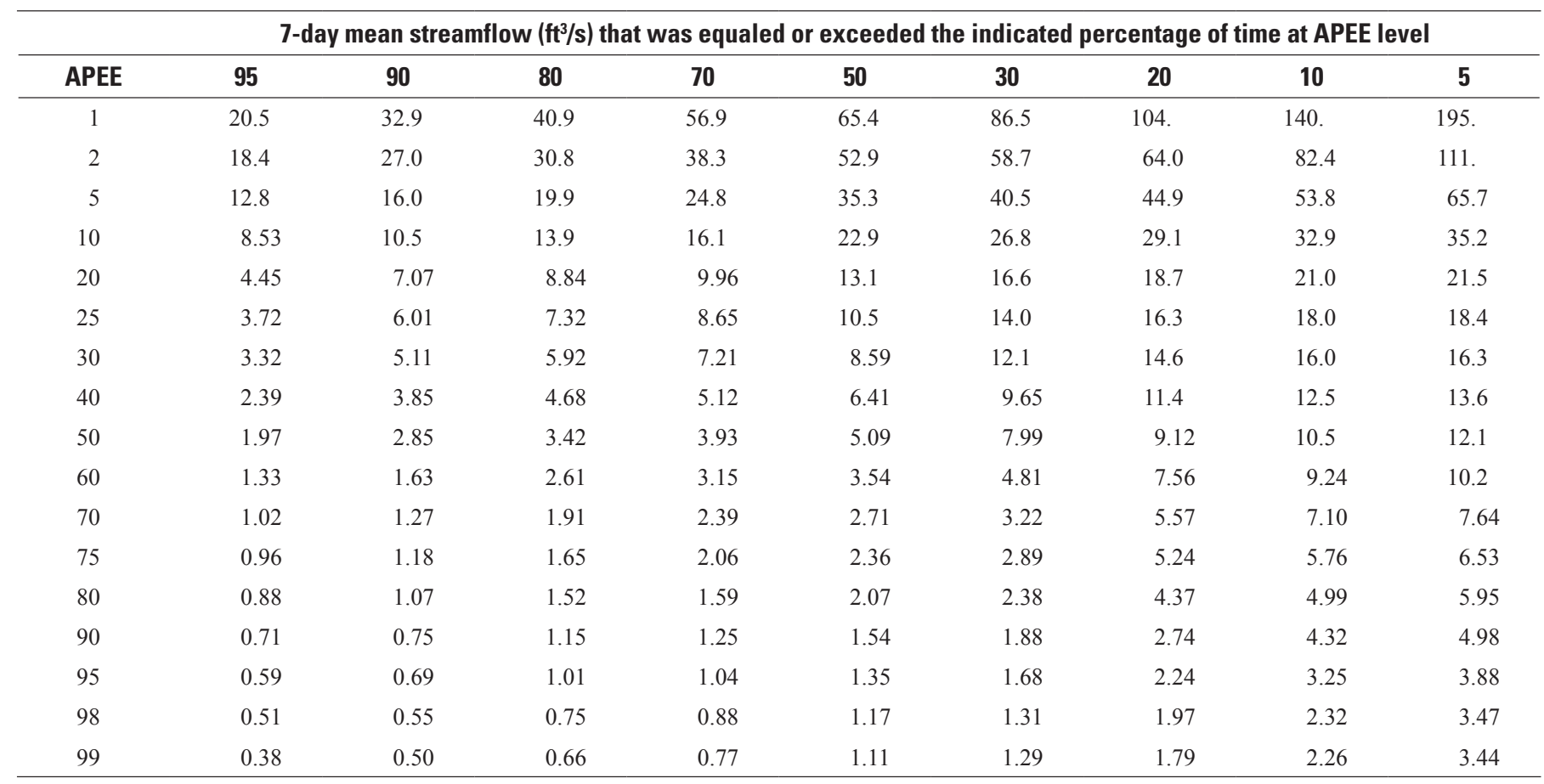




\section{Hocking River at Athens, Ohio}

Years analyzed $=46$

Climatic year range $=1963-2008$

[APEE, annual percentage equaled or exceeded; $\mathrm{ft}^{3} / \mathrm{s}$, cubic foot per second]

\begin{tabular}{|c|c|c|c|c|c|c|c|c|c|}
\hline \multirow[b]{2}{*}{ APEE } & \multicolumn{9}{|c|}{ Daily mean streamflow ( $\left.\mathrm{ft}^{3} / \mathrm{s}\right)$ that was equaled or exceeded the indicated percentage of time at APEE level } \\
\hline & 95 & 90 & 80 & 70 & 50 & 30 & 20 & 10 & 5 \\
\hline 1 & 3940. & 5760. & 6750. & 7320. & 8420. & 9700. & 10600. & 12500 & 14000 \\
\hline 2 & 3360. & 3960. & 5230. & 5890 & 6670. & 7250. & 8290. & 8810. & 10500 . \\
\hline 5 & 1890. & 2240 & 2770 & 3170 & 3960. & 4530. & 4810 & 5560. & 6400. \\
\hline 10 & 1100 & 1390 & 1650 & 2090 & 2370 & 2970. & 3230. & 3860. & 4330. \\
\hline 20 & 601. & 762. & 965. & 1120. & 1350. & 1720 . & 1890. & 2340 & 2590. \\
\hline 25 & 518. & 601. & 779. & 849. & 1140. & 1450. & 1620. & 1960. & 2170 . \\
\hline 30 & 436. & 521. & 635. & 693. & 938. & 1210. & 1380 & 1680 & 1770. \\
\hline 40 & 262. & 306. & 421. & 488. & 619. & 848. & 1060. & 1300 & 1400. \\
\hline 50 & 183. & 218. & 296. & 346. & 412. & 689. & 835. & 1030. & 1060. \\
\hline 60 & 114. & 147. & 216. & 241. & 302. & 497. & 618. & 779. & 855. \\
\hline 70 & 85.0 & 104. & 157. & 182. & 225. & 353. & 452. & 620. & 682. \\
\hline 75 & 74.9 & 91.9 & 134. & 154. & 190. & 263. & 398. & 542. & 604. \\
\hline 80 & 66.9 & 81.2 & 113. & 137. & 157. & 223. & 326. & 445. & 496. \\
\hline 90 & 51.5 & 66.2 & 88.9 & 94.1 & 113. & 163. & 230 & 293. & 367. \\
\hline 95 & 45.6 & 59.7 & 72.5 & 79.3 & 95.1 & 134. & 179. & 238 & 311. \\
\hline 98 & 43.0 & 56.7 & 61.4 & 71.6 & 87.0 & 118. & 145. & 214. & 272. \\
\hline 99 & 40.0 & 55.2 & 59.5 & 68.2 & 85.0 & 107. & 131. & 199. & 242. \\
\hline
\end{tabular}

\begin{tabular}{|c|c|c|c|c|c|c|c|c|c|}
\hline \multirow[b]{2}{*}{ APEE } & \multicolumn{9}{|c|}{ 7-day mean streamflow ( $\left.\mathrm{ft}^{3} / \mathrm{s}\right)$ that was equaled or exceeded the indicated percentage of time at APEE level } \\
\hline & 95 & 90 & 80 & 70 & 50 & 30 & 20 & 10 & 5 \\
\hline 1 & 3130. & 4120. & 4640 & 5110. & 6110 & 7200. & 7950. & 8960. & 13200 . \\
\hline 2 & 2600 & 3330. & 3870 & 4200. & 4980 & 5690. & 6150 & 7250 & 8850. \\
\hline 5 & 2000 & 2120 & 2850 & 3310. & 3780 & 4240. & 4560 & 4970 & 5360. \\
\hline 10 & 1100 & 1510 & 1730 . & 2010 & 2580 & 2980. & 3240 & 3740. & 4380. \\
\hline 20 & 618. & 770. & 1130. & 1220. & 1550 & 1850. & 2070 & 2490 & 2870 . \\
\hline 25 & 527. & 611. & 873. & 960. & 1240 & 1560. & 1810 & 2150 & 2440 . \\
\hline 30 & 437. & 525. & 692. & 761. & 1000 & 1340. & 1570 & 1880. & 2130. \\
\hline 40 & 288. & 345. & 466. & 509. & 681. & 965. & 1180 & 1500 & 1560. \\
\hline 50 & 198. & 222 & 320. & 362. & 474. & 802. & 899. & 1170 & 1220. \\
\hline 60 & 117. & 146. & 222. & 258. & 319. & 579. & 710. & 903. & 1020. \\
\hline 70 & 86.8 & 112. & 163. & 190. & 241. & 387. & 489. & 705. & 800. \\
\hline 75 & 76.8 & 97.4 & 144. & 164. & 211. & 297. & 412. & 595. & 732. \\
\hline 80 & 69.6 & 85.6 & 121. & 139. & 163. & 245. & 337. & 538. & 581. \\
\hline 90 & 53.3 & 66.8 & 94.0 & 97.1 & 116. & 191. & 253. & 322. & 439. \\
\hline 95 & 48.6 & 62.6 & 77.0 & 82.9 & 101. & 145. & 201. & 257. & 362. \\
\hline 98 & 45.7 & 59.3 & 68.8 & 76.4 & 93.6 & 130. & 172. & 230. & 320. \\
\hline 99 & 44.9 & 56.4 & 62.3 & 71.8 & 88.9 & 121. & 158. & 216. & 255. \\
\hline
\end{tabular}


03261500 Great Miami River at Sidney, Ohio

Years analyzed $=82$

Climatic year range $=1927-2008$

[APEE, annual percentage equaled or exceeded; $\mathrm{ft}^{3} / \mathrm{s}$, cubic foot per second]

\begin{tabular}{|c|c|c|c|c|c|c|c|c|c|}
\hline \multirow[b]{2}{*}{ APEE } & \multicolumn{9}{|c|}{ Daily mean streamflow $\left(\mathrm{ft}^{3} / \mathrm{s}\right)$ that was equaled or exceeded the indicated percentage of time at APEE level } \\
\hline & 98 & 95 & 90 & 80 & 50 & 20 & 10 & 5 & 2 \\
\hline 1 & 587. & 1730. & 2520. & 2930. & 4360. & 5660. & 6960. & 7520 & 10100 \\
\hline 2 & 474. & 1200 & 1670 . & 2220 & 3340 & 4240 & 5440. & 5920. & 7360 \\
\hline 5 & 315. & 612. & 1020 . & 1280 . & 1980. & 2860 & 3330. & 3580 . & 4160 . \\
\hline 10 & 243. & 388. & 511. & 703. & 1190. & 1880. & 2030 . & 2230 & 2490. \\
\hline 20 & 148. & 210 & 242. & 351. & 611. & 1000 & 1240 & 1390. & 1500. \\
\hline 25 & 119. & 168. & 193. & 289. & 478. & 782. & 972. & 1110 & 1220. \\
\hline 30 & 98.7 & 139. & 167. & 235 & 387. & 600. & 759. & 915. & 960. \\
\hline 40 & 56.4 & 95.8 & 108. & 146. & 240 & 427. & 547. & 600 & 699. \\
\hline 50 & 45.3 & 65.8 & 77.0 & 105. & 179. & 314. & 379. & 469. & 515. \\
\hline 60 & 39.3 & 51.5 & 59.3 & 71.4 & 127. & 214. & 289. & 357. & 394. \\
\hline 70 & 32.7 & 42.2 & 46.3 & 56.1 & 89.5 & 171. & 225 & 248 & 287. \\
\hline 75 & 30.5 & 38.3 & 43.8 & 50.5 & 79.3 & 151. & 192. & 209. & 259. \\
\hline 80 & 26.7 & 36.1 & 40.3 & 46.6 & 66.1 & 133. & 159. & 180 & 232. \\
\hline 90 & 21.0 & 30.2 & 33.2 & 36.0 & 53.9 & 89.6 & 118. & 128. & 170. \\
\hline 95 & 19.3 & 26.0 & 28.1 & 32.0 & 47.6 & 75.5 & 95.8 & 108. & 146. \\
\hline 98 & 16.7 & 18.4 & 23.2 & 27.3 & 43.8 & 65.8 & 84.4 & 102. & 126. \\
\hline 99 & 14.5 & 15.9 & 21.4 & 26.2 & 41.8 & 63.3 & 75.1 & 97.6 & 123. \\
\hline
\end{tabular}

\begin{tabular}{|c|c|c|c|c|c|c|c|c|c|}
\hline \multirow[b]{2}{*}{ APEE } & \multicolumn{9}{|c|}{ 7-day mean streamflow ( $\mathrm{ft}^{3} / \mathrm{s}$ ) that was equaled or exceeded the indicated percentage of time at APEE level } \\
\hline & 98 & 95 & 90 & 80 & 50 & 20 & 10 & 5 & 2 \\
\hline 1 & 471. & 1280 & 1530. & 2220 & 3250 & 4360 & 5180. & 6090 & 7930. \\
\hline 2 & 370. & 969. & 1340 & 1850 & 2680 & 3360. & 4020 . & 4630 & 6680. \\
\hline 5 & 309. & 630. & 903. & 1270 & 1970. & 2530 & 3060 . & 3400 & 4000 . \\
\hline 10 & 230 & 317. & 552. & 764. & 1220 & 1830. & 2020 . & 2280 & 2520 . \\
\hline 20 & 142. & 230 & 256. & 388. & 665. & 1150 & 1290. & 1440 & 1550. \\
\hline 25 & 122 & 185. & 196. & 306. & 568. & 915. & 1100 & 1180 & 1290. \\
\hline 30 & 99.0 & 154. & 167. & 249. & 442 & 724. & 937. & 1030 & 1080 \\
\hline 40 & 55.5 & 95.7 & 114. & 161. & 292. & 525. & 639. & 756. & 813. \\
\hline 50 & 46.7 & 72.0 & 81.7 & 111. & 198. & 356. & 464. & 547. & 608. \\
\hline 60 & 40.7 & 49.5 & 60.7 & 78.0 & 138. & 261. & 340. & 434. & 486. \\
\hline 70 & 34.2 & 42.6 & 46.8 & 58.1 & 96.9 & 193. & 260 . & 304. & 337. \\
\hline 75 & 31.2 & 38.1 & 43.9 & 53.5 & 81.8 & 172. & 220 & 237. & 285. \\
\hline 80 & 27.7 & 36.7 & 41.2 & 48.2 & 70.7 & 151. & 183. & 199. & 258. \\
\hline 90 & 22.6 & 30.3 & 33.0 & 37.1 & 55.3 & 98.9 & 124. & 136. & 213. \\
\hline 95 & 19.7 & 27.3 & 29.5 & 32.2 & 48.5 & 82.0 & 104. & 116. & 170. \\
\hline 98 & 17.6 & 19.4 & 26.2 & 29.5 & 44.5 & 72.8 & 91.5 & 105. & 141. \\
\hline 99 & 16.7 & 18.6 & 25.4 & 28.7 & 43.0 & 67.1 & 83.6 & 103. & 137. \\
\hline
\end{tabular}




\section{Loramie Creek near Newport, Ohio}

Years analyzed $=44$

Climatic year range $=1965-2008$

[APEE, annual percentage equaled or exceeded; $\mathrm{ft}^{3} / \mathrm{s}$, cubic foot per second]

\begin{tabular}{|c|c|c|c|c|c|c|c|c|c|}
\hline \multirow[b]{2}{*}{ APEE } & \multicolumn{9}{|c|}{ Daily mean streamflow $\left(\mathrm{ft}^{3} / \mathrm{s}\right)$ that was equaled or exceeded the indicated percentage of time at APEE level } \\
\hline & 95 & 90 & 80 & 70 & 50 & 30 & 20 & 10 & 5 \\
\hline 1 & 455. & 953. & 1180. & 1550. & 1750. & 2090. & 2210. & 3160 & 3300. \\
\hline 2 & 365. & 473. & 836. & 1020. & 1330. & 1550 . & 1790 . & 2390 & 2520 . \\
\hline 5 & 202. & 279. & 406. & 548. & 803. & 978. & 1160 & 1290. & 1370 . \\
\hline 10 & 95.1 & 139. & 191. & 247. & 360. & 514. & 577. & 663. & 766. \\
\hline 20 & 35.8 & 55.0 & 72.8 & 98.7 & 155. & 218 & 251. & 294. & 329. \\
\hline 25 & 24.4 & 37.1 & 49.5 & 71.3 & 111. & 164. & 183. & 212. & 236. \\
\hline 30 & 17.5 & 23.0 & 40.2 & 53.5 & 80.1 & 116. & 137. & 168. & 191. \\
\hline 40 & 8.10 & 10.9 & 20.6 & 25.5 & 41.8 & 65.5 & 79.6 & 103. & 122. \\
\hline 50 & 3.90 & 5.18 & 10.0 & 14.0 & 26.0 & 41.0 & 48.5 & 62.0 & 82.4 \\
\hline 60 & 2.40 & 3.65 & 6.20 & 8.52 & 14.4 & 23.5 & 32.0 & 41.5 & 49.0 \\
\hline 70 & 1.67 & 2.55 & 3.30 & 4.41 & 7.23 & 12.4 & 19.0 & 26.0 & 33.7 \\
\hline 75 & 1.38 & 1.58 & 2.80 & 3.38 & 5.30 & 9.15 & 15.0 & 20.5 & 27.6 \\
\hline 80 & 1.02 & 1.26 & 2.30 & 2.65 & 4.25 & 6.95 & 11.0 & 17.0 & 21.8 \\
\hline 90 & 0.49 & 0.83 & 1.00 & 1.69 & 2.33 & 3.56 & 5.42 & 7.37 & 12.2 \\
\hline 95 & 0.27 & 0.55 & 0.78 & 1.12 & 1.75 & 2.70 & 3.33 & 4.56 & 7.27 \\
\hline 98 & 0.24 & 0.45 & 0.68 & 0.84 & 1.32 & 2.42 & 2.70 & 3.62 & 4.24 \\
\hline 99 & 0.22 & 0.33 & 0.59 & 0.73 & 1.17 & 2.10 & 2.47 & 3.23 & 3.77 \\
\hline
\end{tabular}

\begin{tabular}{|c|c|c|c|c|c|c|c|c|c|}
\hline \multirow[b]{2}{*}{ APEE } & \multicolumn{9}{|c|}{ 7-day mean streamflow ( $\mathrm{ft}^{3} / \mathrm{s}$ ) that was equaled or exceeded the indicated percentage of time at APEE level } \\
\hline & 95 & 90 & 80 & 70 & 50 & 30 & 20 & 10 & 5 \\
\hline 1 & 328. & 562. & 775. & 925. & 1160. & 1460. & 1570. & 2130 & 2500 \\
\hline 2 & 271. & 393. & 619. & 744. & 916. & 1130. & 1340 . & 1550 . & 1880. \\
\hline 5 & 191. & 254. & 401. & 502. & 704. & 829. & 928. & 1100. & 1200. \\
\hline 10 & 120. & 160. & 205. & 308. & 399. & 525. & 584. & 635. & 769. \\
\hline 20 & 40.7 & 66.5 & 112. & 144. & 190. & 306. & 331. & 399. & 456. \\
\hline 25 & 27.7 & 49.4 & 77.2 & 101. & 141. & 232. & 266. & 314. & 347. \\
\hline 30 & 20.2 & 26.3 & 56.6 & 72.4 & 105. & 176. & 198. & 247. & 281. \\
\hline 40 & 10.8 & 12.2 & 27.3 & 33.3 & 59.3 & 99.6 & 113. & 152. & 193. \\
\hline 50 & 4.39 & 6.30 & 15.6 & 16.9 & 34.9 & 57.3 & 72.6 & 103. & 129. \\
\hline 60 & 2.91 & 4.31 & 7.46 & 10.6 & 18.0 & 32.0 & 46.0 & 57.1 & 78.4 \\
\hline 70 & 1.95 & 2.49 & 4.04 & 5.19 & 8.80 & 18.0 & 29.2 & 36.9 & 50.7 \\
\hline 75 & 1.49 & 1.75 & 3.02 & 3.94 & 6.32 & 13.3 & 19.7 & 30.1 & 39.5 \\
\hline 80 & 1.16 & 1.27 & 2.33 & 3.25 & 5.14 & 9.67 & 16.4 & 23.4 & 29.6 \\
\hline 90 & 0.71 & 0.93 & 1.42 & 2.06 & 2.68 & 4.53 & 6.70 & 11.4 & 16.1 \\
\hline 95 & 0.35 & 0.58 & 1.04 & 1.37 & 2.01 & 3.17 & 4.47 & 7.00 & 10.6 \\
\hline 98 & 0.26 & 0.51 & 0.82 & 1.00 & 1.73 & 2.84 & 3.41 & 5.18 & 8.10 \\
\hline 99 & 0.24 & 0.49 & 0.81 & 0.88 & 1.49 & 2.56 & 3.10 & 4.29 & 5.52 \\
\hline
\end{tabular}




\section{Loramie Creek at Lockington, Ohio}

Years analyzed $=91$

Climatic year range $=1916-2008$

[APEE, annual percentage equaled or exceeded; $\mathrm{ft}^{3} / \mathrm{s}$, cubic foot per second]

\begin{tabular}{|c|c|c|c|c|c|c|c|c|c|}
\hline \multirow[b]{2}{*}{ APEE } & \multicolumn{9}{|c|}{ Daily mean streamflow $\left(\mathrm{ft}^{3} / \mathrm{s}\right)$ that was equaled or exceeded the indicated percentage of time at APEE level } \\
\hline & 98 & 95 & 90 & 80 & 50 & 20 & 10 & 5 & 2 \\
\hline 1 & 406. & 847. & 1580. & 1900. & 2900. & 3640. & 4340. & 4780 . & 5510. \\
\hline 2 & 311. & 592. & 1200 . & 1420 . & 1980. & 2670 & 3280 . & 4060 . & 4220. \\
\hline 5 & 165. & 296. & 477. & 709. & 1070 & 1530. & 1800 & 2040 . & 2230 \\
\hline 10 & 96.5 & 128 & 207. & 318. & 526. & 820 . & 972. & 1080 & 1250 \\
\hline 20 & 38.0 & 51.7 & 70.1 & 125. & 222. & 338. & 459. & 499. & 573. \\
\hline 25 & 31.3 & 38.0 & 50.0 & 89.9 & 153. & 241. & 336. & 374. & 414. \\
\hline 30 & 21.0 & 30.3 & 40.1 & 66.5 & 114. & 196. & 250 & 292. & 326. \\
\hline 40 & 12.4 & 17.0 & 20.0 & 39.6 & 67.0 & 121. & 148 & 191. & 219. \\
\hline 50 & 8.74 & 10.0 & 13.2 & 21.8 & 44.0 & 76.7 & 103. & 126. & 148. \\
\hline 60 & 6.61 & 7.88 & 8.72 & 15.0 & 25.0 & 55.2 & 68.9 & 83.8 & 100. \\
\hline 70 & 5.54 & 6.92 & 7.53 & 9.78 & 16.8 & 37.6 & 48.8 & 57.4 & 78.2 \\
\hline 75 & 4.48 & 6.51 & 6.86 & 8.35 & 13.0 & 30.0 & 41.8 & 50.8 & 68.7 \\
\hline 80 & 4.25 & 5.41 & 6.12 & 7.44 & 12.0 & 23.8 & 36.8 & 45.8 & 59.3 \\
\hline 90 & 3.32 & 3.99 & 4.55 & 5.68 & 8.76 & 15.6 & 22.5 & 34.2 & 42.1 \\
\hline 95 & 2.49 & 2.97 & 4.02 & 4.60 & 7.50 & 12.0 & 17.3 & 29.0 & 38.6 \\
\hline 98 & 1.54 & 2.58 & 3.22 & 3.94 & 6.83 & 9.81 & 15.0 & 23.8 & 33.2 \\
\hline 99 & 0.91 & 2.08 & 2.88 & 3.70 & 6.27 & 9.42 & 13.0 & 21.9 & 31.1 \\
\hline
\end{tabular}

\begin{tabular}{|c|c|c|c|c|c|c|c|c|c|}
\hline \multirow[b]{2}{*}{ APEE } & \multicolumn{9}{|c|}{ 7-day mean streamflow ( $\mathrm{ft}^{3} / \mathrm{s}$ ) that was equaled or exceeded the indicated percentage of time at APEE level } \\
\hline & 98 & 95 & 90 & 80 & 50 & 20 & 10 & 5 & 2 \\
\hline 1 & 298. & 582. & 935. & 1240. & 1800. & 2520. & 3160. & 3330. & 4270 \\
\hline 2 & 203. & 529. & 762. & 986. & 1480 . & 1930. & 2330 . & 2700 & 3020. \\
\hline 5 & 145. & 288. & 551. & 705. & 980. & 1340 & 1580. & 1870 . & 1960. \\
\hline 10 & 93.2 & 140. & 259. & 379. & 572. & 877. & 987. & 1060. & 1230 \\
\hline 20 & 43.1 & 56.0 & 81.9 & 164. & 293. & 464. & 564. & 687. & 733. \\
\hline 25 & 32.2 & 42.7 & 57.3 & 124. & 219. & 358. & 454. & 514. & 577. \\
\hline 30 & 22.4 & 31.4 & 40.7 & 87.8 & 157. & 274 & 375. & 413. & 472. \\
\hline 40 & 12.3 & 18.7 & 21.7 & 47.4 & 84.7 & 169. & 216. & 266. & 330. \\
\hline 50 & 8.79 & 10.4 & 13.4 & 25.2 & 48.6 & 105. & 133. & 170 . & 214. \\
\hline 60 & 6.80 & 8.00 & 9.44 & 17.4 & 30.3 & 66.4 & 90.4 & 111. & 144. \\
\hline 70 & 5.60 & 6.97 & 7.91 & 10.4 & 18.9 & 46.3 & 65.3 & 76.5 & 103. \\
\hline 75 & 4.65 & 6.57 & 6.92 & 8.73 & 15.2 & 35.2 & 52.5 & 63.5 & 87.7 \\
\hline 80 & 4.24 & 5.36 & 6.19 & 7.53 & 12.4 & 27.1 & 42.4 & 53.5 & 72.7 \\
\hline 90 & 3.28 & 4.17 & 4.83 & 5.67 & 9.04 & 17.7 & 26.3 & 39.7 & 49.4 \\
\hline 95 & 2.59 & 3.37 & 4.11 & 4.77 & 8.04 & 13.3 & 20.6 & 34.5 & 42.2 \\
\hline 98 & 2.47 & 2.92 & 3.84 & 4.19 & 7.29 & 11.1 & 18.3 & 29.4 & 38.0 \\
\hline 99 & 2.40 & 2.78 & 3.71 & 4.01 & 6.84 & 10.1 & 14.9 & 27.6 & 36.2 \\
\hline
\end{tabular}




\section{Great Miami River at Troy, Ohio}

Years analyzed $=46$

Climatic year range $=1963-2008$

[APEE, annual percentage equaled or exceeded; $\mathrm{ft}^{3} / \mathrm{s}$, cubic foot per second]

\begin{tabular}{|c|c|c|c|c|c|c|c|c|c|}
\hline \multirow[b]{2}{*}{ APEE } & \multicolumn{9}{|c|}{ Daily mean streamflow ( $\left.\mathrm{ft}^{3} / \mathrm{s}\right)$ that was equaled or exceeded the indicated percentage of time at APEE level } \\
\hline & 95 & 90 & 80 & 70 & 50 & 30 & 20 & 10 & 5 \\
\hline 1 & 2690. & 4360. & 6370. & 7050. & 8110. & 9860. & 10800. & 12400 & 14200 \\
\hline 2 & 1890. & 3160. & 4120. & 5030 & 5910. & 7260 & 7670 . & 9940. & 11400 . \\
\hline 5 & 1000 & 1490. & 2270 & 3060 . & 3740 . & 4810 . & 5310. & 5910. & 6490. \\
\hline 10 & 598. & 784. & 1260. & 1640 & 2220 . & 2860 . & 3180. & 3560. & 3930. \\
\hline 20 & 338. & 369. & 594. & 767. & 1030. & 1420. & 1730. & 2080 & 2290 . \\
\hline 25 & 272 & 323. & 447. & 586. & 821. & 1150. & 1320. & 1590. & 1830. \\
\hline 30 & 222. & 252. & 394. & 495. & 662. & 911. & 1020 & 1320. & 1480. \\
\hline 40 & 151. & 187. & 274. & 334. & 450. & 639. & 696. & 939. & 1060. \\
\hline 50 & 109. & 129. & 191. & 222. & 350 . & 467. & 531. & 708. & 761. \\
\hline 60 & 79.1 & 92.9 & 143. & 161. & 223. & 349. & 397. & 516. & 587. \\
\hline 70 & 66.3 & 69.2 & 104. & 120. & 154. & 253. & 313. & 383. & 425. \\
\hline 75 & 57.2 & 63.1 & 91.9 & 103. & 123. & 205. & 274 & 314. & 377. \\
\hline 80 & 52.4 & 57.6 & 79.5 & 89.9 & 106. & 189. & 234 & 267. & 327. \\
\hline 90 & 35.7 & 45.5 & 61.4 & 74.3 & 86.3 & 131. & 159. & 205. & 245. \\
\hline 95 & 30.5 & 39.1 & 52.3 & 63.1 & 77.5 & 107. & 134. & 180. & 198. \\
\hline 98 & 28.0 & 33.1 & 44.1 & 53.2 & 69.8 & 97.8 & 114. & 144. & 169. \\
\hline 99 & 24.6 & 30.7 & 39.7 & 49.3 & 67.2 & 91.2 & 106. & 137. & 157. \\
\hline
\end{tabular}

\begin{tabular}{|c|c|c|c|c|c|c|c|c|c|}
\hline \multirow[b]{2}{*}{ APEE } & \multicolumn{9}{|c|}{ 7-day mean streamflow $\left(\mathrm{ft}^{3} / \mathrm{s}\right)$ that was equaled or exceeded the indicated percentage of time at APEE level } \\
\hline & 95 & 90 & 80 & 70 & 50 & 30 & 20 & 10 & 5 \\
\hline 1 & 1980. & 2600 & 4420 & 5120. & 5780. & 6610 & 7690. & 8750. & 11000 . \\
\hline 5 & 1010 & 1430 & 2120 & 2880 & 3410. & 4110. & 4600. & 5430 & 6280. \\
\hline 10 & 626. & 840. & 1390. & 1640. & 2110 & 2820 & 3080 . & 3730. & 4180 . \\
\hline 30 & 216. & 251. & 425. & 564. & 793. & 1150 & 1320. & 1600 & 1720 . \\
\hline 40 & 158. & 195. & 296. & 357. & 541. & 748. & 879. & 1210 & 1270. \\
\hline 50 & 108 & 136. & 201. & 230 & 384. & 544. & 633. & 858. & 927. \\
\hline 60 & 81.4 & 97.6 & 148. & 177. & 245. & 405 & 451. & 626. & 706. \\
\hline 90 & 37.6 & 45.9 & 65.3 & 75.4 & 89.4 & 146. & 178. & 220 & 280. \\
\hline 95 & 31.9 & 38.7 & 54.5 & 64.3 & 80.6 & 112. & 151. & 190. & 228. \\
\hline 98 & 29.9 & 36.0 & 51.0 & 57.9 & 74.1 & 102. & 134. & 161. & 193. \\
\hline 99 & 28.3 & 34.5 & 46.9 & 55.3 & 71.3 & 101. & 126. & 147. & 187. \\
\hline
\end{tabular}


03263000 Great Miami River at Taylorsville, Ohio

Years analyzed $=87$

Climatic year range $=1922-2008$

[APEE, annual percentage equaled or exceeded; $\mathrm{ft}^{3} / \mathrm{s}$, cubic foot per second]

\begin{tabular}{|c|c|c|c|c|c|c|c|c|c|}
\hline \multirow[b]{2}{*}{ APEE } & \multicolumn{9}{|c|}{ Daily mean streamflow $\left(\mathrm{ft}^{3} / \mathrm{s}\right)$ that was equaled or exceeded the indicated percentage of time at APEE level } \\
\hline & 98 & 95 & 90 & 80 & 50 & 20 & 10 & 5 & 2 \\
\hline 1 & 1300. & 3450 & 5240 & 6220. & 9870. & 12600 & 15100. & 17200. & 19700. \\
\hline 2 & 962. & 2270 & 3870. & 4590 . & 7240 & 9660. & 12000 & 13900. & 16600 \\
\hline 5 & 608. & 1370 . & 2020 . & 2590. & 4190. & 6120 & 7090 . & 7470 . & 8200 . \\
\hline 10 & 475 . & 801. & 1030. & 1410 . & 2380 & 3640. & 3990. & 4490 . & 4720 . \\
\hline 20 & 313. & 420. & 506. & 793. & 1220. & 1900. & 2400 & 2650 & 2910. \\
\hline 25 & 269. & 353. & 421. & 618. & 958. & 1510 & 1930. & 2140 & 2350 . \\
\hline 30 & 188. & 289. & 347. & 503. & 794. & 1260. & 1490 . & 1790. & 1960. \\
\hline 40 & 123. & 192. & 229. & 365. & 554. & 894. & 1080 & 1290. & 1410 . \\
\hline 50 & 91.5 & 140 . & 168. & 243. & 388. & 665. & 825. & 980. & 1030. \\
\hline 60 & 82.9 & 106. & 121. & 170. & 275 & 500. & 594. & 766. & 824. \\
\hline 70 & 74.1 & 88.8 & 94.5 & 128. & 191. & 386. & 488. & 573. & 647. \\
\hline 75 & 68.3 & 80.4 & 88.2 & 110. & 166. & 343. & 417. & 479. & 568. \\
\hline 80 & 65.3 & 72.1 & 79.9 & 100. & 146. & 283 & 382. & 399. & 509. \\
\hline 90 & 47.2 & 62.2 & 69.0 & 78.4 & 120. & 200. & 261. & 308. & 388. \\
\hline 95 & 41.3 & 54.6 & 60.6 & 66.7 & 100 & 168 & 223. & 260. & 354. \\
\hline 98 & 38.4 & 46.3 & 54.0 & 61.2 & 93.0 & 142. & 185. & 213. & 325. \\
\hline 99 & 37.1 & 42.7 & 51.9 & 57.0 & 87.6 & 136. & 176. & 203. & 320 . \\
\hline
\end{tabular}

\begin{tabular}{|c|c|c|c|c|c|c|c|c|c|}
\hline \multirow[b]{2}{*}{ APEE } & \multicolumn{9}{|c|}{ 7-day mean streamflow ( $\mathrm{ft}^{3} / \mathrm{s}$ ) that was equaled or exceeded the indicated percentage of time at APEE level } \\
\hline & 98 & 95 & 90 & 80 & 50 & 20 & 10 & 5 & 2 \\
\hline 1 & 1060. & 2600 & 3380. & 4600 . & 6900. & 9570. & 10600. & 13800 & 15300. \\
\hline 2 & 794. & 2180 & 2880 & 3860 . & 5520. & 7230 & 9010. & 10600 . & 14300 \\
\hline 5 & 568. & 1380 & 1940. & 2540 & 3950 . & 5580. & 6390. & 7550 . & 8110 . \\
\hline 10 & 444. & 712. & 1090. & 1570 . & 2530 & 3710 & 4220 . & 4610 . & 4830 . \\
\hline 20 & 299. & 429. & 551. & 837. & 1430 & 2230 & 2610. & 2850 & 3360 . \\
\hline 25 & 267. & 356. & 401. & 647. & 1150 & 1810 & 2230 & 2390 . & 2690. \\
\hline 30 & 179. & 301. & 352. & 554. & 868. & 1490 & 1910. & 2120 & 2210 . \\
\hline 40 & 129. & 187. & 252. & 393. & 591. & 1010. & 1300 . & 1560 . & 1640. \\
\hline 50 & 96.1 & 140. & 187. & 260 & 421. & 737. & 945. & 1140. & 1250. \\
\hline 60 & 83.3 & 105. & 123. & 181. & 293. & 566. & 674. & 850 . & 992. \\
\hline 70 & 74.7 & 89.1 & 98.5 & 135. & 207. & 433. & 569. & 637. & 743. \\
\hline 75 & 69.6 & 81.5 & 90.1 & 114. & 178. & 374. & 483. & 542. & 679. \\
\hline 80 & 65.9 & 72.7 & 80.9 & 103. & 151. & 311. & 395. & 444. & 625. \\
\hline 90 & 50.6 & 64.2 & 68.6 & 80.3 & 123. & 206. & 284. & 360 . & 497. \\
\hline 95 & 41.8 & 56.2 & 60.5 & 69.9 & 106. & 180. & 246. & 286. & 383. \\
\hline 98 & 39.8 & 49.5 & 55.7 & 66.0 & 99.0 & 150. & 202. & 258. & 354. \\
\hline 99 & 39.2 & 45.6 & 53.5 & 61.5 & 94.0 & 145. & 187. & 234. & 329. \\
\hline
\end{tabular}




\section{Stillwater River at Englewood, Ohio}

Years analyzed $=83$

Climatic year range $=1926-2008$

[APEE, annual percentage equaled or exceeded; $\mathrm{ft}^{3} / \mathrm{s}$, cubic foot per second]

\begin{tabular}{|c|c|c|c|c|c|c|c|c|c|}
\hline \multirow[b]{2}{*}{ APEE } & \multicolumn{9}{|c|}{ Daily mean streamflow ( $\left.\mathrm{ft}^{3} / \mathrm{s}\right)$ that was equaled or exceeded the indicated percentage of time at APEE level } \\
\hline & 98 & 95 & 90 & 80 & 50 & 20 & 10 & 5 & 2 \\
\hline 1 & 642. & 1790. & 2920. & 4230. & 6000. & 7280. & 7740. & 8940. & 9570. \\
\hline 2 & 483. & 1040. & 2200 & 3040 . & 4900 & 5930. & 6870. & 7930. & 9040. \\
\hline 5 & 294. & 668. & 1070. & 1550. & 2980 . & 4320. & 4880 & 5080 & 5870. \\
\hline 10 & 224. & 366. & 503. & 701. & 1450. & 2390 . & 2960. & 3140. & 3460 . \\
\hline 20 & 128. & 220. & 258. & 369. & 700 . & 1080. & 1290. & 1490. & 1560. \\
\hline 25 & 111. & 188. & 211. & 288. & 540. & 849. & 1040 & 1140. & 1240. \\
\hline 30 & 98.4 & 131. & 185. & 235. & 419. & 661. & 824. & 929. & 1000. \\
\hline 40 & 59.2 & 82.8 & 126. & 152. & 292. & 454. & 544. & 604. & 689. \\
\hline 50 & 42.7 & 51.8 & 80.6 & 112. & 209. & 331. & 398. & 459. & 500. \\
\hline 60 & 34.8 & 43.4 & 59.6 & 81.6 & 146. & 250 & 307. & 358. & 399. \\
\hline 70 & 29.1 & 37.7 & 45.2 & 64.0 & 106. & 182. & 236. & 266. & 331. \\
\hline 75 & 27.0 & 31.2 & 41.2 & 52.9 & 88.5 & 159. & 200. & 243. & 290. \\
\hline 80 & 24.5 & 27.1 & 36.2 & 46.2 & 73.0 & 121. & 167. & 227. & 266. \\
\hline 90 & 16.0 & 21.4 & 23.8 & 31.8 & 57.0 & 90.2 & 111. & 166. & 220. \\
\hline 95 & 12.7 & 16.5 & 18.1 & 25.2 & 48.3 & 74.7 & 92.5 & 126. & 183. \\
\hline 98 & 10.8 & 13.1 & 15.0 & 21.3 & 41.3 & 66.5 & 76.7 & 115. & 152. \\
\hline 99 & 7.44 & 10.1 & 12.7 & 19.3 & 38.0 & 62.4 & 73.9 & 110. & 141. \\
\hline
\end{tabular}

\begin{tabular}{|c|c|c|c|c|c|c|c|c|c|}
\hline \multirow[b]{2}{*}{ APEE } & \multicolumn{9}{|c|}{ 7-day mean streamflow ( $\left.\mathrm{ft}^{3} / \mathrm{s}\right)$ that was equaled or exceeded the indicated percentage of time at APEE level } \\
\hline & 98 & 95 & 90 & 80 & 50 & 20 & 10 & 5 & 2 \\
\hline 1 & 431. & 1500 & 1820 & 2750 & 4490. & 5610. & 6250 & 8080 & 9080. \\
\hline 5 & 289. & 617. & 1060 & 1690. & 2480 & 3360 & 3820 & 4350 & 5130. \\
\hline 10 & 219. & 401. & 528. & 898. & 1600 & 2340 & 2720 & 2940. & 3200 . \\
\hline 30 & 93.9 & 134. & 187. & 243. & 494. & 842. & 995. & 1230 & 1270. \\
\hline 40 & 51.6 & 81.3 & 125. & 163. & 330. & 568. & 728. & 867. & 899. \\
\hline 50 & 41.1 & 53.1 & 84.2 & 114. & 219. & 404. & 504. & 587. & 651. \\
\hline 60 & 34.1 & 42.3 & 58.3 & 87.5 & 152. & 285 & 357. & 438. & 517. \\
\hline 90 & 20.6 & 22.7 & 25.3 & 33.1 & 58.0 & 97.4 & 127. & 180. & 254. \\
\hline 95 & 16.0 & 19.3 & 21.0 & 28.3 & 50.3 & 81.6 & 101. & 130. & 218. \\
\hline 98 & 14.3 & 15.3 & 17.6 & 24.5 & 44.6 & 72.1 & 82.1 & 121. & 174. \\
\hline 99 & 12.4 & 13.5 & 16.2 & 23.8 & 43.6 & 67.8 & 76.4 & 115. & 154. \\
\hline
\end{tabular}


03267900 Mad River at St Paris Pike at Eagle City, Ohio

Years analyzed $=36$

Climatic year range $=1966-2008$

[APEE, annual percentage equaled or exceeded; $\mathrm{ft}^{3} / \mathrm{s}$, cubic foot per second]

\begin{tabular}{|c|c|c|c|c|c|c|c|c|c|}
\hline \multirow[b]{2}{*}{ APEE } & \multicolumn{9}{|c|}{ Daily mean streamflow $\left(\mathrm{ft}^{3} / \mathrm{s}\right)$ that was equaled or exceeded the indicated percentage of time at APEE level } \\
\hline & 95 & 90 & 80 & 70 & 50 & 30 & 20 & 10 & 5 \\
\hline 1 & 765. & 825. & 1010 & 1300. & 1850. & 2100. & 2340 & 2790. & 3810. \\
\hline 2 & 606. & 637. & 742. & 949. & 1330. & 1590. & 1620. & 2030. & 2190. \\
\hline 5 & 409. & 428. & 509. & 655. & 812. & 919. & 1080 & 1180. & 1270. \\
\hline 10 & 302. & 323. & 368. & 475. & 553. & 697. & 793. & 822. & 856. \\
\hline 20 & 218. & 233. & 263. & 325. & 403. & 482. & 554. & 577. & 595. \\
\hline 25 & 195. & 205. & 238 & 298. & 356. & 435. & 479. & 511. & 542. \\
\hline 30 & 174. & 188. & 223. & 270. & 324. & 399. & 430. & 459. & 508. \\
\hline 40 & 154. & 164. & 196. & 216. & 286. & 330. & 363. & 384. & 442. \\
\hline 50 & 138. & 144. & 170. & 184. & 243. & 297. & 309. & 340. & 394. \\
\hline 60 & 123. & 129. & 145. & 169. & 214. & 249. & 272. & 314. & 340. \\
\hline 70 & 110. & 120. & 130. & 154. & 185. & 215. & 234. & 284. & 303. \\
\hline 75 & 107. & 114. & 123. & 142. & 172. & 199. & 219. & 267. & 280. \\
\hline 80 & 102. & 110. & 118. & 131. & 164. & 188. & 202. & 247. & 262. \\
\hline 90 & 92.0 & 98.8 & 109. & 114. & 149. & 160. & 171. & 220. & 241. \\
\hline 95 & 81.9 & 94.4 & 105. & 111. & 137. & 151. & 162. & 209. & 227. \\
\hline 98 & 75.1 & 88.6 & 100. & 109. & 132. & 142. & 150. & 199. & 212. \\
\hline 99 & 73.9 & 87.5 & 98.5 & 108. & 129. & 140. & 148. & 194. & 208. \\
\hline
\end{tabular}

\begin{tabular}{|c|c|c|c|c|c|c|c|c|c|}
\hline \multirow[b]{2}{*}{ APEE } & \multicolumn{9}{|c|}{ 7-day mean streamflow ( $\left.\mathrm{ft}^{3} / \mathrm{s}\right)$ that was equaled or exceeded the indicated percentage of time at APEE level } \\
\hline & 95 & 90 & 80 & 70 & 50 & 30 & 20 & 10 & 5 \\
\hline 1 & 501. & 644. & 832. & 938. & 1230 & 1500. & 1710 & 1970. & 2150. \\
\hline 5 & 375. & 451 & 488. & 637. & 796. & 935. & 1010 & 1180 & 1310. \\
\hline 10 & 324. & 333. & 391. & 462. & 586. & 731. & 791. & 842. & 886. \\
\hline 30 & 182. & 191. & 235 & 283. & 353. & 434. & 466. & 516. & 548. \\
\hline 40 & 157. & 169. & 204. & 228. & 299. & 358. & 385. & 447. & 476. \\
\hline 50 & 138. & 147. & 173 & 189. & 252. & 306. & 325. & 366. & 425. \\
\hline 60 & 120. & 129. & 146. & 168. & 222. & 262. & 281. & 335. & 379. \\
\hline 90 & 95.5 & 100 & 111. & 116. & 151. & 161. & 177. & 226. & 250. \\
\hline 95 & 81.5 & 95.6 & 106. & 113. & 139. & 156. & 164. & 215. & 238. \\
\hline 98 & 76.1 & 92.2 & 104 & 111. & 133. & 146. & 154. & 205. & 223. \\
\hline 99 & 75.1 & 89.0 & 103. & 110. & 132. & 145. & 151. & 199. & 215. \\
\hline
\end{tabular}




\section{Buck Creek at Springfield, Ohio}

Years analyzed $=30$

Climatic year range $=1915-48$

[APEE, annual percentage equaled or exceeded; $\mathrm{ft}^{3} / \mathrm{s}$, cubic foot per second]

\begin{tabular}{|c|c|c|c|c|c|c|c|c|c|}
\hline \multirow[b]{2}{*}{ APEE } & \multicolumn{9}{|c|}{ Daily mean streamflow $\left(\mathrm{ft}^{3} / \mathrm{s}\right)$ that was equaled or exceeded the indicated percentage of time at APEE level } \\
\hline & 95 & 90 & 80 & 70 & 50 & 30 & 20 & 10 & 5 \\
\hline 1 & 167. & 415. & 746. & 770. & 893. & 1330. & 1480. & 1680 & 2100 \\
\hline 2 & 120. & 265. & 469. & 528. & 588. & 805. & 923. & 1050 & 1220 \\
\hline 5 & 92.2 & 125. & 257. & 278. & 318. & 412. & 449. & 538. & 692. \\
\hline 10 & 69.5 & 98.2 & 138. & 168. & 219. & 253. & 273. & 282. & 391. \\
\hline 20 & 49.5 & 72.3 & 101. & 112. & 153. & 167. & 179. & 211. & 237. \\
\hline 25 & 43.8 & 63.7 & 88.0 & 100 . & 129. & 149. & 165. & 188. & 200 \\
\hline 30 & 39.3 & 55.2 & 74.2 & 90.7 & 117. & 132. & 152. & 169. & 190. \\
\hline 40 & 32.1 & 34.9 & 59.4 & 75.9 & 97.5 & 112. & 129. & 140 . & 158. \\
\hline 50 & 26.1 & 32.5 & 49.4 & 59.0 & 74.5 & 90.7 & 101. & 131. & 141. \\
\hline 60 & 22.0 & 30.7 & 41.6 & 49.2 & 62.0 & 80.6 & 84.8 & 115. & 123. \\
\hline 70 & 19.5 & 28.0 & 35.4 & 42.2 & 52.9 & 72.2 & 82.4 & 98.5 & 117. \\
\hline 75 & 17.6 & 27.0 & 30.8 & 37.9 & 50.0 & 66.8 & 70.8 & 89.4 & 105 \\
\hline 80 & 16.2 & 21.4 & 28.6 & 34.0 & 47.1 & 59.4 & 65.8 & 82.6 & 94.8 \\
\hline 90 & 11.3 & 13.8 & 24.0 & 29.0 & 40.3 & 56.5 & 63.2 & 70.9 & 80.7 \\
\hline 95 & 7.72 & 11.8 & 21.8 & 26.1 & 37.0 & 47.7 & 52.8 & 60.0 & 74.1 \\
\hline 98 & 6.26 & 6.91 & 17.1 & 22.9 & 31.0 & 46.3 & 51.2 & 54.7 & 71.5 \\
\hline 99 & 5.73 & 6.52 & 16.4 & 19.8 & 27.0 & 43.7 & 51.2 & 52.0 & 66.8 \\
\hline
\end{tabular}

\begin{tabular}{|c|c|c|c|c|c|c|c|c|c|}
\hline \multirow[b]{2}{*}{ APEE } & \multicolumn{9}{|c|}{ 7-day mean streamflow $\left(\mathrm{ft}^{3} / \mathrm{s}\right)$ that was equaled or exceeded the indicated percentage of time at APEE level } \\
\hline & 95 & 90 & 80 & 70 & 50 & 30 & 20 & 10 & 5 \\
\hline 1 & 121. & 226. & 359. & 466. & 582. & 712. & 860. & 1130 & 1480 \\
\hline 2 & 110 & 180. & 300. & 350. & 477. & 565. & 666. & 728. & 1090. \\
\hline 5 & 92.2 & 145. & 268. & 276. & 351. & 407. & 505. & 579. & 613. \\
\hline 10 & 77.5 & 103. & 177. & 208. & 246. & 292. & 317. & 362. & 423. \\
\hline 20 & 54.5 & 70.1 & 113. & 127. & 168. & 192. & 223. & 247. & 311. \\
\hline 25 & 46.0 & 64.7 & 102. & 109. & 142. & 172. & 180. & 217. & 267. \\
\hline 30 & 41.1 & 51.2 & 85.6 & 97.0 & 126. & 156. & 166. & 195. & 225 \\
\hline 40 & 32.0 & 34.6 & 62.0 & 81.2 & 104. & 127. & 144. & 157. & 184. \\
\hline 50 & 25.8 & 32.7 & 54.0 & 65.0 & 77.8 & 101. & 112. & 133. & 159. \\
\hline 60 & 23.9 & 31.0 & 44.9 & 53.8 & 65.5 & 86.7 & 97.5 & 124. & 137. \\
\hline 70 & 20.0 & 28.3 & 39.8 & 43.5 & 54.7 & 76.0 & 80.3 & 109. & 123. \\
\hline 75 & 18.3 & 27.3 & 32.2 & 38.1 & 50.9 & 71.0 & 76.3 & 96.7 & 115. \\
\hline 80 & 16.9 & 21.4 & 30.0 & 36.3 & 47.9 & 66.3 & 71.0 & 86.5 & 107. \\
\hline 90 & 11.5 & 15.0 & 24.5 & 29.8 & 41.6 & 56.1 & 63.2 & 71.9 & 83.8 \\
\hline 95 & 7.75 & 12.2 & 22.9 & 27.0 & 37.8 & 50.9 & 56.8 & 62.8 & 77.2 \\
\hline 98 & 6.52 & 8.72 & 19.5 & 25.0 & 36.0 & 47.8 & 52.0 & 56.9 & 73.2 \\
\hline 99 & 5.96 & 6.97 & 17.5 & 24.3 & 30.2 & 46.9 & 52.0 & 55.7 & 71.3 \\
\hline
\end{tabular}


03269500 Mad River near Springfield, Ohio

Years analyzed $=96$

Climatic year range $=1904-2008$

[APEE, annual percentage equaled or exceeded; $\mathrm{ft}^{3} / \mathrm{s}$, cubic foot per second]

\begin{tabular}{|c|c|c|c|c|c|c|c|c|c|}
\hline \multirow[b]{2}{*}{ APEE } & \multicolumn{9}{|c|}{ Daily mean streamflow $\left(\mathrm{ft}^{3} / \mathrm{s}\right)$ that was equaled or exceeded the indicated percentage of time at APEE level } \\
\hline & 98 & 95 & 90 & 80 & 50 & 20 & 10 & 5 & 2 \\
\hline 1 & 661. & 1230. & 1300. & 1970. & 3010 & 4300. & 5060. & 5670. & 7980. \\
\hline 2 & 576. & 879. & 1040. & 1360. & 2040 & 2710 & 3500 . & 3680 & 4690 . \\
\hline 5 & 513. & 584. & 693. & 825. & 1210 & 1590. & 1920. & 2280 & 2390. \\
\hline 10 & 360. & 442. & 531. & 590. & 897. & 1150 & 1340. & 1530 & 1580. \\
\hline 20 & 285 & 323. & 356. & 423. & 613. & 822. & 921. & 1000 & 1060. \\
\hline 25 & 254 & 293. & 322. & 379. & 535. & 726. & 828. & 885. & 966. \\
\hline 30 & 211. & 266. & 291. & 339. & 491. & 667. & 753. & 802. & 859. \\
\hline 40 & 173. & 209. & 242. & 280. & 407. & 557. & 616. & 674. & 723. \\
\hline 50 & 155. & 175. & 206. & 247. & 346. & 480. & 534. & 574. & 664. \\
\hline 60 & 141. & 160. & 191. & 220. & 286. & 407. & 449. & 507. & 567. \\
\hline 70 & 131. & 152. & 171. & 196. & 252. & 356. & 386. & 443. & 485. \\
\hline 75 & 131. & 144. & 164. & 185. & 243. & 327. & 365. & 414. & 459. \\
\hline 80 & 128 & 136. & 155. & 178. & 224 & 311. & 344. & 397. & 431. \\
\hline 90 & 114. & 128. & 141. & 157. & 200 & 263. & 296. & 337. & 378. \\
\hline 95 & 105 & 119. & 131. & 151. & 193. & 243. & 271. & 305. & 346. \\
\hline 98 & 94.8 & 107. & 126. & 142. & 184. & 228. & 256. & 294. & 315. \\
\hline 99 & 89.9 & 103. & 122. & 137. & 179. & 223. & 253. & 286. & 294. \\
\hline
\end{tabular}

\begin{tabular}{|c|c|c|c|c|c|c|c|c|c|}
\hline \multirow[b]{2}{*}{ APEE } & \multicolumn{9}{|c|}{ 7-day mean streamflow ( $\mathrm{ft}^{3} / \mathrm{s}$ ) that was equaled or exceeded the indicated percentage of time at APEE level } \\
\hline & 98 & 95 & 90 & 80 & 50 & 20 & 10 & 5 & 2 \\
\hline 1 & 573. & 856. & 984. & 1370. & 1960. & 2880 . & 3700. & 4310 & 6000. \\
\hline 2 & 519. & 758. & 876. & 1090. & 1610. & 2260. & 2710. & 3070 . & 4250 . \\
\hline 5 & 500 & 594. & 672. & 857. & 1240. & 1630. & 2020. & 2260 & 2630 . \\
\hline 10 & 368 & 432. & 515. & 626. & 970. & 1210. & 1430. & 1530 & 1560. \\
\hline 20 & 302. & 330. & 363. & 443. & 655. & 886. & 1010. & 1110 & 1180. \\
\hline 25 & 260 & 284 & 329. & 391. & 581. & 789. & 927. & 970. & 1050 \\
\hline 30 & 200 & 259. & 300. & 355. & 518. & 701. & 836. & 881. & 961. \\
\hline 40 & 173. & 211. & 243. & 291. & 421. & 599. & 676. & 738. & 759. \\
\hline 50 & 157. & 184. & 213. & 253. & 361. & 501. & 560. & 623. & 706. \\
\hline 60 & 139. & 161. & 194. & 228. & 309. & 432. & 483. & 548. & 611. \\
\hline 70 & 134. & 154. & 177. & 200. & 263. & 374. & 411. & 471. & 512. \\
\hline 75 & 131. & 149. & 167. & 192. & 246. & 337. & 381. & 448. & 491. \\
\hline 80 & 129. & 143. & 157. & 184. & 231. & 321. & 359. & 422. & 458. \\
\hline 90 & 116. & 129. & 144. & 166. & 206. & 274. & 305. & 359. & 401. \\
\hline 95 & 110 & 120. & 135. & 154. & 197. & 257. & 284. & 312. & 357. \\
\hline 98 & 101. & 109. & 132. & 147. & 191. & 242. & 266. & 303. & 340. \\
\hline 99 & 100. & 105. & 127. & 142. & 187. & 237. & 259. & 296. & 327. \\
\hline
\end{tabular}




\section{Mad River near Dayton, Ohio}

Years analyzed $=90$

Climatic year range $=1915-2008$

[APEE, annual percentage equaled or exceeded; $\mathrm{ft}^{3} / \mathrm{s}$, cubic foot per second]

\begin{tabular}{|c|c|c|c|c|c|c|c|c|c|}
\hline \multirow[b]{2}{*}{ APEE } & \multicolumn{9}{|c|}{ Daily mean streamflow ( $\left.\mathrm{ft}^{3} / \mathrm{s}\right)$ that was equaled or exceeded the indicated percentage of time at APEE level } \\
\hline & 98 & 95 & 90 & 80 & 50 & 20 & 10 & 5 & 2 \\
\hline 1 & 858. & 1610. & 1810. & 2410. & 3870. & 5500. & 6610. & 7900. & 10000 \\
\hline 2 & 664. & 1220 & 1350. & 1850. & 2750 . & 3940. & 4570 . & 4940. & 6200. \\
\hline 5 & 573. & 752. & 924. & 1100. & 1710 . & 2210 . & 2690. & 3030. & 3310. \\
\hline 10 & 411. & 567. & 692. & 785. & 1180. & 1580. & 1880. & 2030. & 2300 . \\
\hline 20 & 332. & 399. & 448. & 582. & 801. & 1090. & 1210 & 1370 . & 1460. \\
\hline 25 & 292. & 346. & 418. & 505. & 705. & 959. & 1110 & 1200. & 1280. \\
\hline 30 & 254. & 296. & 358. & 441. & 642. & 860. & 981. & 1050. & 1120. \\
\hline 40 & 194. & 239. & 281. & 352. & 541. & 707. & 800. & 892. & 968. \\
\hline 50 & 173. & 206. & 253. & 306. & 460. & 598. & 683. & 758. & 837. \\
\hline 60 & 163. & 179. & 221. & 264. & 398. & 519. & 576. & 670. & 709. \\
\hline 70 & 154. & 168. & 202. & 234 & 334. & 438. & 491. & 582. & 637. \\
\hline 75 & 149. & 164. & 195. & 222. & 307. & 404. & 458. & 549. & 602. \\
\hline 80 & 147. & 160. & 185. & 213. & 279. & 378. & 425 & 517. & 564. \\
\hline 90 & 127. & 138. & 161. & 188. & 241. & 328. & 373. & 444. & 484. \\
\hline 95 & 120. & 130. & 150. & 172. & 223. & 309. & 341. & 397. & 437. \\
\hline 98 & 107. & 124. & 141. & 164. & 213. & 293. & 322. & 347. & 405. \\
\hline 99 & 104. & 124. & 138. & 158. & 204. & 282. & 303. & 330. & 392. \\
\hline
\end{tabular}

\begin{tabular}{|c|c|c|c|c|c|c|c|c|c|}
\hline \multirow[b]{2}{*}{ APEE } & \multicolumn{9}{|c|}{ 7-day mean streamflow ( $\left.\mathrm{ft}^{3} / \mathrm{s}\right)$ that was equaled or exceeded the indicated percentage of time at APEE level } \\
\hline & 98 & 95 & 90 & 80 & 50 & 20 & 10 & 5 & 2 \\
\hline 1 & 648. & 1090. & 1290. & 1760. & 2600 & 3780 & 4370 . & 6260. & 7410 . \\
\hline 5 & 537. & 726. & 891. & 1160. & 1650 & 2260 & 2610. & 3110. & 3400. \\
\hline 10 & 433. & 564. & 664. & 854. & 1230 & 1650. & 1910. & 2050 & 2150. \\
\hline 30 & 239. & 290. & 369. & 451. & 687. & 903. & 1110. & 1150 & 1280. \\
\hline 40 & 194. & 240. & 285. & 357. & 571. & 756. & 878. & 948. & 1050. \\
\hline 50 & 177. & 212. & 257. & 311. & 479. & 618. & 737. & 816. & 923. \\
\hline 60 & 166. & 177. & 224. & 270. & 399. & 538. & 635. & 717. & 790. \\
\hline 90 & 128. & 140. & 165. & 193. & 252 & 340. & 389. & 461. & 525. \\
\hline 95 & 121. & 132. & 150. & 176. & 228. & 317. & 359. & 402. & 457. \\
\hline 98 & 109. & 129. & 143. & 170. & 222. & 297. & 332. & 378. & 429. \\
\hline 99 & 108. & 128. & 141. & 164. & 216. & 294. & 330. & 361. & 419. \\
\hline
\end{tabular}




\section{Great Miami River at Dayton, Ohio}

Years analyzed $=96$

Climatic year range $=1913-2008$

[APEE, annual percentage equaled or exceeded; $\mathrm{ft}^{3} / \mathrm{s}$, cubic foot per second]

\begin{tabular}{|c|c|c|c|c|c|c|c|c|c|}
\hline \multirow[b]{2}{*}{ APEE } & \multicolumn{9}{|c|}{ Daily mean streamflow $\left(\mathrm{ft}^{3} / \mathrm{s}\right)$ that was equaled or exceeded the indicated percentage of time at APEE level } \\
\hline & 98 & 95 & 90 & 80 & 50 & 20 & 10 & 5 & 2 \\
\hline 1 & 2500 & 7590. & 10200. & 12900. & 18700. & 24000 . & 28300 & 34400 . & 36200 . \\
\hline 2 & 2170 & 5470. & 7870 . & 9650. & 14100 . & 18800 . & 21700 & 26500 & 30600 . \\
\hline 5 & 1580. & 3090. & 4010 . & 5510. & 9020. & 13100. & 14300 . & 14900. & 16200 \\
\hline 10 & 1070. & 1930. & 2210 & 3210 & 5040. & 7740 . & 9000. & 10100. & 10500 \\
\hline 20 & 800. & 1070. & 1360 . & 1880. & 2740 . & 4300. & 4860 . & 5540 . & 6020. \\
\hline 25 & 750. & 864. & 1170 . & 1510 . & 2310 . & 3520 . & 4090 . & 4710 . & 5000. \\
\hline 30 & 609. & 719. & 956. & 1250 & 1970. & 2880 . & 3470 . & 3930. & 4280 . \\
\hline 40 & 354. & 528. & 672. & 912. & 1530. & 2140. & 2500 & 2900. & 3190 . \\
\hline 50 & 300. & 396. & 520. & 647. & 1130. & 1630. & 1970. & 2250 & 2480 . \\
\hline 60 & 258 & 311. & 406. & 496. & 849. & 1330. & 1620 . & 1850. & 1980. \\
\hline 70 & 231. & 255. & 358. & 408. & 650. & 997. & 1270 & 1400 & 1580. \\
\hline 75 & 219. & 237. & 330. & 365. & 564. & 887. & 1110 & 1270 & 1400. \\
\hline 80 & 209. & 219. & 297. & 336. & 492. & 796. & 983. & 1070. & 1260. \\
\hline 90 & 164. & 189. & 215 & 283. & 396. & 598. & 708. & 847. & 1030. \\
\hline 95 & 145. & 163. & 187. & 255. & 361. & 523. & 625. & 780. & 889. \\
\hline 98 & 131. & 139. & 165. & 233. & 324. & 461. & 549. & 697. & 790. \\
\hline 99 & 119. & 131. & 154. & 224. & 316. & 445. & 527. & 661. & 757. \\
\hline
\end{tabular}

\begin{tabular}{|c|c|c|c|c|c|c|c|c|c|}
\hline APEE & \multicolumn{9}{|c|}{ 7-day mean streamflow ( $\mathrm{ft}^{3} / \mathrm{s}$ ) that was equaled or exceeded the indicated percentage of time at APEE level } \\
\hline 1 & 1980. & 5620. & 7080. & 9600. & 14500. & 18400. & 21700. & 27000. & 29500. \\
\hline 5 & 1490. & 2790. & 4090. & 5110. & 7950. & 11000. & 13300 . & 13800 . & 17200 \\
\hline 10 & 1050 & 2060. & 2540 & 3450. & 5260. & 7750 . & 8840. & 9680. & 10200 \\
\hline 30 & 531. & 723. & 1010 & 1350. & 2130 & 3400 . & 3820. & 4560 . & 5080. \\
\hline 40 & 354. & 532. & 709. & 950. & 1620. & 2480 & 2940. & 3390. & 3660. \\
\hline 50 & 303. & 414. & 533. & 660. & 1230. & 1850 & 2280 . & 2470 & 2980. \\
\hline 60 & 269. & 311. & 405. & 505. & 873. & 1500. & 1770. & 1920. & 2380 \\
\hline 90 & 180. & 196. & 209. & 299. & 421. & 625. & 738. & 941. & 1150. \\
\hline 95 & 151. & 166. & 195. & 267. & 375. & 555. & 662. & 803. & 976. \\
\hline 98 & 138. & 149. & 183. & 247. & 352. & 484. & 579. & 743. & 898. \\
\hline 99 & 131. & 139. & 169. & 241. & 338. & 468. & 539. & 704. & 870 . \\
\hline
\end{tabular}


03271500 Great Miami River at Miamisburg, Ohio

Years analyzed $=52$

Climatic year range $=1925-94$

[APEE, annual percentage equaled or exceeded; $\mathrm{ft}^{3} / \mathrm{s}$, cubic foot per second]

\begin{tabular}{|c|c|c|c|c|c|c|c|c|c|}
\hline \multirow[b]{2}{*}{ APEE } & \multicolumn{9}{|c|}{ Daily mean streamflow $\left(\mathrm{ft}^{3} / \mathrm{s}\right)$ that was equaled or exceeded the indicated percentage of time at APEE level } \\
\hline & 98 & 95 & 90 & 80 & 50 & 20 & 10 & 5 & 2 \\
\hline 1 & 2760 & 4310. & 9590. & 13000 & 18500 . & 24200 & 28400 & 35300. & 40100. \\
\hline 2 & 2050 & 3340. & 6180. & 9200. & 14300 . & 18500. & 20800 & 26300 & 34400 . \\
\hline 5 & 1470 & 2660 & 3600 . & 5310. & 9420. & 12600 & 14100 & 15500. & 16300. \\
\hline 10 & 1070 & 1510 . & 2320. & 3050 . & 5350. & 8320. & 9840. & 10400 & 11100 \\
\hline 20 & 616. & 1080 . & 1300. & 1800 & 2970. & 4540 . & 5410. & 6160. & 6230. \\
\hline 25 & 536. & 964. & 1070. & 1550 . & 2500 & 3670 . & 4540 . & 5010. & 5370 . \\
\hline 30 & 475 & 764. & 932. & 1380. & 2160 & 3130 . & 3850 . & 4420 . & 4630. \\
\hline 40 & 400 & 582. & 752. & 976. & 1570. & 2370 & 3000 . & 3290. & 3680 . \\
\hline 50 & 341. & 483. & 563. & 743. & 1210 & 1920. & 2420 & 2550 & 2940. \\
\hline 60 & 304. & 397. & 486. & 598. & 931. & 1620. & 1870 & 2160 & 2460 . \\
\hline 70 & 277. & 334. & 417. & 526. & 763. & 1220. & 1540. & 1730. & 2050. \\
\hline 75 & 266. & 318. & 376. & 485. & 677. & 1100. & 1380. & 1510 & 1860. \\
\hline 80 & 253. & 309. & 349. & 462. & 616. & 1000. & 1220. & 1310. & 1720. \\
\hline 90 & 232. & 265. & 295. & 377. & 500. & 752. & 863. & 1050 & 1320. \\
\hline 95 & 209. & 245. & 279. & 333. & 456. & 670. & 793. & 983. & 1140. \\
\hline 98 & 181. & 221. & 250. & 293. & 431. & 608. & 715. & 858. & 969. \\
\hline 99 & 168. & 210. & 240. & 280 & 421. & 598. & 689. & 824. & 917. \\
\hline
\end{tabular}

7-day mean streamflow $\left(\mathrm{ft}^{3} / \mathrm{s}\right)$ that was equaled or exceeded the indicated percentage of time at APEE level

\begin{tabular}{|c|c|c|c|c|c|c|c|c|c|}
\hline APEE & 98 & 95 & 90 & 80 & 50 & 20 & 10 & 5 & 2 \\
\hline 1 & 2060 & 3070 & 6280. & 9610. & 13700 . & 20100 & 23800 & 29600. & 33700 . \\
\hline 2 & 1690. & 2860 & 5330. & 7310 . & 11600 . & 15400 . & 17400 & 22000 & 28500 . \\
\hline 5 & 1390. & 2380 & 3390. & 4740 . & 8350. & 11300 & 12500 & 13800 . & 17600 \\
\hline 10 & 1070. & 1530. & 2410 . & 3120. & 5690. & 7960. & 8970. & 10000 . & 10800 \\
\hline 20 & 614. & 1080. & 1320. & 1950. & 3420 . & 5050. & 6250 & 6990. & 7540. \\
\hline 25 & 541. & 936. & 1090. & 1620. & 2860. & 4420 . & 5320. & 5690. & 6250. \\
\hline 30 & 478. & 724. & 937. & 1320. & 2320 . & 3820. & 4510. & 5120. & 5420. \\
\hline 40 & 399. & 584. & 763. & 979. & 1690. & 2640 & 3460. & 3640. & 4220 . \\
\hline 50 & 351. & 470. & 581. & 762. & 1270 & 2070 & 2640 & 2810 . & 3380 . \\
\hline 60 & 313. & 399. & 493. & 617. & 985. & 1740. & 2130 & 2350 . & 2690 . \\
\hline 70 & 279. & 331. & 437. & 542. & 802. & 1390. & 1710 & 1870. & 2060. \\
\hline 75 & 268. & 323. & 387. & 516. & 705. & 1210 & 1500 & 1630. & 1900. \\
\hline 80 & 252. & 308. & 358. & 465. & 639. & 1100. & 1280. & 1390. & 1770. \\
\hline 90 & 237. & 275 & 305. & 389. & 525. & 795. & 902. & 1090. & 1490. \\
\hline 95 & 224 & 243. & 286. & 340. & 472. & 688. & 850. & 1020. & 1260. \\
\hline 98 & 204. & 237. & 269. & 308. & 445. & 624. & 782. & 949. & 1160. \\
\hline 99 & 190. & 229. & 264. & 301. & 438. & 615. & 720. & 907. & 1050. \\
\hline
\end{tabular}




\section{Twin Creek near Germantown, Ohio}

Years analyzed $=84$

Climatic year range $=1921-2008$

[APEE, annual percentage equaled or exceeded; $\mathrm{ft}^{3} / \mathrm{s}$, cubic foot per second]

\begin{tabular}{|c|c|c|c|c|c|c|c|c|c|}
\hline \multirow[b]{2}{*}{ APEE } & \multicolumn{9}{|c|}{ Daily mean streamflow $\left(\mathrm{ft}^{3} / \mathrm{s}\right)$ that was equaled or exceeded the indicated percentage of time at APEE level } \\
\hline & 98 & 95 & 90 & 80 & 50 & 20 & 10 & 5 & 2 \\
\hline 1 & 414. & 1300. & 1550. & 2200. & 3520. & 4980. & 5500. & 5640. & 6500 \\
\hline 2 & 232. & 620. & 917. & 1230 & 2260 & 3490 . & 4120 . & 4570 . & 5320. \\
\hline 5 & 115. & 325. & 439. & 639. & 1080 & 1650. & 2050 . & 2410 . & 2760 \\
\hline 10 & 77.7 & 203. & 242. & 350 . & 593. & 875. & 1000 & 1260 . & 1360. \\
\hline 20 & 43.3 & 86.0 & 113. & 169. & 293. & 463. & 507. & 622. & 711. \\
\hline 25 & 35.3 & 61.5 & 97.5 & 131. & 232. & 344. & 411. & 478. & 547. \\
\hline 30 & 28.3 & 45.0 & 76.0 & 109. & 192. & 279. & 326. & 406. & 458. \\
\hline 40 & 16.6 & 24.3 & 43.5 & 71.6 & 130. & 200. & 246. & 279. & 316. \\
\hline 50 & 13.8 & 17.3 & 25.8 & 46.0 & 91.5 & 135. & 174. & 205. & 234. \\
\hline 60 & 9.05 & 11.5 & 15.0 & 28.0 & 61.5 & 95.0 & 126. & 150. & 179. \\
\hline 70 & 6.62 & 9.25 & 12.5 & 18.0 & 36.9 & 58.1 & 81.3 & 122 & 134. \\
\hline 75 & 6.27 & 8.64 & 11.5 & 15.0 & 28.8 & 46.5 & 70.3 & 103. & 117. \\
\hline 80 & 5.82 & 6.78 & 9.70 & 13.0 & 24.5 & 37.0 & 60.7 & 85.9 & 102. \\
\hline 90 & 4.11 & 4.81 & 6.55 & 9.10 & 15.0 & 26.6 & 34.4 & 55.2 & 74.3 \\
\hline 95 & 3.78 & 3.95 & 5.22 & 7.23 & 12.0 & 22.3 & 28.6 & 40.0 & 60.9 \\
\hline 98 & 3.12 & 3.47 & 4.13 & 5.70 & 11.0 & 19.0 & 23.5 & 33.7 & 45.5 \\
\hline 99 & 2.82 & 3.17 & 3.85 & 5.30 & 10.0 & 17.7 & 22.5 & 31.4 & 41.7 \\
\hline
\end{tabular}

\begin{tabular}{|c|c|c|c|c|c|c|c|c|c|}
\hline \multirow[b]{2}{*}{ APEE } & \multicolumn{9}{|c|}{ 7-day mean streamflow ( $\mathrm{ft}^{3} / \mathrm{s}$ ) that was equaled or exceeded the indicated percentage of time at APEE level } \\
\hline & 98 & 95 & 90 & 80 & 50 & 20 & 10 & 5 & 2 \\
\hline 1 & 179. & 789. & 902. & 1300 & 2090 & 3030. & 3460. & 3920. & 4940. \\
\hline 2 & 144. & 572. & 729. & 1040 & 1570 & 2270 & 2610 & 3160. & 4320 \\
\hline 5 & 122 & 387. & 500. & 709. & 1080 & 1530 & 1770 . & 2120 & 2460 \\
\hline 10 & 91.1 & 212. & 266. & 398. & 699. & 1050 & 1220. & 1370 . & 1500 \\
\hline 20 & 50.3 & 85.1 & 124. & 217. & 378. & 577. & 713. & 787. & 980. \\
\hline 25 & 34.8 & 60.5 & 97.7 & 156. & 291. & 452. & 567. & 627. & 808. \\
\hline 30 & 28.9 & 42.6 & 81.7 & 119. & 234. & 357. & 458. & 531. & 636. \\
\hline 40 & 18.9 & 24.2 & 47.1 & 78.7 & 154. & 242. & 306. & 365. & 474. \\
\hline 50 & 14.1 & 17.5 & 26.1 & 51.7 & 103. & 156. & 204. & 261. & 291. \\
\hline 60 & 9.55 & 11.7 & 15.4 & 28.3 & 67.6 & 114. & 147. & 181. & 228 \\
\hline 70 & 6.76 & 9.68 & 12.6 & 18.2 & 41.4 & 72.1 & 94.9 & 144. & 180. \\
\hline 75 & 6.43 & 8.39 & 11.6 & 16.3 & 31.0 & 55.7 & 75.1 & 123. & 147. \\
\hline 80 & 6.03 & 7.31 & 10.4 & 14.6 & 25.6 & 43.7 & 63.8 & 104. & 126. \\
\hline 90 & 4.26 & 5.06 & 6.51 & 9.19 & 15.8 & 28.1 & 36.8 & 70.8 & 88.7 \\
\hline 95 & 3.83 & 4.20 & 5.45 & 7.60 & 12.8 & 23.7 & 30.7 & 47.5 & 68.3 \\
\hline 98 & 3.31 & 3.59 & 4.49 & 6.36 & 11.6 & 20.9 & 26.3 & 39.9 & 51.6 \\
\hline 99 & 2.93 & 3.31 & 4.07 & 6.10 & 10.8 & 19.1 & 25.2 & 37.2 & 47.0 \\
\hline
\end{tabular}




\section{Great Miami River at Hamilton, Ohio}

Years analyzed $=78$

Climatic year range $=1931-2008$

[APEE, annual percentage equaled or exceeded; $\mathrm{ft}^{3} / \mathrm{s}$, cubic foot per second]

\begin{tabular}{|c|c|c|c|c|c|c|c|c|c|}
\hline \multirow[b]{2}{*}{ APEE } & \multicolumn{9}{|c|}{ Daily mean streamflow $\left(\mathrm{ft}^{3} / \mathrm{s}\right)$ that was equaled or exceeded the indicated percentage of time at APEE level } \\
\hline & 98 & 95 & 90 & 80 & 50 & 20 & 10 & 5 & 2 \\
\hline 1 & 6340 & 11900. & 13500 . & 16900 & 26600 & 33700 . & 38500. & 45100. & 57000. \\
\hline 2 & 4420 . & 8460 . & 10900. & 12400 & 19900. & 26300 & 30000 . & 36000. & 45300 . \\
\hline 5 & 3170 . & 4370 . & 6140. & 7860 . & 13400 . & 17400 & 20600 . & 22100 & 23500 . \\
\hline 10 & 1860. & 3010 . & 3700 . & 4880 . & 7950. & 11300 & 13400 & 14000 . & 15200 . \\
\hline 20 & 1250 & 1710 . & 1980. & 2880 & 4460 . & 6360. & 7490 . & 8260 & 9400. \\
\hline 25 & 1050 & 1420 . & 1610. & 2300 & 3680 . & 5370 . & 6200 & 6830. & 7640. \\
\hline 30 & 789. & 1210 & 1400. & 1930. & 3120 . & 4330 & 5400 . & 6050 & 6430. \\
\hline 40 & 560. & 785. & 1070 . & 1340. & 2430. & 3250 . & 4150 & 4400 & 4910. \\
\hline 50 & 460. & 624. & 834. & 997. & 1810. & 2530 & 3190. & 3460. & 3810. \\
\hline 60 & 408 & 461. & 637. & 802. & 1360. & 1990. & 2370 & 2770 . & 3200. \\
\hline 70 & 362. & 420. & 506. & 667. & 993. & 1560. & 1840. & 2350 & 2610. \\
\hline 75 & 342. & 402. & 448. & 589. & 847. & 1330. & 1590. & 2130 & 2360 . \\
\hline 80 & 328. & 379. & 419. & 536. & 756. & 1160. & 1460. & 1960. & 2180. \\
\hline 90 & 286. & 328. & 358. & 468. & 605. & 888. & 1070 & 1430. & 1780 . \\
\hline 95 & 258. & 306. & 320. & 406. & 556. & 796. & 979. & 1230. & 1610. \\
\hline 98 & 220 & 272. & 287. & 377. & 493. & 735 . & 865. & 1060. & 1330. \\
\hline 99 & 201. & 253. & 279. & 354. & 475. & 699. & 826. & 1000 & 1260. \\
\hline
\end{tabular}

7-day mean streamflow $\left(\mathrm{ft}^{3} / \mathrm{s}\right)$ that was equaled or exceeded the indicated percentage of time at APEE level

\begin{tabular}{|c|c|c|c|c|c|c|c|c|c|}
\hline APEE & 98 & 95 & 90 & 80 & 50 & 20 & 10 & 5 & 2 \\
\hline 1 & 4150 & 8320. & 10200 . & 12300 & 19100. & 26800 & 29500. & 38300. & 47500 . \\
\hline 2 & 4000 & 7450 & 8460. & 10100 & 15700 . & 21600 & 25400 & 28500 . & 42700 . \\
\hline 5 & 2720 & 4900. & 6040. & 6940. & 11800 . & 15900. & 19300. & 21700 & 23700 . \\
\hline 10 & 1900. & 3030 . & 3780 . & 4920. & 8130. & 11000 & 12800 & 14600 . & 15300 \\
\hline 20 & 1240 & 1780. & 2060. & 3080 . & 4910. & 7080 . & 7940. & 9090. & 10000 \\
\hline 25 & 1030 & 1420 . & 1660. & 2500 & 3940. & 5880. & 6820. & 7920. & 8620 . \\
\hline 30 & 769. & 1240 & 1460. & 1950. & 3330. & 5100. & 6010 & 6830. & 7340 . \\
\hline 40 & 563. & 815. & 1120. & 1400 & 2550 . & 3690. & 4560 & 5220. & 5500. \\
\hline 50 & 473. & 644. & 871. & 997. & 1880. & 2750 & 3440. & 4010. & 4440 . \\
\hline 60 & 404. & 451. & 668. & 832. & 1370 . & 2170 & 2610 . & 3100 . & 3740 . \\
\hline 70 & 362. & 415. & 507. & 674. & 1060. & 1660. & 2010 . & 2580. & 2870 . \\
\hline 75 & 343. & 403. & 468. & 603. & 904. & 1420. & 1750 & 2220. & 2640. \\
\hline 80 & 328. & 390. & 425. & 550. & 782. & 1280 & 1490. & 2030. & 2430 . \\
\hline 90 & 295. & 331. & 365. & 467. & 651. & 943. & 1140. & 1700. & 1950. \\
\hline 95 & 270 & 318. & 336. & 422. & 596. & 828. & 1030 & 1360. & 1750. \\
\hline 98 & 238 & 290. & 310. & 386. & 520. & 761. & 951. & 1290. & 1570. \\
\hline 99 & 220 & 282. & 308. & 378. & 505. & 732. & 868. & 1240. & 1400 . \\
\hline
\end{tabular}




\section{Maumee River at Antwerp, Ohio}

Years analyzed $=56$

Climatic year range $=1922-81$

[APEE, annual percentage equaled or exceeded; $\mathrm{ft}^{3} / \mathrm{s}$, cubic foot per second]

\begin{tabular}{|c|c|c|c|c|c|c|c|c|c|}
\hline \multirow[b]{2}{*}{ APEE } & \multicolumn{9}{|c|}{ Daily mean streamflow $\left(\mathrm{ft}^{3} / \mathrm{s}\right)$ that was equaled or exceeded the indicated percentage of time at APEE level } \\
\hline & 98 & 95 & 90 & 80 & 50 & 20 & 10 & 5 & 2 \\
\hline 1 & 4860. & 5440. & 5920. & 8690. & 11700 & 16300 & 17800 & 20900. & 23700 \\
\hline 2 & 3240 & 4510 . & 5170. & 7010 . & 10600. & 13400 & 15100 . & 16900. & 21100 \\
\hline 5 & 1620. & 2650 . & 3850 . & 4980. & 7210 . & 9240. & 11300. & 13600. & 14100 \\
\hline 10 & 1080. & 1590. & 2020 . & 2760 & 4770 . & 5740. & 7150 & 8020 . & 9580. \\
\hline 20 & 538. & 692. & 1170 . & 1410 . & 2410 & 3540 . & 4540 . & 5090. & 5780 . \\
\hline 25 & 428. & 501. & 866. & 1060. & 1840. & 2740 & 3660 . & 4030 . & 4460. \\
\hline 30 & 336. & 422. & 679. & 818. & 1480 . & 2290 & 2970 . & 3430 . & 3500 . \\
\hline 40 & 220 & 267. & 315. & 504. & 946. & 1530. & 1850 & 2170 & 2570 . \\
\hline 50 & 181. & 197. & 223. & 353. & 629. & 1010. & 1320 & 1560 & 1810. \\
\hline 60 & 154. & 162. & 171. & 243. & 413. & 749. & 950. & 1120. & 1360. \\
\hline 70 & 126. & 135. & 151. & 192. & 304. & 527. & 708. & 769. & 947. \\
\hline 75 & 111. & 120. & 134. & 176. & 259. & 438. & 548. & 650. & 755. \\
\hline 80 & 98.6 & 104. & 125. & 154. & 235 & 388 & 436. & 550. & 600. \\
\hline 90 & 77.5 & 81.4 & 99.9 & 121. & 167. & 254 & 309. & 352. & 450. \\
\hline 95 & 58.4 & 71.3 & 87.7 & 103. & 141. & 208 & 256. & 291. & 388. \\
\hline 98 & 49.0 & 62.2 & 71.0 & 85.4 & 122. & 176. & 204. & 253. & 327. \\
\hline 99 & 38.3 & 55.4 & 65.9 & 77.7 & 116. & 164. & 188. & 241. & 277. \\
\hline
\end{tabular}

\begin{tabular}{|c|c|c|c|c|c|c|c|c|c|}
\hline \multirow[b]{2}{*}{ APEE } & \multicolumn{9}{|c|}{ 7-day mean streamflow ( $\mathrm{ft}^{3} / \mathrm{s}$ ) that was equaled or exceeded the indicated percentage of time at APEE level } \\
\hline & 98 & 95 & 90 & 80 & 50 & 20 & 10 & 5 & 2 \\
\hline 1 & 3970. & 4590. & 4950. & 7000 & 10200. & 14600 & 16100. & 19000. & 22600 \\
\hline 2 & 2900. & 3430 & 4290. & 6210 & 8980. & 11800 & 14300 & 17200 . & 18500 \\
\hline 5 & 1520. & 2370 & 3240. & 5070 . & 6530. & 8760 & 9650. & 11500 & 12900 \\
\hline 10 & 1000 & 1550. & 1950. & 2670 . & 4570 . & 5650 & 7080 . & 7720 . & 8620 . \\
\hline 20 & 512. & 662. & 1200 & 1460 . & 2540 . & 3600. & 4590 . & 5020. & 5340. \\
\hline 25 & 420. & 476. & 888. & 1100 & 2100. & 2950 . & 3810 . & 4110. & 4390. \\
\hline 30 & 335. & 403. & 701. & 946. & 1590. & 2530 & 3060. & 3370 . & 3720. \\
\hline 40 & 223. & 265. & 320 . & 557. & 960. & 1640. & 2070 . & 2570 & 2800 . \\
\hline 50 & 181. & 188. & 229. & 359. & 647. & 1090. & 1490. & 1730 & 2030. \\
\hline 60 & 157. & 164. & 171. & 250 & 432. & 798. & 1060 . & 1200 & 1490. \\
\hline 70 & 123. & 138. & 151. & 198. & 320 . & 574. & 764. & 857. & 1050. \\
\hline 75 & 111. & 123. & 142. & 179. & 279. & 472. & 652. & 744. & 914. \\
\hline 80 & 106. & 110. & 131. & 159. & 242. & 406. & 508. & 645. & 664. \\
\hline 90 & 81.8 & 88.8 & 104. & 124. & 184. & 291. & 338. & 406. & 471. \\
\hline 95 & 68.9 & 73.4 & 94.2 & 110. & 158. & 229. & 284. & 317. & 419. \\
\hline 98 & 59.5 & 64.2 & 76.8 & 95.7 & 143. & 199. & 257. & 283. & 350. \\
\hline 99 & 48.4 & 61.3 & 73.1 & 88.1 & 134. & 181. & 235. & 274. & 315. \\
\hline
\end{tabular}


04185000 Tiffin River at Stryker, Ohio

Years analyzed $=74$

Climatic year range $=1922-2008$

[APEE, annual percentage equaled or exceeded; $\mathrm{ft}^{3} / \mathrm{s}$, cubic foot per second]

\begin{tabular}{|c|c|c|c|c|c|c|c|c|c|}
\hline \multirow[b]{2}{*}{ APEE } & \multicolumn{9}{|c|}{ Daily mean streamflow $\left(\mathrm{ft}^{3} / \mathrm{s}\right)$ that was equaled or exceeded the indicated percentage of time at APEE level } \\
\hline & 98 & 95 & 90 & 80 & 50 & 20 & 10 & 5 & 2 \\
\hline 1 & 770. & 1510. & 1630. & 1890. & 2590. & 3700. & 4230. & 4530 & 5780. \\
\hline 2 & 651. & 989. & 1320. & 1520. & 2090. & 2980 . & 3220 & 3750 . & 4130. \\
\hline 5 & 335. & 534. & 714. & 967. & 1430. & 1960. & 2130 & 2280 & 2420 . \\
\hline 10 & 220 & 337. & 414. & 629. & 983. & 1340. & 1490. & 1560. & 1680 \\
\hline 20 & 93.5 & 177. & 209. & 279 & 481. & 744. & 900. & 950. & 1000. \\
\hline 25 & 62.5 & 137. & 169. & 210 & 369. & 536. & 668. & 738. & 789. \\
\hline 30 & 50.0 & 106. & 124. & 155. & 293. & 413. & 478. & 546. & 612. \\
\hline 40 & 29.5 & 45.0 & 67.6 & 102. & 207. & 279. & 336. & 354. & 392. \\
\hline 50 & 24.0 & 29.8 & 46.0 & 64.0 & 138. & 187. & 248. & 270 & 298. \\
\hline 60 & 18.5 & 23.5 & 35.7 & 46.0 & 92.9 & 140. & 185. & 203. & 230 \\
\hline 70 & 14.5 & 20.1 & 28.5 & 36.0 & 58.9 & 104. & 131. & 141. & 164. \\
\hline 75 & 12.8 & 18.5 & 24.9 & 28.0 & 49.5 & 77.0 & 111. & 116. & 142. \\
\hline 80 & 11.5 & 18.3 & 20.5 & 26.4 & 41.0 & 63.4 & 87.6 & 100. & 131. \\
\hline 90 & 8.70 & 9.78 & 14.0 & 18.0 & 24.8 & 42.6 & 52.3 & 74.9 & 99.0 \\
\hline 95 & 6.39 & 6.91 & 9.88 & 15.0 & 20.0 & 36.0 & 41.0 & 52.2 & 73.4 \\
\hline 98 & 4.30 & 5.76 & 8.14 & 12.0 & 17.7 & 31.6 & 37.2 & 43.8 & 59.7 \\
\hline 99 & 3.62 & 5.12 & 7.26 & 11.0 & 16.5 & 31.0 & 34.8 & 40.2 & 57.0 \\
\hline
\end{tabular}

\begin{tabular}{|c|c|c|c|c|c|c|c|c|c|}
\hline \multirow[b]{2}{*}{ APEE } & \multicolumn{9}{|c|}{ 7-day mean streamflow ( $\mathrm{ft}^{3} / \mathrm{s}$ ) that was equaled or exceeded the indicated percentage of time at APEE level } \\
\hline & 98 & 95 & 90 & 80 & 50 & 20 & 10 & 5 & 2 \\
\hline 1 & 684. & 1170. & 1260. & 1580. & 2260. & 2890. & 3490. & 3730. & 4900 \\
\hline 2 & 400 & 931. & 1090. & 1270 & 1830 & 2510 & 2670 & 3070 & 3710. \\
\hline 5 & 276 & 459. & 756. & 923. & 1360 & 1800 & 1960. & 2150 & 2320 \\
\hline 10 & 185. & 305. & 405 & 620. & 910. & 1270 & 1430. & 1510. & 1570 . \\
\hline 20 & 92.5 & 185. & 224 & 312. & 508. & 726. & 909. & 937. & 1030. \\
\hline 25 & 61.7 & 143. & 171. & 219 & 405. & 613. & 727. & 783. & 823. \\
\hline 30 & 50.9 & 102. & 127. & 168. & 321. & 485. & 590. & 654. & 699. \\
\hline 40 & 29.9 & 46.1 & 71.6 & 102. & 227. & 316. & 409. & 455. & 490. \\
\hline 50 & 24.5 & 29.5 & 46.8 & 68.6 & 142. & 206. & 277. & 312. & 345. \\
\hline 60 & 19.4 & 24.0 & 36.1 & 48.6 & 99.5 & 153. & 207. & 233. & 263. \\
\hline 70 & 15.1 & 20.0 & 29.8 & 38.3 & 66.9 & 112. & 149. & 156. & 191. \\
\hline 75 & 13.4 & 18.8 & 24.9 & 29.3 & 53.3 & 82.6 & 122. & 128. & 154. \\
\hline 80 & 11.7 & 18.0 & 21.1 & 28.0 & 43.1 & 67.5 & 99.6 & 107. & 142. \\
\hline 90 & 8.85 & 11.1 & 14.5 & 18.3 & 27.0 & 46.3 & 56.5 & 78.9 & 110. \\
\hline 95 & 6.62 & 7.41 & 10.8 & 15.3 & 20.4 & 37.4 & 44.7 & 56.3 & 81.2 \\
\hline 98 & 4.66 & 6.03 & 8.90 & 13.1 & 18.5 & 33.0 & 39.6 & 50.0 & 65.6 \\
\hline 99 & 4.14 & 5.77 & 8.48 & 12.1 & 18.0 & 32.7 & 37.2 & 46.9 & 61.9 \\
\hline
\end{tabular}




\section{Ottawa River at Allentown, Ohio}

Years analyzed $=48$

Climatic year range $=1924-80$

[APEE, annual percentage equaled or exceeded; $\mathrm{ft}^{3} / \mathrm{s}$, cubic foot per second]

\begin{tabular}{|c|c|c|c|c|c|c|c|c|c|}
\hline \multirow[b]{2}{*}{ APEE } & \multicolumn{9}{|c|}{ Daily mean streamflow $\left(\mathrm{ft}^{3} / \mathrm{s}\right)$ that was equaled or exceeded the indicated percentage of time at APEE level } \\
\hline & 95 & 90 & 80 & 70 & 50 & 30 & 20 & 10 & 5 \\
\hline 1 & 432. & 637. & 1030. & 1120. & 1430. & 2010. & 2210. & 2320 & 2480 \\
\hline 2 & 291. & 427. & 668. & 802. & 1090. & 1370 . & 1470 . & 1730 . & 1820. \\
\hline 5 & 121. & 209. & 296. & 385. & 547. & 782. & 828. & 909. & 1040. \\
\hline 10 & 63.8 & 77.2 & 143. & 183. & 244. & 378. & 459. & 556. & 618. \\
\hline 20 & 35.7 & 37.9 & 63.2 & 80.0 & 104. & 158. & 190. & 297. & 316. \\
\hline 25 & 26.0 & 30.0 & 44.2 & 63.9 & 78.5 & 111. & 139. & 202. & 236. \\
\hline 30 & 21.1 & 27.8 & 35.6 & 49.0 & 62.5 & 83.4 & 107. & 149. & 195. \\
\hline 40 & 17.4 & 22.9 & 27.8 & 36.1 & 45.6 & 54.5 & 72.0 & 103. & 118. \\
\hline 50 & 15.6 & 20.0 & 22.8 & 27.0 & 35.0 & 42.3 & 47.6 & 68.2 & 75.8 \\
\hline 60 & 13.8 & 18.0 & 20.8 & 22.7 & 28.0 & 34.0 & 36.8 & 47.5 & 52.9 \\
\hline 70 & 11.4 & 14.0 & 18.0 & 19.7 & 24.0 & 28.3 & 31.0 & 37.3 & 41.1 \\
\hline 75 & 11.0 & 13.8 & 16.0 & 18.9 & 22.0 & 26.3 & 29.1 & 35.0 & 38.0 \\
\hline 80 & 9.47 & 12.8 & 15.8 & 18.7 & 20.5 & 24.0 & 28.1 & 32.3 & 35.1 \\
\hline 90 & 7.47 & 9.98 & 13.6 & 16.0 & 18.0 & 21.2 & 24.8 & 28.1 & 31.5 \\
\hline 95 & 6.48 & 9.26 & 11.8 & 15.0 & 17.0 & 19.3 & 23.1 & 26.2 & 29.1 \\
\hline 98 & 5.92 & 8.81 & 11.0 & 12.7 & 16.0 & 18.0 & 21.2 & 25.1 & 27.0 \\
\hline 99 & 5.64 & 8.61 & 9.75 & 11.7 & 15.0 & 17.8 & 20.0 & 24.0 & 25.0 \\
\hline
\end{tabular}

\begin{tabular}{|c|c|c|c|c|c|c|c|c|c|}
\hline \multirow[b]{2}{*}{ APEE } & \multicolumn{9}{|c|}{ 7-day mean streamflow ( $\mathrm{ft}^{3} / \mathrm{s}$ ) that was equaled or exceeded the indicated percentage of time at APEE level } \\
\hline & 95 & 90 & 80 & 70 & 50 & 30 & 20 & 10 & 5 \\
\hline 1 & 293. & 376. & 657. & 794. & 1010 & 1210 & 1440 & 1740 & 1770 \\
\hline 2 & 236. & 306. & 487. & 622. & 747. & 927. & 1030. & 1270 & 1310 \\
\hline 5 & 119. & 221. & 357. & 430 & 541. & 673. & 723. & 851. & 978. \\
\hline 10 & 66.0 & 86.7 & 181. & 230. & 313. & 444. & 501. & 551. & 593. \\
\hline 20 & 33.5 & 39.1 & 67.9 & 98.2 & 128 & 208. & 283. & 352. & 397. \\
\hline 25 & 29.5 & 33.9 & 55.8 & 71.3 & 98.9 & 158. & 201. & 273. & 324. \\
\hline 30 & 26.9 & 28.9 & 40.9 & 60.1 & 77.5 & 118. & 144. & 218. & 261. \\
\hline 40 & 19.9 & 23.9 & 29.7 & 40.0 & 53.0 & 64.4 & 89.6 & 136. & 173. \\
\hline 50 & 16.4 & 21.0 & 24.7 & 30.3 & 40.0 & 48.2 & 54.2 & 86.7 & 117. \\
\hline 60 & 14.9 & 18.9 & 22.1 & 24.2 & 30.6 & 37.3 & 42.1 & 61.3 & 67.4 \\
\hline 70 & 12.5 & 15.3 & 18.5 & 20.9 & 24.9 & 31.1 & 34.5 & 43.6 & 50.0 \\
\hline 75 & 11.7 & 14.3 & 17.7 & 20.1 & 23.6 & 29.0 & 32.6 & 37.2 & 42.5 \\
\hline 80 & 11.0 & 13.5 & 16.8 & 19.5 & 22.5 & 27.5 & 30.1 & 34.9 & 39.1 \\
\hline 90 & 8.95 & 11.2 & 13.9 & 16.7 & 19.6 & 23.0 & 26.2 & 29.6 & 33.7 \\
\hline 95 & 6.95 & 10.5 & 12.2 & 15.6 & 18.1 & 20.9 & 24.8 & 28.3 & 30.5 \\
\hline 98 & 6.18 & 9.18 & 11.3 & 13.9 & 17.5 & 20.3 & 23.0 & 27.4 & 27.9 \\
\hline 99 & 5.87 & 8.93 & 11.0 & 13.1 & 17.0 & 19.4 & 22.1 & 26.8 & 27.6 \\
\hline
\end{tabular}




\section{Auglaize River near Defiance, Ohio}

Years analyzed $=92$

Climatic year range $=1917-2008$

[APEE, annual percentage equaled or exceeded; $\mathrm{ft}^{3} / \mathrm{s}$, cubic foot per second]

\begin{tabular}{|c|c|c|c|c|c|c|c|c|c|}
\hline \multirow[b]{2}{*}{ APEE } & \multicolumn{9}{|c|}{ Daily mean streamflow ( $\left.\mathrm{ft}^{3} / \mathrm{s}\right)$ that was equaled or exceeded the indicated percentage of time at APEE level } \\
\hline & 98 & 95 & 90 & 80 & 50 & 20 & 10 & 5 & 2 \\
\hline 1 & 4370. & 8250. & 9420. & 13000. & 18800. & 25200. & 29500. & 32600 . & 36600 . \\
\hline 2 & 3390. & 6430. & 7650 & 9750. & 14500 . & 18700 & 22800 & 26200 . & 30500 . \\
\hline 5 & 2270 & 3330. & 4190. & 5850. & 8940. & 11700 & 14400 . & 15700 . & 18400 . \\
\hline 10 & 836. & 1590. & 2330 & 2810 & 5020. & 7150 . & 8670. & 10400. & 12000 . \\
\hline 20 & 365. & 619. & 896. & 1180. & 2160. & 3410 . & 4410. & 4960. & 5760 . \\
\hline 25 & 283. & 448. & 646. & 836. & 1550. & 2680 & 3290. & 3540 . & 4560. \\
\hline 30 & 218. & 301. & 490. & 611. & 1190. & 2020 . & 2570 & 2690 & 3480 . \\
\hline 40 & 113. & 137. & 280 & 352. & 702. & 1140. & 1520. & 1720 . & 2010. \\
\hline 50 & 42.9 & 73.6 & 129. & 217. & 477. & 779. & 982. & 1180. & 1380. \\
\hline 60 & 34.9 & 39.0 & 65.7 & 135. & 292. & 511. & 662. & 829. & 1010. \\
\hline 70 & 30.0 & 33.8 & 50.0 & 75.9 & 185. & 325. & 448. & 589. & 717. \\
\hline 75 & 26.9 & 31.3 & 40.6 & 53.8 & 138. & 231. & 339. & 512. & 652. \\
\hline 80 & 24.9 & 30.0 & 32.8 & 40.6 & 93.1 & 191. & 255. & 460. & 561. \\
\hline 90 & 8.30 & 20.0 & 25.0 & 31.6 & 49.0 & 99.6 & 163. & 267. & 387. \\
\hline 95 & 4.72 & 13.6 & 20.0 & 25.6 & 36.6 & 79.2 & 94.4 & 168. & 292. \\
\hline 98 & 3.75 & 9.51 & 17.0 & 20.4 & 31.5 & 52.8 & 73.9 & 123. & 167. \\
\hline 99 & 3.42 & 7.62 & 12.4 & 19.0 & 29.8 & 46.5 & 62.2 & 77.2 & 139. \\
\hline
\end{tabular}

\begin{tabular}{|c|c|c|c|c|c|c|c|c|c|}
\hline \multirow[b]{2}{*}{ APEE } & \multicolumn{9}{|c|}{ 7-day mean streamflow $\left(\mathrm{ft}^{3} / \mathrm{s}\right)$ that was equaled or exceeded the indicated percentage of time at APEE level } \\
\hline & 98 & 95 & 90 & 80 & 50 & 20 & 10 & 5 & 2 \\
\hline 1 & 4160. & 6210. & 6940. & 8430. & 14300 & 19300. & 23300 & 25500 & 29500. \\
\hline 5 & 1760. & 3430 & 4300 & 5360. & 8320 & 10900. & 12400 & 14200 & 17000 . \\
\hline 10 & 704. & 1540. & 2210 & 3140 . & 5120. & 7250 & 8340. & 9500. & 10900. \\
\hline 30 & 219. & 315. & 548. & 718. & 1420 & 2370 & 3200 . & 3810. & 4280 . \\
\hline 40 & 145. & 156. & 305. & 387. & 812. & 1420. & 2070 . & 2500 & 3060 . \\
\hline 50 & 90.4 & 100 & 138. & 237. & 572. & 935. & 1300 & 1570 & 1960. \\
\hline 60 & 56.8 & 71.0 & 87.1 & 165. & 334. & 595. & 820. & 1080. & 1300. \\
\hline 90 & 11.3 & 26.0 & 33.3 & 46.7 & 80.8 & 132. & 194. & 324. & 492. \\
\hline 95 & 5.31 & 19.4 & 27.5 & 34.8 & 65.0 & 99.0 & 152. & 220. & 392. \\
\hline 98 & 4.61 & 13.4 & 21.9 & 27.9 & 47.1 & 79.9 & 106. & 191. & 298. \\
\hline 99 & 3.62 & 11.7 & 19.7 & 24.4 & 42.4 & 70.2 & 92.4 & 166. & 270. \\
\hline
\end{tabular}




\section{Maumee River near Defiance, Ohio}

Years analyzed $=75$

Climatic year range $=1925-2008$

[APEE, annual percentage equaled or exceeded; $\mathrm{ft}^{3} / \mathrm{s}$, cubic foot per second]

\begin{tabular}{|c|c|c|c|c|c|c|c|c|c|}
\hline \multirow[b]{2}{*}{ APEE } & \multicolumn{9}{|c|}{ Daily mean streamflow $\left(\mathrm{ft}^{3} / \mathrm{s}\right)$ that was equaled or exceeded the indicated percentage of time at APEE level } \\
\hline & 98 & 95 & 90 & 80 & 50 & 20 & 10 & 5 & 2 \\
\hline 1 & 12700 & 15800 & 20900. & 25800 & 35900. & 47700 & 57300. & 61100 & 69800. \\
\hline 2 & 9730. & 12900. & 16800. & 19500. & 29600. & 37500 . & 41200. & 47400 . & 59700. \\
\hline 5 & 4880. & 8060 . & 10300 . & 13000 . & 20100 . & 25800 & 29600. & 33300 . & 37500 . \\
\hline 10 & 2680 & 3880 . & 5680. & 7550 . & 12200 . & 17800 & 21000 & 22300 & 24800 . \\
\hline 20 & 1040. & 1800. & 2670 . & 3420 . & 6440. & 9580. & 11700 . & 13200. & 13500 . \\
\hline 25 & 779. & 1250. & 2090 . & 2530 . & 5040. & 7580 & 9160. & 10400. & 11200. \\
\hline 30 & 588. & 813. & 1550 . & 1920. & 3660 . & 6070 & 7340 . & 8200 . & 9080. \\
\hline 40 & 449. & 485. & 814. & 1260. & 2360 & 3680. & 4680 & 5180. & 6310. \\
\hline 50 & 269. & 312. & 457. & 746. & 1560. & 2490 & 3190. & 3620. & 4150. \\
\hline 60 & 191. & 238 & 297. & 514. & 1070 . & 1650. & 2250 & 2570 & 2890. \\
\hline 70 & 142. & 192. & 240 & 347. & 700 . & 1180. & 1590. & 2060 . & 2080. \\
\hline 75 & 126. & 175. & 220 & 305. & 600. & 911. & 1400 & 1720. & 1880. \\
\hline 80 & 114. & 135. & 190. & 251. & 474. & 716. & 1230. & 1380. & 1540. \\
\hline 90 & 68.9 & 94.4 & 128. & 172. & 302. & 467. & 781. & 931. & 1070. \\
\hline 95 & 54.4 & 68.0 & 97.1 & 135. & 255. & 367. & 529. & 709. & 764. \\
\hline 98 & 30.1 & 56.4 & 76.6 & 103. & 208. & 313. & 426. & 558. & 652. \\
\hline 99 & 16.7 & 49.8 & 71.1 & 90.4 & 180. & 290. & 376. & 505. & 560. \\
\hline
\end{tabular}

\begin{tabular}{|c|c|c|c|c|c|c|c|c|c|}
\hline \multirow[b]{2}{*}{ APEE } & \multicolumn{9}{|c|}{ 7-day mean streamflow ( $\mathrm{ft}^{3} / \mathrm{s}$ ) that was equaled or exceeded the indicated percentage of time at APEE level } \\
\hline & 98 & 95 & 90 & 80 & 50 & 20 & 10 & 5 & 2 \\
\hline 1 & 10400 & 13700. & 16100. & 19300. & 26800 & 38800 . & 49200. & 52300. & 61100. \\
\hline 2 & 8340. & 9970. & 14800 . & 16800 & 23900 . & 30900. & 36200 . & 39900. & 49900. \\
\hline 5 & 4370. & 7970. & 10200 . & 12100 & 18000 & 23400 . & 25900. & 29200 . & 35500 . \\
\hline 10 & 2400 & 3370 . & 5530. & 7390. & 11900. & 16700. & 19800. & 20600 . & 23600 \\
\hline 20 & 979. & 1870 . & 3070 . & 3840 . & 7110. & 10700 . & 12400 & 13600. & 15000 \\
\hline 25 & 733. & 1280 & 2260 & 2850. & 5620. & 8820. & 10100 . & 11200 & 12700 \\
\hline 30 & 563. & 831. & 1660 & 2080. & 4320. & 6700. & 8210 . & 9530. & 10700 \\
\hline 40 & 442. & 477. & 889. & 1300. & 2760 . & 4360. & 5580. & 6440. & 7030 . \\
\hline 50 & 280. & 324. & 473. & 804. & 1810. & 2880 . & 3810. & 4580 . & 5260 . \\
\hline 60 & 193. & 246. & 303. & 544. & 1210. & 1940. & 2720 . & 3160 . & 3600 . \\
\hline 70 & 155. & 204. & 250 & 380. & 757. & 1290. & 1870 . & 2270 & 2510 . \\
\hline 75 & 138. & 190. & 222 & 317. & 647. & 1040. & 1630 & 2050 & 2190. \\
\hline 80 & 119. & 170. & 204. & 280. & 508. & 802. & 1420 & 1630. & 1860. \\
\hline 90 & 77.1 & 103. & 139. & 193. & 316. & 511. & 878. & 1070 . & 1300. \\
\hline 95 & 66.2 & 71.2 & 98.8 & 150. & 268. & 418. & 628. & 837. & 981. \\
\hline 98 & 49.6 & 62.9 & 83.8 & 119. & 232. & 360. & 492. & 696. & 773. \\
\hline 99 & 37.9 & 59.5 & 75.2 & 110. & 216. & 319. & 433. & 614. & 706. \\
\hline
\end{tabular}




\section{Maumee River at Waterville, Ohio}

Years analyzed $=85$

Climatic year range $=1899-2008$

[APEE, annual percentage equaled or exceeded; $\mathrm{ft}^{3} / \mathrm{s}$, cubic foot per second]

\begin{tabular}{|c|c|c|c|c|c|c|c|c|c|}
\hline \multirow[b]{2}{*}{ APEE } & \multicolumn{9}{|c|}{ Daily mean streamflow ( $\left.\mathrm{ft}^{3} / \mathrm{s}\right)$ that was equaled or exceeded the indicated percentage of time at APEE level } \\
\hline & 98 & 95 & 90 & 80 & 50 & 20 & 10 & 5 & 2 \\
\hline 1 & 16900. & 19100. & 23000. & 29100. & 40800. & 54600. & 64900. & 67500 & 77400. \\
\hline 2 & 12400. & 15900. & 18300. & 22000 & 32900 . & 44000. & 47400 & 54500. & 67300. \\
\hline 5 & 6420. & 9380. & 11800. & 15000 & 22400 & 29400. & 32800. & 36200. & 44500 . \\
\hline 10 & 3030 . & 5380. & 6880. & 9030. & 14000. & 19700. & 22400 & 24100 & 28100 . \\
\hline 20 & 1340. & 2400 . & 3130. & 4390. & 7280. & 10900. & 13300. & 15000. & 15600. \\
\hline 25 & 986. & 1610. & 2430. & 2980. & 5560. & 8090. & 10800 & 11700. & 12800. \\
\hline 30 & 722. & 1060. & 1790. & 2300 & 4210. & 6280 & 8270. & 9410. & 10300. \\
\hline 40 & 475. & 573. & 922. & 1410 & 2600. & 3870. & 5420. & 5830. & 6480. \\
\hline 50 & 322. & 363. & 505. & 850. & 1780. & 2690. & 3680. & 4000 & 4630. \\
\hline 60 & 256. & 268. & 322. & 490. & 1170. & 1900. & 2560 & 2920. & 3460 . \\
\hline 70 & 108. & 170. & 225. & 308. & 780. & 1280. & 1850. & 2150 & 2460 . \\
\hline 75 & 86.4 & 106. & 196. & 264. & 616. & 990. & 1530. & 1850. & 2110. \\
\hline 80 & 74.3 & 78.0 & 142. & 229. & 469. & 779. & 1250. & 1660. & 1870. \\
\hline 90 & 25.8 & 62.0 & 91.4 & 159. & 310. & 507. & 807. & 1050. & 1320. \\
\hline 95 & 15.8 & 57.5 & 70.0 & 121. & 238. & 408. & 587. & 787. & 982. \\
\hline 98 & 8.60 & 50.6 & 62.5 & 91.2 & 186. & 346. & 461. & 639. & 743. \\
\hline 99 & 5.00 & 36.9 & 50.0 & 78.2 & 163. & 321. & 417. & 573. & 641. \\
\hline
\end{tabular}

7-day mean streamflow ( $\left.\mathrm{ft}^{3} / \mathrm{s}\right)$ that was equaled or exceeded the indicated percentage of time at APEE level

\begin{tabular}{|c|c|c|c|c|c|c|c|c|c|}
\hline APEE & 98 & 95 & 90 & 80 & 50 & 20 & 10 & 5 & 2 \\
\hline 1 & 13300 . & 15300 . & 18700 . & 22400 & 32300 . & 44800. & 54600. & 59000. & 70600 \\
\hline 2 & 11000 & 12400 & 16200 & 19100. & 26700 & 35200. & 39800. & 47100 . & 57500. \\
\hline 5 & 5950. & 9980. & 11200 . & 14400 & 20000 . & 26400 & 29000. & 33000 . & 40000 . \\
\hline 10 & 2830 & 5000 . & 6840. & 8730. & 13800 . & 18200. & 22000 & 22800 & 25500 . \\
\hline 20 & 1290. & 2400 & 3470 . & 4750 . & 8080 . & 11200 & 13700 & 15300 . & 17300 . \\
\hline 25 & 925. & 1690. & 2590. & 3530. & 6200. & 9480. & 11300 & 12500 . & 14600 . \\
\hline 30 & 661. & 1040. & 1990. & 2450 & 4690. & 7390 . & 9580. & 10300 . & 12100 \\
\hline 40 & 466. & 571. & 961. & 1530. & 2980 . & 4600 . & 6520 & 7300. & 7690. \\
\hline 50 & 338. & 381. & 519. & 862. & 2040 . & 3190. & 4410 & 4950. & 5380. \\
\hline 60 & 266. & 291. & 333. & 511. & 1230. & 2300 & 2970 . & 3360 . & 3970 . \\
\hline 70 & 134. & 190. & 239. & 373. & 855. & 1430. & 2160 & 2570. & 2770 . \\
\hline 75 & 92.2 & 130. & 202. & 280 & 688. & 1140. & 1860 & 2040. & 2500 . \\
\hline 80 & 78.3 & 92.5 & 172. & 242 & 526. & 867. & 1560. & 1770. & 2230 . \\
\hline 90 & 36.9 & 67.3 & 103. & 183. & 338. & 534. & 890. & 1300. & 1540. \\
\hline 95 & 25.9 & 58.7 & 85.1 & 138. & 264. & 454. & 722. & 898. & 1220. \\
\hline 98 & 17.9 & 57.3 & 70.6 & 112. & 225. & 389. & 499. & 718. & 1020. \\
\hline 99 & 15.8 & 55.3 & 66.7 & 108. & 199. & 359. & 477. & 661. & 892. \\
\hline
\end{tabular}


04195500 Portage River at Woodville, Ohio

Years analyzed $=57$

Climatic year range $=1952-2008$

[APEE, annual percentage equaled or exceeded; $\mathrm{ft}^{3} / \mathrm{s}$, cubic foot per second]

\begin{tabular}{|c|c|c|c|c|c|c|c|c|c|}
\hline \multirow[b]{2}{*}{ APEE } & \multicolumn{9}{|c|}{ Daily mean streamflow $\left(\mathrm{ft}^{3} / \mathrm{s}\right)$ that was equaled or exceeded the indicated percentage of time at APEE level } \\
\hline & 98 & 95 & 90 & 80 & 50 & 20 & 10 & 5 & 2 \\
\hline 1 & 1500. & 1570. & 2120. & 2650. & 4200. & 5450. & 6910. & 7830 & 8210 \\
\hline 2 & 817. & 1190. & 1380. & 1930. & 3190. & 3980. & 4700. & 5420. & 6380 \\
\hline 5 & 437. & 673. & 918. & 1020 & 1740 & 2410 & 2950. & 3300 . & 4340. \\
\hline 10 & 148. & 277. & 400 & 489. & 879. & 1300 . & 1600. & 2040 & 2200 \\
\hline 20 & 57.1 & 125. & 156. & 221. & 372. & 586. & 730 . & 926. & 1050. \\
\hline 25 & 39.3 & 91.9 & 110. & 158. & 270 & 430 . & 539. & 685. & 725. \\
\hline 30 & 32.8 & 62.7 & 79.8 & 118. & 203. & 318. & 403. & 537. & 612. \\
\hline 40 & 21.2 & 31.5 & 44.7 & 72.6 & 122. & 202. & 251. & 335. & 382. \\
\hline 50 & 12.3 & 14.9 & 20.4 & 44.4 & 83.0 & 128. & 176. & 234. & 265. \\
\hline 60 & 8.24 & 10.0 & 13.1 & 27.8 & 51.8 & 86.6 & 118. & 162. & 170. \\
\hline 70 & 6.28 & 7.68 & 10.8 & 19.2 & 34.0 & 54.5 & 79.0 & 118. & 123. \\
\hline 75 & 5.56 & 6.99 & 9.74 & 15.0 & 24.0 & 44.2 & 67.2 & 100 & 105. \\
\hline 80 & 4.79 & 6.30 & 9.42 & 12.6 & 20.0 & 36.4 & 58.7 & 87.5 & 91.7 \\
\hline 90 & 3.32 & 4.68 & 7.03 & 9.00 & 12.0 & 23.6 & 38.1 & 51.5 & 62.1 \\
\hline 95 & 2.96 & 3.66 & 4.90 & 6.98 & 9.77 & 17.4 & 22.8 & 35.5 & 42.7 \\
\hline 98 & 2.39 & 2.68 & 4.31 & 5.80 & 8.00 & 12.1 & 18.0 & 24.4 & 30.3 \\
\hline 99 & 2.13 & 2.48 & 4.00 & 5.32 & 7.37 & 11.0 & 15.1 & 20.4 & 26.8 \\
\hline
\end{tabular}

\begin{tabular}{|c|c|c|c|c|c|c|c|c|c|}
\hline \multirow[b]{2}{*}{ APEE } & \multicolumn{9}{|c|}{ 7-day mean streamflow ( $\mathrm{ft}^{3} / \mathrm{s}$ ) that was equaled or exceeded the indicated percentage of time at APEE level } \\
\hline & 98 & 95 & 90 & 80 & 50 & 20 & 10 & 5 & 2 \\
\hline 1 & 871. & 952. & 1240 & 1780 & 2660 & 4020 . & 4960. & 5610. & 6000 \\
\hline 2 & 635. & 786. & 1050 & 1340 & 2160 & 3080 . & 3450 . & 3730. & 4790. \\
\hline 5 & 430 & 626 & 693. & 1000 & 1530. & 2120 & 2450 & 2690 & 3190. \\
\hline 10 & 160. & 293. & 423. & 602. & 999. & 1360. & 1790. & 1930. & 2470 \\
\hline 20 & 53.4 & 137. & 158. & 295. & 499. & 771. & 1050. & 1250 & 1330. \\
\hline 25 & 38.2 & 105 & 128. & 183. & 366. & 598. & 785 & 982. & 1060 \\
\hline 30 & 34.5 & 81.7 & 90.1 & 132. & 267. & 451. & 617. & 752. & 821. \\
\hline 40 & 23.1 & 32.4 & 49.5 & 76.6 & 153. & 295. & 346. & 487. & 580. \\
\hline 50 & 14.2 & 17.1 & 20.7 & 47.0 & 102. & 178. & 238 & 335. & 369. \\
\hline 60 & 8.47 & 10.3 & 14.5 & 30.1 & 61.4 & 107. & 164. & 232. & 248. \\
\hline 70 & 6.56 & 8.04 & 11.0 & 20.7 & 38.4 & 64.1 & 106. & 167. & 173. \\
\hline 75 & 6.01 & 7.22 & 10.4 & 16.9 & 28.7 & 50.2 & 81.1 & 140. & 144. \\
\hline 80 & 5.03 & 6.47 & 9.78 & 14.3 & 20.9 & 38.7 & 69.6 & 106. & 115. \\
\hline 90 & 3.55 & 5.03 & 7.72 & 9.55 & 13.2 & 26.6 & 48.9 & 83.3 & 84.4 \\
\hline 95 & 3.02 & 3.84 & 5.52 & 7.84 & 11.0 & 20.3 & 28.3 & 54.3 & 66.2 \\
\hline 98 & 2.64 & 2.95 & 4.75 & 6.65 & 9.13 & 13.6 & 21.4 & 28.9 & 45.6 \\
\hline 99 & 2.39 & 2.62 & 4.46 & 6.15 & 8.19 & 12.5 & 17.3 & 25.8 & 38.2 \\
\hline
\end{tabular}




\section{Sandusky River near Bucyrus, Ohio}

Years analyzed $=51$

Climatic year range $=1926-2008$

[APEE, annual percentage equaled or exceeded; $\mathrm{ft}^{3} / \mathrm{s}$, cubic foot per second]

\begin{tabular}{|c|c|c|c|c|c|c|c|c|c|}
\hline \multirow[b]{2}{*}{ APEE } & \multicolumn{9}{|c|}{ Daily mean streamflow $\left(\mathrm{ft}^{3} / \mathrm{s}\right)$ that was equaled or exceeded the indicated percentage of time at APEE level } \\
\hline & 98 & 95 & 90 & 80 & 50 & 20 & 10 & 5 & 2 \\
\hline 1 & 252. & 466. & 677. & 796. & 1240. & 1530. & 1750. & 1920. & 2460 \\
\hline 2 & 148. & 307. & 405. & 493. & 850. & 1130. & 1290 & 1480 . & 1730. \\
\hline 5 & 77.6 & 131. & 203. & 243. & 406. & 583. & 697. & 843. & 935. \\
\hline 10 & 46.3 & 70.2 & 92.8 & 130. & 200 & 292. & 348. & 383. & 459. \\
\hline 20 & 15.4 & 31.2 & 43.9 & 56.8 & 90.0 & 143. & 163. & 168. & 195. \\
\hline 25 & 8.78 & 21.8 & 30.8 & 44.0 & 69.0 & 111. & 119. & 126. & 134. \\
\hline 30 & 4.63 & 15.7 & 24.2 & 33.4 & 55.0 & 85.6 & 98.8 & 106. & 117. \\
\hline 40 & 2.35 & 8.80 & 12.4 & 20.2 & 35.0 & 55.4 & 69.4 & 73.4 & 81.9 \\
\hline 50 & 1.62 & 4.52 & 7.89 & 14.0 & 24.0 & 38.8 & 47.8 & 52.2 & 53.0 \\
\hline 60 & 1.27 & 2.94 & 4.61 & 9.32 & 16.4 & 25.6 & 33.2 & 38.8 & 41.9 \\
\hline 70 & 1.13 & 2.14 & 3.64 & 5.57 & 10.0 & 16.5 & 20.6 & 27.4 & 33.8 \\
\hline 75 & 1.12 & 1.88 & 3.16 & 4.68 & 8.65 & 13.8 & 17.6 & 24.0 & 28.8 \\
\hline 80 & 1.12 & 1.74 & 2.80 & 3.84 & 7.40 & 11.0 & 13.0 & 17.9 & 25.7 \\
\hline 90 & 1.02 & 1.46 & 2.02 & 2.70 & 4.50 & 7.27 & 8.96 & 10.7 & 14.9 \\
\hline 95 & 0.90 & 1.12 & 1.53 & 2.12 & 3.47 & 5.47 & 6.83 & 8.76 & 11.0 \\
\hline 98 & 0.80 & 0.98 & 1.20 & 1.68 & 2.80 & 4.56 & 5.13 & 7.05 & 8.19 \\
\hline 99 & 0.67 & 0.79 & 0.94 & 1.43 & 2.60 & 4.12 & 4.59 & 5.65 & 6.86 \\
\hline
\end{tabular}

\begin{tabular}{|c|c|c|c|c|c|c|c|c|c|}
\hline \multirow[b]{2}{*}{ APEE } & \multicolumn{9}{|c|}{ 7-day mean streamflow ( $\mathrm{ft}^{3} / \mathrm{s}$ ) that was equaled or exceeded the indicated percentage of time at APEE level } \\
\hline & 98 & 95 & 90 & 80 & 50 & 20 & 10 & 5 & 2 \\
\hline 1 & 128 & 296. & 398. & 461. & 642. & 841. & 943. & 1200 & 1980. \\
\hline 2 & 98.6 & 222. & 324. & 377. & 562. & 704. & 893. & 1010 & 1100 \\
\hline 5 & 81.2 & 141. & 222 & 280 & 398. & 512. & 595. & 749. & 941. \\
\hline 10 & 57.6 & 89.0 & 125. & 161. & 254. & 336. & 397. & 433. & 592. \\
\hline 20 & 19.5 & 31.6 & 59.3 & 83.4 & 120. & 188. & 246. & 269. & 311. \\
\hline 25 & 7.96 & 23.8 & 39.3 & 62.1 & 90.6 & 162. & 195. & 221. & 228 \\
\hline 30 & 4.14 & 18.7 & 28.4 & 43.4 & 73.1 & 131. & 155. & 173. & 190. \\
\hline 40 & 2.72 & 9.42 & 14.8 & 22.1 & 45.7 & 79.5 & 99.0 & 114. & 137. \\
\hline 50 & 1.99 & 5.09 & 9.33 & 15.5 & 30.4 & 53.5 & 62.7 & 71.3 & 80.0 \\
\hline 60 & 1.62 & 3.36 & 5.41 & 9.48 & 20.1 & 33.5 & 42.0 & 47.5 & 57.1 \\
\hline 70 & 1.37 & 2.67 & 3.71 & 6.47 & 12.5 & 20.7 & 27.2 & 34.7 & 40.7 \\
\hline 75 & 1.24 & 2.26 & 3.44 & 5.65 & 9.79 & 16.6 & 20.2 & 30.9 & 37.0 \\
\hline 80 & 1.19 & 1.97 & 3.06 & 4.84 & 8.39 & 13.7 & 15.7 & 26.8 & 32.6 \\
\hline 90 & 1.09 & 1.67 & 2.22 & 3.35 & 5.26 & 9.18 & 10.5 & 14.4 & 20.5 \\
\hline 95 & 1.03 & 1.26 & 1.71 & 2.55 & 4.42 & 7.20 & 7.80 & 10.3 & 12.8 \\
\hline 98 & 0.95 & 1.17 & 1.41 & 2.06 & 4.01 & 5.83 & 6.59 & 8.65 & 11.0 \\
\hline 99 & 0.93 & 1.13 & 1.26 & 1.82 & 3.39 & 5.08 & 5.92 & 7.69 & 9.88 \\
\hline
\end{tabular}




\section{Tymochtee Creek at Crawford, Ohio}

Years analyzed $=36$

Climatic year range $=1973-2008$

[APEE, annual percentage equaled or exceeded; $\mathrm{ft}^{3} / \mathrm{s}$, cubic foot per second]

\begin{tabular}{|c|c|c|c|c|c|c|c|c|c|}
\hline \multirow[b]{2}{*}{ APEE } & \multicolumn{9}{|c|}{ Daily mean streamflow $\left(\mathrm{ft}^{3} / \mathrm{s}\right)$ that was equaled or exceeded the indicated percentage of time at APEE level } \\
\hline & 95 & 90 & 80 & 70 & 50 & 30 & 20 & 10 & 5 \\
\hline 1 & 688. & 833. & 1390. & 1860. & 2120. & 2600. & 3140. & 3870 . & 4330 \\
\hline 2 & 435. & 641. & 1060. & 1380 . & 1660 & 1950. & 2220 & 2930. & 3520 . \\
\hline 5 & 236. & 361. & 652. & 811. & 1010 & 1310 . & 1450 . & 1790 . & 2350 \\
\hline 10 & 114. & 164. & 308. & 373. & 521. & 773. & 953. & 1110 & 1140 . \\
\hline 20 & 44.6 & 59.8 & 102. & 154. & 199. & 318. & 424. & 468. & 494. \\
\hline 25 & 30.3 & 41.8 & 70.1 & 112. & 148. & 206. & 302. & 329. & 345. \\
\hline 30 & 21.9 & 28.1 & 53.6 & 80.2 & 111. & 156. & 216. & 244. & 264. \\
\hline 40 & 9.10 & 14.4 & 31.5 & 40.8 & 64.0 & 90.0 & 136. & 152. & 161. \\
\hline 50 & 4.46 & 9.16 & 19.4 & 25.1 & 40.3 & 57.3 & 81.6 & 100 & 109. \\
\hline 60 & 2.75 & 5.02 & 9.20 & 13.1 & 22.5 & 36.8 & 50.2 & 68.0 & 76.2 \\
\hline 70 & 1.29 & 2.20 & 4.09 & 5.40 & 13.8 & 20.9 & 31.6 & 47.9 & 53.9 \\
\hline 75 & 0.84 & 1.91 & 2.68 & 3.56 & 9.90 & 17.9 & 25.9 & 39.8 & 44.6 \\
\hline 80 & 0.56 & 1.38 & 1.70 & 2.44 & 6.70 & 16.7 & 20.1 & 32.3 & 34.5 \\
\hline 90 & 0.21 & 0.41 & 0.73 & 1.33 & 3.09 & 8.04 & 10.6 & 19.5 & 20.9 \\
\hline 95 & 0.08 & 0.13 & 0.42 & 0.51 & 1.70 & 5.77 & 7.35 & 10.1 & 15.4 \\
\hline 98 & 0.02 & 0.08 & 0.19 & 0.35 & 1.10 & 3.73 & 5.25 & 6.74 & 11.3 \\
\hline 99 & 0.02 & 0.04 & 0.14 & 0.26 & 0.82 & 3.18 & 4.25 & 5.88 & 9.61 \\
\hline
\end{tabular}

\begin{tabular}{|c|c|c|c|c|c|c|c|c|c|}
\hline \multirow[b]{2}{*}{ APEE } & \multicolumn{9}{|c|}{ 7-day mean streamflow ( $\mathrm{ft}^{3} / \mathrm{s}$ ) that was equaled or exceeded the indicated percentage of time at APEE level } \\
\hline & 95 & 90 & 80 & 70 & 50 & 30 & 20 & 10 & 5 \\
\hline 1 & 433. & 630. & 901. & 1220 & 1530 & 1850 & 2100 & 2630 & 3070 \\
\hline 2 & 332. & 566. & 781. & 953. & 1200 & 1510 & 1800. & 2220 & 2380 \\
\hline 5 & 224 & 364. & 602. & 772 & 901. & 1090. & 1320. & 1620 & 1890 \\
\hline 10 & 138. & 170 & 345. & 459. & 560. & 723. & 855. & 1020. & 1350 \\
\hline 20 & 53.7 & 76.4 & 134. & 199. & 292. & 419. & 546. & 577. & 731. \\
\hline 25 & 33.0 & 49.2 & 86.3 & 152. & 199. & 296. & 408 & 466. & 563. \\
\hline 30 & 24.5 & 31.8 & 63.0 & 109. & 154. & 216. & 313. & 376. & 461. \\
\hline 40 & 12.0 & 17.3 & 36.3 & 52.7 & 91.4 & 127. & 177. & 218 & 243. \\
\hline 50 & 4.89 & 9.54 & 20.8 & 27.9 & 51.6 & 77.0 & 112 & 142. & 161. \\
\hline 60 & 2.97 & 5.41 & 9.46 & 14.8 & 27.9 & 50.5 & 64.0 & 95.8 & 103. \\
\hline 70 & 1.66 & 2.41 & 3.93 & 5.55 & 15.3 & 29.5 & 43.4 & 66.4 & 75.0 \\
\hline 75 & 0.92 & 1.79 & 2.92 & 3.67 & 11.9 & 21.4 & 37.5 & 54.5 & 61.5 \\
\hline 80 & 0.62 & 1.45 & 1.79 & 2.72 & 7.34 & 19.3 & 27.0 & 42.8 & 47.8 \\
\hline 90 & 0.31 & 0.50 & 0.80 & 1.48 & 3.02 & 9.38 & 12.5 & 25.0 & 25.9 \\
\hline 95 & 0.11 & 0.16 & 0.47 & 0.58 & 2.02 & 6.45 & 8.80 & 16.1 & 16.9 \\
\hline 98 & 0.05 & 0.10 & 0.25 & 0.44 & 1.28 & 4.74 & 6.65 & 10.3 & 11.6 \\
\hline 99 & 0.04 & 0.08 & 0.20 & 0.35 & 1.08 & 4.23 & 5.01 & 8.23 & 10.4 \\
\hline
\end{tabular}




\section{Black River at Elyria, Ohio}

Years analyzed $=64$

Climatic year range $=1945-2008$

[APEE, annual percentage equaled or exceeded; $\mathrm{ft}^{3} / \mathrm{s}$, cubic foot per second]

\begin{tabular}{|c|c|c|c|c|c|c|c|c|c|}
\hline \multirow[b]{2}{*}{ APEE } & \multicolumn{9}{|c|}{ Daily mean streamflow $\left(\mathrm{ft}^{3} / \mathrm{s}\right)$ that was equaled or exceeded the indicated percentage of time at APEE level } \\
\hline & 98 & 95 & 90 & 80 & 50 & 20 & 10 & 5 & 2 \\
\hline 1 & 1410. & 1760. & 2340. & 3240. & 4450. & 5550. & 6810. & 7590. & 8770. \\
\hline 2 & 994. & 1240 . & 1640. & 2200 & 2920 . & 4030 . & 4510 & 5570. & 6940. \\
\hline 5 & 421. & 681. & 839. & 1020. & 1480 & 2260 . & 2570 & 3160 . & 3630. \\
\hline 10 & 223. & 283. & 399. & 512. & 767. & 1290. & 1490. & 1590. & 1930. \\
\hline 20 & 69.3 & 138. & 164. & 214. & 359. & 594. & 687. & 762. & 841. \\
\hline 25 & 48.5 & 97.1 & 118. & 149. & 271. & 419. & 536. & 592. & 648. \\
\hline 30 & 36.6 & 71.2 & 87.8 & 109. & 212. & 317. & 415. & 493. & 510. \\
\hline 40 & 24.9 & 38.6 & 46.5 & 64.2 & 134. & 208. & 259. & 319. & 347. \\
\hline 50 & 18.3 & 19.5 & 30.0 & 40.0 & 87.0 & 130. & 172. & 226. & 254. \\
\hline 60 & 11.9 & 14.1 & 20.0 & 27.4 & 56.5 & 93.0 & 121. & 162. & 170. \\
\hline 70 & 8.69 & 11.0 & 13.9 & 19.0 & 33.5 & 58.0 & 76.4 & 101. & 124. \\
\hline 75 & 7.46 & 9.08 & 11.5 & 16.0 & 25.3 & 46.0 & 62.3 & 77.0 & 93.3 \\
\hline 80 & 6.04 & 7.57 & 10.2 & 13.0 & 20.1 & 36.2 & 46.2 & 63.8 & 69.4 \\
\hline 90 & 3.83 & 5.62 & 7.10 & 8.46 & 13.0 & 22.0 & 25.9 & 32.2 & 42.4 \\
\hline 95 & 2.57 & 4.43 & 5.45 & 6.50 & 9.68 & 17.0 & 20.0 & 23.7 & 29.2 \\
\hline 98 & 1.94 & 3.48 & 4.25 & 5.10 & 7.87 & 12.3 & 16.2 & 17.2 & 21.4 \\
\hline 99 & 1.65 & 3.05 & 3.83 & 4.70 & 6.85 & 12.0 & 14.2 & 14.7 & 18.5 \\
\hline
\end{tabular}

\begin{tabular}{|c|c|c|c|c|c|c|c|c|c|}
\hline \multirow[b]{2}{*}{ APEE } & \multicolumn{9}{|c|}{ 7-day mean streamflow ( $\mathrm{ft}^{3} / \mathrm{s}$ ) that was equaled or exceeded the indicated percentage of time at APEE level } \\
\hline & 98 & 95 & 90 & 80 & 50 & 20 & 10 & 5 & 2 \\
\hline 1 & 906. & 1110 & 1560. & 1740. & 2710 & 3580. & 4040 & 4510 & 5540. \\
\hline 2 & 834. & 891. & 1190. & 1490. & 2070 & 2860 & 3300. & 3920. & 4330. \\
\hline 5 & 431. & 587. & 801. & 1080 & 1440 & 1880. & 2430 & 2780 & 3390. \\
\hline 10 & 268. & 350. & 427. & 614. & 906. & 1370 . & 1510 & 1840. & 2150 \\
\hline 20 & 70.2 & 158. & 215. & 284 & 472. & 810. & 966. & 1060 & 1180. \\
\hline 25 & 54.3 & 110. & 135. & 208. & 366. & 641. & 761. & 847. & 928. \\
\hline 30 & 38.0 & 88.0 & 101. & 146. & 280. & 493. & 591. & 654. & 771. \\
\hline 40 & 26.0 & 41.5 & 51.1 & 73.0 & 173. & 312. & 353. & 452. & 517. \\
\hline 50 & 18.2 & 21.0 & 30.7 & 46.0 & 109. & 194. & 249. & 328. & 377. \\
\hline 60 & 12.1 & 15.1 & 21.8 & 32.0 & 63.9 & 115 & 167. & 215. & 253. \\
\hline 70 & 9.00 & 12.0 & 15.3 & 21.7 & 40.9 & 68.0 & 109. & 151. & 160. \\
\hline 75 & 6.90 & 9.89 & 11.9 & 17.6 & 31.2 & 53.0 & 86.6 & 104. & 135. \\
\hline 80 & 6.22 & 8.44 & 10.7 & 14.6 & 24.0 & 41.4 & 62.9 & 82.3 & 99.3 \\
\hline 90 & 3.78 & 6.19 & 7.78 & 9.44 & 13.7 & 26.6 & 31.4 & 45.7 & 51.3 \\
\hline 95 & 3.00 & 4.82 & 6.12 & 7.43 & 10.5 & 19.4 & 25.0 & 30.3 & 37.7 \\
\hline 98 & 2.58 & 4.33 & 4.99 & 6.20 & 9.35 & 16.4 & 19.7 & 23.2 & 30.3 \\
\hline 99 & 2.47 & 4.13 & 4.66 & 5.80 & 8.79 & 14.1 & 17.3 & 20.4 & 23.2 \\
\hline
\end{tabular}


04201500 Rocky River near Berea, Ohio

Years analyzed $=76$

Climatic year range $=1924-2008$

[APEE, annual percentage equaled or exceeded; $\mathrm{ft}^{3} / \mathrm{s}$, cubic foot per second]

\begin{tabular}{|c|c|c|c|c|c|c|c|c|c|}
\hline \multirow[b]{2}{*}{ APEE } & \multicolumn{9}{|c|}{ Daily mean streamflow $\left(\mathrm{ft}^{3} / \mathrm{s}\right)$ that was equaled or exceeded the indicated percentage of time at APEE level } \\
\hline & 98 & 95 & 90 & 80 & 50 & 20 & 10 & 5 & 2 \\
\hline 1 & 1050. & 1730. & 1960. & 2390. & 3390. & 4670. & 5180. & 5630. & 6620 \\
\hline 2 & 811. & 1070 . & 1350. & 1690. & 2400 & 3200 & 3610 . & 4100 . & 4350. \\
\hline 5 & 339. & 452. & 783. & 908. & 1220 & 1790 . & 2000 . & 2190. & 2540 \\
\hline 10 & 205. & 258. & 354. & 456. & 668. & 945. & 1190. & 1230 & 1460. \\
\hline 20 & 71.5 & 101. & 155. & 213. & 315. & 462. & 580. & 619. & 719. \\
\hline 25 & 48.4 & 74.0 & 121. & 152. & 253. & 377. & 443. & 473. & 555. \\
\hline 30 & 34.6 & 56.4 & 88.4 & 124. & 208. & 308. & 362. & 392. & 453. \\
\hline 40 & 20.2 & 29.1 & 49.1 & 81.7 & 136. & 208. & 244. & 278. & 315. \\
\hline 50 & 14.4 & 19.4 & 25.0 & 52.0 & 92.8 & 151. & 186. & 218 & 239. \\
\hline 60 & 8.97 & 11.7 & 17.0 & 33.4 & 60.5 & 109. & 147. & 163. & 176. \\
\hline 70 & 5.28 & 7.90 & 11.9 & 19.4 & 43.9 & 75.6 & 102. & 118. & 130. \\
\hline 75 & 3.84 & 6.36 & 9.15 & 14.7 & 37.0 & 65.7 & 82.8 & 104. & 108 \\
\hline 80 & 2.85 & 4.93 & 6.42 & 12.4 & 32.0 & 54.4 & 67.8 & 77.9 & 93.6 \\
\hline 90 & 1.09 & 2.36 & 3.98 & 7.34 & 20.3 & 36.2 & 42.0 & 54.3 & 59.3 \\
\hline 95 & 0.55 & 1.91 & 3.22 & 5.70 & 15.3 & 29.0 & 35.0 & 40.7 & 46.7 \\
\hline 98 & 0.41 & 1.32 & 2.45 & 4.06 & 10.7 & 23.7 & 29.5 & 32.7 & 35.5 \\
\hline 99 & 0.39 & 0.80 & 2.34 & 3.69 & 9.20 & 22.6 & 27.1 & 28.7 & 30.3 \\
\hline
\end{tabular}

\begin{tabular}{|c|c|c|c|c|c|c|c|c|c|}
\hline \multirow[b]{2}{*}{ APEE } & \multicolumn{9}{|c|}{ 7-day mean streamflow ( $\mathrm{ft}^{3} / \mathrm{s}$ ) that was equaled or exceeded the indicated percentage of time at APEE level } \\
\hline & 98 & 95 & 90 & 80 & 50 & 20 & 10 & 5 & 2 \\
\hline 1 & 616. & 933. & 1160 & 1290. & 1920. & 2490 & 2800 & 2990. & 3330. \\
\hline 2 & 537. & 720 . & 914. & 1110 & 1530 & 2180 & 2410 & 2610 & 3090 \\
\hline 5 & 371. & 561. & 737. & 817. & 1080 & 1440 & 1630 & 1980. & 2290 \\
\hline 10 & 224. & 305. & 432. & 543. & 766. & 1050 & 1200. & 1310 & 1530. \\
\hline 20 & 77.1 & 141. & 201. & 279. & 429. & 694. & 771. & 879. & 938. \\
\hline 25 & 51.0 & 88.8 & 156. & 196. & 334. & 538. & 651. & 706. & 805. \\
\hline 30 & 35.0 & 65.0 & 109. & 151. & 258 & 445. & 545. & 597. & 683. \\
\hline 40 & 21.7 & 29.8 & 54.4 & 97.5 & 164. & 291. & 361. & 431. & 491. \\
\hline 50 & 14.7 & 19.8 & 30.5 & 57.8 & 119. & 192. & 259. & 309. & 366. \\
\hline 60 & 9.12 & 11.8 & 18.8 & 36.4 & 76.1 & 133. & 180. & 237. & 278 \\
\hline 70 & 4.96 & 8.11 & 12.1 & 20.9 & 53.3 & 97.8 & 140. & 153. & 179. \\
\hline 75 & 4.09 & 7.01 & 9.57 & 16.8 & 47.1 & 83.0 & 109. & 132. & 159. \\
\hline 80 & 3.16 & 5.40 & 6.97 & 13.6 & 39.4 & 69.7 & 84.0 & 104. & 133. \\
\hline 90 & 1.52 & 3.64 & 4.54 & 8.71 & 25.5 & 44.7 & 55.9 & 76.1 & 88.3 \\
\hline 95 & 0.74 & 2.49 & 3.59 & 7.00 & 17.9 & 33.5 & 42.1 & 50.8 & 58.1 \\
\hline 98 & 0.49 & 1.95 & 2.85 & 4.99 & 13.9 & 28.5 & 34.6 & 39.3 & 44.5 \\
\hline 99 & 0.40 & 1.55 & 2.50 & 4.05 & 11.4 & 26.4 & 31.6 & 36.1 & 39.7 \\
\hline
\end{tabular}




\section{Cuyahoga River at Independence, Ohio}

Years analyzed $=77$

Climatic year range $=1922-2008$

[APEE, annual percentage equaled or exceeded; $\mathrm{ft}^{3} / \mathrm{s}$, cubic foot per second]

\begin{tabular}{|c|c|c|c|c|c|c|c|c|c|}
\hline \multirow[b]{2}{*}{ APEE } & \multicolumn{9}{|c|}{ Daily mean streamflow $\left(\mathrm{ft}^{3} / \mathrm{s}\right)$ that was equaled or exceeded the indicated percentage of time at APEE level } \\
\hline & 98 & 95 & 90 & 80 & 50 & 20 & 10 & 5 & 2 \\
\hline 1 & 2270. & 3090. & 3400. & 3860. & 5340. & 6490. & 7210. & 8010. & 9520. \\
\hline 2 & 1830. & 2250 & 2830 & 3060 . & 4200 . & 5180. & 5790. & 6150. & 7150. \\
\hline 5 & 1250 & 1550 . & 1870 . & 2180 & 2770 & 3550 . & 3970. & 4180. & 4690. \\
\hline 10 & 881. & 1090. & 1180. & 1500. & 1960. & 2710 . & 2960. & 3150 . & 3280 . \\
\hline 20 & 487. & 576. & 728. & 891. & 1250 & 1690. & 1950. & 2110. & 2210. \\
\hline 25 & 356. & 429. & 579. & 730. & 1040 . & 1420. & 1640. & 1780. & 1860. \\
\hline 30 & 282. & 328. & 481. & 591. & 898. & 1230. & 1380. & 1600. & 1610. \\
\hline 40 & 193. & 225. & 297. & 392. & 647. & 930. & 1090. & 1230. & 1300. \\
\hline 50 & 146. & 158. & 230 & 256. & 486. & 719. & 880. & 1030. & 1070. \\
\hline 60 & 108. & 113. & 167. & 212. & 381. & 552. & 729. & 850. & 882. \\
\hline 70 & 86.4 & 95.6 & 129. & 174. & 301. & 444. & 554. & 635. & 715. \\
\hline 75 & 79.4 & 91.0 & 118. & 155. & 257. & 406. & 484. & 564. & 637. \\
\hline 80 & 69.9 & 83.0 & 111. & 141. & 232 & 367. & 424. & 474. & 569. \\
\hline 90 & 55.0 & 65.8 & 84.2 & 114. & 188. & 277. & 328. & 362. & 409. \\
\hline 95 & 45.9 & 52.1 & 73.6 & 101. & 157. & 238. & 281. & 308. & 344. \\
\hline 98 & 36.6 & 45.3 & 68.3 & 93.6 & 141. & 218. & 241. & 281. & 303. \\
\hline 99 & 30.7 & 39.0 & 64.8 & 87.1 & 135. & 205. & 233. & 264. & 292. \\
\hline
\end{tabular}

\begin{tabular}{|c|c|c|c|c|c|c|c|c|c|}
\hline \multirow[b]{2}{*}{ APEE } & \multicolumn{9}{|c|}{ 7-day mean streamflow ( $\left.\mathrm{ft}^{3} / \mathrm{s}\right)$ that was equaled or exceeded the indicated percentage of time at APEE level } \\
\hline & 98 & 95 & 90 & 80 & 50 & 20 & 10 & 5 & 2 \\
\hline 1 & 1680. & 2210. & 2410. & 2780. & 3960. & 4800. & 5370. & 5800. & 6900. \\
\hline 2 & 1490. & 1970. & 2000 & 2510 & 3430 . & 4100. & 4510. & 4750. & 6290. \\
\hline 5 & 1190. & 1510 . & 1660. & 1970. & 2480 . & 3280 . & 3600. & 3840 . & 4840 . \\
\hline 10 & 842. & 1060 . & 1150. & 1530. & 1920. & 2550 . & 2750 & 2840. & 3030 . \\
\hline 20 & 449. & 533. & 773. & 885. & 1300 . & 1830. & 2030 & 2180. & 2330. \\
\hline 25 & 334. & 435. & 586. & 760. & 1110. & 1530. & 1750 & 1970. & 2060. \\
\hline 30 & 266. & 306. & 483. & 636. & 956. & 1320. & 1560. & 1780. & 1860. \\
\hline 40 & 197. & 221. & 332. & 419. & 696. & 994. & 1230. & 1410. & 1470. \\
\hline 50 & 157. & 162. & 230 . & 274 & 521. & 783. & 972. & 1180. & 1220. \\
\hline 60 & 107. & 115. & 185. & 221. & 411. & 621. & 818. & 972. & 1030. \\
\hline 70 & 83.1 & 99.9 & 141. & 182. & 325. & 518. & 618. & 713. & 829. \\
\hline 75 & 78.1 & 92.7 & 127. & 157. & 292. & 449. & 530. & 626. & 755. \\
\hline 80 & 73.4 & 88.2 & 119. & 149. & 270 & 410. & 498. & 554. & 672. \\
\hline 90 & 62.9 & 76.0 & 99.0 & 125. & 208. & 321. & 379. & 422. & 503. \\
\hline 95 & 55.9 & 62.9 & 89.6 & 111. & 180. & 269. & 325. & 371. & 388. \\
\hline 98 & 48.9 & 59.5 & 86.4 & 99.6 & 149. & 241. & 288 & 324. & 347. \\
\hline 99 & 44.8 & 57.6 & 82.3 & 97.8 & 148. & 231. & 275 & 298. & 327. \\
\hline
\end{tabular}


04209000 Chagrin River at Willoughby, Ohio

Years analyzed $=69$

Climatic year range $=1926-2008$

[APEE, annual percentage equaled or exceeded; $\mathrm{ft}^{3} / \mathrm{s}$, cubic foot per second]

\begin{tabular}{|c|c|c|c|c|c|c|c|c|c|}
\hline \multirow[b]{2}{*}{ APEE } & \multicolumn{9}{|c|}{ Daily mean streamflow $\left(\mathrm{ft}^{3} / \mathrm{s}\right)$ that was equaled or exceeded the indicated percentage of time at APEE level } \\
\hline & 98 & 95 & 90 & 80 & 50 & 20 & 10 & 5 & 2 \\
\hline 1 & 1520. & 2010. & 2100. & 2430. & 3380. & 4120. & 4610. & 5220. & 7020 . \\
\hline 2 & 1070 & 1380 . & 1530. & 1760 . & 2270 & 2870 & 3340 . & 4280 & 5100. \\
\hline 5 & 564. & 671. & 893. & 1000 . & 1340 & 1690. & 1940. & 2040 . & 2910. \\
\hline 10 & 367. & 394. & 484. & 594. & 795. & 1020. & 1080 . & 1450 & 2130 \\
\hline 20 & 194. & 213. & 284. & 323. & 438. & 580. & 638. & 747. & 1010. \\
\hline 25 & 155. & 172. & 218. & 253. & 339. & 470 . & 520. & 578. & 748. \\
\hline 30 & 124. & 147. & 162. & 207. & 286. & 396. & 432. & 463. & 584. \\
\hline 40 & 79.4 & 103. & 110. & 143. & 214. & 284. & 319. & 347. & 389. \\
\hline 50 & 55.0 & 66.0 & 80.0 & 99.0 & 160. & 220. & 241. & 265. & 279. \\
\hline 60 & 40.6 & 51.0 & 56.0 & 71.0 & 118. & 162. & 188. & 202. & 221. \\
\hline 70 & 30.3 & 36.0 & 39.0 & 52.2 & 80.8 & 124. & 147. & 155. & 171. \\
\hline 75 & 26.6 & 29.5 & 35.0 & 44.0 & 70.0 & 109. & 126. & 135. & 144. \\
\hline 80 & 22.8 & 26.0 & 31.0 & 37.0 & 58.0 & 97.0 & 108. & 115. & 120. \\
\hline 90 & 14.6 & 19.0 & 22.0 & 27.0 & 44.0 & 72.6 & 83.0 & 89.0 & 98.0 \\
\hline 95 & 10.2 & 14.7 & 18.3 & 25.0 & 35.0 & 59.0 & 68.0 & 75.2 & 84.4 \\
\hline 98 & 7.24 & 12.2 & 16.0 & 20.3 & 28.0 & 52.3 & 61.0 & 66.8 & 73.6 \\
\hline 99 & 5.85 & 8.32 & 14.0 & 18.7 & 27.0 & 47.0 & 58.0 & 64.8 & 70.4 \\
\hline
\end{tabular}

\begin{tabular}{|c|c|c|c|c|c|c|c|c|c|}
\hline \multirow[b]{2}{*}{ APEE } & \multicolumn{9}{|c|}{ 7-day mean streamflow ( $\mathrm{ft}^{3} / \mathrm{s}$ ) that was equaled or exceeded the indicated percentage of time at APEE level } \\
\hline & 98 & 95 & 90 & 80 & 50 & 20 & 10 & 5 & 2 \\
\hline 1 & 865. & 1050 & 1240 & 1510 . & 1890. & 2550 & 2990. & 3180. & 3700 . \\
\hline 2 & 698. & 876. & 998. & 1210 & 1610 & 1930. & 2140 & 2390. & 3340. \\
\hline 5 & 567. & 687. & 799. & 924. & 1200 & 1480 & 1540 & 1670 . & 2680 \\
\hline 10 & 394. & 451. & 496. & 636. & 842. & 1080 . & 1230. & 1370 . & 1960. \\
\hline 20 & 213. & 245. & 314. & 390. & 534. & 733. & 792. & 893. & 1110 \\
\hline 25 & 173. & 198. & 274. & 314. & 440 & 624. & 677. & 767. & 964. \\
\hline 30 & 141. & 155. & 193. & 251. & 362. & 498. & 568. & 664. & 830 . \\
\hline 40 & 85.1 & 112. & 129. & 161. & 266. & 350 . & 425. & 481. & 587. \\
\hline 50 & 60.3 & 75.4 & 83.6 & 113. & 185. & 261. & 308. & 360. & 440 \\
\hline 60 & 39.3 & 51.1 & 57.3 & 78.6 & 131. & 204. & 238. & 278. & 339. \\
\hline 70 & 31.1 & 36.0 & 41.0 & 54.1 & 94.6 & 146. & 182. & 200 . & 239. \\
\hline 75 & 27.9 & 31.6 & 39.3 & 47.1 & 81.6 & 130. & 155 & 172. & 207. \\
\hline 80 & 25.2 & 27.7 & 31.3 & 39.1 & 68.1 & 119. & 135. & 154. & 164. \\
\hline 90 & 17.8 & 21.3 & 24.2 & 29.7 & 51.4 & 84.1 & 94.1 & 106. & 113. \\
\hline 95 & 12.2 & 16.0 & 21.0 & 26.4 & 37.0 & 64.4 & 78.9 & 88.6 & 100. \\
\hline 98 & 9.30 & 12.8 & 17.4 & 23.4 & 32.7 & 56.0 & 69.6 & 80.7 & 90.0 \\
\hline 99 & 7.65 & 11.8 & 15.9 & 21.4 & 29.6 & 53.9 & 65.3 & 77.2 & 83.3 \\
\hline
\end{tabular}




\section{Mill Creek near Jefferson, Ohio}

Years analyzed $=32$

Climatic year range $=1942-73$

[APEE, annual percentage equaled or exceeded; $\mathrm{ft}^{3} / \mathrm{s}$, cubic foot per second]

\begin{tabular}{|c|c|c|c|c|c|c|c|c|c|}
\hline \multirow[b]{2}{*}{ APEE } & \multicolumn{9}{|c|}{ Daily mean streamflow $\left(\mathrm{ft}^{3} / \mathrm{s}\right)$ that was equaled or exceeded the indicated percentage of time at APEE level } \\
\hline & 95 & 90 & 80 & 70 & 50 & 30 & 20 & 10 & 5 \\
\hline 1 & 760. & 885. & 1040. & 1140. & 1290. & 1430. & 1700. & 2050 & 2380 \\
\hline 2 & 539. & 622. & 736. & 877. & 959. & 1090. & 1130 & 1270 . & 1350. \\
\hline 5 & 245 & 295. & 401. & 472. & 558. & 633. & 661. & 747. & 769. \\
\hline 10 & 133. & 156. & 199. & 224 & 291. & 339. & 365. & 393. & 428. \\
\hline 20 & 62.9 & 71.4 & 85.2 & 93.0 & 127. & 156. & 183. & 202. & 206. \\
\hline 25 & 38.3 & 50.0 & 62.7 & 71.0 & 86.8 & 107. & 130. & 152. & 158. \\
\hline 30 & 25.6 & 35.6 & 44.5 & 51.5 & 62.6 & 80.5 & 93.5 & 115. & 123. \\
\hline 40 & 12.5 & 17.4 & 22.0 & 26.0 & 33.8 & 46.5 & 55.5 & 72.8 & 78.1 \\
\hline 50 & 3.45 & 5.45 & 7.08 & 9.14 & 16.8 & 26.6 & 35.4 & 46.8 & 49.0 \\
\hline 60 & 0.10 & 0.26 & 1.14 & 2.59 & 6.24 & 13.1 & 21.4 & 28.6 & 31.1 \\
\hline 70 & 0.00 & 0.02 & 0.10 & 0.10 & 1.93 & 5.32 & 8.02 & 16.0 & 18.4 \\
\hline 75 & 0.00 & 0.00 & 0.00 & 0.10 & 1.09 & 2.68 & 4.14 & 9.89 & 12.0 \\
\hline 80 & 0.00 & 0.00 & 0.00 & 0.10 & 0.40 & 1.34 & 2.48 & 4.58 & 6.96 \\
\hline 90 & 0.00 & 0.00 & 0.00 & 0.00 & 0.10 & 0.21 & 0.42 & 0.87 & 2.33 \\
\hline 95 & 0.00 & 0.00 & 0.00 & 0.00 & 0.05 & 0.10 & 0.10 & 0.27 & 1.11 \\
\hline 98 & 0.00 & 0.00 & 0.00 & 0.00 & 0.03 & 0.10 & 0.10 & 0.10 & 0.50 \\
\hline 99 & 0.00 & 0.00 & 0.00 & 0.00 & 0.03 & 0.10 & 0.10 & 0.10 & 0.38 \\
\hline
\end{tabular}

\begin{tabular}{|c|c|c|c|c|c|c|c|c|c|}
\hline \multirow[b]{2}{*}{ APEE } & \multicolumn{9}{|c|}{ 7-day mean streamflow ( $\mathrm{ft}^{3} / \mathrm{s}$ ) that was equaled or exceeded the indicated percentage of time at APEE level } \\
\hline & 95 & 90 & 80 & 70 & 50 & 30 & 20 & 10 & 5 \\
\hline 1 & 358. & 518. & 607. & 683. & 795. & 982. & 1010. & 1110 & 1170. \\
\hline 2 & 306. & 441. & 498. & 528. & 614. & 726. & 785. & 872. & 973. \\
\hline 5 & 218 & 274. & 356. & 427. & 455. & 525. & 553. & 607. & 652. \\
\hline 10 & 141. & 162. & 250 & 281. & 339. & 356. & 386. & 436. & 456. \\
\hline 20 & 72.6 & 84.0 & 134. & 163. & 186. & 210 & 233. & 268. & 306. \\
\hline 25 & 56.7 & 65.4 & 95.0 & 112. & 149. & 170 & 196. & 221. & 242. \\
\hline 30 & 34.0 & 47.7 & 67.8 & 78.7 & 108. & 136. & 157. & 177. & 194. \\
\hline 40 & 13.4 & 25.1 & 31.4 & 38.9 & 53.4 & 76.3 & 89.1 & 115. & 132. \\
\hline 50 & 4.31 & 5.92 & 9.72 & 13.1 & 23.5 & 34.3 & 50.8 & 84.2 & 85.3 \\
\hline 60 & 0.09 & 0.32 & 1.59 & 2.78 & 10.7 & 19.2 & 30.9 & 45.1 & 47.9 \\
\hline 70 & 0.00 & 0.07 & 0.13 & 0.23 & 2.71 & 8.64 & 14.4 & 22.1 & 26.8 \\
\hline 75 & 0.00 & 0.00 & 0.05 & 0.10 & 1.20 & 3.82 & 8.04 & 15.8 & 19.4 \\
\hline 80 & 0.00 & 0.00 & 0.00 & 0.10 & 0.70 & 2.20 & 3.11 & 8.69 & 13.2 \\
\hline 90 & 0.00 & 0.00 & 0.00 & 0.00 & 0.11 & 0.36 & 0.69 & 1.67 & 3.84 \\
\hline 95 & 0.00 & 0.00 & 0.00 & 0.00 & 0.10 & 0.10 & 0.23 & 0.35 & 1.22 \\
\hline 98 & 0.00 & 0.00 & 0.00 & 0.00 & 0.05 & 0.10 & 0.10 & 0.23 & 0.80 \\
\hline 99 & 0.00 & 0.00 & 0.00 & 0.00 & 0.05 & 0.10 & 0.10 & 0.14 & 0.65 \\
\hline
\end{tabular}


Appendix 6. Annual Seasonal-Period Flow-Duration Results for Sites with Streamflows that are Subject to Regulation 
03086500 Mahoning River at Alliance, Ohio

Years analyzed $=38$

Climatic year range $=1955-92$

[APEE, annual percentage equaled or exceeded; $\mathrm{ft}^{3} / \mathrm{s}$, cubic foot per second; Sep, September; Nov, November]

\begin{tabular}{|c|c|c|c|c|c|c|c|c|c|}
\hline \multirow[b]{2}{*}{ APEE } & \multicolumn{9}{|c|}{ Sep-Nov daily mean streamflow ( $\left.\mathrm{ft}^{3} / \mathrm{s}\right)$ that was equaled or exceeded the indicated percentage of time at APEE level } \\
\hline & 95 & 90 & 80 & 70 & 50 & 30 & 20 & 10 & 5 \\
\hline 1 & 10.0 & 26.7 & 64.8 & 86.5 & 223. & 373. & 508. & 711. & 799. \\
\hline 2 & 9.82 & 24.3 & 55.9 & 70.8 & 174. & 329. & 423. & 580. & 719. \\
\hline 5 & 9.01 & 23.0 & 40.1 & 48.7 & 107. & 188. & 303. & 423. & 530. \\
\hline 10 & 8.34 & 19.2 & 29.4 & 38.8 & 57.2 & 123. & 183. & 312. & 328. \\
\hline 20 & 6.95 & 16.0 & 20.6 & 25.8 & 35.7 & 76.0 & 121. & 141. & 184. \\
\hline 25 & 6.93 & 11.9 & 16.8 & 23.4 & 31.3 & 61.9 & 98.6 & 117. & 126. \\
\hline 30 & 6.92 & 9.96 & 16.0 & 21.0 & 29.1 & 47.0 & 77.1 & 97.9 & 109. \\
\hline 40 & 6.87 & 8.85 & 14.2 & 17.7 & 24.8 & 38.0 & 60.6 & 72.1 & 81.7 \\
\hline 50 & 5.32 & 7.22 & 9.16 & 14.7 & 20.5 & 30.5 & 41.9 & 52.8 & 57.6 \\
\hline 60 & 4.18 & 5.51 & 8.30 & 10.7 & 17.0 & 25.4 & 29.8 & 41.0 & 54.4 \\
\hline 70 & 3.52 & 4.73 & 7.24 & 9.14 & 13.3 & 21.6 & 26.0 & 32.7 & 49.2 \\
\hline 75 & 3.52 & 4.10 & 6.36 & 7.93 & 11.5 & 18.9 & 24.0 & 30.3 & 46.3 \\
\hline 80 & 2.76 & 3.63 & 5.36 & 7.14 & 9.81 & 15.9 & 21.2 & 28.5 & 46.1 \\
\hline 90 & 0.00 & 1.95 & 3.63 & 4.99 & 6.96 & 12.7 & 15.2 & 20.1 & 34.7 \\
\hline 95 & 0.00 & 1.26 & 3.22 & 4.37 & 6.01 & 11.0 & 12.6 & 16.2 & 25.7 \\
\hline 98 & 0.00 & 0.90 & 2.86 & 3.59 & 5.50 & 8.78 & 12.2 & 13.2 & 25.6 \\
\hline 99 & 0.00 & 0.90 & 2.70 & 3.47 & 4.90 & 8.78 & 12.0 & 13.0 & 25.5 \\
\hline
\end{tabular}

03093000 Eagle Creek at Phalanx Station, Ohio

Years analyzed $=80$

Climatic year range $=1926-2008$

[APEE, annual percentage equaled or exceeded; $\mathrm{ft}^{3} / \mathrm{s}$, cubic foot per second; Sep, September; Nov, November]

\begin{tabular}{|c|c|c|c|c|c|c|c|c|c|}
\hline \multirow[b]{2}{*}{ APEE } & \multicolumn{9}{|c|}{ Sep-Nov daily mean streamflow (ft $\left.{ }^{3} / \mathrm{s}\right)$ that was equaled or exceeded the indicated percentage of time at APEE level } \\
\hline & 98 & 95 & 90 & 80 & 50 & 20 & 10 & 5 & 2 \\
\hline 1 & 20.6 & 30.1 & 42.0 & 89.6 & 331. & 893. & 1470 & 2240 & 3540 \\
\hline 2 & 20.6 & 30.0 & 38.0 & 72.3 & 268. & 616. & 1080 & 1470 . & 2500 \\
\hline 5 & 18.0 & 21.9 & 30.2 & 46.1 & 122. & 369. & 546. & 729. & 1140 \\
\hline 10 & 14.9 & 20.1 & 23.1 & 34.7 & 78.0 & 222. & 336. & 496. & 689. \\
\hline 20 & 12.6 & 18.0 & 19.1 & 26.4 & 46.5 & 113. & 163. & 219. & 262. \\
\hline 25 & 12.6 & 15.1 & 18.0 & 24.0 & 40.0 & 93.6 & 128 & 162. & 227. \\
\hline 30 & 11.2 & 13.5 & 17.0 & 22.0 & 35.5 & 76.3 & 114. & 147. & 189. \\
\hline 40 & 9.76 & 12.1 & 15.0 & 18.0 & 29.3 & 53.4 & 83.9 & 104. & 132. \\
\hline 50 & 8.81 & 9.34 & 13.0 & 16.0 & 23.0 & 43.2 & 59.7 & 87.6 & 112. \\
\hline 60 & 8.06 & 8.76 & 12.0 & 14.2 & 20.0 & 31.8 & 46.7 & 74.2 & 90.4 \\
\hline 70 & 7.28 & 8.04 & 10.1 & 13.0 & 16.1 & 27.6 & 38.3 & 51.5 & 74.6 \\
\hline 75 & 6.80 & 7.73 & 9.63 & 12.0 & 15.5 & 26.8 & 35.7 & 49.0 & 70.3 \\
\hline 80 & 6.38 & 7.59 & 9.00 & 11.0 & 14.4 & 24.0 & 33.9 & 46.4 & 65.9 \\
\hline 90 & 5.08 & 6.22 & 7.16 & 8.90 & 12.0 & 20.8 & 24.9 & 38.0 & 42.2 \\
\hline 95 & 4.17 & 5.03 & 6.04 & 7.96 & 11.0 & 18.0 & 21.6 & 28.9 & 35.6 \\
\hline 98 & 2.99 & 4.06 & 5.21 & 6.62 & 10.4 & 17.0 & 19.8 & 26.0 & 30.1 \\
\hline 99 & 2.17 & 3.81 & 4.55 & 6.02 & 9.90 & 17.0 & 19.0 & 24.9 & 30.1 \\
\hline
\end{tabular}


03097500 Meander Creek at Mineral Ridge, Ohio

Years analyzed $=22$

Climatic year range $=1929-50$

[APEE, annual percentage equaled or exceeded; $\mathrm{ft}^{3} / \mathrm{s}$, cubic foot per second; Sep, September; Nov, November]

\begin{tabular}{|c|c|c|c|c|c|c|c|c|c|}
\hline \multirow[b]{2}{*}{ APEE } & \multicolumn{9}{|c|}{ Sep-Nov daily mean streamflow $\left(\mathrm{ft}^{3} / \mathrm{s}\right)$ that was equaled or exceeded the indicated percentage of time at APEE level } \\
\hline & 95 & 90 & 80 & 70 & 50 & 30 & 20 & 10 & 5 \\
\hline 1 & 1.42 & 1.53 & 1.76 & 2.07 & 2.60 & 6.67 & 66.2 & 162. & 412. \\
\hline 2 & 1.34 & 1.43 & 1.68 & 1.95 & 2.47 & 5.99 & 55.0 & 137. & 370. \\
\hline 5 & 0.90 & 1.09 & 1.34 & 1.54 & 1.85 & 4.92 & 37.4 & 105 & 261. \\
\hline 10 & 0.82 & 0.90 & 1.05 & 1.24 & 1.65 & 2.22 & 5.28 & 59.0 & 147. \\
\hline 20 & 0.70 & 0.73 & 0.80 & 0.98 & 1.43 & 1.88 & 2.54 & 19.8 & 28.1 \\
\hline 25 & 0.70 & 0.70 & 0.80 & 0.89 & 1.40 & 1.82 & 2.48 & 10.6 & 15.4 \\
\hline 30 & 0.61 & 0.70 & 0.80 & 0.80 & 1.20 & 1.81 & 2.44 & 8.55 & 11.6 \\
\hline 40 & 0.60 & 0.60 & 0.70 & 0.70 & 1.06 & 1.62 & 2.28 & 5.47 & 8.28 \\
\hline 50 & 0.50 & 0.53 & 0.66 & 0.70 & 0.95 & 1.60 & 1.82 & 4.76 & 6.41 \\
\hline 60 & 0.48 & 0.49 & 0.56 & 0.69 & 0.84 & 1.39 & 1.64 & 3.79 & 5.08 \\
\hline 70 & 0.40 & 0.43 & 0.56 & 0.60 & 0.70 & 1.32 & 1.54 & 2.75 & 4.66 \\
\hline 75 & 0.40 & 0.40 & 0.50 & 0.60 & 0.70 & 1.21 & 1.54 & 2.44 & 4.33 \\
\hline 80 & 0.32 & 0.40 & 0.46 & 0.50 & 0.65 & 1.20 & 1.48 & 2.37 & 3.96 \\
\hline 90 & 0.22 & 0.31 & 0.37 & 0.40 & 0.65 & 1.10 & 1.26 & 1.99 & 2.98 \\
\hline 95 & 0.12 & 0.27 & 0.30 & 0.40 & 0.50 & 0.96 & 1.26 & 1.92 & 2.60 \\
\hline 98 & 0.10 & 0.16 & 0.30 & 0.38 & 0.50 & 0.78 & 1.13 & 1.81 & 2.49 \\
\hline 99 & 0.10 & 0.13 & 0.30 & 0.30 & 0.50 & 0.70 & 1.04 & 1.81 & 2.49 \\
\hline
\end{tabular}

03097550 Mahoning River at Ohio Edison P PIt at Niles, Ohio

Years analyzed $=22$

Climatic year range $=1987-2008$

[APEE, annual percentage equaled or exceeded; $\mathrm{ft}^{3} / \mathrm{s}$, cubic foot per second; Sep, September; Nov, November]

\begin{tabular}{|c|c|c|c|c|c|c|c|c|c|}
\hline \multirow[b]{2}{*}{ APEE } & \multicolumn{9}{|c|}{ Sep-Nov daily mean streamflow ( $\mathrm{ft}^{3} / \mathrm{s}$ ) that was equaled or exceeded the indicated percentage of time at APEE leve } \\
\hline & 95 & 90 & 80 & 70 & 50 & 30 & 20 & 10 & 5 \\
\hline 1 & 546. & 701. & 896. & 987. & 1320. & 3370 . & 4230. & 5330. & 7350. \\
\hline 2 & 448. & 568. & 732. & 817. & 1190. & 2850 . & 3980. & 4540 . & 6460. \\
\hline 5 & 387. & 455. & 513. & 589. & 1020 . & 1990. & 3240. & 3920. & 4310. \\
\hline 10 & 374. & 403. & 432. & 504. & 847. & 1390. & 2440 . & 2670 . & 3180. \\
\hline 20 & 338. & 363. & 380 & 401. & 665. & 966. & 1920. & 2240 . & 2310. \\
\hline 25 & 329. & 341. & 363. & 374. & 583. & 913. & 1770. & 2160 . & 2260 \\
\hline 30 & 321. & 326. & 346. & 363. & 497. & 880 . & 1630. & 1950. & 2060. \\
\hline 40 & 292. & 310. & 328. & 337. & 444. & 770. & 1190. & 1610. & 1730 \\
\hline 50 & 247 & 298. & 306. & 313. & 407. & 648. & 970. & 1300 . & 1350. \\
\hline 60 & 234. & 264. & 285 & 292. & 376. & 565. & 773. & 1110. & 1180 \\
\hline 70 & 222 & 247. & 262. & 273. & 330 . & 456. & 628. & 902. & 1020. \\
\hline 75 & 216. & 233. & 250 & 268 & 320. & 433. & 573. & 813. & 941. \\
\hline 80 & 211. & 228 & 242 & 258 & 310. & 390. & 529. & 722 & 858. \\
\hline 90 & 201. & 207. & 224 & 234 & 284. & 380 . & 438. & 594. & 658. \\
\hline 95 & 196. & 201. & 213. & 227. & 271. & 344. & 399. & 454. & 560. \\
\hline 98 & 189. & 189. & 205. & 224 & 254 & 330. & 378. & 440. & 551. \\
\hline 99 & 185. & 187. & 202. & 219. & 249. & 329. & 374. & 433. & 545. \\
\hline
\end{tabular}


03116000 Tuscarawas River at Clinton, Ohio

Years analyzed $=39$

Climatic year range $=1939-77$

[APEE, annual percentage equaled or exceeded; $\mathrm{ft}^{3} / \mathrm{s}$, cubic foot per second; Sep, September; Nov, November]

\begin{tabular}{|c|c|c|c|c|c|c|c|c|c|}
\hline \multirow[b]{2}{*}{ APEE } & \multicolumn{9}{|c|}{ Sep-Nov daily mean streamflow $\left(\mathrm{ft}^{3} / \mathrm{s}\right)$ that was equaled or exceeded the indicated percentage of time at APEE level } \\
\hline & 95 & 90 & 80 & 70 & 50 & 30 & 20 & 10 & 5 \\
\hline 1 & 61.0 & 73.0 & 134. & 152. & 200. & 388. & 600. & 782. & 1000 \\
\hline 2 & 59.3 & 66.5 & 115. & 132. & 187. & 324. & 491. & 694. & 899. \\
\hline 5 & 51.0 & 59.4 & 84.0 & 91.8 & 135. & 209. & 274. & 409. & 577. \\
\hline 10 & 49.0 & 56.0 & 71.6 & 75.0 & 102. & 138. & 175. & 236. & 316. \\
\hline 20 & 46.0 & 54.0 & 62.4 & 66.6 & 81.6 & 95.2 & 125. & 163. & 178. \\
\hline 25 & 44.0 & 53.0 & 57.0 & 65.0 & 75.0 & 86.0 & 117. & 141. & 159. \\
\hline 30 & 43.4 & 49.8 & 53.0 & 61.8 & 70.4 & 82.4 & 109. & 130. & 155. \\
\hline 40 & 43.0 & 46.0 & 52.4 & 58.0 & 66.0 & 77.0 & 90.2 & 118. & 142. \\
\hline 50 & 42.0 & 44.0 & 49.0 & 55.0 & 63.0 & 75.0 & 80.0 & 100. & 130. \\
\hline 60 & 41.0 & 42.0 & 47.8 & 52.0 & 59.0 & 70.8 & 74.0 & 85.0 & 118. \\
\hline 70 & 37.6 & 40.0 & 44.0 & 48.0 & 57.0 & 66.0 & 70.0 & 79.0 & 111. \\
\hline 75 & 37.0 & 40.0 & 43.0 & 47.0 & 57.0 & 64.0 & 69.0 & 77.0 & 85.0 \\
\hline 80 & 35.8 & 39.0 & 42.0 & 47.0 & 56.0 & 64.0 & 67.0 & 76.0 & 82.4 \\
\hline 90 & 33.0 & 38.0 & 39.4 & 41.2 & 52.0 & 61.2 & 66.0 & 71.2 & 73.2 \\
\hline 95 & 31.6 & 34.0 & 37.0 & 40.0 & 50.6 & 59.6 & 63.0 & 70.0 & 73.0 \\
\hline 98 & 28.7 & 34.0 & 36.0 & 37.0 & 47.5 & 56.8 & 60.0 & 69.5 & 72.0 \\
\hline 99 & 27.0 & 32.0 & 35.0 & 37.0 & 46.0 & 56.0 & 60.0 & 67.0 & 72.0 \\
\hline
\end{tabular}

\section{Chippewa Creek at Easton, Ohio}

Years analyzed $=21$

Climatic year range $=1960-80$

[APEE, annual percentage equaled or exceeded; $\mathrm{ft}^{3} / \mathrm{s}$, cubic foot per second; Sep, September; Nov, November]

\begin{tabular}{|c|c|c|c|c|c|c|c|c|c|}
\hline \multirow[b]{2}{*}{ APEE } & \multicolumn{9}{|c|}{ Sep-Nov daily mean streamflow $\left(\mathrm{ft}^{3} / \mathrm{s}\right)$ that was equaled or exceeded the indicated percentage of time at APEE level } \\
\hline & 95 & 90 & 80 & 70 & 50 & 30 & 20 & 10 & 5 \\
\hline 1 & 24.4 & 38.2 & 78.8 & 115. & 210. & 535. & 591. & 1280 & 3720. \\
\hline 2 & 21.7 & 33.8 & 62.8 & 102. & 144. & 356. & 498. & 1260. & 2450 \\
\hline 5 & 14.4 & 18.0 & 40.6 & 53.5 & 82.8 & 211. & 266. & 883. & 1000. \\
\hline 10 & 11.4 & 15.4 & 23.5 & 34.0 & 46.8 & 100. & 180. & 322. & 854. \\
\hline 20 & 10.2 & 14.2 & 17.6 & 23.8 & 32.2 & 53.7 & 104. & 132. & 443. \\
\hline 25 & 9.76 & 13.2 & 16.2 & 21.2 & 30.0 & 44.4 & 81.4 & 110. & 408 \\
\hline 30 & 9.76 & 13.2 & 15.0 & 19.4 & 26.0 & 41.4 & 69.2 & 103. & 302. \\
\hline 40 & 9.30 & 12.0 & 13.0 & 14.6 & 22.2 & 31.3 & 54.1 & 82.8 & 214. \\
\hline 50 & 8.84 & 11.0 & 12.0 & 13.2 & 20.0 & 27.6 & 40.2 & 71.0 & 167. \\
\hline 60 & 8.32 & 9.52 & 10.8 & 12.6 & 17.8 & 25.6 & 33.5 & 56.6 & 116. \\
\hline 70 & 7.92 & 9.02 & 10.3 & 11.0 & 15.0 & 22.2 & 28.9 & 48.1 & 92.2 \\
\hline 75 & 7.87 & 8.52 & 9.76 & 11.0 & 15.0 & 20.8 & 24.2 & 44.6 & 84.8 \\
\hline 80 & 7.19 & 7.62 & 9.13 & 9.92 & 14.0 & 20.0 & 21.4 & 40.0 & 72.1 \\
\hline 90 & 6.22 & 6.56 & 8.06 & 9.06 & 13.0 & 16.9 & 20.6 & 28.2 & 35.4 \\
\hline 95 & 5.09 & 5.98 & 7.13 & 8.72 & 12.0 & 16.0 & 18.6 & 25.8 & 33.8 \\
\hline 98 & 4.47 & 5.22 & 6.68 & 8.28 & 10.8 & 14.4 & 17.1 & 25.5 & 32.2 \\
\hline 99 & 4.22 & 4.64 & 6.58 & 7.94 & 10.0 & 14.4 & 16.6 & 24.0 & 31.3 \\
\hline
\end{tabular}


03117000 Tuscarawas River at Massillon, Ohio

Years analyzed $=71$

Climatic year range $=1938-2008$

[APEE, annual percentage equaled or exceeded; $\mathrm{ft}^{3} / \mathrm{s}$, cubic foot per second; Sep, September; Nov, November]

\begin{tabular}{|c|c|c|c|c|c|c|c|c|c|}
\hline \multirow[b]{2}{*}{ APEE } & \multicolumn{9}{|c|}{ Sep-Nov daily mean streamflow $\left(\mathrm{ft}^{3} / \mathrm{s}\right)$ that was equaled or exceeded the indicated percentage of time at APEE level } \\
\hline & 98 & 95 & 90 & 80 & 50 & 20 & 10 & 5 & 2 \\
\hline 1 & 124. & 214 & 300. & 449. & 958. & 2290 & 2880 & 3540. & 6600. \\
\hline 2 & 118. & 159. & 257. & 354. & 728. & 1980. & 2440 & 3300 . & 6010. \\
\hline 5 & 103. & 119. & 198. & 254. & 453. & 1180. & 2010 & 2660. & 3970. \\
\hline 10 & 89.9 & 107. & 149. & 200. & 317. & 671. & 1130 & 1800. & 2260 . \\
\hline 20 & 86.0 & 99.6 & 134. & 150. & 215. & 421. & 572. & 987. & 1160. \\
\hline 25 & 83.9 & 98.4 & 127. & 142. & 190. & 359. & 484. & 704. & 991. \\
\hline 30 & 82.5 & 96.2 & 113. & 132. & 176. & 323. & 432. & 589. & 854. \\
\hline 40 & 78.8 & 93.4 & 105. & 123. & 153. & 257. & 355. & 456. & 608. \\
\hline 50 & 75.6 & 88.2 & 96.2 & 115. & 137. & 211. & 315. & 390. & 505. \\
\hline 60 & 73.6 & 84.0 & 92.0 & 107. & 125. & 186. & 267. & 334. & 399. \\
\hline 70 & 71.5 & 75.4 & 87.2 & 98.8 & 115. & 172. & 233. & 269. & 342. \\
\hline 75 & 70.8 & 74.6 & 85.2 & 94.4 & 112. & 166. & 210. & 247. & 319. \\
\hline 80 & 67.7 & 71.2 & 78.8 & 89.2 & 109. & 160. & 198. & 237. & 302. \\
\hline 90 & 60.3 & 67.1 & 70.4 & 81.2 & 101. & 141. & 167. & 202. & 268. \\
\hline 95 & 57.4 & 61.4 & 68.0 & 76.0 & 96.2 & 133. & 151. & 183. & 217. \\
\hline 98 & 53.4 & 58.1 & 63.9 & 71.7 & 93.0 & 127. & 147. & 176. & 196. \\
\hline 99 & 52.3 & 57.0 & 62.0 & 70.4 & 90.0 & 125. & 145. & 171. & 191. \\
\hline
\end{tabular}

\section{Middle Branch Nimishillen Creek at Canton, Ohio}

Years analyzed $=68$

Climatic year range $=1941-2008$

[APEE, annual percentage equaled or exceeded; $\mathrm{ft}^{3} / \mathrm{s}$, cubic foot per second; Sep, September; Nov, November]

\begin{tabular}{|c|c|c|c|c|c|c|c|c|c|}
\hline \multirow[b]{2}{*}{ APEE } & \multicolumn{9}{|c|}{ Sep-Nov daily mean streamflow $\left(\mathrm{ft}^{3} / \mathrm{s}\right)$ that was equaled or exceeded the indicated percentage of time at APEE level } \\
\hline & 98 & 95 & 90 & 80 & 50 & 20 & 10 & 5 & 2 \\
\hline 1 & 2.74 & 5.32 & 20.8 & 35.4 & 82.0 & 234. & 322 & 418 & 785. \\
\hline 2 & 2.64 & 4.91 & 16.1 & 25.2 & 62.0 & 176. & 259 & 353. & 534. \\
\hline 5 & 2.28 & 3.93 & 7.01 & 16.8 & 40.9 & 112. & 183. & 193. & 216. \\
\hline 10 & 2.17 & 3.45 & 5.66 & 11.7 & 24.3 & 67.7 & 119. & 144. & 180. \\
\hline 20 & 1.78 & 2.92 & 4.93 & 7.52 & 16.3 & 38.4 & 60.1 & 72.3 & 85.5 \\
\hline 25 & 1.72 & 2.67 & 4.64 & 6.08 & 14.0 & 33.4 & 48.6 & 60.6 & 70.1 \\
\hline 30 & 1.64 & 2.64 & 4.03 & 5.39 & 13.0 & 26.8 & 41.2 & 51.1 & 65.2 \\
\hline 40 & 1.44 & 2.38 & 3.18 & 4.55 & 10.0 & 20.4 & 34.4 & 41.7 & 56.8 \\
\hline 50 & 1.14 & 1.99 & 2.78 & 3.88 & 9.05 & 17.2 & 27.4 & 35.2 & 48.3 \\
\hline 60 & 1.06 & 1.80 & 2.29 & 3.69 & 7.88 & 15.2 & 24.2 & 31.1 & 42.4 \\
\hline 70 & 0.95 & 1.60 & 2.09 & 2.92 & 6.85 & 14.0 & 22.1 & 25.5 & 37.9 \\
\hline 75 & 0.93 & 1.55 & 1.99 & 2.88 & 6.75 & 13.2 & 21.1 & 23.5 & 35.3 \\
\hline 80 & 0.92 & 1.47 & 1.79 & 2.72 & 6.33 & 12.2 & 20.0 & 21.5 & 34.1 \\
\hline 90 & 0.82 & 1.30 & 1.42 & 2.31 & 5.65 & 11.0 & 17.3 & 19.0 & 30.0 \\
\hline 95 & 0.56 & 1.04 & 1.35 & 1.88 & 4.94 & 10.2 & 14.3 & 17.8 & 21.2 \\
\hline 98 & 0.43 & 0.88 & 1.15 & 1.58 & 4.54 & 10.0 & 12.2 & 16.6 & 19.1 \\
\hline 99 & 0.36 & 0.69 & 0.90 & 1.58 & 4.40 & 9.60 & 12.2 & 16.1 & 18.6 \\
\hline
\end{tabular}


03118500 Nimishillen Creek at North Industry, Ohio

Years analyzed $=88$

Climatic year range $=1921-2008$

[APEE, annual percentage equaled or exceeded; $\mathrm{ft}^{3} / \mathrm{s}$, cubic foot per second; Sep, September; Nov, November]

\begin{tabular}{|c|c|c|c|c|c|c|c|c|c|}
\hline \multirow[b]{2}{*}{ APEE } & \multicolumn{9}{|c|}{ Sep-Nov daily mean streamflow $\left(\mathrm{ft}^{3} / \mathrm{s}\right)$ that was equaled or exceeded the indicated percentage of time at APEE level } \\
\hline & 98 & 95 & 90 & 80 & 50 & 20 & 10 & 5 & 2 \\
\hline 1 & 92.3 & 105. & 147. & 262. & 504. & 1070. & 1450 & 2220. & 3530. \\
\hline 2 & 86.0 & 93.0 & 119. & 195. & 400 & 909. & 1120 & 1700. & 2070. \\
\hline 5 & 61.3 & 67.3 & 86.9 & 128. & 258. & 511. & 751. & 896. & 1180. \\
\hline 10 & 49.3 & 60.0 & 67.8 & 96.8 & 167. & 322. & 492. & 575. & 707. \\
\hline 20 & 38.1 & 49.5 & 61.8 & 73.8 & 118. & 218 & 266. & 363. & 399. \\
\hline 25 & 36.7 & 47.9 & 57.9 & 70.0 & 107. & 201. & 240 & 303. & 351. \\
\hline 30 & 35.5 & 44.5 & 56.0 & 65.6 & 102. & 170 & 221. & 270. & 295. \\
\hline 40 & 33.5 & 40.3 & 54.7 & 61.0 & 90.7 & 133. & 197. & 219. & 247. \\
\hline 50 & 29.3 & 36.4 & 48.7 & 56.8 & 80.5 & 122. & 172. & 188. & 217. \\
\hline 60 & 27.6 & 34.0 & 45.8 & 54.8 & 74.7 & 117. & 158. & 170. & 194. \\
\hline 70 & 25.8 & 31.9 & 44.3 & 52.0 & 71.3 & 110. & 139. & 152. & 174. \\
\hline 75 & 25.6 & 31.3 & 43.7 & 50.0 & 69.0 & 106. & 131. & 143. & 166. \\
\hline 80 & 24.6 & 30.1 & 42.5 & 48.8 & 67.5 & 103. & 126. & 138. & 162. \\
\hline 90 & 23.3 & 26.5 & 35.7 & 44.0 & 64.7 & 96.0 & 114. & 131. & 147. \\
\hline 95 & 21.7 & 24.7 & 33.1 & 41.8 & 62.0 & 89.3 & 105. & 124. & 133. \\
\hline 98 & 18.3 & 23.4 & 29.2 & 40.0 & 59.3 & 86.9 & 99.9 & 118. & 128. \\
\hline 99 & 16.8 & 21.4 & 29.0 & 39.8 & 57.5 & 85.0 & 97.2 & 114. & 126. \\
\hline
\end{tabular}

03145000 South Fork Licking River near Hebron, Ohio

Years analyzed $=50$

Climatic year range $=1939-2008$

[APEE, annual percentage equaled or exceeded; $\mathrm{ft}^{3} / \mathrm{s}$, cubic foot per second; Sep, September; Nov, November]

\begin{tabular}{|c|c|c|c|c|c|c|c|c|c|}
\hline \multirow[b]{2}{*}{ APEE } & \multicolumn{9}{|c|}{ Sep-Nov daily mean streamflow (ft $\left.{ }^{3} / \mathrm{s}\right)$ that was equaled or exceeded the indicated percentage of time at APEE level } \\
\hline & 98 & 95 & 90 & 80 & 50 & 20 & 10 & 5 & 2 \\
\hline 1 & 35.4 & 85.8 & 139. & 296. & 603. & 1210. & 1540 & 2160 & 4460 \\
\hline 2 & 23.0 & 85.4 & 134. & 211. & 477. & 931. & 1330. & 2070 . & 2840 \\
\hline 5 & 15.7 & 64.2 & 98.9 & 178. & 299. & 512. & 814. & 1360. & 1950. \\
\hline 10 & 10.8 & 11.4 & 51.3 & 110 . & 212. & 391. & 474. & 746. & 1510. \\
\hline 20 & 7.87 & 9.02 & 21.3 & 32.8 & 103. & 255. & 338. & 436. & 445. \\
\hline 25 & 7.20 & 7.59 & 9.86 & 22.6 & 74.0 & 171. & 274 & 323. & 405 \\
\hline 30 & 6.31 & 7.07 & 9.26 & 18.5 & 47.4 & 134. & 238. & 273. & 363. \\
\hline 40 & 5.31 & 5.96 & 7.16 & 12.0 & 30.8 & 60.2 & 122. & 188. & 324. \\
\hline 50 & 3.72 & 5.07 & 5.73 & 9.14 & 20.5 & 39.8 & 73.1 & 134. & 242 \\
\hline 60 & 3.52 & 4.46 & 4.92 & 7.14 & 15.0 & 31.2 & 44.8 & 84.5 & 153. \\
\hline 70 & 3.20 & 3.73 & 4.43 & 6.23 & 12.3 & 21.0 & 34.4 & 57.8 & 81.7 \\
\hline 75 & 3.10 & 3.37 & 3.93 & 5.30 & 10.5 & 19.0 & 27.8 & 49.1 & 66.9 \\
\hline 80 & 3.00 & 3.06 & 3.45 & 4.93 & 9.66 & 17.7 & 24.3 & 39.1 & 51.8 \\
\hline 90 & 2.00 & 2.18 & 2.73 & 3.99 & 7.76 & 15.2 & 19.9 & 26.3 & 41.0 \\
\hline 95 & 0.97 & 1.84 & 2.43 & 3.62 & 6.71 & 14.5 & 17.9 & 22.8 & 30.7 \\
\hline 98 & 0.78 & 1.34 & 2.09 & 3.57 & 6.14 & 13.8 & 14.9 & 20.6 & 24.8 \\
\hline 99 & 0.70 & 1.30 & 2.01 & 3.42 & 5.95 & 13.8 & 14.9 & 19.8 & 24.0 \\
\hline
\end{tabular}


03156000 Hunters Run at Lancaster, Ohio

Years analyzed $=23$

Climatic year range $=1956-78$

[APEE, annual percentage equaled or exceeded; $\mathrm{ft}^{3} / \mathrm{s}$, cubic foot per second; Sep, September; Nov, November]

\begin{tabular}{|c|c|c|c|c|c|c|c|c|c|}
\hline \multirow[b]{2}{*}{ APEE } & \multicolumn{9}{|c|}{ Sep-Nov daily mean streamflow $\left(\mathrm{ft}^{3} / \mathrm{s}\right)$ that was equaled or exceeded the indicated percentage of time at APEE level } \\
\hline & 95 & 90 & 80 & 70 & 50 & 30 & 20 & 10 & 5 \\
\hline 1 & 1.56 & 1.96 & 5.28 & 8.00 & 27.0 & 37.4 & 61.4 & 113. & 120. \\
\hline 2 & 1.46 & 1.76 & 4.05 & 7.33 & 15.1 & 33.9 & 55.1 & 83.7 & 92.5 \\
\hline 5 & 1.30 & 1.43 & 2.65 & 4.36 & 7.00 & 18.5 & 27.0 & 41.1 & 53.2 \\
\hline 10 & 1.10 & 1.26 & 2.19 & 3.13 & 5.34 & 9.16 & 14.0 & 21.6 & 25.1 \\
\hline 20 & 1.00 & 1.12 & 1.66 & 2.24 & 3.40 & 6.18 & 7.38 & 12.6 & 14.3 \\
\hline 25 & 1.00 & 1.12 & 1.58 & 2.22 & 2.90 & 4.94 & 6.32 & 9.26 & 12.3 \\
\hline 30 & 0.95 & 1.12 & 1.48 & 2.02 & 2.64 & 4.50 & 5.96 & 8.02 & 10.4 \\
\hline 40 & 0.82 & 1.02 & 1.37 & 1.92 & 2.24 & 3.74 & 4.86 & 6.05 & 6.40 \\
\hline 50 & 0.80 & 0.96 & 1.28 & 1.72 & 2.00 & 3.12 & 3.66 & 5.10 & 6.22 \\
\hline 60 & 0.78 & 0.94 & 1.20 & 1.52 & 1.80 & 2.68 & 3.18 & 4.73 & 5.46 \\
\hline 70 & 0.70 & 0.86 & 1.20 & 1.27 & 1.60 & 2.29 & 2.51 & 4.42 & 5.20 \\
\hline 75 & 0.62 & 0.82 & 1.10 & 1.20 & 1.40 & 2.04 & 2.42 & 4.30 & 4.86 \\
\hline 80 & 0.54 & 0.82 & 1.10 & 1.12 & 1.40 & 1.97 & 2.22 & 4.00 & 4.40 \\
\hline 90 & 0.51 & 0.58 & 0.96 & 1.00 & 1.22 & 1.68 & 1.92 & 3.05 & 3.76 \\
\hline 95 & 0.31 & 0.51 & 0.78 & 0.91 & 1.00 & 1.38 & 1.74 & 2.75 & 3.46 \\
\hline 98 & 0.22 & 0.46 & 0.68 & 0.89 & 1.00 & 1.25 & 1.57 & 2.56 & 3.26 \\
\hline 99 & 0.22 & 0.46 & 0.68 & 0.80 & 0.95 & 1.20 & 1.50 & 2.48 & 3.24 \\
\hline
\end{tabular}

\section{Hocking River at Athens, Ohio}

Years analyzed $=47$

Climatic year range $=1962-2008$

[APEE, annual percentage equaled or exceeded; $\mathrm{ft}^{3} / \mathrm{s}$, cubic foot per second; Sep, September; Nov, November]

\begin{tabular}{|c|c|c|c|c|c|c|c|c|c|}
\hline \multirow[b]{2}{*}{ APEE } & \multicolumn{9}{|c|}{ Sep-Nov daily mean streamflow $\left(\mathrm{ft}^{3} / \mathrm{s}\right)$ that was equaled or exceeded the indicated percentage of time at APEE leve } \\
\hline & 95 & 90 & 80 & 70 & 50 & 30 & 20 & 10 & 5 \\
\hline 1 & 123. & 199. & 398. & 817. & 1700. & 3800. & 5670. & 7680. & 11200. \\
\hline 2 & 118. & 178. & 321. & 670. & 1440. & 2730 & 5070. & 6860 & 9490. \\
\hline 5 & 112. & 156. & 282. & 377. & 885. & 1750. & 3410 . & 4710 & 6670. \\
\hline 10 & 105. & 134. & 214. & 256. & 511. & 1100 & 1910. & 2990 . & 3340 . \\
\hline 20 & 82.1 & 112. & 151. & 185. & 325. & 685. & 897. & 1620 & 1850. \\
\hline 25 & 76.4 & 104. & 142. & 167. & 287. & 512. & 710. & 1280 & 1630. \\
\hline 30 & 72.1 & 98.1 & 132. & 159. & 244. & 442. & 627. & 1120 & 1510. \\
\hline 40 & 65.8 & 87.8 & 116. & 136. & 187. & 303. & 503. & 855. & 1070. \\
\hline 50 & 62.8 & 75.0 & 103. & 113. & 151. & 246. & 394. & 611. & 825. \\
\hline 60 & 56.0 & 68.5 & 94.0 & 95.6 & 126. & 203. & 309. & 503. & 684. \\
\hline 70 & 51.5 & 64.9 & 83.4 & 89.6 & 108. & 180. & 253. & 444. & 584. \\
\hline 75 & 49.4 & 60.0 & 78.8 & 87.0 & 102. & 168. & 232. & 404. & 542. \\
\hline 80 & 47.7 & 59.8 & 72.8 & 85.1 & 98.0 & 151. & 221. & 371. & 481. \\
\hline 90 & 45.0 & 57.8 & 65.8 & 76.2 & 92.0 & 135. & 211. & 307. & 341. \\
\hline 95 & 43.4 & 55.6 & 61.6 & 73.2 & 87.0 & 121. & 158. & 238. & 302. \\
\hline 98 & 40.7 & 53.5 & 60.6 & 72.3 & 83.5 & 111. & 146. & 217. & 268. \\
\hline 99 & 40.2 & 52.0 & 60.6 & 71.4 & 83.0 & 111. & 136. & 211. & 250. \\
\hline
\end{tabular}


03261500 Great Miami River at Sidney, Ohio

Years analyzed $=83$

Climatic year range $=1926-2008$

[APEE, annual percentage equaled or exceeded; $\mathrm{ft}^{3} / \mathrm{s}$, cubic foot per second; Sep, September; Nov, November]

\begin{tabular}{|c|c|c|c|c|c|c|c|c|c|}
\hline \multirow[b]{2}{*}{ APEE } & \multicolumn{9}{|c|}{ Sep-Nov daily mean streamflow $\left(\mathrm{ft}^{3} / \mathrm{s}\right)$ that was equaled or exceeded the indicated percentage of time at APEE level } \\
\hline & 98 & 95 & 90 & 80 & 50 & 20 & 10 & 5 & 2 \\
\hline 1 & 61.1 & 98.2 & 117. & 181. & 576. & 2750. & 4220 & 6060. & 6710. \\
\hline 2 & 56.1 & 92.0 & 106. & 180. & 563. & 2190. & 3930. & 5440. & 6410. \\
\hline 5 & 51.7 & 73.2 & 87.8 & 120. & 324. & 1360. & 2330 & 3790. & 4430. \\
\hline 10 & 45.2 & 51.0 & 68.5 & 91.6 & 256. & 779. & 1510 . & 2110. & 2970. \\
\hline 20 & 36.3 & 45.2 & 53.6 & 68.9 & 152. & 372. & 706. & 1180. & 1540. \\
\hline 25 & 35.1 & 43.6 & 49.0 & 64.0 & 124. & 309. & 536. & 783. & 1260. \\
\hline 30 & 33.4 & 42.4 & 47.4 & 58.8 & 97.0 & 273 & 392. & 640. & 1030. \\
\hline 40 & 30.0 & 40.0 & 42.4 & 50.8 & 81.0 & 181. & 275. & 430. & 763. \\
\hline 50 & 27.8 & 37.0 & 39.0 & 46.0 & 68.0 & 138. & 223. & 362. & 523. \\
\hline 60 & 24.7 & 34.0 & 35.7 & 41.8 & 61.6 & 118. & 191. & 268. & 330. \\
\hline 70 & 23.6 & 31.2 & 33.0 & 36.8 & 54.8 & 110. & 165. & 197. & 301. \\
\hline 75 & 22.7 & 29.0 & 32.0 & 35.0 & 52.0 & 102. & 151. & 174. & 283. \\
\hline 80 & 21.6 & 26.8 & 30.0 & 33.0 & 50.8 & 96.5 & 132. & 149. & 256. \\
\hline 90 & 18.9 & 21.6 & 26.5 & 29.2 & 45.0 & 76.2 & 101. & 117. & 224. \\
\hline 95 & 16.2 & 18.6 & 23.4 & 28.8 & 42.0 & 69.2 & 92.8 & 109. & 214. \\
\hline 98 & 11.7 & 14.8 & 22.1 & 26.7 & 40.8 & 65.2 & 86.8 & 109. & 198. \\
\hline 99 & 10.0 & 14.4 & 21.8 & 26.0 & 40.0 & 64.2 & 85.6 & 109. & 190. \\
\hline
\end{tabular}

\section{Loramie Creek near Newport, Ohio}

Years analyzed $=45$

Climatic year range $=1964-2008$

[APEE, annual percentage equaled or exceeded; $\mathrm{ft}^{3} / \mathrm{s}$, cubic foot per second; Sep, September; Nov, November]

\begin{tabular}{|c|c|c|c|c|c|c|c|c|c|}
\hline \multirow[b]{2}{*}{ APEE } & \multicolumn{9}{|c|}{ Sep-Nov daily mean streamflow ( $\left.\mathrm{ft}^{3} / \mathrm{s}\right)$ that was equaled or exceeded the indicated percentage of time at APEE level } \\
\hline & 95 & 90 & 80 & 70 & 50 & 30 & 20 & 10 & 5 \\
\hline 1 & 9.12 & 13.8 & 30.2 & 66.8 & 259. & 1050. & 1620 & 2240 & 2870 \\
\hline 2 & 8.57 & 11.5 & 22.7 & 52.6 & 248 & 792. & 1430. & 1880. & 2470 \\
\hline 5 & 6.06 & 7.19 & 12.6 & 20.6 & 127. & 420. & 846. & 1290. & 1440. \\
\hline 10 & 4.80 & 5.48 & 7.14 & 11.9 & 47.0 & 224 & 366. & 502. & 729. \\
\hline 20 & 3.13 & 3.64 & 4.42 & 6.33 & 17.2 & 84.6 & 154. & 222. & 256. \\
\hline 25 & 2.83 & 3.36 & 3.82 & 4.98 & 13.0 & 49.0 & 97.6 & 169. & 187. \\
\hline 30 & 2.56 & 2.80 & 3.42 & 4.47 & 11.0 & 26.7 & 85.0 & 124. & 136. \\
\hline 40 & 1.54 & 1.96 & 2.56 & 3.24 & 7.80 & 15.8 & 44.8 & 74.0 & 84.3 \\
\hline 50 & 1.16 & 1.36 & 1.80 & 2.70 & 5.50 & 10.6 & 34.2 & 46.4 & 59.1 \\
\hline 60 & 0.93 & 1.10 & 1.44 & 2.30 & 3.46 & 7.60 & 23.8 & 34.0 & 41.9 \\
\hline 70 & 0.61 & 0.94 & 1.26 & 1.87 & 3.02 & 5.31 & 15.5 & 26.8 & 33.7 \\
\hline 75 & 0.52 & 0.83 & 1.04 & 1.70 & 2.50 & 5.06 & 11.6 & 21.0 & 30.8 \\
\hline 80 & 0.39 & 0.74 & 1.02 & 1.55 & 2.34 & 4.27 & 9.12 & 15.8 & 26.9 \\
\hline 90 & 0.25 & 0.45 & 0.84 & 1.08 & 1.80 & 2.65 & 3.80 & 8.50 & 10.3 \\
\hline 95 & 0.23 & 0.35 & 0.69 & 0.84 & 1.26 & 2.43 & 2.94 & 5.76 & 7.56 \\
\hline 98 & 0.21 & 0.31 & 0.57 & 0.69 & 1.10 & 1.99 & 2.73 & 4.29 & 6.03 \\
\hline 99 & 0.20 & 0.28 & 0.50 & 0.64 & 1.00 & 1.92 & 2.58 & 3.90 & 5.85 \\
\hline
\end{tabular}




\section{Loramie Creek at Lockington, Ohio}

Years analyzed $=94$

Climatic year range $=1915-2008$

[APEE, annual percentage equaled or exceeded; $\mathrm{ft}^{3} / \mathrm{s}$, cubic foot per second; Sep, September; Nov, November]

\begin{tabular}{|c|c|c|c|c|c|c|c|c|c|}
\hline \multirow[b]{2}{*}{ APEE } & \multicolumn{9}{|c|}{ Sep-Nov daily mean streamflow $\left(\mathrm{ft}^{3} / \mathrm{s}\right)$ that was equaled or exceeded the indicated percentage of time at APEE level } \\
\hline & 98 & 95 & 90 & 80 & 50 & 20 & 10 & 5 & 2 \\
\hline 1 & 6.46 & 11.8 & 20.0 & 37.0 & 341. & 1750. & 2910 . & 3670 & 4340 \\
\hline 2 & 6.46 & 11.1 & 17.9 & 34.4 & 226. & 1540 & 2420 & 3050 . & 3640. \\
\hline 5 & 6.10 & 9.38 & 13.7 & 20.0 & 97.6 & 820 . & 1310 . & 1900. & 2460 \\
\hline 10 & 5.72 & 8.75 & 10.9 & 14.8 & 48.4 & 346. & 651. & 818. & 1280 \\
\hline 20 & 4.50 & 7.48 & 8.08 & 10.0 & 23.0 & 155. & 248 & 312. & 785. \\
\hline 25 & 4.38 & 6.85 & 7.70 & 10.0 & 19.5 & 109. & 200 . & 245 & 641. \\
\hline 30 & 4.17 & 6.58 & 7.19 & 9.00 & 17.4 & 75.0 & 136. & 180. & 494. \\
\hline 40 & 3.70 & 5.89 & 6.60 & 7.74 & 13.5 & 53.2 & 94.0 & 126. & 353. \\
\hline 50 & 3.37 & 5.18 & 5.95 & 6.90 & 12.0 & 39.0 & 68.5 & 91.5 & 230. \\
\hline 60 & 3.35 & 4.80 & 5.39 & 6.06 & 10.0 & 27.0 & 53.4 & 68.1 & 105. \\
\hline 70 & 3.10 & 4.09 & 4.65 & 5.20 & 9.00 & 22.0 & 43.5 & 56.2 & 74.1 \\
\hline 75 & 2.67 & 3.85 & 4.35 & 5.00 & 8.60 & 18.0 & 40.0 & 49.5 & 66.1 \\
\hline 80 & 2.53 & 3.59 & 4.15 & 4.74 & 8.14 & 17.0 & 31.0 & 44.6 & 59.2 \\
\hline 90 & 1.83 & 2.65 & 3.63 & 4.20 & 7.06 & 14.0 & 21.6 & 37.7 & 44.2 \\
\hline 95 & 1.15 & 2.20 & 3.11 & 3.96 & 6.65 & 12.6 & 19.5 & 33.6 & 42.8 \\
\hline 98 & 0.68 & 2.17 & 2.70 & 3.80 & 6.00 & 10.8 & 17.7 & 28.4 & 36.0 \\
\hline 99 & 0.50 & 1.63 & 2.65 & 3.70 & 5.80 & 10.0 & 14.0 & 24.3 & 33.0 \\
\hline
\end{tabular}

\section{Great Miami River at Troy, Ohio}

Years analyzed $=47$

Climatic year range $=1962-2008$

[APEE, annual percentage equaled or exceeded; $\mathrm{ft}^{3} / \mathrm{s}$, cubic foot per second; Sep, September; Nov, November]

\begin{tabular}{|c|c|c|c|c|c|c|c|c|c|}
\hline \multirow[b]{2}{*}{ APEE } & \multicolumn{9}{|c|}{ Sep-Nov daily mean streamflow ( $\left.\mathrm{ft}^{3} / \mathrm{s}\right)$ that was equaled or exceeded the indicated percentage of time at APEE level } \\
\hline & 95 & 90 & 80 & 70 & 50 & 30 & 20 & 10 & 5 \\
\hline 1 & 141. & 184. & 259. & 394. & 1080. & 3800. & 6270 & 10000 & 12000 \\
\hline 2 & 137. & 178. & 242. & 373. & 1000. & 3270 . & 5600. & 9370. & 11100 \\
\hline 5 & 117. & 151. & 207. & 281. & 589. & 2080 & 3720. & 5480. & 7370 . \\
\hline 10 & 78.5 & 115. & 145. & 226. & 399. & 837. & 1930. & 2850 & 3560 . \\
\hline 20 & 63.9 & 91.4 & 106. & 143. & 245. & 485. & 842. & 1410 . & 1740 . \\
\hline 25 & 57.4 & 83.0 & 102. & 122. & 190. & 324. & 609. & 980. & 1310. \\
\hline 30 & 56.0 & 80.6 & 94.2 & 110. & 177. & 266. & 511. & 804. & 1040. \\
\hline 40 & 52.8 & 71.8 & 79.3 & 90.5 & 113. & 229. & 327. & 614. & 747. \\
\hline 50 & 50.2 & 64.0 & 72.6 & 84.8 & 105. & 177. & 250. & 511. & 566. \\
\hline 60 & 47.3 & 60.2 & 67.5 & 76.4 & 95.8 & 155. & 219. & 389. & 441. \\
\hline 70 & 44.1 & 48.9 & 61.0 & 70.8 & 86.2 & 143. & 193. & 296. & 347. \\
\hline 75 & 40.4 & 44.6 & 59.6 & 67.4 & 83.0 & 136. & 182. & 268. & 328. \\
\hline 80 & 38.0 & 40.9 & 57.0 & 64.3 & 78.8 & 131. & 173. & 232. & 266. \\
\hline 90 & 34.0 & 38.6 & 52.0 & 56.9 & 72.2 & 112. & 141. & 183. & 225 . \\
\hline 95 & 31.6 & 37.5 & 46.5 & 50.5 & 68.6 & 105. & 129. & 155. & 211. \\
\hline 98 & 28.4 & 34.0 & 40.0 & 48.0 & 64.7 & 100 & 114. & 146. & 195. \\
\hline 99 & 26.4 & 32.8 & 38.8 & 46.6 & 64.0 & 95.8 & 111. & 144. & 186. \\
\hline
\end{tabular}


03263000 Great Miami River at Taylorsville, Ohio

Years analyzed $=88$

Climatic year range $=1921-2008$

[APEE, annual percentage equaled or exceeded; $\mathrm{ft}^{3} / \mathrm{s}$, cubic foot per second; Sep, September; Nov, November]

\begin{tabular}{|c|c|c|c|c|c|c|c|c|c|}
\hline \multirow[b]{2}{*}{ APEE } & \multicolumn{9}{|c|}{ Sep-Nov daily mean streamflow $\left(\mathrm{ft}^{3} / \mathrm{s}\right)$ that was equaled or exceeded the indicated percentage of time at APEE level } \\
\hline & 98 & 95 & 90 & 80 & 50 & 20 & 10 & 5 & 2 \\
\hline 1 & 108. & 192. & 232. & 299. & 1240. & 4840. & 10300 & 12400 & 14900. \\
\hline 2 & 92.6 & 181. & 219. & 293. & 952. & 3980. & 8890. & 10700. & 13100. \\
\hline 5 & 87.9 & 145. & 179. & 238 & 664. & 2510. & 5280. & 8080 & 10300. \\
\hline 10 & 82.8 & 99.8 & 148. & 181. & 408. & 1480. & 2720 & 3980. & 5200. \\
\hline 20 & 72.6 & 85.2 & 118. & 138. & 284. & 823. & 1530. & 2000 & 3180 . \\
\hline 25 & 69.2 & 83.5 & 109. & 126. & 246. & 616. & 1070. & 1600. & 2690. \\
\hline 30 & 66.9 & 80.1 & 104. & 116. & 215. & 519. & 952. & 1370 . & 2410. \\
\hline 40 & 63.2 & 75.0 & 90.4 & 102. & 169. & 396. & 670. & 1030. & 1830. \\
\hline 50 & 62.5 & 70.3 & 82.7 & 95.6 & 148. & 285. & 507. & 748. & 1290. \\
\hline 60 & 59.5 & 66.9 & 75.6 & 88.8 & 130. & 256. & 405. & 631. & 781. \\
\hline 70 & 54.9 & 64.9 & 68.0 & 82.8 & 118. & 240. & 350. & 516. & 663. \\
\hline 75 & 53.4 & 60.9 & 64.9 & 79.8 & 116. & 221. & 332. & 496. & 615. \\
\hline 80 & 53.0 & 57.4 & 62.0 & 74.6 & 108. & 209. & 259. & 396. & 575. \\
\hline 90 & 45.5 & 53.5 & 57.8 & 66.6 & 96.2 & 170. & 203. & 321. & 497. \\
\hline 95 & 39.9 & 51.0 & 54.5 & 62.8 & 91.7 & 157. & 194. & 255. & 463. \\
\hline 98 & 37.6 & 47.2 & 52.6 & 56.0 & 84.1 & 140. & 184. & 206. & 432. \\
\hline 99 & 35.6 & 45.9 & 51.9 & 55.8 & 82.5 & 135. & 179. & 197. & 384. \\
\hline
\end{tabular}

03266000 Stillwater River at Englewood, Ohio

Years analyzed $=84$

Climatic year range $=1925-2008$

[APEE, annual percentage equaled or exceeded; $\mathrm{ft}^{3} / \mathrm{s}$, cubic foot per second; Sep, September; Nov, November]

\begin{tabular}{|c|c|c|c|c|c|c|c|c|c|}
\hline \multirow[b]{2}{*}{ APEE } & \multicolumn{9}{|c|}{ Sep-Nov daily mean streamflow $\left(\mathrm{ft}^{3} / \mathrm{s}\right)$ that was equaled or exceeded the indicated percentage of time at APEE level } \\
\hline & 98 & 95 & 90 & 80 & 50 & 20 & 10 & 5 & 2 \\
\hline 1 & 42.9 & 57.5 & 88.5 & 149. & 837. & 3920. & 6050 & 6520 & 7490. \\
\hline 2 & 42.1 & 55.3 & 83.4 & 131. & 545. & 3260 . & 5850. & 6400 & 7380 . \\
\hline 5 & 40.7 & 48.8 & 76.6 & 95.4 & 258. & 1490. & 4260 & 5230. & 6340. \\
\hline 10 & 38.2 & 43.8 & 62.2 & 79.8 & 193. & 961. & 1700 & 3150 . & 3570 . \\
\hline 20 & 30.7 & 37.1 & 50.7 & 65.0 & 126. & 411. & 664. & 1200 & 1890. \\
\hline 25 & 29.7 & 34.3 & 47.0 & 61.0 & 115. & 320. & 479. & 987. & 1450. \\
\hline 30 & 29.1 & 33.4 & 42.9 & 58.4 & 100. & 250 & 427. & 736. & 1180. \\
\hline 40 & 26.5 & 31.2 & 38.0 & 52.4 & 87.0 & 190. & 324. & 541. & 830. \\
\hline 50 & 22.8 & 26.0 & 33.5 & 46.0 & 72.5 & 150. & 274. & 412. & 547. \\
\hline 60 & 19.8 & 23.0 & 30.0 & 40.0 & 65.9 & 122. & 208. & 301. & 477. \\
\hline 70 & 14.9 & 20.1 & 22.6 & 36.6 & 56.9 & 106. & 166. & 266. & 451. \\
\hline 75 & 13.4 & 19.3 & 21.0 & 35.0 & 53.0 & 95.0 & 155. & 253. & 429. \\
\hline 80 & 12.8 & 18.0 & 19.5 & 32.0 & 51.4 & 88.8 & 139. & 244. & 404. \\
\hline 90 & 11.0 & 15.4 & 17.0 & 25.0 & 43.6 & 76.4 & 118. & 230 & 284. \\
\hline 95 & 8.66 & 12.2 & 15.0 & 23.0 & 40.1 & 66.6 & 109. & 185. & 261. \\
\hline 98 & 5.53 & 9.92 & 13.8 & 19.8 & 36.2 & 63.5 & 100. & 166. & 247. \\
\hline 99 & 5.22 & 8.65 & 12.0 & 19.0 & 36.0 & 61.0 & 99.5 & 159. & 239. \\
\hline
\end{tabular}


03267900 Mad River at St Paris Pike at Eagle City, Ohio

Years analyzed $=38$

Climatic year range $=1965-2008$

[APEE, annual percentage equaled or exceeded; $\mathrm{ft}^{3} / \mathrm{s}$, cubic foot per second; Sep, September; Nov, November]

\begin{tabular}{|c|c|c|c|c|c|c|c|c|c|}
\hline \multirow[b]{2}{*}{ APEE } & \multicolumn{9}{|c|}{ Sep-Nov daily mean streamflow $\left(\mathrm{ft}^{3} / \mathrm{s}\right)$ that was equaled or exceeded the indicated percentage of time at APEE leve } \\
\hline & 95 & 90 & 80 & 70 & 50 & 30 & 20 & 10 & 5 \\
\hline 1 & 141. & 152. & 212. & 286. & 548. & 968. & 1500. & 2110 & 2790. \\
\hline 2 & 139. & 145 & 206. & 238. & 442. & 716. & 1370. & 1810 & 2370 . \\
\hline 5 & 129. & 136. & 170 . & 201. & 274. & 418. & 807. & 965. & 1540. \\
\hline 10 & 121. & 130. & 153. & 177. & 217. & 306. & 444. & 614. & 887. \\
\hline 20 & 119. & 123. & 135. & 145. & 186. & 237. & 301. & 400 & 498. \\
\hline 25 & 118. & 122. & 131. & 137. & 179. & 219. & 269. & 372. & 436. \\
\hline 30 & 115 & 118. & 127. & 132. & 171. & 210 & 250. & 355. & 416. \\
\hline 40 & 105. & 115. & 118. & 127. & 160. & 199. & 236. & 290. & 374. \\
\hline 50 & 100 & 112. & 116. & 125. & 156. & 182. & 222. & 272 & 350. \\
\hline 60 & 99.0 & 109. & 113. & 118. & 149. & 172. & 209. & 240 & 336. \\
\hline 70 & 97.1 & 105 & 110 . & 113. & 143. & 167. & 201. & 232. & 314. \\
\hline 75 & 97.1 & 99.8 & 107. & 113. & 141. & 161. & 188. & 230. & 280. \\
\hline 80 & 95.5 & 97.9 & 105 & 112. & 137. & 156. & 174. & 207. & 238. \\
\hline 90 & 85.7 & 96.4 & 102. & 110. & 132. & 148. & 158. & 198. & 226. \\
\hline 95 & 83.6 & 94.4 & 99.3 & 109. & 129. & 143 & 157. & 196. & 222. \\
\hline 98 & 81.7 & 93.3 & 96.8 & 106. & 126. & 140 & 155. & 195. & 218. \\
\hline 99 & 81.7 & 92.5 & 95.6 & 105. & 126. & 139. & 155. & 193. & 218. \\
\hline
\end{tabular}

\section{Buck Creek at Springfield, Ohio}

Years analyzed $=33$

Climatic year range $=1914-73$

[APEE, annual percentage equaled or exceeded; $\mathrm{ft}^{3} / \mathrm{s}$, cubic foot per second; Sep, September; Nov, November]

\begin{tabular}{|c|c|c|c|c|c|c|c|c|c|}
\hline \multirow[b]{2}{*}{ APEE } & \multicolumn{9}{|c|}{ Sep-Nov daily mean streamflow $\left(\mathrm{ft}^{3} / \mathrm{s}\right)$ that was equaled or exceeded the indicated percentage of time at APEE level } \\
\hline & 95 & 90 & 80 & 70 & 50 & 30 & 20 & 10 & 5 \\
\hline 1 & 51.0 & 67.0 & 86.0 & 148. & 225. & 486. & 743. & 880. & 915. \\
\hline 2 & 49.0 & 62.2 & 72.4 & 108. & 163. & 385. & 446. & 700 . & 767. \\
\hline 5 & 35.1 & 47.3 & 57.6 & 79.2 & 106. & 218. & 296. & 464. & 507. \\
\hline 10 & 29.2 & 38.1 & 47.2 & 63.4 & 87.0 & 114. & 197. & 234. & 316. \\
\hline 20 & 25.6 & 32.6 & 40.0 & 50.2 & 65.0 & 88.8 & 126. & 161. & 191. \\
\hline 25 & 24.6 & 31.6 & 39.4 & 44.0 & 64.0 & 80.0 & 103. & 136. & 180. \\
\hline 30 & 24.6 & 30.2 & 36.0 & 40.2 & 60.8 & 78.4 & 101. & 125 & 159. \\
\hline 40 & 21.1 & 27.0 & 34.4 & 37.6 & 53.0 & 68.9 & 84.8 & 99.0 & 127. \\
\hline 50 & 18.7 & 25.8 & 29.8 & 36.0 & 51.0 & 67.0 & 76.8 & 93.6 & 117. \\
\hline 60 & 15.3 & 23.8 & 28.0 & 32.4 & 46.0 & 60.0 & 75.4 & 87.5 & 105. \\
\hline 70 & 13.8 & 23.8 & 26.0 & 30.2 & 46.0 & 58.1 & 68.6 & 80.8 & 99.5 \\
\hline 75 & 13.2 & 21.8 & 25.8 & 28.6 & 45.0 & 56.8 & 62.4 & 77.0 & 97.5 \\
\hline 80 & 11.9 & 20.4 & 24.8 & 28.6 & 43.0 & 55.4 & 61.4 & 76.4 & 94.8 \\
\hline 90 & 11.0 & 17.8 & 21.0 & 27.7 & 36.0 & 51.4 & 59.2 & 74.1 & 86.4 \\
\hline 95 & 10.8 & 16.4 & 19.8 & 26.7 & 35.0 & 49.3 & 53.2 & 68.2 & 82.7 \\
\hline 98 & 9.33 & 16.0 & 18.4 & 24.9 & 34.0 & 47.4 & 52.0 & 66.4 & 78.1 \\
\hline 99 & 7.14 & 16.0 & 18.4 & 23.6 & 34.0 & 46.0 & 52.0 & 58.0 & 74.2 \\
\hline
\end{tabular}




\section{Mad River near Springfield, Ohio}

Years analyzed $=97$

Climatic year range $=1904-2008$

[APEE, annual percentage equaled or exceeded; $\mathrm{ft}^{3} / \mathrm{s}$, cubic foot per second; Sep, September; Nov, November]

\begin{tabular}{|c|c|c|c|c|c|c|c|c|c|}
\hline \multirow[b]{2}{*}{ APEE } & \multicolumn{9}{|c|}{ Sep-Nov daily mean streamflow ( $\left.\mathrm{ft}^{3} / \mathrm{s}\right)$ that was equaled or exceeded the indicated percentage of time at APEE level } \\
\hline & 98 & 95 & 90 & 80 & 50 & 20 & 10 & 5 & 2 \\
\hline 1 & 172. & 223. & 271. & 346. & 754. & 2130. & 3300 & 4130. & 5690. \\
\hline 2 & 170. & 200 & 244. & 310. & 628. & 1660. & 2430 & 3010. & 3640. \\
\hline 5 & 158. & 175. & 224. & 261. & 438. & 950. & 1420. & 1590. & 2420 . \\
\hline 10 & 148. & 163. & 193. & 241. & 352. & 619. & 901. & 1150. & 1430. \\
\hline 20 & 144. & 156. & 171. & 212. & 284 & 452. & 618. & 846. & 1250. \\
\hline 25 & 142. & 152. & 168. & 206. & 269. & 416. & 540. & 719. & 958. \\
\hline 30 & 134. & 152. & 166. & 201. & 261. & 388. & 481. & 641. & 886. \\
\hline 40 & 132. & 147. & 161. & 190. & 245. & 350. & 410. & 572. & 760. \\
\hline 50 & 128. & 145. & 157. & 183. & 237. & 320. & 373. & 516. & 621. \\
\hline 60 & 128. & 140. & 152. & 179. & 227. & 301. & 352. & 462. & 591. \\
\hline 70 & 115. & 135. & 148. & 173. & 212. & 290. & 343. & 408. & 560. \\
\hline 75 & 115 & 131. & 144. & 169. & 209. & 283. & 332. & 386. & 542. \\
\hline 80 & 110. & 123. & 144. & 165. & 204. & 277. & 323. & 368. & 526. \\
\hline 90 & 102. & 123. & 137. & 155. & 194. & 260. & 306. & 336. & 479. \\
\hline 95 & 99.8 & 118. & 132. & 146. & 189. & 250. & 296. & 318. & 445. \\
\hline 98 & 89.3 & 115. & 125. & 141. & 184. & 245. & 280 & 306. & 382. \\
\hline 99 & 85.7 & 113. & 123. & 140. & 183. & 242. & 270 & 297. & 380. \\
\hline
\end{tabular}

\section{Mad River near Dayton, Ohio}

Years analyzed $=91$

Climatic year range $=1915-2008$

[APEE, annual percentage equaled or exceeded; $\mathrm{ft}^{3} / \mathrm{s}$, cubic foot per second; Sep, September; Nov, November]

\begin{tabular}{|c|c|c|c|c|c|c|c|c|c|}
\hline \multirow[b]{2}{*}{ APEE } & \multicolumn{9}{|c|}{ Sep-Nov daily mean streamflow ( $\left.\mathrm{ft}^{3} / \mathrm{s}\right)$ that was equaled or exceeded the indicated percentage of time at APEE level } \\
\hline & 98 & 95 & 90 & 80 & 50 & 20 & 10 & 5 & 2 \\
\hline 1 & 205. & 250. & 351. & 404. & 992. & 2690. & 3820. & 4570. & 5630. \\
\hline 2 & 190. & 239. & 299. & 367. & 824. & 2050. & 3180. & 3880. & 4760 . \\
\hline 5 & 175. & 203. & 240 . & 302. & 602. & 1310. & 1940. & 2370 . & 2970. \\
\hline 10 & 160. & 187. & 213. & 274. & 460. & 858. & 1120. & 1600. & 1900. \\
\hline 20 & 157. & 173. & 203. & 252. & 359. & 628. & 722. & 1040. & 1390. \\
\hline 25 & 154. & 169. & 198. & 237. & 346. & 541. & 656. & 896. & 1230. \\
\hline 30 & 154. & 167. & 195. & 235 & 331. & 503. & 590. & 833. & 1020. \\
\hline 40 & 150. & 162. & 190. & 223. & 297. & 448. & 537. & 708. & 833. \\
\hline 50 & 147. & 158. & 182. & 216. & 283. & 402. & 488. & 675. & 770. \\
\hline 60 & 139. & 155. & 180. & 206. & 275. & 382. & 457. & 623. & 711. \\
\hline 70 & 128. & 145. & 177. & 198. & 265. & 360. & 432. & 556. & 666. \\
\hline 75 & 127. & 141. & 163. & 193. & 257. & 347. & 412. & 484. & 638. \\
\hline 80 & 122. & 131. & 159. & 190. & 242. & 334. & 400 & 473. & 606. \\
\hline 90 & 109. & 129. & 147. & 176. & 229. & 317. & 379. & 426. & 563. \\
\hline 95 & 106. & 125. & 144. & 170 . & 219. & 310. & 352. & 412. & 508. \\
\hline 98 & 103. & 124. & 138. & 166. & 214. & 293. & 333. & 385. & 491. \\
\hline 99 & 103. & 123. & 137. & 162. & 209. & 291. & 327. & 368. & 484. \\
\hline
\end{tabular}




\section{Great Miami River at Dayton, Ohio}

Years analyzed $=96$

Climatic year range $=1913-2008$

[APEE, annual percentage equaled or exceeded; $\mathrm{ft}^{3} / \mathrm{s}$, cubic foot per second; Sep, September; Nov, November]

\begin{tabular}{|c|c|c|c|c|c|c|c|c|c|}
\hline \multirow[b]{2}{*}{ APEE } & \multicolumn{9}{|c|}{ Sep-Nov daily mean streamflow ( $\left.\mathrm{ft}^{3} / \mathrm{s}\right)$ that was equaled or exceeded the indicated percentage of time at APEE level } \\
\hline & 98 & 95 & 90 & 80 & 50 & 20 & 10 & 5 & 2 \\
\hline 1 & 270. & 486. & 631. & 853. & 2470. & 10800. & 19800. & 21200 & 27500 . \\
\hline 2 & 269. & 467. & 597. & 776. & 2310 . & 9220. & 16400 & 18500 . & 22200 . \\
\hline 5 & 260. & 336. & 512. & 630. & 1520. & 6410 & 10100. & 14300 . & 19300. \\
\hline 10 & 247. & 296. & 435. & 537. & 1170. & 3240. & 5860. & 8310. & 10300 . \\
\hline 20 & 210 & 250 & 382. & 452. & 795. & 1910. & 2930. & 3310. & 5370. \\
\hline 25 & 207. & 239. & 367. & 436. & 705. & 1440. & 2200 & 2810 & 4860 . \\
\hline 30 & 204 & 232. & 350. & 405. & 652. & 1240. & 2010. & 2420 & 4240 . \\
\hline 40 & 196. & 223. & 321. & 371. & 550. & 964. & 1640. & 2010 & 3280 . \\
\hline 50 & 190. & 219. & 294. & 344. & 501. & 830. & 1280. & 1740 . & 2210. \\
\hline 60 & 184. & 212. & 278. & 320. & 449. & 743. & 1140. & 1370 . & 1980. \\
\hline 70 & 173. & 189. & 215. & 295. & 410. & 689. & 860. & 1180. & 1770 . \\
\hline 75 & 158. & 184. & 205. & 286. & 395. & 641. & 814. & 1100 . & 1700. \\
\hline 80 & 153. & 167. & 199. & 268 & 381. & 611. & 789. & 969. & 1640. \\
\hline 90 & 140. & 146. & 182. & 248 & 347. & 536. & 663. & 797. & 1450. \\
\hline 95 & 131. & 138. & 176. & 232 & 331. & 498. & 625 & 700 . & 1380. \\
\hline 98 & 124. & 135. & 160. & 220 & 313. & 469. & 568. & 671. & 1300 . \\
\hline 99 & 119. & 130. & 155. & 216. & 311. & 463. & 561. & 631. & 1100. \\
\hline
\end{tabular}

\section{Great Miami River at Miamisburg, Ohio}

Years analyzed $=54$

Climatic year range $=1924-94$

[APEE, annual percentage equaled or exceeded; $\mathrm{ft}^{3} / \mathrm{s}$, cubic foot per second; Sep, September; Nov, November]

\begin{tabular}{|c|c|c|c|c|c|c|c|c|c|}
\hline \multirow[b]{2}{*}{ APEE } & \multicolumn{9}{|c|}{ Sep-Nov daily mean streamflow $\left(\mathrm{ft}^{3} / \mathrm{s}\right)$ that was equaled or exceeded the indicated percentage of time at APEE leve } \\
\hline & 98 & 95 & 90 & 80 & 50 & 20 & 10 & 5 & 2 \\
\hline 1 & 344. & 521. & 643. & 935. & 2590. & 10700. & 21500 & 23500. & 27000 \\
\hline 2 & 344. & 494. & 598. & 844. & 2440 & 8470 . & 18300 . & 21200 & 23700 \\
\hline 5 & 333. & 368. & 553. & 692. & 1830 & 6450 & 13000 . & 16400 . & 18900. \\
\hline 10 & 300. & 342 . & 519. & 590. & 1420 & 3770 . & 6730. & 9670. & 12700 \\
\hline 20 & 284 & 328. & 463. & 517. & 1020 & 2210 & 3060 . & 4390 . & 8450 . \\
\hline 25 & 277. & 320. & 451. & 497. & 907. & 1610. & 2610. & 3740 . & 7760 . \\
\hline 30 & 273. & 318. & 423. & 480 . & 787. & 1410. & 2390 . & 3220 & 6730. \\
\hline 40 & 261. & 307. & 396. & 459. & 658. & 1060 & 2090. & 2590 . & 5400. \\
\hline 50 & 249 & 300. & 355. & 440. & 597. & 979. & 1770 & 2160 & 3830. \\
\hline 60 & 245. & 286. & 328. & 431. & 547. & 900. & 1380 . & 1830. & 3090. \\
\hline 70 & 240 & 265. & 294. & 418. & 513. & 828. & 1060 & 1650 & 2670 . \\
\hline 75 & 236. & 256. & 289. & 411. & 504. & 780 & 1010 & 1500 & 2530 . \\
\hline 80 & 231. & 249. & 282. & 396. & 465. & 757. & 950. & 1320 . & 2350 . \\
\hline 90 & 221. & 227. & 262. & 377. & 436. & 662. & 829. & 1110. & 2130. \\
\hline 95 & 201. & 220 & 249 & 338. & 423. & 650 & 798. & 1020 & 1880 \\
\hline 98 & 167. & 195. & 224. & 291. & 405. & 638. & 768. & 958. & 1760 . \\
\hline 99 & 149. & 164. & 210 & 278. & 394. & 623. & 758. & 920. & 1760. \\
\hline
\end{tabular}




\section{Twin Creek near Germantown, Ohio}

Years analyzed $=86$

Climatic year range $=1920-2008$

[APEE, annual percentage equaled or exceeded; $\mathrm{ft}^{3} / \mathrm{s}$, cubic foot per second; Sep, September; Nov, November]

\begin{tabular}{|c|c|c|c|c|c|c|c|c|c|}
\hline \multirow[b]{2}{*}{ APEE } & \multicolumn{9}{|c|}{ Sep-Nov daily mean streamflow $\left(\mathrm{ft}^{3} / \mathrm{s}\right)$ that was equaled or exceeded the indicated percentage of time at APEE level } \\
\hline & 98 & 95 & 90 & 80 & 50 & 20 & 10 & 5 & 2 \\
\hline 1 & 9.27 & 11.4 & 26.8 & 48.6 & 442. & 2090. & 4100 & 5150. & 5970. \\
\hline 2 & 8.78 & 11.4 & 22.2 & 42.1 & 307. & 1420. & 3020 . & 4240 . & 4850 \\
\hline 5 & 7.44 & 11.2 & 18.7 & 29.9 & 141. & 671. & 1380 . & 2030 . & 2540 \\
\hline 10 & 7.05 & 10.1 & 16.0 & 23.3 & 85.1 & 351. & 709. & 1020. & 1250 \\
\hline 20 & 6.59 & 8.94 & 12.9 & 18.4 & 48.0 & 138. & 322. & 415. & 523. \\
\hline 25 & 6.39 & 8.28 & 12.7 & 17.4 & 39.5 & 96.8 & 220 & 339. & 422. \\
\hline 30 & 6.24 & 7.69 & 11.7 & 16.6 & 32.0 & 81.4 & 192. & 274. & 381. \\
\hline 40 & 5.63 & 6.94 & 10.0 & 14.4 & 27.0 & 53.7 & 107. & 188. & 220 \\
\hline 50 & 4.87 & 6.39 & 9.05 & 11.4 & 23.5 & 41.6 & 87.6 & 149. & 177. \\
\hline 60 & 4.27 & 5.49 & 7.04 & 9.54 & 19.5 & 35.0 & 76.2 & 124. & 146. \\
\hline 70 & 4.07 & 4.81 & 5.94 & 8.54 & 17.5 & 30.2 & 49.4 & 98.2 & 125. \\
\hline 75 & 3.97 & 4.54 & 5.89 & 8.14 & 15.0 & 28.0 & 38.9 & 86.6 & 113. \\
\hline 80 & 3.78 & 4.15 & 5.29 & 7.79 & 14.0 & 23.0 & 33.1 & 78.6 & 96.6 \\
\hline 90 & 3.30 & 3.55 & 4.55 & 6.80 & 12.0 & 20.6 & 29.2 & 56.9 & 85.5 \\
\hline 95 & 2.89 & 3.33 & 3.97 & 6.28 & 11.0 & 18.6 & 28.7 & 46.2 & 67.4 \\
\hline 98 & 2.54 & 2.84 & 3.67 & 5.78 & 10.0 & 17.4 & 26.2 & 35.2 & 59.9 \\
\hline 99 & 2.37 & 2.84 & 3.54 & 5.44 & 10.0 & 16.6 & 25.3 & 35.2 & 57.3 \\
\hline
\end{tabular}

03274000 Great Miami River at Hamilton, Ohio

Years analyzed $=79$

Climatic year range $=1930-2008$

[APEE, annual percentage equaled or exceeded; $\mathrm{ft}^{3} / \mathrm{s}$, cubic foot per second; Sep, September; Nov, November]

\begin{tabular}{|c|c|c|c|c|c|c|c|c|c|}
\hline \multirow[b]{2}{*}{ APEE } & \multicolumn{9}{|c|}{ Sep-Nov daily mean streamflow ( $\left.\mathrm{ft}^{3} / \mathrm{s}\right)$ that was equaled or exceeded the indicated percentage of time at APEE level } \\
\hline & 98 & 95 & 90 & 80 & 50 & 20 & 10 & 5 & 2 \\
\hline 1 & 469. & 676. & 908. & 1210. & 4330. & 14100. & 26700 & 29900. & 31700. \\
\hline 2 & 459. & 608. & 806. & 1130. & 3530. & 12000 & 22400 & 25600 & 26400 . \\
\hline 5 & 422. & 515. & 679. & 896. & 2590. & 8950. & 14600 & 18400 . & 22100 . \\
\hline 10 & 393. & 472. & 583. & 747. & 1800 & 4530 . & 8930. & 10600. & 12000 . \\
\hline 20 & 356. & 399. & 532. & 647. & 1310. & 2520 & 3610. & 5080. & 6450. \\
\hline 25 & 346. & 380 . & 499. & 626. & 1160 & 1910. & 3130. & 4030 . & 5680. \\
\hline 30 & 344. & 372. & 491. & 613. & 1030. & 1700. & 2920 . & 3410. & 4680 . \\
\hline 40 & 324. & 363. & 469. & 574. & 841. & 1310. & 2210 & 2890. & 3730. \\
\hline 50 & 312. & 355. & 445. & 531. & 748. & 1180. & 1980. & 2480 . & 2950. \\
\hline 60 & 297. & 340 . & 399. & 509. & 675. & 1120. & 1380. & 1950. & 2630. \\
\hline 70 & 281. & 330 . & 366. & 466. & 652. & 958. & 1220 & 1700 . & 2320 . \\
\hline 75 & 277. & 325. & 352. & 462. & 620. & 945. & 1190. & 1550. & 2160. \\
\hline 80 & 268. & 310. & 337. & 438. & 593. & 859. & 1140. & 1490. & 2090. \\
\hline 90 & 250 & 288. & 308. & 414. & 508. & 765. & 947. & 1290. & 1880. \\
\hline 95 & 217. & 269. & 294. & 392. & 487. & 713. & 897. & 1170. & 1680. \\
\hline 98 & 191. & 258. & 289. & 378. & 463. & 696. & 851. & 1060. & 1560. \\
\hline 99 & 177. & 246. & 267. & 357. & 458. & 683. & 846. & 974. & 1470. \\
\hline
\end{tabular}




\section{Maumee River at Antwerp, Ohio}

Years analyzed $=57$

Climatic year range $=1921-81$

[APEE, annual percentage equaled or exceeded; $\mathrm{ft}^{3} / \mathrm{s}$, cubic foot per second; Sep, September; Nov, November]

\begin{tabular}{|c|c|c|c|c|c|c|c|c|c|}
\hline \multirow[b]{2}{*}{ APEE } & \multicolumn{9}{|c|}{ Sep-Nov daily mean streamflow ( $\left.\mathrm{ft}^{3} / \mathrm{s}\right)$ that was equaled or exceeded the indicated percentage of time at APEE level } \\
\hline & 98 & 95 & 90 & 80 & 50 & 20 & 10 & 5 & 2 \\
\hline 1 & 231. & 277. & 376. & 530. & 2260. & 5950. & 9500. & 11500. & 13100. \\
\hline 2 & 199. & 218. & 334. & 451. & 2140 . & 5400 . & 8670. & 11200. & 12900. \\
\hline 5 & 164. & 170. & 261. & 390. & 1310. & 3830. & 6000 & 9580. & 10600 . \\
\hline 10 & 143. & 160. & 218 & 307. & 823. & 2520. & 4150. & 5930. & 7730 . \\
\hline 20 & 120. & 126. & 178. & 225. & 513. & 1360. & 2430 & 2850 & 5740. \\
\hline 25 & 112. & 119. & 165. & 206. & 410. & 1070 . & 1670 & 2440 & 4560 . \\
\hline 30 & 107. & 113. & 156. & 190. & 375. & 870. & 1310 & 2100 & 4050 . \\
\hline 40 & 96.1 & 103. & 140. & 163. & 306. & 550. & 854. & 1590. & 2810 . \\
\hline 50 & 91.6 & 95.9 & 122. & 143. & 275 & 426. & 585. & 1160 & 2320 . \\
\hline 60 & 85.1 & 88.9 & 110. & 130. & 212. & 353. & 456. & 870. & 1650. \\
\hline 70 & 75.2 & 84.3 & 103. & 121. & 189. & 303. & 377. & 666. & 1300. \\
\hline 75 & 75.0 & 82.7 & 94.4 & 116. & 175. & 282. & 352. & 594. & 1110. \\
\hline 80 & 68.4 & 76.0 & 90.0 & 112. & 164. & 241. & 325. & 507. & 911. \\
\hline 90 & 53.9 & 63.2 & 74.1 & 98.2 & 140. & 204. & 265. & 430. & 701. \\
\hline 95 & 48.1 & 59.7 & 68.1 & 85.7 & 121. & 196. & 240 & 415. & 657. \\
\hline 98 & 32.4 & 51.0 & 64.1 & 75.6 & 110. & 174. & 221. & 372. & 443. \\
\hline 99 & 30.8 & 50.8 & 61.6 & 72.8 & 110. & 163. & 208. & 335. & 429. \\
\hline
\end{tabular}

\section{Tiffin River at Stryker, Ohio}

Years analyzed $=76$

Climatic year range $=1921-2008$

[APEE, annual percentage equaled or exceeded; $\mathrm{ft}^{3} / \mathrm{s}$, cubic foot per second; Sep, September; Nov, November]

\begin{tabular}{|c|c|c|c|c|c|c|c|c|c|}
\hline \multirow[b]{2}{*}{ APEE } & \multicolumn{9}{|c|}{ Sep-Nov daily mean streamflow $\left(\mathrm{ft}^{3} / \mathrm{s}\right)$ that was equaled or exceeded the indicated percentage of time at APEE level } \\
\hline & 98 & 95 & 90 & 80 & 50 & 20 & 10 & 5 & 2 \\
\hline 1 & 23.2 & 36.7 & 53.0 & 137. & 735. & 1240. & 1570. & 2090. & 3100 \\
\hline 2 & 22.8 & 35.2 & 50.4 & 123. & 673. & 1200 & 1530. & 1960. & 3040 . \\
\hline 5 & 21.2 & 32.9 & 42.9 & 77.2 & 366. & 938. & 1190. & 1720 . & 2290 \\
\hline 10 & 19.2 & 29.1 & 35.3 & 63.0 & 221. & 648. & 989. & 1190. & 1670 \\
\hline 20 & 17.2 & 26.6 & 30.7 & 48.2 & 122. & 332. & 560. & 664. & 962. \\
\hline 25 & 15.2 & 24.9 & 29.7 & 40.4 & 103. & 258. & 339. & 450 . & 820. \\
\hline 30 & 15.2 & 24.9 & 28.4 & 38.8 & 95.4 & 202. & 317. & 376. & 657. \\
\hline 40 & 11.1 & 22.1 & 26.0 & 32.6 & 70.0 & 148. & 226. & 270 & 382. \\
\hline 50 & 10.0 & 19.5 & 22.7 & 27.4 & 52.5 & 113. & 169. & 204. & 266. \\
\hline 60 & 9.62 & 16.7 & 20.0 & 23.4 & 43.2 & 85.5 & 134. & 158. & 204. \\
\hline 70 & 8.50 & 12.1 & 15.9 & 19.0 & 35.1 & 64.6 & 98.9 & 141. & 168. \\
\hline 75 & 7.86 & 10.7 & 13.7 & 17.0 & 29.5 & 57.2 & 86.0 & 110. & 154. \\
\hline 80 & 7.19 & 8.82 & 11.7 & 16.2 & 27.2 & 46.8 & 68.0 & 96.1 & 141. \\
\hline 90 & 5.82 & 6.92 & 10.0 & 14.0 & 20.1 & 37.6 & 54.2 & 66.3 & 116. \\
\hline 95 & 5.50 & 5.87 & 9.37 & 12.6 & 19.0 & 35.1 & 45.6 & 56.9 & 105. \\
\hline 98 & 4.49 & 5.14 & 7.95 & 11.8 & 17.9 & 33.2 & 41.5 & 54.5 & 99.3 \\
\hline 99 & 3.81 & 5.13 & 7.88 & 11.0 & 17.5 & 33.2 & 38.6 & 53.7 & 96.9 \\
\hline
\end{tabular}




\section{Ottawa River at Allentown, Ohio}

Years analyzed $=52$

Climatic year range $=1923-81$

[APEE, annual percentage equaled or exceeded; $\mathrm{ft}^{3} / \mathrm{s}$, cubic foot per second; Sep, September; Nov, November]

\begin{tabular}{|c|c|c|c|c|c|c|c|c|c|}
\hline \multirow[b]{2}{*}{ APEE } & \multicolumn{9}{|c|}{ Sep-Nov daily mean streamflow $\left(\mathrm{ft}^{3} / \mathrm{s}\right)$ that was equaled or exceeded the indicated percentage of time at APEE level } \\
\hline & 98 & 95 & 90 & 80 & 50 & 20 & 10 & 5 & 2 \\
\hline 1 & 23.5 & 34.6 & 42.1 & 63.8 & 150. & 953. & 1570 & 2230 & 2320 \\
\hline 2 & 21.8 & 31.4 & 35.2 & 42.8 & 102. & 646. & 1310 . & 1960. & 2040 . \\
\hline 5 & 15.4 & 19.9 & 24.0 & 29.4 & 65.3 & 300 . & 576. & 1080. & 1510. \\
\hline 10 & 13.9 & 15.0 & 18.6 & 24.0 & 47.1 & 119. & 208. & 501. & 856. \\
\hline 20 & 10.1 & 12.5 & 16.2 & 20.6 & 34.8 & 55.0 & 104. & 250. & 457. \\
\hline 25 & 9.78 & 11.7 & 16.0 & 19.0 & 31.0 & 46.6 & 82.5 & 172. & 345. \\
\hline 30 & 9.53 & 11.3 & 14.6 & 18.6 & 28.5 & 38.3 & 67.1 & 146. & 289. \\
\hline 40 & 9.06 & 10.7 & 14.6 & 18.0 & 24.0 & 34.1 & 49.0 & 90.6 & 153. \\
\hline 50 & 8.68 & 10.0 & 12.3 & 16.6 & 21.0 & 30.4 & 39.0 & 62.6 & 101. \\
\hline 60 & 8.28 & 9.73 & 11.3 & 15.0 & 20.0 & 28.9 & 35.6 & 47.5 & 74.0 \\
\hline 70 & 7.96 & 9.17 & 11.0 & 13.6 & 18.8 & 27.4 & 32.5 & 40.7 & 59.1 \\
\hline 75 & 7.85 & 8.86 & 10.6 & 13.6 & 18.5 & 26.8 & 31.5 & 37.7 & 55.0 \\
\hline 80 & 7.66 & 8.73 & 10.3 & 12.6 & 18.5 & 25.4 & 30.8 & 34.7 & 50.4 \\
\hline 90 & 7.06 & 8.23 & 9.55 & 12.0 & 16.6 & 23.8 & 28.1 & 32.3 & 41.6 \\
\hline 95 & 6.83 & 7.76 & 9.34 & 11.0 & 16.0 & 22.2 & 25.0 & 30.7 & 34.4 \\
\hline 98 & 6.04 & 6.98 & 8.86 & 9.87 & 14.4 & 20.7 & 23.6 & 28.2 & 30.7 \\
\hline 99 & 5.60 & 6.64 & 8.56 & 9.66 & 14.0 & 20.4 & 23.0 & 27.7 & 29.9 \\
\hline
\end{tabular}

\section{Auglaize River near Defiance, Ohio}

Years analyzed $=94$

Climatic year range $=1915-2008$

[APEE, annual percentage equaled or exceeded; $\mathrm{ft}^{3} / \mathrm{s}$, cubic foot per second; Sep, September; Nov, November]

\begin{tabular}{|c|c|c|c|c|c|c|c|c|c|}
\hline \multirow[b]{2}{*}{ APEE } & \multicolumn{9}{|c|}{ Sep-Nov daily mean streamflow $\left(\mathrm{ft}^{3} / \mathrm{s}\right)$ that was equaled or exceeded the indicated percentage of time at APEE level } \\
\hline & 98 & 95 & 90 & 80 & 50 & 20 & 10 & 5 & 2 \\
\hline 1 & 135. & 259. & 514. & 879. & 3260. & 11600. & 14000 & 17700 . & 22300 . \\
\hline 2 & 132. & 239. & 386. & 674. & 3020. & 10100. & 12600 . & 15000 . & 22100 . \\
\hline 5 & 97.1 & 163. & 239. & 341. & 1480 & 6860. & 9860. & 11000 . & 16000 . \\
\hline 10 & 59.6 & 102. & 138. & 238 & 933. & 2910 . & 6150. & 7600 . & 12700 . \\
\hline 20 & 43.8 & 60.3 & 84.1 & 118. & 401. & 1260. & 2550 . & 4320 . & 8920. \\
\hline 25 & 38.3 & 44.8 & 59.0 & 102. & 288. & 833. & 1780. & 2620 . & 6680. \\
\hline 30 & 34.5 & 39.4 & 50.9 & 79.2 & 229. & 681. & 1220. & 1870 . & 5720. \\
\hline 40 & 29.6 & 33.6 & 37.2 & 56.8 & 153. & 473. & 848. & 1200. & 2990. \\
\hline 50 & 22.7 & 30.0 & 35.0 & 48.0 & 117. & 322. & 634. & 912. & 2110. \\
\hline 60 & 21.4 & 30.0 & 31.3 & 39.6 & 82.4 & 224. & 416. & 816. & 1240. \\
\hline 70 & 18.5 & 21.8 & 29.3 & 34.0 & 56.9 & 175. & 283. & 536. & 869. \\
\hline 75 & 14.0 & 19.8 & 27.0 & 30.0 & 48.5 & 165. & 257. & 452. & 696. \\
\hline 80 & 13.4 & 19.3 & 24.0 & 29.0 & 44.5 & 113. & 215. & 428. & 629. \\
\hline 90 & 7.28 & 14.8 & 20.0 & 25.2 & 37.3 & 71.0 & 147. & 243. & 385. \\
\hline 95 & 5.06 & 10.2 & 15.5 & 21.6 & 33.0 & 57.8 & 104. & 180. & 245. \\
\hline 98 & 1.87 & 6.67 & 9.66 & 20.0 & 30.0 & 47.8 & 62.4 & 102. & 140. \\
\hline 99 & 1.49 & 5.65 & 9.20 & 19.0 & 29.0 & 46.0 & 58.5 & 88.5 & 119. \\
\hline
\end{tabular}




\section{Maumee River near Defiance, Ohio}

Years analyzed $=77$

Climatic year range $=1924-2008$

[APEE, annual percentage equaled or exceeded; $\mathrm{ft}^{3} / \mathrm{s}$, cubic foot per second; Sep, September; Nov, November]

\begin{tabular}{|c|c|c|c|c|c|c|c|c|c|}
\hline \multirow[b]{2}{*}{ APEE } & \multicolumn{9}{|c|}{ Sep-Nov daily mean streamflow ( $\left.\mathrm{ft}^{3} / \mathrm{s}\right)$ that was equaled or exceeded the indicated percentage of time at APEE level } \\
\hline & 98 & 95 & 90 & 80 & 50 & 20 & 10 & 5 & 2 \\
\hline 1 & 248. & 370. & 750. & 1100. & 9120. & 22000. & 27900 & 31700 & 42900. \\
\hline 2 & 244 & 358. & 627. & 866. & 7970. & 20800 & 26400 & 30700 . & 39500. \\
\hline 5 & 196. & 256. & 492. & 640. & 4650. & 15300. & 20100. & 24500 & 28600 . \\
\hline 10 & 165. & 241. & 386. & 520. & 2700 . & 8170 & 12900. & 18900. & 24500 . \\
\hline 20 & 131. & 188. & 299. & 404. & 1390. & 4080 & 7280 . & 9840. & 17100 . \\
\hline 25 & 123. & 178 & 244. & 342. & 1130. & 2790 & 5040 . & 7390. & 14400 . \\
\hline 30 & 114. & 165 & 227. & 314. & 943. & 2410 & 4200 & 5830. & 11800 . \\
\hline 40 & 99.7 & 154. & 189. & 284. & 675. & 1340. & 2520 & 3810. & 8510 . \\
\hline 50 & 78.2 & 138. & 165. & 232 & 506. & 959. & 1740. & 2880 & 5630. \\
\hline 60 & 69.3 & 123. & 157. & 207. & 398. & 744. & 1380. & 2020 & 3870 . \\
\hline 70 & 62.1 & 93.5 & 117. & 182. & 339. & 574. & 1050. & 1640. & 2550. \\
\hline 75 & 57.0 & 81.6 & 114. & 170. & 303. & 526. & 906. & 1570 . & 2140 . \\
\hline 80 & 55.2 & 75.3 & 105. & 160. & 273. & 455. & 831. & 1440 & 1770. \\
\hline 90 & 48.6 & 62.4 & 75.6 & 128. & 234. & 376. & 643. & 927. & 1230. \\
\hline 95 & 21.3 & 61.2 & 69.6 & 111. & 215. & 338. & 557. & 830. & 994. \\
\hline 98 & 11.6 & 47.9 & 58.0 & 84.6 & 198. & 294. & 467. & 640. & 811. \\
\hline 99 & 4.68 & 41.8 & 53.4 & 84.6 & 170. & 280 & 393. & 609. & 745. \\
\hline
\end{tabular}

\section{Maumee River at Waterville, Ohio}

Years analyzed $=87$

Climatic year range $=1899-2008$

[APEE, annual percentage equaled or exceeded; $\mathrm{ft}^{3} / \mathrm{s}$, cubic foot per second; Sep, September; Nov, November]

\begin{tabular}{|c|c|c|c|c|c|c|c|c|c|}
\hline \multirow[b]{2}{*}{ APEE } & \multicolumn{9}{|c|}{ Sep-Nov daily mean streamflow ( $\left.\mathrm{ft}^{3} / \mathrm{s}\right)$ that was equaled or exceeded the indicated percentage of time at APEE level } \\
\hline & 98 & 95 & 90 & 80 & 50 & 20 & 10 & 5 & 2 \\
\hline 1 & 423. & 629. & 865. & 1250. & 9090. & 24900. & 30700 . & 36300. & 47000. \\
\hline 2 & 361. & 526. & 813. & 1150 & 8550. & 22900 & 28200 & 35500 . & 43200 . \\
\hline 5 & 299. & 416. & 570. & 781. & 5530. & 16700 & 21500 & 29400 . & 34100 . \\
\hline 10 & 246. & 291. & 442. & 601. & 3150. & 8770. & 13800 & 20900 . & 28300 . \\
\hline 20 & 158. & 202. & 253. & 399. & 1540. & 4900 & 7480 . & 9950. & 20300 . \\
\hline 25 & 102. & 113. & 199. & 353. & 1180. & 3000 . & 4740 & 7570 . & 16500 . \\
\hline 30 & 77.6 & 96.0 & 182. & 320. & 1000. & 2280 & 4090 & 5720 . & 12600 . \\
\hline 40 & 70.4 & 89.4 & 159. & 275. & 737. & 1480 & 2680 . & 3830 . & 8780 . \\
\hline 50 & 64.1 & 80.0 & 126. & 202. & 574. & 1080 & 1960. & 2960. & 6280. \\
\hline 60 & 35.2 & 70.0 & 89.2 & 169. & 425. & 870. & 1690. & 2070. & 3640. \\
\hline 70 & 32.8 & 53.1 & 70.0 & 150. & 348. & 618. & 1320. & 1640. & 2760 . \\
\hline 75 & 16.4 & 47.2 & 70.0 & 145. & 295. & 544. & 1060 & 1510. & 2310 . \\
\hline 80 & 16.4 & 44.9 & 67.5 & 128. & 263. & 494. & 923. & 1340 . & 1990. \\
\hline 90 & 8.80 & 35.4 & 63.2 & 100. & 213. & 410. & 662. & 1020. & 1300. \\
\hline 95 & 8.80 & 30.2 & 52.4 & 88.9 & 180. & 356. & 584. & 860. & 999. \\
\hline 98 & 5.00 & 26.8 & 38.6 & 68.8 & 154. & 303. & 492. & 601. & 933. \\
\hline 99 & 5.00 & 24.4 & 33.0 & 64.2 & 146. & 281. & 458. & 548. & 914. \\
\hline
\end{tabular}




\section{Portage River at Woodville, Ohio}

Years analyzed $=58$

Climatic year range $=1951-2008$

[APEE, annual percentage equaled or exceeded; $\mathrm{ft}^{3} / \mathrm{s}$, cubic foot per second; Sep, September; Nov, November]

\begin{tabular}{|c|c|c|c|c|c|c|c|c|c|}
\hline \multirow[b]{2}{*}{ APEE } & \multicolumn{9}{|c|}{ Sep-Nov daily mean streamflow $\left(\mathrm{ft}^{3} / \mathrm{s}\right)$ that was equaled or exceeded the indicated percentage of time at APEE level } \\
\hline & 98 & 95 & 90 & 80 & 50 & 20 & 10 & 5 & 2 \\
\hline 1 & 13.4 & 16.9 & 47.8 & 82.8 & 1090. & 2720. & 3790. & 5250 & 5950. \\
\hline 2 & 12.3 & 14.9 & 43.3 & 66.6 & 841. & 2450 & 3330. & 4720 . & 5090. \\
\hline 5 & 11.1 & 13.9 & 34.3 & 43.6 & 363. & 1330. & 1770 . & 3440 . & 4320 . \\
\hline 10 & 9.19 & 10.9 & 25.1 & 32.0 & 157. & 612. & 1010 & 1820. & 2310 \\
\hline 20 & 7.92 & 9.90 & 15.6 & 22.3 & 53.9 & 242. & 416. & 998. & 1180. \\
\hline 25 & 6.95 & 9.67 & 14.0 & 19.6 & 44.5 & 153. & 318. & 658. & 963. \\
\hline 30 & 6.10 & 9.50 & 12.0 & 17.6 & 35.6 & 114. & 286. & 442. & 720 . \\
\hline 40 & 5.23 & 8.58 & 10.9 & 14.2 & 27.5 & 76.4 & 158. & 337. & 541. \\
\hline 50 & 4.42 & 7.61 & 9.24 & 12.0 & 22.0 & 56.8 & 96.0 & 235. & 336. \\
\hline 60 & 4.02 & 6.03 & 7.16 & 10.0 & 18.0 & 42.8 & 73.1 & 173. & 213. \\
\hline 70 & 3.37 & 4.92 & 6.21 & 9.10 & 14.9 & 30.8 & 59.6 & 106. & 151. \\
\hline 75 & 3.23 & 4.28 & 5.60 & 8.70 & 13.5 & 28.0 & 50.7 & 88.5 & 124. \\
\hline 80 & 2.97 & 3.87 & 5.37 & 8.13 & 12.0 & 21.8 & 43.2 & 80.7 & 105 \\
\hline 90 & 2.65 & 3.56 & 4.59 & 6.72 & 10.0 & 18.0 & 27.5 & 49.1 & 78.9 \\
\hline 95 & 2.23 & 3.17 & 4.33 & 6.04 & 8.88 & 15.7 & 22.0 & 39.6 & 71.4 \\
\hline 98 & 2.07 & 2.29 & 3.46 & 5.18 & 8.04 & 14.2 & 18.6 & 32.4 & 63.1 \\
\hline 99 & 1.82 & 2.09 & 3.46 & 5.04 & 7.75 & 14.0 & 16.3 & 32.4 & 63.1 \\
\hline
\end{tabular}

04196000 Sandusky River near Bucyrus, Ohio

Years analyzed $=56$

Climatic year range $=1925-2008$

[APEE, annual percentage equaled or exceeded; $\mathrm{ft}^{3} / \mathrm{s}$, cubic foot per second; Sep, September; Nov, November]

\begin{tabular}{|c|c|c|c|c|c|c|c|c|c|}
\hline \multirow[b]{2}{*}{ APEE } & \multicolumn{9}{|c|}{ Sep-Nov daily mean streamflow $\left(\mathrm{ft}^{3} / \mathrm{s}\right)$ that was equaled or exceeded the indicated percentage of time at APEE level } \\
\hline & 98 & 95 & 90 & 80 & 50 & 20 & 10 & 5 & 2 \\
\hline 1 & 12.7 & 19.5 & 26.2 & 48.0 & 277. & 855. & 1270 & 1420 & 1420 \\
\hline 2 & 6.72 & 12.7 & 23.1 & 38.4 & 210 & 646. & 875. & 1120. & 1350 \\
\hline 5 & 3.05 & 8.37 & 11.8 & 18.4 & 112. & 275. & 568. & 732. & 750 . \\
\hline 10 & 2.16 & 5.30 & 8.20 & 11.7 & 53.1 & 132. & 237. & 258 & 397. \\
\hline 20 & 1.61 & 3.50 & 5.29 & 7.50 & 25.7 & 65.5 & 108. & 154. & 176. \\
\hline 25 & 1.55 & 3.18 & 4.27 & 6.28 & 17.0 & 48.6 & 68.2 & 108. & 138. \\
\hline 30 & 1.47 & 3.15 & 3.78 & 5.94 & 14.2 & 39.8 & 58.7 & 88.6 & 118. \\
\hline 40 & 1.31 & 2.61 & 3.28 & 5.00 & 10.0 & 26.3 & 44.7 & 59.9 & 85.8 \\
\hline 50 & 1.20 & 2.06 & 2.87 & 4.24 & 8.20 & 19.0 & 31.8 & 46.0 & 64.0 \\
\hline 60 & 1.17 & 1.77 & 2.54 & 3.04 & 6.66 & 14.0 & 24.2 & 38.4 & 48.6 \\
\hline 70 & 1.06 & 1.49 & 1.95 & 2.64 & 5.38 & 11.4 & 17.0 & 28.2 & 38.8 \\
\hline 75 & 1.04 & 1.38 & 1.64 & 2.54 & 4.75 & 10.0 & 14.3 & 25.6 & 36.7 \\
\hline 80 & 1.00 & 1.26 & 1.50 & 2.32 & 4.20 & 9.78 & 12.0 & 24.4 & 33.5 \\
\hline 90 & 0.90 & 1.16 & 1.30 & 1.95 & 3.33 & 6.50 & 8.72 & 14.6 & 23.9 \\
\hline 95 & 0.81 & 0.90 & 1.17 & 1.54 & 3.01 & 5.50 & 7.92 & 12.1 & 19.6 \\
\hline 98 & 0.60 & 0.70 & 0.95 & 1.33 & 2.68 & 4.69 & 5.95 & 8.27 & 14.3 \\
\hline 99 & 0.60 & 0.69 & 0.87 & 1.20 & 2.40 & 4.20 & 5.33 & 6.95 & 14.2 \\
\hline
\end{tabular}




\section{Tymochtee Creek at Crawford, Ohio}

Years analyzed $=37$

Climatic year range $=1972-2008$

[APEE, annual percentage equaled or exceeded; $\mathrm{ft}^{3} / \mathrm{s}$, cubic foot per second; Sep, September; Nov, November]

\begin{tabular}{|c|c|c|c|c|c|c|c|c|c|}
\hline \multirow[b]{2}{*}{ APEE } & \multicolumn{9}{|c|}{ Sep-Nov daily mean streamflow $\left(\mathrm{ft}^{3} / \mathrm{s}\right)$ that was equaled or exceeded the indicated percentage of time at APEE level } \\
\hline & 95 & 90 & 80 & 70 & 50 & 30 & 20 & 10 & 5 \\
\hline 1 & 6.78 & 8.48 & 27.0 & 57.2 & 516. & 1070. & 1880 & 2570 & 2710 \\
\hline 2 & 5.08 & 6.64 & 25.3 & 52.2 & 424. & 978. & 1570 . & 2330 & 2530 \\
\hline 5 & 4.23 & 5.43 & 15.6 & 34.9 & 202. & 648. & 978. & 1360 . & 1620. \\
\hline 10 & 2.91 & 4.16 & 13.8 & 25.2 & 63.6 & 291. & 564. & 981. & 1260. \\
\hline 20 & 1.84 & 3.31 & 6.07 & 17.2 & 24.6 & 103. & 208. & 403. & 629. \\
\hline 25 & 1.50 & 3.06 & 4.76 & 13.0 & 20.0 & 63.6 & 162. & 268. & 467. \\
\hline 30 & 1.26 & 2.49 & 4.09 & 8.22 & 17.0 & 47.4 & 116. & 198. & 291. \\
\hline 40 & 1.05 & 1.80 & 2.73 & 4.25 & 11.2 & 32.2 & 76.0 & 102. & 180. \\
\hline 50 & 0.77 & 0.88 & 1.84 & 3.20 & 6.60 & 26.4 & 47.0 & 81.2 & 101. \\
\hline 60 & 0.56 & 0.61 & 1.09 & 2.07 & 5.30 & 15.9 & 36.5 & 50.0 & 70.3 \\
\hline 70 & 0.20 & 0.40 & 0.85 & 1.29 & 4.06 & 12.4 & 25.8 & 32.8 & 59.3 \\
\hline 75 & 0.15 & 0.32 & 0.62 & 0.98 & 2.40 & 11.6 & 19.6 & 26.4 & 52.4 \\
\hline 80 & 0.11 & 0.25 & 0.48 & 0.83 & 1.90 & 10.1 & 13.4 & 22.0 & 48.2 \\
\hline 90 & 0.08 & 0.10 & 0.30 & 0.50 & 1.30 & 5.42 & 8.70 & 15.4 & 28.7 \\
\hline 95 & 0.06 & 0.07 & 0.18 & 0.39 & 0.94 & 3.90 & 5.80 & 12.9 & 25.2 \\
\hline 98 & 0.01 & 0.02 & 0.14 & 0.34 & 0.81 & 2.52 & 4.74 & 11.6 & 22.0 \\
\hline 99 & 0.01 & 0.02 & 0.09 & 0.30 & 0.68 & 2.02 & 4.34 & 11.4 & 22.0 \\
\hline
\end{tabular}

\section{Black River at Elyria, Ohio}

Years analyzed $=65$

Climatic year range $=1944-2008$

[APEE, annual percentage equaled or exceeded; $\mathrm{ft}^{3} / \mathrm{s}$, cubic foot per second; Sep, September; Nov, November]

\begin{tabular}{|c|c|c|c|c|c|c|c|c|c|}
\hline \multirow[b]{2}{*}{ APEE } & \multicolumn{9}{|c|}{ Sep-Nov daily mean streamflow $\left(\mathrm{ft}^{3} / \mathrm{s}\right)$ that was equaled or exceeded the indicated percentage of time at APEE level } \\
\hline & 98 & 95 & 90 & 80 & 50 & 20 & 10 & 5 & 2 \\
\hline 1 & 11.6 & 30.3 & 56.2 & 82.0 & 1050. & 2420. & 3570. & 4560. & 6390. \\
\hline 2 & 11.1 & 26.0 & 47.0 & 65.4 & 840 . & 2030 & 3040 . & 3660 . & 5320. \\
\hline 5 & 10.4 & 19.3 & 33.2 & 45.8 & 378. & 1170 & 1860. & 2330 & 3340. \\
\hline 10 & 9.51 & 17.9 & 24.5 & 36.2 & 179. & 535. & 843. & 1440 . & 2270 \\
\hline 20 & 7.64 & 12.5 & 17.0 & 28.6 & 74.0 & 207. & 345 . & 710 . & 1040 \\
\hline 25 & 6.78 & 11.9 & 15.0 & 23.2 & 58.0 & 158. & 263. & 533. & 800 \\
\hline 30 & 5.44 & 11.3 & 13.6 & 18.6 & 47.8 & 122. & 222. & 409. & 621. \\
\hline 40 & 4.23 & 9.67 & 11.6 & 15.2 & 31.2 & 72.2 & 138. & 232. & 406. \\
\hline 50 & 3.98 & 6.77 & 9.36 & 14.0 & 23.0 & 50.4 & 101. & 155. & 296. \\
\hline 60 & 3.04 & 6.20 & 8.47 & 10.8 & 18.0 & 35.8 & 72.9 & 107. & 204. \\
\hline 70 & 2.47 & 4.82 & 7.28 & 8.52 & 15.0 & 27.8 & 57.8 & 88.8 & 151. \\
\hline 75 & 2.26 & 3.96 & 6.68 & 7.56 & 13.0 & 25.0 & 52.4 & 80.0 & 128 \\
\hline 80 & 2.07 & 3.59 & 5.90 & 7.40 & 11.0 & 22.3 & 46.4 & 66.3 & 106. \\
\hline 90 & 1.52 & 2.72 & 4.87 & 5.76 & 9.22 & 18.0 & 26.6 & 34.3 & 46.8 \\
\hline 95 & 1.20 & 2.47 & 4.11 & 5.04 & 7.60 & 13.9 & 18.6 & 22.8 & 28.7 \\
\hline 98 & 0.91 & 1.98 & 3.60 & 4.58 & 6.20 & 12.0 & 14.7 & 18.5 & 24.4 \\
\hline 99 & 0.82 & 1.58 & 2.94 & 3.82 & 5.70 & 11.8 & 14.4 & 17.4 & 22.4 \\
\hline
\end{tabular}


04201500 Rocky River near Berea, Ohio

Years analyzed $=78$

Climatic year range $=1923-2008$

[APEE, annual percentage equaled or exceeded; $\mathrm{ft}^{3} / \mathrm{s}$, cubic foot per second; Sep, September; Nov, November]

\begin{tabular}{|c|c|c|c|c|c|c|c|c|c|}
\hline \multirow[b]{2}{*}{ APEE } & \multicolumn{9}{|c|}{ Sep-Nov daily mean streamflow $\left(\mathrm{ft}^{3} / \mathrm{s}\right)$ that was equaled or exceeded the indicated percentage of time at APEE level } \\
\hline & 98 & 95 & 90 & 80 & 50 & 20 & 10 & 5 & 2 \\
\hline 1 & 19.6 & 29.9 & 60.5 & 250. & 1350. & 3380. & 4190. & 4810 & 8610. \\
\hline 2 & 19.2 & 25.6 & 51.1 & 163. & 988. & 2300 & 3210 & 3890 . & 4670 . \\
\hline 5 & 16.6 & 17.4 & 36.8 & 103. & 454. & 1070 . & 1860. & 2420 & 2910 . \\
\hline 10 & 11.7 & 14.8 & 19.9 & 70.3 & 236. & 579. & 824. & 1360. & 1730. \\
\hline 20 & 8.88 & 11.9 & 16.3 & 37.9 & 115. & 267. & 395. & 589. & 1010. \\
\hline 25 & 7.43 & 9.92 & 15.5 & 28.6 & 92.5 & 187. & 278 & 456. & 815. \\
\hline 30 & 5.87 & 8.93 & 14.5 & 25.6 & 75.3 & 160. & 226. & 349. & 614. \\
\hline 40 & 4.28 & 6.98 & 11.2 & 19.0 & 56.5 & 111. & 146. & 238. & 445. \\
\hline 50 & 3.40 & 5.90 & 6.91 & 15.8 & 45.0 & 81.2 & 117. & 187. & 317. \\
\hline 60 & 2.32 & 4.09 & 5.48 & 12.6 & 36.4 & 63.4 & 90.8 & 152. & 216. \\
\hline 70 & 1.51 & 3.37 & 4.75 & 8.60 & 27.5 & 51.2 & 70.2 & 130. & 164. \\
\hline 75 & 1.43 & 3.00 & 4.19 & 7.98 & 24.6 & 47.4 & 62.3 & 108. & 147. \\
\hline 80 & 1.14 & 2.59 & 4.05 & 7.06 & 22.6 & 43.7 & 54.8 & 85.7 & 121. \\
\hline 90 & 0.81 & 1.87 & 3.35 & 5.80 & 18.1 & 34.7 & 40.3 & 53.3 & 91.6 \\
\hline 95 & 0.63 & 1.85 & 2.65 & 4.77 & 13.6 & 28.6 & 34.7 & 41.2 & 58.0 \\
\hline 98 & 0.44 & 1.17 & 2.46 & 3.71 & 12.4 & 24.7 & 28.5 & 36.0 & 39.9 \\
\hline 99 & 0.26 & 1.17 & 2.38 & 3.56 & 11.0 & 24.0 & 28.5 & 36.0 & 38.0 \\
\hline
\end{tabular}

\section{Cuyahoga River at Independence, Ohio}

Years analyzed $=80$

Climatic year range $=1921-2008$

[APEE, annual percentage equaled or exceeded; $\mathrm{ft}^{3} / \mathrm{s}$, cubic foot per second; Sep, September; Nov, November]

\begin{tabular}{|c|c|c|c|c|c|c|c|c|c|}
\hline \multirow[b]{2}{*}{ APEE } & \multicolumn{9}{|c|}{ Sep-Nov daily mean streamflow $\left(\mathrm{ft}^{3} / \mathrm{s}\right)$ that was equaled or exceeded the indicated percentage of time at APEE level } \\
\hline & 98 & 95 & 90 & 80 & 50 & 20 & 10 & 5 & 2 \\
\hline 1 & 158. & 285 & 381. & 708. & 2430 & 4450. & 5620. & 7430 & 12800 \\
\hline 2 & 149. & 250. & 333. & 622. & 1840 & 3460 . & 4390. & 5900. & 9140. \\
\hline 5 & 124. & 184. & 246. & 477. & 1150 & 2340. & 3270 & 3750 & 4330. \\
\hline 10 & 108. & 142. & 199. & 357. & 858. & 1660. & 2030 & 2540 & 3040. \\
\hline 20 & 94.1 & 130. & 160. & 273. & 519. & 1010. & 1270 & 1730. & 2140. \\
\hline 25 & 88.5 & 122. & 147. & 247. & 432. & 874. & 1190. & 1600 & 1840. \\
\hline 30 & 83.2 & 116. & 138. & 211. & 368. & 751. & 1000 & 1240 & 1510. \\
\hline 40 & 75.0 & 112. & 128. & 161. & 325. & 590. & 848. & 1070 & 1220. \\
\hline 50 & 69.5 & 104. & 118. & 139. & 280. & 509. & 705. & 855. & 1040. \\
\hline 60 & 62.0 & 97.8 & 107. & 129. & 232 & 437. & 539. & 725. & 876. \\
\hline 70 & 57.1 & 82.5 & 99.1 & 115. & 208. & 388. & 442. & 524. & 726. \\
\hline 75 & 53.5 & 80.3 & 90.5 & 109. & 197. & 354. & 414. & 490. & 644. \\
\hline 80 & 50.0 & 76.1 & 84.5 & 106. & 178. & 315. & 390. & 440. & 610. \\
\hline 90 & 43.1 & 69.2 & 74.8 & 97.6 & 149. & 249. & 327. & 382. & 509. \\
\hline 95 & 34.1 & 60.7 & 69.7 & 85.0 & 138. & 228. & 279 & 312. & 332. \\
\hline 98 & 30.3 & 52.8 & 62.4 & 74.3 & 127. & 216. & 271. & 293. & 326. \\
\hline 99 & 24.9 & 45.0 & 58.1 & 72.2 & 124. & 208. & 267. & 289. & 323. \\
\hline
\end{tabular}


04209000 Chagrin River at Willoughby, Ohio

Years analyzed $=73$

Climatic year range $=1925-2008$

[APEE, annual percentage equaled or exceeded; $\mathrm{ft}^{3} / \mathrm{s}$, cubic foot per second; Sep, September; Nov, November]

\begin{tabular}{|c|c|c|c|c|c|c|c|c|c|}
\hline \multirow[b]{2}{*}{ APEE } & \multicolumn{9}{|c|}{ Sep-Nov daily mean streamflow $\left(\mathrm{ft}^{3} / \mathrm{s}\right)$ that was equaled or exceeded the indicated percentage of time at APEE level } \\
\hline & 98 & 95 & 90 & 80 & 50 & 20 & 10 & 5 & 2 \\
\hline 1 & 98.4 & 124. & 248. & 344. & 1660. & 3260. & 4250 & 5420. & 8660 \\
\hline 2 & 86.1 & 118. & 215. & 290. & 1180. & 2540 & 2960. & 3840. & 6300 \\
\hline 5 & 70.0 & 96.2 & 141. & 176. & 746. & 1350. & 1500. & 2080 . & 2950 \\
\hline 10 & 54.8 & 74.9 & 93.5 & 145. & 386. & 775. & 1010. & 1250. & 1580 \\
\hline 20 & 41.9 & 53.0 & 59.6 & 98.4 & 217. & 446. & 531. & 652. & 880. \\
\hline 25 & 39.4 & 46.4 & 53.0 & 86.0 & 186. & 356. & 421. & 528. & 738. \\
\hline 30 & 35.4 & 42.7 & 49.3 & 67.4 & 138. & 288. & 370. & 469. & 647. \\
\hline 40 & 30.9 & 35.2 & 41.8 & 55.4 & 110. & 206. & 273 & 327. & 481. \\
\hline 50 & 25.0 & 28.7 & 36.2 & 46.8 & 98.0 & 155. & 212. & 244. & 388. \\
\hline 60 & 23.0 & 27.0 & 31.9 & 39.3 & 78.0 & 127. & 165. & 195. & 276. \\
\hline 70 & 20.3 & 24.1 & 27.2 & 33.0 & 61.6 & 106. & 136. & 145. & 229. \\
\hline 75 & 20.0 & 22.4 & 26.0 & 30.0 & 53.0 & 102. & 129. & 135. & 183. \\
\hline 80 & 18.4 & 20.7 & 23.4 & 27.8 & 47.4 & 97.5 & 116. & 123. & 164. \\
\hline 90 & 14.7 & 16.8 & 19.1 & 25.8 & 39.0 & 68.6 & 87.2 & 102. & 115. \\
\hline 95 & 10.8 & 13.8 & 16.6 & 20.6 & 32.6 & 58.0 & 73.0 & 92.2 & 99.9 \\
\hline 98 & 6.91 & 12.7 & 15.3 & 19.8 & 30.8 & 53.7 & 66.9 & 77.5 & 94.5 \\
\hline 99 & 6.82 & 12.1 & 14.4 & 18.8 & 30.0 & 52.0 & 61.8 & 76.2 & 93.8 \\
\hline
\end{tabular}

\section{Mill Creek near Jefferson, Ohio}

Years analyzed $=33$

Climatic year range $=1942-74$

[APEE, annual percentage equaled or exceeded; $\mathrm{ft}^{3} / \mathrm{s}$, cubic foot per second; Sep, September; Nov, November]

\begin{tabular}{|c|c|c|c|c|c|c|c|c|c|}
\hline \multirow[b]{2}{*}{ APEE } & \multicolumn{9}{|c|}{ Sep-Nov daily mean streamflow $\left(\mathrm{ft}^{3} / \mathrm{s}\right)$ that was equaled or exceeded the indicated percentage of time at APEE level } \\
\hline & 95 & 90 & 80 & 70 & 50 & 30 & 20 & 10 & 5 \\
\hline 1 & 6.04 & 15.0 & 97.2 & 256. & 728. & 1100 & 1220. & 1950. & 2470 \\
\hline 2 & 4.16 & 8.19 & 48.6 & 172. & 549. & 951. & 1010. & 1100 & 1380 \\
\hline 5 & 0.27 & 2.52 & 18.7 & 47.6 & 183. & 397. & 570. & 667. & 877. \\
\hline 10 & 0.24 & 0.61 & 1.88 & 10.6 & 74.4 & 206. & 276. & 408. & 507. \\
\hline 20 & 0.00 & 0.00 & 0.10 & 2.32 & 41.0 & 70.0 & 140. & 261. & 282. \\
\hline 25 & 0.00 & 0.00 & 0.10 & 1.26 & 17.0 & 50.6 & 99.2 & 216 & 224. \\
\hline 30 & 0.00 & 0.00 & 0.00 & 0.16 & 8.74 & 36.3 & 81.8 & 152. & 171. \\
\hline 40 & 0.00 & 0.00 & 0.00 & 0.14 & 2.40 & 13.7 & 58.9 & 67.5 & 114. \\
\hline 50 & 0.00 & 0.00 & 0.00 & 0.10 & 0.75 & 6.34 & 29.0 & 36.8 & 52.6 \\
\hline 60 & 0.00 & 0.00 & 0.00 & 0.01 & 0.10 & 1.74 & 9.78 & 20.7 & 35.0 \\
\hline 70 & 0.00 & 0.00 & 0.00 & 0.01 & 0.10 & 0.40 & 3.29 & 13.4 & 25.9 \\
\hline 75 & 0.00 & 0.00 & 0.00 & 0.00 & 0.10 & 0.34 & 2.34 & 8.64 & 22.1 \\
\hline 80 & 0.00 & 0.00 & 0.00 & 0.00 & 0.10 & 0.10 & 1.29 & 5.98 & 16.3 \\
\hline 90 & 0.00 & 0.00 & 0.00 & 0.00 & 0.05 & 0.10 & 0.12 & 1.68 & 5.25 \\
\hline 95 & 0.00 & 0.00 & 0.00 & 0.00 & 0.05 & 0.10 & 0.10 & 1.14 & 3.38 \\
\hline 98 & 0.00 & 0.00 & 0.00 & 0.00 & 0.05 & 0.10 & 0.10 & 0.50 & 2.64 \\
\hline 99 & 0.00 & 0.00 & 0.00 & 0.00 & 0.05 & 0.10 & 0.10 & 0.50 & 2.32 \\
\hline
\end{tabular}






\section{敢}

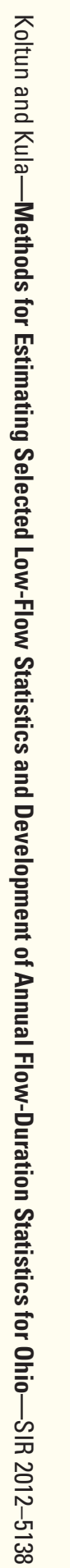

